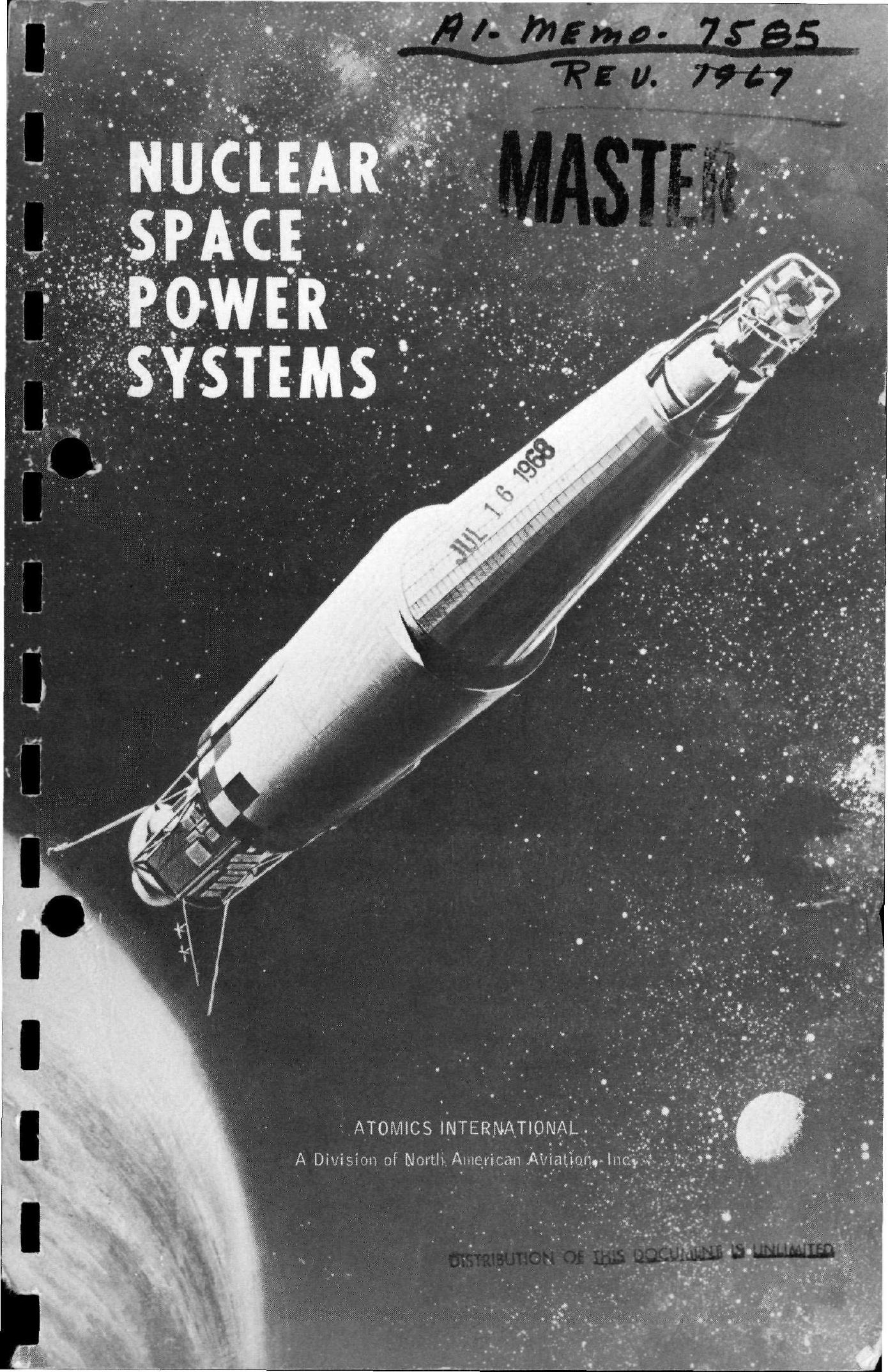




\section{DISCLAIMER}

This report was prepared as an account of work sponsored by an agency of the United States Government. Neither the United States Government nor any agency Thereof, nor any of their employees, makes any warranty, express or implied, or assumes any legal liability or responsibility for the accuracy, completeness, or usefulness of any information, apparatus, product, or process disclosed, or represents that its use would not infringe privately owned rights. Reference herein to any specific commercial product, process, or service by trade name, trademark, manufacturer, or otherwise does not necessarily constitute or imply its endorsement, recommendation, or favoring by the United States Government or any agency thereof. The views and opinions of authors expressed herein do not necessarily state or reflect those of the United States Government or any agency thereof. 


\section{DISCLAIMER}

Portions of this document may be illegible in electronic image products. Images are produced from the best available original document. 


\title{
NUCLEAR SPACE POWER SYSTEMS
}

\author{
H. M. DIECKAMP \\ Vice President, Engineering
}

\section{ACKNOWLEDGMENT}

The author acknowledges the valuable contributions of Mr's WV Botts and $V$. Keshishıan and the assistance of the Al staff

\section{ATOMICS INTERNATIONAL}

A Division of North American Aviation, Inc.

P. 0. Box 309

Canoga Park, California

ISSUED: SEPTEMBER 1967 
BLANK 


\section{CONTENTS}

Page

I. Nuclear Space Power Systems ........... 19

A. Introduction.................... 19

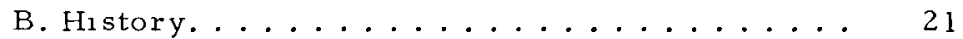

C. Development Status............. 27

a. Nuclear Heat Sources........... 27

b. Power Conversion ............. 27

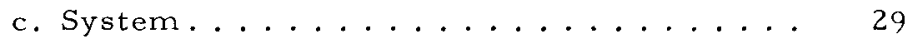

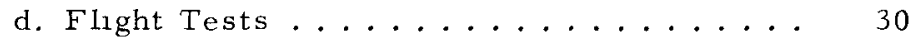

D. Operational Factors............ 31

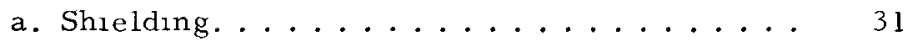

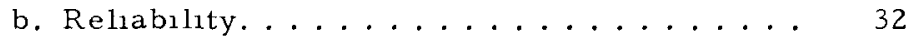

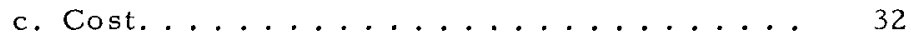

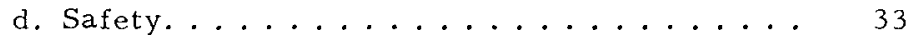

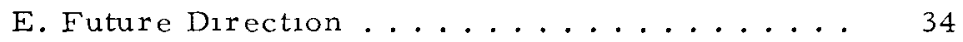

F. Competitive Position............. 37

G. Applications ................. 43

II. Reactor Space Power Systems ........... 45

1. The Reactor Heat Source............ 45

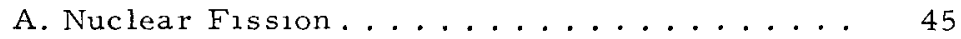

a. Fission Process.............. 45

b. Release of Neutrons............ 46

c. Fission Energy ............. 49

d. Energy Distribution ............... 51

e. Energy Equıvalents............ 52

B. The Chain Reaction............. 53

a. Conditions for Self-Sustaining....... 53

b. Neutron Balance............. 54

c. Multiplication Factor for Thermal

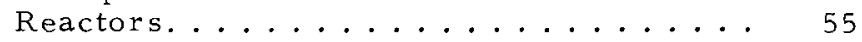

d. Leakage of Neutrons........... 59

e. Critical Size of Reactor.......... 60

f. Reactor Flux and Power Distribution..... 66 
Page

g. Reflector ................. 67

h. Reactor Kinetics ............. 69

1. Reactor Control.............. 71

C. Reactor Design .............. 72

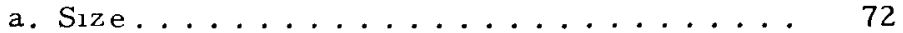

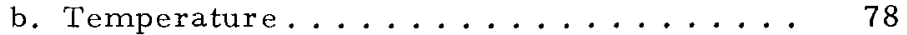

c. Control.................. 82

d. Power Density.............. 83

e. Energy Density .............. 90

f. Summary Example ............ 95

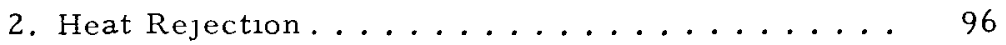

A. Temperature and Efficiency .......... 96

B Radiant Heat Transfer.............. 100

a. Heat Transfer Surfaces.......... 100

b. Analysis of Isothermal Heat Transfer

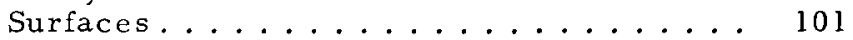

c. Analysis of Nonisothermal Heat Transfer

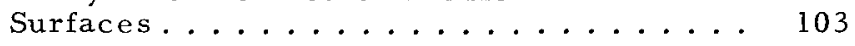

d. Analysis of Extended Surfaces ....... 105

e. Sink Temperature............. 110

f. Emissivity .......................... 111

C. Meteorold Protection ............. 112

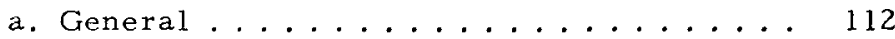

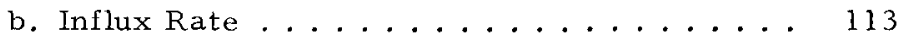

c. Mass and Density..................... 113

d. Flux vs Mass................. 114

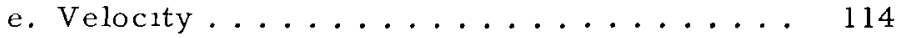

f. Penetration Theory............. 114

g. Meteorold Damage............. 118

h. Weight Penalty.................. 121

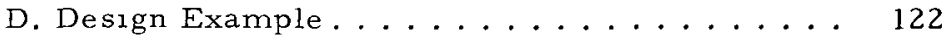




\section{CONTENTS}

Page

3. System Design. . . . . . . . . . . . 127

A. System Considerations . . . . . . . . . 127

B. Turboelectric Systems . . . . . . . . . . 128

a. Rankine Cycle Power Conversion . . . . . . 128

b. Brayton Cycle Power Conversion ...... 133

c. Reactor - Turboelectric Systems ...... 135

C. Thermoelectric Systems . . . . . . . . . . 142

a. Thermoelectric Power Conversion ..... 142

b. Thermoelectric Converters . . . . . . . . 149

c. Reactor Thermoelectric Systems ...... 151

D. Thermionic Systems . . . . . . . . . 153

a. Thermionic Power Conversion ....... 153

b. Reactor - Thermionic System. . . . . . 155

E. System Comparison Summary. . . . . . . . 165

4. Vehicle Integration. . . . . . . . . . 168

A. Introduction. . . . . . . . . . . . 168

B. Shıelding. . . . . . . . . . . . . . . . . . . 169

a. Source Strength . . . . . . . . . . . . . 169

b. Radiaton Effects. . . . . . . . . . 172

c. Radiation Attenuation . . . . . . . . . 173

d. Shield Geometry. . . . . . . . . . . . . 177

e. Sh1eldıng of Manned Systems ........ 181

C. Safety. . . . . . . . . . . . . . 183

a. Criteria ................. 183

b. Shipment and Integration Period ...... 186

c. Launch Pad Operations Period ........ 187

d. Launch-to-Orbit Period. . . . . . . . . . 187

e. Reentry Perrod ............... 188

D. Factory-to-Flight Sequence . . . . . . . 189

5. SNAP Systems ................... 193

A. SNAP Hydride Reactor . . . . . . . . . . 193

a. Concept ................. 193 


\section{CONTENTS}

Page

b. Development Experience ........... 193

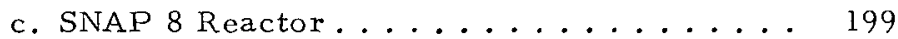

d. SNAP 10A Reactor...............203

e. SNAP Hydride Reactor Summary. ..... 209

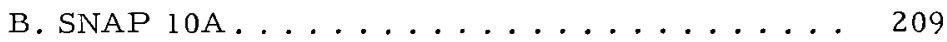

a. System Description. ........... 209

b. SNAPSHOT Flight Experience........ 225

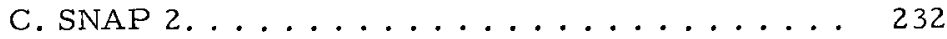

D. $\operatorname{SNAP} 8 \ldots \ldots \ldots \ldots . \ldots \ldots 243$

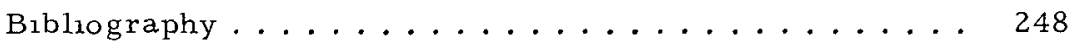

III. Radiorsotope Space Power .............. 255

1. Physics of Radiorsotopes............. 255

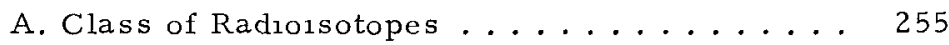

a. Beta Emitters ............... 255

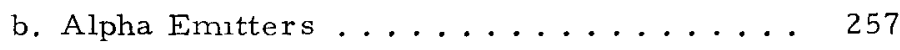

c. Gamma Emitters ............. 257

B. Formation of Radioisotopes ......... 257

a. Fission Products .............. 257

b. Reactor Produced Fuels ............ 258

C. Sources of Radiation................ 258

a. Alpha-Neutron Reaction........... 261

b. Importance of Isotopic Purity........ 262

c. Criticality of $\mathrm{Pu}^{238}$ and $\mathrm{Cm}^{244} \ldots \ldots 265$

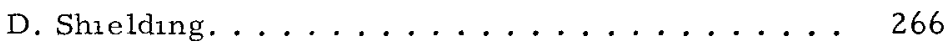

a. Radiation Sources for Shrelding Analysis.. 267

b. Shleld Analysis .............. 267

c. Shıeld Example ............ 275

E. Criteria for Radioisotope Selection ...... 275

a. Half-Life .................. 275

b. Power Density............... 276

c. Fuel Composition............ 276 


\section{CONTENTS}

Page

d. Physical Properties ........... 276

e. Shielding Requirement. ........ 276

f. Cost and Availability. .......... 276

g. Nuclear Safety Considerations ....... 277

F. Nuclear Safety............... 277

a. Fuel Capsule Fabrication......... 278

b. Transportation to Launch Site....... 278

c. Prelaunch Activities.......... 278

d. Flight Safety............... 278

2. Radiorsotope Capsule Design .......... 281

A. Thermal Environment ........... 283

1. Normal Operation.............. 283

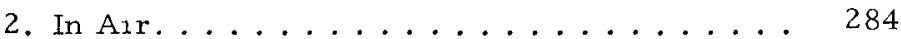

3. Earth Burral ............. 284

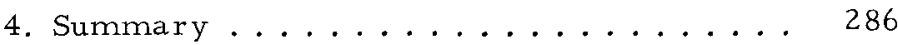

B. Pressure Buildup............. 286

C. Temperature and Pressure vs Trme ..... 288

D. Fuel Fraction ................. 289

E. Container Material Evaluation and Selection . . 292

1. Structural Container........... 292

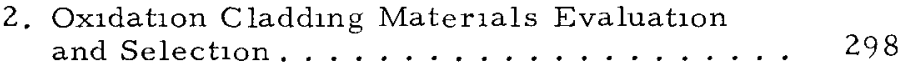

F. Capsule Structural Design .......... 301

G. Impact Analysis................ 301

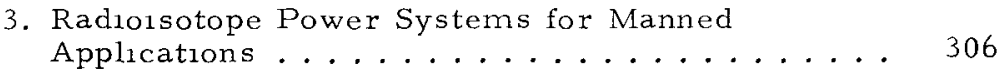

A. General Characteristics .......... 306

B. Isotope Fuel Selection........... 307

a. Short-Lived Isotopes........... 308

b. Long-Lived Isotopes ............. 309

c. Fuel Selection Summary ......... 310 


\section{CONTENTS}

Page

C. Heat Source Concepts ............ 311

a. Individual Capsule Reentry......... 312

b. Reentry of Lightweight Sheets....... 315

c. Reentry of Lenticular Lifting Body ..... 316

d. Controlled Reentry of Complete Heat

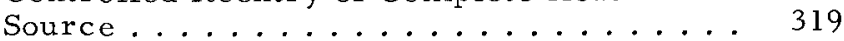

e. Effect of Reentry Mode on Heat Source

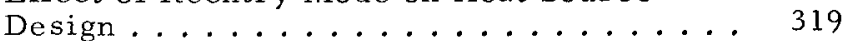

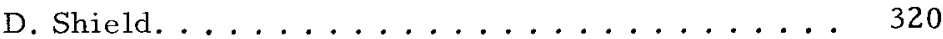

E. Thermoelectric Systems .......... 322

a. SiGe Thermoelectric Systems....... 323

b. PbTe Thermoelectric Systems ....... 331

c. Cascaded Thermoelectric System ...... 339

F. Mercury Rankine System ........... 341

a. State of Development........... 345

b. System Performance............ 346

c. Summary of Characteristics......... 347

G. Manned Brayton Systems ........... 352

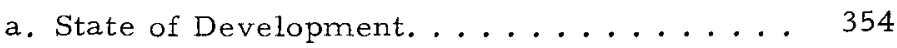

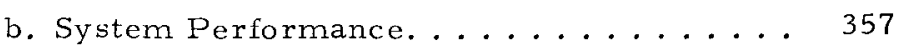

H. Radioisotope Systems Summary ....... 363

a. Thermoelectric Conversion ........ 364

b. Dynamic Power Conversion ........ 367

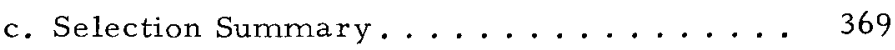

d. Mission Applications............. 369

4. Unmanned Radioisotope Systems . . . . . . . 373

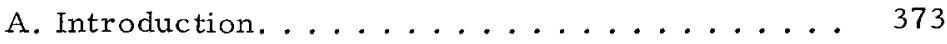

B. Radioisotope Thermoelectric Systems ..... 374

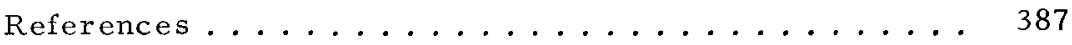




\section{TABLES}

Page

I-1. SNAP Reactor System Development Programs .. . 24

I-2. Continuing SNAP Reactor Programs ........ 25

I-3. SNAP Radioisotope Systems ........... 26

II-1. Average Number of Neutrons Liberated per

Thermal Neutron Absorbed in Fission ........ 46

II-2. Liberation of Heat Due to Fission . . . . . . . 52

II-3. Heat Liberated by $1 \mathrm{lb}$ of Fissionable Material . . 53

II-4. Thermal Neutron Cross Sections ......... 56

II-5. Buckling of Bare Reactors ........... 62

II-6. Moderator Properties ............. 64

II-7. Minimum Size of Bare Homogeneous

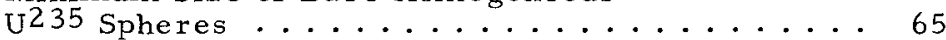

II-8. Flux Distribution in Bare Critical Reactors . . . . 66

II-9. Peak-to-Average Power for Bare Reactors .... 66

II-10. Physical Properties of Some Selected

Liquid Metals ................. 84

II-11. Fuel Properties................ 87

II- I2. Properties of Radiator Fin Materials ....... 109

II-13. Comparison of Meteoroid Penetration Models . . . 117

II-14. Meteoroid Armor Material Properties ...... 120

II-15. Typical Thermionic Converter Performance . . . 155

II-16. Radiation Attenuation Characteristics ...... 176

II-17. Properties of Lithium Hydride .......... 176

II-18. Radiation Tolerance of Man . . . . . . . . . 182

II-19. SNAP Hydride Reactors ............ 208

II-20. SNAP Reactor Operating Experience ....... 210

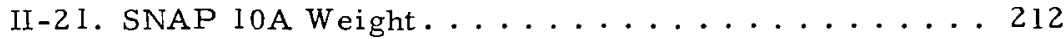

III-1. Characteristics of Radioisotopic Heat Sources . . 256

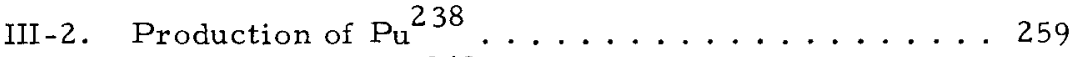

III-3. Production of $\mathrm{Cm}^{242} \ldots \ldots . \ldots 259$

III-4. Production of $\mathrm{Cm}^{244} \ldots \ldots . \ldots 260$

III-5. Decay Chain of $\mathrm{Pu}^{236} \ldots \ldots \ldots . \ldots 263$

III-6. Gamma Ray Source Spectrum . . . . . . . . . . 268 


\section{TABLES}

Page

III-7. Neutron Source Strength . . . . . . . . 267

III-8. Shield Requirement for a 5000-watt Source and $5 \mathrm{mrem} / \mathrm{hr}$ at 10 feet ........... 275

III-9. Isotope Fuel Characteristics .......... 311

III-10. Summary of Reentry Body Concepts . . . . . . 313

III-11. 2.I-kwe Isotope SiGe Systems . . . . . . . . . . . . 332

III-12. 2.1-kwe Isotope PbTe Systems . . . . . . . 338

III-13. Cascaded TE System Performance . . . . . . . 340

III-14. MRP Experience Summary . . . . . . . . . . 345

III-15. 5-kwe Net Module Design Point . . . . . . . 346

III-16. $\begin{aligned} 5 \text {-kwe Mercury Rankine Power System } \\ \text { Performance Characteristics ......... } 348\end{aligned}$

III-17. 5 -kwe Net Mercury Rankine System $\ldots \ldots \ldots \ldots . \ldots 349$

III-18. System Design Parameters . . . . . . . . 361

III-19. PBC System Weight Summary . . . . . . . 362

III-20. Radioisotope Systems Performance Summary. . . 365

III-2 1. Characteristics of SNAP 3B 7, 8, and SNAP 9A . . 377

III-22. SNAP 27 System Summary $\ldots \ldots \ldots \ldots \ldots 38$ 


\section{FIGURES}

Page

I-1. Power and Duration Regions for Various

Energy Sources ............... 20 20

I-2. Specific Weight vs Power Output for Nuclear

Power Systems ................... 36

I-3. Comparison of Weight of Solar Cell and

Nuclear APU's as a Function of Power ...... 38

I-4. Area Requirements for Solar Cell Arrays

and Nuclear Systems as a Function of Power ....440

I-5. Cost Comparison Between Nuclear and Solar

Cell Systems as a Function of Power ........441

I-6. Nuclear Space Applications . . . . . . . . . . . . 42

II-1. Neutron Yield from the Fissioning of $U^{235} \ldots 47$

II-2. Energy Distribution of Prompt Fission

Neutrons ................... 48

II-3. Time Dependence of Neutron Emission from

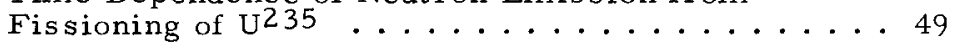

II-4. Neutron Economy for an Infinite Medium

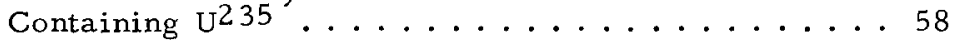

II-5. Neutron Economy for a Finite System

Containing $\mathrm{U}^{2} 35 \ldots \ldots 61$

II-6. Effect of a Reflector on Neutron Flux

Distribution and Effective Reactor Size . . . . . . 67

II-7. Effect of Reflector Thickness on Reactor

Core Size and Reflector Savings as a

Function of Thickness ............. 68

II-8. Volume of Bare 100\% Dense Spherical Reactors

with Critical Mass in the Range of 2.5 to $20 \mathrm{~kg}$

as a Function of Fermi Age ........... 74

II-9. Critical Mass of Bare 100\% Dense Homogeneous Spheres of U235 Moderated by Water and by Be

as a Function of Radius and Moderator to

U Atom Ratio .................... 75

II-10. Weight of Bare 100\% Dense Spherical Reactors as a Function of Moderato $r$ to Uranium Atom

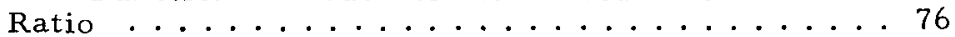

II-11. Hydrogen Density vs Temperature for Zirconium Hydride at 1 Atmosphere Dissociation Pressure and Water at the Critical Pressure ........ 77 


\section{FIGURES}

Page

II-12. Weight of Bare Spherical Fast Reactors

as a Function of Uranium Density ........ 78

II-13. System Lifetime vs Operating Temperatures .... 80

II-14. Number of Power Reactors in Operation or

Planned for Immediate Future as a Function

of Operating Temperature ............. 80

II-15. Weight of Bare 100\% Dense Spherical Reactors as a Function of Operating Temperature ......8 81

II-16. Reactor Power Density as a Function of Fuel Element Diameter and as a Function of Surface and Volumetric Heat Transfer Limits ... 88

II-17. Burnup Performance of Stainless Steel- $\mathrm{UO}_{2}$ Cermet Fuel Specimens ............. 92

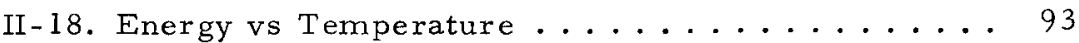

II-19. Reactor Volume Requirements as a Function of Rod Diameter, Criticality, Heat-Transfer Limit, and Burnup Limit ............. 94

II-20. Optimum Carnot Efficiency for Minimum Radiator Area as a Function of Conversion

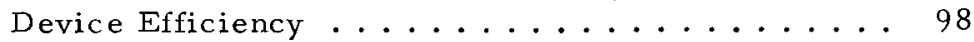

II-21. Radiator Area vs Carnot Efficiency ........ 99

II-22. Typical Space Radiator Surfaces .......... 100

II-23. Radiation Heat Flux for Isothermal Surfaces with Unit Emissivity .............. 102

II-24. Flat Plate Radiator ............... 103

II-25. Radiative Heat Rejection Correction for a Nonisothermal Surface ................ 105

II-26. Extended Fin Radiator ............. 106

II-27. Fin Effectiveness vs Dimensionless Fin

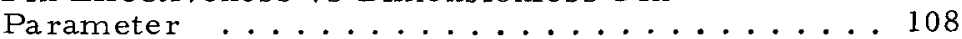

II-28. Relative Fin Weight vs Fin Effectiveness for Rectangular and Triangular Fins ........ 109

II-29. Thermal Conductivity Over Density Ratio for Radiator Fin Materials ............. 110

II-30. Emissivity of Some Typical Ceramic Materials vs Temperature ............ 112

II-31. Meteoroid Flux vs Mass ............. 115 


\section{FIGURES}

Page

II-32. Meteoroid Armor Thickness Requirements ....

II-33. Example Performance Penalty for Micrometeorite Armor ............ 122

II-34. Typical Radiator Geometry ........... 123

II-35. Tube and Fin Detail ............... 124

II-36. Radiator Werght vs Number of Tubes ....... 125

II-37. Radiator Weight vs Fin Effectiveness ........ 126

II-38. Weight Distribution for Nuclear Turboelectric Systems ................. 128

II-39. Rankıne Cycle Thermodynamic Diagram

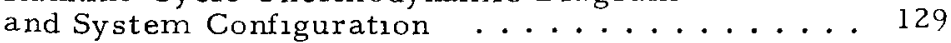

II-40. Vapor Pressure vs Temperature for Potentıal Rankıne Cycle Working Fluıds ....... 130

II-4I. Radiator Area per Kilowatt as a Function of Boiling Temperature for Rankine Cycle Workıng Fluıds.................. 132

II-42. Brayton Cycle Thermodynamic Diagram and System Configuration ............. 134

II-43. Radiator Area and Source Temperature Comparison for Brayton and Rankıne Cycles . . . 136

II-44. Rankine Cycle System Configuration ....... 137

II-45. Reactor Thermal-Power and SourceTemperature Requir ements as a Function of Electrical Power Output and Avaslable Radiator Area for Rankine Turboelectric

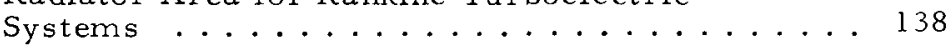

II-46. Reactor Plus Radiator Weight as a Function of Power for Various Reactor-Cycle

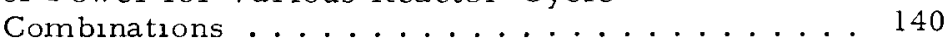

II-47. Tradeoff Between Reactor Weight and Radiator Weight as a Function of Operating Temperature ............143

II-48. Ideal Thermocouple . ............. 144

II-49. Ideal Thermoelectric Materials Efficiency .... 146

II-50. Radiator Area per Kilowatt as a Function of Hot Junction T emperature for Currently Available PbTe and Future Materials ........ 148 


\section{FIGURES}

Page

II-51. Reactor Thermal Power and Source

Temperature Requirements for Thermo-

electric Systems as a Function of Elec-

trical Power and Radiator Area ......... 150

II-52. Thermoelectric System Configuration ....... 152

II-53. Ideal Thermionic Converter ......... 154

II-54. Optimum Parameters for the Ideal

Thermionic Converter ............ 156

II-55. Survey of Thermionic Converter

Demonstrated Performance ......... 157

II-56. Thermionic System Configurations ........ 158

II-57. Thermionic Conversion Fuel Element . . . . . 160

II-58. Core Volume and Radiator Area as a

Function of Core Temperature for

a Thermionic Reactor ............. 162

II-59. Thermionic Reactor.............. 164

II-60. Space Reactor Power Systems ........... 166

II-6I. Approximate Radiation Source Strength

for Unshielded Space Reactors ......... 170

II-62. Survey of Radiation Damage Resistance of Electronic Components ........... 174

II-63. Degradation of Current Gain with Neutron

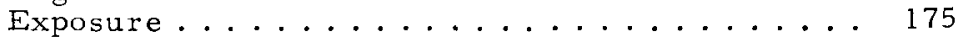

II-64. Variation in LiH Thickness with Separation

Distance for Given Dose Limits ......... 178

II-65. Space Reactor - Payload Shadow Shield

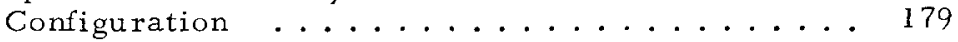

II-66. Example Shadow Shield Weight as a Function of Reactor Size and Power ........... 180

II-67. Shield Weight vs Separation Distance . . . . . 182

II-68. MORL Reactor/Shadow Shield Assembly . . . . . 184

II-69. Parametric Shielding Data for the Manrated SNAP 8 Reactor .............. 185

II-70. Factory-to-Flight Sequence .......... 190

II-71. SNAP 10A in the Final Phase of Launch

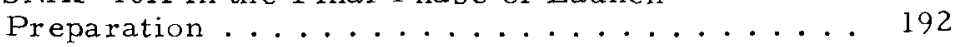

II-72. SNAP 2 Fuel Element .............. 194 


\section{FIGURES}

Page

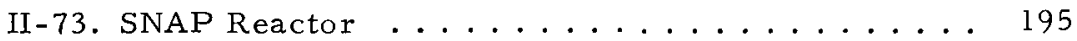

II-74. SNAP I0A/2 Experimental Reactor ....... 196

II-75. SNAP 2 Development Reactor . . . . . . . . . 197

II-76. Internal View of the SNAP 8 Core........ 198

II-77. SNAP 8 Experimental Reactor Test Assembly

Being Lowered into the Test Facility ....... 200

II-78. Current SNAP 8 Reactor Performance

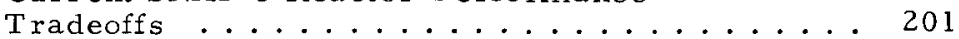

II-79. SNAP 8 Reactor and Shield ........... 202

II-80. SNAP 10A/2 Reactor ............. 204

II-81. Launch Shock and Vibration Environment

Qualification of the SNAP 10A Reactor ......2206

II-82. SNAP 10A FS-3 Converter Power Output . . . . . 207

II-83. SNAP 10 A System .............. 211

II-84. SNAP 10A Performance Characteristics . . . . . 212

II-85. Exploded View of SNAP 10A/2 Reactor

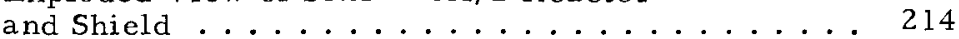

II-86. SNAP 10A Thermoelectric Powered

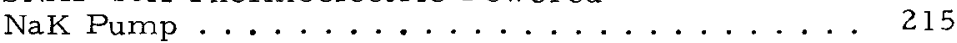

II-87. SNAP 10A Thermoelectric Converter Module . . . 216

II-88. SNAP 10A Thermoelectric Converter Module . . . 217

II-89. SNAP 10A Titanium Structure ......... 218

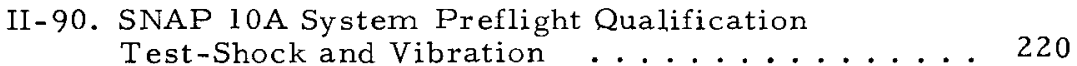

II-91. SNAP 10A Thermal Performance Test ...... 221

II-92. SNAP 10A Flight System ........... . 222

II-93. SNAP IOA NPU Integration with Atlas-Agena . . . 223

II-94. SNAP I0A System Electrical Simulator
Compatibility Test with an Agena Mockup . . . . 224

II-95. SNAP I0A Orbital Startup Sequence . . . . . 228

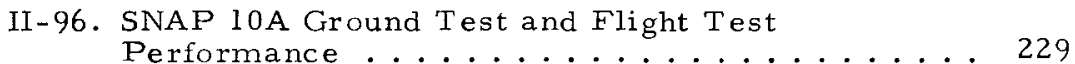

II-97. 5-kwe Mercury Rankine Schematic ....... 233

II-98. 5-kwe Mercury Rankine System . . . . . . . . 234 


\section{FIGURES}

Page

II-99. Combined Rotating Unit - V .......... 236

II-100. Combined Rotating Unit Hardware - CRU V . . 237

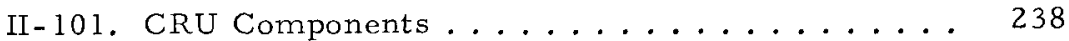

II-102. Developmental Mercury Rankine Radiator

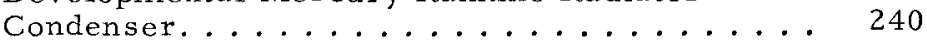

II-103. Mercury Rankine Performance Test System . . . 241

II-104. SNAP 2 Startup and Test........... 242

II-105. SNAP 8 Electric Generating System. . . . . . 244

II-106. SNAP 8 Turbine Assembly . . . . . . . . 246

II-107. SNAP 8 Power Conversion System - G. . . . 247

III-1. Unshielded Dose Rate as a Function of Time from $1.0 \mathrm{ppm}$ of $\mathrm{Pu}_{2} 36$ in $\mathrm{Pu}^{2} 38 \ldots 264$

III-2. Critical Mass of Unreflected $\mathrm{Cm}^{244}$ and

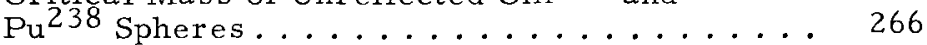

III-3. Effect of Self-Shielding on Gamma Ray Dose Rate for Capsules.............. 269

III-4. Parametric Shielding for $\mathrm{Co}^{60} \ldots . . \ldots 270$

III-5. Parametric Shielding for $\mathrm{Sr}^{90}\left(\mathrm{SrTiO}_{3}\right) \ldots 270$

III-6. Parametric Shielding for $\mathrm{Pm}^{147}$ with $0.25 \mathrm{ppm}$

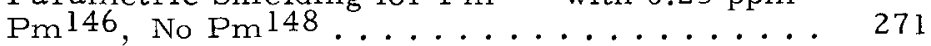

III-7. Parametric Shielding for Po $210 \ldots 271$

III-8. Parametric Shielding for $\mathrm{Pu}^{238} \ldots \ldots 272$

III-9. Typical Capsule.............. 280

III-10. Prototype Refractory Alloy Capsule........ 281

III-11. Capsule Temperature as a Function of Surface

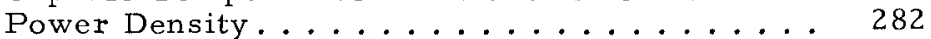

III-12. Normalized Temperature Distribution Around Infinitely Buried Capsule - Computer Results . . 285

III-13. Time Dependent Behavior of a Radiatively Cooled Reactor ............... 290

III-14. Temperature and Pressure Dependence on Fuel Fraction ................

III-15. Ultimate Tensile Strengths of Cb-752, $\mathrm{TZM}$, and $\mathrm{T}-222 \ldots \ldots \ldots \ldots$ 


\section{FIGURES}

Page

III-16. Larson-Miller Relationship for Stress

Rupture of TZM $(\mathrm{C}-20) \ldots \ldots 294$

III-17. Larson-Miller Relationship for Stress

Rupture of Tantalum Alloys (C-15) .......295

III-18. Rupture-Stress-to-Density Ratio for TZM

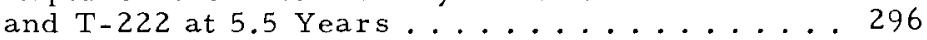

III-19. Air Oxidation of Platinum and Rhodium . . . . . 299

III-20. Specific Weight vs Vold-to-Fuel-Volume Ratio,

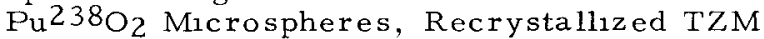

Cladding.................... 300

III-21. Specific Power vs Vold-to-Fuel-Volume Ratio for $\mathrm{Pu}^{2} 38 \mathrm{O}_{2} \mathrm{M} 1 \mathrm{crospheres,} \mathrm{TZM} \mathrm{Stress-}$

Relieved Cladding............... 302

III-22. Typical Test Specimen, Containment Capsule

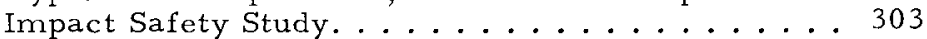

III-23. Velocity vs Proportion Ruptured, Sl Capsules . . . 304

III-24. L/D Ratıo vs Wall Thickness, Probit Analysıs .. 304

III-25. Refractory Metal Capsule Specimens........ 305

III-26. Reentry Clad Polonıum Isotope Capsule . . . . . 314

III-27, Lightwelght Sheet............. 316

III-28. Lenticular Liftıng Body, Concept 3 ....... 317

III-29. 45-kwt Reentry Package Heat Source, Pu 238

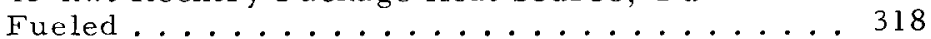

III-30. Po 210 Shield Weight vs Beginning-vs-Life

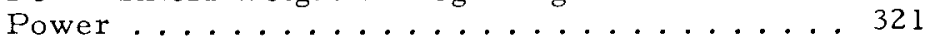

HI- 31. Po ${ }^{210}$ SiGe System Schematic.......... 323

III-32. SiGe Compact Converter Module......... 324

III-33. Po ${ }^{210} \mathrm{SiGe}$ System Performance vs Carnot

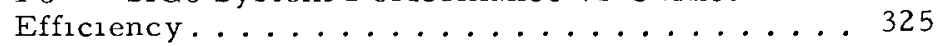

III-34. Pu ${ }^{238} S_{1} G e$ System Performance vs Carnot

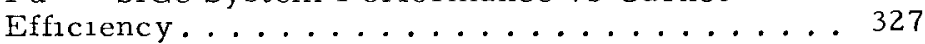

III-35. Po ${ }^{210} \mathrm{SiGe}$ System Weight vs Power....... 328

III-36. $\mathrm{Pu}^{238}$ SiGe System Weight vs Power....... 329

III-37. PbTe Converter Submodule .......... 333

III-38. Po $210 \mathrm{PbTe}$ System Performance vs Carnot

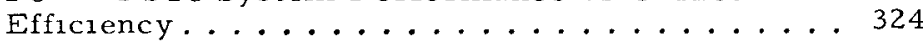




\section{FIGURES}

Page

III-39. $\mathrm{Pu}^{238} \mathrm{PbTe}$ System Performance vs Carnot

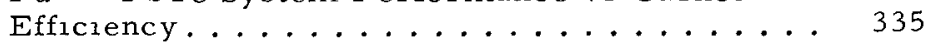

III-40. Po ${ }^{210}$ PbTe System Weight vs Power. ..... 336

III-41. $\mathrm{Pu}^{238} \mathrm{PbTe}$ System Welght vs Power. . . . . . 337

III-42. Cascaded System Schematıc.......... 339

III-43. Cascaded System Performance......... 340

III-44. Multiple PCS Mercury Rankine System with I

Isotope Heat Source ............. 342

III-45. Turboalternator Assembly - CRU-V ...... 344

III-46. Effect of PCS Redundancy on Total System

Reliabilıty, 20-kwe System ............. 350

III-47. Brayton Cycle System Schematic......... 353

III-48. Pu ${ }^{238}$ Brayton Cycle System ......... 354

III-49. PCS Weight as a Function of Power Rating . . . 356

III-50. Compressor Inlet Temperature vs Overall

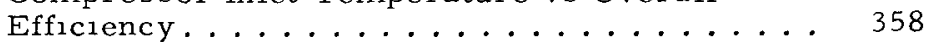

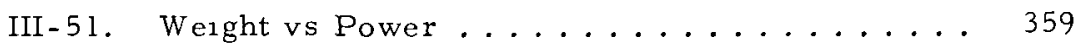

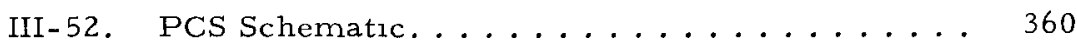

III-53. Power Conversion Selection and Avarlability . . 366

III-54. Radioisotope System Performance Comparison . 368

III-55. Manned Orbitıng Research Laboratory...... 370

III-56. Manned Orbitıng Laboratory.......... 372

III-57. Typical One-Loop Convection System...... 375

III-58. SNAP 3B Fuel Capsule Drawing ........ 376

III-59. Cross Section of SNAP 3B Generator...... 378

III-60. Exterior Drawing of the SNAP 9A Generator . . 379

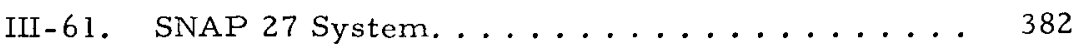




\section{CHAPTER I \\ NUCLEAR SPACE POWER SYSTEMS}

\section{A. INTRODUCTION}

The significant peaceful utilization of space will demand large quantities of reliable and long-lived power. The first 10 years of the space age have seen an ever-increasing number of satellites and space vehicles of growing complexity and sophistication. All spacecraft require electrical power for instrumentation, electronic data handling, guidance, and com-

- munication. To date, the electrical power has been supplied primarily by batteries, fuel cells, and solar cells. The power level has been of the order of a few watts up to a few hundred watts. The first nuclear power system in space was SNAP 3B, a 2.7-watt radioisotope unit fueled by $P \mathrm{u}^{238}$, which was launched in June 1961. The first reactor power system in space was SNAP 10A, a 500-watt unit, which was launched in April 1965. To date, five radioisotope units with power levels up to 25 watts and one 500-watt reactor unit have been used in the U.S. space program.

In the near future large booster systems will have the capability of placing large vehicles and payloads into space. The successful utilization of these large payloads will only be realized with the concurrent availability of lightweight, long-lived, high power, reliable, electrical generating systems. The avail ability of large boosters coupled with nuclear auxiliary power sources will allow the space program to progress beyond the current era of space exploration. The future era of space utilization will reap untold benefits to all of mankind through worldwide communications, weather forecasts, navigational aids, etc. As nuclear power sources grow in power output and reliability, the promising ion and plasma forms of electric propulsion will allow interplanetary exploration without the fantastic size and cost of pure chemical systems. 


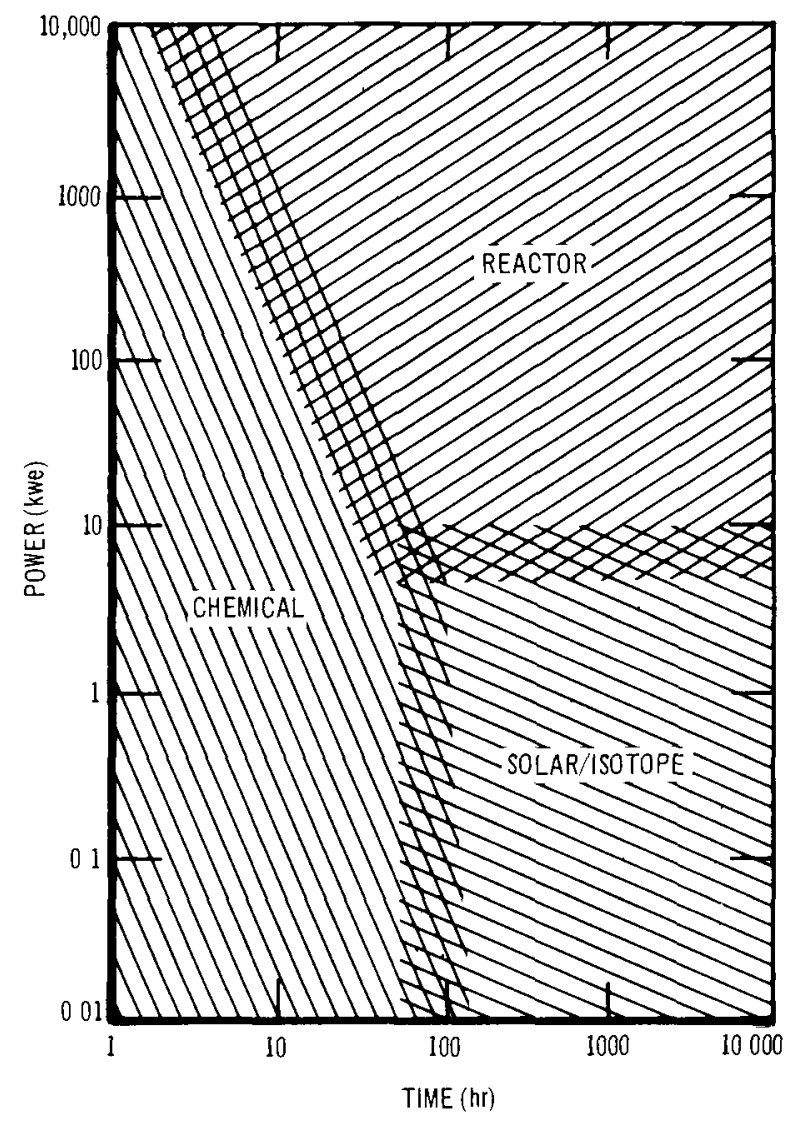

$7 \cdot 57 \cdot 192-1$

Figure I-1. Power and Duration Regions for Various

Energy Sources. (Chemical includes storage batteries, fuel cells, and cryogenic $\mathrm{H}_{2}-\mathrm{O}_{2}$ engine. Solar includes both photovoltaic as well as solar mirror systems.) 
For significant electrical power loads, which are required for missions in excess of several days, only solar and nuclear systems can be considered. Batteries and other chemical systems are ruled out on the basis of the large weights associated with these systems. The applicable range of various power systems is illustrated in Figure I- 1. At power levels of the order of a few kilowatts, the various solar and nuclear radioisotope or reactor power systems offer their own specific advantages and disadvantages. The selection of a particular solar or nuclear power system can only be accomplished in the context of specific mission requirements, payload considerations, reliability, costs, etc. In this evaluation, the nuclear system offers definite advantages of ruggedness, high power per unit area, no collector deployment, or orientation, continuous power, minimum power storage requirements, etc. In many cases, the added power availability of a nuclear system should offer significant operational flexibility and improved reliability through application of more conventional circuitry and instrumentation and through active redundancy. As the power requirements are increased to the order of tens of kilowatts, the nuclear reactor systems have an increasingly favorable weight, size, and cost advantage over any of the presently envisioned solar power systems. For power levels in the hundreds of kilowatts, and above, a nuclear reactor system is the only one which appears at all feasible. In recognition of this superiority, the U.S. Atomic Energy Commission initiated the SNAP (Systems for Nuclear Auxiliary Power) program for the development and demonstration of a spectrum of units to fill future needs.

\section{B. HISTORY}

The difficulty of supplying power for the useful application of space and the unique energy density advantage of nuclear sources were recognized in the early 1950s. Atomics International, a division of North American Aviation, Inc., conducted studies on the relative merits of radioisotope and fission heat 
sources in 1953. It was immediately recognized that significant power in space would require a reactor heat source. System conceptual design studies identified the mutually dependent reactor heat source, power conversion requirements, and concept selection criteria. By 1955, these studies resulted in the identification of a reactor and power conversion concept for the 0.5 to 10 -kwe power range. The hydride reactor was chosen on the basis of minimum reactor weight, applicable operating temperature, and a reasonable extrapolation of reactor and materials state of the art. A mercury rankine cycle power conversion subsystem was selected on the basis of minimum radiative heat rejection area requirement within the temperature constraint implied by reactor heat source development cost and schedule. Concurrent studies by the Martin Company evaluated radioisotope heat sources and power conversion concepts and culminated in the definition of a 500-watt system, fueled with cesium-144 and employing a mercury rankine cycle for power conversion. These studies formed the basis for the establishment of the Systems for Nuclear Auxiliary Power (SNAP) program by the Atomic Energy Commission in 1956.

In the SNAP reactor system program, specific objectives for a 3-kwe mercury rankine system, SNAP 2, were established in 1956 and system development was initiated. Reactor concept verification by means of a critical assembly was achieved concurrent with the start of the space age in October 1957. Interest in the thermoelectric direct power conversion for space systems was initiated in 1958. The specific objectives of the SNAP 10A 500-watt system were established in December 1960. This system progressed through the complete developmental cycle from concept identification to flight demonstration in orbit in the spring of 1965.

SNAP 8, a joint AEC/NASA program to develop a 35-kwe system, based on a hydride reactor and mercury rankine cycle, was initiated in May 1960. The development of a high-powered, 300-kwe, fast reactor potassium rankine cycle system, SNAP 50, 
was initiated in 1962 but reduced to a reactor technology program in 1965. Currently SNAP 8 is the only active reactor power system program. Reactor technology programs have been established to provide the basis for an advanced hydride reactor, a high temperature gas cooled reactor (710), a high temperature liquid metal cooled reactor, and an in-core thermionic reactor. The SNAP Reactor System programs are summarized in Table I- 1 , and the continuing reactor technology programs are listed in Table I-2.

In the SNAP radioisotope system program, specific objectives were established in 1956 for SNAP 1, a 500-watt cesium-144 powered unit employing a mercury rankine cycle for power conversion. Subsequently, the power objectıve was lowered to 125 watts and the power conversion was changed to PbTe thermoelectrics. The SNAP 1A program was dropped in 1960 in favor of reactor heat sources for the greater than 250-watt power range. The SNAP 3 system using $\mathrm{Po}^{210}$ and $\mathrm{PbTe}$ was initiated in 1959, and achieved the first demonstration of an integral nuclear space power unit in 1960. Modified SNAP 3A units fueled with $P u^{238}$ were flown in June and November of 1961 . The larger 25-watt SNAP 9A development started in 1961, and three units were flown in navigation satellites in the 1963-64 time period. In 1967, the program has progressed to the point of having integrated two 25-watt SNAP 19 units into the Nimbus weather satellite for subsequent flight, delivered the first 50watt SNAP 27 unit designed for lunar surface application, initiated development of the 400 to 500-watt SNAP 29 unit, and initiated development of a large radioisotope heat source for use with a Brayton cycle to provide 5 to $10 \mathrm{kwe}$ for manned spacecraft. The SNAP radiolsotope systems, past and present, are summarized in Table I- 3 . 
TAB LE I- 1

SNAP REACTOR SYSTEM DEVELOPMENT PROGRAMS

\begin{tabular}{|c|c|c|c|c|}
\hline & SNAP 10A & SNAP 2 & NASA SNAP 8 & SPUR SNAP 50 \\
\hline POWER (kwe) & 0.5 & 5 & 35 TO 50 & 350 \\
\hline REACTOR POWER (kwt) & 30 & 55 & 600 & 2500 \\
\hline EFFICIENCY $(\%)$ & 1.6 & 9 & 8 & 14 \\
\hline REACTOR OUTLET TEMPERATURE ( $\left.{ }^{\circ} \mathrm{F}\right)$ & 1000 & 1200 & 1300 & $\sim 2000$ \\
\hline REACTOR & $\mathrm{U}-\mathrm{ZrH}_{\mathrm{x}}$ THERMAL & $\mathrm{U}-\mathrm{ZrH}_{\mathrm{x}}$ THERMAL & $\mathrm{U}-\mathrm{ZrH}_{\mathrm{x}}$ THERMAL & UC FAST \\
\hline PRIMARY COOLANT & NaK -78 & $\mathrm{NaK}-78$ & NaK-78 & LITHIUM \\
\hline POWER CONVERSION & Ge-Si THERMOELECTRIC & Hg RANKINE & Hg RANKINE & K RANKINE \\
\hline BOILING TEMPERATURE $\left({ }^{\circ} \mathrm{F}\right)$ & - & 930 & 1070 & - \\
\hline TURBINE INLET TEMPERATURE $\left({ }^{\circ} \mathrm{F}\right)$ & - & 1150 & 1250 & 1950 \\
\hline CONDENSING TEMPERATURE $\left({ }^{\circ} \mathrm{F}\right)$ & - & 600 & 700 & 1300 T0 1400 \\
\hline HOT JUNCTION TEMPERATURE (OF) & 930 & - & - & - \\
\hline COLD JUNCTION TEMPERATURE $\left({ }^{\circ} \mathrm{F}\right)$ & 615 & - & - & - \\
\hline RADIATOR TEMPERATURE ( $\left.{ }^{\circ} F\right)$ & 615 & 600 & 580 & 1300 T0 1400 \\
\hline RADIATOR AREA $\left(\mathrm{ft}^{2}\right)$ & 62.5 & 120 & 1800 & 700 \\
\hline$\left(\mathrm{ft}^{2} / \mathrm{kwe}\right)$ & 125 & 40 & 45 & 2 \\
\hline SYSTEM UNSHIELDED WEIGHT ( $b$ ) & 650 & 1200 & 10,000 & 6000 (EST) \\
\hline$(\mathrm{l} b / \mathrm{kwe})$ & 1300 & 240 & 300 & 10 TO 20 \\
\hline AVAILABLE & 1965 & & 1972 & \\
\hline DEVELOPMENT AGENCY & AEC & $A E C$ & AEC/NASA & $\mathrm{AEC} / \mathrm{AF}$ \\
\hline FLIGHT TEST AGENCY & $A E C(A F)$ & $\star \star$ & * & * \\
\hline SYSTEM CONTRACTOR & ATOMICS INTERNATIONAL & ATOMICS INTERNATIONAL & AEROJET GENERAL & PRATT AND WHITNEY \\
\hline POWER CONVERSION CONTRACTOR & RADIO CORP OF AMERICA & THOMPSON RAMO WOOLDRIDGE & AEROJET GENERAL & AIRESEARCH \\
\hline REACTOR CONTRACTOR & ATOMICS INTERNATIONAL & ATOMICS INTERNATIONAL & ATOMICS INTERNATIONAL & PRATT AND WHITNEY \\
\hline FLIGHT TEST CONTRACTOR & LOCKHEED & & & \\
\hline STATUS & COMPLETE & CANCELLED & CONTINUING & CANCELLED \\
\hline
\end{tabular}

*FLIGHT TEST PLANS CURRENTLY UNDEFINED 
TAB LE I-2

CONTINUING SNAP REAC TOR PROGRAMS

\begin{tabular}{|c|c|c|c|c|c|c|c|}
\hline REACTOR TYPE & $\begin{array}{c}\text { PROGRAM } \\
\text { DESIGNATION }\end{array}$ & $\begin{array}{c}\text { ELECTRICAL } \\
\text { POWER } \\
\text { RANGE }\end{array}$ & $\begin{array}{c}\text { APPLICABLE } \\
\text { POWER } \\
\text { CONVERSION }\end{array}$ & $\begin{array}{l}\text { REACTOR } \\
\text { OUTLET } \\
\text { TEMP }\end{array}$ & $\begin{array}{c}\text { FUEL } \\
\text { MATERIAL }\end{array}$ & $\begin{array}{l}\text { FUEL CLAD } \\
\text { MATERIAL }\end{array}$ & COOLANT \\
\hline \multirow{2}{*}{$\begin{array}{l}\text { ZIRCONIUM } \\
\text { HYDRIDE } \\
\text { THERMAL }\end{array}$} & SNAP 8 & 50 Kwe & Hg RANKINE & $1300^{\circ} \mathrm{F}$ & $(U-Z r) H_{x}$ & HASTELLOY N & $\mathrm{NaK}-78$ \\
\hline & $\begin{array}{l}\text { REACTOR } \\
\text { IMPROVEMENT }\end{array}$ & 1-100 Kwe & $\begin{array}{l}\text { PbTe TE } \\
\text { SiGe TE } \\
\text { ORGANIC RAN- } \\
\text { KINE } \\
\text { Hg RANKINE } \\
\text { BRAYTON }\end{array}$ & $1300^{\circ} \mathrm{F}$ & $(\mathrm{U}-\mathrm{Zr}) \mathrm{H}_{\mathrm{x}}$ & HASTELLOY N & $\mathrm{NaK}-78$ \\
\hline \multirow{3}{*}{$\begin{array}{l}\text { ADVANCED } \\
\text { HIGH } \\
\text { TEMPERATURE } \\
\text { FAST }\end{array}$} & $\begin{array}{l}\text { LIQUID } \\
\text { METAL } \\
\text { COOLED }\end{array}$ & $0.3-10 \mathrm{Mwe}$ & $\begin{array}{l}\text { POTASSIUM } \\
\text { RANKINE } \\
\text { BRAYTON }\end{array}$ & $>2000^{\circ} \mathrm{F}$ & UN & TUNGSTEN & LITHIUM \\
\hline & THERMIONIC & $0.3-10 \mathrm{Mwe}$ & THERMIONIC & $3-4000^{\circ} \mathrm{F}$ & OPEN & TUNGSTEN & $\begin{array}{l}\text { SODIUM OR } \\
\text { LITHIUM }\end{array}$ \\
\hline & $\begin{array}{l}\text { GAS } \\
\text { COOLED } \\
710\end{array}$ & $\sim 200$ Kwe & BRAYTON & $>2000^{\circ} \mathrm{F}$ & $\begin{array}{l}\mathrm{W}-\mathrm{UO}_{2} \\
\text { CERMET }\end{array}$ & $\begin{array}{l}\text { REFRACTORY } \\
\text { ALLOY }\end{array}$ & $\begin{array}{l}\text { INERT } \\
\text { GAS }\end{array}$ \\
\hline
\end{tabular}


TAB LE I- 3

SNAP RADIOISOTOPE SYSTEMS

\begin{tabular}{|c|c|c|c|c|c|c|c|}
\hline DESIGNATION & APPLICATION & CONTRACTOR & $\begin{array}{l}\text { OUTPUT } \\
\text { (watts) }\end{array}$ & $\underset{(y r)}{D}$ & $\begin{array}{l}\text { WEIGHT } \\
\text { (Ib) }\end{array}$ & $\begin{array}{c}\text { FUEL } \\
\text { (ISOTOPE AND QUANTITY) }\end{array}$ & REMARKS \\
\hline SNAP-3A & $\begin{array}{l}\text { TRANSIT 4A } \\
\text { NAVIGATION SATELLITE }\end{array}$ & MARTIN & 2.7 & 5 & 5 & $\mathrm{Pu}^{238}, 95$ grams & $\begin{array}{l}\text { LAUNCHED JUNE } 1961 \text { OPERAT- } \\
\text { ING AT AN UNDETERMINED LOWER } \\
\text { POWER LEVEL }\end{array}$ \\
\hline \multirow[t]{2}{*}{ SNAP-3A } & $\begin{array}{l}\text { TRANSIT 4B } \\
\text { NAVFGATION SATELLITE }\end{array}$ & MARTIN & 27 & 5 & 5 & $\mathrm{Pu}^{238}, 95$ grams & $\begin{array}{l}\text { LAUNCHED NOV } 1961 \text { SHUTDOWN } \\
\text { BY POWER SYSTEM FAILURE IN } \\
\text { JUNE } 1962\end{array}$ \\
\hline & NAVIGATION SATELLITE & MARTIN & 25 & 5 & 27 & $\mathrm{Pu}^{238},-1$ kılogram & $\begin{array}{l}\text { LAUNCHED SEPT } 1963 \text { OPERAT- } \\
\text { ING AT AN UNDISCLOSED LOWER } \\
\text { POWER LEVEL }\end{array}$ \\
\hline SNAP-9A & NAVIGATION SATELLITE & MARTIN & 25 & 5 & 27 & $\mathrm{Pu}^{238}, \sim 1$ ktlogram & $\begin{array}{l}\text { LAUNCHED DEC 1963. OPERAT- } \\
\text { ING AT AN UNDISCLOSED LOWER } \\
\text { POWER LEVEL }\end{array}$ \\
\hline SNAP-9A & NAVIGATION SATELLITE & MARTIN & 25 & 5 & 27 & $\mathrm{Pu}^{238}, \sim 1$ kılogram & $\begin{array}{l}\text { LAUNCHED APR 1964. BURNED } \\
\text { UP ON REENTRY }\end{array}$ \\
\hline SNAP-11 & LUNAR SURFACE USE & MARTIN & 25 & 0.3 & 30 & $\mathrm{Cm}^{242}$ & $\begin{array}{l}\text { FUELED GENERATOR SCHEDULED } \\
\text { TO BE TESTED AT OAK RIDGE } \\
\text { NATIONAL LABORATORY }\end{array}$ \\
\hline SNAP-I7A & DEVELOPMENTAL & MARTIN & 30 & 3-5 & 30 & $\mathrm{Sr}^{90}$ & $\begin{array}{l}\text { WORK NOW LIMITED TO TE MODULE } \\
\text { TESTING, WITH THE RESULTS TO BE } \\
\text { APPLIED TO FUTURE SNAP GENER- } \\
\text { ATOR PROJECTS }\end{array}$ \\
\hline SNAP-17B & DEVELOPMENTAL & $\begin{array}{l}\text { GENERAL } \\
\text { ELECTRIC }\end{array}$ & 30 & $3-5$ & 30 & $\mathrm{Sr}^{90}$ & \\
\hline SNAP-19 & $\begin{array}{l}\text { NIMBUS-B WEATHER } \\
\text { SATELLITE }\end{array}$ & MARTIN & 30 & 5 & 30 & $\mathrm{Pu}^{238}$ & $\begin{array}{l}\text { SCHEDULED FOR FLIGHT IN LATE } \\
1967\end{array}$ \\
\hline SNAP-27 & $\begin{array}{l}\text { APOLLO LUNAR SURFACE } \\
\text { EXPERIMENT PKG(ALSEP) }\end{array}$ & $\begin{array}{l}\text { GENERAL } \\
\text { ELECTRIC }\end{array}$ & 50 & 1 & 40 & $\mathrm{Pu}^{238}$ & $\begin{array}{l}\text { UNDER DEVELOPMENT, WITH FIRST } \\
\text { FUELED UNITS TO BE DELIVERED IN } \\
\text { APRIL AND FLIGHT HARDWARE TO } \\
\text { FOLLOW IN JULY } 1967\end{array}$ \\
\hline SNAP-29 & SPACE APPLICATION & MARTIN & 400 & 0.25 & 400 & $\mathrm{Pu}^{210}$ & UNDER DEVELOPMENT \\
\hline LRHS & MANNED SPACE MISSIONS & Al & 5-10 kwe & 7 & $-25 \mathrm{lb} / \mathrm{kwt}$ & $\mathrm{Pu}^{238}$ & $\begin{array}{l}\text { HEAT SOURCE TO BE USEO WITH } \\
\text { BRAYTON CYCLE POWER CON- } \\
\text { VERSION }\end{array}$ \\
\hline
\end{tabular}




\section{DEVELOPMENT STATUS \\ a. Nuclear Heat Sources}

The radioisotope heat sources to date have been low temperature, 1000 to $1200^{\circ} \mathrm{F}$, single capsules coupled to the thermoelectric power conversion by conduction and radiation. SNAP 19 and 27 are typical of these sources, which are constructed of conventional super-alloy materials. Work has been initiated on a larger system, SNAP 29, which requires an assembly of capsules with radiative coupling to the converter. The Large Radioisotope Heat Source (LRHS) program is developing high-temperature, $2000^{\circ} \mathrm{F}$, refractory metal capsules for use in a 25 to 50 thermal kilowatt heat source which will probably require a liquid metal heat transfer loop to couple the heat source to the intended Brayton cycle power conversion subsystem.

The SNAP hydride reactors used in SNAP $10 A$ and SNAP 8 have been tested extensively. To date five reactors have operated about 35,000 hours, including a 10,000-hour uninterrupted SNAP IOA ground test and a SNAP $10 \mathrm{~A}$ test in space. The SNAP 8 reactor has operated for 1 year at $1300^{\circ} \mathrm{F}$ in the range of 400 to $600 \mathrm{kwt}$ and produced the integral energy requirement of the SNAP 8 system. No reactor test has been terminated by a malfunction. A second generation SNAP 8 reactor is scheduled to begin operation in the spring of 1968 .

The advanced reactor technology programs, 710 , the liquid cooled reactor, and the thermionic reactor, have not progressed to the point of integral reactor tests. These programs are concentrating on high temperature fuel and structural materials selection and evaluation.

\section{b. Power Conversion}

Lead telluride thermoelectric conversion has been used exclusively in the radioisotope systems to date. Typically the $\mathrm{PbTe}$ is operated below about $1100^{\circ} \mathrm{F}$ hot junction temperature and achieves about $5 \%$ efficiency. In the low power radioisotope 
systems, radiator area is purposefully increased to obtain improved efficiency and fuel savings.

The SNAP I0Asystem employed silicon-germanium alloy materials for thermoelectric direct power conversion. The lower figure of merit, $s^{2}$ ipk of the Si-Ge alloys, was accepted in order to achieve better fabricability and higher temperature capability than the more familiar $\mathrm{Pb}-\mathrm{Te}$. The SNAP 10A converter operated at a peak temperature of $1000^{\circ} \mathrm{F}$ with an eff 1 crency of about $1.5 \%$ and produced 9 watts/sq $\mathrm{ft}$ of radiator. The SNAP I0A-type direct radiating SiGe converter has been uprated to $1300^{\circ} \mathrm{F}$ operation and tested to an equivalent 5-year life. The efficiency is improved to 3 to $4 \%$, depending upon radiator area, and the power density is 15 to 20 watts $/ \mathrm{ft}^{2}$.

Compact thermoelectric converters which operate between a liquid metal hot and cold loop are under development for use with either radioisotope or reactor heat sources.

The Hg Rankıne cycle turbomachinery development for SNAP 2 has overcome the structural and thermal distortion problems that were limiting the reliability of the Hg lubricated bearıngs. Recent rotatıng machinery endurance accomplishments on SNAP 2 (30,000 machine-hr) have demonstrated the complete engineering feasibility of a hermetic machine with working fluid lubrication. The SNAP 2 rotating machine operates at a turbine inlet temperature of $1150^{\circ} \mathrm{F}$ and achieves an overall machine efficiency of $40 \%$ of the ideal cycle efficiency which results in about 40 watts/sq ft of radiator area.

In a 1962 redirection of the SNAP 8 program, the advantages of the SNAP 2 concept were abandoned. The NASA feels that the redirection to utılize rotating shaft seals and organic lubricants will allow a separation of development variables and will provide a closer relationship to existing technology for the SNAP 8 rotating machinery. In comparison to SNAP 2, which employed direct condensation of the $\mathrm{Hg}$ in a combination condenser-radiator, SNAP 8 now includes a compact condenser with a liquid metal 
heat transfer loop coupling the condenser to the radiator. In addition, SNAP 8 includes an organic bearing lubricant, coolant, and radiator loop. The SNAP 8 turbomachinery development will reveal the practicability of attempting to apply conventional conversion machinery technology to space power.

Development of a Brayton cycle power conversion system in the 5 to $10 \mathrm{kwe}$ region has been initiated by NASA. The machinery is intended for use with the LRHS to provide power for extended manned space missions. The engineering feasibility of the requisite high efficiency turbine and compressor and of the gas bearings have been demonstrated at the component level. Integral machine testing is programmed.

Small organic rankine cycle machines have been tested and show promise for use with isotope heat sources. The organic cycle is not as efficient as the Brayton cycle but it does not require a high temperature heat source and it offers a backup to the potentially difficult Brayton cycle gas bearing requirement.

Work is continuing on components for an advanced potassium rankine system, including the turbine, bearings, and boiler. A complete turbogenerator remains to be designed.

There is growing interest in thermionic conversion which requires power at a source temperature of about $3500^{\circ} \mathrm{F}$. Considerable test data has been accumulated on diodes out-of-pile and in-pile, including series connected arrays. About 80,000 hours of diode testing have been achieved to date. The fuel, cathode material, and the required insulators all constitute formidable materials development problems to satisfy the required temperature and radiation environmental conditions. The detailed method of integrating a reliable converter into a reactor core remains to be developed.

\section{c. System}

The isotope systems to date have all been small integral units of minimal complexity and only minor spacecraft integration 
problems. As the isotope systems grow larger, SNAP 29 and LRHS, the added complexity of liquid metal heat transfer loops and extended radiator surfaces will impose more critical system design and vehicle integration requirements and constraints.

The solution of the detailed system design and vehicle integration for the 500-watt, $1000-1 b$, SNAP $10 \mathrm{~A}$ reactor system and the necessary ground qualification, factory-to-flight sequence, and space testing have been demonstrated. The SNAP 10A program which successfully completed the entire cycle from definıtion to flight provides ample confidence that reactor thermoelectrıc systems can meet the requirements of a space power supply.

The SNAP 2 system development proceeded through electrically heated mockup system testing, system structural tests, and a successful series of automatic startup tests in a configuration designed to approximate zero gravity conditions on the ground. The SNAP 2 experience demonstrated that the boiling and condensing two-phase flow conditions inherent in a rankine cycle can be solved by straightforward engineering and ground testing.

The SNAP 8 program is still proceeding with independent reactor and power conversion subsystems testing. The integration and ground testing of a complete system is in the planning stage.

System development efforts do not now exist for a large potassium rankine or a thermionic system.

d. Flight Tests

A total of four small radioisotope generators at power levels up to 25 watts have been successfully flown. Two SNAP 19A generators are scheduled for flight in late 1967 and SNAP 27 is scheduled to accompany the Apollo lunar landing. No specific flight test plans have been established for the next generation of larger radioisotope systems. 
SNAP 10A was operated successfully in space in the spring of 1965 and demonstrated the first reactor power unit in space. The space test was prematurely terminated after 43 days by a spacecraft electrical malfunction not associated with the SNAP 10A unit. At this time no flight test plans exist for SNAP 8 or any of the advanced reactor power system concepts.

\section{OPERATIONAL FACTORS}

a. Shrelding

The shield weight is a stronger function of the mission and allowable integration configuration than it is of the power unit. In the case of payloads comprised of semiconductor devices, dose levels below $10^{11} \mathrm{nvt}$ and $10^{6} \mathrm{r}$ probably require very minor restrictions on component selection. Payload hardening for $10^{12} \mathrm{nvt}$ and $10^{7} \mathrm{r}$ can be readily accommodated if properly considered from the outset of payload design. These levels require shields with reactor systems and no shield for alphaemitting radioisotope sources. However, the scientific missions of interest to small radioisotope systems often impose severe radiation restrictions to avoid interference with spacecraft sensors.

The unmanned application shield weight for simple conical shadow shield geometry can be in the region of 200 to $500 \mathrm{lb}$ for SNAP 10A, 2, and 8. In the case of manner applications, the reactor shield weight varies from 3000 to $7000 \mathrm{lb}$ for a simple conical shadow shield configuration of a small (10 ft diameter) space station to 15,000 to $20,000 \mathrm{lb}$ for a large (150 ft diameter) toroidal station. The low shielding requirement of alphaemitting radioisotopes poses only a minor penalty in manned systems. In fact, it permits the flexible integration of kilowatt size systems into existing space hardware which is a major advantage. 


\section{b. Reliability}

The high-energy density advantage of nuclear heat source directly implies that long life is a necessary requirement to achleve the full advantage of nuclear power systems. The only unıque, self-ımposed, environment that could influence reliability is radiation. The more important influences of high temperature, corrosion, creep, high vacuum, micrometeors, etc., are shared by other approaches to high performance space power. The major problem becomes apparent when one considers the unreasonable time and cost associated with a statistical demonstration of reliability or with the corollary identification of failure modes with confidence. This basic dilemma is shared by many other aspects of the space program. The ultimate solution must rely upon simplicity, basic phenomenological understanding, and sound engineering. Basic system development must progress to a level which allows a valid judgment of inherent reliabılity and considerable experience will have to come from interim usage.

The uninterruptible energy output of a radioisotope power supply would seem to offer some reliability advantage over reactor systems which require controls for startup and operation. However, the ever-present heat in a radioisotope source poses some disadvantage during system assembly, launch operations, and accident conditions. As radioisotope systems grow into the hundreds to thousands of watts region, the added requirements for pad cooling, power flattening, heat transfer loops, etc., make it difficult to ascribe any net reliability advantage to a radio1sotope heat source.

\section{c. Cost}

The SNAP 10A and 8 programs have progressed to the point where reasonably accurate cost estimates can be made. The basic cost of a SNAP 10A unit is estimated at about 1 million dollars and the SNAP 8 unit should cost between 3 to 5 million dollars. 
Short-lived radioisotope units fueled with $\mathrm{Po}^{210}$ at under $\$ 100$ per thermal watt will cost about $\$ 1000$ to $\$ 2000$ per electrical watt. Long-lived $\mathrm{Pu}^{238}$ fueled system cost is dominated by the fuel cost which is estimated at $\$ 500$ to $\$ 1000$ per thermal watt for a system cost of about $\$ 5$ to 10 million per electrical kilowatt. Recovery and reuse of the $\mathrm{Pu}{ }^{238}$, which seems quite reasonable for manned applications, can significantly reduce the effective cost.

\section{d. Safety}

The SNAP program is demonstrating that nuclear safety need not impede the use of nuclear power in space. The AEC has established an Aerospace Safety Program for the specific purpose of developing the technology necessary to minimize any nuclear safety problems. The SNAP systems have been designed to meet operational factory-to-flight sequence requirements. In general, the nuclear powered SNAP unit can be transported, stored, installed, checked-out, etc., without nuclear hazard or personnel exposure.

The use of a reactor powered unit need not perturb the normal launch operations. During launch, the normal chemical exclusion radius is adequate to protect launch personnel from any unlikely nuclear hazard introduced by a vehicle malfunction or abort. During the entire prelaunch and launch sequence a reactor is basically inert and contains a negligible inventory of radioactivity. After startup and operation in orbit, the system can be shut down and the accumulated radioactivity will decay to a safe level during the remaining time in orbit prior to reentry.

The early small radioisotope systems were designed for reentry burnup and dispersal in the upper atmosphere. As systems become larger, it is desirable to return the 1sotope capsules intact to the earth from low short-lived orbital applications or from launch aborts. In this manner, the use of radioisotopes will not be impeded by a buildup in atmospheric contamination. This approach is possible with alpha-emitting 
isotopes since the external radiation from a contained source is a hazard only through extended intimate contact. Designs for intact reentry include ablative coatings and reentry bodies. After design for reentry survival, the conditions imposed by mishandling or by a launch pad abort are not limiting.

\section{E. FUTURE DIRECTION}

The key to improved performance (watts/lb or watts $/ \mathrm{ft}^{2}$ ) of all space power systems, including the SNAP units, is heat source temperature. In general, the performance advancements will be paced by the avallability of the materials technology required by a given operating temperature.

In the reactor, temperature and fuel material selection will determine the useful power output of the reactor before fallure due to fission product induced fuel swelling. The future high temperature reactors look toward UC, $(U-Z r) C$, and $U N$ for the fuel and refractory metals and alloys for the cladding. In order to achieve minimum size, these reactors will operate with a fast neutron spectrum. In general, reactor size and weight and the resultant shield weight will increase with increasing operating temperature.

In the radioisotope heat sources the Large Radioisotope Heat Source (LRHS) program is pointing toward a $2000^{\circ} \mathrm{F}$ heat source with 5-year life. Refractory alloys and noble metal oxidation barriers are required. The LRHS objectives are quite compatible with a Brayton cycle power conversion system which is especially advantageous because of the potential of $20 \%$ efficiency. Since the isotope systems will be limited to about $10 \mathrm{kwe}$ by cost and avalability of long-lived alpha emitters, the required radiator area does not present a sufficient problem to warrant higher temperature operation. In the remainder of the system, temperature and material selection will limit life due to corrosion, creep, sublimation, etc. It is clear that temperature is the key to improved performance; however, it may be detrimental to system life and reliability. 
It should be remembered that the prime incentive for a high temperature heat source, reactor or isotope, is to reduce radiator area which is the major weight constituent in a high power system. In general, the heat source itself will weigh more as operatıng temperatures increase. In the case of large manned systems wherein the shield can be about $50 \%$ of the system weight, the performance improvement to be achieved through higher temperature and thus lower radiator area is more difficult to justify than it is for unmanned systems.

The performance of thermoelectric systems improves rapidly with source temperature. The watts $/ \mathrm{ft}^{2}$ of radiator area are proportional to the fifth power of the source temperature. $\mathrm{PbTe}$ is limited to about $1200^{\circ} \mathrm{F}$, however, $\mathrm{SiGe}$ is capable of operation at temperatures up to about $1800^{\circ} \mathrm{F}$. The low efficiency disadvantage of thermoelectric systems will probably be offset by the inherent reliability of static power conversion up to the tens of kilowatts power level.

The performance of the Hg rankine cycles is limited by the practical working pressure limit and the thermodynamic properties of $\mathrm{Hg}$. The major improvement in rankine cycles occurs with the change in working fluid which unfortunately involves a discrete temperature step of about 600 to $800^{\circ} \mathrm{F}$ to the SNAP 50 conditions. In the future, $\mathrm{Hg}$ systems will probably be used beyond their region of optimum size because of the more immediate avallability of the lower temperature technology. Thus, large $\mathrm{Hg}$ rankine systems or multıple smaller systems may well be used in the hundred kilowatt power range.

The discrete temperature applicability of rankıne cycles introduces a significant aspect of the Brayton cycle. The cycle requires a larger radiator area at a given heat source temperature limit, but the cycle and the machinery are probably more versatile in accepting the increased heat source temperature capability that time and technological improvements will yield. The potential of a more continuous performance growth 


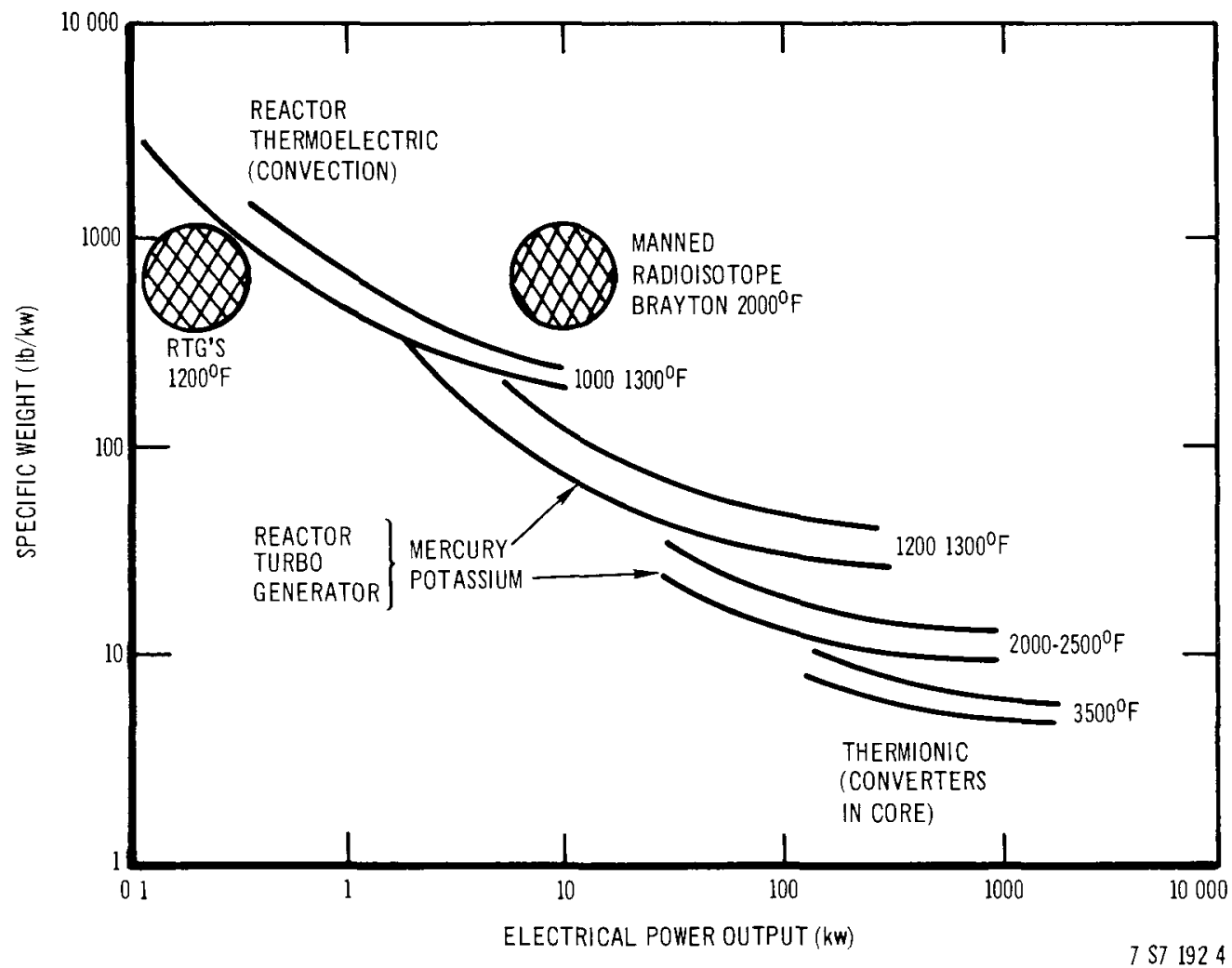

Figure I-2. Specific Weight vs Power Output for Nuclear Power Systems (shielded for unmanned missions except as noted) 
could have a significant influence on long-term reliability achievement.

A promising system of the future that offers potentially high performance, $10 \mathrm{lb} / \mathrm{kw}$, is the reactor thermionic system. Even though this concept is being studied, no system development program has been establıshed. The prime approach places the diodes directly in the core as an integral subassembly of the fuel element. The nuclear material operates at the cathode temperature of the space charge neutralized diode of about $3000^{\circ} \mathrm{F}$, while the remaining reactor structure, control, coolant, pumps, etc., operate at the anode temperature of about $1500^{\circ} \mathrm{F}$. There have been many independent and government sponsored research efforts in the field of thermionic conversion. The basic phenomenon is reasonably well understood as evidenced by demonstrated conversion efficiency and power density accomplishments. The problems of materials selection for the environment and for useful diode life are less well understood. The technology necessary for the selection of a reactor fuel material with appropriate physical properties and high energy output capability is currently far from the status required to support serious system design and development.

A performance summary of current and future reactor space power systems is shown in Figure I- 2 .

\section{F. COMPETITIVE POSITION}

A partial appreciation of the comparison between nuclear power plants and the alternate energy sources can be derived from the following figures. The selection of a power system is greatly dependent upon the mission requirements and constraints. These considerations must include an overall assessment of weight, area, drag, cost, reliability, vehicle integration, mission interaction, state of the art, etc. The following comparisons, therefore, cannot provide a complete assessment of the competitive position of nuclear power but can orient the reader 


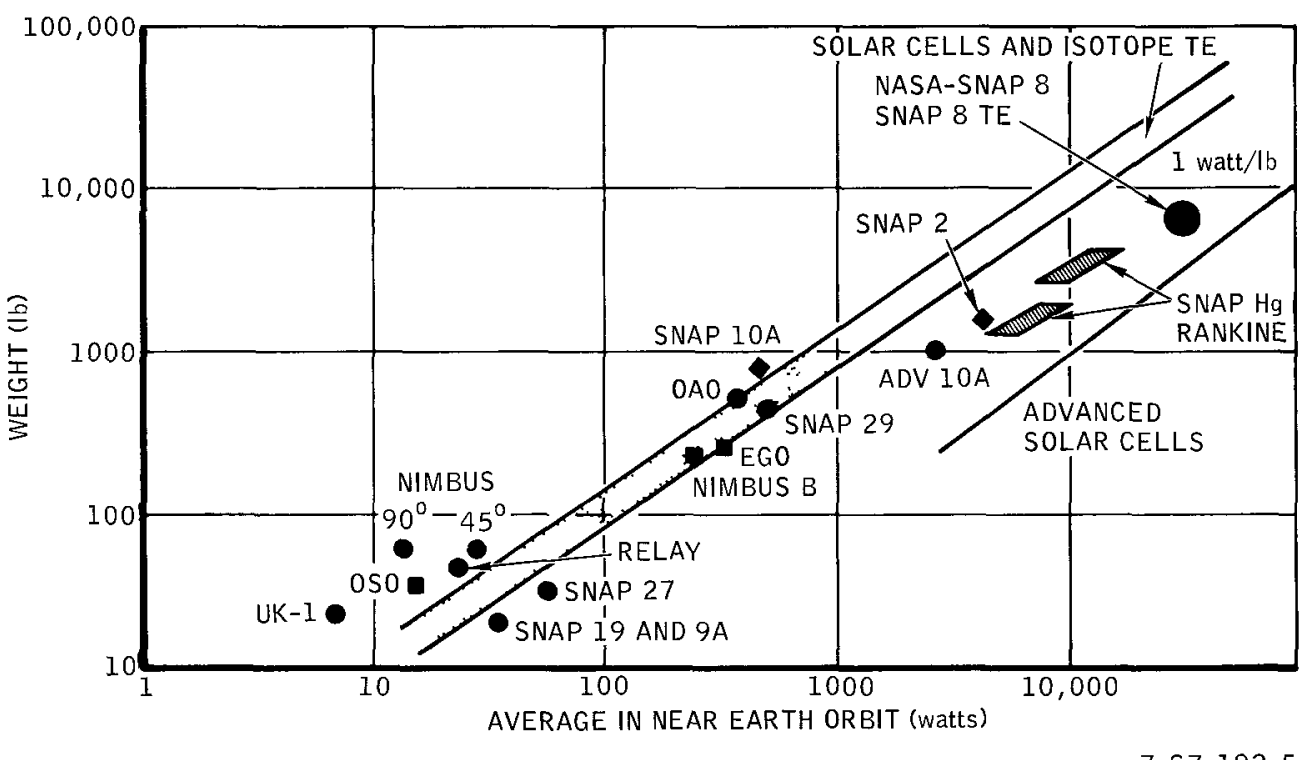

Figure I-3. Comparison of Weight of Solar Cell and Nuclear APU's as a Function of Power. (The solar cell weights do not include the weight of orientation propellant for long durations. The nuclear system weights include shielding for electronic payloads, $10^{12}$ nvt. Solar cell data: Cherry, Wm. R., Astronautics and Aerospace Engineering, May 1963) 
with respect to relative or comparative performance on ind vidual factors.

Radiolsotope systems in the kilowatt power range are of major interest for extending the lifetime of manned orbital missions. If one compares the radiorsotope system performance of 1 to 2 watts/lb with the best possible fuel cell performance of about 1000 watts $-\mathrm{hr} / \mathrm{lb}$, it is apparent that the radioisotope system will have a weight advantage beyond 500 to 1000 hours. Figure I- 3 compares the weight of the current nuclear power plants with the approximate weight of solar cell systems as a function of power. Reactor systems, shrelded for manned applications, can compete with solar systems in lowearth orbit where there is a significant dark time and energy storage requirement as well as a propellant requirement for drag compensation. Reactor systems are also advantageous on the lunar surface because of the extended lunar night. Since weight alone is not the complete picture, Figure I- 4 compares the required solar cell area with the nuclear power plant radiator area requirements. It should be remembered that the nuclear power plant requires no continuous orientation and no energy storage to cover operation while in the earth's shadow. The area comparison further favors nuclear power when one considers far planet operations where the solar intensity is significantly lower. From a dollar-cost point of view, SNAP 10A will cost about 1 million dollars which is competitive with an equivalent solar cell system. At high power levels, the reactor systems will have a significant cost advantage (see Figure I-5).

The high cost of long-lived alpha emitters tends to restrict the use of radioisotope systems to low power applications, high cost and high priority scientific missions for which solar cells are inappropriate, and long duration manned orbital missions wherein the 1 sotope cost can be effectively reduced through the return and reuse of the isotope inventory. 


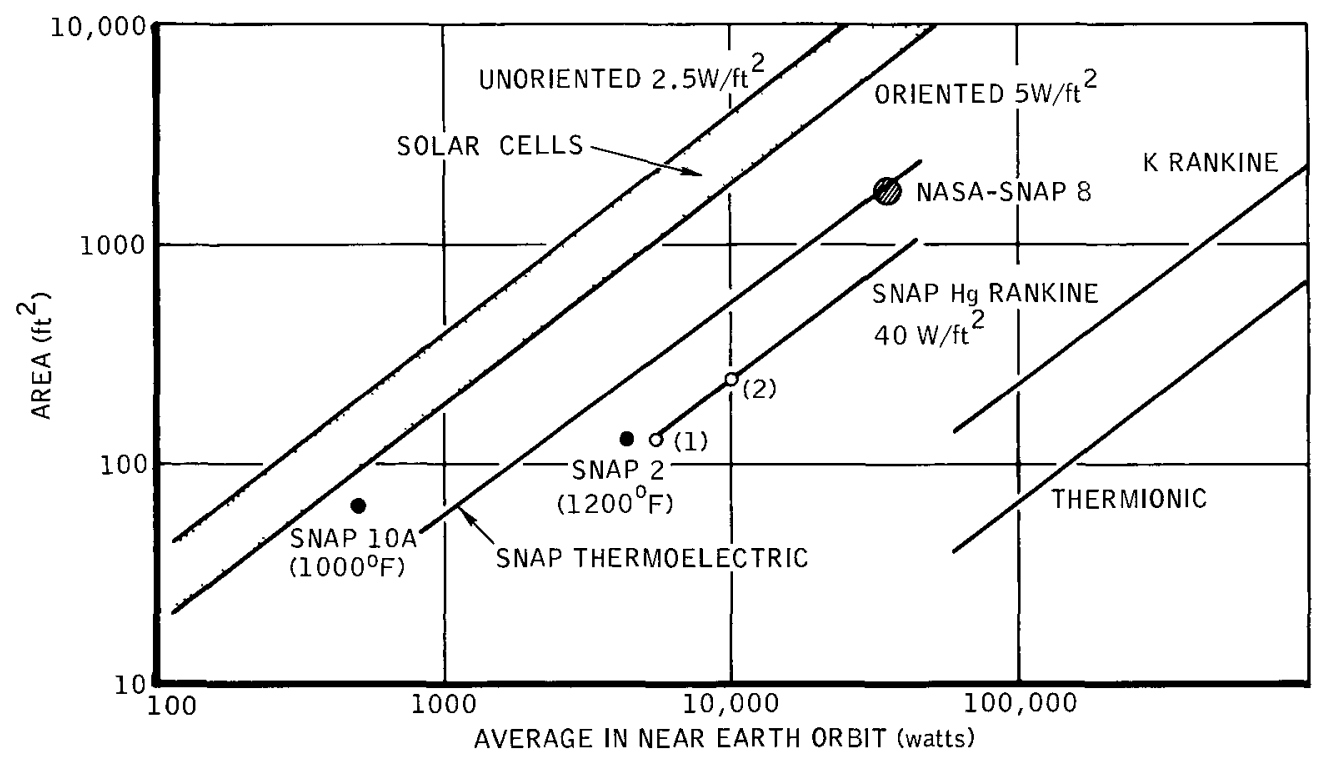

7-S7-192-6

Figure I-4. Area Requirements for Solar Cell Arrays and Nuclear Systems as a Function of Power 


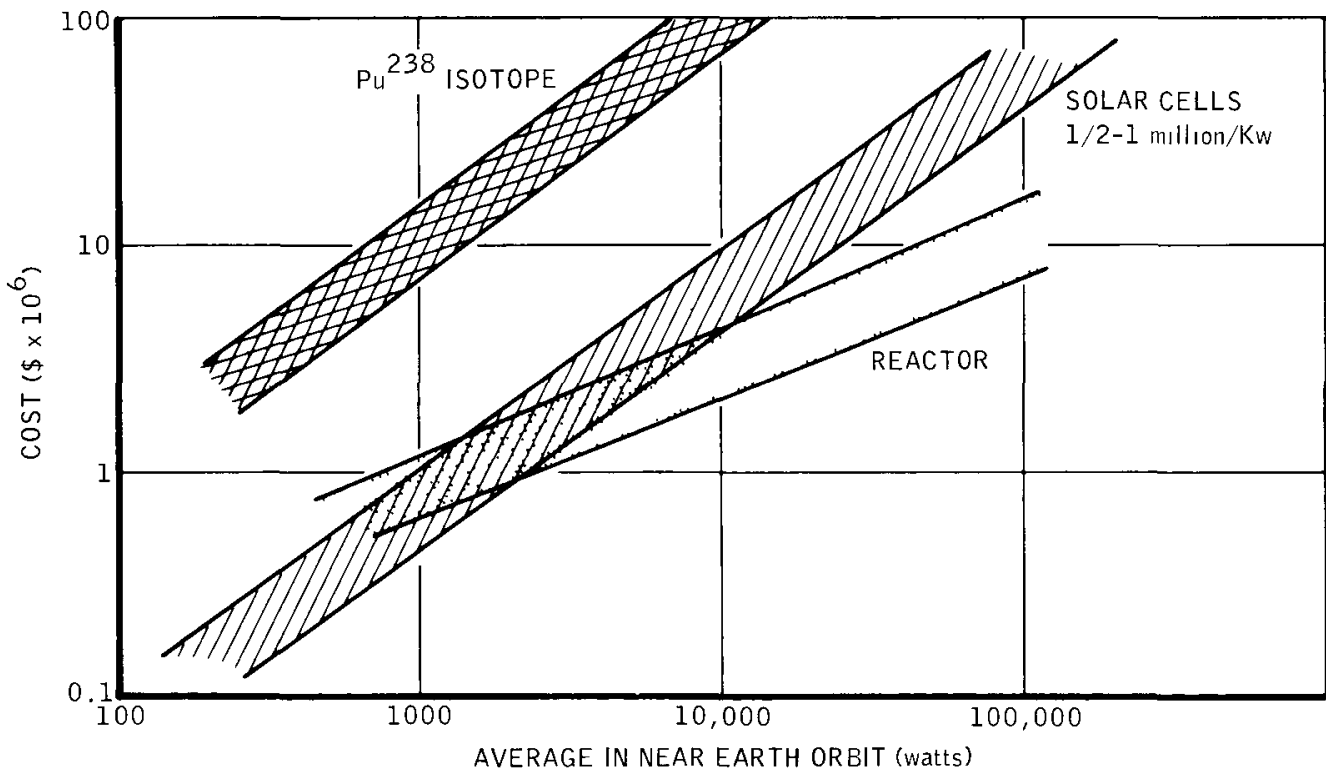

7-57-192-7

Figure I-5. Cost Comparison Between Nuclear and Solar Cell Systems as a Function of Power 


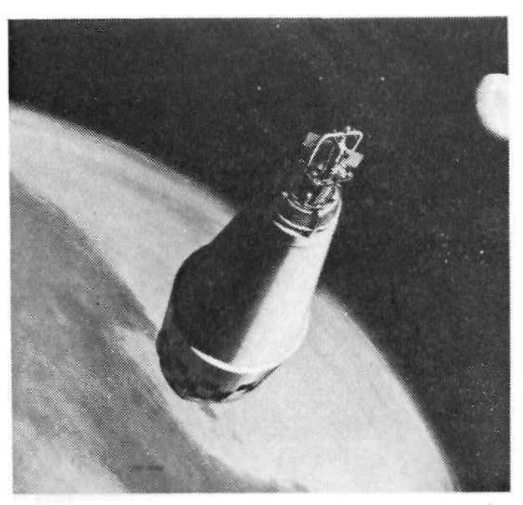

WORLD WIDE T.V. SATELLITE

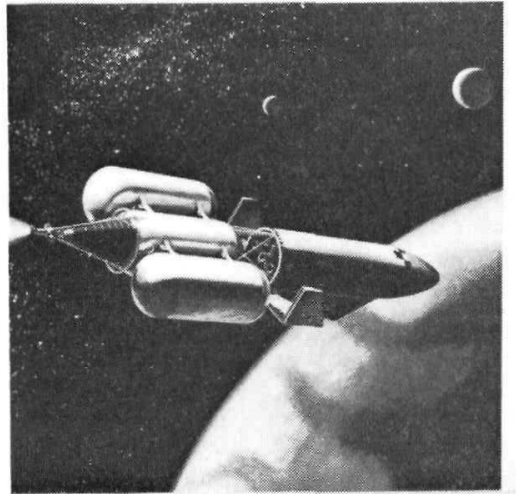

MARS EXPLORATION - 2000

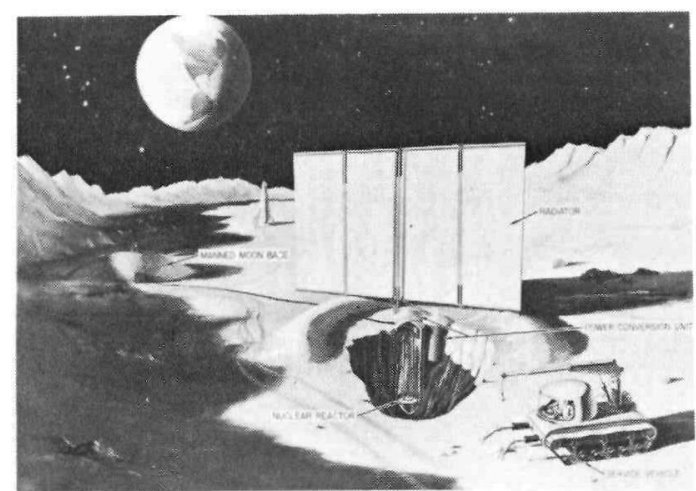

LUNAR BASE - 1980

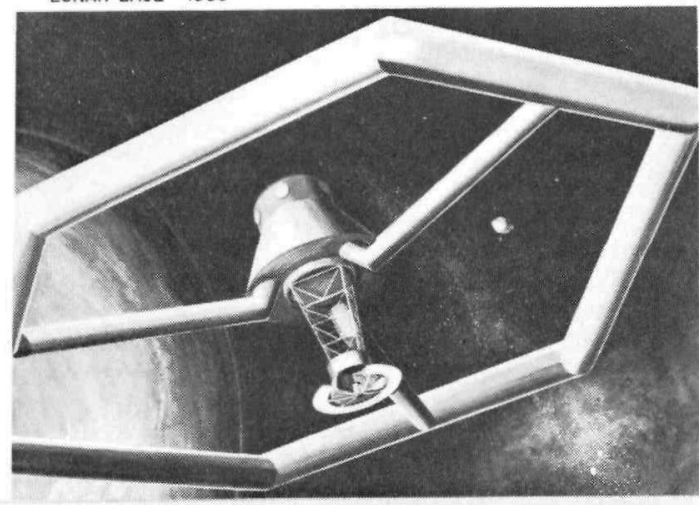

MANNED SPACE STATION-1975

Figure I-6. Nuclear Space Applications 


\section{G. APPLICATIONS}

As the utilization of space increases and as man's exploration extends beyond our own planet to the outer regions of our solar system, reactor power systems will assume a role of major importance. Experience on earth has clearly shown that technological advancement always requires more power. Even when the power demand per function is reduced, the number of functions increases more rapidly with the end result of an ever increasing total power requirement. This same experience will be even more valid in space because of the hostile nature of the environment. Thus, to a large extent, the availability of large amounts of reliable and long lived power will control our space aspirations. Some of these long range objectives are illustrated in Figure I-6. Future communications satellites broadcasting several channels of television directly to the individual home from a synchronous orbit will require power levels up to about 100 kwe with 3 to 5 years lifetime. A large 20 to 40 man orbital space station will provide a base for earth oriented services like communications, navigational aids, meteorological observations, etc., and for space research. The power demand of such a station can readily exceed $100 \mathrm{kwe}$. A probable future objective will be the establishment of a semipermanent base on the moon. Studies of the requirements and problems of such a base are already underway and revealing power needs of 100 to $1000 \mathrm{kwe}$. Beyond the moon, the exploration of Mars will probably be accomplished before the end of this century. The power system will have to use a nuclear source and lifetime and reliability will be an extremely demanding requirement. Missions beyond Mars will use electric propulsion which is only feasible with high temperature and long lived nuclear power supplies. In conclusion, the "space age" will rely heavily on the future application of products of the "atomic age." 


\section{BLANK}




\section{CHAPTER II \\ REACTOR SPACE POWER SYSTEMS \\ I. THE REACTOR HEAT SOURCE}

\section{A. NUCLEAR FISSION \\ a. Fission Process}

From the point of view of the utilization of nuclear energy, the importance of fission lies in two facts. First, the process is associated with the release of considerable amounts of energy, and second, the reaction initiated by neutrons is also accompanied by the liberation of neutrons. It 1 s thus possible, under proper conditions, for the process to be self-sustaining and for energy to be generated continuously, once the fission reaction has been started.

In the fission process the nucleus absorbs a neutron and the resulting compound nucleus is so unstable that it immediately breaks up into two parts of more or less equal mass, called fission fragments. Most of the fragments are radioactive, decaying at different rates, with the emission of negative beta particles and gamma radiation, to form products which are themselves usually radioactive. In the fission of $\mathrm{U}^{235}$, for example, there are formed more than 80 primary products, with mass numbers ranging from 72 to 160. Each of these undergoes, on the average, three stages of radioactive decay before being converted into a stable nucleus. As a result, there are over 200 radioactive isotopes of 30 or more different elements present among the fission products after a short time.

Nuclear fission as a result of neutron capture occurs only with the heaviest elements. Whereas certain isotopes, notably $\mathrm{U}^{233}, \mathrm{U}^{235}$, and $\mathrm{Pu}^{239}$ undergo fission with thermal (low-energy) neutrons as well as with fast (high-energy) neutrons, others, such as $\mathrm{Th}^{232}$ and $\mathrm{U}^{238}$ require fast neutrons to cause fission. In general, the cross section (neutron capture probability) is 
largest for thermal neutrons, it then decreases with increasing neutron energy, according to (velocity) ${ }^{-1}$, and becomes relatively small for fast neutrons.

\section{b. Release of Neutrons}

Most of the nuclear fragments formed when fission occurs have too many neutrons for stability, and so there is a tendency for some of them to expel neutrons almost instantaneously. These are the neutrons which appear to accompany the fission process. The average number, $\nu$, of neutrons liberated for each thermal neutron absorbed in a fission reaction by $\mathrm{U}^{235}, \mathrm{U}^{233}$, and $\mathrm{Pu}^{239}$ is given in Table II- 1 . It will be noted that the average numbers of neutrons liberated are not integers. Although the number of neutrons expelled in any individual act of fission must be integral, the average over a large number of fissions is not necessarily a whole number. A typical distribution of the number of neutrons per fission of $\mathrm{U}^{235}$ is shown in Figure II- 1 .

The neutrons emitted as a result of the fission process can be divided into two categories, namely prompt neutrons and delayed neutrons. The prompt neutrons, which constitute over $99 \%$ of the fission neutrons, are released within an extremely short interval of time, probably about $10^{-14} \mathrm{sec}$ (or less), of the instant of fission. The energy of these neutrons covers a considerable range, from over $10 \mathrm{Mev}$ down to quite small

TAB LE II- 1

AVERAGE NUMBER OF NEUTRONS LIBERATED PER THERMAL NEUTRON ABSORBED IN FISSION

\begin{tabular}{c|c}
\hline \hline Fissionable Nucleus & $\begin{array}{c}\text { Number of Neutrons } \\
(\nu)\end{array}$ \\
\hline $\mathrm{U}^{233}$ & $2.41 \pm 0.03$ \\
$\mathrm{U}^{235}$ & $2.51 \pm 0.03$ \\
$\mathrm{Pu}^{239}$ & $2.91 \pm 0.04$ \\
\hline
\end{tabular}




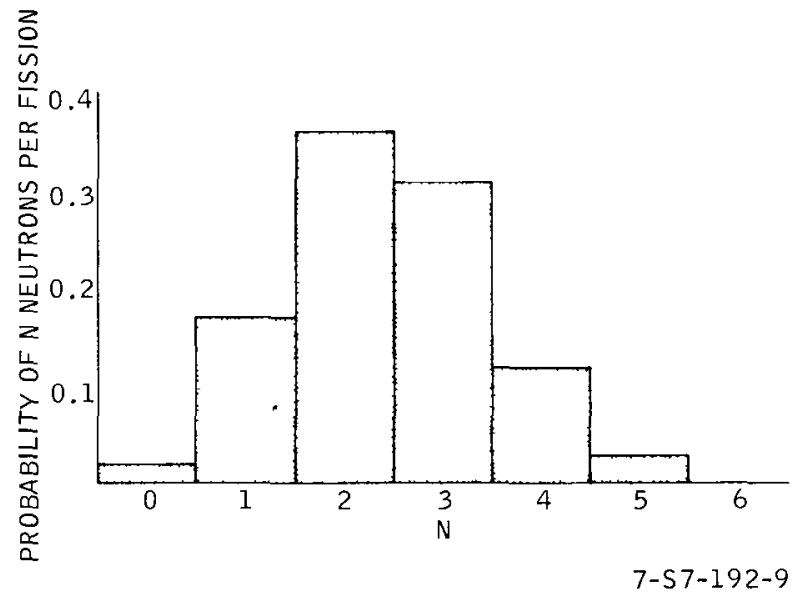

Figure II-1. Neutron Yield from the Fissioning of $\mathrm{U}^{235}$. (Even though the number of neutrons from any one fission event is an integer, the average over a large number is not.)

values; the average energy of the prompt neutrons is about 2 Mev. The energy distribution for $U^{235}$ fission neutrons is shown in Figure II-2. The energy of the prompt fission neutrons is an insignificant fraction of the total fission energy. However, the neutron energy is very significant to the subsequent fission that a neutron can induce. In a "fast" reactor the prompt neutrons are utilized near their energy of origin. In the "thermal reactor" the neutrons must be slowed down from millions of electron volts to energies less than 1/10 of an electron volt before they can enter into a subsequent fission event. It will be seen later that this neutron slowing-down process has a strong influence on the size and composition of a thermal reactor.

The delayed neutrons, as their name implies, are emitted over a period of a few seconds to minutes, the intensity falling off rapidly with time. The delayed neutrons accompanying fission fall into unique groups according to characteristic delay times. The rate of decay of the intensity in each delayed-neutron 


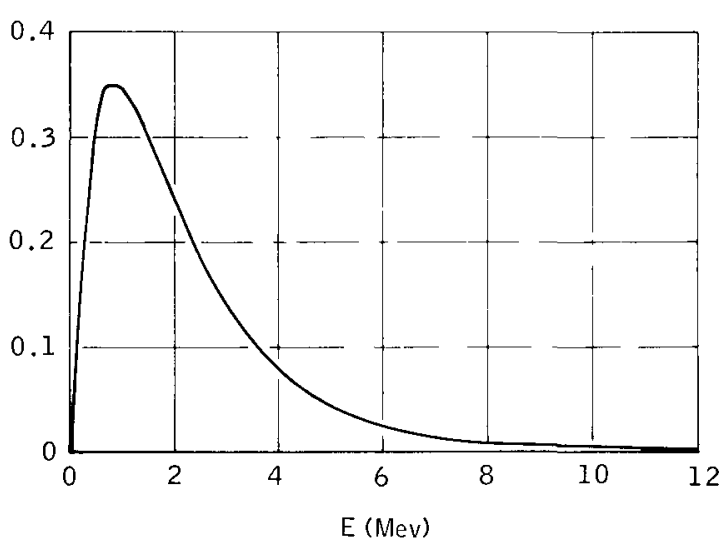

7-57-192-10

Figure II-2. Energy Distribution of Prompt Fission Neutrons

group is exponential, as it is for radioactive decay. Because the delayed neutrons are a result of nuclear decay of fission fragments, they fall into the same groups for different fissioning nuclei. The six generally accepted delayed neutron groups have half lives of $54,22,5.6,2.12,0.45$, and $0.15 \mathrm{sec}$. In a time perıod equal to one half-life, one-half of the neutrons remaining at the start of that period are emitted. The fraction of delayed neutrons is a function of the fissioning nucleus and Is $0.3,0.75$, and $0.23 \%$ for $\mathrm{U}^{233}, \mathrm{U}^{235}$, and $\mathrm{Pu}^{239}$, respectively. The energy of the delayed neutrons falls in the region of 0.25 to $0.6 \mathrm{Mev}$. Figure II-3 shows the fraction of fission neutrons remaining to be emitted as a function of time for $\mathrm{U}^{235}$. The delayed neutrons have an important bearing on the timedependent behavior of nuclear reactors. Were it not for these neutrons, the safe control of nuclear reactors would be much more difficult than it is. 


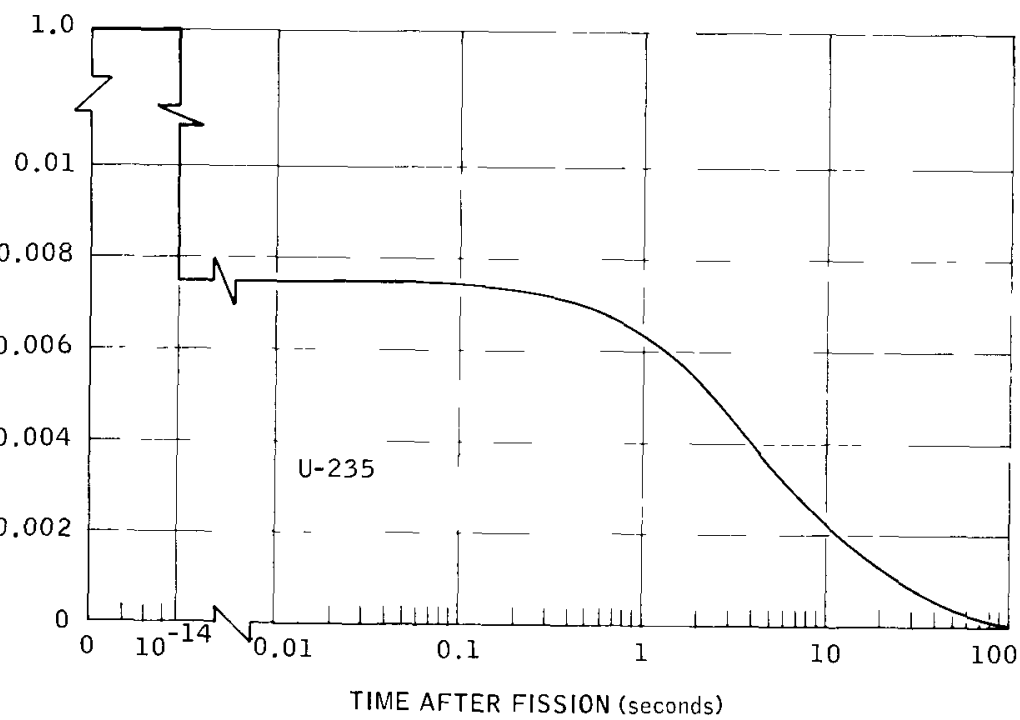

$7-57-192-11$

Figure II-3. Time Dependence of Neutron Emission from Fissioning of $\mathrm{U}^{235}$. (The fact that $3 / 4$ of $1 \%$ of the U235 fission neutrons are delayed by a significant time greatly simplifies reactor control.)

\section{c. Fission Energy}

The fission process is remarkable for the magnitude of the energy released; it is about $200 \mathrm{Mev}$ for each nucleus undergoing fission, which may be compared with a few electron volts for each atom reacting in chemical processes, such as the combustion of coal or oil, or up to $20 \mathrm{Mev}$ for nonfission nuclear reactions. The large energy release in fission is associated with the fact that the products of the reaction have an appreciably smaller total mass than that of the nucleus undergoing fission plus the neutron causing fission. Because of the equivalence of mass and energy, the considerable decrease in mass in the fission reaction must be accompanied by the liberation of a large amount of energy. 
The relationship between energy $E$ and the equivalent mass $m$ is given by the Einstein equation

$$
\mathrm{E}=\mathrm{mc} \mathrm{c}^{2}
$$

where $c$ is the velocity of light. If $m$ is in grams, and $c$ in $\mathrm{cm} / \mathrm{sec}, 1 . \mathrm{e}, 3 \times 10^{10} \mathrm{~cm} / \mathrm{sec}$, then $\mathrm{E}$ will be in ergs. For the present purpose it is more useful to express $m$ in atomic mass units, the equation then becomes

$$
\mathrm{E}(\mathrm{ergs})=\mathrm{m}(\mathrm{amu}) \times 1.49 \times 10^{-3} \text {. }
$$

$1 \mathrm{Mev}=1.60 \times 10^{-6} \mathrm{erg}$, and so the energy equivalent $1 \mathrm{~s} \mathrm{ex}-$ pressed in Mev by

$$
E(\mathrm{Mev})=\mathrm{m}(\mathrm{amu}) \times 931 .
$$

The magnitude of the energy released in nuclear fission will be estimated for the fission of $U^{235}$, making the simplifying assumption that the products are nuclei with mass number 95 and 139, since these are known to be obtained in greatest amount. In order to balance the mass numbers, it is evident that two fission neutrons are liberated in this case, as may be seen from the following equation

$$
\mathrm{U}^{235}+\mathrm{n}^{1} \rightarrow \mathrm{X}^{95}+\mathrm{Y}^{139}+2 \mathrm{n}^{1}
$$

The neutron on the left is the one which initiates fission, and the two on the right are formed as a result of fission.

The mass of the $\mathrm{U}^{235}$ atom is known to be $235.124 \mathrm{amu}$, whereas that of the neutron is $1.00897 \mathrm{amu}$, which may be approximated to 1.009 for the present purpose. By comparison with known stable species, the masses of the two fission products postulated above will be 94.945 and $138.955 \mathrm{amu}$, respectively. Hence the following balance sheet of the masses before and after fission may be drawn up. 
Masses Before Fission

$\mathrm{u}^{235}$

1 neutron

Total
235.124

$\frac{1.009}{236.133}$
Masses After Fission

Mass number-95

94.945

Mass number-139

138.955

2 neutrons

Total 2.018 235.918

Mass converted into energy $=236.133-235.918=0.215 \mathrm{amu}$. As seen above, 1 amu is equivalent to $931 \mathrm{Mev}$, and so the energy released per fission is given by

Energy released per fission $=(0.215)(931)=198 \mathrm{Mev}$.

Although this calculation was made for one particular mode of fission, it may be regarded as quite typical. While there are slight variations from one mode to another, it appears, on the whole, that an estimate of about $200 \mathrm{Mev}$ of energy released per $U^{235}$ nucleus undergoing fission is satisfactory. The same value may also be taken as applying to the fission of $\mathrm{U}^{233}$ and $\mathrm{Pu}^{239}$.

\section{d. Energy Distribution}

The major proportion - over $80 \%$ - of the energy of fission appears as kinetic energy of the fission fragments, and this immediately manifests itself as heat within less than $0.01 \mathrm{~mm}$ of the point of fission. Part of the remalning $20 \%$ or so is liberated in the form of instantaneous gamma rays and as kinetic energy of the fission neutrons. The rest is released gradually as energy carried by the beta particles and gamma rays emitted by the radioactive fission products as they decay over a period of time.

The approximate distribution of the fission energy, which may be regarded as applying to all three of the important fissionable species, is given in Table II-2. Of the heat liberated instantaneously, about 90\%, 1.e., $168 \mathrm{Mev}$, will be produced at (or near) the point of fission, and only $17 \mathrm{Mev}$ elsewhere. 
TAB LE II-2

LIBERATION OF HEAT DUE TO FISSION

Instantaneous

$\frac{\mathrm{Mev}}{168}$

Energy of fission fragments

Energy of fission neutrons

Instantaneous gamma rays

5

Capture gamma rays

Delayed

Beta particles from fission products

Gamma rays from fission products

Radiation from capture products

But of the delayed heat, the two amounts are approximately equal, i. e., about $7 \mathrm{Mev}$ at the place where fission occurs and $8 \mathrm{Mev}$ at a distance.

\section{e. Energy Equivalents}

In order to convert the fission energy into practical units, it should be recalled that $1 \mathrm{Mev}$ is equal to $1.60 \times 10^{13}$ watt-sec. Hence the total energy ( $200 \mathrm{Mev}$ ) available per fission is about $3.2 \times 10^{-11}$ watt-sec, so that it requires $3.1 \times 10^{10}$ fissions to release 1 watt-sec of energy. In other words, fissions at the rate of $3.1 \times 10^{10}$ per sec produce 1 watt of power, provided the reactor has been operating for some time.

One gram atom of an element, i.e., the atomic weight expressed in grams, of any element, contains Avogardo number of individual nuclei $\left(6.02 \times 10^{23}\right)$; if all of these undergo fission, the energy liberated would be $\left(6.02 \times 10^{23}\right)$ times $\left(3.2 \times 10^{-11}\right)$ $=1.9 \times 10^{13}$ watt-sec, or $5.3 \times 10^{6} \mathrm{kwh}$. This is the amount of heat that would be released by the complete fission of $233 \mathrm{grams}$ of $\mathrm{U}^{233}$, or $235 \mathrm{grams}$ of $\mathrm{U}^{235}$, or $239 \mathrm{grams}$ of $\mathrm{Pu}^{239}$. Neglecting the relatively small differences between these weights, 
the results in Table II- 3 may be regarded as applying to the heat produced by the fission of $1 \mathrm{lb}$ of any of these materials. One pound of uranium is a cube which is 1.1 in. on a side. A useful fact to remember is that the power production corresponding to the fission of 1 gram of material per day would be roughly $10^{6}$ watts or $1 \mathrm{Mw}$.

TAB LE II - 3

HEAT LIBERATED BY 1 lb OF FISSIONAB LE MATERIAL

$0.9 \times 10^{13} \mathrm{cal}$

$1.0 \times 10^{7} \mathrm{kwh}$

$2.8 \times 10^{13} \mathrm{ft}-1 \mathrm{~b}$

$3.6 \times 10^{10} \mathrm{Btu}$

B. THE CHAIN-REAC TION

a. Conditions for Self-Sustainıng

If a chain reaction $1 \mathrm{~s}$ to be maintained, the minimum condition is that for each nucleus capturing a neutron and undergoing fission there shall be produced, on the average, at least one neutron which causes the fission of another nucleus. This condition can conveniently be expressed in terms of a multiplication factor or reproduction factor, defined as the ratio of the number of neutrons of any one generation to the number of corresponding neutrons of the immediately preceding generation. If the multiplication factor, represented by $k$, is exactly equal to or slightly greater than unity, a chain reaction will be possible. But if $k$ is less than unity, even by a very small amount, the chain cannot be maintained.

Suppose, for example, a particular generation starts with 100 neutrons, if the multiplication factor is unity, there will be 100 corresponding neutrons at the beginning of the second generation, 100 at the third, and so on. Once it has started, the 
fission will continue at the same rate. For practical purposes, however, it is necessary that $k$ be capable of exceeding unity, if power production is to be appreciable. The simplest way in which a required power level can be attained is for the multiplication factor to exceed unity, the number of neutrons present and, hence, the fission rate will then increase until the desired rate is reached.

b. Neutron Balance

The magnitude of the multiplication factor in any system containing fissionable material depends on the relative extents to which the neutrons take part in four main processes. These are (1) complete loss or escape of neutrons from the system, generally referred to as leakage, (2) nonfission capture by the fuel, (3) nonfission capture, sometimes called parasitic capture, by the various extraneous substances ("polsons") such as structural materials, coolant, fission products, and impurities in the uranium, and finally (4) fission capture of slow or of fast neutrons by the fuel.

In each of these four processes neutrons are removed from the system, but in the fourth process, 1.e., in the fission reaction, other neutrons are generated to replace them. Hence, if the number of neutrons produced in the latter process is just equal to (or exceeds) the total number lost by escape and by fission and nonfission capture, the multiplication factor will equal (or exceed) unity and a chain reaction should be possible.

An illustration of the type of neutron balance that might exist in a system for which the multiplication factor is exactly unity is depicted below. It is assumed that fission results only from the capture of slow neutrons, and it is supposed, for simplicity, that exactly two neutrons are produced, on the average, in each fission process. 
100 slow neutrons absorbed by $\mathrm{U}^{235}$ to cause fission $+$

200 fission neutrons

$\longmapsto 60$ leak out during slowıng down

140 neutrons slowed down

$\downarrow 10$ leak out as slow neutrons

130 slow neutrons avallable for absorption

$\downarrow-30$ absorbed by moderator, structure, poisons, etc. 100 slow neutrons (absorbed by $\mathrm{U}^{235}$ to cause fission)

Since 100 slow neutrons are absorbed in fission processes at the beginning, and 100 are avalable for similar absorption at the end of the generation, the conditions for a self-sustaining chain are satisfied.

\section{c. Multiplication Factor for Thermal Reactors}

For the present, in order to avoud the problem of the loss of neutrons by leakage, it will be postulated that the multiplying system is infinite in extent. Suppose that, at a given instant representing the initiation of a generation, there are avalable $n$ thermal neutrons which are captured in fuel. Let $\eta$ be the average number of fast fission neutrons emitted as a result of the capture of one thermal neutron in fuel material. Then, due to the absorption of the $n$ thermal neutrons $n \eta$ fast neutrons w1ll be produced. It should be noted that since the neutrons captured in fuel do not all necessarily lead to fission, the value of $\eta$ differs, in general, from the average number (see Table II- I) of fast neutrons released per slow neutron fission. If the latter number is represented by $\nu$, then

$$
\eta=\frac{\sigma_{f}}{\sigma_{f}+\sigma_{c}} \nu,
$$

where $\sigma_{f}$ is the macroscopic cross section for slow neutron fission, and $\sigma_{c}$ is the cross section for absorption of thermal neutrons by nonfission processes in the fuel material (see Table II-4). 
TAB LE II- 4

THERMAL NEUTRON CROSS SECTIONS

\begin{tabular}{|c|c|c|c|c|c|}
\hline \multirow[b]{2}{*}{ Fuel } & \multicolumn{3}{|c|}{$\begin{array}{c}\text { Cross Sections } \\
\text { (Barns) }\end{array}$} & \multicolumn{2}{|c|}{ Neutron Yield } \\
\hline & $\begin{array}{c}\text { Fission } \\
\sigma_{f}\end{array}$ & $\begin{array}{c}\text { Radiatıve } \\
\text { Capture } \\
\sigma_{c}\end{array}$ & $\begin{array}{l}\text { Absorption } \\
\sigma_{\mathrm{a}}-\sigma_{\mathrm{f}}+\sigma_{\mathrm{c}}\end{array}$ & $\begin{array}{c}\text { Per } \\
\text { Fission } \\
\nu\end{array}$ & $\begin{array}{c}\text { Per } \\
\underset{\eta}{\text { Absorption }}\end{array}$ \\
\hline $\mathrm{U}^{233}$ & 532 & 59 & 591 & 2.51 & 2.29 \\
\hline $\mathrm{U}^{235}$ & 579 & 118 & 697 & 2.47 & 2.07 \\
\hline $\mathrm{Pu}^{239}$ & 740 & 285 & 1025 & 2.91 & 2.09 \\
\hline
\end{tabular}

Before the $\mathrm{n} \eta$ fast neutrons have slowed down appreciably some will be captured by, and cause fission of, the fuel. Since more than one neutron is produced on the average in each fission, there will be an increase in the number of fast neutrons avalable. Allowance for this effect may be made by introducing the fast fission factor denoted by $\epsilon$, and defined as the ratio of the total number of fast neutrons produced by fissions due to neutrons of all energies to the number resulting from thermal-neutron fissions.

$$
\epsilon=\frac{\text { number of neutrons produced by all fissions }}{\text { number of neutrons produced by thermal fissions }}
$$

Consequently, as a result of the capture of $n$ thermal neutrona in fuel, $n \eta \in$ fast neutrons will be formed.

As a result of collisions, mainly elastic, with the moderator, the fast neutrons will ultimately be slowed down or thermalized. However, during the slowing down process some of the neutrons are captured in nonfission processes, so that not all of the n $\eta \boldsymbol{\epsilon}$ fast neutrons reach thermal energies. The fraction of the fast (fission) neutrons which escape capture while being slowed down is called the resonance escape probability, and is represented by $p$. 
$\mathrm{p}=\frac{\text { number of neutrons which escape capture during slowing down }}{\text { total number of fast neutrons produced }}$

Consequently, the number of neutrons which become thermalized 1s $\mathrm{n} \eta \in \mathrm{p}$.

When the energy of the neutrons has been reduced to the thermal region, they will diffuse for some time, the energy distribution remaining essentially constant until they are ultımately absorbed by fuel, moderator, or such poisons as may be present. Of the thermal neutrons, therefore, a fraction $f$, called the thermal utilization, will be absorbed in fuel material; the value of $f$ is represented by

$$
\mathrm{f}=\frac{\text { thermal neutrons absorbed in fuel }}{\text { total thermal neutrons absorbed }} \text {, }
$$

where the denominator is the total number of thermal neutrons absorbed by fuel, moderator, and other materials present in the reactor. The number of thermal neutrons captured in fuel is consequently $n \eta \in \mathrm{pf}$.

For the present purpose, since the multiplication factor may be defined as the ratio of the total number of thermal neutrons absorbed, on the average, in one generation to the number of thermal neutrons absorbed in the preceding generation, on the average, in an infinite medium, it follows that

$$
\mathrm{k}_{\infty}=\frac{\mathrm{n} \eta \epsilon \mathrm{pf}}{\mathrm{n}}=\eta \in \mathrm{pf}
$$

where

$$
\begin{aligned}
& k_{\infty} \text { - infinite medium multiplication factor } \\
& \eta \text { - neutron yield per fuel capture } \\
& \epsilon=\text { fast effect factor } \\
& p=\text { resonance escape probability } \\
& f=\text { thermal utilization. }
\end{aligned}
$$


This result is sometimes referred to as the four factor formula. As seen above, the condition for a self-sustaining chain reaction in a system is that the multiplication factor should be unity; the criterion for an infinite system is, therefore, that $\eta \epsilon \mathrm{pf}=1$. The neutron economy of an infinite medium is summarized in the diagram of Figure II- 4 .

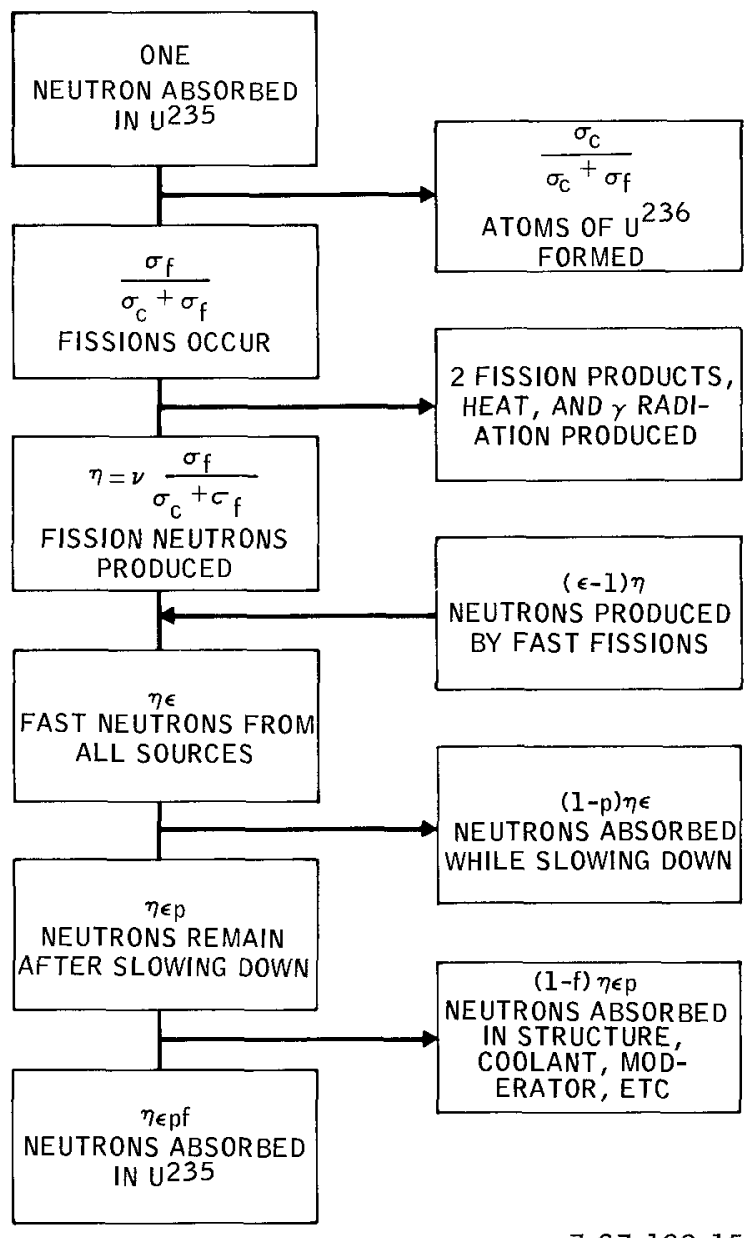

7-57-192-15

Figure II-4. Neutron Economy for an Infinite Medium Containing $\mathrm{U}^{2} 35$ 
In the case of a reactor in which the fuel material contains only $\mathrm{U}^{235}$ and no $\mathrm{U}^{238}$, both the fast fission factor, $\epsilon$, and the resonance escape probability, p, will be virtually unity. Such a reactor can be made critical with a small proportion of fuel relative to moderator. In these circumstances,

$$
\mathrm{k}_{\infty}=\eta f\left(\mathrm{U}^{235} \text { system }\right) .
$$

\section{d. Leakage of Neutrons}

For a reactor of finite size the condition that the infinite medium multiplication factor should be unity is no longer aderuate for a self-sustaining chain reaction. It is required, in a finite system, that for every thermal neutron absorbed in fuel there shall be produced, on the average, one thermal neutron in addition to those lost by leakage from the reactor. If $\mathrm{P}$ is the total nonleakage probability, 1.e., the probability that a neutron will not escape either during the slowing down process or while it diffuses as a thermal neutron, then the condition for a chain reaction to be maintained is

$$
k_{\infty} P=1 .
$$

where

$$
\begin{aligned}
& P=P_{f} P_{t} \\
& P_{f}=\text { fast neutron nonleakage probability } \\
& P_{t}=\text { slow neutron nonleakage probability. }
\end{aligned}
$$

Only for the infinite system is the nonleakage probability unity, and then $k_{\infty}=1$, satisfies the condition for the chain reaction. For a finite reactor, $P$ is less than unity, and hence the infinite multiplication factor must exceed unity if the nuclear chain reaction is to be maintained.

The proportion of neutrons lost by escape from a finite reactor can be diminished by increasing the size of the system. The escape of neutrons occurs at the exterior, but absorption, 
leading to fission and neutron production, occurs throughout the whole of the interior of the reactor. The number of neutrons lost by escape thus depends on the external surface area, while the number formed is determined by the volume. To minimize the loss of neutrons and thereby increase the nonleakage probability, it is necessary to decrease the ratio of area to volume, this can be done by increasing the size of the reactor. The critical size is that for which the nonleakage probability $P$ is such that $k_{\infty} P$ is just equal to unity. Since the area-to-volume ratio depends on the geometrical shape, the nonleakage probability $w 11$ be determined by the shape of the reactor. For a given volume, a sphere has the smallest ratio of a rea to volume, hence, leakage from a spherıcal reactor will be less than for any other shape. The critical volume of such a reactor wall consequently also be less.

As indicated above, the value of $k_{\infty}$ is determined by the composition of the system, 1. c., by the nature of the fuel and the proportion of moderator, and also by the arrangement of the material. Hence, if these are specified, a chain reaction will be possible only if $P$ is large enough to make $k_{\infty} P$ equal to or greater than unity. The neutron economy of a finite $U^{235}$ system is summarised in the diagram of Figure II- 5 .

e. Critical Size of Reactor

The finite system must satısfy a neutron continuity equation which states

$$
+ \text { Production - Leakage - Absorption }=\frac{d n}{d t} \text {. }
$$

The solution of this equation is dependent upon the model used to describe the slowing down of neutrons. For the case of the Fermi-Age or "continuous slowing down" model, it is assumed that a neutron loses a constant fraction of its incident energy with each collision. The physical quantity of principal interest is the average distance traveled by a neutron in the process of 


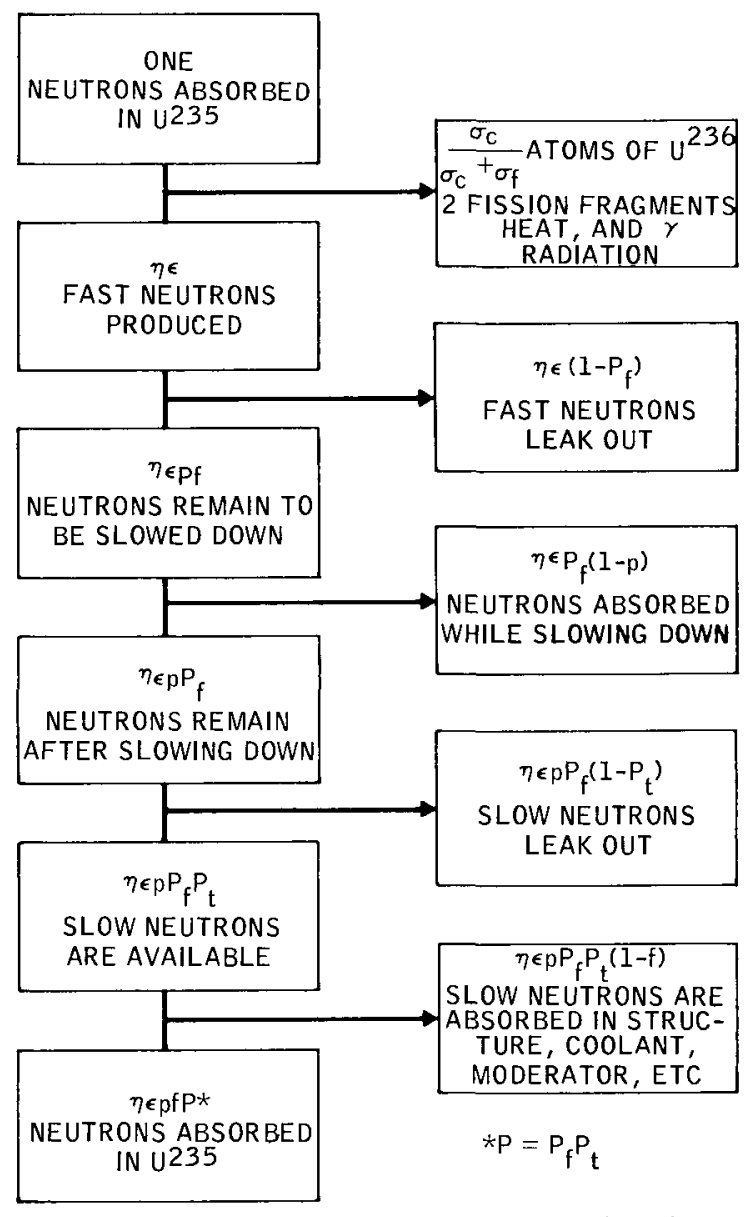

7-57-192-16

Figure II-5. Neutron Economy for a Finite System Containing U235

slowing down because the relationship between this distance and the reactor dimensions determines the fast leakage. In like manner the relationship between the distance traveled by a slow neutron prior to absorption and the reactor dimensions determines the slow leakage. 
Solution of the continuity equation results in the following statement of the criticality condition

$$
\text { Criticality Equation } \quad \frac{k_{\infty} e^{-B^{2} r}}{1+L^{2} B^{2}}=1 \text {. }
$$

In the previous equation $\mathrm{B}^{2}$ (called geometrical buckling) is related to the reactor shape and dimensions and is determined by the boundary conditions in the solution of the neutron diffusion equation

$$
\nabla^{2} \phi+B^{2} \phi \quad 0
$$

The value of $\mathrm{B}^{2}$ for various reactor configurations is given in Table II- 5 .

TAB LE II- 5

BUCKLING OF BARE REACTORS

\begin{tabular}{l|c|c}
\hline \multicolumn{1}{c|}{ Geometry } & Buckling (B $\left.{ }^{2}\right)$ & $\begin{array}{c}\text { Minimum } \\
\text { Volume }\end{array}$ \\
\hline Sphere & $\left(\frac{\pi}{R}\right)^{2}$ & $\frac{130}{B^{3}}$ \\
\hline Rectangular parallelepiped & $\left(\frac{\pi}{\mathrm{a}}\right)^{2}+\left(\frac{\pi}{\mathrm{b}}\right)^{2}+\left(\frac{\pi}{\mathrm{c}}\right)^{2}$ & $\frac{161}{\mathrm{~B}^{3}}$ \\
\hline Cylinder & $\left(\frac{2405}{\mathrm{R}}\right)^{2}+\left(\frac{\pi}{\mathrm{H}}\right)^{2}$ & $\frac{148}{\mathrm{~B}^{3}}$ \\
\hline
\end{tabular}

$\begin{array}{ll}\mathrm{R}=\text { radius } & \mathrm{a} \quad \text { length } \\ \mathrm{H}=\text { cylinder height } & \mathrm{b}=\text { width }\end{array}$

In the criticality equation above $e^{-B^{2} \Upsilon}$ is the fast neutron nonleakage probability. The Fermi Age, $\Upsilon$, is one sixth the mean square (crow flight) distance traveled by a neutron from the time of its emission to the time that it is slowed down. 
In the criticality equation above $1 /\left(1+L^{2} B^{2}\right)$ is the thermal neutron nonleakage probability. The square of the diffusion length $L^{2}$ is one-sixth the mean square (crow flight) distance a neutron travels after slowing down and before absorption. In summary, then

$$
\begin{aligned}
& \text { Fast neutron nonleakage probability }-P_{f}-e^{-B^{2} \Upsilon} ; \\
& \text { Slow neutron nonleakage probability }-P_{t}=\frac{1}{1+L^{2} B^{2}} \text {, } \\
& \text { Total nonleakage probability }-P-P_{f} P_{t}=\frac{e^{-B^{2} \Upsilon}}{1+L^{2} B^{2}} .
\end{aligned}
$$

The effective multiplication factor $k_{\text {eff }}$ for a finite system is then

$$
k_{e f f}=\frac{k_{\infty} e^{-B^{2} \Upsilon}}{1+L^{2} B^{2}}
$$

and the criticality condition is

$$
\mathrm{k}_{\text {eff }}=1 \text {. }
$$

If $B^{2} \Upsilon$ is small enough that the expansion $e^{-B^{2} \Upsilon}=\left(1-B^{2} \Upsilon\right)$ $=\left(1+B^{2} \Upsilon\right)^{-1}$ is a good approximation, then the criticality condition can be reduced to

$$
k_{\text {eff }}-\frac{k_{o o}}{\left(1+L^{2} B^{2}\right)\left(1+B^{2} \Upsilon\right)}=1 \text {, }
$$

or

$$
\frac{k_{\infty}}{\left[1+B^{2}\left(L^{2}+r\right)\right]}=1 \text {. }
$$


If $\left(L^{2}+\Upsilon\right)$ is replaced by a quantity $M^{2}$ called the "migration area," then the criticality equation reduces to

$$
k_{\text {eff }}=\frac{k_{\omega O}}{1+M^{2} B^{2}}-1 \text {, }
$$

or

$$
\frac{\eta \in \mathrm{pf}}{1+\mathrm{M}^{2} \mathrm{~B}^{2}}=1 \text {. }
$$

The Fermi Age $\Upsilon$ is a function of the moderator scattering cross section and mass number. The diffusion length (L) is a function of the neutron absorption cross section of the reactor. Some typical values of $L^{2}, \Upsilon$, and $M^{2}$ are given in Table II-6.

TABLE II- 6

MODERATOR PROPERTIES

\begin{tabular}{c|c|c|c}
\hline Moderator & $\begin{array}{c}\text { Diffusion } \\
\text { Length } \\
\text { Lm }(\mathrm{cm})\end{array}$ & $\begin{array}{c}\text { Fermi Age } \\
\Upsilon\left(\mathrm{cm}^{2}\right)\end{array}$ & $\begin{array}{c}\text { Migration } \\
\text { Length } \\
\text { M (cm) }\end{array}$ \\
\hline $\mathrm{H}_{2} \mathrm{O}$ & 2.88 & 33 & 6.43 \\
$\mathrm{D}_{2} \mathrm{O}$ & 100. & 120 & 101. \\
$\mathrm{Be}$ & 23.6 & 98 & 25.8 \\
$\mathrm{C}$ & 50.2 & 350 & 53.6 \\
\hline
\end{tabular}

$$
M=\sqrt{L^{2}+\Upsilon}
$$

The addition of fuel to the moderator does not significantly influence $\Upsilon$ except to the degree of dilution, but it increases the neutron-absorption probability which decreases the distance a thermal neutron can wander about before being absorbed. Thus $L$ is a function of fuel concentration. The effective $L$ for the reactor medium can be expressed

$$
\mathrm{L}_{\text {Reactor }}=(1-\mathrm{f}) \mathrm{L}_{\text {Moderator }}
$$

where $f$ is the thermal utilization. 
It can be seen then that when $f$ approaches unity, 1.e., when practically all neutrons are absorbed in fuel, $M^{2}$ approaches $\Upsilon$. In a $100 \%$ enriched $U^{235}$ reactor $\eta \cong 2$ and $\epsilon \mathrm{p} \cong 1$, therefore from

$$
\frac{\eta \in \mathrm{pf}}{1+\mathrm{M}^{2} \mathrm{~B}^{2}}=1 \text {, }
$$

becomes

$$
\frac{2}{1+B^{2} \Upsilon} \cong 1
$$

or

$$
\mathrm{B}^{2} \Upsilon \cong 1
$$

and since for a sphere of radius $R$

$$
\begin{gathered}
\mathrm{B}^{2}\left(\frac{\pi}{\mathrm{R}}\right)^{2}, \\
\left(\frac{\pi}{\mathrm{R}}\right)^{2} \Upsilon \cong 1, \\
\mathrm{R}_{\text {Critical }} \cong \pi \sqrt{\Upsilon} .
\end{gathered}
$$

Based on this approximation, the minimum size of various homogeneous $\mathrm{U}^{235}$ spheres is shown in Table II-7.

TAB LE II- 7

MINIMUM SIZE OF BARE HOMOGENEOUS U ${ }^{235}$ SPHERES

\begin{tabular}{c|c|c|c}
\hline Moderator & $\pi \sqrt{\Upsilon}(\mathrm{cm})$ & $\begin{array}{c}\mathrm{R} \text { Critical } \\
(\mathrm{cm})\end{array}$ & $\begin{array}{c}\text { Volume } \\
\left(\mathrm{ft}^{3}\right)\end{array}$ \\
\hline $\mathrm{H}_{2} \mathrm{O}$ & 18 & 16 & 0.65 \\
$\mathrm{D}_{2} \mathrm{O}$ & 34.5 & 32 & 5. \\
$\mathrm{Be}$ & 31 & 29 & 3.6 \\
$\mathrm{C}$ & 59 & 55 & 23 \\
\hline
\end{tabular}

Adjusted for boundary condition (extrapolation distance). 


\section{f. Reactor Flux and Power Distribution}

The neutron flux distribution in a reactor is determined by the solution of the neutron diffusion equation

$$
\nabla^{2} \phi+B^{2} \phi=0
$$

for appropriate coordinate systems and boundary conditions. The results for the common geometric shapes are given in Table II- 8 . These solutions apply only to a critical reactor.

TAB LE II- 8

FLUX DISTRIBUTION IN BARE CRITICAL REAC TORS

\begin{tabular}{l|c}
\hline \multicolumn{1}{c|}{ Geometry } & Critical Flux Distribution \\
\hline Sphere & $\phi(x)=\frac{A}{r} \sin \frac{\pi r}{R}$ \\
Rectangular Parallelopiped & $\phi(x, y, z)=A \cos \frac{\pi x}{\mathrm{a}} \cos \frac{\pi y}{\mathrm{~b}} \cos \frac{\pi \mathrm{z}}{\mathrm{C}}$ \\
Finite Cylinder & $\phi(\mathrm{r}, \mathrm{z})=\mathrm{A} \mathrm{J}_{\mathrm{O}} \frac{2.405 \mathrm{r}}{\mathrm{R}} \cos \frac{\pi \mathrm{z}}{\mathrm{H}}$ \\
\hline
\end{tabular}

A comparison of the three functions, 1. e., $\frac{1}{r} \sin \frac{\pi r}{R}, \cos \frac{\pi x}{a}$, and $J_{0}\left(\frac{2.405 r}{R}\right)$ will reveal that a cosine function is a good approximation for all.

In a homogeneous reactor, wherein the fuel is uniformly dispersed throughout the reactor volume, the power distribution is the same as the flux distribution. The resulting ratio of maximum to average power in bare reactors is given in Table II-9.

TAB LE II-9

PEAK-TO-AVERAGE POWER FOR BARE REACTORS

\begin{tabular}{l|c}
\hline \multicolumn{1}{c|}{ Geometry } & $P_{\max } / P_{a v g}$ \\
\hline Sphere & 3.29 \\
Rectangular Parallelopiped & 3.87 \\
Finite Cylinder & 3.64 \\
\hline
\end{tabular}


The maximum-to-average power ratio must be considered in the detailed heat-transfer and fuel burnup design of a reactor.

\section{g. Reflector}

In small reactors, criticality is a strong function of neutron leakage. The overall neutron economy of a reactor can be significantly improved by the addition of a nonfissionable blanket of material whose function is to reflect neutrons back into the reactor core and thus decrease the leakage. The best reflecting materials in general are those with high scattering cross sections and low neutron absorption characteristics. Whereas in the bare reactor the neutron flux approaches zero at the core boundary, in the reflected reactor the flux is increased at the core boundary and does not approach zero until some distance into the reflector region. The net result is that the effective reactor size is increased beyond the extent of the core. The flux distribution and effective core size change are illustrated in Figure $\amalg-6$.

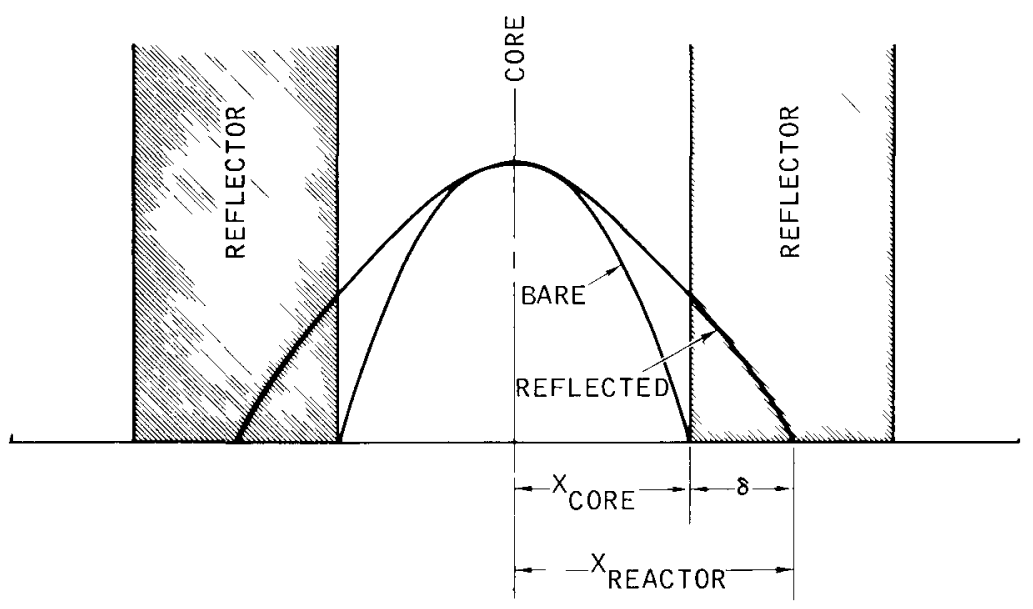

7-57-192-22

Figure II-6 Effect of a Reflector on Neutron Flux Distribution and Effective Reactor Size 
The increase in effective reactor size is called the "reflector savings," $\delta$. The magnitude of $\delta$ is a function of the reflector thickness and the nuclear properties of the core and reflector regions. For small reflector thickness $\delta$ is a fraction of the thickness. For large reflector thicknesses $\delta$ approaches a constant limit. This asymptotic value of $\delta$ is practically achieved when the reflector thickness is between 1.5 and 2 times the neutron-diffusion length in the reflector material. Therefore, as reflector thickness is increased the effective reactor dimensions increase or the core size can be decreased to maintain the same effective size. When the reflector is thick enough to yield the asymptotic value of $\delta$, no further benefit results from increased reflector thickness. This point is illustrated in Figure II- 7 .

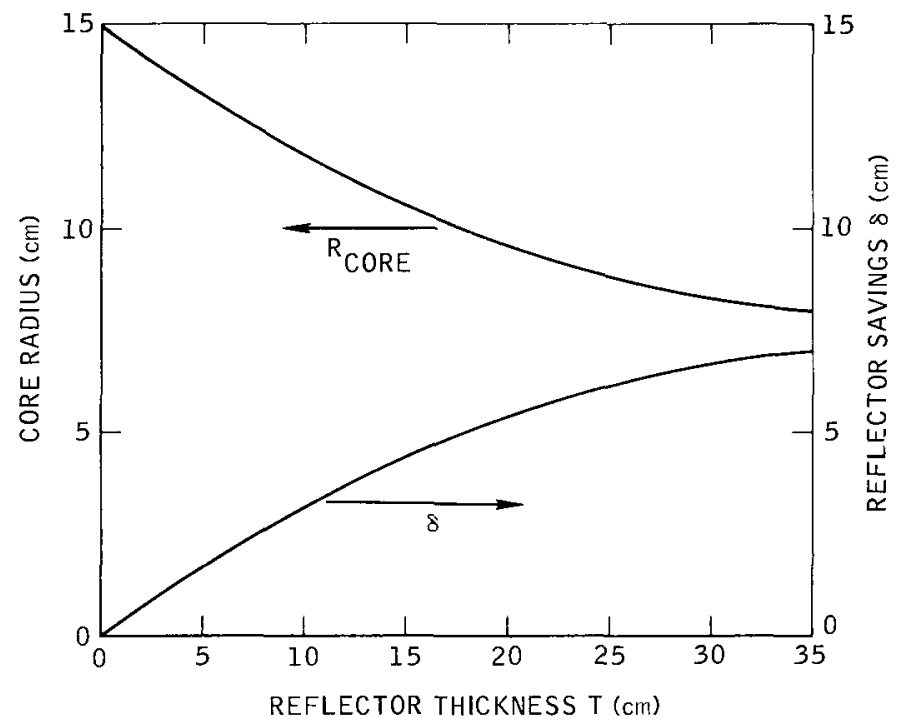

7-57-192-23

Figure II-7. Effect of Reflector Thickness on Reactor Core Size and Reflector Savings as a Function of Thickness 
The effective reactor dimension is then

$$
\mathrm{x}_{\text {Reactor }}=\mathrm{x}_{\text {Core }}+\delta
$$

This effective dimension is now used in calculating $B^{2}$ and leakage and also in describing the flux or power distribution.

For compact reactors the reflector has several significant advantages.

1) The critical mass or uranium inventory is decreased as a result of the smaller allowable core.

2) For certain material combinations the overall weight of the core plus reflector can be made less than the weight of the bare reactor by choosing an optimum reflector thickness.

3) The increased neutron flux at the core boundary decreases the peak-to-average power distribution in the reactor. In a compact reactor a decrease factor of 2 is reasonable.

4) Variations in reflector thickness can be used as an effective way to change leakage and thus control the reactor. This is especially true for compact reactors wherein $\delta$ can be an appreciable fraction of the effective reactor dimensions.

\section{h. Reactor Kinetics}

The multiplication factor $k$ is effectively the number of neutrons present at the end of a neutron generation for each neutron present at the beginning of that generation. Since one neutron is required to maintain the chain reaction, the number of neutrons will increase by $k$ - 1 in a generation. Thus, if there are $n$ neutrons present initially, the rate of increase will be $n(k-1)$ per generation. If $\ell$ is the average time between successive neutron generations in the system under consideration, then,

$$
\frac{\mathrm{dn}}{\mathrm{dt}}=\frac{\mathrm{n}(\mathrm{k}-\mathrm{I})}{\ell}=\frac{\mathrm{nk} \mathrm{ex}}{\ell},
$$


where $k_{\text {ex }}$ is defined by

$$
\mathrm{k}_{\mathrm{ex}} \equiv \mathrm{k}-1
$$

Upon integration of above, it is seen that,

$$
n=n_{0} e^{t\left(k_{e x} / \ell\right)}
$$

where $n_{0}$ is the initial number of neutrons and $n$ is the number after the lapse of time $t$. It is seen, therefore, that if the multiplication factor is greater than unity, the number of neutrons will increase exponentially with time.

The generation time, $\ell$, varies from about $10^{-9} \mathrm{sec}$ for a fast reactor to $10^{-3} \mathrm{sec}$ for very large reactors. If for example $k=1.005$ and $\ell=10^{-3} \mathrm{sec}$, the neutron increase per second equals $e^{5}$ or a factor of $150 / \mathrm{sec}$.

The above calculation of the rate of neutron increase in a reactor with a multiplication factor exceeding unity gives the correct rate of neutron increase only if all the fission neutrons are released promptly, i.e., essentially at the instant of fission. About $0.75 \%$ of the fission neutrons are delayed, and this affects the calculation of the rate of neutron increase (or decrease).

The mean lives of the delayed neutrons range from about 0.6 to $80 \mathrm{sec}$. By weighting the values appropriately, according to the fraction in each group, the mean delay time, averaged over all the fission neutrons, is about $0.1 \mathrm{sec}$. The average time between the fission capture of a neutron in two successive generations is, consequently, about $0.1+\ell \mathrm{sec}$, the first term is the average time elapsing between fission and the complete release of the neutron, whereas the second is that between release and capture in a fission process. In other words, the effective lifetime $\bar{l}$ of a neutron 1 s roughly $0.1 \mathrm{sec}$. 
Using the value $0.1 \mathrm{sec}$ for $\bar{l}$, and taking $\mathrm{k}$ to be 1.005 , as before, it is found that the number of neutrons actually increases by a factor of $\mathrm{e}^{0.05}$, 1. e., about $1.05 / \mathrm{sec}$, as compared with a factor of $150 / \mathrm{sec}$ if all the neutrons were prompt. Clearly, the effect of the delayed fission neutrons, when the multiplication factor exceeds unity, 15 to make the rate of neutron increase much slower than it would have been had all the neutrons been released promptly.

When the effective multiplication factor 1 s equal to 1.0075 , the condition of a reactor is described as prompt critical, since the nuclear fission chain can be maintained by means of the prompt neutrons alone. If $k$ exceeds this value, multiplication will occur due to the prompt neutrons, irrespective of those delayed, and the neutron density will increase rapidly right from the commencement. In this condition, a reactor is $\mathrm{d}_{1} \mathrm{ff}_{1-}$ cult to control and hence it is avoided in practice.

Just as the delayed fission neutrons affect the rate of in crease of neutrons when the effective multiplication factor exceeds unity, so they influence the decay in the neutron density when the reactor is made subcritical, 1 .e., when it is being shut down. The delayed neutrons continue to be emitted for some time, and this maintains a fission rate that is considerably higher than would be the case if all the fission neutrons were prompt. The ultimate rate at which the neutron flux in a thermal reactor decreases after shutdown is determined essentially by the most delayed group of neutrons, 1. e., by those with a mean life of $80 \mathrm{sec}$.

\section{i. Reactor Control}

For practical operation, a reactor must be constructed so that it is appreciably greater than the critical size. One reason is that an effective multiplication factor exceeding unity provides the only feasible means of increasing the number of neutrons, and hence the fission rate, up to the point where the required power level is attained. Once this has been reached, it is 
necessary to decrease the effective multiplication factor to unity, and then the reactor will remain in a steady state, neutrons being produced just as fast as they are used up by leakage and capture.

The adjustment of the multiplication of neutrons in a thermal reactor is achieved by the insertion of control rods of cadmium or boron steel. Both cadmium and boron have large capture cross sections for slow neutrons, hence, by varying the positions of the control rods the effective multiplication factor can be made to vary over a suitable range. In order to shut down the reactor, the control rods are inserted to an extent that permits them to absorb additional neutrons. The system now loses neutrons faster than they are formed by fission, the effective multiplication factor sinks below unity, and the chain reaction dies out.

The insertion of poison or control rods changes the effective multiplication factor, $k_{\text {eff }}$, by influencing the thermal utilization, f. A reactor can also be controlled by means of variation in the neutron leakage probability. In a reflected reactor this type of control called reflector control is achieved by moving portions of the reflector in order to vary the neutron leakage probability and thus the effective multiplication factor.

\section{REACTOR DESIGN \\ a. Size}

In the case of space reactors, wherein size and weight are of extreme importance, the dominant factor in the determination of reactor size is the leakage probability. In any reactor, whether it be thermal, epithermal, or fast, the leakage is dependent on a relationship between the reactor's physical size and the distance between succeeding fission events. For a thermal or nearly thermal reactor the distance between fission events is controlled by the number of neutron collisions or the distance necessary to slow the fission neutrons down from fission energy to the thermal energy at which they are captured by 
the fuel, and results in the succeeding fission. This slowing down distance is a function of the moderator material scattering cross section and atomic mass. A moderator material property, known as the Fermi age $(\Upsilon)$, is related to the mean square slowing down distance and has units of $\mathrm{cm}^{2}$. The influence of slowing down distance is revealed in Figure II- 8 which shows the critical volume of $100 \%$ dense bare spheres with critical mass in the range of 2.5 to $20 \mathrm{~kg}$ of $U^{235}$ as a function of Fermi age with the common moderator materials indicated on the abscissa. It is obvious that $\mathrm{H}_{2} \mathrm{O}$ or hydrogenous moderation permits the smallest thermal reactor. The exceptional ability of water is a result of the hydrogen content. Since the hydrogen atom has the same mass as the neutron, a neutron can transfer up to all of its kinetic energy to the hydrogen atom in one collision. Thus, hydrogenous materials are very effective neutron moderators.

A more detailed survey of the relationship between critical mass and size of $100 \%$ dense bare spherical reactors is shown in Figure II-9 for mixtures of $\mathrm{U}^{235}$ with $\mathrm{H}_{2} \mathrm{O}$ and $\mathrm{Be}$. Both curves begin at the zero moderator point which corresponds to the critical mass and size of a $\mathrm{U}^{235}$ fast reactor. In the region to the left of the minimum critical mass point, the reactors are said to be "under moderated" and the average neutron energy causing fission is epithermal (greater than KT). In the region to the right of the critical mass minimum the reactors are over moderated and thermal. Figure II- 10 shows the weight of $U^{235}$ $\mathrm{Be}, \mathrm{U}^{235}-\mathrm{H}_{2} \mathrm{O}$, and $\mathrm{U}^{235}-\mathrm{ZrH}_{\mathrm{x}}$ reactors as a function of moderator to uranium ratio. Zirconium hydride $\left(\mathrm{ZrH}_{\mathrm{x}}\right)$ has been included because it is a high temperature $\left(1400^{\circ} \mathrm{F}\right)$ form of hydrogenous moderator. $Z_{r H}$ can have the same hydrogen density as cold water at temperatures of about $1200^{\circ} \mathrm{F}$ with a dissociation pressure of only 1 atmosphere (see Figure II-11). The U-ZrH ${ }_{x}$ calculations were based on the critical size of a $\mathrm{H}_{2} \mathrm{O}$ reactor but the difference arises from the fact that $\mathrm{ZrH}_{\mathrm{x}}$ has a density of about 5.6. 


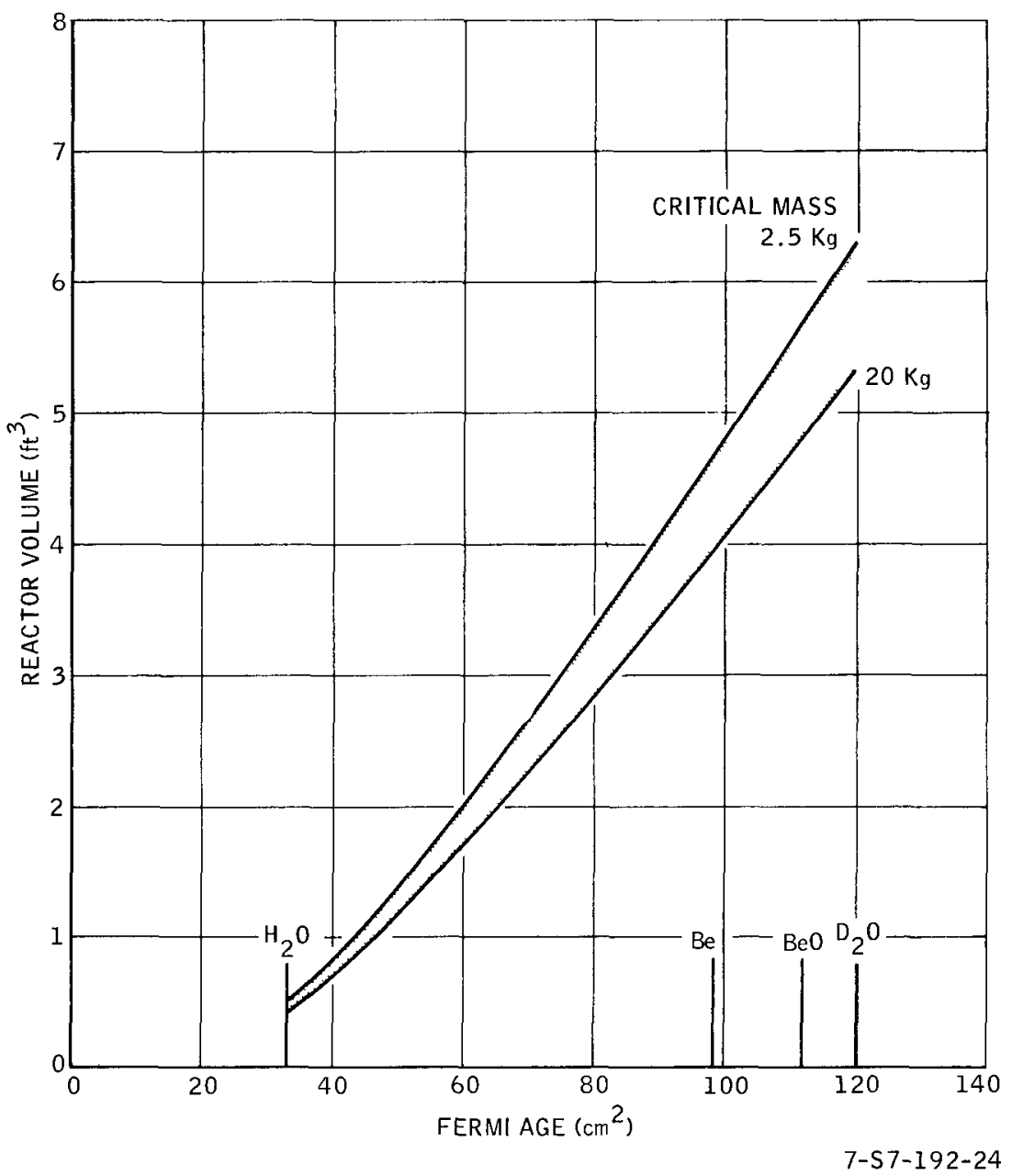

Figure II-8. Volume of Bare 100\% Dense Spherical Reactors with Critical Mass in the Range of 2.5 to $20 \mathrm{~kg}$ as a Function of Fermi Age 


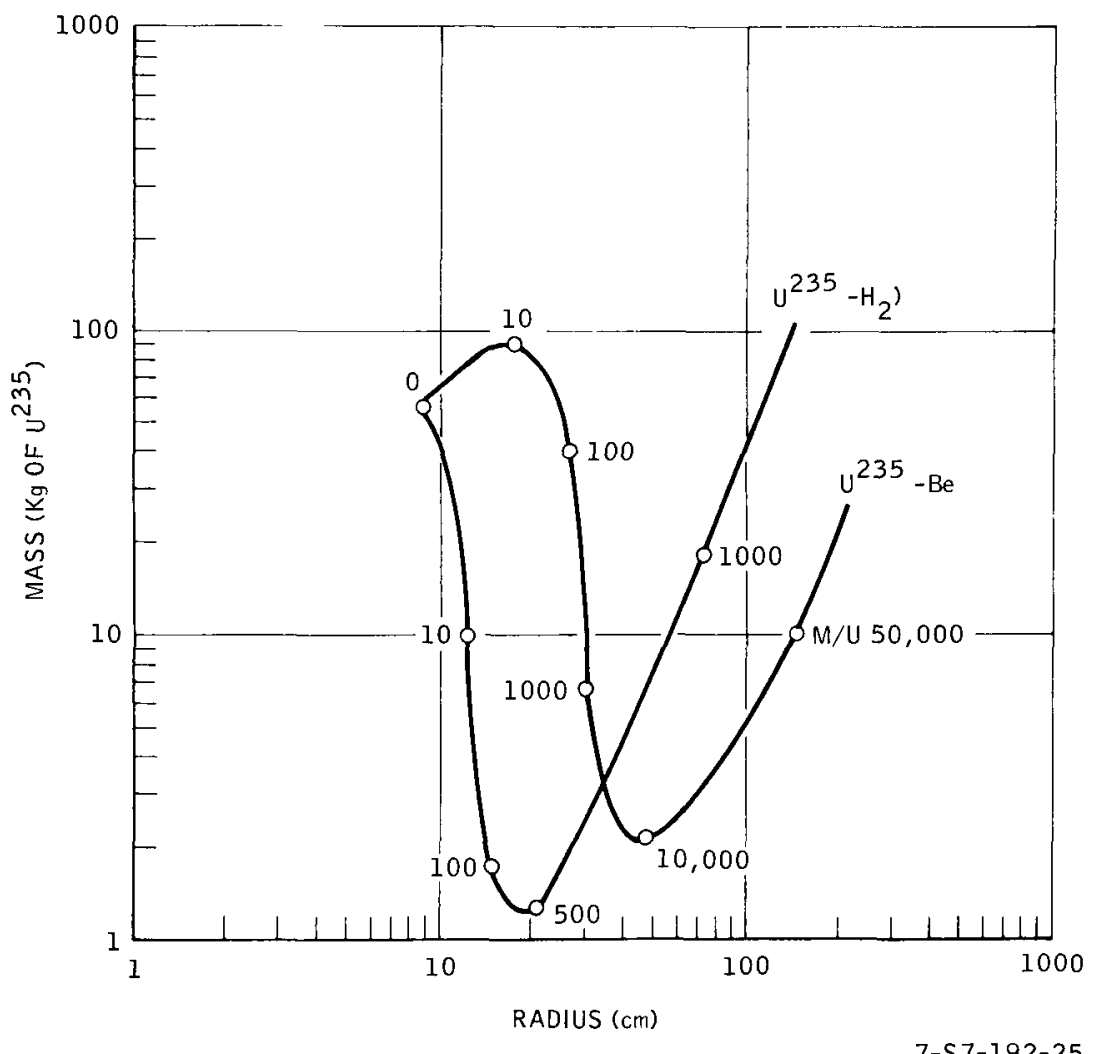

Figure II-9. Critical Mass of Bare 100\% Dense Homogeneous Spheres of $\mathrm{U}^{235}$ Moderated by Water and by Be as a Function of Radius and Moderator to U Atom Ratio 


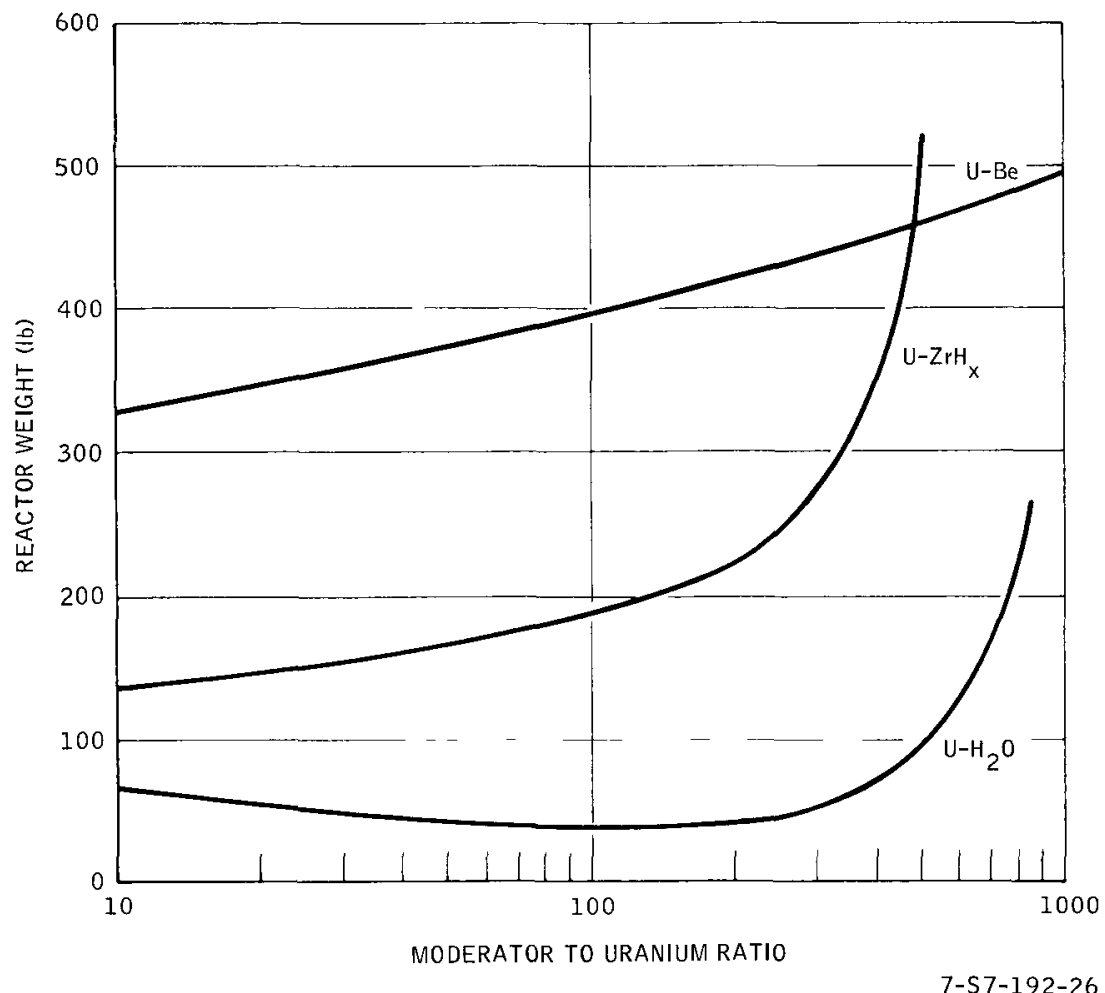

Figure II-10. Weight of Bare 100\% Dense Spherical

Reactors as a Function of Moderator to

Uranium Atom Ratio 


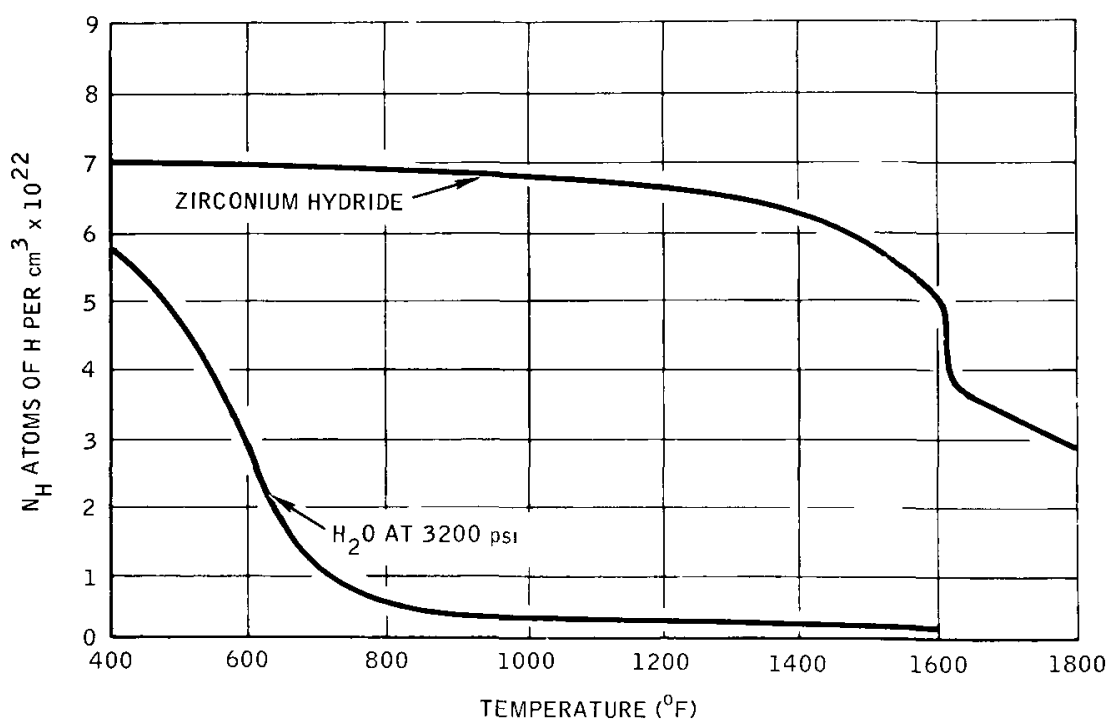

$7-57-192-27$

Figure II-11. Hydrogen Density vs Temperature for Zirconium Hydride at 1 Atmosphere

Dissociation Pressure and Water at the Critical Pressure

The information discussed thus far has been for reactors of $100 \%$ density. A useful reactor must contain coolant passages for the removal of the reactor heat. The presence of such voids effectively decreases the density of the reactor. In order to maintain criticality the dimensions of a reactor must vary inversely as the density of the core material (fuel moderator). Therefore, since the volume varies as (density) ${ }^{-3}$ weight will be proportional to (density) ${ }^{-2}$. The result of density dependence is shown in Figure II- 12 which gives the weight of bare spherical fast reactors as a function of density. This type of density dependence holds for all reactors. 


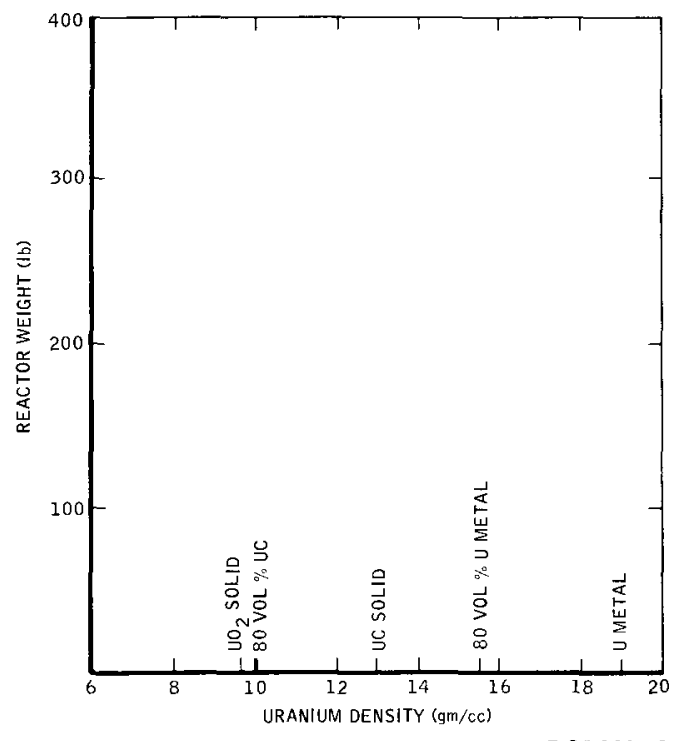

$7-57-192-28$

Figure II- 12. Weight of Bare Spherical Fast Reactors as a Function of Uranium Density

In addition to satisfying the basic criticality requirements for maintaining a chain reaction the reactor size is influenced by:

1) Operating temperature

2) Excess reactivity requirements for compensation of fuel depletion, fission product poisons, etc.

3) Power density or heat transfer limitations

4) Energy density or fuel burnup limitations.

\section{b. Temperature}

The size of a reactor is somewhat influenced by the operating temperature because the fuel and moderator material nuclear properties are a function of temperature. In addition, as stated above, the reactor size is inversely proportional to density which varies with temperature. The most significant temperature limitations of a reactor are the materials strength, corrosion, compatibility, etc., limitations of the structural materials. 
Even though a reactor can theoretically produce power at any temperature, the engineering limitations of the fuel, moderator, and structure limit the available reactor outlet temperature. Even though it cannot be explicity defined there is an intuitive relationship between operating temperature and lifetime. Figure II- 13 shows the lifetime-temperature relationship between current accomplishment and development objectives in nuclear power. This figure implies a state-of-the-art boundary for perhaps the next 10 to 20 years. This state-of-the-art boundary strongly influences the reactor concept selection for immediate development and emphasizes the fact that high temperature materials development will pace the development of high temperature and high performance space systems of the future. A similar conclusion can be drawn about the state of the art of hightemperature reactors from Figure II- 14 which shows the number of operating and planned reactors as a function of the reactor outlet temperature. Of course the cholce of power reactor temperatures is greatly influenced by economics which may be considered as not applicable for space power. However, in effect, cost is probably one of the best direct measures of a state of the art.

The approximate minimum welght of various reactors is shown as a function of temperature in Figure II-15. The weights shown are for $100 \%$ dense spheres. Even though these weights could be reduced by the addition of a Be reflector, the inclusion of a void fraction for the reactor coolant would, in general, more than offset the weight reduction. Therefore, these weights can be considered representative, but by no means exact.

The aqueous homogeneous solution-type reactor is very light at low temperature but the moderator density decreases rapidly with temperature and the pressure necessary to suppress bolling soon becomes inconsistent with the objective of a lightweight reactor (the curve does not include the core vessel weight). The $\mathrm{U}^{235}$ metal fast reactor is quite small, however, It must be $11 \mathrm{mited}$ to below $1200^{\circ} \mathrm{F}$ because of a large density 


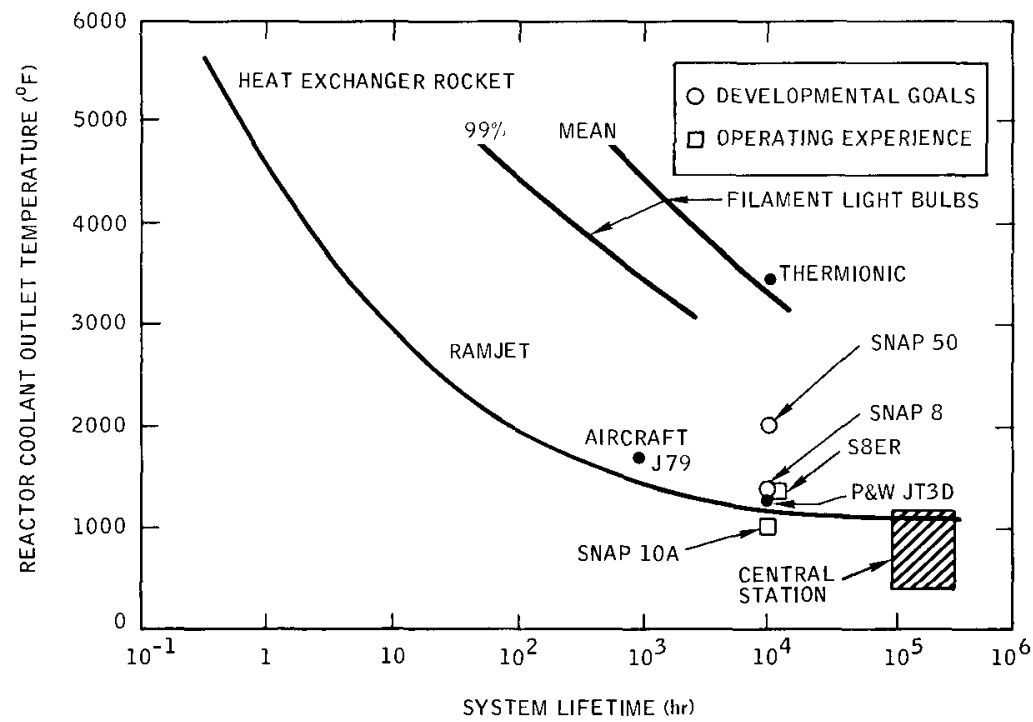

7 A4 $17624 \mathrm{~A}$

Figure II-13. System Lifetime vs Operating Temperatures

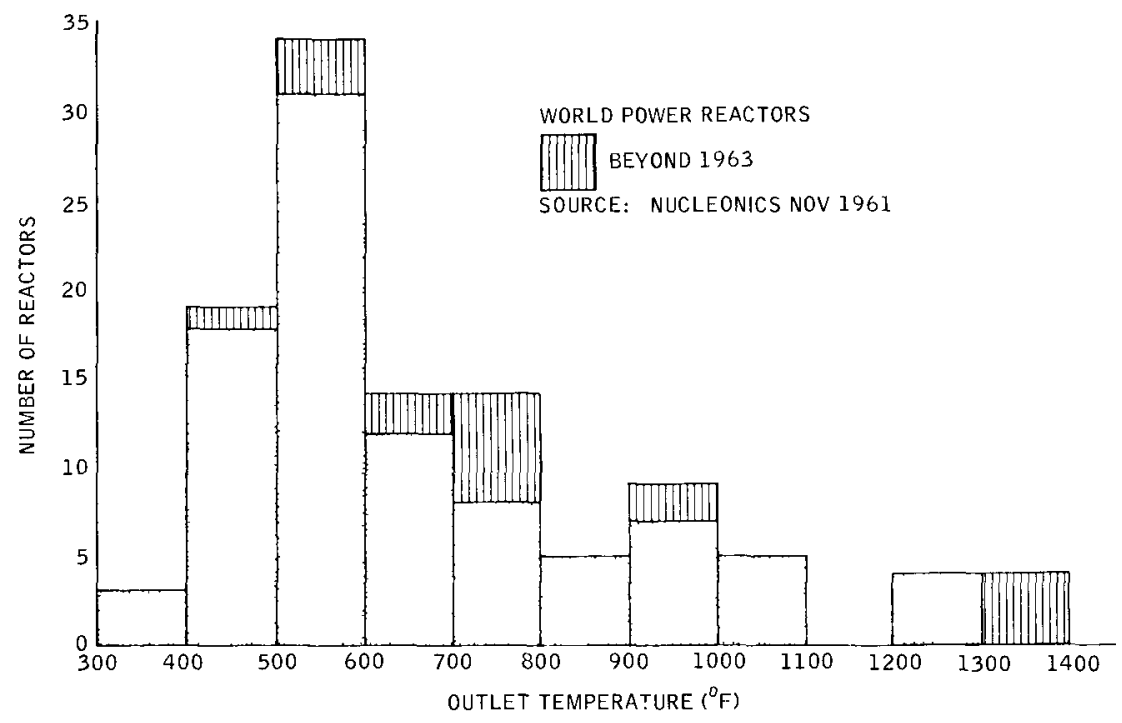

$7-57-192-29$

Figure II-14. Number of Power Reactors in Operation or Planned for Immediate Future as a Function of Operating Temperature 


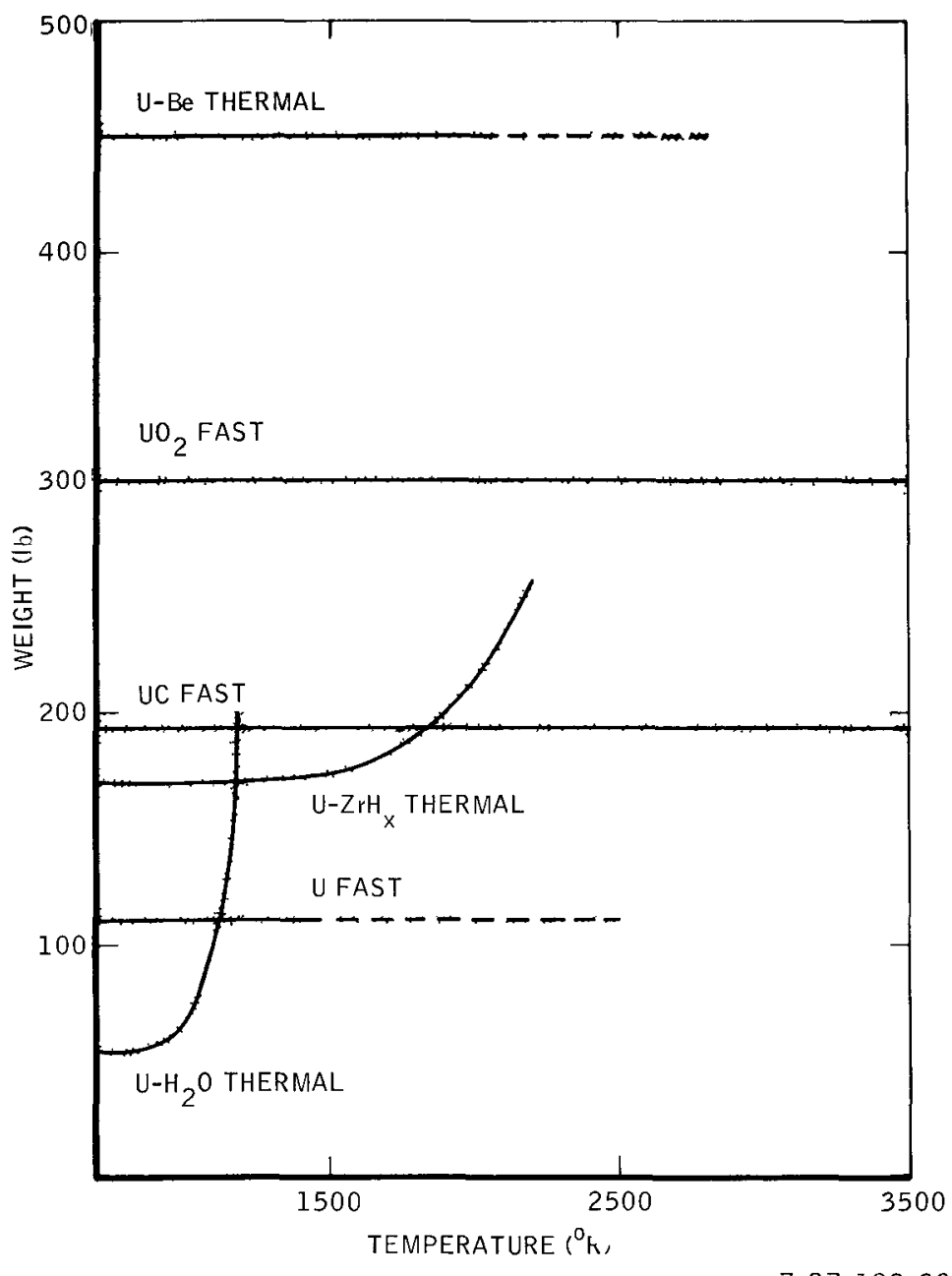

7-57-192-30

Figure II-15. Weight of Bare 100\% Dense Spherical Reactors as a Function of Operating Temperature 
change that accompanies a phase change at $1224^{\circ} \mathrm{F}$. The $\mathrm{U}-\mathrm{ZrH}_{\mathrm{x}}$ system is similar in size to the $\mathrm{H}_{2} \mathrm{O}$ moderated reactor because it can have the same hydrogen density as water below about $1200^{\circ} \mathrm{F}$. Beyond $1200^{\circ} \mathrm{F}$ the size and weight increase because the hydrogen density must be decreased in order to control the thermal dissociation pressure of the compound. The UC and the $\mathrm{UO}_{2}$ fast reactors are capable of up to 4200 and $5100^{\circ} \mathrm{F}$, respectively, the U-Be thermal reactor is limited in its temperature capability because of radiation damage induced swelling of $\mathrm{Be}$ at temperatures above about $1500^{\circ} \mathrm{F}$.

\section{c. Control}

In addition to the excess reactivity required to overcome the general reactivity decrease resulting from temperature, high power systems must have sufficient excess reactivity, and thus increased size, to compensate for burnup or depletion of the initial uraniurn inventory and the buildup of fission products of which some have high neutron absorption cross sections. The major fission product poisons are samarium and xenon. The most troublesome of these is xenon since the amount of poisoning is largest and since the poisoning increases rapidly and goes through a maximum after reactor shutdown or power decrease. This behavior imposes a serious limit on the allowable duty cycle of a high power density thermal reactor. Therefore it is very desirable to maintain reactor power constant. Fast reactors are not as greatly influenced by fission product poisons in general because the absorption cross sections are much lower at the neutron energy of the fast reactor.

These excess reactivity requirements impose a reactor control problem. In order to control the reactor from shutdown to operation throughout its lifetime the reactor must have more control worth than excess reactivity requirement. The amount of control that can be provided is a function of reactor size. Large reactors must be controlled by poison rods since leakage is small and provides little range for variation. Small reactors 
can be leakage controlled by varying the effective reflector thickness. However, the total amount of control avallable will decrease with increasing reactor size because the leakage decreases with size. Therefore, control requirements determine the appropriate control method and can limit the power density or energy content of a given reactor design.

\section{d. Power Density}

In addition to the fuel depletion and fission product poison considerations reactor size is a function of power density because (1) the reactor density is decreased by the inclusion of coolant passages, and (2) surface or volumetric heat transfer and stress liriltations can limit the power density.

In order to minimize both the reactor coolant fraction and the coolant pumping power, high temperature and high heat capacity coolants are desired. These conditions are best met by liquid metals. The properties of selected liquid metal coolants are shown in Table II-10. It must be remembered that the reactor materials and the coolant must be compatible from a corrosion standpoint at the operating temperature.

If the reactor fuel element design or the coolant impose a heat flux limitation, then the allowable reactor power density will be a function of the heat transfer surface per unit volume. It is therefore desirable for the reactor to contain the maximum amount of heat transfer surface consistent with the needs. The core materials of a thermal reactor can be arranged in two ways. The heterogeneous reactor has the fuel separated from the moderator. Since a thermal reactor has only a few volume percent fuel and since practically all of the power is generated in the fuel, only a few volume percent are therefore avalable to provide or contain heat transfer surface. The homogeneous reactor has the fuel and moderator intimately mixed and power is produced throughout the entire core volume. Therefore the entire core volume of a homogeneous reactor is avallable for heat transfer surface. Since the core of a fast reactor contains 
TAB LE II- 10

PHYSICAL PROPERTIES OF SOME SELECTED LIQUID METALS

\begin{tabular}{|c|c|c|c|c|c|c|}
\hline & Sodium & $\begin{array}{c}\mathrm{NaK} \\
44 \% \mathrm{~K} \\
\end{array}$ & $\mathrm{Hg}$ & $\mathrm{Pb}$ & $\begin{array}{c}\mathrm{Pb}-\mathrm{B}_{1} \\
\text { Eutectic }\end{array}$ & $\mathrm{L}_{1}$ \\
\hline Melting point, ${ }^{\circ} \mathrm{F}$ & 208 & 65 & -37 & 622 & 257 & 354 \\
\hline Bolling point, ${ }^{\circ} \mathrm{F}(147 \mathrm{ps})$ & 1621 & 1518 & 675 & 3270 & 3038 & 2403 \\
\hline Liquid density, $\mathrm{gm} / \mathrm{cm}^{3}$ & $0 \quad 928-0 \quad 78$ & $0886-0742$ & $135-123$ & 102 & 10 & $0 \quad 507-0 \quad 441$ \\
\hline Specific heat, Btu/lb- ${ }^{\circ} \mathbf{F}$ & $033-0 \quad 30$ & $0269-0253$ & 0033 & $0 \quad 034$ & 0035 & 10 \\
\hline Thermal conductivity, Btu/ft ${ }^{2}$ & $498-345$ & $148-167$ & $5-9$ & 8 & $53-65$ & 22 \\
\hline Heat transfer coefficient, Btu/hr- ${ }^{\circ} \mathrm{F}-\mathrm{ft}^{2}$ & 6400 & 3500 & 5700 & 4100 & 3700 & 5800 \\
\hline Pumping power (water -100 ) & 0925 & G 925 & 131 & 115 & 115 & 05 \\
\hline Probable temperature range, ${ }^{\circ} \mathrm{F}$ & $250-1500$ & $100-1400$ & $-37-1000$ & $650-1700$ & $300-1700$ & $400-2300$ \\
\hline
\end{tabular}

At $10 \mathrm{ft} / \mathrm{sec}$ in 1 in diameter tube 
fuel only, it has the same advantage as a homogeneous reactor Thus, for minimum weight, high power density reactors the homogeneous arrangement of core materials is far superior

If we consider a reactor whose core 15 composed of a bundle of mutually tangent cylindrical fuel elements, the heat transfer surface per unit volume is

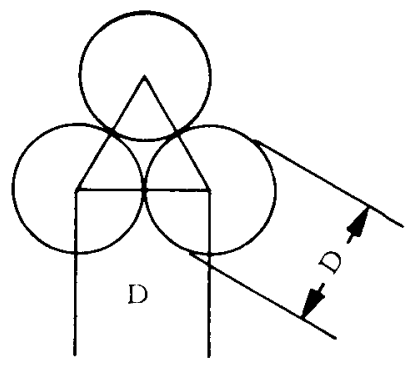

$$
\begin{aligned}
& \frac{\text { Surface }}{\text { Volume }}=\frac{1 / 2 \pi D}{1 / 2 D \frac{\sqrt{3}}{2} D} \\
&=\frac{2 \pi}{\sqrt{3} D} \\
& \frac{\text { Surface }\left(\mathrm{ft}^{2}\right)}{\text { Volume }\left(\mathrm{ft}^{3}\right)}=\frac{436}{D(\mathrm{in})}
\end{aligned}
$$

If the limiting heat transfer flux is $Q / A$ then

$$
Q / V=\frac{A}{V} \frac{Q}{A}
$$

and

$$
\frac{Q}{V}\left(\frac{M w}{f t^{3}}\right)=\frac{12.8}{D(1 \mathrm{n} .)} \frac{Q}{A}\left(\frac{10^{6} \mathrm{Btu}}{\mathrm{hr}-\mathrm{ft}}\right) .
$$

The maxımum temperature drop across a given fuel element may be limited by maximum temperature considerations resulting from the fuel material melting point, phase change, etc., or it may be limited by a maximum allowable stress.

The temperature drop across a cylinder with uniform surface cooling and uniform volumetric heat generation is

$$
\Delta T=\frac{(Q / V) D^{2}}{16 K}
$$


Therefore for a given $\Delta \mathrm{T}$ limit

$$
\begin{gathered}
\frac{Q}{V}=\frac{16 K \Delta T}{D^{2}}, \\
\frac{Q}{V}\left(\frac{M w}{f t^{3}}\right)=\frac{0.000675}{D^{2}\left(\mathrm{~m}^{2}\right)}(K \Delta T) .
\end{gathered}
$$

where $\mathrm{K}$ is the thermal conductivity in $\mathrm{Btu} / \mathrm{hr}-\mathrm{ft}-{ }^{\circ} \mathrm{F}$ and $\Delta \mathrm{T}$ is in ${ }^{\circ} \mathrm{F}$. If there exists a $\Delta \mathrm{T}$ limit, the power density is a function of the fuel element dimensions and $K \Delta T$ which is a material property. For example, uranium metal undergoes a phase change at about $1200^{\circ} \mathrm{F}$ which results in a $5 \%$ density decrease. This phase change must be avolded for metallurgical as well as reactivity reasons. If a uranium metal fast reactor 1 s operating at a fuel surface temperature of $1000^{\circ} \mathrm{F}$ then the maximum $\Delta \mathrm{T}$ can be only $200^{\circ} \mathrm{F}$. The thermal conductivity of uranium metal is about 15 and therefore $\mathrm{K} \Delta \mathrm{T}=3000$. The allowable power density for $1 / 4-1$. diameter fuel rods is then $Q / V=\left[0.000675 /(1 / 4)^{2}\right] 3000 \cong$ $32 \mathrm{Mw} / \mathrm{ft}^{3}$. The volume of such a reactor might be about $1 / 10 \mathrm{ft}^{3}$. Thus, 1 could produce $3.2 \mathrm{Mw}$. If a higher power were desired the surface temperature would have to be reduced or the fuel rod diameter reduced, or the reactor made larger for heat transfer purposes. We might say the reactor size is criticality limited below $3.2 \mathrm{Mw}$ and heat transfer limited beyond $3.2 \mathrm{Mw}$.

The temperature drop across a material leads to a stress. In the case of a cylinder the maximum tensile stress $1 \mathrm{~s}$

$$
\sigma=\frac{E \alpha \Delta T}{2(1-\nu)}
$$

where

$$
\begin{aligned}
E & =\text { Young's modulus }\left(\mathrm{ps}_{1}\right) \\
\alpha & =\text { linear coefficient of expansion }\left({ }^{\circ} \mathrm{F}\right)^{-1} \\
\nu & =\text { Poisson's ratio } \cong 1 / 3 \\
\sigma_{\max } & =\text { allowable stress }(\mathrm{ps} 1) .
\end{aligned}
$$


If the stress leads to brittle fracture in the material and the fracture influences heat transfer or mechanical integrity, then the stress must be kept below the fracture stress and the allowable $\Delta \mathrm{T}$ is

$$
\Delta \mathrm{T}_{\max }=\frac{4}{3} \frac{\sigma_{\mathrm{max}}}{\mathrm{E} \alpha},
$$

and from above

$$
\frac{Q}{V} \quad \frac{0.0009}{D^{2}} \frac{K \sigma_{\max }}{E} \text {. }
$$

The physical properties of two potential high temperature, high power density fast reactor core materials are given in Table II- 11.

TAB LE II - 11

\begin{tabular}{|c|c|c|c|c|}
\hline Property & Metal & $\mathrm{UO}_{2}$ & $\mathrm{UC}$ & $\mathrm{UN}$ \\
\hline Density (gm/cc) & 19.0 & 10.96 & 13.63 & - \\
\hline U Content ( $\mathrm{gm} / \mathrm{cc})$ & - & 9.66 & 12.97 & 13.52 \\
\hline Melting Point $\left({ }^{\circ} \mathrm{F}\right)$ & 2070 & 5100 & 4200 & 5230 \\
\hline $\begin{array}{l}\text { Thermal Conductivity } \\
\left(\mathrm{Btu} / \mathrm{hr}-\mathrm{ft}-{ }^{\circ} \mathrm{F}\right)\end{array}$ & 15 & 1 & 14 & 12 \\
\hline $\begin{array}{l}\text { Linear Coefficient of } \\
\text { Expansion } \times 10^{6}\end{array}$ & $\sim 20$ & 4 & 6.7 & - \\
\hline $\begin{array}{l}\text { Modulus of Elasticity } \\
\left(\text { psi } \times 10^{-6}\right)\end{array}$ & 20 & 25 & 30 & - \\
\hline $\begin{array}{l}\text { Modulus of Rupture } \\
\left(\text { psi } \times 10^{-3}\right)\end{array}$ & - & 25 & 25 & - \\
\hline$\sigma \mathrm{K} / \mathrm{E} \boldsymbol{\alpha}=\mathrm{K} \Delta \mathrm{T}_{\max }$ & - & $1 / 4 \times 10^{3}$ & $5 / 3 \times 10^{3}$ & - \\
\hline
\end{tabular}

FUEL PROPER TIES

Anisotropic

For example, consider a fast reactor which uses $\mathrm{UO}_{2}$ for the fuel material and has 1/4-1n, diameter fuel elements. Since 


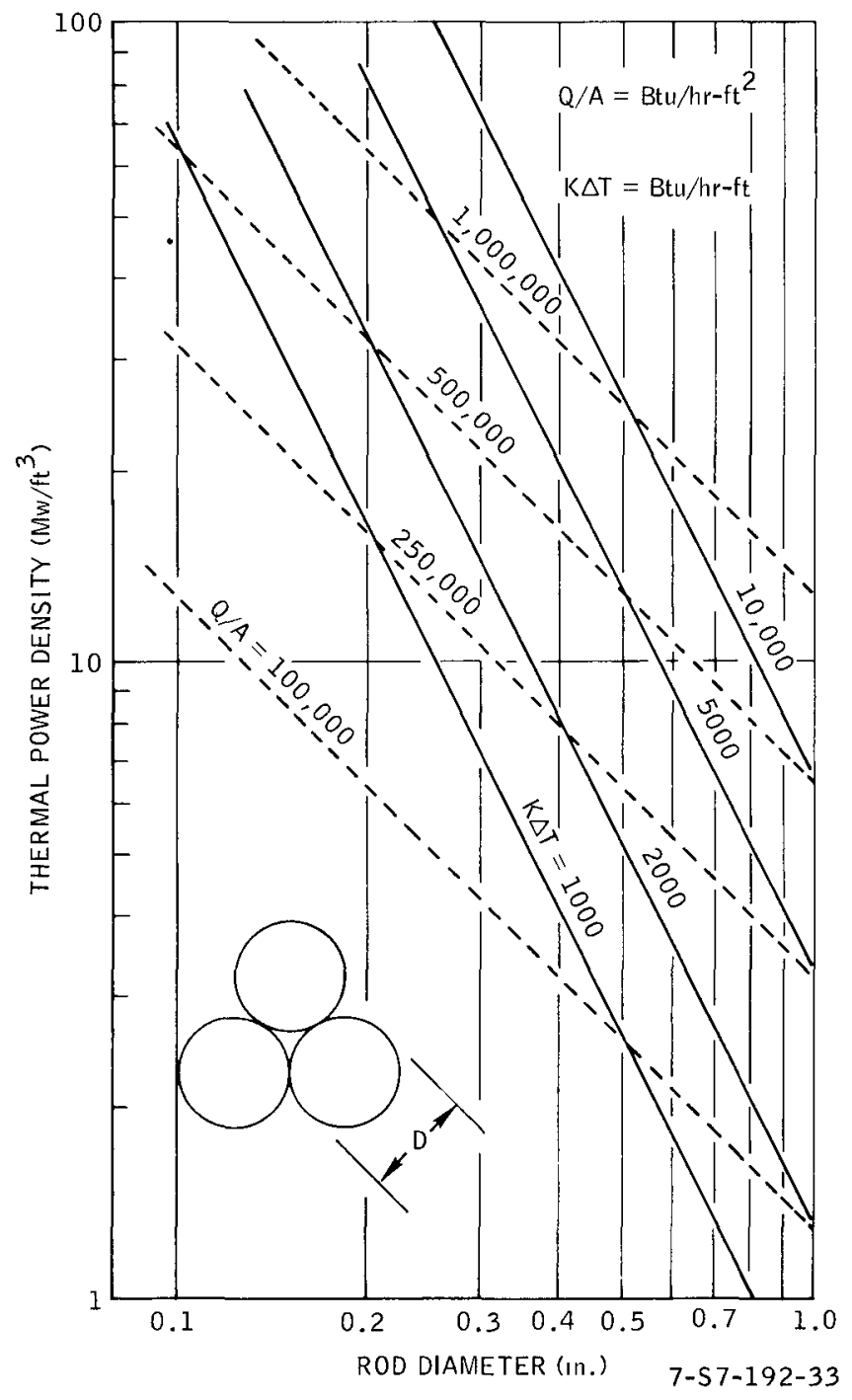

Figure II-16. Reactor Power Density as a Function of Fuel Element Diameter and as a Function of Surface and Volumetric Heat Transfer Limits 
the stress limited $\mathrm{K} \triangle \mathrm{T}$ for $\mathrm{UO}_{2} 1 \mathrm{~s}$ about $1 / 4 \times 10^{3}$, this reactor would be stress limited as a power density of

$$
\frac{Q}{V} \quad \frac{0.0009}{(1 / 4)^{2}}\left(1 / 4 \times 10^{3}\right)=3.6 \frac{\mathrm{Mw}}{\mathrm{ft}^{3}} \text {. }
$$

If, however, it is determined that thermal stress induced fracture of the $\mathrm{UO}_{2}$ does not impede heat transfer or mechanical integrity but that the fuel must be limited by central melting, then $\Delta T_{\max }$ becomes $T_{M}-T_{C}$, where $T_{M}$ - melting temperature and $T_{C}=$ surface temperature, and

$$
\frac{Q}{V}-\frac{0.000675}{(1 / 4)^{2}} K\left(T_{M}-T_{C}\right)
$$

since $K=1$ and $T_{M}=5100^{\circ} \mathrm{F}$,

$\begin{array}{cc}{ }^{\mathrm{T}_{\mathrm{C}}} & \mathrm{Q} / \mathrm{V} \\ \frac{\left.{ }^{\circ} \mathrm{F}\right)}{1000} & \frac{\left(\mathrm{Mw} / \mathrm{ft}^{3}\right)}{24} \\ 2000 & 33 \\ 3000 & 22\end{array}$

It is readily seen that the maximum power density under the above conditions is a function of the reactor operating temperature.

Figure II- 16 shows the relationship between power density, fuel element diameter, heat flux limit, and $\Delta T$ or stress limits for a homogeneous system with mutually tangent cylindrical fuel elements. Note that the above calculations a re based totally on average values. No consideration has been given to the detailed power and temperature distribution in the reactor. These calculations are intended only to indicate the reactor design considerations and limitations. 


\section{e. Energy Density}

Burnup and resulting fuel depletion which are related to energy density impose control requirements and can limit reactor life. However, radiation damage is generally the most significant energy density limitation. Even though the fissioning of $1 \mathrm{gm}$ of uranium per day releases $1 \mathrm{Mw}$ of power, we have seen that reactor criticality demands anywhere from a few to a hundred kilograms in order to utilize the energy of fission. Furthermore, only a few percent of the uranium inventory or critical mass of the reactor can be utilized because of radiation damage.

Fission results in the replacement of each fissioned uranium atom with two fission products. Thus, as the fissioning of uranium metal, for example, proceeds the metal lattice is being stuffed with one extra atom for each fission. These extra atoms plus the fact that the fission product may not comfortably fill a uranium site result in internal strains in the material. In addition, the energetic particles and fission products collide with the atoms of the parent lattice and disrupt it which causes further strain. The parent lattice can only accommodate so much of this strain until it must yield or distort in order to relieve the fission induced strains. This resulting material distortion and expansion is radiation damage. It can be argued on intuitive grounds that a given material can accommodate some maximum fractional increase in the normal atom density before unacceptable damage results. In other words, some fraction of the total number of atoms per unit volume can be fissioned. Experience shows that radiation damage 1 s indeed well correlated on a total atom percent burnup basis. However, the amount of physical distortion or swelling is a function primarily of the parent lattice or material that is absorbing the fission damage and the temperature at which the material is operating durıng fission. The above is a gross simplification; however, sufficlent data and experience for a quantitative description of the radiation damage limits for all 
materials do not exist. In fact, it is an extremely difficult, costly, and time-consuming task to establish such limits. Figure II- 17 shows the results of many years of investigation of the $\mathrm{UO}_{2}$ stainless steel system. This system is of no interest for space reactors but the data illustrate the kind of burnup vs temperature limitations that exist for all materials. Such detailed information is not yet available for the materials and temperatures of interest for space reactors. However, past experience has shown that few materials can absorb more than 1 to 2 at. $\%$ burnup without loss of fuel element physical integrity.

If we assume that a material contains $5 \times 10^{22}$ atoms/cc, which is about the atom density of $U$ metal and we further as sume that $1 \%$ of the atoms can be fissioned with acceptable radiation damage, then the material can sustain the fissioning of $5 \times 10^{20}$ atoms/cc. The fissioning of $5 \times 10^{20}$ atoms/cc results in the release of

$$
\text { lat. } \% \cong \frac{5 \times 10^{20}}{6 \times 10^{23}} \times 5.3 \times 10^{6} \mathrm{kwh} / \mathrm{mole}-4.4 \mathrm{Mwh} / \mathrm{cc}
$$

Therefore, the fissioning of 1 at. \% releases about $15 \mathrm{Mw}$-years per cubic foot of reactor core material. Since the atom density of most materials is near the above assumption, this figure is a reasonable approximation.

The temperature-dependence of the burnup limit is of extreme importance when maximum temperatures are desired as is the case for space systems. Even in the case of terrestrial reactor systems where low temperature heat sinks are available there is a tendency to strive for high temperatures in order to maximize Carnot. If, however, one wants maximum net energy output per core either for economic reasons or for endurance reasons, the significant parameter is the product of allowable burnup times Carnot efficiency. This quantity is proportional to the net avallable energy that can be delivered by one core. Since burnup capability decreases with temperature and Carnot increases 


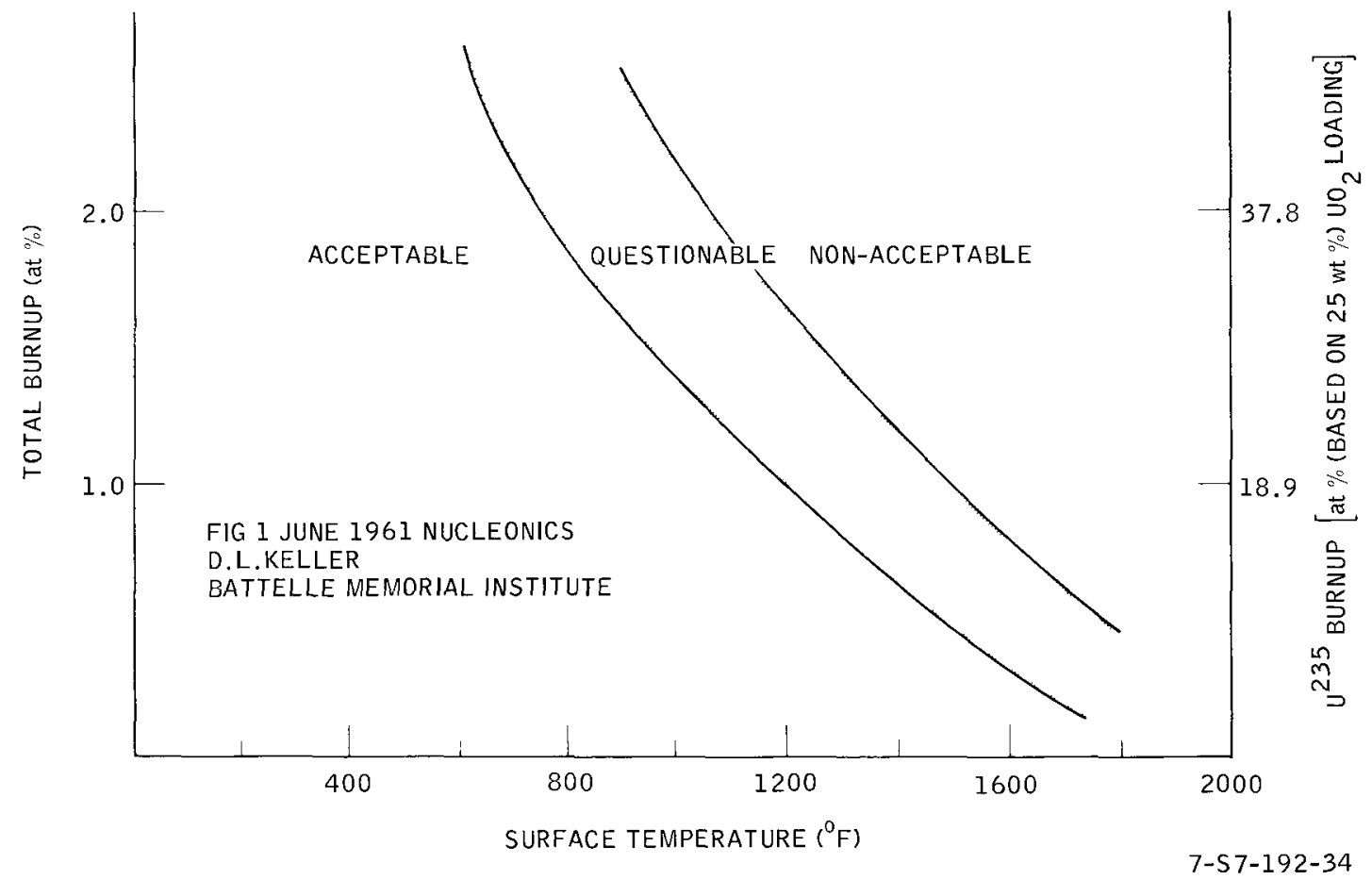

Figure II-17. Burnup Performance of Stainless Steel-UO $\mathrm{UO}_{2}$ Cermet Fuel Specimens 
with source temperature (for a fixed sink temperature), there is an optimum temperature for maximum system endurance. Figure II- 18 illustrates this point for a system having the burnup limits of Figure II- 17 and having a fixed sink temperature of $100^{\circ} \mathrm{F}$. The $\mathrm{UO}_{2}$ stainless steel cermet is generally considered a good "high-temperature" fuel. However, it can be readily seen from Figure II- 18 that the optimum temperature for maximum endurance is quite low in comparison to the normally considered temperature limits of such a material.

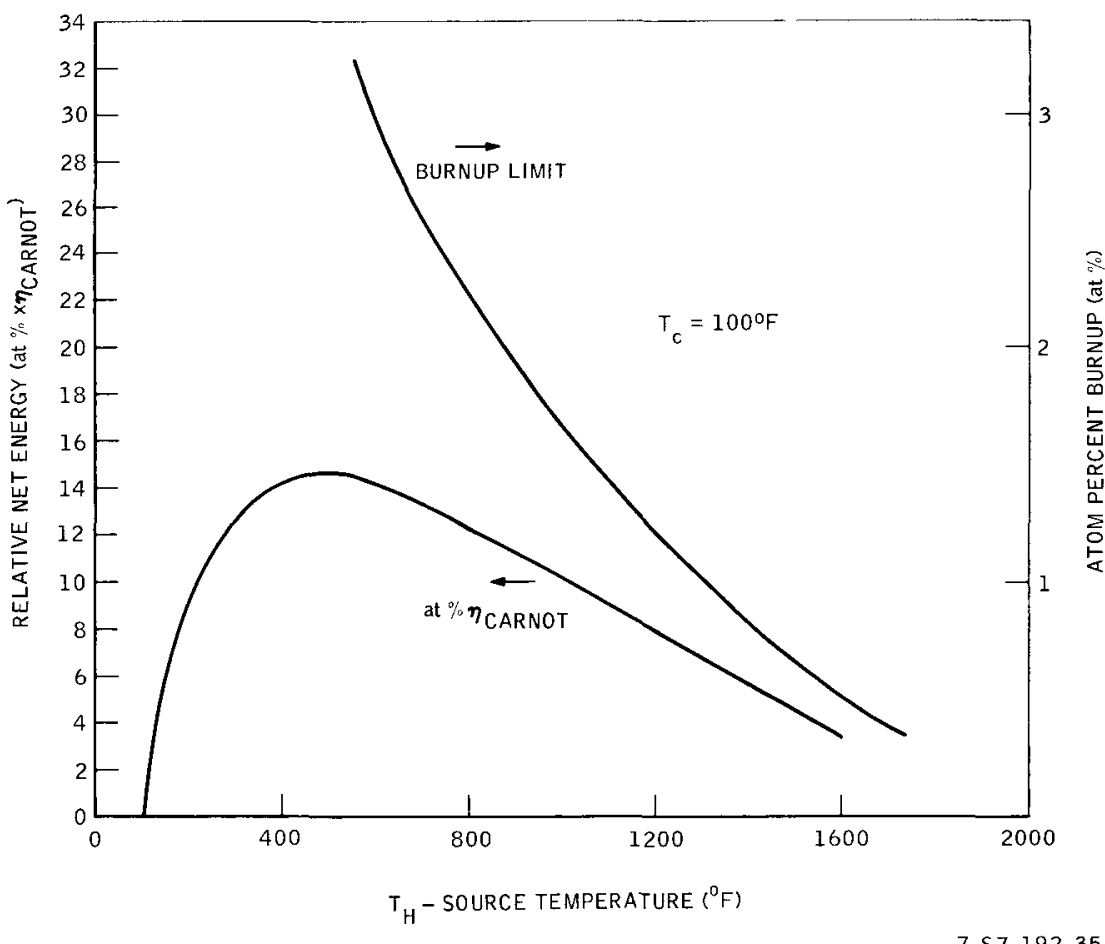

Figure II-18. Energy vs Temperature 


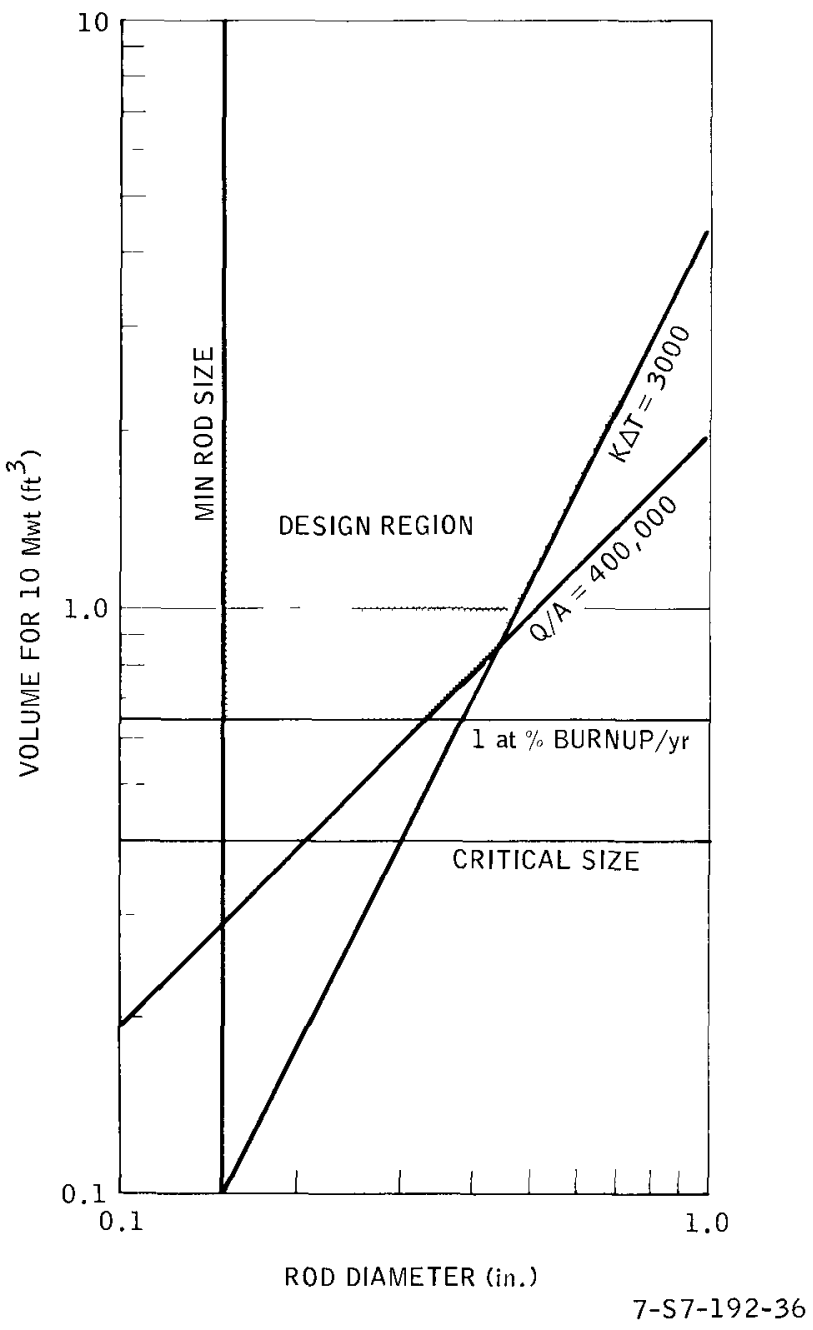

Figure II-19. Reactor Volume Requirements as a Function of Rod Diameter, Criticality, Heat-Transfer Limit, and Burnup Limit 


\section{f. Summary Example}

The relative importance of the various reactor size limitations above can be shown by an example. Assume the following conditions

1) Reactor critical volume $=0.4 \mathrm{ft}^{3}$

2) Heat flux limit $400,000 \mathrm{Btu} / \mathrm{hr}-\mathrm{ft}^{2}$

3) Fuel thermal conductivity $=15 \mathrm{Btu} / \mathrm{hr}-\mathrm{ft}-{ }^{\circ} \mathrm{F}$

4) Fuel $\Delta \mathrm{T}_{\max } 200^{\circ} \mathrm{F}$

5) Fuel burnup limit 1 at. $\%$

6) Fuel element minimum diameter - $0.15 \mathrm{in}$.

7) Reactor power $10 \mathrm{Mwt}$

8) Lifetime - 1 year

Figure II- 19 shows the reactor volume as a function of fuel element diameter for the various limits. If fuel element cladding thickness had been included, the vold fraction would increase with decreasing rod diameter and the criticality limit and the burnup limit would not be independent of rod diameter.

A probable design point for this example would lie on the burnup limit in order to minimize reactor volume and weight and would also lie on the heat flux limit in order to minimize the number of fuel elements and thus the manufacturing cost.

It should be noted that the above treatment has been highly qualitative for illustrative purposes. Throughout, the power distribution in the reactor and the temperature distribution detalls have been completely neglected. The quantities used have been average values. In general, reactor power varies as a chopped cosine function in all directions. In small reactors this leads to a power distribution with a peak to average value in the range of 1.5 to 2 In a rigorous design analysis, all limits are placed on the maximum temperature, heat flux, power density, or burnup. 


\section{HEAT REJECTION}

\section{A. TEMPERATURE AND EFFICIENCY}

The basic requirement of a space power system to maintain the thermodynamic cycle cold temperature by the radiative rejection of heat from an extended surface or radiator introduces unique considerations and constraints. It is immediately obvious that high Carnot efficiency requires minimum cycle cold temperature which increases the radiator area requirement because of the $\mathrm{T}^{4}$ dependence of the radiator area. Since radiator size will have a significant influence on system design criteria, concept selection, and weight, it is important to evaluate and understand the tradeoff between system efficiency and radiator area. In the general case

$$
\eta_{\mathrm{O}}=\eta_{\mathrm{D}} \eta_{\mathrm{C}}
$$

where

$$
\begin{aligned}
& \eta_{\mathrm{O}}=\text { overall conversion efficiency } \\
& \eta_{\mathrm{C}}-\text { Carnot efficiency } \\
& \eta_{\mathrm{D}}=\text { conversion device efficiency. }
\end{aligned}
$$

The system heat input, $Q$, is

$$
Q=\frac{P}{\eta_{D} \eta_{C}}
$$

where $P$ is the electrical power output.

The heat rejected, $Q_{R}$, is

$$
Q_{R}=Q-P-P\left(\frac{1}{\eta_{D} \eta_{C}}-1\right) \text {. }
$$

If

$$
\begin{aligned}
\mathrm{T}_{\mathrm{H}} & =\text { source temperature } \\
\mathrm{T}_{\mathrm{C}} & =\text { cycle cold temperature }
\end{aligned}
$$


then

$$
\eta_{\mathrm{C}}-1-\frac{\mathrm{T}_{\mathrm{C}}}{\mathrm{T}_{\mathrm{H}}}
$$

and

$$
\mathrm{Q}_{\mathrm{R}}=\sigma \epsilon \mathrm{AT}_{\mathrm{C}}^{4}-\sigma \epsilon \mathrm{A}\left(1-\eta_{\mathrm{C}}\right)^{4} \mathrm{~T}_{\mathrm{H}}^{4}
$$

where

$$
\begin{aligned}
& \sigma \text { - Stefan-Boltzmann constant } \\
& \epsilon-\text { surface emissivity } \\
& \text { A - surface area }
\end{aligned}
$$

and

$$
\frac{\mathrm{P}}{\mathrm{A}}=\frac{\sigma \epsilon \mathrm{T}_{\mathrm{H}}^{4}\left(1-\eta_{\mathrm{C}}\right)^{4}}{\left(\frac{1}{\eta_{\mathrm{D}} \eta_{\mathrm{C}}}-1\right)}
$$

The maximum power per unit area or the minimum area for a given power at a fixed $T_{H}$ can be determined by solving for $\eta_{C}$ in

$$
\frac{\partial}{\partial \eta_{C}}\left(\frac{P}{A}\right)=0
$$

The result is

$$
\left.\eta_{\mathrm{C}}\right|_{\text {min. arca }}-\frac{2}{5 \pm \sqrt{25-16 \eta_{\mathrm{D}}}}
$$

This expression has only a minor dependence on the conversion device efficiency $\eta_{D}$. This dependence is shown in Figure II- 20. As $\eta_{\mathrm{D}}$ varies from 0 to $100 \%$ the optimum Carnot efficiency for minimum area varies only from 0.8 to 0.75 . 


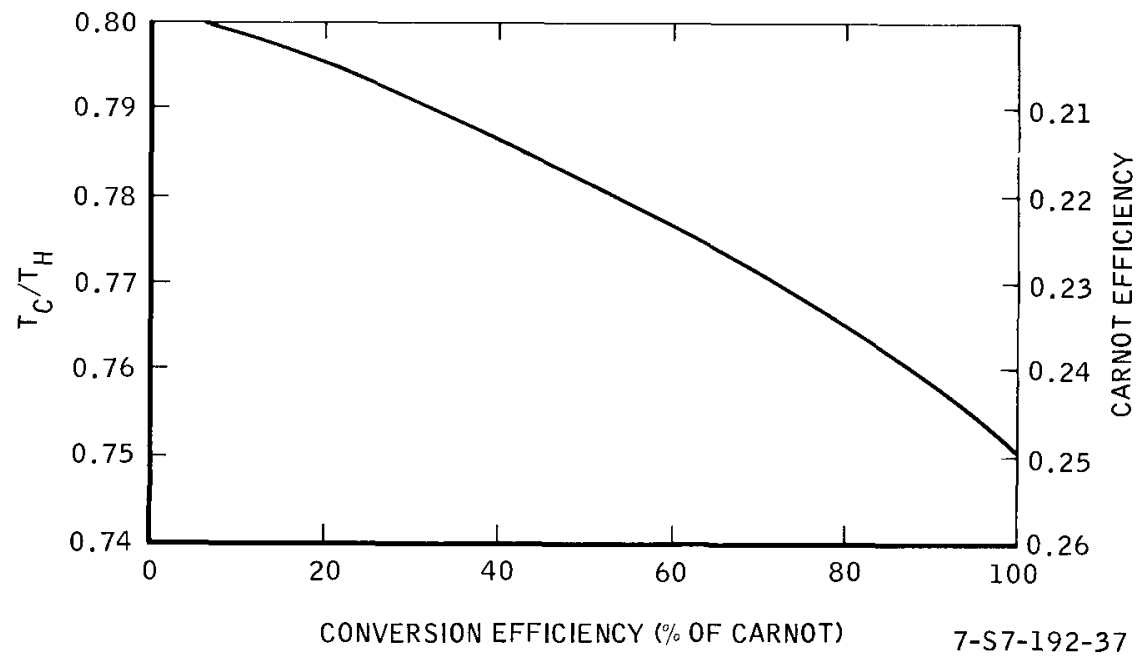

Figure II-20. Optimum Carnot Efficiency for Minimum Radiator Area as a Function of Conversion

Device Efficiency

Because of the high energy content of a nuclear reactor, nuclear space power system designs generally tend toward the optimum Carnot condition. However, it is not necessarily true that minimum werght occurs at minimum area. At the same time the direct physical size and the potential structural awkwardness of the radiator area are distinct considerations.

If there is a desire to shift toward higher efficiency which can be motivated by heat source limitations, especially in low efficiency systems, the generalized relationship between area and efficiency is shown in Figure II-2l. For illustrative purposes a median value of $\eta_{D}$ was chosen. It can be seen that the efflciency can be increased by a factor of two by increasing radiator area by a factor of about 1.7 and to increase the efficiency by a factor of 3 requires 6.5 times as much area.

The net result of the radiative heat rejection requirement is to force space power systems to accept much lower efficiencies than are generally used on the ground and to place a high premium on high source temperature to minimize the radiator area and weight. 


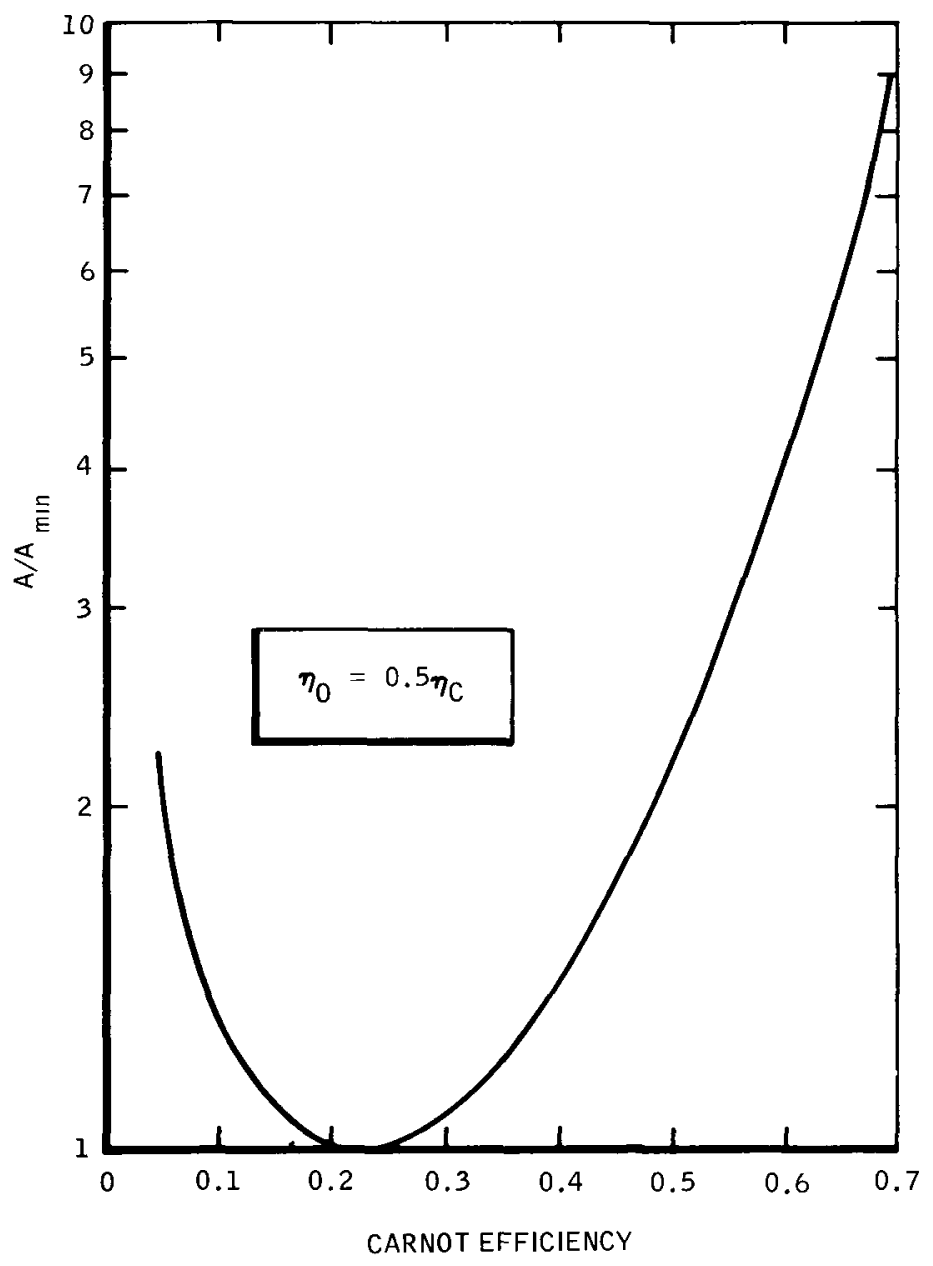

$7-57-192-38$

Figure II-21. Radiator Area vs Carnot Efficiency 
B. RADIANT HEAT TRANSFER

a. Heat Transfer Surfaces

Some of the more common types of heat transfer surfaces employed in space heat rejection systems are illustrated in Figure II-22. The selection of any particular type of surface or construction will be dependent on many factors, such as use of the surface as an integral part of the space vehicle, or as a load carrying member, etc., this discussion, however, will be limited to analyzing the thermal characteristics of the radiator only.

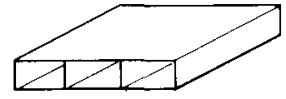

(A)

FLAT PLATE

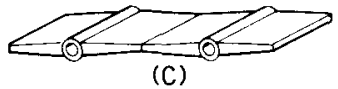

TUBE AND FIN (TAPERED FIN)

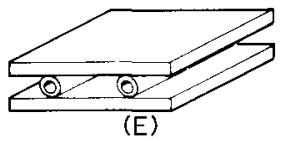

TUBE AND FIN (DOUBLE FIN SURFACE)

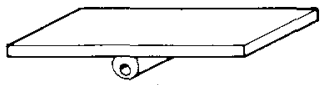

(G)

TUBE AND FIN

METEOROID PROTECTION ON ONE SIDE ONLY

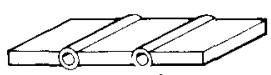

(B)

TUBE AND FIN (CONSTANT THICKNESS FIN)

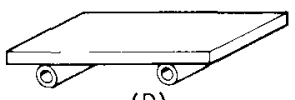

(D)

TUBE AND FIN

(SINGLE FIN SURFACE)

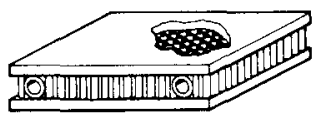

(F)

TUBE AND FIN (HONEYCOMB CONSTRUCTION)

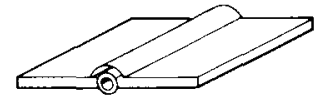

(H)

TUBE AND FIN

METEOROID ARMOUR SHEET

(ONE SIDE ONLY)

7-5 7-192-39

Figure II-22. Typical Space Radiator Surfaces 


\section{b. Analysis of Isothermal Heat Transfer Surfaces}

The basic equation that governs radiant heat transfer from a surface is the famıliar Stefan-Boltzmann law

$$
Q=\epsilon_{T} \sigma A\left(T^{4}-T_{s}^{4}\right)
$$

where

$$
\begin{aligned}
Q= & \text { power radiated }(\mathrm{kw}) \\
\epsilon_{\mathrm{T}}= & \text { surface emissivity for thermal radiatıon } \\
\sigma= & \text { Stefan-Boltzmann constant }=5.02 \times 10^{-13} \\
& \left(\mathrm{kw} / \mathrm{ft}^{2}-{ }^{\circ} \mathrm{R}^{4}\right) \\
\mathrm{A}= & \text { radiatıng surface area }\left(\mathrm{ft}^{2}\right) \\
\mathrm{T}= & \text { radiating surface temperature }\left({ }^{\circ} \mathrm{R}\right) \\
\mathrm{T}_{\mathrm{S}}- & \text { radiative sink temperature }\left({ }^{\circ} \mathrm{R}\right) .
\end{aligned}
$$

The quantity $\sigma \mathrm{T}^{4}$ is plotted in Figure II-23 such that the heat rejection rate per unit area can be conveniently calculated from

$$
\frac{\mathrm{Q}}{\mathrm{A}}=\epsilon\left(\sigma \mathrm{T}^{4}-\sigma \mathrm{T}_{\mathrm{s}}^{4}\right)
$$

In many situations of interest, it is permissible to neglect the sink temperature term. This is generally acceptable for the high-temperature space power systems. For situations where $\mathrm{T}^{4}>\mathrm{T}_{\mathrm{s}}^{4}$

$$
Q=\epsilon_{T} \sigma T^{4}
$$

The error in this simplification becomes cvident when the radiation equation is rewritten in the form

$$
\frac{Q}{A}=\epsilon \sigma T^{4}\left[1-\left(\frac{T_{s}}{T}\right)^{4}\right] \text {. }
$$




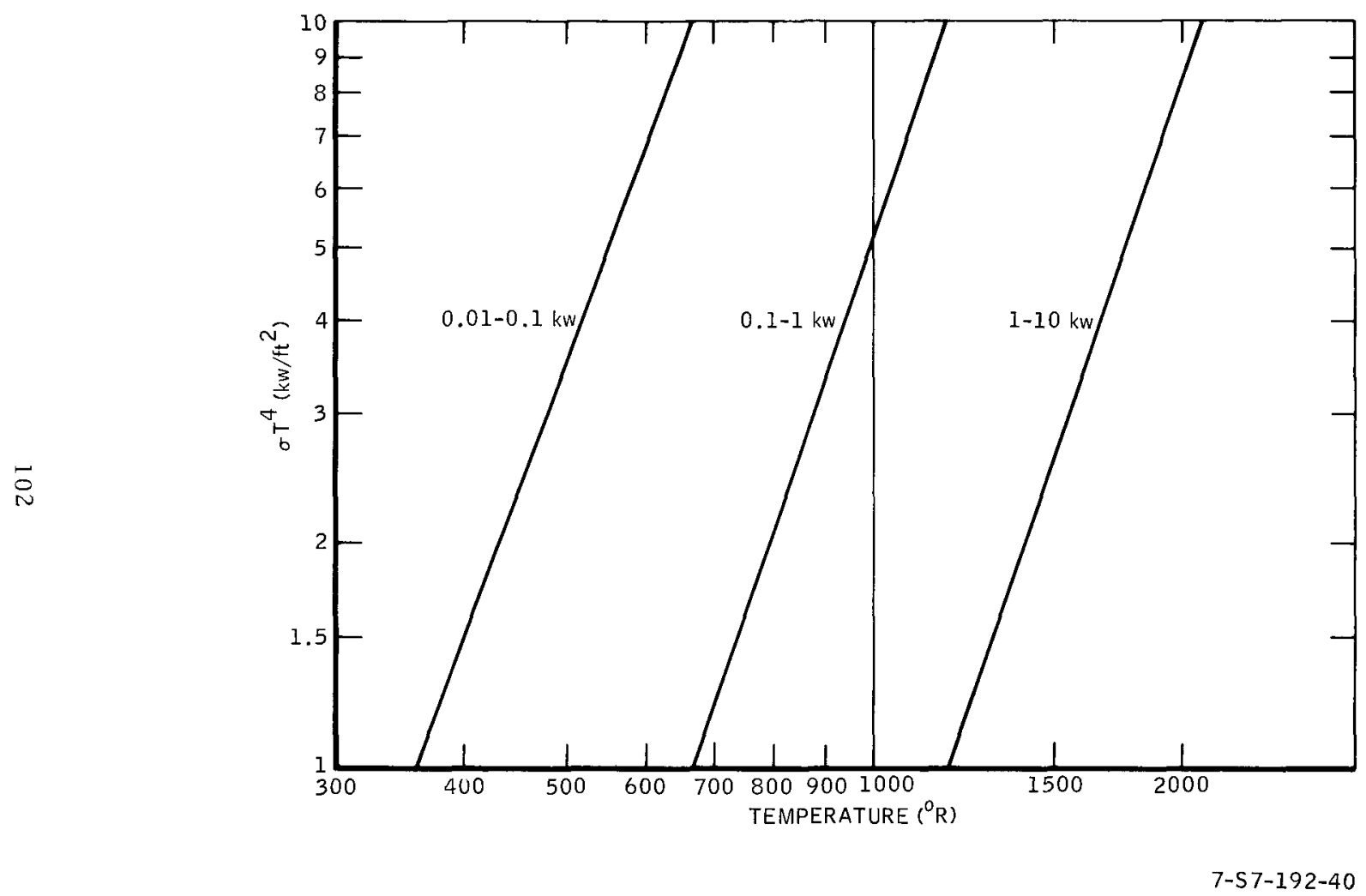

Figure II-23. Radiation Heat Flux for Isothermal Surfaces with Unit Emissivity 
Values of $T_{S} / T$ less than 0.316 result in less than $1 \%$ error and values less than 0.57 result in less than $10 \%$ error.

\section{c. Analysis of Nonisothermal Heat Transfer Surfaces}

Consider the case of the parallel or flat plate radiator shown in Figure II-24. A fluid at a weight flow or W(1b/hr) enters the radiator at $T_{1}$ and exits at $T_{2}$. Radiation is assumed to take place from one side only. Neglecting the film drop between the fluid and the surface and neglecting the sink temperature, a solution for this case is developed as follows the heat transfer from the elemental area is given by

$$
d Q-\epsilon \sigma T^{4} y d x
$$

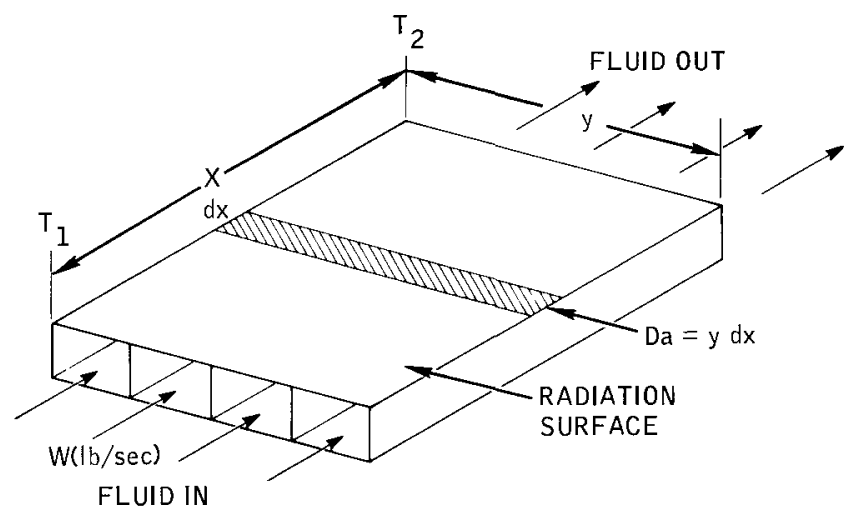

7-S7-192-41

Figure II-24. Flat Plate Radiator

Assuming thermal equilibrium between the flund and the surface, that $1 \mathrm{~s}$, the heat lost by the fluid equals the heat radiated from the surface, then

$$
\mathrm{dQ}=\mathrm{C}_{\mathrm{P}} \mathrm{WdT}
$$

where $C_{P}$ is the fluid specific heat. Therefore

$$
-C_{P} W d T-\epsilon \sigma T^{4} y d x
$$


rearranging terms and indicating the appropriate integration limits,

$$
-\int_{T_{1}}^{T_{2}} \frac{d T}{T^{4}}-\int_{0}^{x} \frac{\epsilon \sigma}{C_{P} W} y d x
$$

which integrates to

$$
\left(\frac{1}{3 T^{3}}\right)_{T_{1}}^{T_{2}}=\frac{\epsilon \sigma(y x)}{C_{P} W}
$$

and since the product (xy) equals the surface area, the equation for the area required is given by

$$
A=\frac{C_{P} W}{3 \epsilon \sigma}\left(\frac{1}{T_{2}^{3}}-\frac{1}{T_{1}^{3}}\right)
$$

where $T_{1}$ and $T_{2}$ are the fluid temperatures into and out of the radiator respectively.

Since $Q-C_{P} W \Delta T$ and $T_{2}=T_{1}-\Delta T$, where $\Delta T$ is the fluid temperature drop through the radiator, the result can be put in the following form

$$
\frac{Q}{A}-\epsilon \sigma T_{1}^{4} 3 \frac{\Delta T}{T_{1}} \frac{1}{\left[\frac{1}{\left(1-\Delta T / T_{1}\right)^{3}}-1\right]} \text {. }
$$

The correction factor necessary to compensate for a fluid temperature difference between radiator inlet and outlet is plotted as a function of $\Delta \mathrm{T} / \mathrm{T}_{1}$ in Figure II-25. The above equation assumes a sink temperature of zero. The error in neglecting the sink temperature is less than the indicated error when evaluated on the basis of the outlet temperature, $\mathrm{T}_{2}$, 1.e., if the whole surface were at $\mathrm{T}_{2}$. 


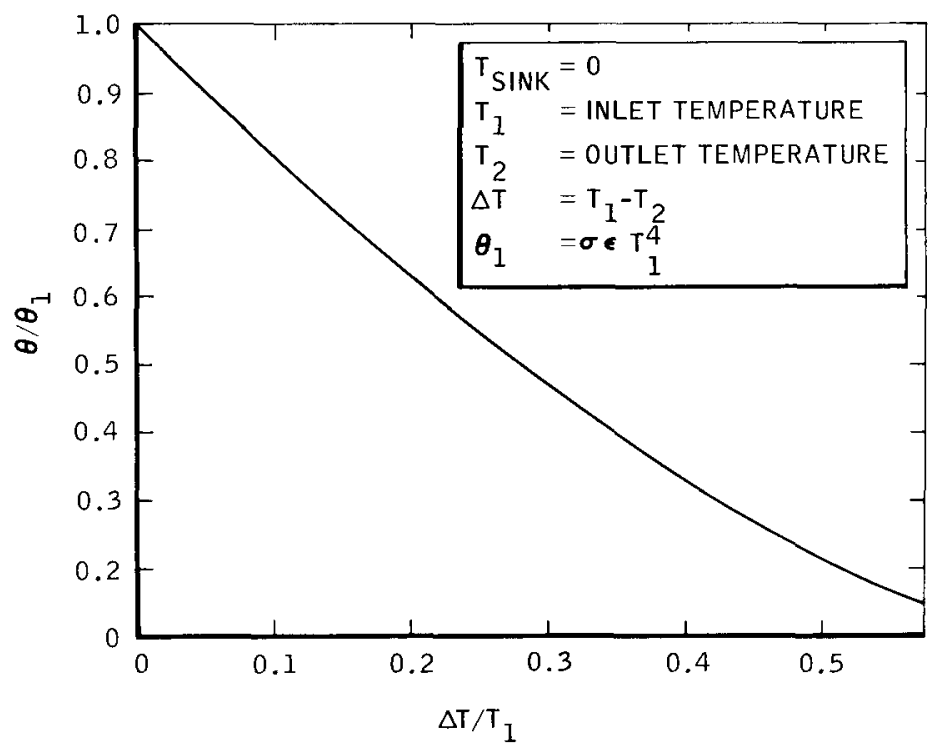

$7-57-192-42$

Figure II-25. Radiative Heat Rejection Correction for a Nonisothermal Surface

d. Analysis of Extended Surfaces

In most cases extended surface or finned radiators are advantages to (1) reduce vulnerable area and thus micrometeorite armor weight, (2) to increase vapor velocities for positive condenser operation in $0-\mathrm{g}$, and (3) to reduce heat transfer fluid weight in liquid radiators and subcoolers, etc. The effectiveness of a finned radiator must be less than $100 \%$ and 1 s determined by the physical dimensions and the properties of the fin material. In order to evaluate finned radiators, the derivation of the heat transfer from a simple extended surface is outlined in Figure II-26.

Combining

$$
\frac{\mathrm{d}^{2} \mathrm{~T}(\mathrm{y})}{\mathrm{dy^{2 }}}=\frac{\sigma\left(\epsilon_{\mathrm{A}}+\epsilon_{\mathrm{B}}\right)}{\mathrm{Kt} \mathrm{t}_{\mathrm{o}}} \mathrm{T}(\mathrm{y})^{4}
$$




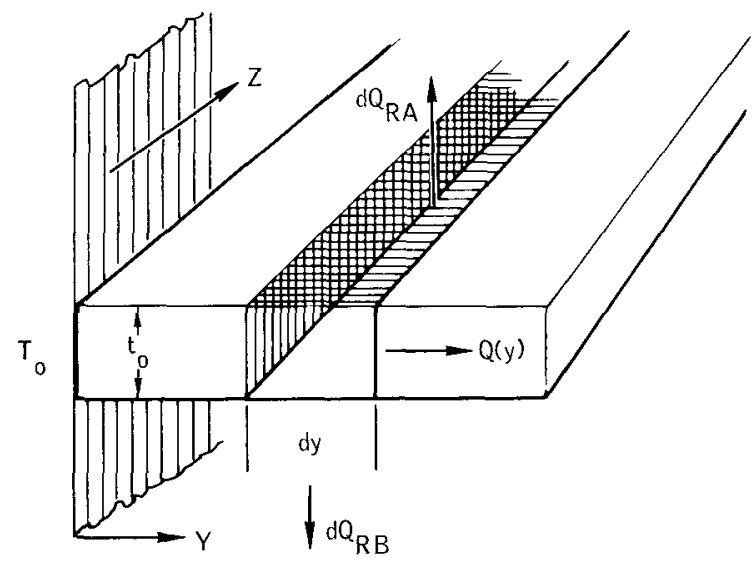

$7-57-192-43$

Figure II-26. Extended Fin Radiator

put into dimensionless form by substituting

$$
Y=\frac{y}{L} \quad T=\frac{T(y)}{T_{0}}
$$

results in

$$
\frac{\mathrm{d}^{2} \mathrm{~T}}{\mathrm{dy} \mathrm{y}^{2}}=\frac{\sigma\left(\epsilon_{\mathrm{A}}+\epsilon_{\mathrm{B}}\right) \mathrm{L}^{2} \mathrm{~T}_{\mathrm{o}}^{3}}{K \mathrm{t}_{\mathrm{O}}} \mathrm{T}^{4} .
$$

The boundary conditions are

$$
\begin{aligned}
\frac{\mathrm{d} T}{\mathrm{dy}}=0 & \text { at } & \mathrm{Y}=1 \\
\mathrm{~T}=1 & \text { at } & \mathrm{Y}=0
\end{aligned}
$$

The fin effectiveness is defined as the ratio of the heat radiated from the fin to the heat that could be radiated from the fin area at the base temperature 


$$
\eta_{F} \equiv \frac{\sigma \epsilon \int_{0}^{\mathrm{L}} \mathrm{T}(\mathrm{y})^{4} \mathrm{dy}}{\mathrm{LT}_{\mathrm{O}}^{4}}
$$

The results for rectangular and triangular fins have been obtained by computer. (57) The fin effectiveness as a function of the dimensionless parameter

$$
\frac{\sigma\left(\epsilon_{\mathrm{A}}+\epsilon_{\mathrm{B}}\right) \mathrm{L}^{2} \mathrm{~T}_{\mathrm{o}}^{3}}{K t_{\mathrm{o}}}
$$

is plotted in Figure II-27. Through the application of the fin effeciveness function, a generalized plot of fin weight vs area can be obtained. It can be seen from Figure II- 28 that rectangular fins have a minımum weight for a given heat transfer at a fin effectiveness of 0.57 . The minimum fin weight condition incurs a significant increase in radiator area. The weight of the fin base heat source, micrometeorite armor and other weight penalties such as structure which are associated with the radiator area will generally cause the minimum weight of a practical radiator to occur at higher fin effectiveness. It 15 also important to note that the minimum weight of a triangular fin is only $65 \%$ of the minimum rectangular fin. Or the fin effectiveness can be increased from 0.57 to about 0.8 without an increase in fin weight by the use of triangular rather than rectangular fins.

The physical properties of some applicable fin materials are given in Table II-12. Since the fin effectiveness depends on the thermal conductivity and the weight on density, the ratio of $\mathrm{K} / \rho$ is a measure of material effectivness. Values of $\mathrm{K} / \rho$ are plotted as a function of temperature in Figure II-29. It can be seen from Table II- 12 and from Figure II-29 that aluminum is the best low temperature fin material. Beryllium suffers from cost and avalability while magnesium probably has too high a vapor pressure. Even though Be has a much higher melting point than 


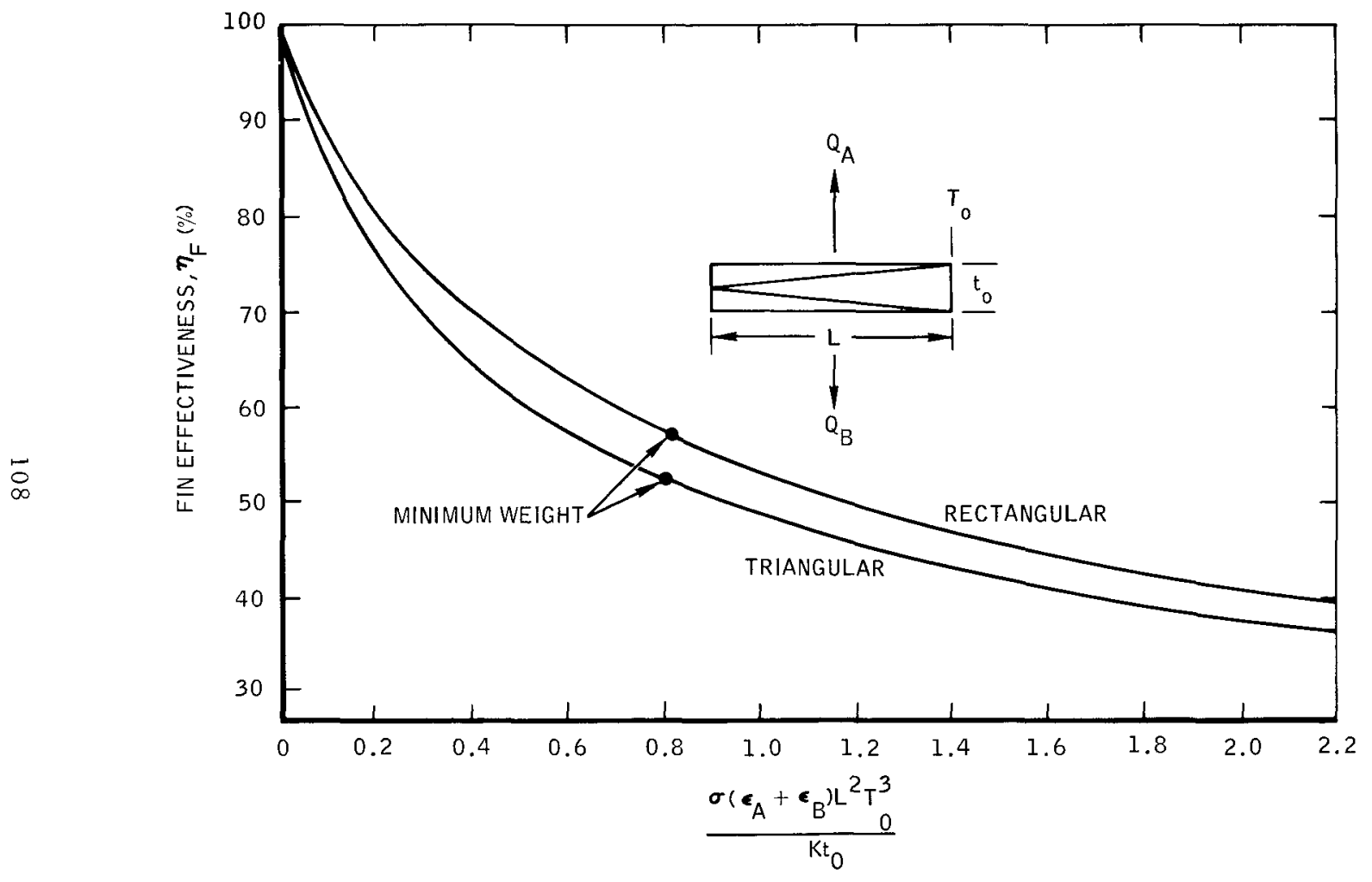

7-57-192-44

Figure II-27. Fin Effectiveness vs Dimensionless Fin Parameter 


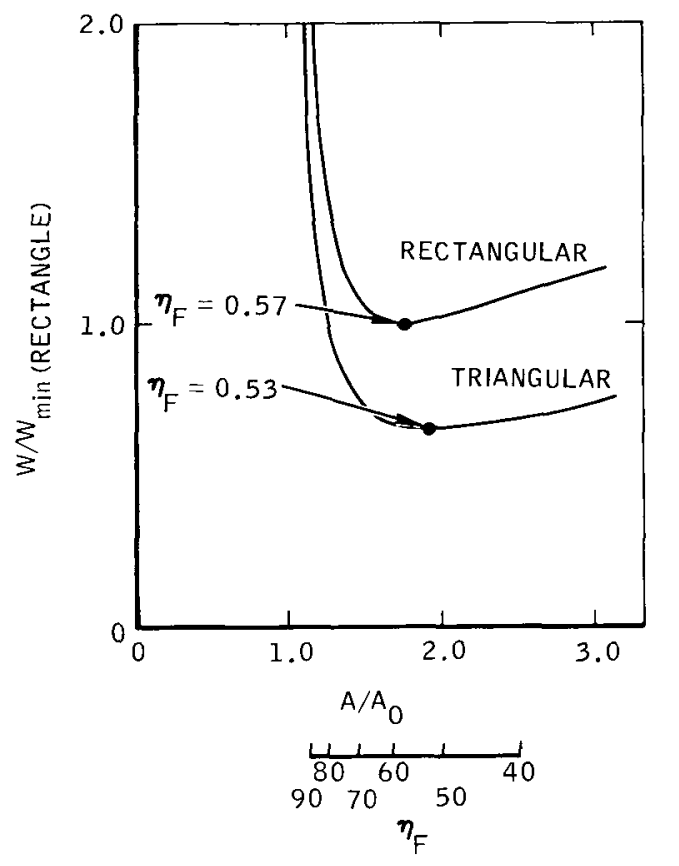

$$
\text { 7-\$ 7-192-45 }
$$

Figure II-28. Relative Fin Weight vs Fin Effectiveness for Rectangular and Triangular Fins

TAB LE II - 12

PROPERTIES OF RADIATOR FIN MATERIALS

\begin{tabular}{|c|c|c|c|c|c|}
\hline & $\begin{array}{c}\text { Thermal } \\
\text { Conductivity } \\
\text { at } 1000^{\circ} \mathrm{R} \\
\left(\mathrm{Btu}^{\mathrm{I}} \mathrm{s} / \mathrm{hr}-\mathrm{ft}-{ }^{\circ} \mathrm{F}\right)\end{array}$ & $\begin{array}{l}\text { Density } \\
\text { at RT } \\
\left(1 \mathrm{~b} / \mathrm{ft}^{3}\right)\end{array}$ & $\frac{K}{\rho}$ & $\begin{array}{c}\text { Melting } \\
\text { Point } \\
\left({ }^{\circ} \mathrm{R}\right)\end{array}$ & $\begin{array}{c}\text { Vapor Pressure } \\
10-8 \mathrm{~mm} \text { of } \mathrm{Hg} \\
\text { at Temperature } \\
\left({ }^{\circ} \mathrm{R}\right)\end{array}$ \\
\hline Aluminum & 116 & 169 & 0.686 & 1678 & 1710 \\
\hline Beryllium & 73 & 116 & 0.63 & 2790 & 1770 \\
\hline Copper & 216 & 558 & 0.387 & 2440 & 1980 \\
\hline Magnesium & 77 & 109 & 0.706 & 1661 & 840 \\
\hline Molybdenum & 70 & 640 & 0.109 & 5212 & 3350 \\
\hline
\end{tabular}




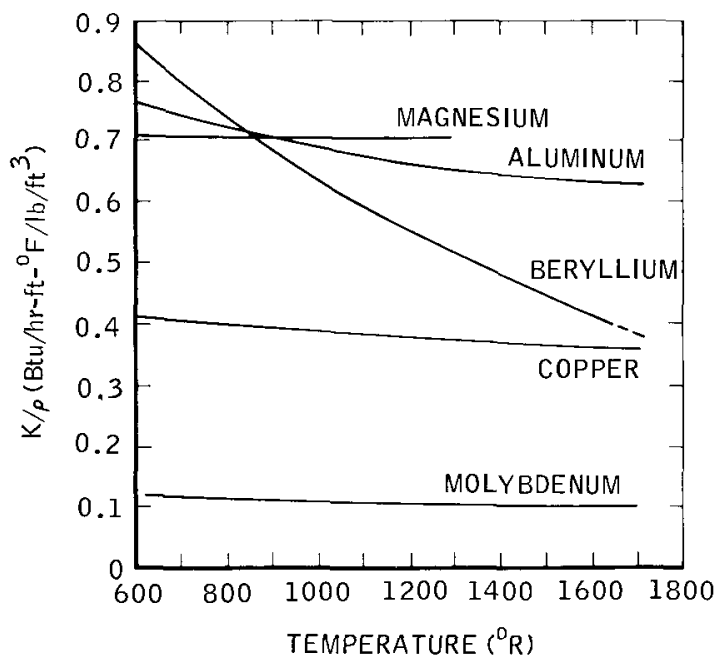

$7-57-192-46$

F1gure II-29. Thermal Conductivity Over Density Ratio for Radiator Fin Materials

aluminum, it does not seem to offer a significant advantage in vapor pressure at high temperature. As a result the preferable high temperature fin material is copper.

e. Sink Temperature

If there were no net heat radiated from the surface then

$$
Q=\epsilon_{T} \sigma A\left(T^{4}-T_{s}^{4}\right) \quad 0
$$

and the temperature of the surface would come to equal the sink temperature, 1. e., $\mathrm{T}-\mathrm{T}_{\mathrm{S}}$. Converscly then, the sink temperature can be defined as that temperature reached by the surface (A) when the surface is in thermal equilibrium with its environment. With this definition, the sink temperature is not a physical temperature in the usual sense, bccause of its dependence upon $\epsilon$, but is an effective temperature. The effective sink temperature is that surface tempcrature nccessary to re-radiate the total incident energy from the principle external sources, 
1.e., sun, solar reflection from the earth, and infrared radiation from the earth.

It is readily seen that the effective sink temperature is a function of the location and orientation of the specific surface in question with respect to the principal sources. Because of the relatıvely high radiator temperature of most nuclear space power systems of interest the sink temperature is not a significant parameter. Thus, the reader is referred to References 55-58. For near-earth (few hundred miles) orbits, the average sink temperature is about $0^{\circ} \mathrm{F}$. The sink temperature in such cases affects the radiator by less than $10 \%$ in area for radiator temperatures in excess of $340^{\circ} \mathrm{F}$.

\section{f. Emissivity}

In general, materials selected for construction of radiator surfaces will not have the desired emissivity or absorptivity characteristics. Since the area required is inversely proportional to the emissivity, it is desirable to have high values of emissivity at the wavelength corresponding to thermal emission and low values of absorptivity for the incident energy wavelengths. As a result, various coatings are usually applied to the radiation surfaces $w h i c h$ have appropriate values of $\left(\epsilon_{T} / \alpha_{I}\right)$, where $\epsilon_{T}$ is the value of emissivity for the thermal radiation and $\alpha_{I}$ is the absorptivity of the surface for the incident radiation. Some typical ceramic coatings of high emissivities are given in Figure II-30 as a function of temperature. Since the solar spectrum approximates ablach body radiatıng at $10,000^{\circ} \mathrm{R}$, it is seen from this figure that these compounds do have low values of absorptivity (since $\epsilon_{T}=\alpha_{T}$ ) in this range. In general, coatings specifically developed for space radiators will have values of $\epsilon_{\mathrm{T}}=0.8$ to 0.95 and $\alpha_{\mathrm{I}}=0.1$ to 0.25 when low temperature radiators are involved. For high temperature systems only high emissivity is important. 


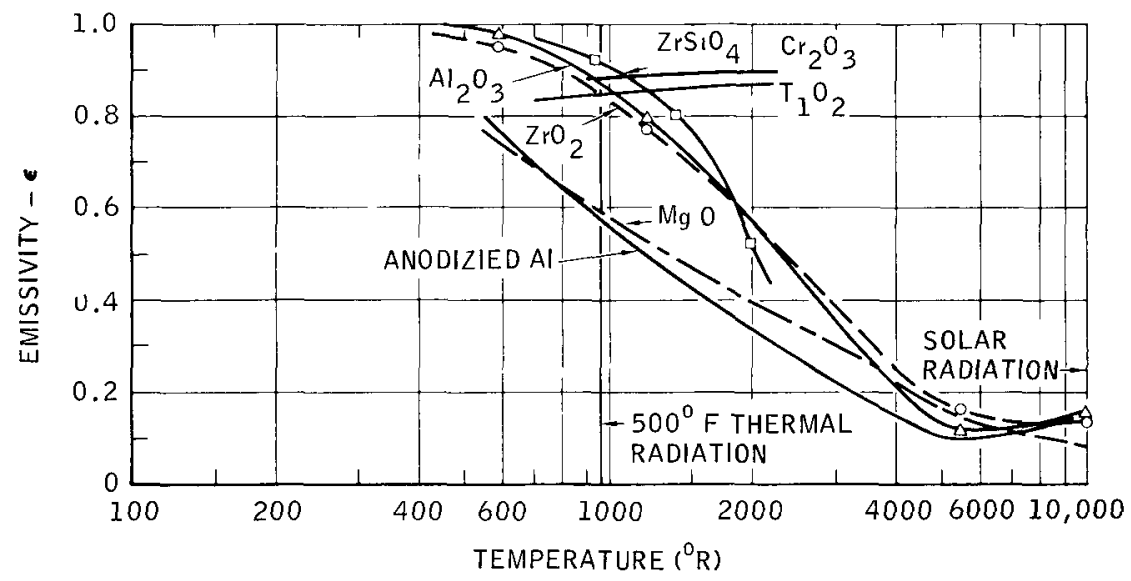

7-57-192-48

Figure II-30. Emissivity of Some Typical Ceramic Materials vs Temperature

\section{METEOROID PROTECTION}

a. General

Meteorold is the name given to the general class of material particles found in outer space. When these particles enter the earth's atmosphere, they are referred to as meteors (shooting stars) and the remnants of those that reach the ground are referred to as meteorites. There are large areas of ignorance of the meteorold environment in space, and only fragmentary knowledge is available to establish protection criteria. There are three principal mechanisms by which meteoroids may cause fallure of space radiator (1) puncture of fluid-carrying passages, with the resultant loss of either working fluid or coolant, (2) spalling, whereby sizable pieces of metal are expelled from the inside of the fluid carrying passage and find their way to the turbine or bearings, with catastrophic results, and (3) erosion of emissivity surface coatings. Spalling and penetration are two distinctly different phenomena. The amount of armor required to protect against damage is sensitive, of course, to the damage mechanism, and to the nature and distribution of meteoroids in space. 


\section{b. Influx Rate}

By far the greatest amount of information on meteorolds is based on earth observations. Both optical and radar methods have been employed to determine relationships between the frequency of the particles and the magnitude or luminosity produced as the meteors traverse the atmosphere. Reduction of this data to estimates of meteoritic mass has been much more difficult because of the uncertanties of the particle drag characteristics and the luminous efficiency of the process.

The influx rate of meteors in the earth's atmosphere has been well established to the +5 visual magnitude by photographic surveys and is becoming well established to the +12 magnitude by radar techniques. (67) Based on a total of 90 million meteors brighter than a visual magnitude of +5 entering the earth's atmosphere in 24 hours, and a frequency increase of a factor of 3.4 per magnıtude, the cumulatıve flux magnitude relationship can be expressed as

$$
\left.\phi=4.5 \times 10^{-15} \times(3.4)^{M} \text { (number } / \mathrm{m}^{2}-\mathrm{sec}\right)
$$

c. Mass and Density

According to recent data from the Harvard College ${ }^{(65)}$ and Smithsonian Astrophysical Observatories, ${ }^{(61,62)}$ an improved basis for estimating masses and densities at photographic magnitudes is now available. The new basis is a series of experimental determinations of luminous efficiency being made from photographs of artifically injected meteors. The results of the first successful experiment of this type are now avalable. The value of luminous efficiency obtained from this first artificial iron meteor experiment results in an estimate of $0.4 \mathrm{~g} / \mathrm{cc}$ for the density of meteors of cometary origin, which make up the great majority of all meteors, and a mass of $0.4 \mathrm{~g}$ for meteors of zero visual magnitude. The relationship between mass and visual magnitude is then

$$
\mathrm{m}=0.4 \times 10^{-0.4 \mathrm{M}}
$$




\section{d. Elux vs Mass}

These equations may be combined to give the following cumulative flux as a function of mass $m$ or greater.

$$
\phi=1.3 \times 10^{-15} \mathrm{~m}^{-4 / 3}
$$

It is based on the following

1) Frequency at the photographic magnitudes and the rate of change of frequency with magnitude as reported by Hawkins and Upton in 1958 (60) $^{(60)}$

2) An extrapolation of these data through the radar magnitudes as recommended by Hawkins in 1962 (67)

3) A mass of $0.4 \mathrm{~g}$ at $\angle$ ero magnitude as recommended by Cook. (62)

This equation is shown in Figure II-31. Estimates of Whipple ${ }^{(64)}$ in 1958 and Watson ${ }^{(65)}$ in 1956 are shown for comparison purposes, together with satellite data reported by Duberg. ${ }^{(66)}$

\section{e. Velocity}

Whipple $\mathrm{e}^{(64)}$ states that the average velocity of photographic meteors is $28 \mathrm{~km} / \mathrm{sec}$ and that the velocity "undoubtedly falls off for smaller meteorolds," he arbitrarily selected $25 \mathrm{~km} / \mathrm{sec}$ at the +9 magnitude. A velocity of $30 \mathrm{~km} / \mathrm{sec} 1 \mathrm{~s}$ recommended for use in design studies to be on the high side of the applicable range. The uncertainty in the average velocity of a $\mathrm{few} \mathrm{km} / \mathrm{sec}$ is not important to the weight of a radiator.

\section{f. Penetration Theory}

The prediction of the depth of penetration of meteoroids into target materials at extremely high velocity involves great uncertainty. There are nearly as many empirical correlations as experimenters, and theories as theorists. The most widely 


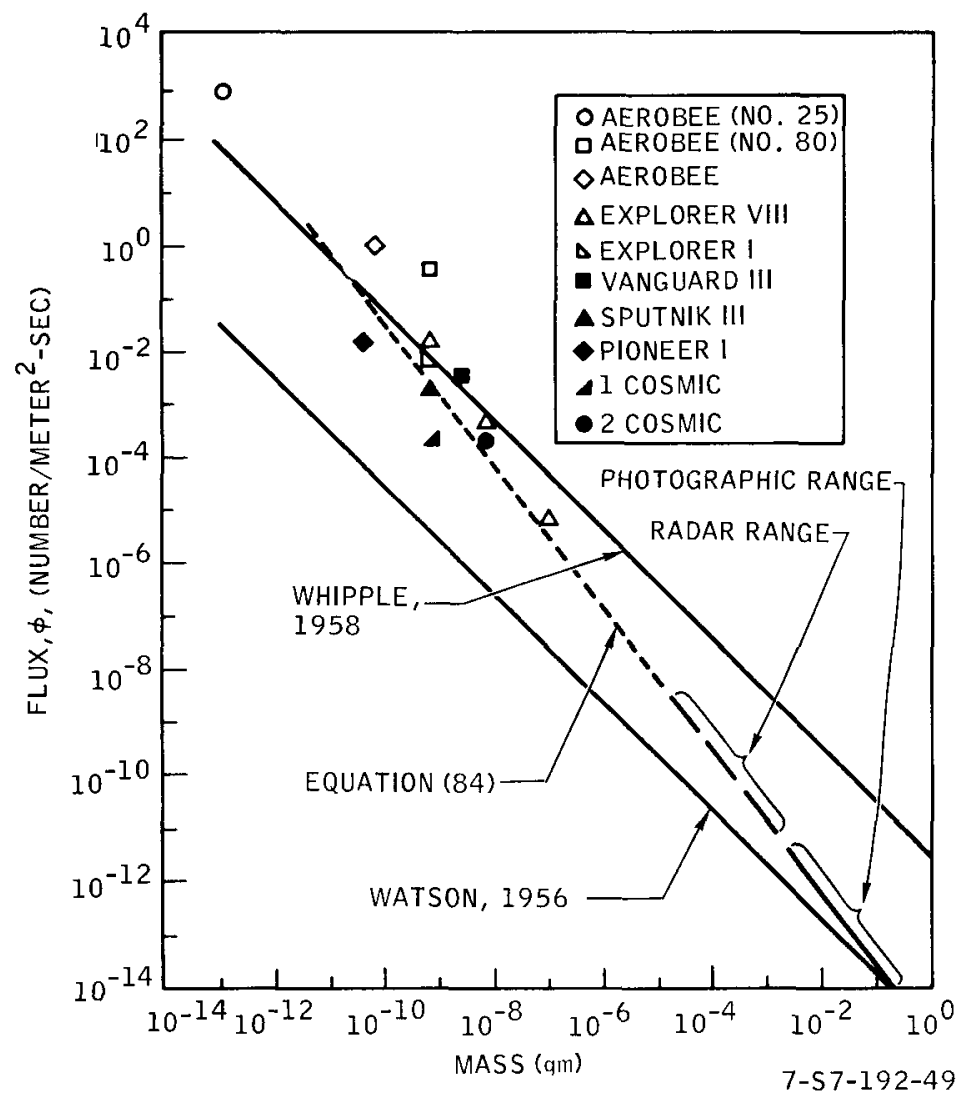

Figure II-31. Meteorold Flux vs Mass

used penetration equation is that of Summers and Charters. (68)

$$
p=2.28\left(\frac{\rho_{\mathrm{m}}}{\rho_{\mathrm{t}}}\right)^{2 / 3}\left(\frac{\mathrm{V}}{\mathrm{C}}\right)^{2 / 3} \mathrm{~d}
$$

where

$$
\begin{aligned}
\mathrm{p} & =\text { penetration distance } \\
\rho_{\mathrm{m}} & =\text { meteoroid density } \\
\rho_{\mathrm{t}} & =\text { target density }
\end{aligned}
$$




$$
\begin{aligned}
& \mathrm{V}=\text { meteoroid velocity } \\
& \mathrm{C}=\text { velocity of sound in target } \\
& \mathrm{d}=\text { meteoroid diameter }
\end{aligned}
$$

Substituting for the velocity of sound in the target material

$$
C=g^{1 / 2}\left(\frac{E}{\rho_{t}}\right)^{I / 2}
$$

where

$$
\begin{aligned}
& g=\text { gravitational constant } \\
& E=\text { target elastic modulus }
\end{aligned}
$$

and for the meteorold diameter

$$
\mathrm{d}=\left(\frac{6}{\pi}\right)^{1 / 3}\left(\frac{\mathrm{m}}{\rho_{\mathrm{m}}}\right)^{1 / 3}
$$

where $\mathrm{m}=$ meteoroid mass

and rearranging results in

$$
p-0.283\left(\frac{\rho_{\mathrm{m}}}{\rho_{\mathrm{t}}}\right)^{1 / 3}\left(\frac{\mathrm{mV}^{2}}{\mathrm{E}}\right)^{1 / 3}
$$

Bjork ${ }^{(69)}$ analyzed the hypervelocity impact phenomenon, treating it as a fluid impact, in which material strength was considered to be of no importance. His result, for iron targets was

$$
p-0.0404\left(\frac{\rho_{\mathrm{m}}}{\rho_{\mathrm{t}}}\right)^{\mathrm{l} / 3}(\mathrm{mV})^{1 / 3}
$$

Eichelberger and Gehring ${ }^{(70)}$ describe the impact phenomenon and argue the importance of target strength, Brinell Hardness Number in particular. They recommend the following penetration equation 


$$
p-0.961 \times 10^{-2}\left(\frac{\mathrm{mV}^{2}}{B H N}\right)^{1 / 3}
$$

where $\mathrm{BHN}=$ Brınell Hardness Number $\left(\mathrm{kg} / \mathrm{mm}^{2}\right)$.

Notice that Equations $A$ and $B$ include density of both meteoroid and target while Equation $C$ is independent of both densities. Further, Equation A includes the target modules of elasticity, Equation $C$ includes target Brinell hardness, while Equation B 15 independent of target mechanical properties. Fortunately all three have the same functional dependence on mass, so they may be compared. Using the values of meteoroid density of $0.4 \mathrm{~g} / \mathrm{cc}$, and velocity of $30 \mathrm{~km} / \mathrm{sec}(98,300 \mathrm{ft} / \mathrm{sec})$, we can compare the room temperature penetration of $0.001 \mathrm{~g}$ meteoroids into a target of a high-strength steel, with the following room temperature properties

$$
\begin{aligned}
\rho_{\mathrm{t}} & -7.67 \mathrm{~g} / \mathrm{cc} \\
\mathrm{E} & =29 \times 10^{6} \mathrm{ps} 1\left(2 \times 10^{6} \mathrm{~kg} / \mathrm{cm}^{2}\right) \\
\mathrm{BHN} & =350 \mathrm{~kg} / \mathrm{mm}^{2} .
\end{aligned}
$$

The penetration equations and the results for a $0.001 \mathrm{~g}$ meteorold are given in Table II- 13 .

TAB LE II - 13

COMPARISON OF METEOROID PENETRATION MODELS

\begin{tabular}{l|c|c}
\hline \multicolumn{1}{c|}{ Researcher } & Correlation & $\begin{array}{c}\text { Penetration of } \\
0.001 \mathrm{~g} \text { Meteoroid }\end{array}$ \\
\hline (A) Summers & $\mathrm{p}=1.75 \mathrm{~m}^{1 / 3}$ & $0.175 \mathrm{~cm}$ \\
(B) Bjork & $p=0.7 \mathrm{~m}^{1 / 3}$ & 0.07 \\
(C) Elchelberger & $p=2.9 \mathrm{~m}^{1 / 3}$ & 0.29 \\
\hline
\end{tabular}




\section{g. Meteoroid Damage}

The remaining analysis is based on the work of Summers and Charters. (68) The penetration theories are based on targets with thickness $t>p$. It is generally agreed that if a meteoroid will penetrate a distance, $p$, into a thick target, it will just puncture a target of thickness $1.5 \mathrm{p}$, and may cause spalling of a thicker target. Thus, to provide armor against meteoroids of mass $\mathrm{m}$, a thickness greater than $\mathrm{p}$ must be provided.

$$
\mathrm{t} \quad \mathrm{Fp}
$$

where $F$, the thin target factor, is 1.5 or greater. A value of F - 1.75 has been recommended by NASA Lewis. Combining,

$$
t=\mathrm{FKm}^{\mathrm{l} / 3}
$$

where $K$ is a function of target and meteoroid properties.

The cumulative meteoroid flux is expressed by an equation of the general form

$$
\phi \quad a m^{-b} .
$$

The penetrating flux $\phi_{\mathrm{p}}$ of micrometeors with mass equal to or greater than the mass required to penetrate a thickness $t$ is

$$
\phi_{\mathrm{p}}=\mathrm{a}\left(\frac{\mathrm{t}}{\mathrm{FK}}\right)^{-3 b} .
$$

The probability of no system fallure due to meteoroid damage is

$$
P(0)=e^{-\phi_{p} A_{V} T}
$$

where

$$
\begin{aligned}
A_{V} & =\text { exposed area }, \\
T & =\text { time }
\end{aligned}
$$


for small values of $P(0)$,

$$
1-P(0) \approx \phi_{p_{v}}{ }^{T} \text {. }
$$

Combining equations we obtain

$$
1-P(0)-a\left(\frac{t}{F K}\right)^{-3 b} A_{V} T
$$

which is rearranged to

$$
t-F K a l / 3 b\left[\frac{T}{1-P(0)}\right]^{1 / 3 b} A_{v}^{1 / 3 b} .
$$

Substituting for

$$
\text { b } 4 / 3
$$

and rewriting

$$
\mathrm{p}=0.283 \mathrm{Fa}^{1 / 4} \mathrm{~K}\left[\frac{\mathrm{AV}_{\mathrm{V}} \mathrm{T}}{1-\mathrm{P}(0)}\right]^{1 / 4} .
$$

The quantity $a^{1 / 4} K$ can be rewritten into the product of a micrometeorite property term and a target material property term

$$
\mathrm{a}^{1 / 4} \mathrm{~K}=\mathrm{a}^{1 / 4}\left(\rho_{\mathrm{M}} \mathrm{V}^{2}\right)^{1 / 3}\left(\rho_{\mathrm{t}} \mathrm{E}\right)^{-1 / 3}
$$

It should be noted at this point that a factor of 10 uncertainty in a, the meteorold flux, results in a factor of 1.78 uncertainty in the meteorold armor requirement, a factor of 10 in $P_{M}$, the meteoroid density, results in a factor of 2.15 uncertainty in the armor. Substituting for

$$
\begin{aligned}
\mathrm{a} & -1.3 \times 10^{-15} \text { number } / \mathrm{m}^{2}-\mathrm{sec} \\
\rho_{\mathrm{M}} & =0.4 \mathrm{~g} / \mathrm{cc} \\
\mathrm{V} & =30 \mathrm{Km} / \mathrm{sec} \\
\mathrm{F} & =1.75
\end{aligned}
$$


and putting $E$ into units of $10^{6} \mathrm{~kg} / \mathrm{cm}^{2}, T$ into years and $A_{v}$ in meters ${ }^{2}$, results

$$
t(\mathrm{~cm})=0.106\left(\rho_{\mathrm{t}} \mathrm{E}^{\prime}\right)^{-1 / 3}\left[\frac{\mathrm{A}_{\mathrm{V}}(\mathrm{m})^{2} \mathrm{~T}(\mathrm{yr})}{1-\mathrm{P}(0)}\right]^{1 / 4}
$$

The micrometeoroid armor weight penalty per unit area is

$$
\rho_{\mathrm{t}} \mathrm{t}=0.106 \rho_{\mathrm{t}}^{2 / 3} \mathrm{E}^{\prime-1 / 3}\left[\frac{\mathrm{A}_{\mathrm{V}} \mathrm{T}}{1-\mathrm{P}(0)}\right]^{1 / 4}
$$

This equation suggests a micrometeoroid armor material figure of merit (MFM).

$$
M F M=\rho_{t}^{2 / 3} E^{\prime-1 / 3}
$$

Room temperature values of $\left(\rho_{\mathrm{t}} \mathrm{E}^{\prime}\right)^{-1 / 3}$ and $\rho_{\mathrm{t}}^{2 / 3} \mathrm{E}^{\prime-1 / 3}$ are given in Table II- 14 for the most appropriate materials.

TAB LE II- 14

METEOROID ARMOR MATERIAL PROPERTIES

\begin{tabular}{l|c|c|c|c}
\hline \multicolumn{1}{c|}{ Material } & $\begin{array}{c}\text { Density } \\
(\mathrm{g} / \mathrm{cc})\end{array}$ & $\begin{array}{c}\mathrm{E} \times 10^{-6} \\
\left(\mathrm{~kg} / \mathrm{cm}^{2}\right)\end{array}$ & $\rho_{\mathrm{t}} \mathrm{E}^{\prime-1 / 3}$ & $\rho_{\mathrm{t}}^{2 / 3} \mathrm{E}^{\prime-1 / 3}$ \\
\hline Beryllium & 1.85 & 2.9 & 0.57 & 1.1 \\
Aluminum & 2.7 & 0.63 & 0.83 & 2.2 \\
Iron & 7.9 & 2.1 & 0.39 & 3.1 \\
Molybdenum & 10.2 & 33 & 0.31 & 3.2 \\
Stainless Steel & 7.9 & 1.9 & 0.4 & 3.2 \\
Copper & 8.9 & 1.1 & 0.46 & 4.1 \\
\multicolumn{1}{l}{$1 \mathrm{~kg} / \mathrm{cm}^{2}=14.4 \mathrm{psi}$} & & \\
\hline
\end{tabular}

A generalized plot of armor thickness $(t)$ vs $\left[\left(A_{V} T\right) / 1-P(0)\right]$ for values of the target property $\left(\rho_{t} E^{\prime}\right)^{-1 / 3} 1$ s given in Figure II- 32 . 


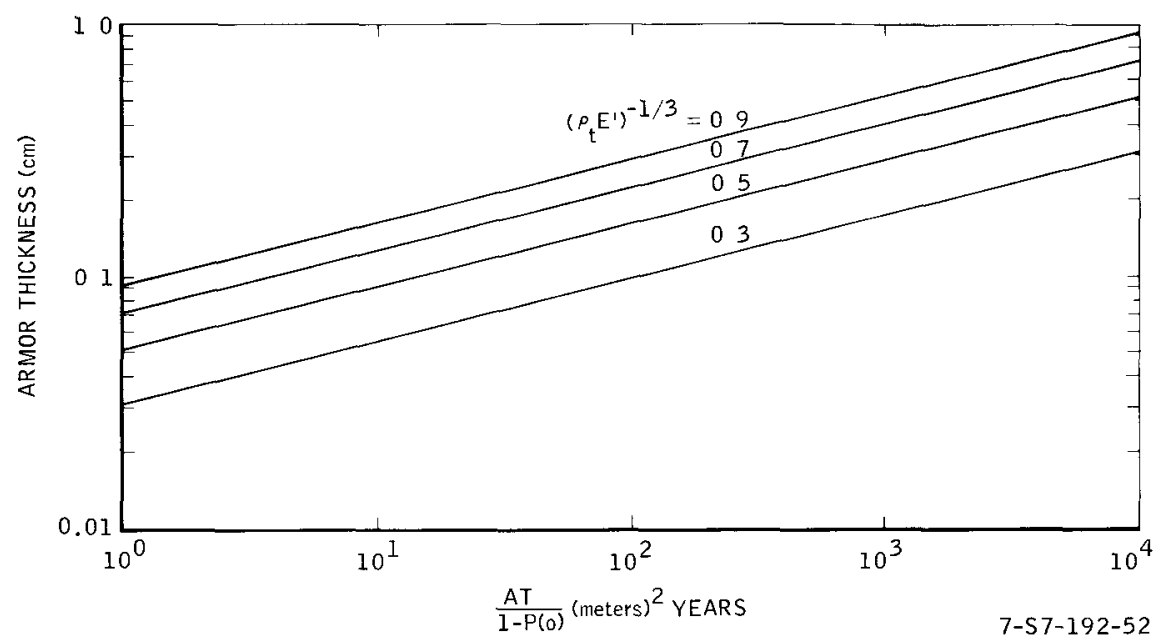

Figure II-32. Meteoroid Armor Thickness Requirements

h. Weight Penalty

In large power systems, the micrometeorite armor requirement imposes a significant weight penalty that provides a strong incentive to reduce the vulnerable area by the use of fins and 0 reduce the radiator area by using a higher temperature power conversion cycle. The armor weight per electrical watt is

$$
\frac{\rho_{t} \mathrm{AA}_{\mathrm{V}}}{\mathrm{P}}=\text { Constant }\left[\frac{\mathrm{T}}{1-\mathrm{P}(0)}\right]^{1 / 4} \frac{\mathrm{A}_{\mathrm{V}}^{5 / 4}}{\mathrm{P}}
$$

If

$$
A_{v}=x A
$$

where $A$ is the total radiator area, and for low efficiency systems

$$
A \simeq \frac{P}{\eta_{O} \sigma \in T_{C}^{4}}
$$

then

$$
\frac{\rho_{\mathrm{t}}^{\mathrm{tA}} \mathrm{v}}{\mathrm{P}}=\text { Constant }\left[\frac{\mathrm{PT}}{\mathrm{l}-\mathrm{P}(0)}\right]^{1 / 4}\left[\frac{\mathrm{x}}{\eta_{\mathrm{O}} \sigma \epsilon}\right]^{5 / 4} \cdot \frac{\mathrm{l}}{\mathrm{T}_{\mathrm{c}}^{5}}
$$


The results are plotted as a function of $\mathrm{T}_{\mathrm{c}}$ in Figure II- 33 for the following example

$$
\begin{aligned}
& P=1 \text { Mwe } \\
& \eta_{\mathrm{O}}-10 \% \\
& \mathrm{x}=0.1
\end{aligned}
$$

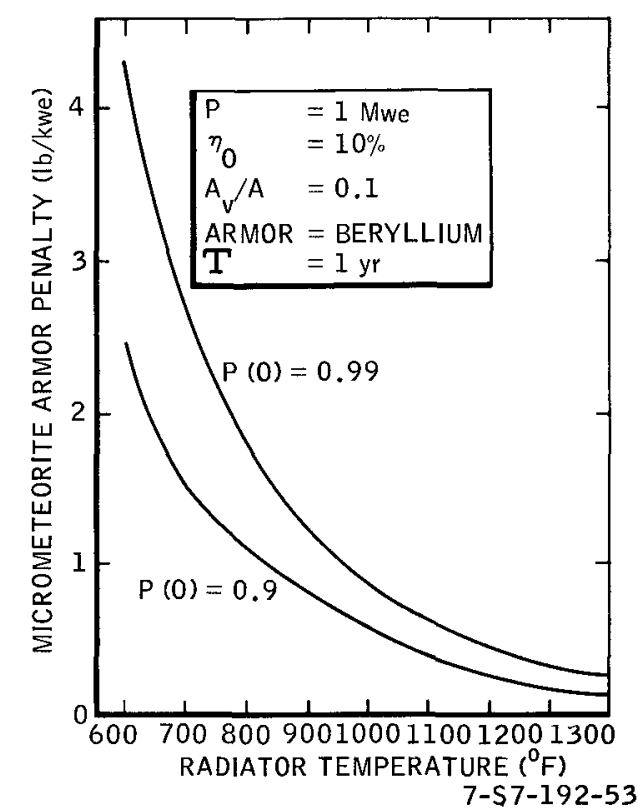

Figure II-33. Example Performance

Penalty for Micrometeorite

Armor

\section{DESIGN EXAMPLE}

Let us assume we have the problem of determining the minimum weight radiator required to reject the waste cycle heat from a mercury rankine cycle power system. The thermal heat to be rejected w11 be assumed to be $50 \mathrm{kwt}$ at an average condensing temperature of $600^{\circ} \mathrm{F}$. The geometry selected is shown in Figure II- 34 and consists of an array of tubes attached to a cylindrical surface. The mercury vapor is distributed to the individual tubes by a manifold. The mercury vapor is 


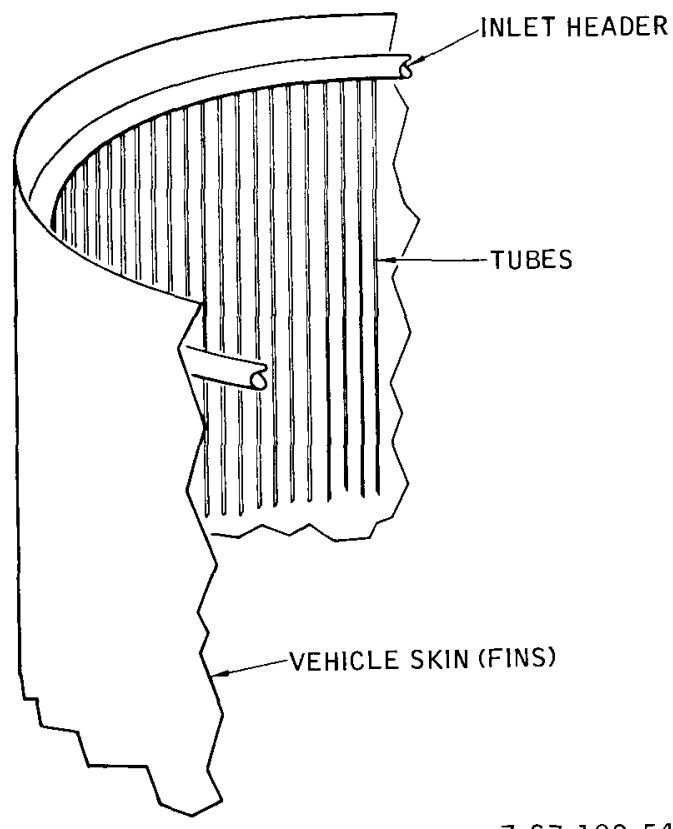

7-S7-192-54

Figure II-34. Typical Radiator Geometry

condensed to a liquid as it flows through the tubes and the heat of condensation is radiated to space from the surface of radiator. The assumed tube and fin detail and materials are shown in Figure II-35. This geometry closely approaches the 1dealized rectangular fin radiatıng from one side. In addition, assume that the cylinder is $5 \mathrm{ft}$ in diameter and that the surface emissivity can be taken as 0.90 . The particular configuration or overall shape that a radiator will assume is usually dictated by the vehicle or application considerations. From Figure II-23, the quantity

$$
\left.\frac{\mathrm{Q}}{\mathrm{A}}\right|_{600}-0.70 \mathrm{kw} / \mathrm{ft}^{2} .
$$




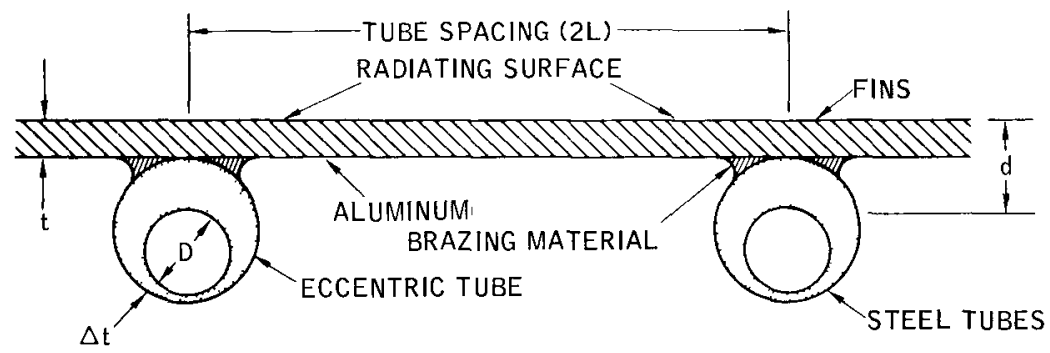

7-S7-192-55

Figure II-35. Tube and Fin Detail

With the values of $\epsilon=0.9$ and $Q=50 \mathrm{kw}$ the absolute minimum radiator area will be $79.5 \mathrm{ft}^{2}$ at a fin effectiveness of 1.0 , which can only be approached. If we arbitrarily assume a fin effectiveness of $\eta_{\mathrm{f}}=0.80$, then the area is $100 \mathrm{ft}^{2}$ and the length of the cylinder is $6.3 \mathrm{ft}$. With the fixed value of fin effectiveness, from Figure II- 27

$$
\frac{\sigma \epsilon \mathrm{T}^{\mathrm{L}^{2} \mathrm{~T}_{\mathrm{o}}^{3}}}{\mathrm{kt}}=0.20 .
$$

$L$, as will be recalled, is the fin length and hence is related to the number of tubes $(N)$ in this example by $L=\pi D / 2 N$. With a fixed value for the number of tubes, the thickness of the fins can be calculated. Also, with the number of tubes and length of tubes fixed, the tube diameter can be calculated to give the desired pressure drop. The vulnerable area, which is the sum of the projected areas of the condenser tubes, can now be calculated. The weight of the radiator then becomes the sum of the weight of the fins, armor, tubes and the mercury fluid holdup. As the number of tubes is increased, with the fin effectiveness held constant, the fin weight decreases, and the tube armor and fluid holdup weights increase. This effect is illustrated in Figure II- 36 and results in a minimum weight solution for some combination 


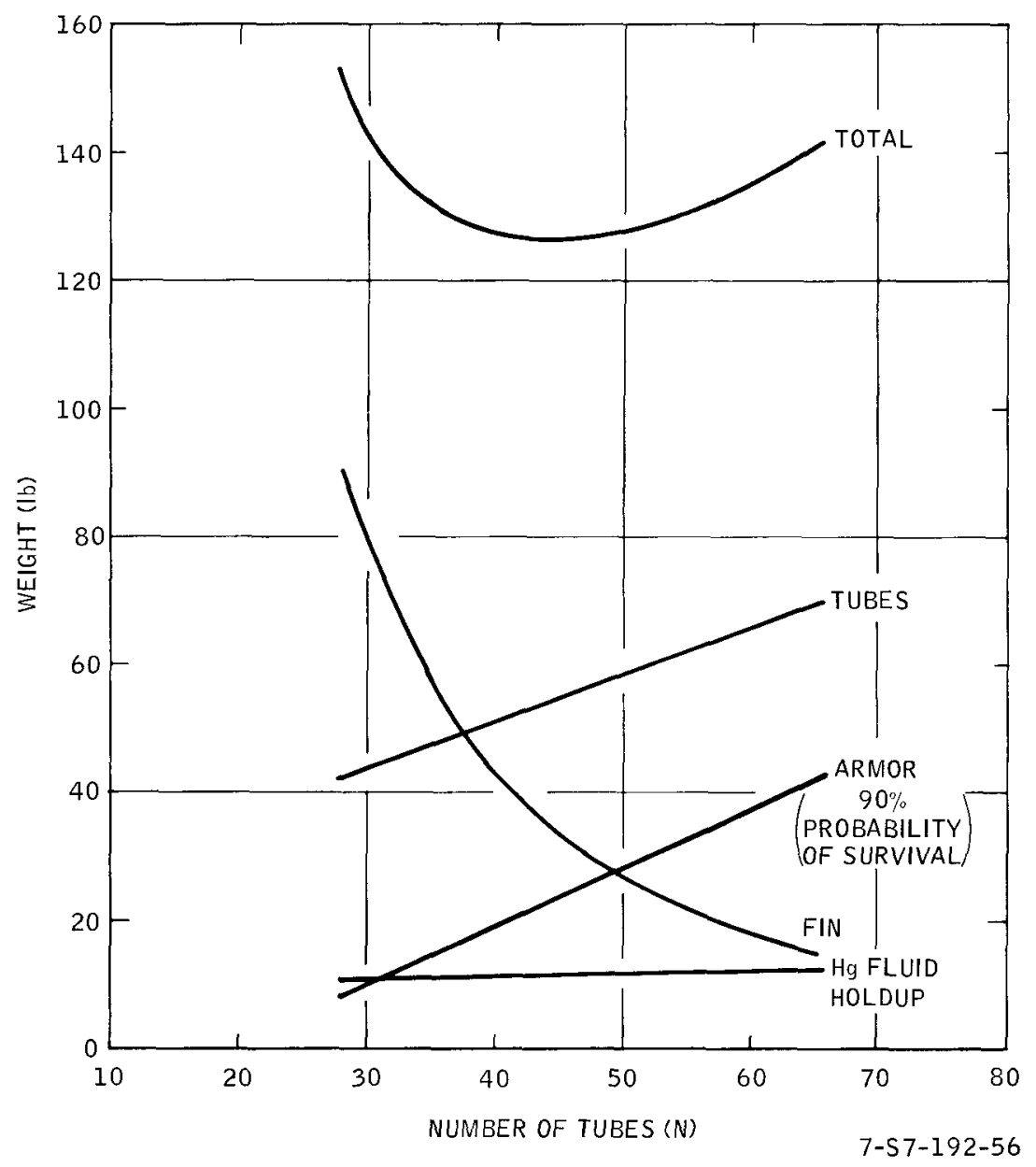

Figure II-36. Radiator Weight vs Number of Tubes

of the four elements. If a series of such curves for different values of fin effectiveness are constructed and the minimum points plotted, an optimum value ( 1 e. , minımum weight) of fin effectiveness can be found, as illustrated in Figure II-37. A minimum weight solution can be discerned, or conversely, the weight penalty associated with operating with either higher or lower radiator area. For this case, the optimum fin effectiveness as shown in Figure II- 37 is about 0.76 , which corresponds 
to an area of $106 \mathrm{ft}^{2}$. It should be noted that the effect of the tube and armor weight was to increase the optimum fin effectiveness over that indicated for minimum fin weight only.

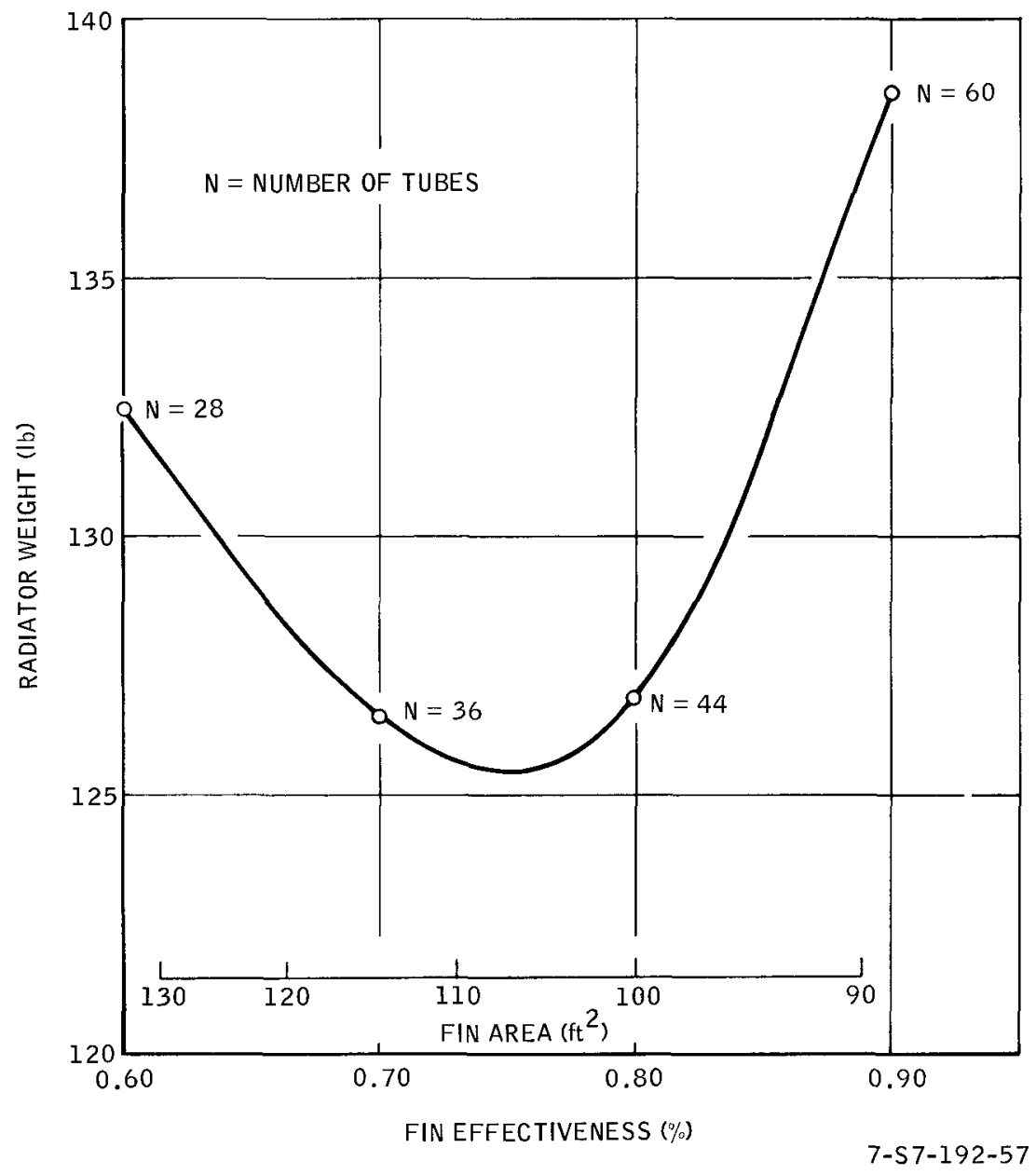

Figure II-37. Radiator Weight vs Fin Effectiveness 


\section{SYSTEM DESIGN}

\section{A. SYSTEM CONSIDERATIONS}

Thus far we have considered the requirements and limitations of reactors. In order to establish the specific reactor requirements for a space power application and to select a reactorpower conversion combination, we must consider the mutual interactions of the three major subsystems for nuclear space power plants. These include (1) the reactor heat source, (2) the power conversion cycle, and (3) the waste heat radiator. The power conversion cycle determines the operating temperature of the other two and its weight is relatively insensitive to temperature. The detalls of the cycle, for example the working fluid, are a function of the operating temperature. The radiator area and weight are, of course, a strong function of the cycle cold temperature. The reactor size, weight, and concept are a function of temperature because of the operating temperature limitations of the applicable core materials. In order to minimize the weight of the heat rejection system, it is necessary that the conversion system operate at a high sink temperature and recover a maximum fraction of Carnot efficiency. Since the waste heat of a cycle must be radiated to space, the area requirement and hence the weight associated with the heat rejection system is, for a given power level, proportioned to the fourth power of the cycle cold temperature and inversely proportional to the cycle conversion efficiency. At low powers, the reactor size is independent of power because of the minimum critical size requirements. Therefore, the conversion efficiency affects only the size of the radiator and the conversion equipment. Since the reactor and shield are the dominant weight of nuclear power systems at low power, the conversion efficiency and the cycle cold temperature are not so important. At higher power levels, however, the radiator becomes the dominant welght 1 tem of the system. Consequently, for the higher power systems there is a great incentive to achieve high efficiency and high heat rejection 
temperatures to minimize the weight of the radiator. This point is illustrated in Figure II- 38 which shows the relative weight contribution of the different system components as the power level is increased at constant radiator temperature.

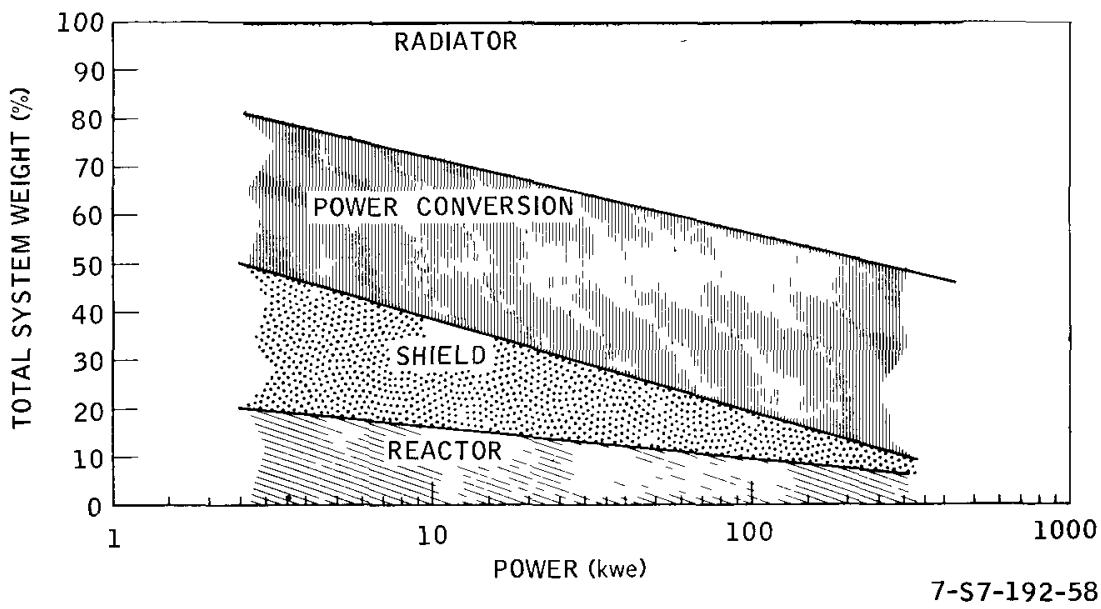

Figure II-38. Weight Distribution for Nuclear Turboelectric Systems

\section{B. TURBOELECTRIC SYSTEMS}

Of the myriad of thermodynamic heat engines that have been invented, the ones of most interest for space application are the Rankine and the Brayton cycles. A very brief description of these cycles is given below.

\section{a. Rankine Cycle Power Conversion}

The cycle diagram and the schematic arrangement of the Rankine cycle are shown in Figure II-39. The Rankine cycle is characterized by a working fluid that changes state. In the cycle, the working fluid is compressed as a liquid and heat is added to raise the liquid to the saturation temperature, i. e., 1-2; heat is added at constant pressure and temperature during a phase change from liquid to vapor, i.e., 2-3; the vapor is expanded through a turbine and work is extracted, i.e., 3-4; and the waste heat is 

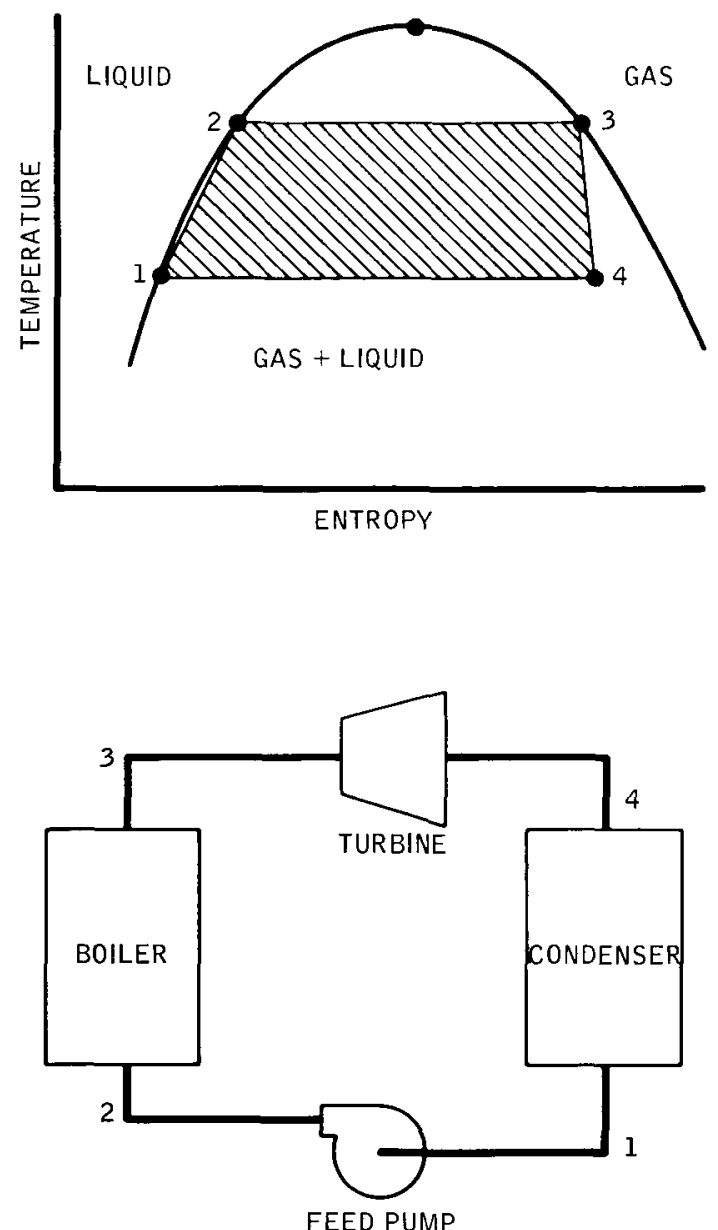

7-57-192-59

Figure II-39. Rankine Cycle Thermodynamic Diagram and System Configuration

rejected at constant pressure and temperature during a phase change from vapor to liquid, 1. e., 4-1.

As a result of the bolling and condensing phase change which allows constant temperature heat addition and extraction, the 1deal Rankıne cycle efficiency approaches the Carnot efficiency. In addition, very little work is required to compress the working fluid in the liquid state. 


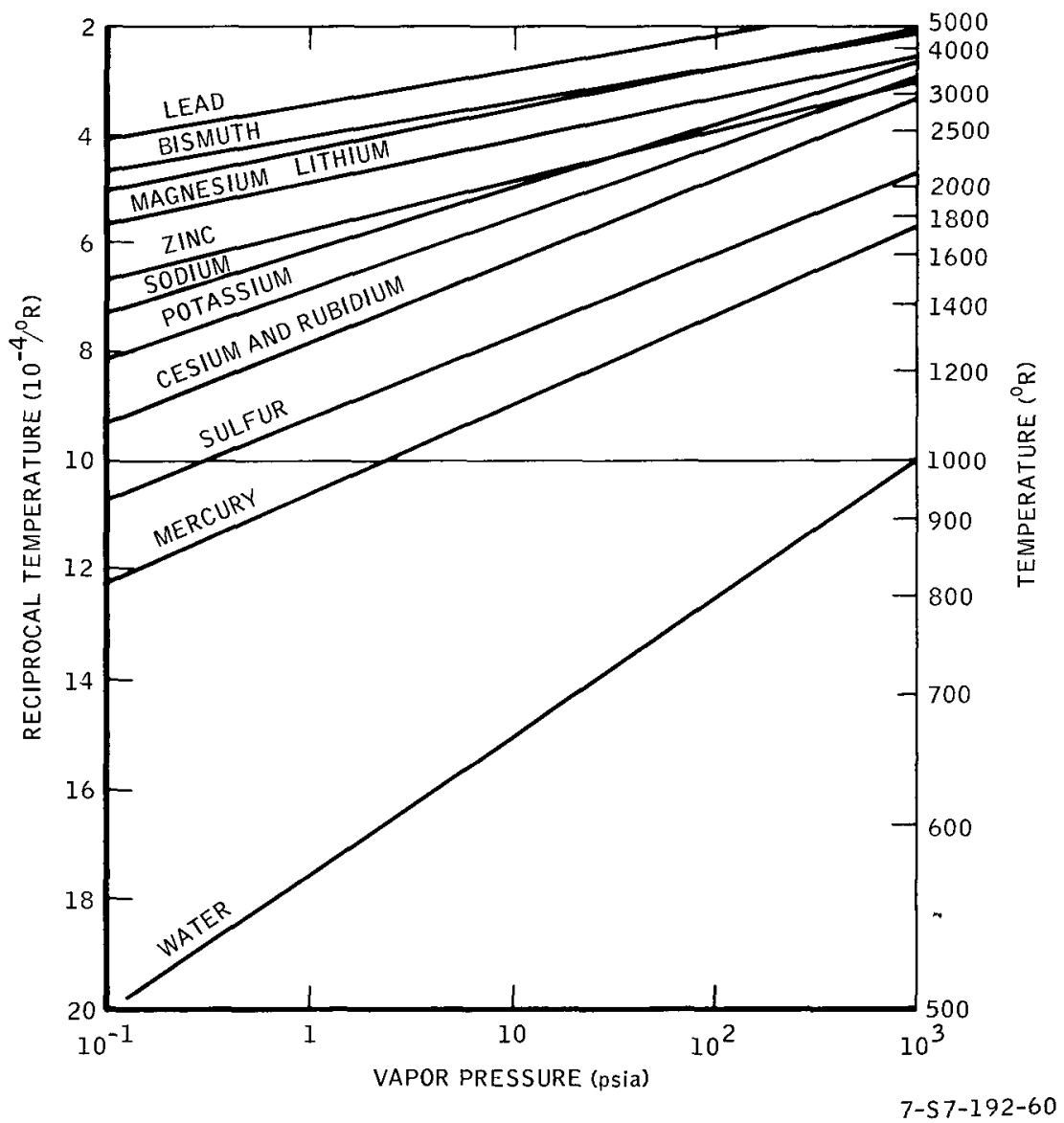

Figure II-40. Vapor Pressure vs Temperature for Potential Rankine Cycle Working Fluids 
The principal advantages of the Rankine Cycle are the high cycle efficiency and the isothermal heat rejection which allow minimum radiator area for a given source temperature.

The principal disadvantages are the inherent corrosion and erosion characteristics of the applicable working fluids.

In the application of a Rankine cycle to space, it is necessary to identify potential working fluids which have the requisite thermodynamic properties for application in the high temperature range demanded by radiator area considerations. The vapor pressure of mercury and the alkali metals 1 s shown in Figure II-40. One thing to note is that the avalable fluids do not provide a continuous coverage of the temperature scale. The thermodynamic properties are such that all of these liquid metals yield an ideal Rankine cycle efficiency that is about 950 of the Carnot efficiency in the range of interest for space.

The optimum heat rejection temperature for a Rankine cycle is determined by the working fluid selected. As a result of balancing Carnot efficiency against the $T^{4}$ law for radiation, the optimum radiator or condensing temperature is about $3 / 4$ of the absolute boiling temperature. The relationship between boiling temperature, radiator area, and cycle working fluid is shown in Figure II-41. The radiator a rea per kilowatt of output is calculated on the basis of an optimum Carnot of $25 \%$ and a conversion efficiency of $40 \%$ of Carnot which results in an overall system efflciency of $10 \%$.

The approximate range of interest for the varlous working fluids is indicated as falling between a bolling pressure of 50 and $300 \mathrm{psia}$. This choice is arbitrary, however, a lower bolling pressure limit results from consideration of pressure ratio across the turbine and vapor specific volume. An upper pressure limit results from consideration of system weight. Another point of concern in cycle temperature selection is the condensing pressure. The 5 psia condensing pressure points for the various fluids are shown in Figure II-41. The condensing pressure must 


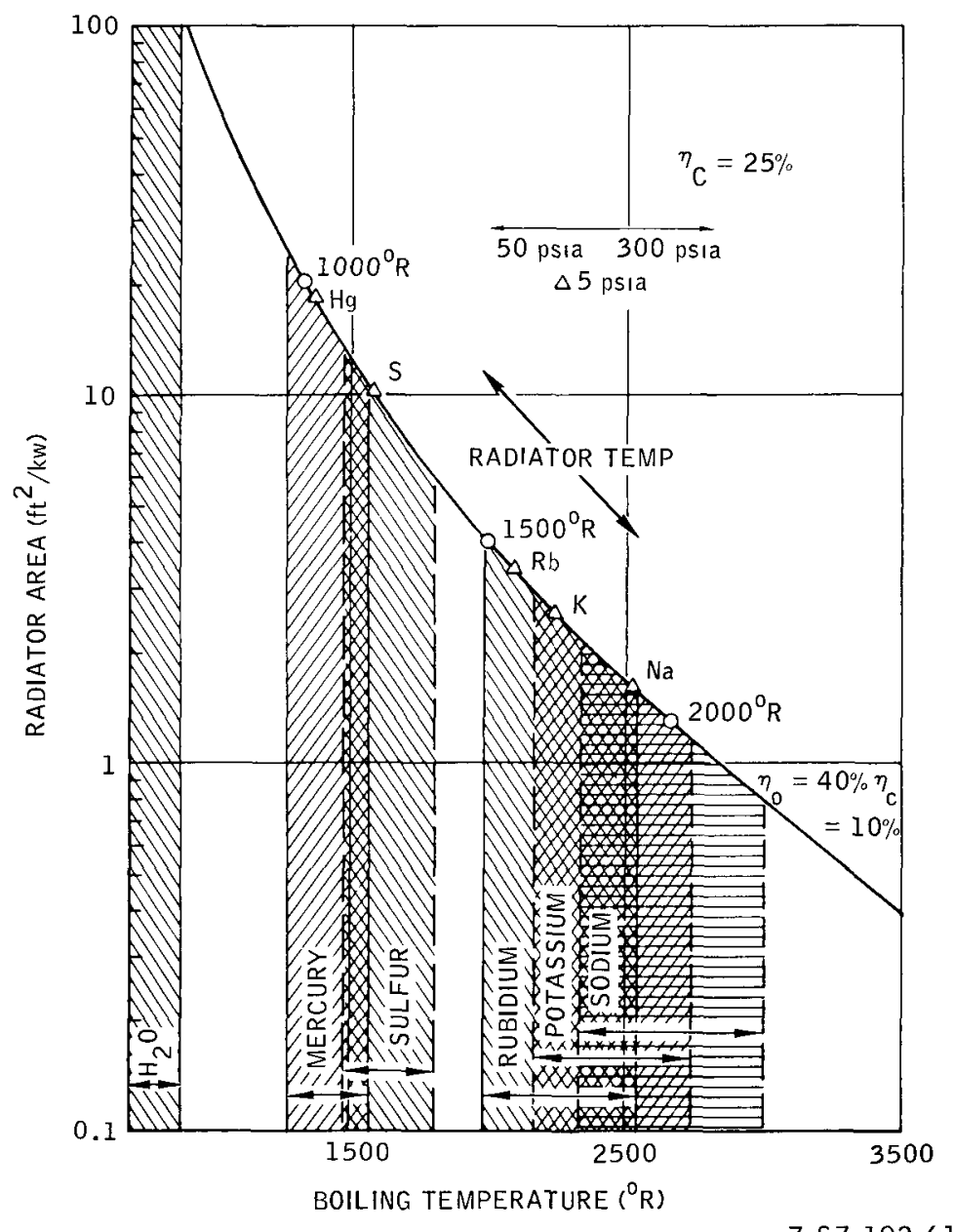

$7-\$ 7-192-61$

Figure II-41. Radiator Area per Kilowatt as a Function of Boiling Temperature for Rankine Cycle Workıng Fluıds 
be high enough to allow for condensing pressure drop while maintaining.an adequate boller feed pump inlet pressure to prevent cavitation.

\section{b. Brayton Cycle Power Conversion}

The cycle dragram and the schematic arrangement of the Brayton cycle are shown in Figure II-42. The working fluid is an inert gas such as helium, neon, argon, etc., or a combination of these gases. In the cycle, the gas is compressed, 1.e., 1-2, heat is added at constant pressure, 1.e., 2-3, the gas is expanded through a turbine and work is extracted, 1.e., 3-4, and waste heat is rejected by a radiator, 1.e., 4-1. Because the working fluid is in a single state and absorbs energy at constant pressure through a rise in temperature, the ideal cycle is inherently limited to a relatively small fraction of the Carnot efficiency. Recent advances in lightweight gas heat exchangers have made it feasible to consider the recuperated Brayton cycle wherein a portion of the otherwise waste heat (4-1) is used in the initial phase of the heat addition (2-3). The recuperated cycle achieves an 1 deal gas cycle efficiency of about 25 to $30 \%$ of Carnot.

Since considerable work is expended in the gas compression (1-2), a large fraction of the work output (3-4) is consumed by the compression stage. Thus, the net cycle performance is quite dependent upon the turbine and compressor efficiency. Again, recent advances in the state of the art of high efficiency and high speed turbines and compressors have contributed to renewed interest in the Brayton cycle.

The advantages of the Brayton cycle are

1) Inherent simplicity of a single loop and a single phase working fluid.

2) The noncorrosive properties of the inert gas working flusd. 

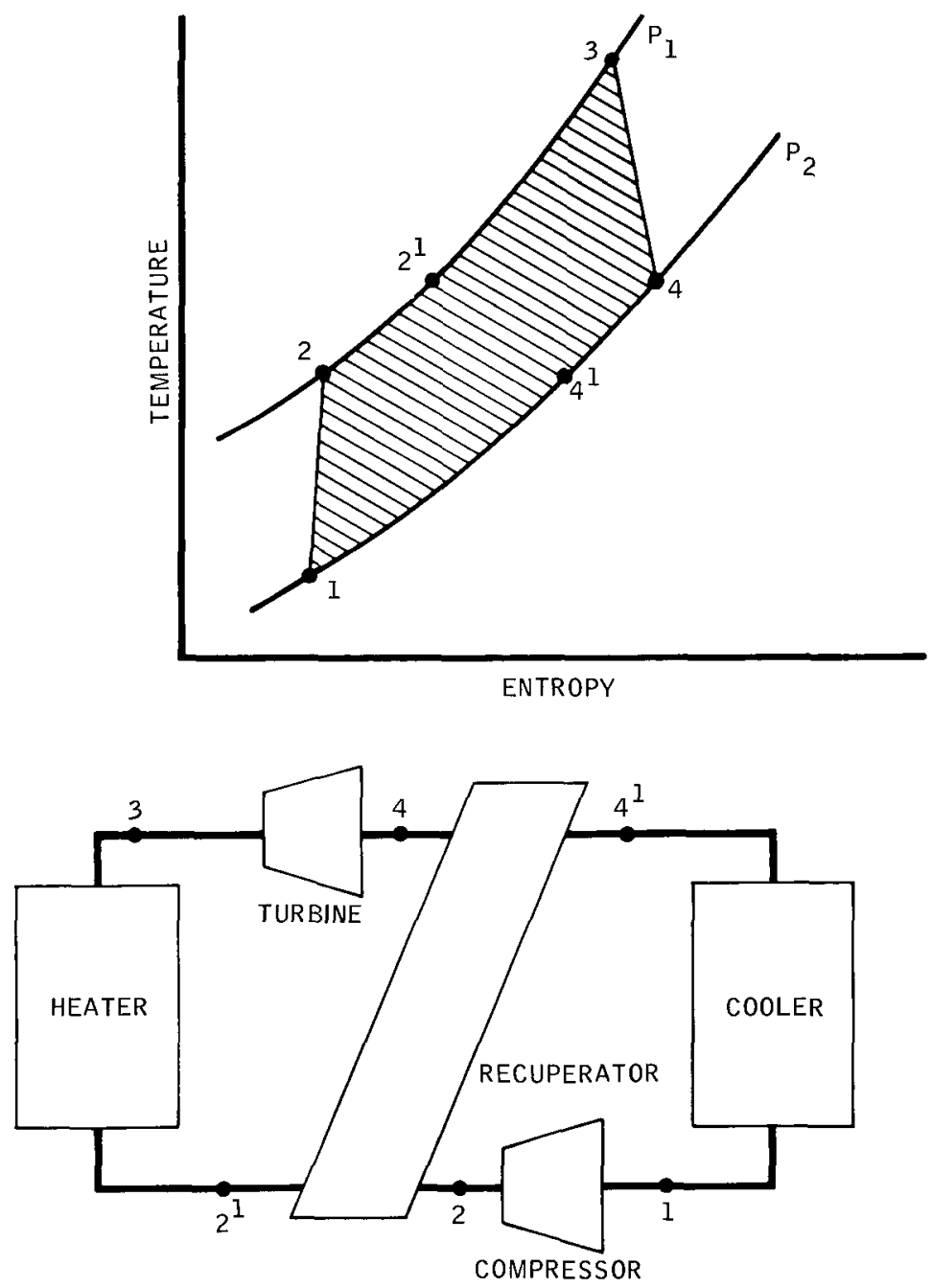

7-S7-192-62

Figure II-42. Brayton Cycle Thermodynamic Diagram and System Configuration 
The disadvantages of the Brayton cycle are

1) The cycle net performance is extremely dependent upon achieving high component efficiencies.

2) The low ideal gas cycle efficiency must be overcome by going to a high Carnot which results in either extreme source temperature requirements or extremely large radiator area.

This latter consideration is probably the dominant factor in the performance evaluation of the Brayton cycle. The detalled parametric optimization of the Brayton cycle efficiencies and temperatures is beyond the scope of this work. However, Figure II-43 shows the results of a detalled study by A1Research, Reference 74 , on a computer optimization of a 35-kwe recuperated Brayton cycle. For comparison purposes, the radiator area/kwe for a Rankine cycle of $40^{\circ}{ }_{0}$ of Carnot efficiency is also shown. It is quite evident that the minimum area Brayton cycle requires a much larger radiator area than a saturated Rankıne cycle operating at the same source temperature. The minimum weight Brayton cycle probably requires more radiator area than that shown in Figure II-43.

\section{c. Reactor - Turboelectric Systems}

A turboelectric nuclear space power system will probably utılıze a Rankine cycle because it offers a high conversion efficiency, operates at relatively low source temperatures, and rejects the waste cycle heat at relatively high temperatures. The Brayton cycle appears unattractive for space application because of its lower fraction of Carnot efficiency, higher reactor temperature requirements, and larger radıator area demands.

There are a number of alternate approaches to the coupling of the reactor heat source, the power conversion, and the heat rejection radiator for a Rankine cycle system. These alternatives 


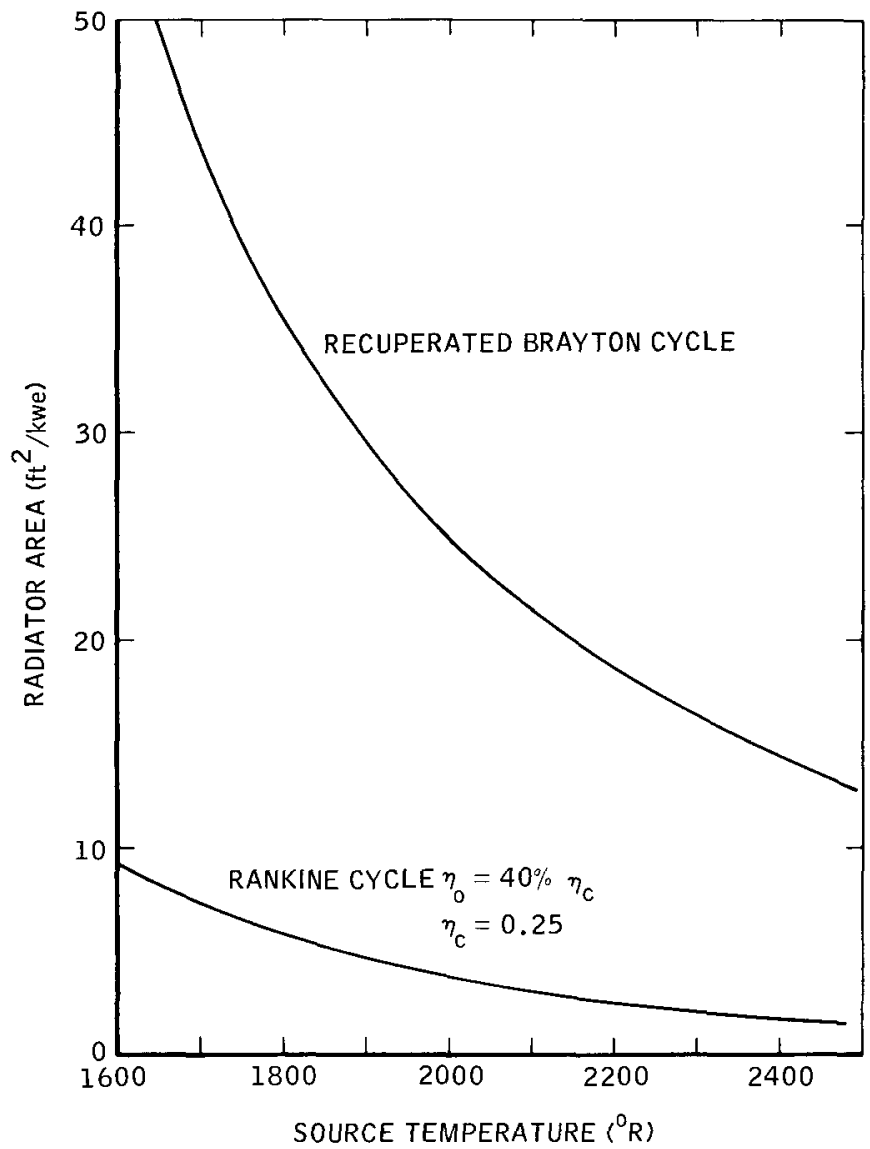

$7-57-192-63$

Figure II-43. Radiator Area and Source Temperature Comparison for Brayton and Rankine Cycles

are shown in Figure II-44. The most direct approach to a saturated cycle is the combination of ab which employs direct boiling of the working fluid in the reactor and direct condensing in the radiator. This combination requires a liquid-vapor separator and a recirculation pump such that the high heat transfer coefficients of low quality bolling can be maintained in the reactor core. The disadvantages of this system are the nuclear interactions between the working fluid and the reactor. 


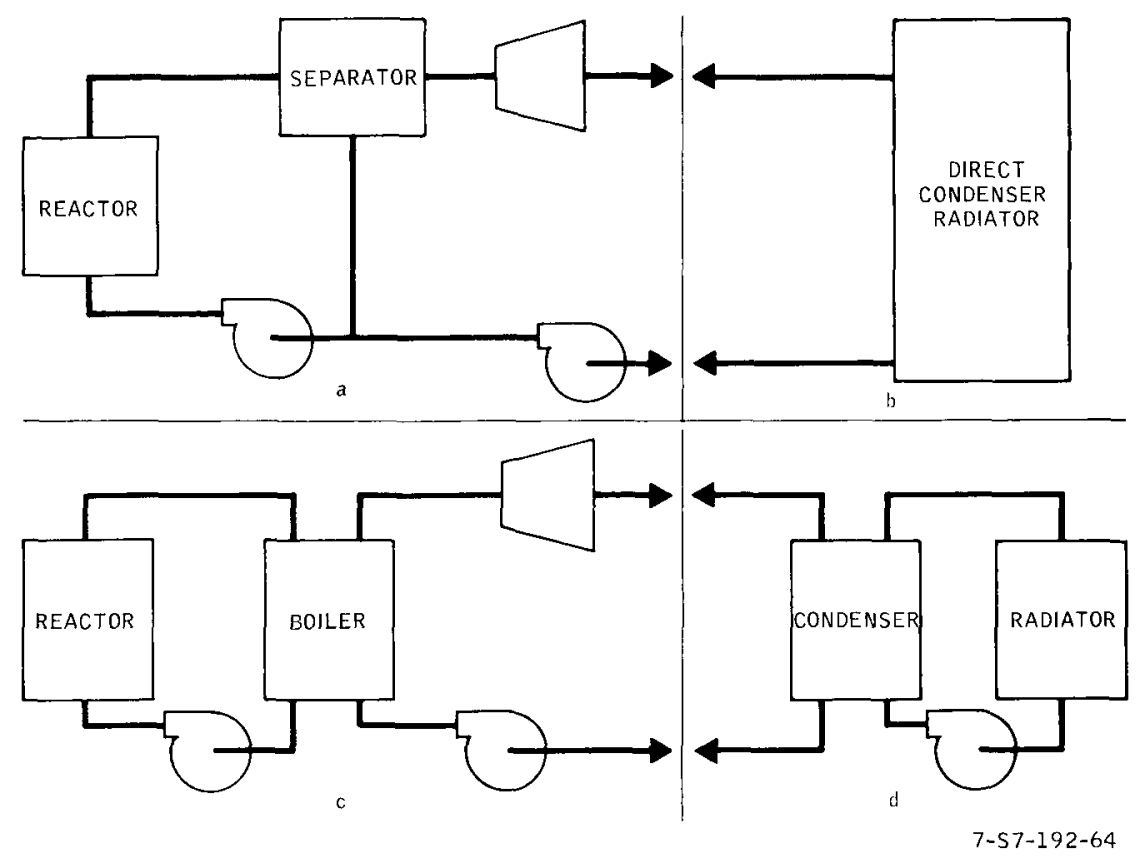

Figure II-44. Rankine Cycle System Configuration

Research on this concept is being conducted by ORNL (Oak Ridge National Laboratory) as a backup to SNAP 50.

The more conventional Rankine cycle employs superheat in order to minimize moisture content in the turbine. The combination of $\mathrm{cb}$ is used in SNAP 2 and SNAP 50. The reactor is coupled to the boiler by a pumped liquid metal heat transfer loop. The working fluid is boiled and superheated in a once-through boiler. The combination $\mathrm{cd}$ is employed in the NASA SNAP 8. The necessary temperature drop in the radiator heat transfer loop increase the radiator requirements over that of the direct condenser-radiator approach. However, the third loop allows greater vehicle integration flexibility which becomes increasingly important at higher power levels.

Figure II-41 defines the area of interest or heat source temperature requirements for varıous Rankine cycle working 


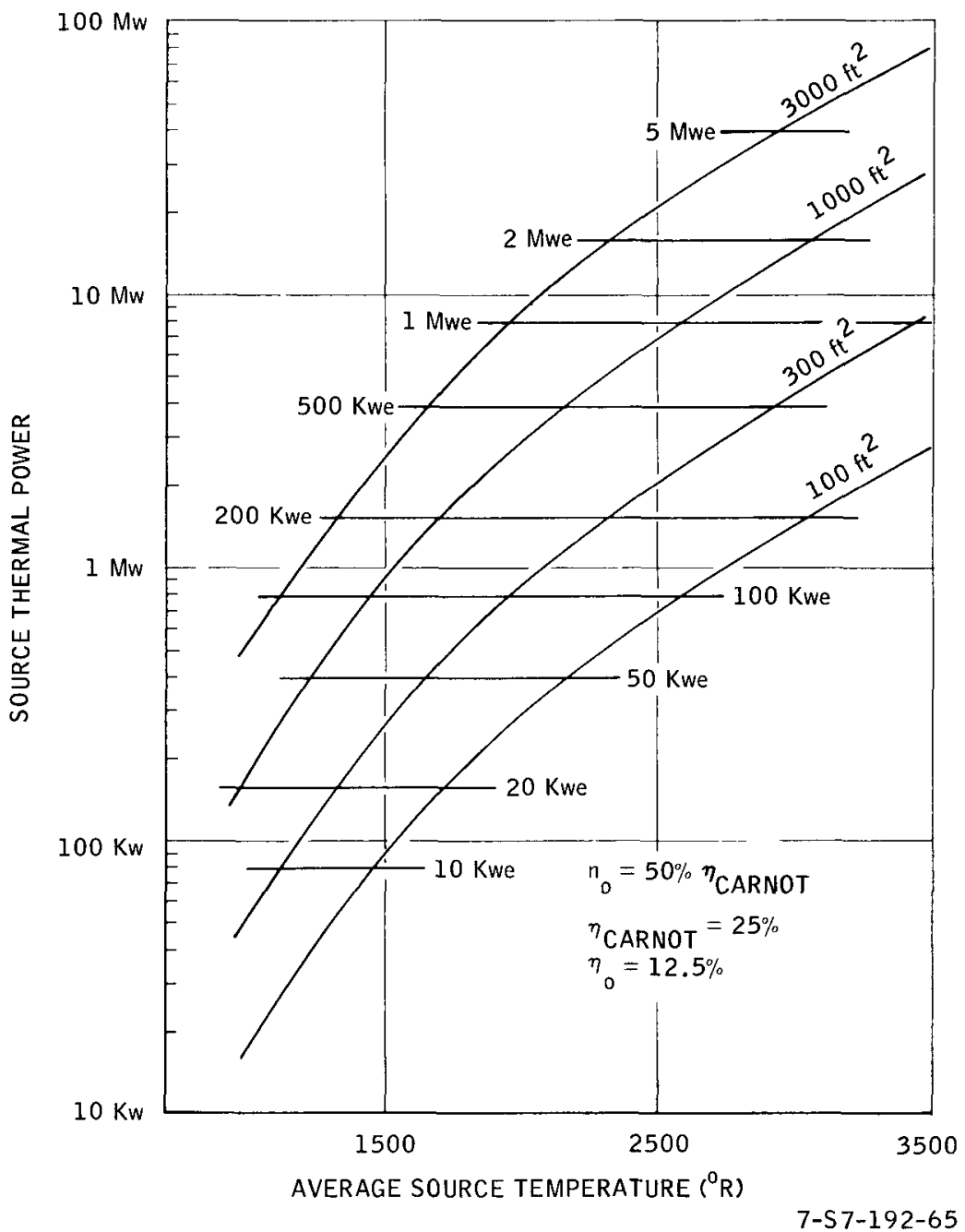

Figure II-45. Reactor Thermal-Power and SourceTemperature Requirements as a Function of Electrical Power Output and Available Radiator Area for Rankine Turboelectric Systems 
fluids. The reactor heat source thermal power and temperature requirements for Rankine cycle systems are shown in Figure II-45 as a function of electrical power output and avallable radiator area. This survey assumed a constant fraction (50\%) of Carnot conversion efficiency and a constant Carnot efficiency of $25 \%$ for minimum radiator area. This latter assumption should not be confused with minimum system weight which can occur at a different value of Carnot. It should be remembered that the actual heat-source temperature requirement is probably several hundred degrees higher than the boiling temperature in order to provide superheat and to allow for temperature drops throughout the system, especially the boiler. This problem can be eliminated by the use of a direct cycle which boils directly in the reactor core to provide saturated vapor. This approach is also attractive from the point of view of eliminating system components and weight. However, direct bolling in the reactor can introduce reactor control problems and a saturated cycle probably demands the complication of moisture separation in the turbine.

Since the reactor weight and the cycle selection are a function of temperature and since the radiator weight is a function of temperature and power, the choice of a reactor-cycle combination is a function of system power level. This point can be illustrated by means of an example which compares the weight of the reactor and radiator for a water reactor-water cycle, for a hydride reactor-mercury cycle, and for a carbide reactor-rubidium cycle (see Figure II-15). The example shown in Figure II-46 assumed a reactor weight increased by $50 \%$ over that shown in Figure II- 15 in order to provide for coolant void and cylindrical geometry. Reactor weights are for this purpose considered independent of power, and radiator area $1 \mathrm{~s}$ assumed at $1 / 2$ to $1 \mathrm{lb} / \mathrm{ft}^{2}$. Figure II-46 indicates that

1) The water system is too limited in its applicable range,

2) The hydride-mercury system has the lower weight between about 2 and $20 \mathrm{kwe}$, and

3) The carbide-rubidium system is better above $20 \mathrm{kwe}$. 


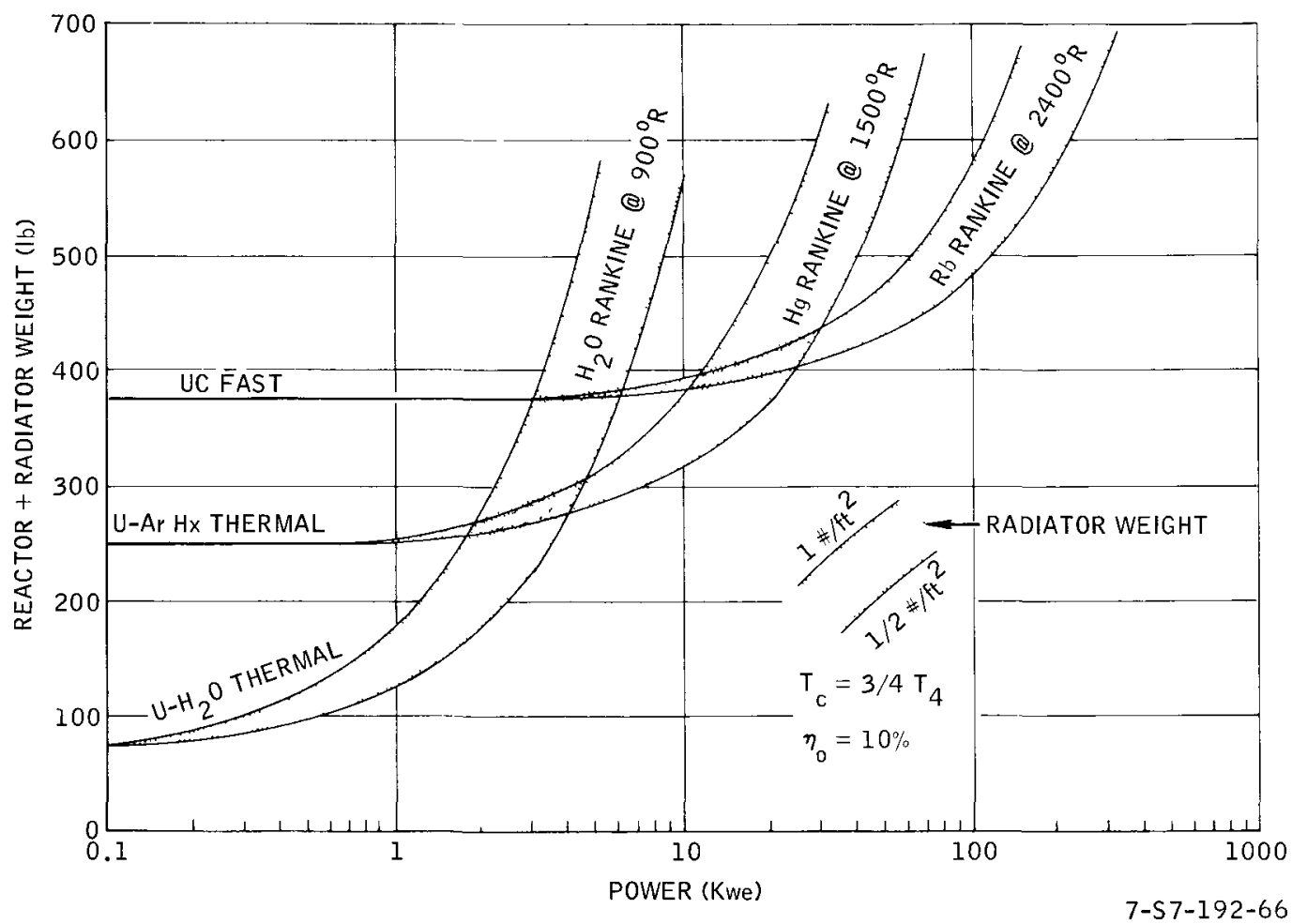

Figure II-46. Reactor Plus Radiator Weight as a Function of Power for Various Reactor-Cycle Combinations. (Reactor weights have been increased by $50 \%$ over Figure II-15 to provide for coolant vold and cylindrical geometry.) 
However, this example does not consider the state-of-the-art difference between a $1500^{\circ} \mathrm{R}$ system and a $2400^{\circ} \mathrm{R}$ system (see Figures II-13 and II-14). In effect, lower radiator weight is achieved in the higher temperature system at the expense of not only increased reactor weight but, more importantly, increased materials problems such as strength, corrosion, sublimation, self-welding, etc. In general, increased temperature is rarely an easy solution to a problem. However, there is no denying that high-power systems will demand increased temperatures and that the necessary technology will be developed. One cannot rule out a system because of temperature except on the basis of development time. As temperatures increase, greatly increased development tımes must be allotted. Avaılability will in effect move the crossover point to a higher temperature system to much higher power levels until the technology exists to make the simple weight consideration independent of state-of-the-art differences. Thus, for example, the hydride-mercury system may well be extended into the 100-kwe range because of technological avallability even though it does not have the lightest weight potential at that power. It is, how ever, clear that the high-temperature systems must ultimately be developed in order to achieve the minimum specific weight (lb/kwe) required for future missions and especially for electric propulsion. As illustrated in Figure II-38, at very high powers the reactor weight per kilowatt becomes small and the radiator weight per kilowatt becomes dominant. Radiator area and weight can only be reduced by increased source temperature. The significance of increased source temperatures for minimum radiator area is even further increased when the weight penalty for meteorite protection is considered.

The influence of operating temperature on the reactor fuel burnup limit should not be forgotten. In the example of Figure II- 18 the effect of temperature on the useful energy from a $\mathrm{UO}_{2}$-stainless steel cermet core was illustrated. In the case of a space system the reward for higher temperature is lower 
radiator area and weight and the penalty is decreased core endurance or a larger core for a given endurance requirement. Thus, if temperature is increased radiator weight will decrease and the reactor weight will increase if the reactor size is determined by a temperature-dependent burnup limit. The following example will illustrate this situation. Assume

1) The reactor core material has the same burnup dependence on temperature as shown in Figure II- 17

2) 1 at $\%=10 \mathrm{Mw}-\mathrm{yr}$

3) Reactor weight $=750 \mathrm{lb} / \mathrm{ft}^{3}$ of core

4) Power = 1 Mwe

5) Efficiency $=10 \%$

6) System life $=1 \mathrm{yr}$

7) Radiator weight $=1 \mathrm{lb} / \mathrm{ft}^{2}$

Figure II-47 shows the reactor and the radiator weight for these assumptions as a function of operating source temperature. The results indicate a minimum reactor plus radiator weight at a source temperature of about $1700^{\circ} \mathrm{F}$. This result should not be taken literally since the reference fuel material, $\mathrm{UO}_{2}$ stainles steel cermet, is not a useful fuel for space systems. However, it could be considered representative of a very stable hightemperature fuel. A significant optimization must await complete burnup vs temperature data for applicable high-temperature fuels like UC.

\section{THERMOELECTRIC SYSTEMS \\ a. Thermoelectric Power Conversion}

Direct conversion of heat to electricity by means of the Seebeck (thermoelectric) effect offers advantages of static operation and high reliability potential. However, the temperature limits and the conversion efficiency of current "stateof-the-art" converter materials limit the usefulness of thermoelectric conversion systems to relatıvely low power levels. 


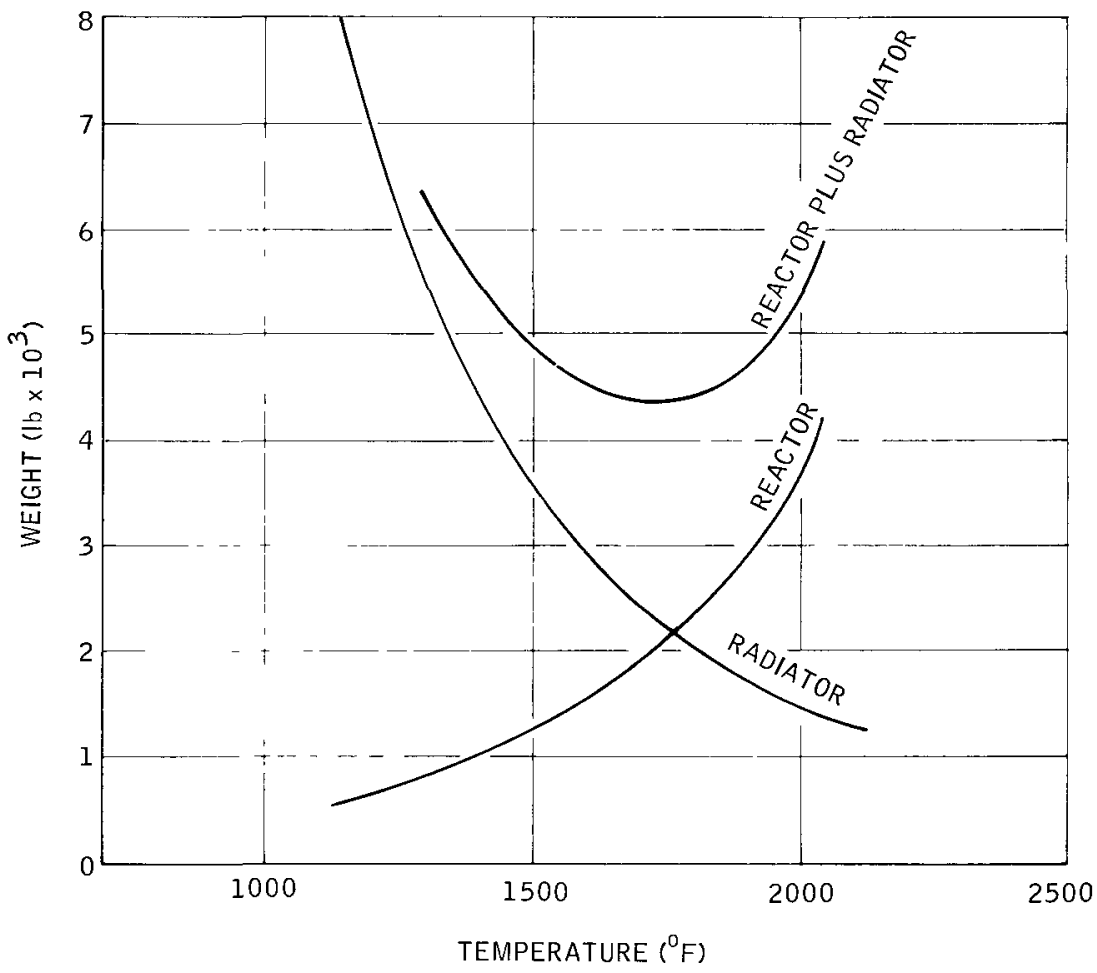

$7-57-192-67$

Figure II-47. Tradeoff Between Reactor Weight and Radiator Weight as a Function of Operating Temperature. (This illustration applies to a reactor which has the burnup limit of Figure II-17.)

The direct conversion of heat to electricity was discovered by Seebeck in 1822. For over 100 years the approach was considered to be of no practical significance because of the low efficiency of known materials. The efficiency of a thermoelectric power conversion device is a function of the material's figure of merit, 2 , which is defined as $s^{2} / \rho \mathrm{K}$ where $s$ is the Seebeck coefficient in volts/C, $\rho$ is the electrical resistivity in $\Omega-\mathrm{cm}$, and $\mathrm{K}$ is the thermal conductivity in watts $/ \mathrm{cm}-{ }^{\circ} \mathrm{C}$. The development of semiconductor materials in the late 1950's resulted in the identification of a number of intermetallic compounds and alloys of low thermal conductivity that could be doped to provide a carrier concentration leading to a maximum $\mathrm{Z}$. 
The basic elements of a thermoelectric converter are illustrated in Figure II-48. Heat flows from a source at temperature $\mathrm{T}_{\mathrm{H}}$ through the thermoelectric material to a sink at temperature $\mathrm{T}_{\mathrm{C}}$. The resulting temperature difference across the thermoelectric material induces a voltage difference between the hot and cold end, $v=S\left(T_{H}-T_{C}\right)$. The two pieces of thermoelectric material are called a couple and are doped $N$ and $P$ such that the Seebeck voltage of one is positive and the other negative. This allows series electrical connection of the thermoelectric elements without the introduction of a heat shunt.

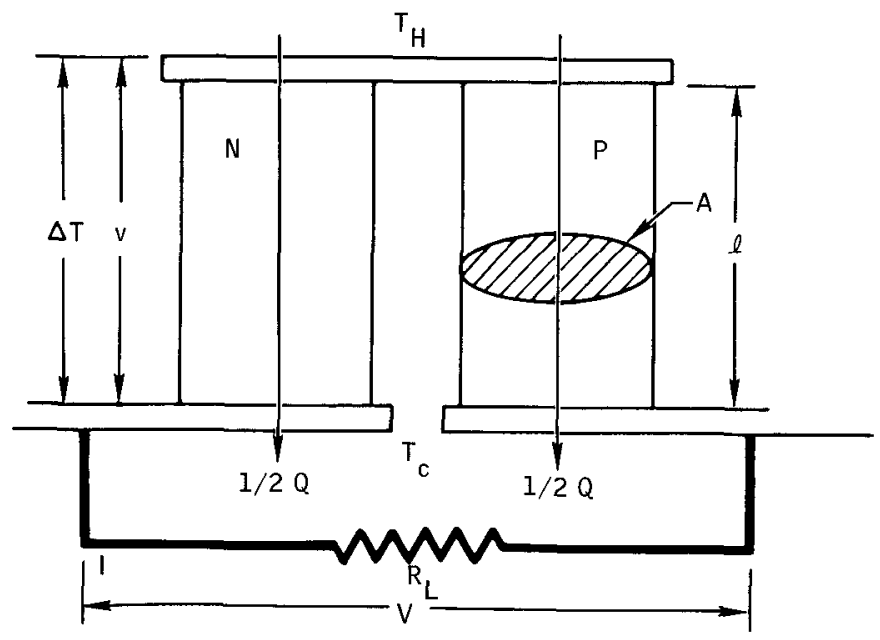

7-S7-192-68

Figure II-48. Ideal Thermocouple

An approximate expression for the ideal thermoelectric converter efficiency can be derived as follows for materials with properties $\pm S, \rho$, and $K$

The heat flow

$$
Q=2 K \frac{A}{\ell} \Delta T
$$


The open crrcuit voltage

$$
V=2 S \Delta T
$$

The generator impedance

$$
R_{g}-2 \rho \frac{\ell}{A}
$$

At matched load

$$
R_{L}-R_{g}
$$

and the power delivered to the load

$$
\begin{gathered}
P=\left(\frac{V}{R_{g}+R_{L}}\right)^{2} R_{L}=\frac{V^{2}}{4 R_{g}} \\
P=\frac{S^{2} \Delta T^{2}}{2 \rho \frac{\ell}{A}} \\
\eta-\frac{P}{Q}-\frac{1}{4} \frac{S^{2}}{\rho K} \Delta T
\end{gathered}
$$

the efficiency equation can be put into a general form of a device efficiency times Carnot

$$
\begin{gathered}
\eta-\eta_{\mathrm{D}} \eta_{\mathrm{C}} \\
\eta=\frac{1}{4} \frac{\mathrm{S}^{2}}{\rho \mathrm{K}} \mathrm{T}_{\mathrm{H}} \cdot \frac{\Delta \mathrm{T}}{\mathrm{T}_{\mathrm{H}}} .
\end{gathered}
$$

The quantity $1 / 4 \mathrm{ZT}_{\mathrm{H}}$ is a function of the materials properties only and is approximately the fraction of Carnot efficiency of an ideal converter.

In a more rigorous derivation which includes $I^{2} R$ heating and the Peltier effect which is the direct transport of heat due to a 

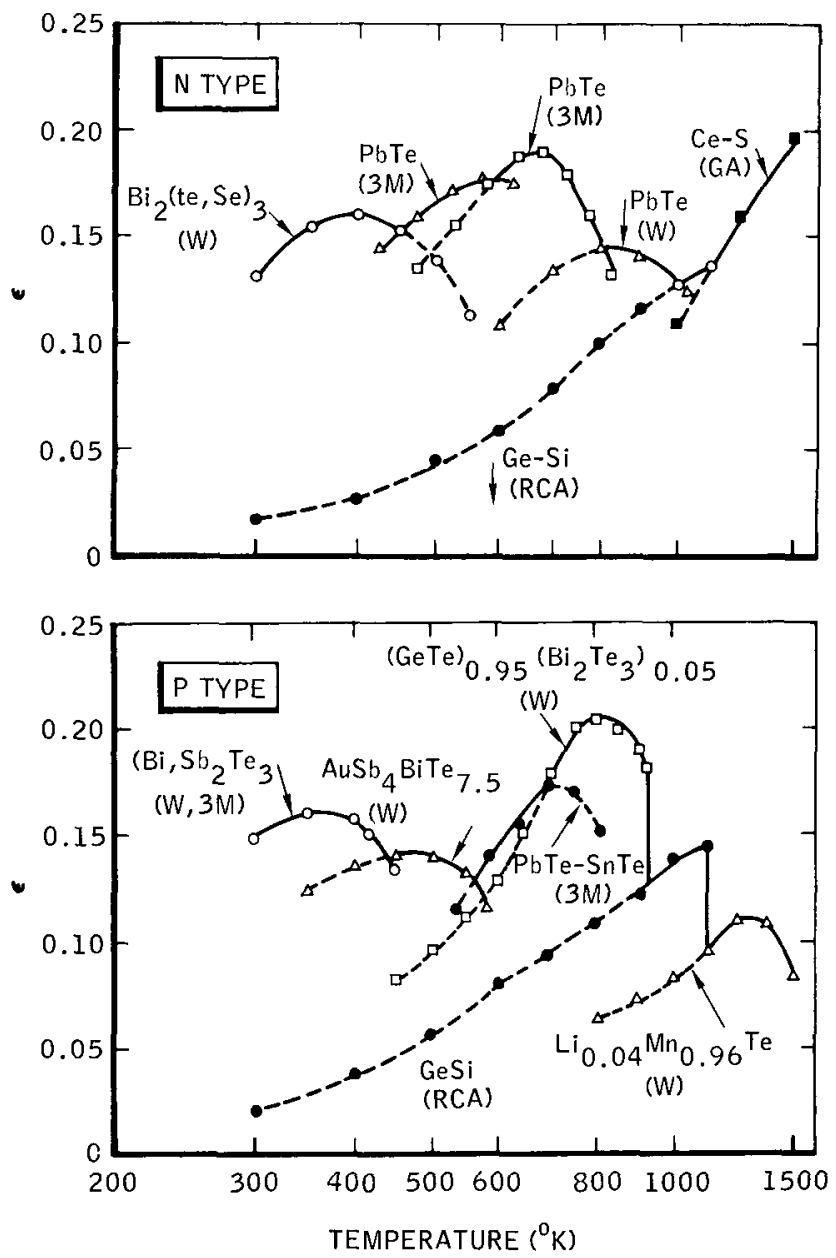

$W$ = WESTINGHOUSE

$3 M=$ MINNESOTA MINING AND MANUFACTURING CO

GA = GENERAL ATOMIC DIV, GENERAL DYNAMICS CORP RCA = RADIO CORPORATION OF AMERICA

Figure II-49. Ideal Thermoelectric Materials Efficiency 
current flow in a thermocouple, the expression for maximum device efficiency is

$$
\eta_{D}=\frac{\sqrt{2 T+1}-1}{\sqrt{2 T+1}+\frac{T_{C}}{T_{H}}}
$$

The ideal device efficiency of the useful materials of interest is shown in Figure II-49. For $\mathrm{ZT}=1$ and $\mathrm{T}_{\mathrm{C}} / \mathrm{T}_{\mathrm{H}}=0.7-0.8$, the ideal materials efficiency is about $19 \%$ which corresponds very nearly to an envelope of the maximum efficlency of all known materials. It should also be noted that at $Z T=1$, the efficiency is about $3 / 4$ of that given by the approximate expression $\eta_{\mathrm{D}}=1 / 4 \mathrm{ZT}$.

In a large space thermoelectric system the influence of $Z$ and temperature on radiator area and thus weight is of more importance than efficiency. If one assumes a constant device efficiency $\eta_{D}$, the approximate heat rejection is

$$
\begin{gathered}
Q_{\mathrm{R}}=\frac{\mathrm{P}_{\mathrm{O}}}{\eta_{\mathrm{D}} \eta_{\mathrm{C}}}=\sigma \epsilon \mathrm{AT}_{\mathrm{C}}^{4} \\
\frac{\mathrm{P}}{\mathrm{A}}=\sigma \epsilon \eta_{\mathrm{D}} \mathrm{T}_{\mathrm{H}}^{4} \eta_{\mathrm{C}}\left(1-\eta_{\mathrm{C}}\right)^{4}
\end{gathered}
$$

where

$$
\begin{aligned}
P_{O} & =\text { electrical power output } \\
\sigma & =\text { Stefan-Boltzmann constant } \\
A & =\text { radiator area } \\
T_{H} & =\text { source temperature } \\
\epsilon & =\text { emissivity } \\
Q_{R} & - \text { heat rejected }
\end{aligned}
$$

Therefore, for $\eta_{\mathrm{D}}=$ Constant, the power per unit radiator area is proportional to the fourth power of the maximum hot temperature 


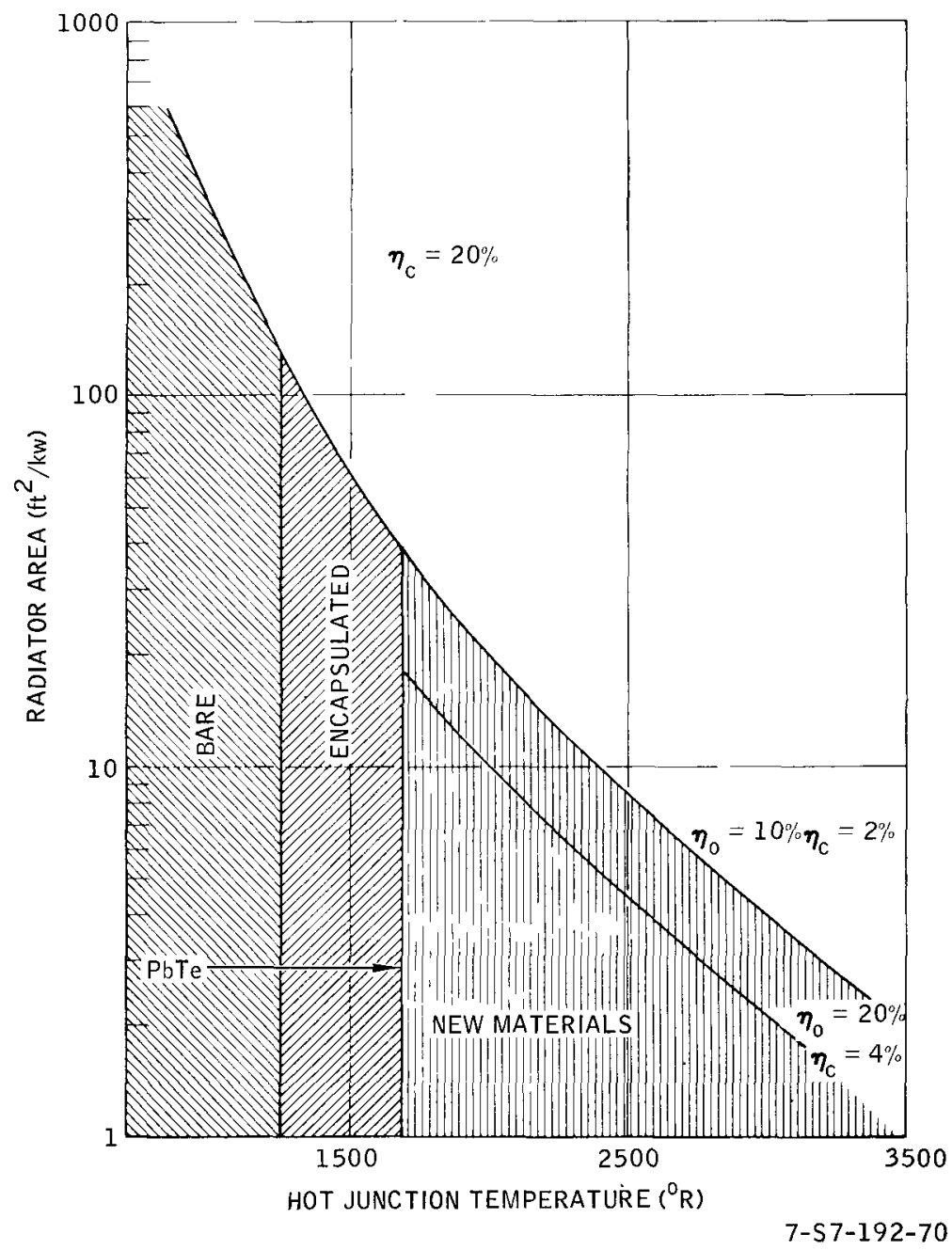

Figure II-50. Radiator Area per Kilowatt as a Function of Hot Junction Temperature for Currently Available $\mathrm{PbTe}$ and Future Materials 
that the thermoelectric material can withstand as determined by metallurgical stability, sublimation, strength, etc. Variations in $\eta_{D}$ can be readily overcome by the $\mathrm{T}_{\mathrm{H}}^{4}$ part of the dependence if the materials are capable of higher temperature operation. Remember that maximum $P / A$ occurs at $\eta_{C}-0.2$ for a low efficiency device Because of converter inefficiencies, fin effectiveness, emissivity, etc, practical devices achieve only about $50 \%$ of the ideal power per unit area. The area per kwe of thermoelectric systems is shown in Figure II-50.

\section{b. Thermoelectric Converters}

The discussion thus far has considered only the ideal converter performance as limited by $Z$ and temperature. A practical consideration is the identification of a converter design capable of capturing a maximum fraction of the available theoretical materials efficiency and the degree to which the physical and metallurgical properties of a given thermoelectric material control the design. It should be remembered that the theoretical materials efficiency is a function of the materials physical properties alone and is completely independent of geometry. The design of a practical thermoelectric converter must provide the following (1) the individual thermocouples must be electrically isolated to allow a series connection to provide a useful output voltage, (2) the transfer of heat from source to radiator must have minimum thermal impedance in series with the thermoelectric material because any loss of avalable $\Delta T$ directly diminishes the converter efficiency, (3) the electrical impedance of all interconnections and contact junctions in series with the thermoelectric material must be minimal because all extraneous $I^{2} R$ losses directly diminish the converter efficiency, and (4) all shunt heat transfer from source to radiator must be minimal.

The physical and metallurgical properties of the thermoelectric material can influence the converter design and efficiency. For example (1) the extraneous converter resistance is strongly 


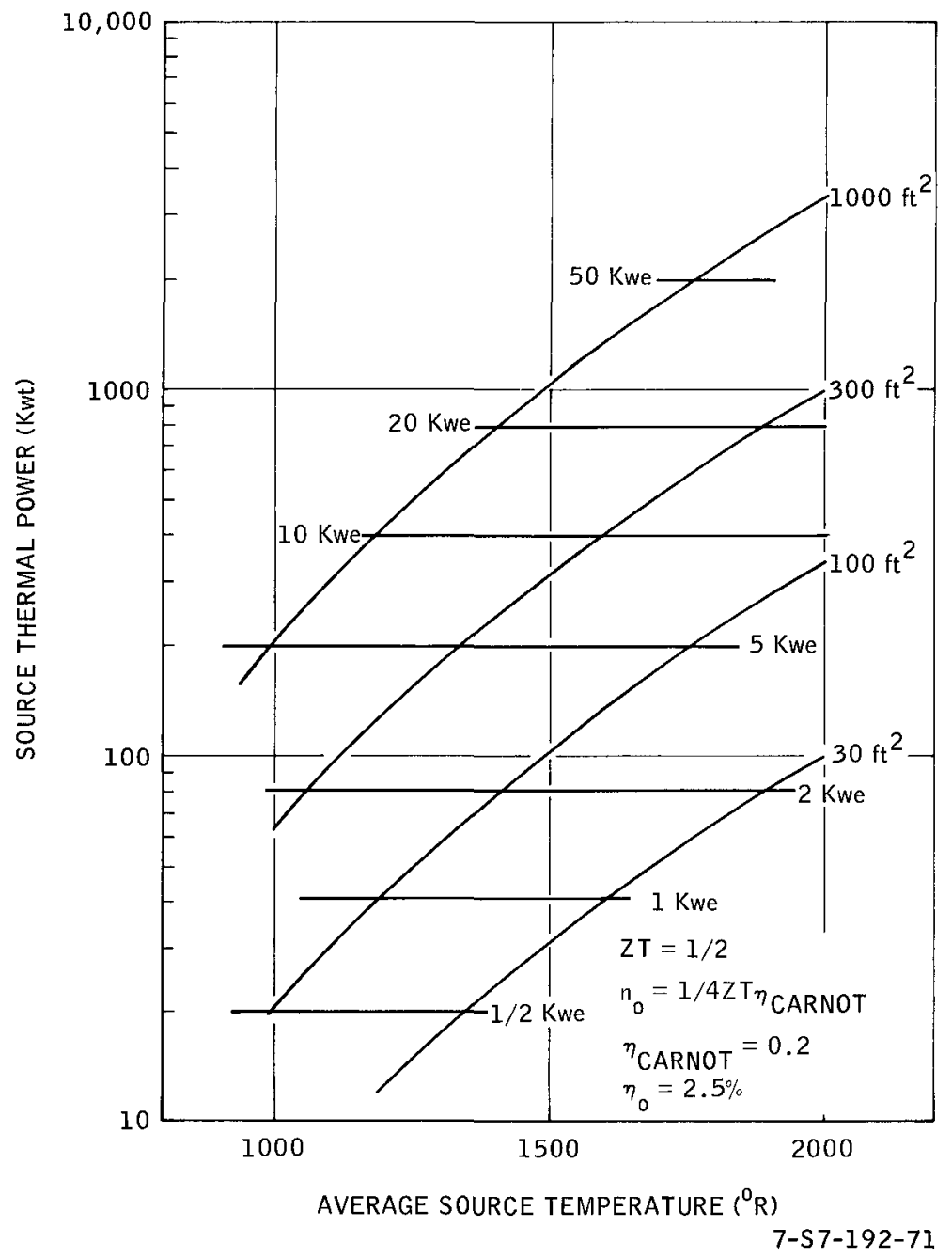

Figure II-51. Reactor Thermal Power and Source Temperature Requirements for Thermoelectric Systems as a Function of Electrical Power and Radiator Area 
influenced by the abılity to make metallurgically stable electrical contacts of minimum resistance to the thermoelectric material, (2) any requirement for a coating or barrıer on the thermoelectric material to suppress sublimation provides a shunt heat path, and (3) the physical strength of the thermoelectric material can determine structural or thermal stress limitations.

\section{c. Reactor Thermoelectric Systems}

As a result of practical engineering limitations, the only materials that can be currently considered for space are PbTe, $\mathrm{Pb}-\mathrm{Sn}-\mathrm{Te}$, and the Ge-Si alloys. The basic PbTe material has a theoretical conversion efficiency of about $15 \%$ of Carnot and 15 limited to a peak hot-junction temperature of about $800^{\circ} \mathrm{F}$ by sublimation. Through converter design the upper temperature limit can be extended somewhat by use of an encapsulant to suppress sublimation. The Ge-Si alloys have a lower conversion efficiency, about 10 to $\mathrm{J} 5 \%$ of Carnot, but are not as limited in operating temperature. Other practical engineering considerations such as thermal impedances and electrical contact resistance limit the net efficiency of practical devices to about $10 \%$ of Carnot.

However, the large energy content of a reactor minimizes the inefficiency penalty which is further offset by the reliability advantages of direct conversion with no moving parts. In practical large space power units converter device design and minımum weight requirements will result in converter net efficiencies of about 3\%. Again, because of the large energy content of a critical reactor, the efficiency is of secondary importance until the system electrical energy output increases to the point of requiring a larger than minimum critical size reactor or of requiring a larger reactor to accommodate heat transfer limitations. Beyond this point reactor weight increases with power and efficrency is of greater concern. In general these problems do not begin to appear until the electrical power output exceeds a few kilowatts. The performance of thermoelectric systems as a function of temperature and area is shown in Figure II-51. 


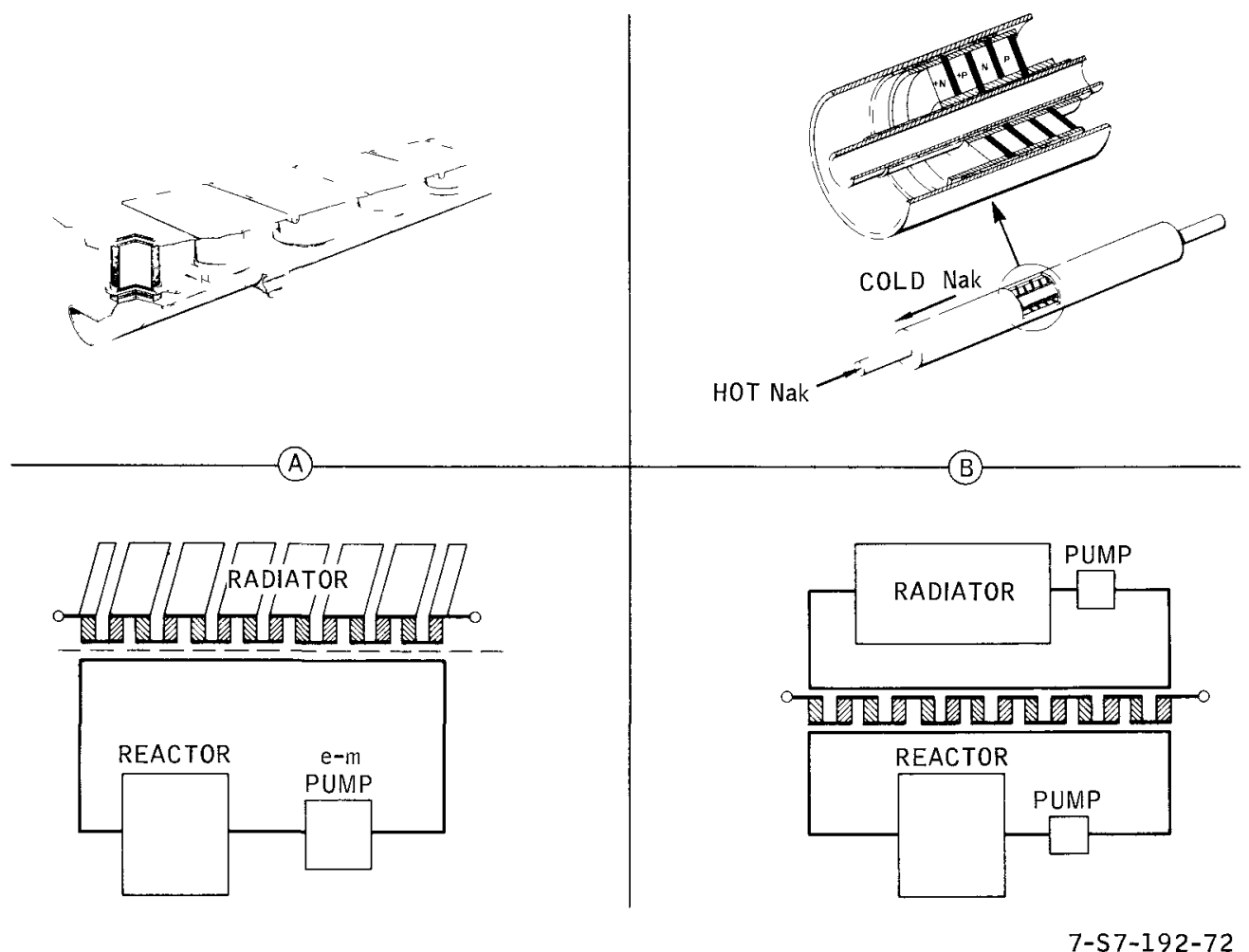

Figure II-52. Thermoelectric System Configuration 
The most appropriate converter and system configurations are shown in Figure II-52. The converter of Figure II-52A is referred to as the direct radiating converter and employs a single liquid metal heat transfer loop for coupling to the reactor heat source. This is the concept of SNAP IOA and is described in detall in Section 5. The converter of Figure II-52B is referred to as a compact converter and requires two heat transfer loops one for coupling to the heat source and the second for coupling to a remote radiator. The principal advantage of this approach is the vehicle integration flexibility afforded by the remote radiator.

The principal weight of a thermoelectric system is the reactor and shield weight. In general, the converter weight is small. In theory the converter weight could be made quite small, however, in practice the device inefficiency resulting from thermal and electrical losses increases with decreasing converter weight. The reactor weight is fixed by criticality limits of the reactor concept necessary to provide the source temperature. The radiator area per unit electrical output is large because of the hot temperature limit and the low conversion efficiency. Thermoelectric systems will have a net efficiency of about $3 \%$. For low-power systems (less than a few kilowatts) the simplicity and reliability advantages of a thermoelectric system can outweigh the disadvantage of large radiator area. However, at large powers radiator weight and size or awkwardness limit the attractiveness of these systems until such time as better materials have been developed and reduced to practice.

\section{THERMIONIC SYSTEMS \\ a. Thermionic Power Conversion}

The thermionic converter is particularly suited for consideration for use in a nuclear space power system. It has a potentially high thermal conversion efficiency, it can operate at a very high heat sink temperature, it is capable of high power density, and is a static operating device. 
The thermionic converter may be pictured as an "electronboiler." It consists of an emitter, a collector, electrical leads, and suitable structure. The emitter (cathode) is heated to a temperature at which it can emit a large current of electrons. A contact potential difference between the anode and the cathode causes the electrons to flow across the interelectrode space and be collected on the second electrode, the collector (anode). The electrons leaving the collector deliver power to an external load as they return through leads to the emitter. A schematic diagram of the thermionic converter is shown in Figure II-53.

Two types of thermonc converters are possible. Each is characterized by its means of neutralizing the interelectrode space charge. In the vacuum diode the electrode spacing is made of the order of a few thousandths of an inch or less in order to limit the negative charge buldup between the electrodes. In the cesium diode, space charge neutralization is accomplished by injecting positive cesium ions into the space between the

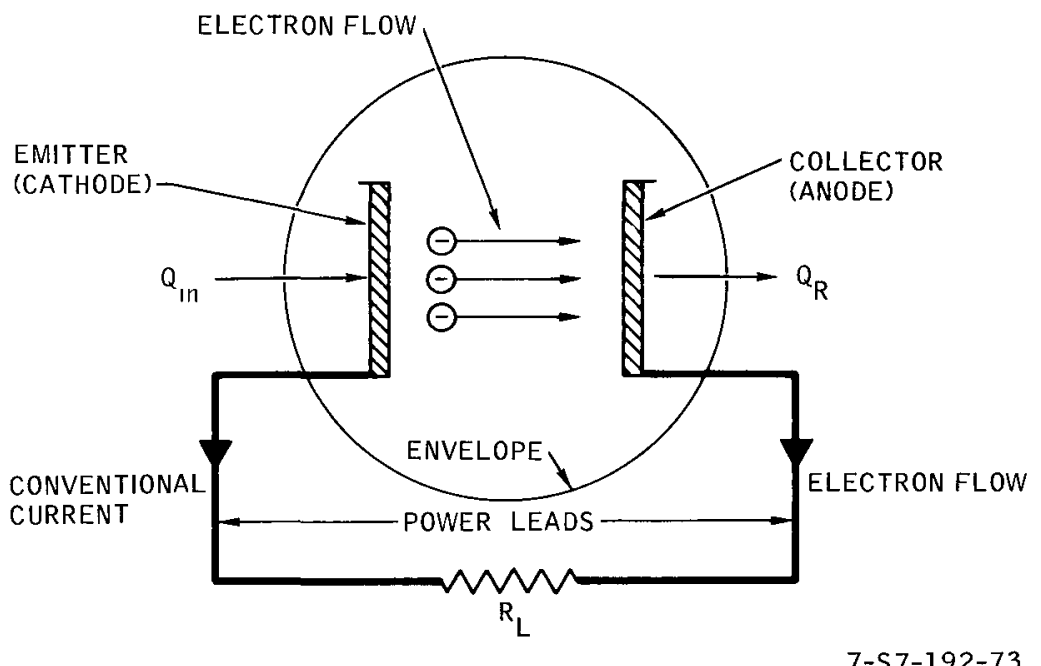

Figure II-53. Ideal Thermionic Converter 
electrodes, and the spacing requirements become far less stringent. Typical temperatures, efficiencies, and power densities for these diodes are listed in Table II- 15

TAB LE II - 15

TYPICAL THERMIONIC CONVERTER PERFORMANCE

\begin{tabular}{l|c|c}
\hline & Vacuum Diode & Cesium Diode \\
\hline Emitter temperature $\left({ }^{\circ} \mathrm{F}\right)$ & 1000 to 1900 & 2600 to 3500 \\
Collector temperature $\left({ }^{\circ} \mathrm{F}\right)$ & 700 to 1100 & 1100 to 1400 \\
Interelectrode spacing $\left(\mathrm{mils}^{\mathrm{s}}\right)$ & 0.3 to 0.5 & 2 to 5 \\
Power density (watts $\left./ \mathrm{cm}^{2}\right)$ & 1 to 2 & 5 to 15 \\
Eff ciency $(\%)$ & 2 to 6 & 6 to 18 \\
\hline
\end{tabular}

Rasor ${ }^{(75)}$ has analyzed the performance of the ideal converter, 1.e., one that is emission-limited only. An ideal converter is limited by the surface emission process. Transport effects which inhibit the interelectrode flow of electrons are assumed not to be present. Thus, the performance of an ideal converter defines an upper limit on performance for all types of thermionic converters. The maximum obtainable efficiency is uniquely determined in the ideal converter by $\mathrm{T}_{e}$, the emitter temperature, $\phi$, the collector work function, $\epsilon$, the emissivity, and $\mathrm{K}_{\mathrm{G}}$, the gas conductivity. If one inserts reasonable values for the independent variables, the ideal or ultimate converter performance can be established. Such results with $\phi-1.7 \mathrm{ev}$ and $\mathrm{K}_{\mathrm{G}}=0$ are shown in Figure II-54. The significant feature is that both optimum efficiency and power density increase with increasing temperature.

Figure II- 55 presents the results of a recent thermionic converter performance survey by Rasor. (75)

b. Reactor - Thermionic System

There are several alternate approaches to the integration of a reactor heat source and the thermionic power conversion for space power. Perhaps the simplest approach is a solid 


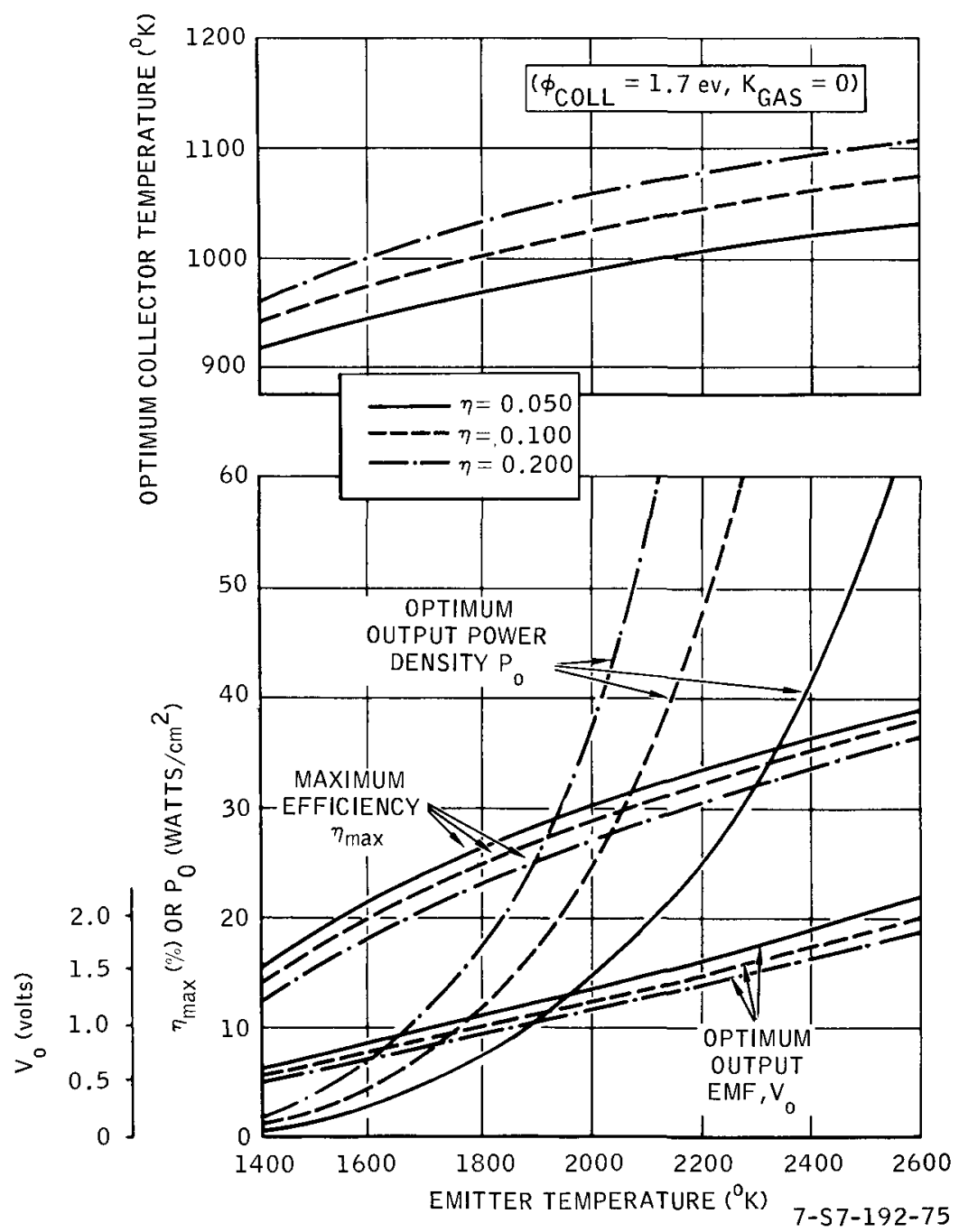

Figure II-54. Optimum Parameters for the Ideal Thermionic Converter $\left(\phi_{\mathrm{coll}}=1.7 \mathrm{ev}, \mathrm{K}_{\mathrm{gas}}=0\right)$ 

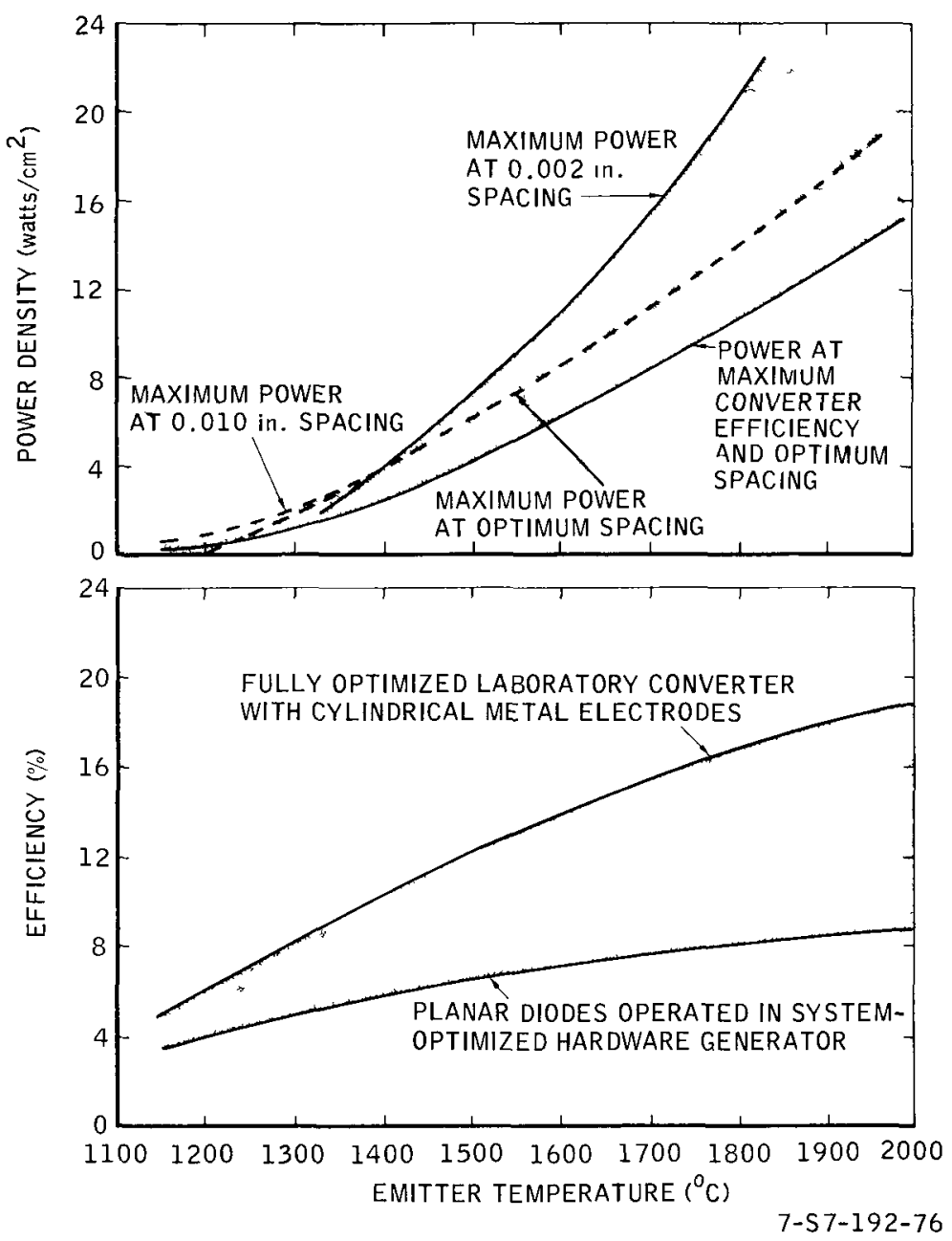

Figure II-55. Survey of Thermionic Converter Demonstrated Performance 

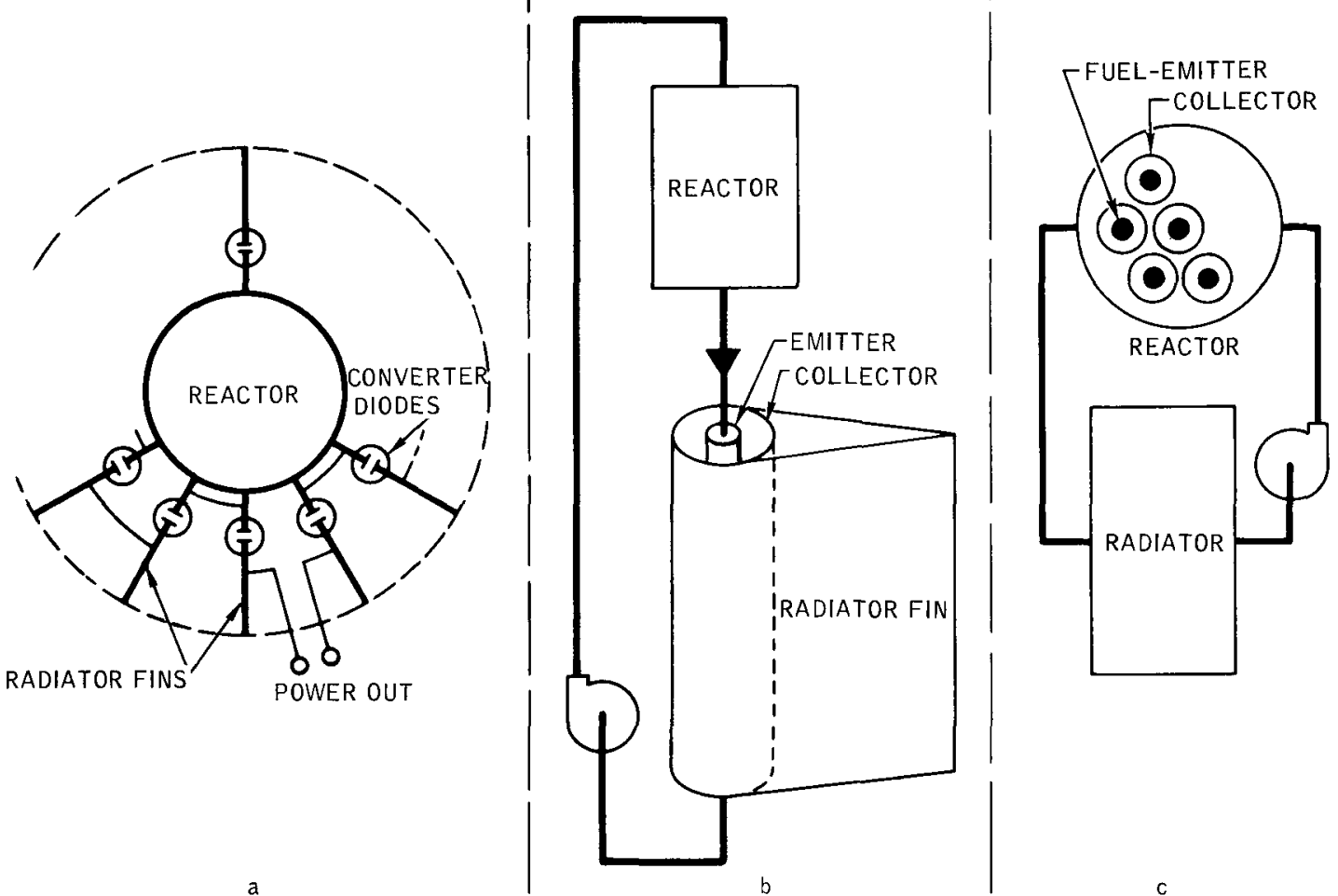

Figure II-56. Thermionic System Configurations 
cylindrical reactor with thermionic diodes located on the outer surface of the reactor and an individual radiator fin for each anode. This concept is shown schematically in Figure II-56a. This concept is inherently limited to small power levels by the peak temperatures required for conduction heat transfer in the core and by the limited radiator area that can be achicved with conductive heat transfer to the extended surface.

The core power density limit and the radiator area restrictions can be removed by transferring heat from the core by means of a liquid metal coolant loop to converter cathodes which are located in an extended array. This concept is shown schematically in Figure II-56b. The major drawback of this concept is that it requires all reactor and coolant loop components to operate at the cathode temperature.

The third and generally favored approach is to assemble the reactor core from a number of integral fuel element converters. The anode temperature is maintained by a liquid metal coolant loop to an extended radiator. This concept is shown schematically in Figure II-56c and described more fully in subsequent paragraphs.

For reactor application the cesium diode appears most practical for the following reasons (1) the efficiency of the cesium diode is considerably greater than that of the vacuum diode, (2) very close electrode spacings are not required, and (3) choice of materials is larger, since cesium vapor helps establish electrode surface characteristics.

Because of the extremely high cathode (emitter) temperature the system requires the cathode to be loaded with fissionable fuel to attain the necessary temperature. Inspection of Figure II- 15 reveals that the most applicable reactor concept for thermionic systems is the UC fast reactor. Only UC and $\mathrm{UO}_{2}$ have the necessary temperature capability. UC has the advantage of higher uranium density and higher thermal conductivity, and thus smaller reactor size and weight. 


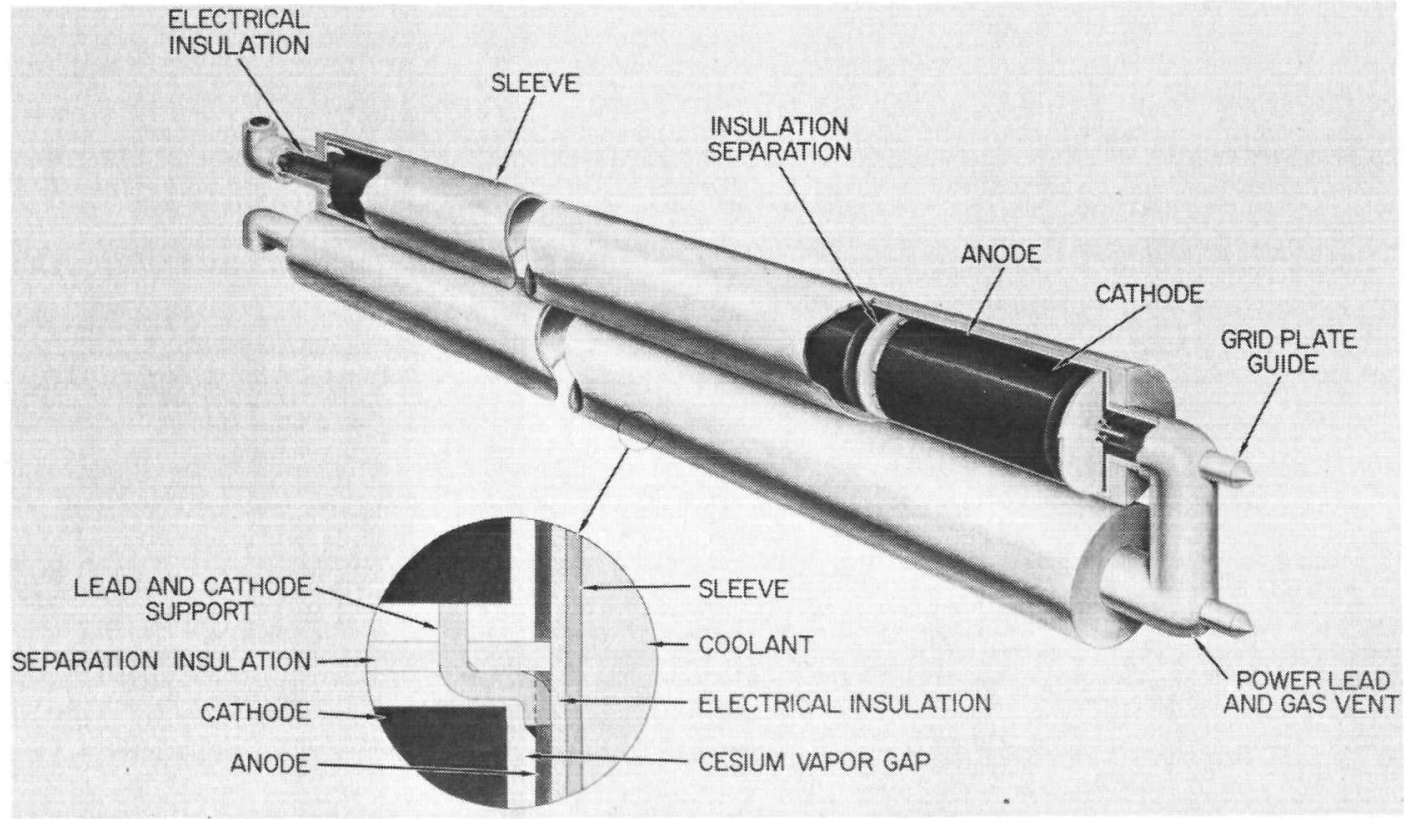

7590-A068

Figure II-57. Thermionic Conversion Fuel Element 
A typical thermionic conversion fuel element is illustrated in Figure II- 57 where a number of thermionic converter cells are shown connected in series. Since each cell is capable of producing a potential of approximately 1 volt, many cells must be connected in series to produce useful high voltage. Each cell consists of a cathode, an anode, an interelectrode space containing cesium vapor, insulation to provide the proper electrical configuration, and an outer sleeve to isolate the entire element from its external surroundings and provide the structural integrity for the assembly.

The cathodes are solid cylinders of fuel material which are held in place by the insulation separators and the electrical leads. Nuclear heat supplies the thermal energy to "lift" free electrons at the cathode surface to an energy level at which they are emitted into the interelectrode space. This electron removal of heat from the cathode is accompanied by other cooling processes including radiation to the anode, and conduction of heat through the electrical leads connecting the anodes to the cathodes. These latter two processes are heat losses and must be accounted for in cell optimizations. It may be that the cathodes will have to be clad to prevent deleterious effects caused by the release of the fission products from the fuel.

The anode is a thin cylindrical shell of metallic conductor surrounding the cathode. The electrons which travel to the

- anode from the cathode are absorbed with the conversion of their kinetıc energy into sensible heat. This heat must be removed from the anode to maintain the electrode temperature difference. For good heat transfer the anode must be in thermal contact with the outer metallic sleeve, which is cooled by a liquid metal. In order that the anodes of the adjacent cells are not electrically shorted, a layer of electrical insulation must be interposed. The annular space between the electrodes contains cesium vapor which is ionized by the high-temperature environment and serves to neutralize the space charge which is produced by the high 


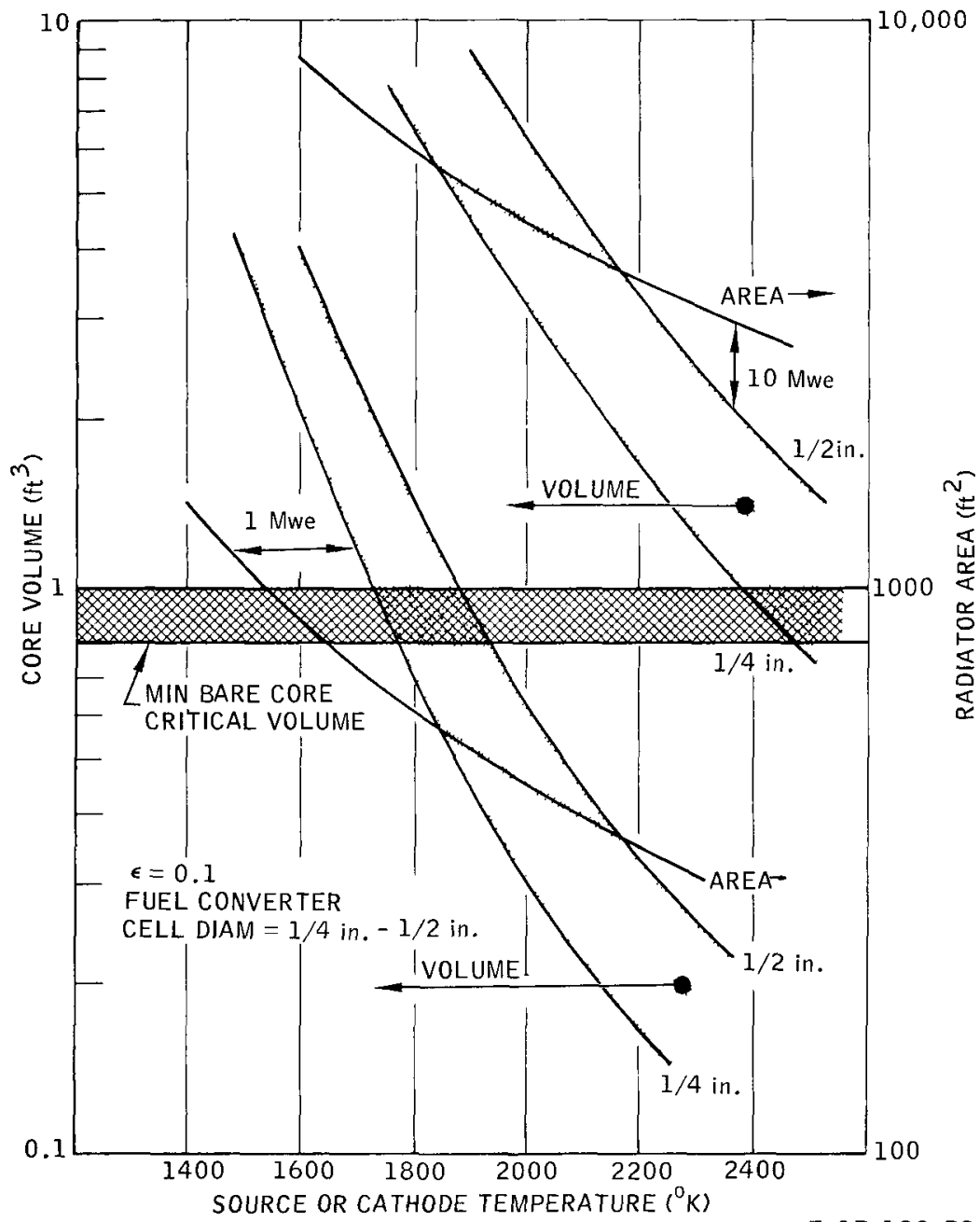

$7-57-192-78$

Figure II-58. Core Volume and Radiator Area as a Function of Core Temperature for a Thermionic Reactor 
electron flux in the interelectrode space. The thermionic fuel elements are assembled into a close spaced hexagonal lattice to form a cylindrical reactor core.

The characteristics of a reactor thermionic space power system can be explored by using the ideal converter performance shown in Figure II-54. The significant feature is that both optimum efficiency and power density increase with increasing temperature. The output power of a combined reactor-thermionic converter will then be a function of the reactor fuel element (cathode) surface area and the operating temperature. The required reactor volume as a function of cathode temperature is shown in Figure II-58 for 1 Mwe and 10 Mwe. The values for $\epsilon=0.1$ in Figure II- 54 were used and the cathode surface area was taken as $3 / 4$ of the maximum for cylindrical fuel-converter cells of $1 / 4 \mathrm{in}$. and $1 / 2 \mathrm{in}$. diameter. It can be seen from Figure II-58 that both reactor volume and radiator area are a strong function of cathode or fuel temperature. It should be noted that this survey assumes constant conditions throughout and makes no allowances for reactor power or temperature distributions. The indicated minimum bare core critıcal volume assumes a UC fuel element that occupies $2 / 3$ of the core volume. The core size could be reduced by the addition of a reflector. It is not clear that the requisite increase in operating temperature is desirable. However, when reactor power distribution is considered in a real case, it is clear that a reflector is mandatory for the purpose of power flattening. From a materials point of view, the minimum operating temperature seems desirable. However, the decreased power density at lower temperatures causes a significant penalty in core volume and weight. From the point of view of minimum system weight, it seems desirable to increase temperature until the core is no longer power-density-limited but becomes reactorcriticality-limited. Depending on the burnup capability of the fuel, it may be necessary to increase core volume for endurance. The very configuration of a thermionic reactor system makes it extremely sensitive to fuel swelling due to fission products. The 
feasibility of a thermionic reactor system remains to be demonstrated. It is obvious that the potential limit to feasibility is fuel swelling. The general hope is that the extreme fuel temperatures will contribute to feasibility by allowing fission products to rapidly diffuse out of the fuel material and thus minimize swelling.

In the previous analysis, the parameters were optimized for maximum efficiency. In general this is not the correct approach for a minimum-weight high-power system. However, if the thermionic reactor concept indeed turns out to be reactor-energycontent-limited, the maximum efficiency approach is probably correct. The increased radiator area and weight do not in any way constitute the same kind of feasibility limit. Figure II-59 presents a conceptual design of a 300-kwe reactor assembly

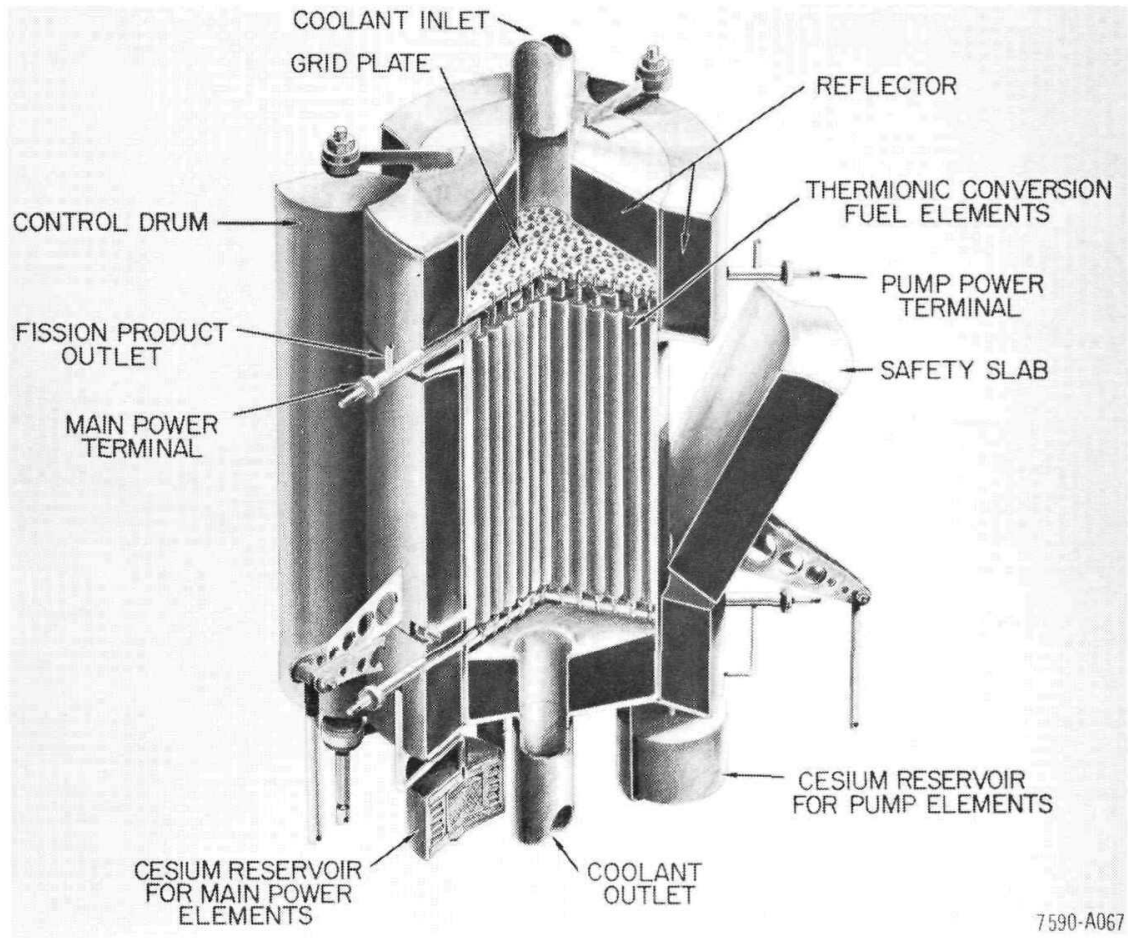

Figure II-59. Thermionic Reactor 
which consists of the core composed of converter fuel elements located by an upper and lower grid plate, a reflector with movable sections for nuclear control, electrical terminals for power output, and cesium circulation system.

\section{E. SYSTEM COMPARISON SUMMARY}

A generalized picture of the performance and applicable power range of the thermoelectrıc, Rankine turboelectric, and thermionic nuclear space power systems is shown in Figure II-60. The most dominant variable in these space power systems is temperature. Each major performance improvement can be characterised by an increase in temperature. Namely,

$\begin{array}{lr}\text { Thermoelectric } & 800-1200^{\circ} \mathrm{F} \\ \text { Hg Rankıne } & 1200-1400^{\circ} \mathrm{F} \\ \text { K or Rb Rankine } & 1800-2300^{\circ} \mathrm{F} \\ \text { Thermionic } & 3000-3500^{\circ} \mathrm{F}\end{array}$

Temperature is not only the key to increased performance, but will, in the end, pace the technological advances necessary to achieve these projected goals.

At low power levels (up to a few kslowatts) the reactor and shield are the dominant weight contributors. The immediate relatively low space power requirements will be filled with the most immediate technology. In effect, performance will be sacrificed for low temperaturc and simple systems. The thermoelectric system fits this description. The reliability advantage of its static operation is very desirable for the low power requirements of early space utilization systems. The lack of orientation requirement, freedom from battery storage, and high resistance to the space environment will make these systems very competitive in the apparent range of solar cells.

At intermediate power levels (a few to hundreds of kilowatts), increased performance is mandatory. The weight and radiator awkwardness of low-temperature and low-efficiency systems is intolerable. The increased performance brings with it the 


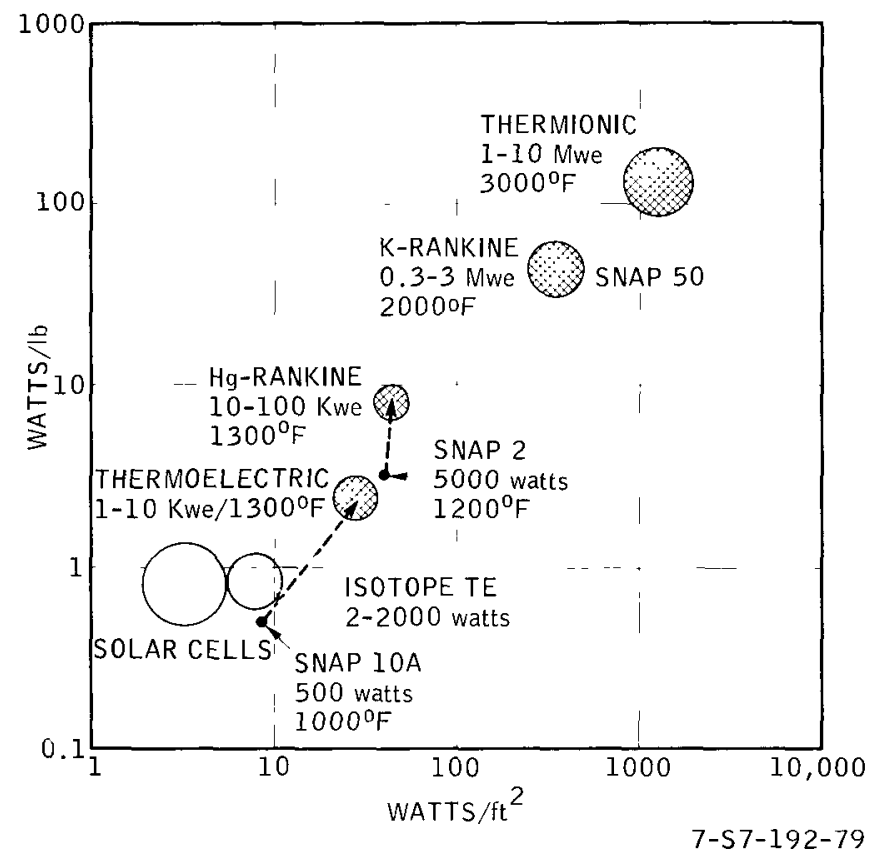

Figure II-60. Space Reactor Power Systems

materials problems of higher temperature, dynamic control, the mechanical perturbations of rotating machinery, and more severe problems in orbital startup and zero-gravity operation. For this power range the ultimate Rankine system will not be selected because of the time scale associated with the required technology. The hydride reactor-mercury Rankine system requires the least extrapolation of the state of the art. It is most probable that this combination will be used at power levels beyond its region of optimum performance because of its availability.

At high powers (hundreds to thousands of kilowatts) the system selection may be more application-dependent. In the case of manned orbital stations or lunar bases the dominant consideration will be reliability. Very probably the increased confidence in and inherent reliability of lower temperature 
systems will outweigh the performance advantages of a higher temperature and newer system. Since the shield weight for a manned system can be in the 10 to 20,000-lb region, the incentive for system weight reduction through the radiator area reduction of a high temperature concept is greatly diminished. In fact the higher temperature reactor will, in general, be larger and thus incur a larger shield weight than a low temperature concept. In the case of electric propulsion, both maximum performance and reliability will be necessary for this form of propulsion to be competitive. The required performance will dictate the highest possible temperature system. The thermionic reactor concept seems very attractive for this application, however, the required temperatures will delay its avallability. For these high-power systems the reactors w1ll be fast and probably fueled by UC or UN. The avallability of extremely high temperature reactor heat sources is totally dependent on currently undemonstrated fuel burnup capability. 


\section{VEHICLE INTEGRATION}

\section{A. INTRODUCTION}

The three major subsystems of a reactor space power unit, 1.e., (1) nuclear reactor heat source, (2) power-conversion system, and (3) heat-rejection system, are closely interrelated in the selection of a power system concept and the subsequent system optimization and design point selection. The unique space limitation of radiative heat rejection results in an extreme premium on high heat source temperature and a system Carnot cycle efficiency optimum $\left(\eta_{c}=0.2\right.$ to 0.25$)$ for minimum heatrejection radiator area. These considerations coupled with the efficiency and temperature requirements of the power-conversion subsystem define the reactor heat source temperature and power. The reactor temperature and power requirements in turn determine the reactor concept, materials, size, and configuration.

In order to integrate the reactor heat source, the power conversion, and the heat rejection subsystem into a complete useful space power system, it is necessary to introduce additional criteria. The most significant of these are

1) One year unattended operation in space with automatic control and without maintenance

2) Operation in the space environment of vacuum and micrometeorites

3) Remote startup in orbit via radio command

4) Capability to operate without subjecting the spacecraft to excessive disturbing torques

5) Design and installation to permit efficiency low weight shadow shrelding of payloads

6) Minimum interference and interaction with basic booster and payload subsystems 
7) Configured within vehicle structural and flight stability criteria

8) Capability to withstand the severe shocks, vibrations, pressure, and temperature transients during vehicle launch

9) Prelaunch installation and checkout with maximum safety and minimum vehicle and facility interference

10) Capable of low cost quantity production with maximum reliability.

The purpose of this section is to discuss those unique aspects of a nuclear space power unit that affect the power unit space system integration. Specifically, they are shielding, safety, and the factory-to-flight sequence.

\section{B. SHIE LDING}

The radiation, gammas and neutrons, that accompanies the fission process introduces the most significant unique consideration in the design and application of nuclear power units. The damage to payload components requires that the payload be either shielded from the damaging radiation or that the payload sensitivity to the radiation be reduced by component selection and/or circuit design. The requirement to provide shielding within a total system weight constraint results in specific configurational influence on the nuclear power unit and its integration with the payload.

The entire science of radiation shielding is highly complex and sophisticated. It is certainly beyond the scope of these notes to treat the subject adequately. However, the limited use of some general results which have had the background benefit of more detailed analysis will allow some insight into the radiation shielding considerations.

\section{a. Source Strength}

As discussed earlier, each fission event results in the release of about 2.5 neutrons, one of which 1 s required to initiate 


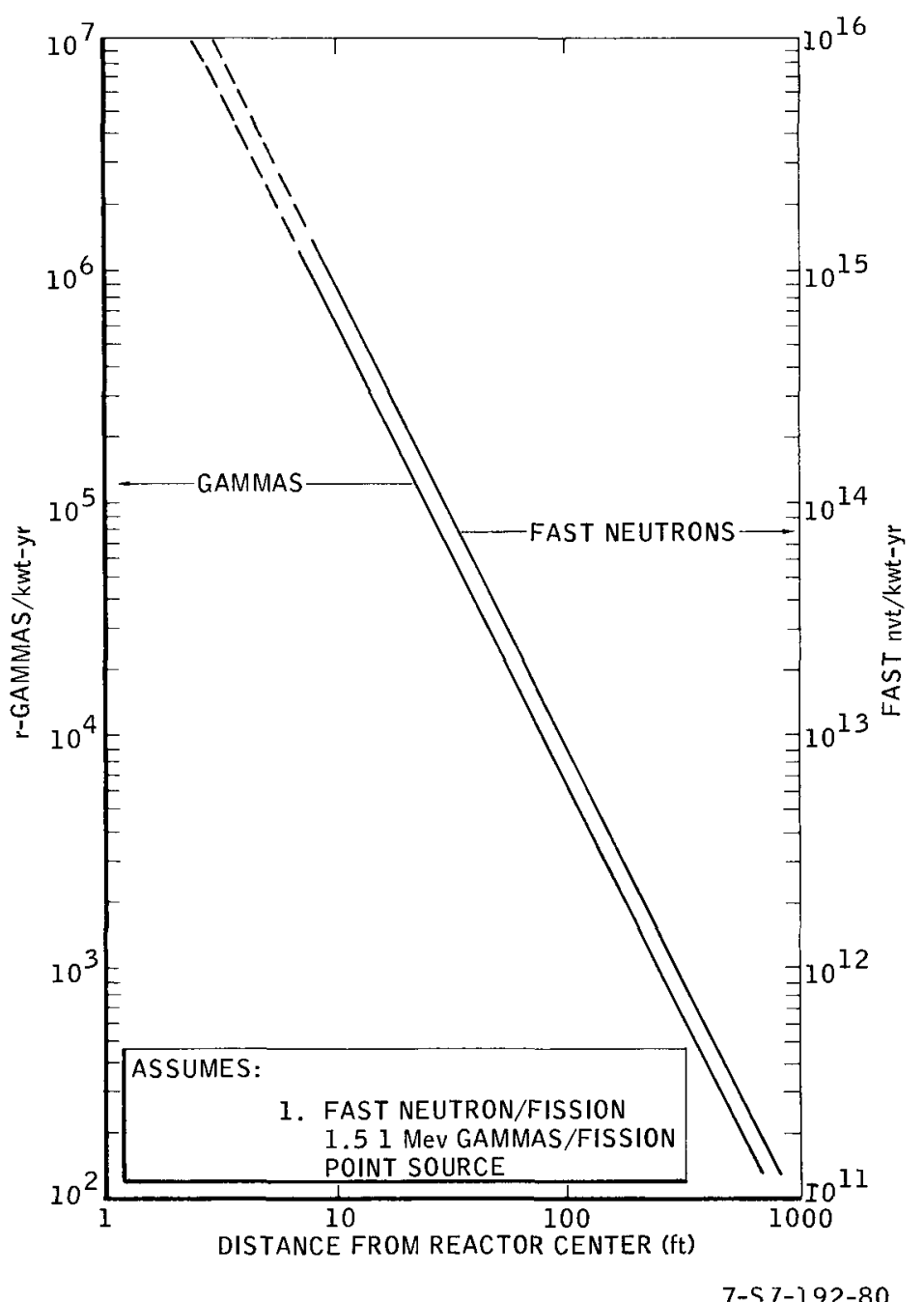

Figure II-61. Approximate Radiation Source Strength for Unshielded Space Reactors 
the subsequent fission event of the self-sustaining chain reaction. In a small reactor, the neutron economy is dominated by leakage. In the case of the SNAP hydride reactors about $40 \%$, or 1 neutron per fission, leak from the reactor. Since the dominant leakage is fast, these neutrons constitute the effective source that must, in general, be attenuated. A leakage of l neutron per fission results in a source strength of about $10^{21}$ neutrons per thermal kllowatt year. The effectıve source strength of a very large space reactor with lower leakage might be not more than a factor of two smaller.

For the distances of practical interest, it is acceptable to consider the source to be located at the reactor's center. The neutron dose then at a distance, $R$, is equal to the source strength divided by the surface area of a sphere of radius $R$. Thus

$$
D_{n}(R) \quad \frac{S}{4 \pi R^{2}}
$$

where

$$
\begin{aligned}
D_{n}(R) & \text { - neutron dose (neutrons/year } / \mathrm{cm}^{2} \text { ) } \\
S & =\text { source strength (neutrons/year) } \\
R & =\text { distance from source center }(\mathrm{cm}) .
\end{aligned}
$$

The neutron flux per thermal kilowatt year as a function of distance is shown in Figure II-6I for the assumed leakage of 1 neutron per fission.

Each fission is accompanied on the average by the equivalent of 5 gamma rays of 1 Mev energy. Again, not all of this radiation leaks from the reactor because of self-absorption by the reactor materials. In the case of the SNAP hydride reactors, the externally effective source is equivalent to about 1.5 gammas of $1 \mathrm{Mev}$ energy per fission or about $1.5 \times 10^{21}$ Mev per thermal kllowatt year. 
The standard unit for gamma ray flux is the roentgen, $r$, which is defined in terms of energy absorption per $\mathrm{cm}^{3}$ of air. For l Mev gammas

$$
1 \mathrm{r}=2.18 \times 10^{9} \mathrm{Mev} / \mathrm{cm}^{2}
$$

Therefore the integrated gamma dose, assuming a point source, at a distance, $R$, is

$$
D_{\gamma}(R)-\frac{S_{\gamma}}{4 \pi R^{2}(2.18) \times 10^{9}}
$$

where

$$
\begin{aligned}
& D_{\gamma}(R) \text { - gamma dose (r/year) } \\
& S_{\gamma} \text { - gamma source (Mev/year) } \\
& R=\text { distance from source }(\mathrm{cm}) .
\end{aligned}
$$

The gamma dose as a function of distance assuming $1.5 \mathrm{Mev} /$ fission is shown in Figure II-61. For larger space reactors and for higher density core materials the dose might be lower by as much as a factor of 5 .

b. Radiation Effects

In order to evaluate the shielding requirements of nuclear power systems, it is necessary to identify the radiation sensitivity of the particular payload. There are two general types of radiation effects

1) Rate Effects - This type of radiation effect is a function of the instantaneous radiation intensity and is usually associated with ionisation and is of principal significance in the electrical properties of insulators and semiconductors.

2) Integral Effects - This type of radiation effect is a function of the integrated radiation dose and is usually a result of semipermanent lattice defects which produce changes in practically all physical properties of materials. 
A very broad indication of the radiation dose required for measurable effects is given in Figure II-62. Since space payloads are predominantly composed of semiconductor devices, the radiation sensitivity of this class of components will generally establish the shielding requirements for unmanned applications. The actual payload sensitivity is also a function of circuit design; however, the radiation toler ance of transistors is a good guide. The general neutron radiation damage behavior of transistors is shown in Figure II-63. The relative sensitivity to gamma radiation is usually considered to be about $10^{5}$ nvt I $r$. The difficulty of semiconductor payload hardening as a function of the integrated fast neutrons per $\mathrm{cm}^{2}$ can be characterized as follows.

$$
\begin{gathered}
<10^{11} \text { - only minor consideration } \\
10^{11}-10^{12}-\text { careful component selection } \\
10^{12}-10^{13}-\text { restricted component usage and special } \\
\quad \text { circuit considerations } \\
>10^{13}-\text { difficult }
\end{gathered}
$$

Unique payload sensors may be more radiation-sensitive than the payload itself. The size of such sensors is usually such that local sensor shelding is the most economical approach.

For high radiation environments, conventional and high temperature ceramic vacuum tubes have very high radiation tolerance. The avallability of larger amounts of power through the use of nuclear power supplies can make these devices quite acceptable for space with the important advantage of high temperature radiative cooling.

\section{c. Radiation Attenuation}

It has already been shown that distance or separation provides a simple means of radiation dose reduction. Separation is probably most practical in the 10 to $100 \mathrm{ft}$ region. Beyond that further attenuation must be achieved through the interposition of materials that absorb the radiation. In the case of neutrons, the attenuation results from the slowing down of the 
$\underset{\sim}{\sim}$

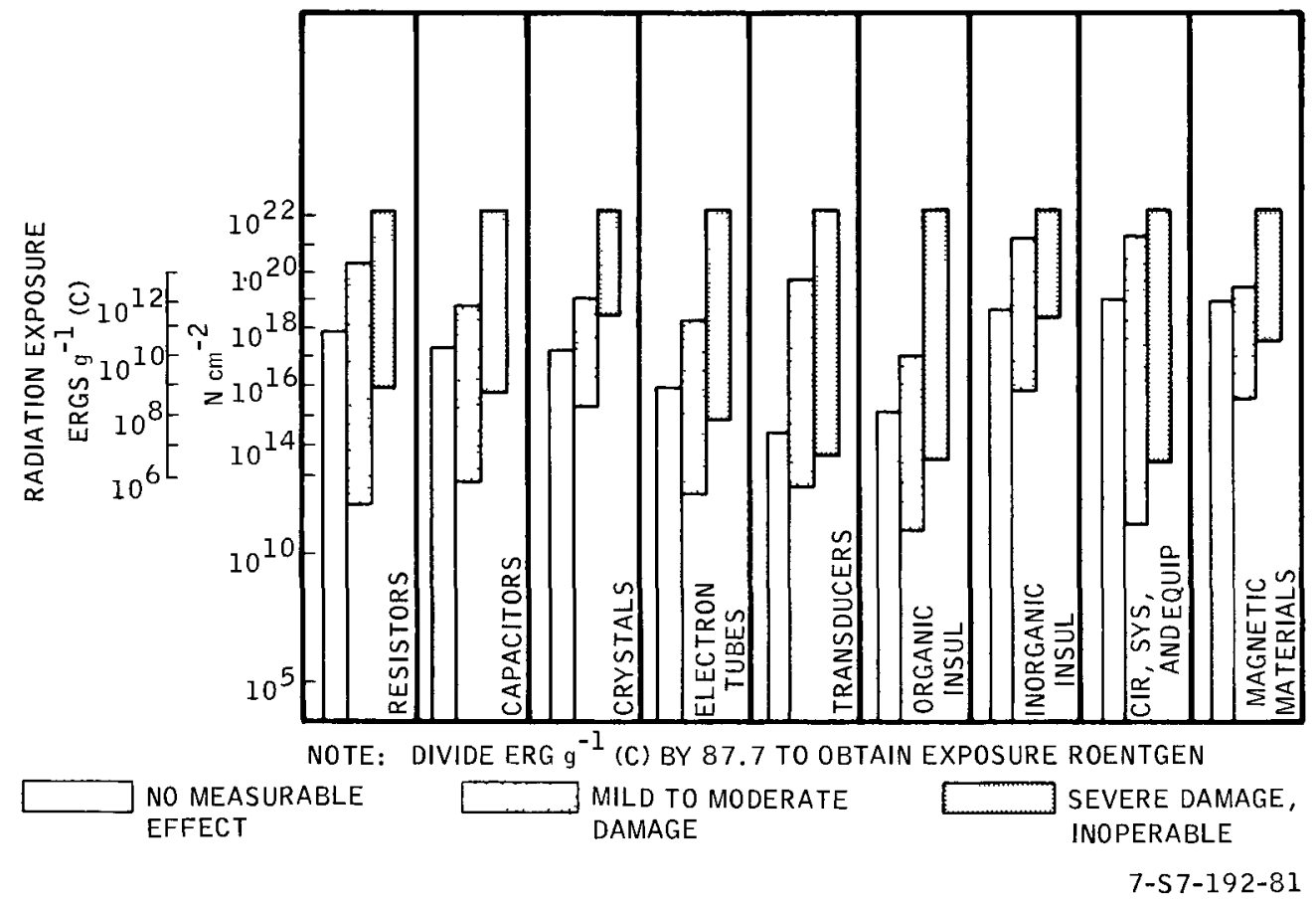

Figure II-62. Survey of Radiation Damage Resistance of Electronic Components 


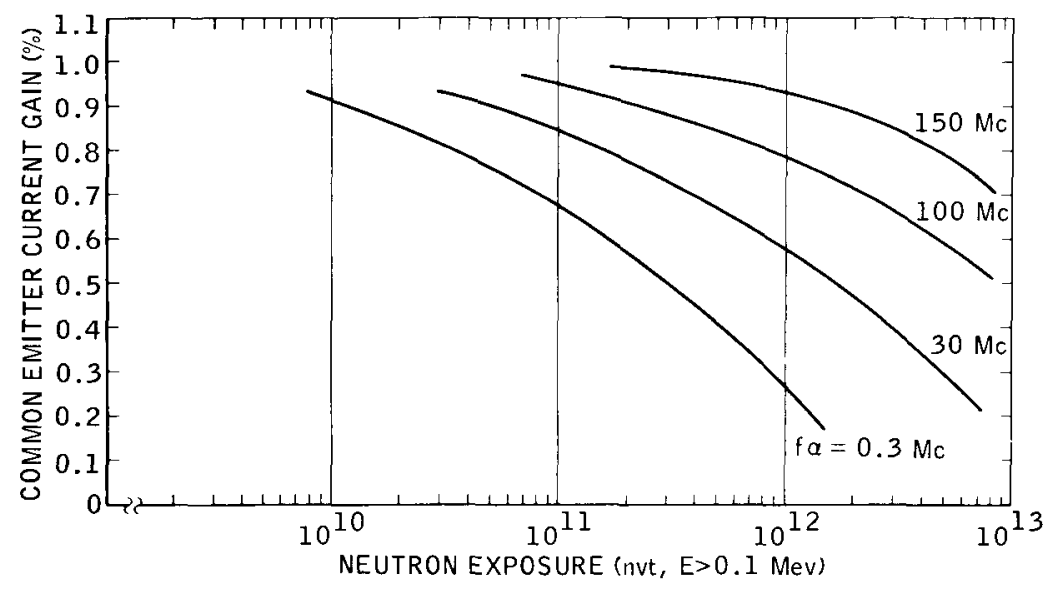

$7-57-192-82$

Figure II-63. Degradation of Current Gain with Neutron Exposure

neutrons until they are captured by an absorber. In fact the dominant consideration in a neutron shielding material is its werght effectiveness for neutron slowing down. It is a simple matter to introduce sufficient absorber to eliminate the neutrons after they have been slowed down. In the case of gamma radiation, attenuation or energy loss is the result of gamma ray interactions and energy exchange with the electrons contained in the shielding material. The best neutron attenuation materials are hydrogenous and the best gamma attenuation materials are heavy metals.

The radiation attenuation capability of a material is described by a removal length and the attenuation is an exponential function of the material thickness in terms of removal lengths, i.e.,

$$
\frac{D(T)}{D(o)}=e^{-T / L}-10^{-T / 2.3 L}
$$


TAB LE II- 16

RADIATION ATTENUATION CHARACTERISTICS

\begin{tabular}{l|c|c|c}
\hline \multirow{2}{*}{ Material } & \multirow{2}{*}{$\begin{array}{c}\text { Density } \\
\text { (gm/cc) }\end{array}$} & $L_{\text {neutrons }}$ & $\begin{array}{c}\text { Removal Length (cm) } \\
(2.5 \mathrm{Mev})\end{array}$ \\
\cline { 3 - 4 } & 0.75 & 6.7 & 34.2 \\
Lithium hydride & 1.00 & 9.71 & 23.0 \\
Water & 0.93 & 8.9 & 23.3 \\
Polyethelyne & 11.4 & 8.55 & 2.06 \\
Lead & 18.9 & 6.44 & 1.24 \\
Tungsten & 18.7 & 5.87 & 1.19 \\
\hline
\end{tabular}

TAB LE II -17

PROPER TIES OF LITHIUM HYDRIDE

Molecular weight

7.948

Density

$0.775 \mathrm{gm} / \mathrm{cc}$

Hydrogen density

$5.9 \times 10^{22}$ atoms/cc

Specific heat

$0.96 \mathrm{Btu} / 1 \mathrm{~b}-{ }^{\circ} \mathrm{F}$ at $68^{\circ} \mathrm{F}$

$1.67 \mathrm{Btu} / \mathrm{lb}-{ }^{\circ} \mathrm{F}$ at $1000^{\circ} \mathrm{F}$

Dissociation pressure

$27 \mathrm{~mm} \mathrm{Hg}$ at $1270^{\circ} \mathrm{F}$

Coefficient of thermal expansion

$\sim 23 \times 10^{-6} \cdot \mathrm{F}^{-1}$

Thermal conductivity

$2.5 \mathrm{Btu} /(\mathrm{hr})-\left(\mathrm{ft}^{2}\right)\left({ }^{\circ} \mathrm{F} / \mathrm{ft}\right)$ 
where

$$
\begin{aligned}
D(T) & =\text { dose penetrating thickness } T \\
D(0) & =\text { incident dose } \\
L & =\text { material removal length } \\
T & - \text { material thickness }
\end{aligned}
$$

The density and removal length of various neutron and gamma shicld materials are given in Table II-16. In general, neutron attenuation is the controlling problem in the shielding of unmanned space payloads. The most efficient and high temperature material is lithium hydride, LiH. The other important physical properties of LiH are contained in Table II- 17.

The attenuation of separation and LiH thickness are combined in Figure II-64 such that the LiH shield thickness for a given dose and separation distance can be read directly. If additional gamma attenuation is required over that provided by separation (Figure II-64) and by the LiH thickness (Table II-16) it can be provided by an additional slab of heavy metal, 1. e.,

$$
\log _{10} \frac{\mathrm{D}_{\gamma}(\mathrm{R})}{\mathrm{D}_{\gamma}(\mathrm{o})}-\frac{\mathrm{T}_{\mathrm{L} 1 \mathrm{H}}}{2.3 \mathrm{~L}_{\gamma \mathrm{L} 1 \mathrm{H}}}+\frac{\mathrm{T}_{\mathrm{M}}}{2.3 \mathrm{~L}_{\gamma \mathrm{M}}}
$$

\section{d. Shield Geometry}

The weight of a space shield is extremely dependent upon geometry. It is obvious that the shield weight will be a direct function of the solid angle that the shield shadow must subtend. In addition the shield geometry must be such that there are no paths or mechanisms by which radiation can bypass or be shunted around the shield. The presence of any structural material positioned such that it is illuminated by the source and can scatter radiation directly into the payload region can drastically reduce the shreld net effectiveness. As a result of this consideration minimum weight shields dictate that the source have a minimum projected area and that all structural members be contained 


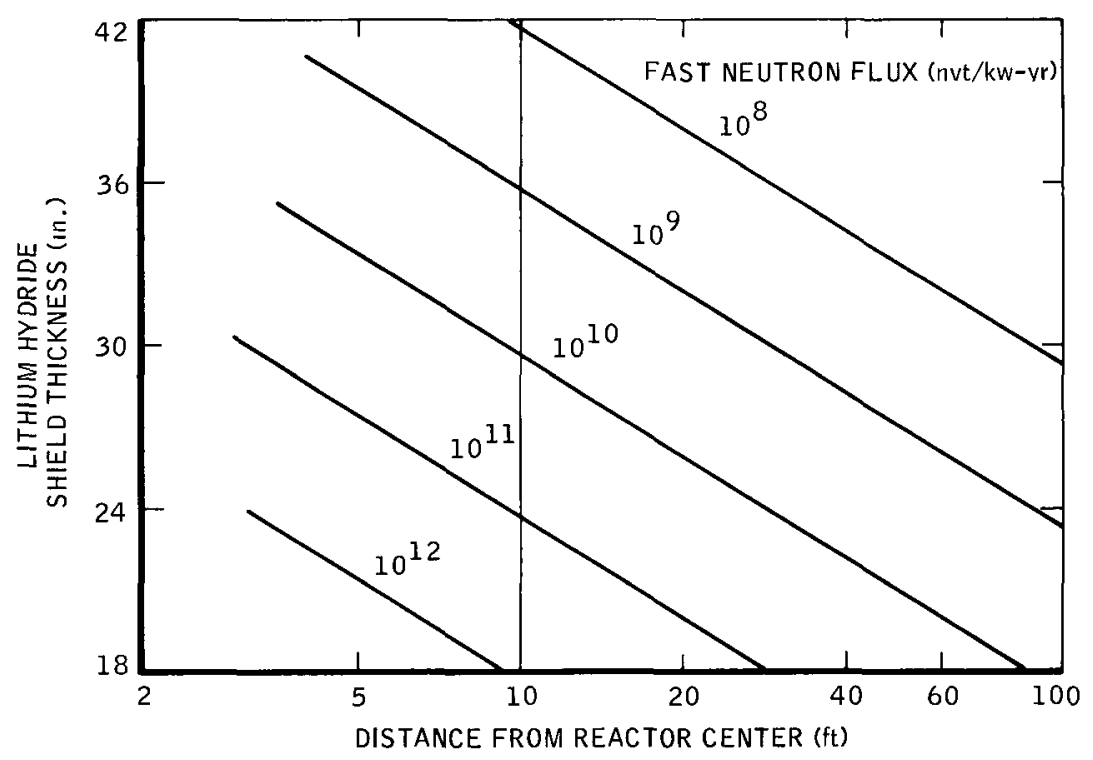

7-57-192-85

Figure II-64. Variation in LiH Thickness with Separation Distance for Given Dose Limits

wathin the conical region defined by the dose plane and the extremities of the source. The resulting ideal geometry is shown in Figure II-65. It is obvious that this consideration has a major influence on the configuration of a nuclear powerplant.

The approximate weight of $\mathrm{LiH}_{1}$ shields to limit the neutron dose to $10^{11}-10^{12}$ over a 5 -ft diameter payload section at a distance of $30 \mathrm{ft}$ is shown in Figure II-66 as a function of reactor size and thermal power. It is apparent that reactor source size is a more important consideration than thermal power. Thus there is a prime incentive to minimize reactor size in order to reduce shield weight. However, a minimum weight reactor does not necessarily lead to a minimum weight reactor-shield combination. 


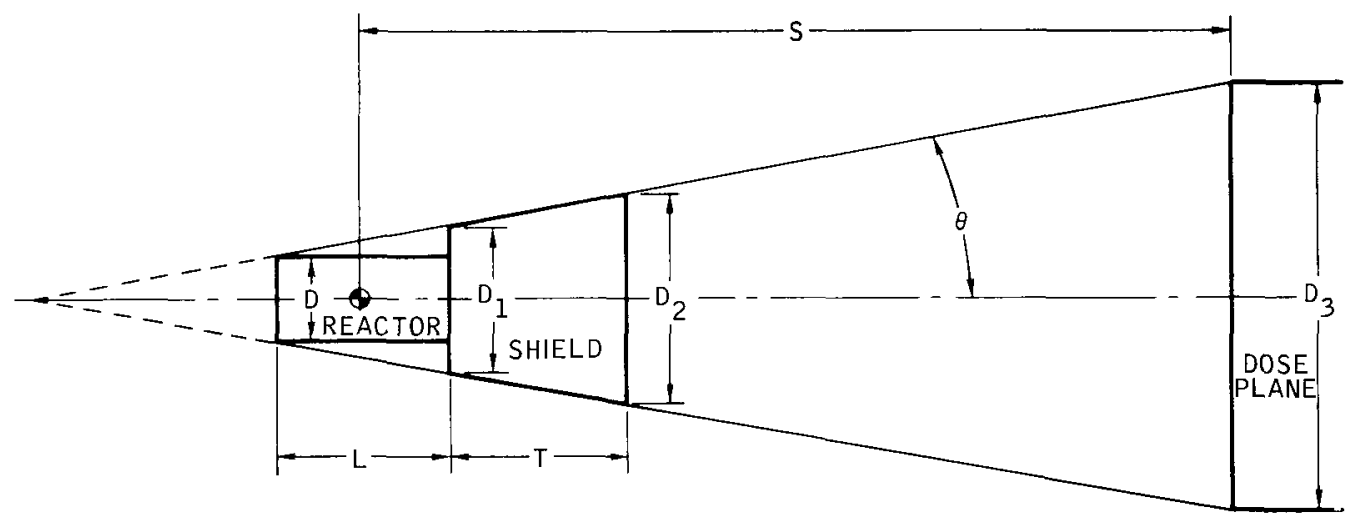

$$
\begin{aligned}
& \tan \theta=\frac{D_{3}-D}{2 S+L} \\
& D_{1}=D+2 L \tan \theta \\
& D_{2}=D_{1}+2 T \tan \theta \quad V_{\text {SHIELD }}=\frac{\pi}{24}\left[D_{2}^{3}-D_{1}^{3}\right] \frac{1}{\tan \theta}
\end{aligned}
$$

Figure II-65. Space Reactor - Payload Shadow Shield Configuration 


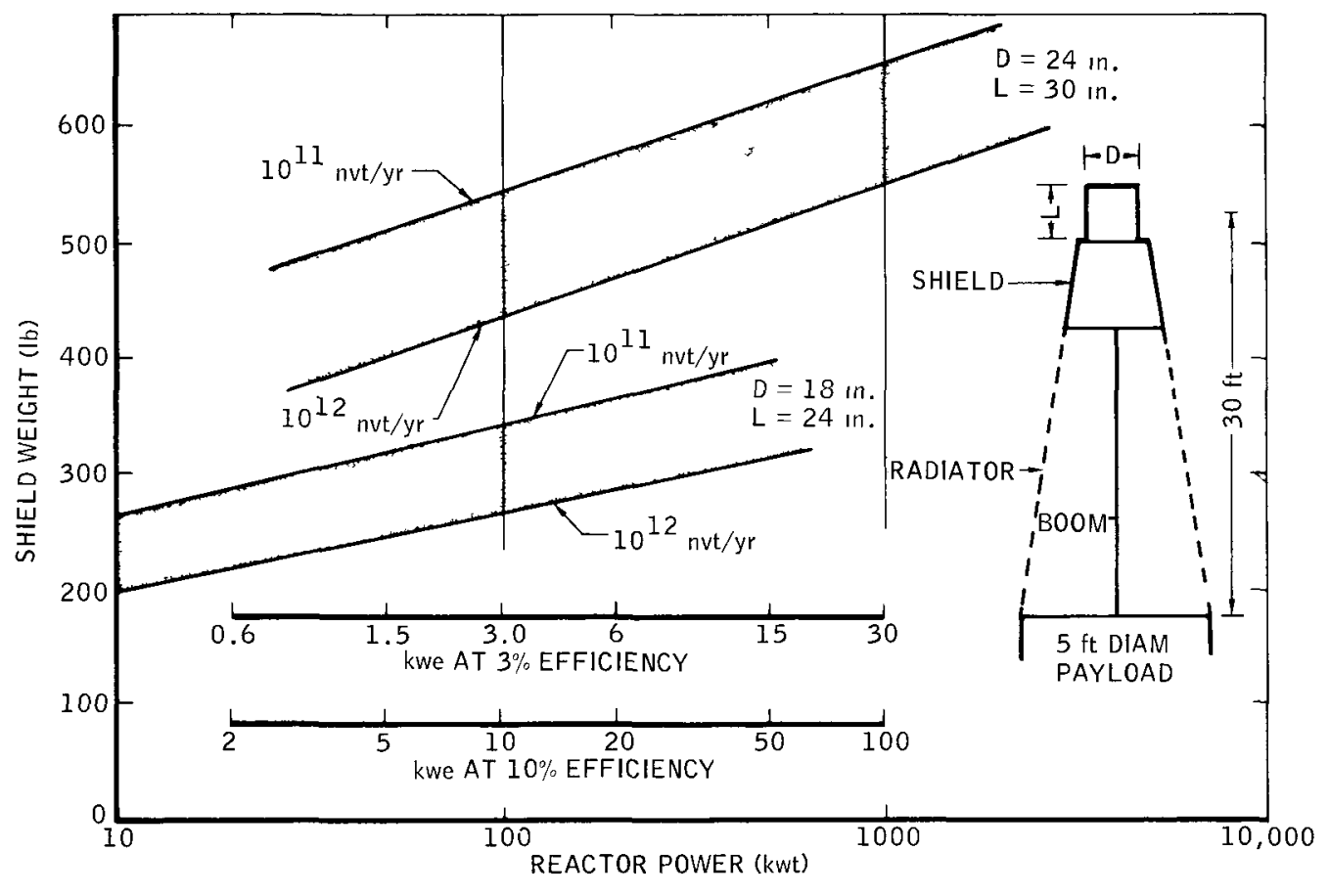

$7-57-192-87$

Figure II-66. Example Shadow Shield Weight as a Function of Reactor Size and Power 
From Figure II- 65 it is apparent that separation distance has a major influence on shield weight. The shield weight of a typical case is shown in Figure II-67 as a function of separation distance. If the separation incurs an added structure, boom, weight and optimum separation distance for minimum shield-boom weight w1ll result. It should be noted that large separation can also be advantageous for gravity gradient stabilization of a satellite.

\section{e. Shreldıng of Manned Systems}

As a result of the much lower radiation tolerance of man, greater attenuation is required. The effects of whole body radiation on man are shown in Table II-18. The simple shrelding analysis applicable to unmanned systems is not valid for large attenuations because such effects as gamma spectrum hardening and neutron capture gammas become significant contributors to the total dose.

In addition to the more sophisticated shielding analysis it is also necessary to make special provision for shielding the primary reactor loop radioactive coolant and to prevent neutron activation of a power conversion cycle working fluid which could present a large source in a radiator. A shield for a manned system involves the same geometry considerations shown in Figure II-65. In general a manned system shield will consist of five regions

1) A heavy metal gamma shield to reduce the core fission gammas to a level similar to that imposed by the radioactive primary loop heat exchangers, etc.

2) A L1H neutron shield of sufficient attenuation to prevent activation of the secondary or working fluid

3) A compartment to house the primary to secondary heat transfer, boiler, and pump, components 


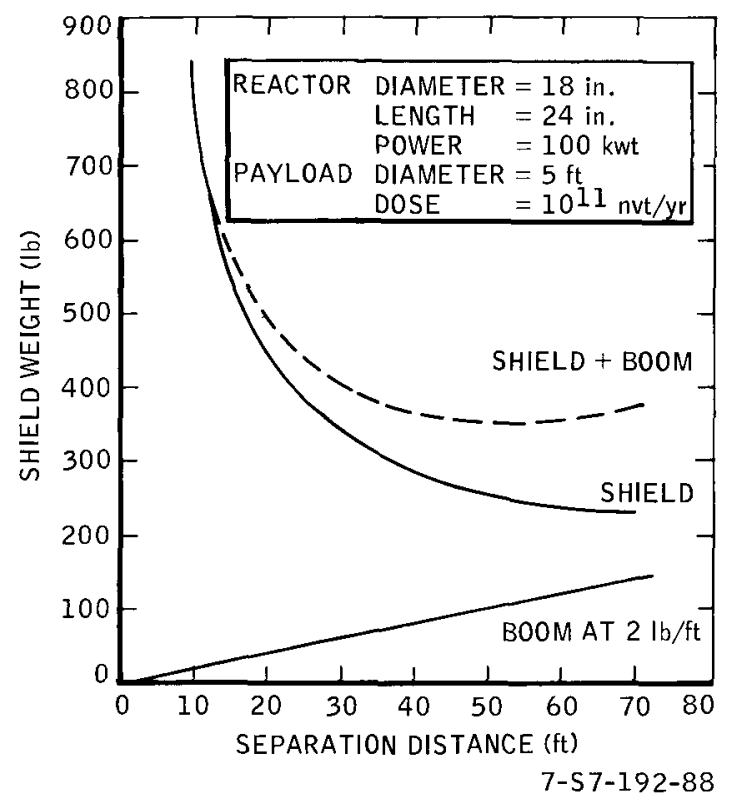

Figure II-67. Shield Weight vs

Separation Distance

TAB LE II - 18

RADIATION TOLERANCE OF MAN

\begin{tabular}{|c|c|c|c|}
\hline & Dose & \multirow{2}{*}{$\begin{array}{l}\text { Gammas } \\
(2.5 \mathrm{Mev})\end{array}$} & \multirow{2}{*}{$\begin{array}{l}\text { Fast Neutrons } \\
\text { (RBE } \approx 8) \\
(2.5 \mathrm{Mev})\end{array}$} \\
\hline & rem & & \\
\hline AEC occupational limit & $3 \mathrm{rem} / 13 \mathrm{wk}$ & $3.75 \times 10^{3} \frac{\mathrm{Mev}}{\mathrm{cm}^{2}-\mathrm{sec}}$ & $46.2 \frac{\mathrm{n}}{\mathrm{cm}^{2}-\mathrm{sec}}$ \\
\hline $\begin{array}{l}\text { Threshhold of clinzcal } \\
\text { detection }\end{array}$ & 25 rem & $5.85 \times 10^{10} \frac{\mathrm{Mev}}{\mathrm{cm}^{2}}$ & $7.34 \times 10^{8} \mathrm{nvt}\left(\frac{\mathrm{n}}{\mathrm{cm}}\right)$ \\
\hline Acute sickness & 125 rem & $2.93 \times 10^{11} \frac{\mathrm{Mev}}{\mathrm{cm}^{2}}$ & $3.67 \times 10^{9} \mathrm{nvt}\left(\frac{\mathrm{n}}{\mathrm{cm}^{2}}\right)$ \\
\hline $50 \%$ probability of death & $400 \mathrm{rem}$ & $9.36 \times 10^{11} \frac{\mathrm{Mev}}{\mathrm{cm}^{2}}$ & $1.27 \times 10^{10} \mathrm{nvt}\left(\frac{\mathrm{n}}{\mathrm{cm}^{2}}\right)$ \\
\hline
\end{tabular}


4) A heavy metal gamma shield to attenuate the radioactive primary loop gammas and any capture gammas from the first $\mathrm{L}_{1} \mathrm{H}$ region.

5) A final LiH shield to provide neutron attenuation.

A typical manned shicld is shown in Figure II-68. The detailed analysis of such a shield is beyond the scope of these notes. However, the results of a survey of shields for the SNAP 8 reactor are shown in Figure II-69. These results are presented in a convenient manner for scalıng to a wide variety of configurations, power, doses, and space station siles.

\section{SAFETY}

a. Criteria

Throughout the design and development of SNAP reactors and systems, safety has provided the basis for many design decisions. In order to satisfy the objective of maximum possible safety of the SNAP space reactor systems, specific evaluation of the system design is necessary in order to obtain an appropriate balance between the safety of the system and the operational characteristics of reliability. simplicity, and weight. The general safety design criteria that have been used for the SNAP space reactor systems arc outlined below

1) Safety and Ease of Handling - The reactor system will be designed so that personnel can handle, install, and repair the system before launch with safety.

2) Prevention of Accidental Criticality - The reactor sys tem will be designed to prevent criticality of the reactor under any condition except controlled operation.

3) Inherent Shutdown - The reactor system will have inherent shutdown characteristics (1. c., a negatıve temperature coefficient and full utilisation of any fundamental energy release limiting mechanism). 


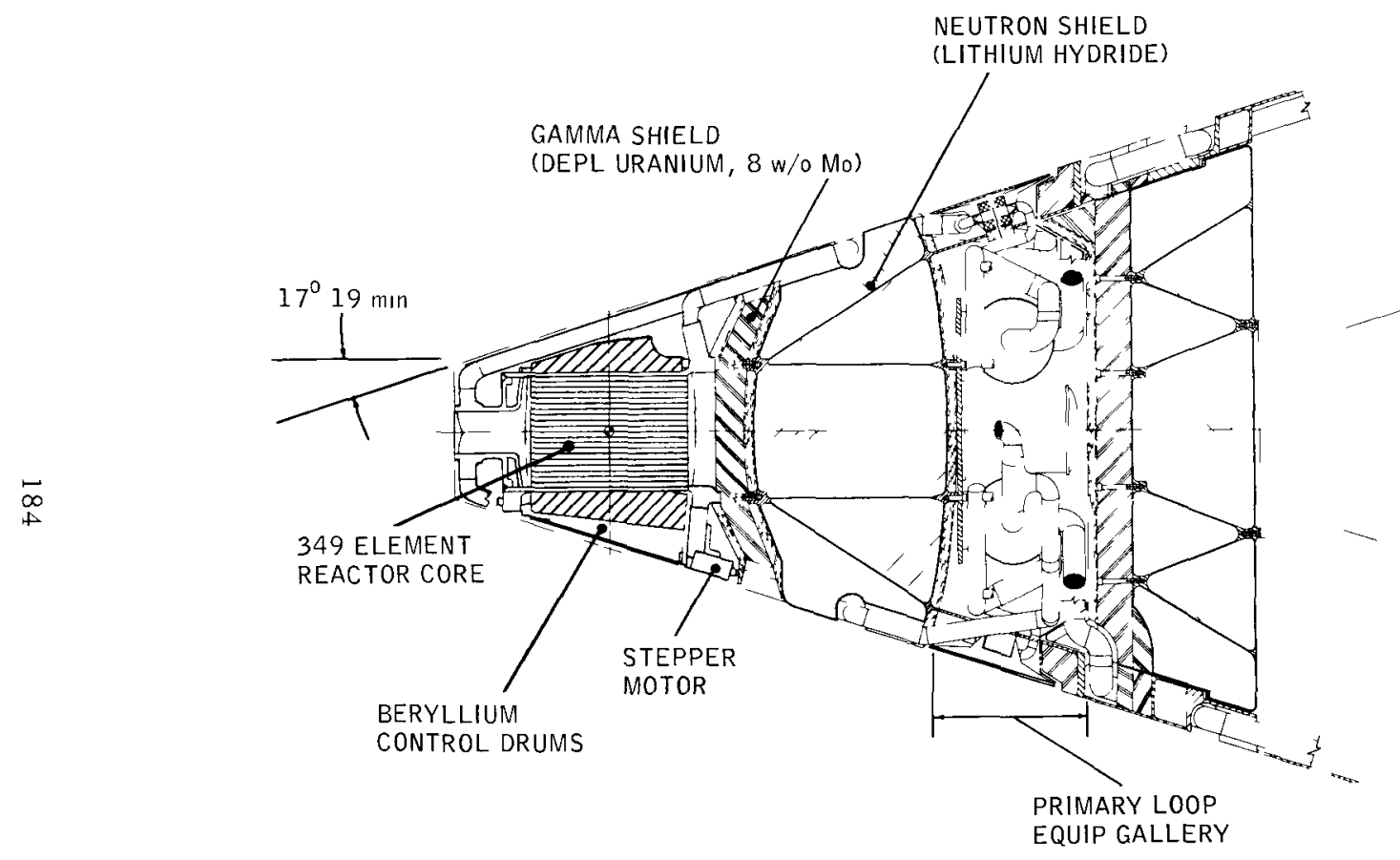

$77695-098141 \mathrm{~A}$

Figure II-68 MORL Reactor/Shadow Shield Assembly 


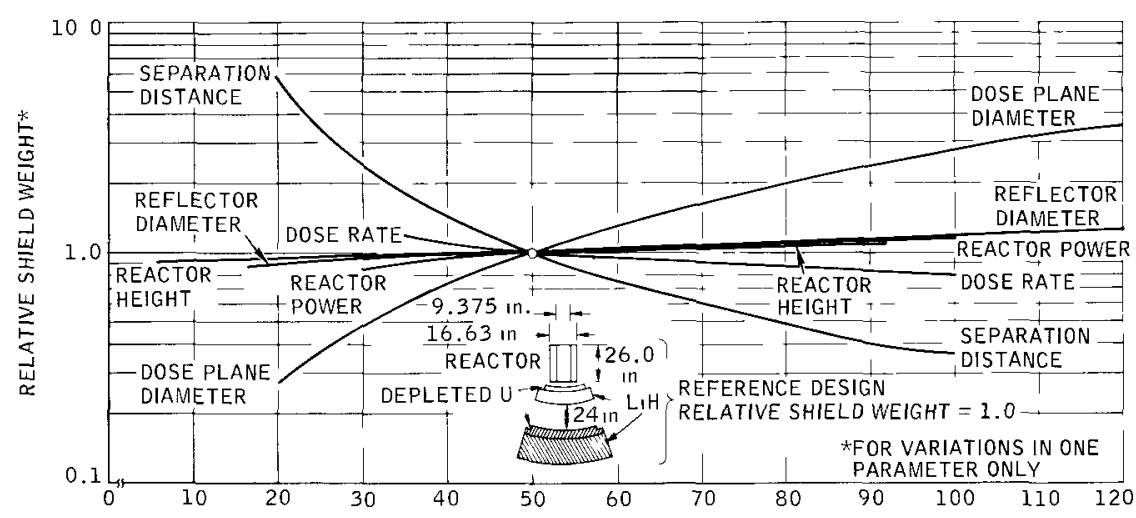

SEPARATION DISTANCE (fE)

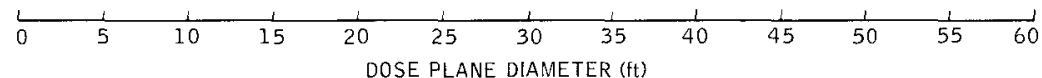

\begin{tabular}{|c|c|c|c|c|c|c|c|c|c|c|}
\hline 0 & 100 & 200 & 300 & 400 & 500 & 600 & 700 & 800 & $\frac{1}{900}$ & 1000 \\
\hline
\end{tabular}

\begin{tabular}{|c|c|}
\hline \multicolumn{2}{|c|}{ REFERENCE DESIGN } \\
\hline SEPARATION & $=50 \mathrm{ft}$ \\
\hline DOSE PLANE & $=25 \mathrm{ft}$ \\
\hline REACTOR POWER & $=300 \mathrm{kwt}$ \\
\hline DOSE RATE & $=4 \mathrm{mrem} / \mathrm{hr}$ \\
\hline SHIELD WEIGHT & $=6500 \mathrm{lb}$ \\
\hline
\end{tabular}

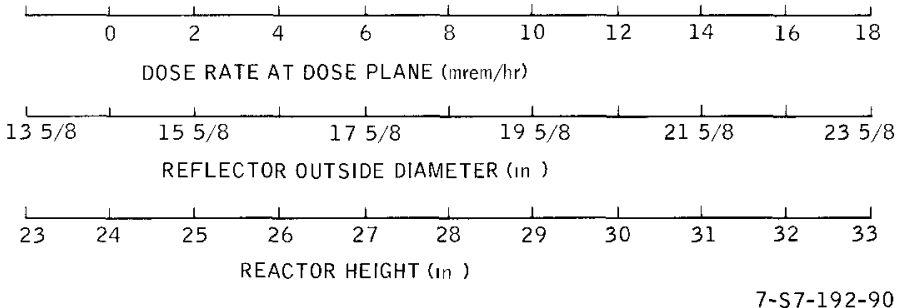

Figure II-69. Parametric Shielding Data for the Manrated SNAP 8 Reactor 
4) Orbital Startup - Reactor system full power operation need not begin until after a suitably safe orbit has been established. Thus, there is no radioactivity hazard with prelaunch accident or preorbit abort.

5) Orbital Shutdown - After the mission has been completed and prior to reentry, the reactor is designed to be shut down by a redundant combination of on board and command actuated devices.

6) Reentry Burnup - Design of the reactor system and components will enhance the probability of high altitude reentry burnup and dispersal of SNAP reactor components.

The safety aspects are conveniently categorized on the basis of the operational sequence.

b. Shipment and Integration Period

During the shipment and integration of the Nuclear Auxiliary Power Unit into its payload and launch system, the possibility of accidental criticality and an uncontrolled power excursion must be prevented. The SNAP reactor is specifically designed to allow the removal of the reactor's beryllium reflector and thus greatly increase the safety margin that must be overcome for accidental criticality. During shipment and integration the reflector is replaced with a poisoned aluminum jacket such that accidental immersion in water, liquid hydrogen, or kerosene cannot cause criticality. Likewise, the proximity of personnel cannot cause accidental criticality. During the shipment and integration period the radioactivity remaining in the core from the factory checkout operations will have decayed to a sufficiently low level that personnel working on or around the power unit will be subjected to radiation levels below the AEC established occupational dose rate. By supplementing these physical constraints with carefully planned procedures and trained personnel, the potential of accidental criticality and personnel injury during the shipment and integration period is even further reduced. 


\section{c. Launch Pad Operations Period}

The SNAP unit is never operated at full power on nuclear heat prior to space. The reactor is subcritical during all launch pad checkout operations. Accidental criticality from extraneous neutron reflecting media is prevented by the interposition of neutron poison materials. No as semblage of people around the reactor can cause criticality. Motion of the reflector control around the reactor can cause criticality. Motion of the reflector control mechanisms is physically blocked and the intimate insertion of reactivity by a liquid reflecting medium is eliminated by vold fillers which remain in place until all personnel are evacuated for vehicle fueling and launch. In the case of a major chemical accident accompanied by an accidental power excursion, analysis indicates only minor hazards outside the normal launch complex exclusion radius. Deposition of radioactivity within the exclusion radius could lead to temporary evacuation, but the combination of decay time and emergency decontamination procedures can readily restore the launch pad area to usefulness.

\section{d. Launch-to-Orbit Period}

The SNAP unit is not operating during the launch phase. The significant problem during the launch to orbit period is the possible chemical explosion of a launch vehicle abort accompanied by a reactor power excursion. Because of the location of U.S. launching sites, the missile path passes over land only during the early stages of launch. For this period, the hazards discussion for the launch pad period which indicated only minor hazards outside the normal exclusion radius is applicable. After liftoff the dispersal and dilution factors for the altitudes associated with the missile path over land will further decrease these minor hazards. The remainder of the abort conditions for the launch phase will exist over an open ocean, nonpopulated areas, and far from islands or major citıes. The potential hazard to the general populace from a personnel as well as a contamination standpoint is negligible over the complete range of possible abort conditions. 


\section{e. Reentry Period}

In the first three periods considered, the hazards are at all times subject to control through site selection, meteorological limitations, emergency procedures, range safety, etc. The unique problem associated with reactor reentry results from the unpredictable location of reentry and the fact that radiation is not inherently detectable by the general populace. The objective of the SNAP development program is to design for fuel element high altitude burnup and dispersal to result from reentry heating. Preliminary calculations supplemented by arc-jet experiments and ballistic reentry tests indicate that this objective can be achieved. In the event of reactor reentry from orbit prior to nuclear operation, the potential effects of reentry heating, disassembly, and partial burnup coupled with core disfigurement on earth impact virtually eliminates any nuclear consequences from the reentry In order to evaluate the world wide significance of contributing fission products to the earth's environment through reentry burnup and dispersal of SNAP systems, the resultant buldup of $\mathrm{Sr}^{90}$ has been considered. The analysis indicates that the equilibrium level of $\mathrm{Sr}^{90}$ resulting from the annual reentry dispersal of 100 SNAP $10 A$ units would add about $0.1 \%$ to the dose received from the earth's natural radioactive background. The design of the SNAP 10A unit incorporates both ground command and "on board" shutdown devices such that a decay period in space can be provided prior to reentry. Orbital lifetime and thus decay time in excess of 250 years reduces the total SNAP IOA fission product inventory at reentry to less than l curie. The capability of orbital startup and the design shutdown devices can be coupled with long-lived orbits to provide an added margin of safety until complete reentry burnup and high altitude dispersal has been proven.

In conclusion, radiological hazards need not limit the use of nuclear power in space. The prelaunchand launch period hazards 
can be controlled through operational procedures and appropriate facilities and equipment. Reentering systems with high altitude burnup and dispersal can be used in large numbers without appreciably contributing to the contamination of the earth's surface or atmosphere. The use of high altitude orbits and orbital startup minimize the reentry hazard by allowing long decay times prior to reentry.

\section{FACTORY-TO-F LIGHT SEQUENCE}

Nuclear power units can be designed to impose virtually no interference with the normal space vehicle launching operations. The SNAP IOA unit has been designed with this objective in mind and the resulting operational sequence is a good example.

The operational factory-to-flight sequence of SNAP $10 \mathrm{~A}$ is lllustrated in Figure II-70. After assembly of the SNAP 10A unit, the fuel elements are loaded into the core in a conventional critical assembly. The anticipated excess reactivity requirement is adjusted by varying the fuel loading at a nominal reflector thickness. After welding the core vessel top head and the NaK pump into place, the unit is charged with NaK. The NaK is circulated through an external filtering and cold trapping device until the oxygen content of the $\mathrm{NaK}$ circuit has been reduced to about $10 \mathrm{ppm}$. The external $\mathrm{NaK}$ auxiliaries are then pinched off and the NaK circuit is sealed. There is no subsequent requirement to breach the integrity of the core and NaK circuit. With the ald of electrical heat applied to the NaK cırcuit, the entire SNAP $10 A$ unit is raised to operating temperature while the reactor is maintained critical at low power to monitor excess reactivity. Final adjustment of the excess reactivity required to operate at design temperature and power is accomplished by reflector thickness variation. After the hot nuclear calibration the unit is ready for shipment to the launch site. The reflector mechanism is removed and replaced with a safety sleeve to prevent accidental criticality. Within two weeks the corefission product radioactivity has decayed to a negligible quantity. The 


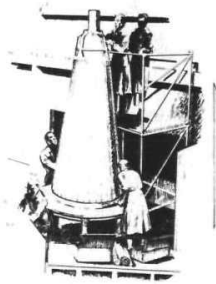

ASSEMBLY

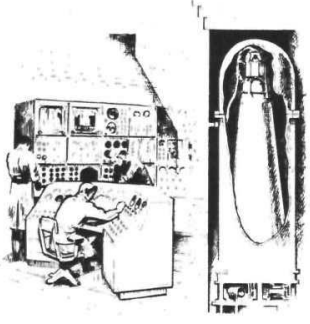

ACCEPTANCE TESTING

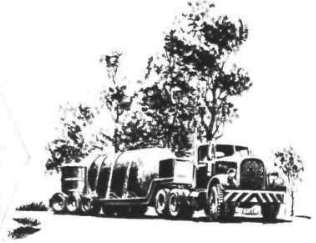

SHIPMENT

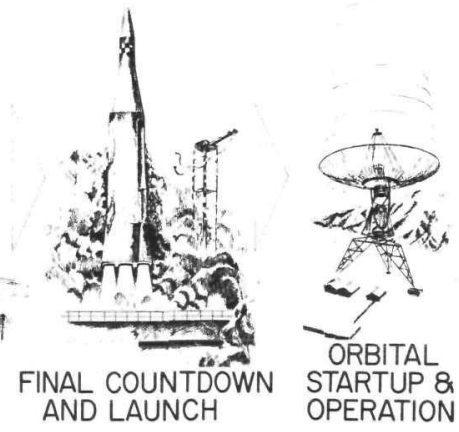

Figure II-70. Factory-to-Flight Sequence 
integral SNAP 10A unit and its reflector subassembly are separately shipped to the launch site where the core and reflector assembly are reunited, but poison sleeves, void filler blocks, and physical constraints are applied to prevent accidental criticality during the remaining spacecraft mating, and checkout operations. These safety devices are not removed until all installation and checkout activities are complete. At this time the gantry is removed and the only remaining prelaunch activity is the remote fueling of the launch vehicle. After fueling and final countdown the spacecraft is launched. A nose cone aerodynamic fairing is ejected at the time of first and second stage operation. After injection into orbit, the spacecraft is tracked in order to ascertain the acceptability of the orbit. During this period the power unit radiator is covered by a heat shield which reduces the heat losses to space sufficiently to prevent freezing of the NaK. A radio command to the spacecraft initiates power system startup. After the power unit has heated up to a few hundred degrees, the heat shield is ejected. When the output temperature of the reactor heat source achieves the design condition, the insertion of reactivity is interrupted. Reactor control based on outlet temperature continues for 72 hours until thermal and short time fission product poison equilibrium is attained.

The actual factory-to-flight sequence of SNAP IOA is shown in Figure II-71. 


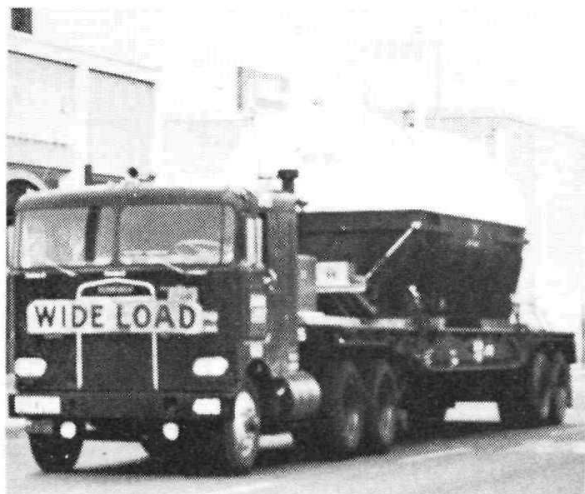

SHIPMENT

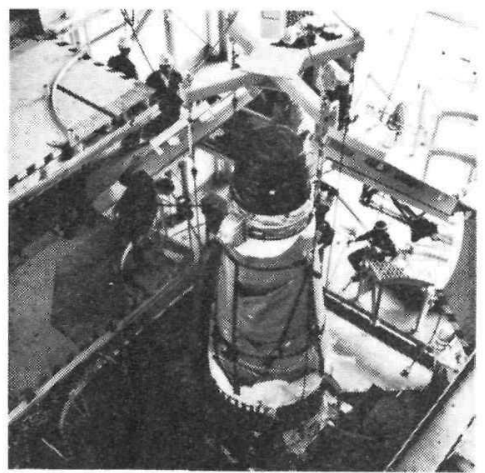

MATING

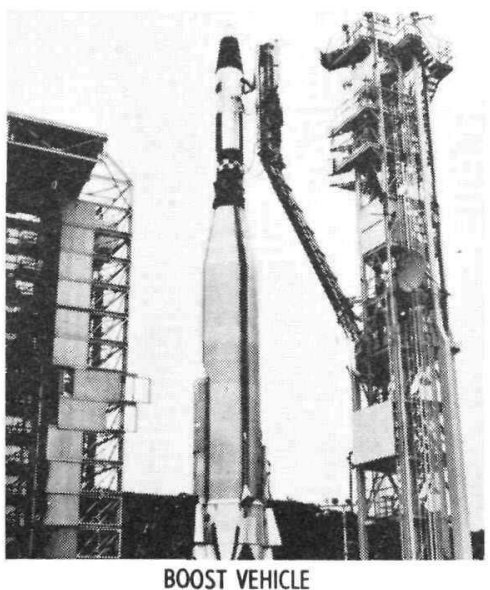

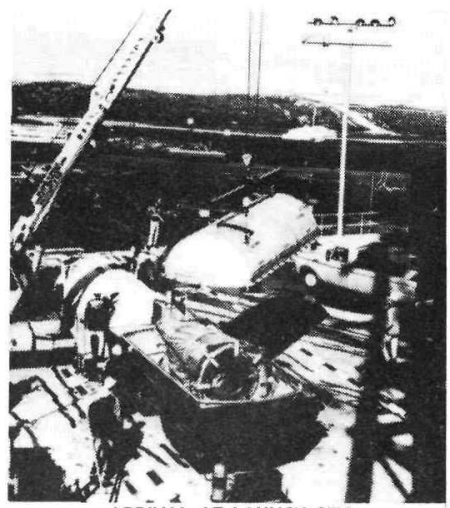

ARRIVAL AT LAUNCH SITE
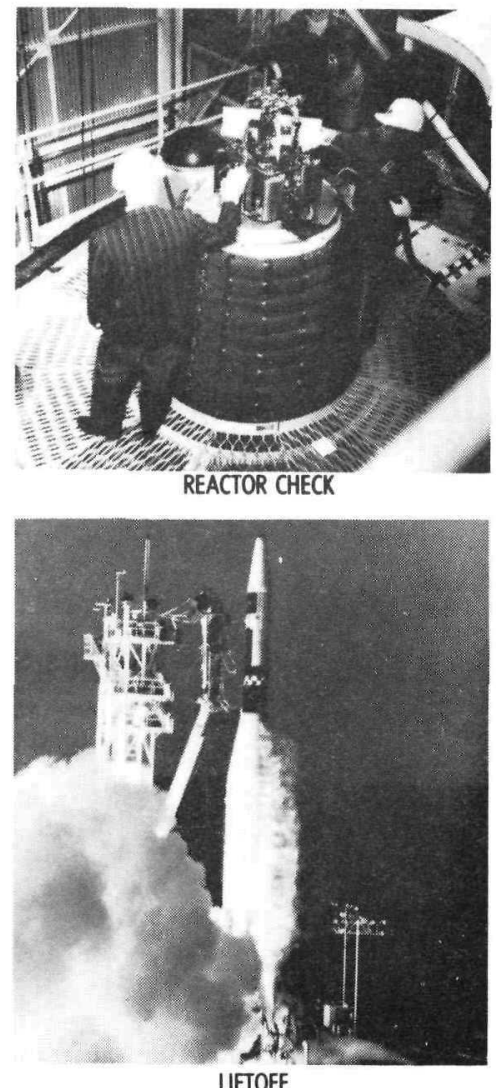

7561-02854

Figure II-71. SNAP I0A in the Final Phase of Launch Preparation 


\section{SNAP SYSTEMS}

A. SNAP HYDRIDE REACTOR

a. Concept

SNAP 2, 8, and $10 \mathrm{~A}$ all use the same reactor concept as a heat source (see Table I-1). The reactor concept employs a homogeneous fuel moderator of zirconium hydride containing $\mathrm{U}^{235}$. For minimum weight, the reactor is reflected by beryllium and controlled by variation of the effective reflector thickness by means of angular rotation of semicylindrical beryllium drums. The core is composed of a bundle of cylindrical fuelmoderator elements, see Figure II-72. Each fuel element is clad in a thin wall steel tube for liquid metal exclusion. The fuel elements are contained in a steel core vessel, with the beryllium radial reflector outside the vessel. The reflector is completely separable from the core for safe reactor shutdown during handling (Figure II-73). The thermal output is removed by the flow of NaK - 78 axially through the core within the interstitial passages between the fuel elements.

\section{b. Development Experience}

Immediately after formal program initiation in early 1957, demonstration and verification of the reactor physics of the chosen concept was established as a prime goal. This objective was achieved with the correlation of analysis and experimental results from a critical assembly which first achieved criticality in October 1957. The core consisted of a pseudo spherical assembly of cold pressed blocks of a $\mathrm{UO}_{2}$ and $\mathrm{ZrH}_{x}$ powder mixture containing 10 wt \% uranium. In addition to a criticality verification, the assembly provided significant information on reflector effectiveness and the control worth of reflector geometry variations.

The next major objective was the power, temperature, and life demonstration of the reactor concept. The final feasibility 


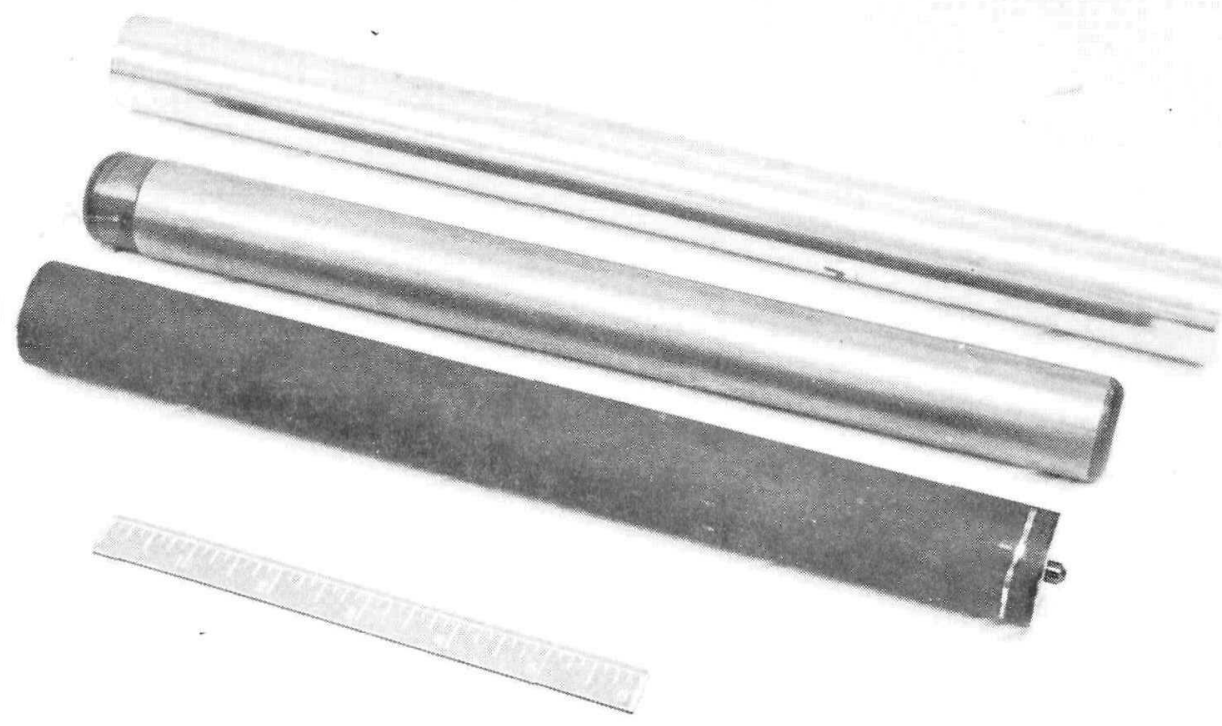

Figure II-72. SNAP 2 Fuel Element 


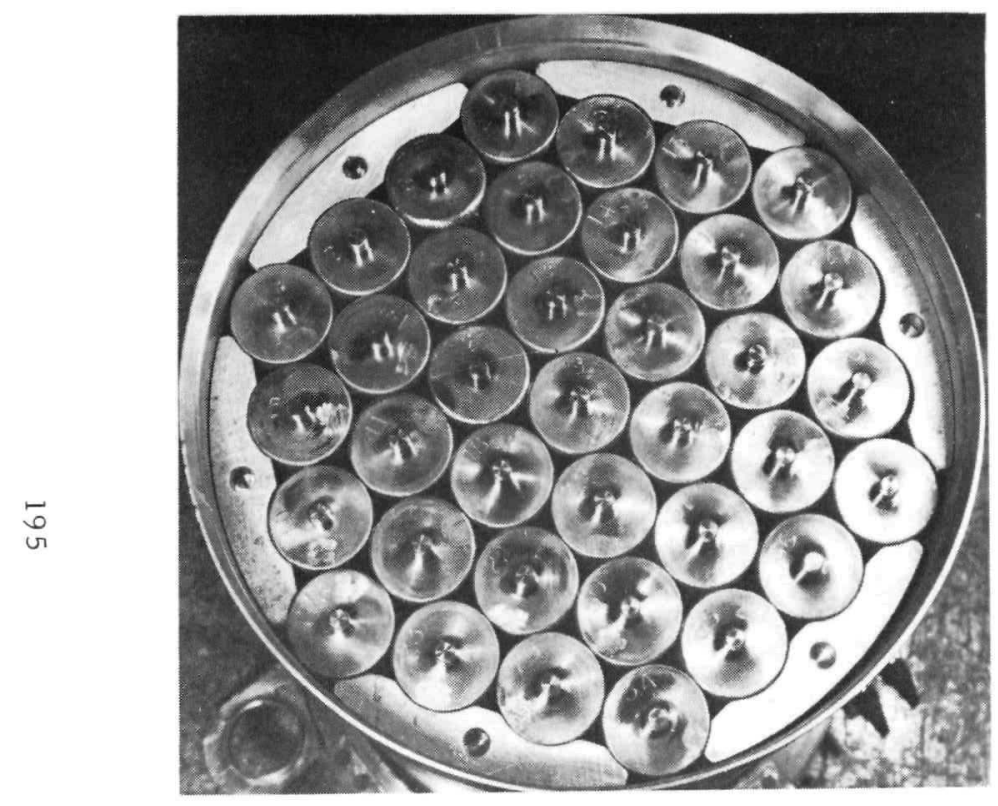

$7-5-63$

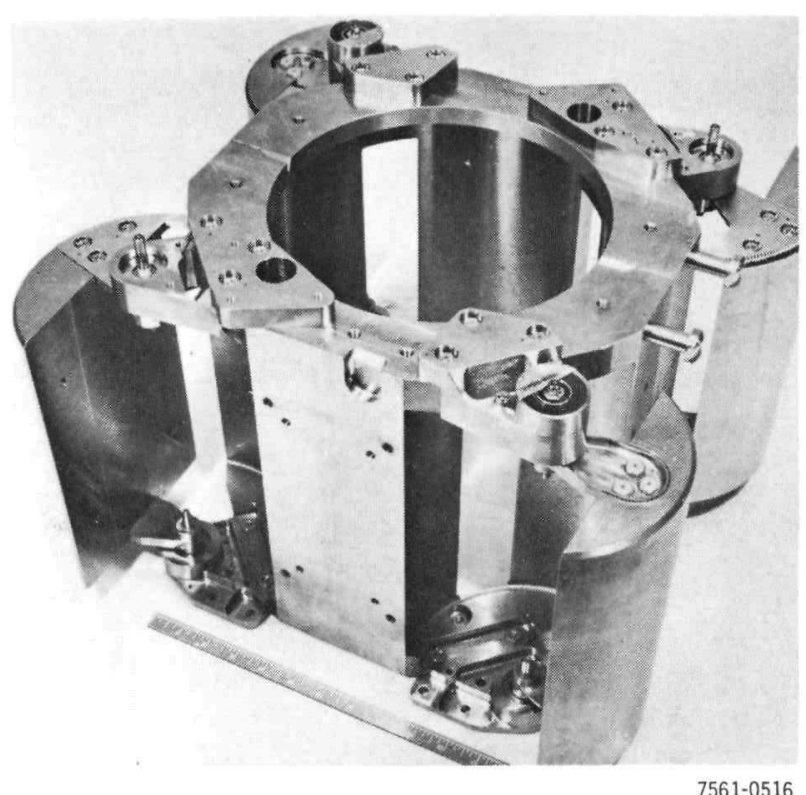

b. SNAP 10A/2 Reactor Beryllium Control Subassembly a. Internal View of the SNAP 10A/2 Core 
of the concept rested on demonstrating the required hydrogen retention ability of the fuel cladding under the reactor conditions of power, temperature, and radiation. The SNAP Experimental Reactor (SER) was designed and fabricated toward this purpose. The SER began operation in September 1959 and was operated until the conclusion of the necessary test program in November 1960 (see Figure II-74). The SER embodied all of the features of the SNAP reactor concept. The fuel elements contained 7 wt $\%$ uranium and $6 \times 10^{23}$ atoms/cc of $\mathrm{H}_{2}$ in the form of $\mathrm{ZrH}_{\mathrm{x}}$. The core contained sixty-one 1 -in. diameter fuel moderator elements on a triangular matrix contained in a 9-in. diameter core vessel. The reactor was cooled by the flow of NaK-78. Reactor control was accomplished by means of reflector variation external to the

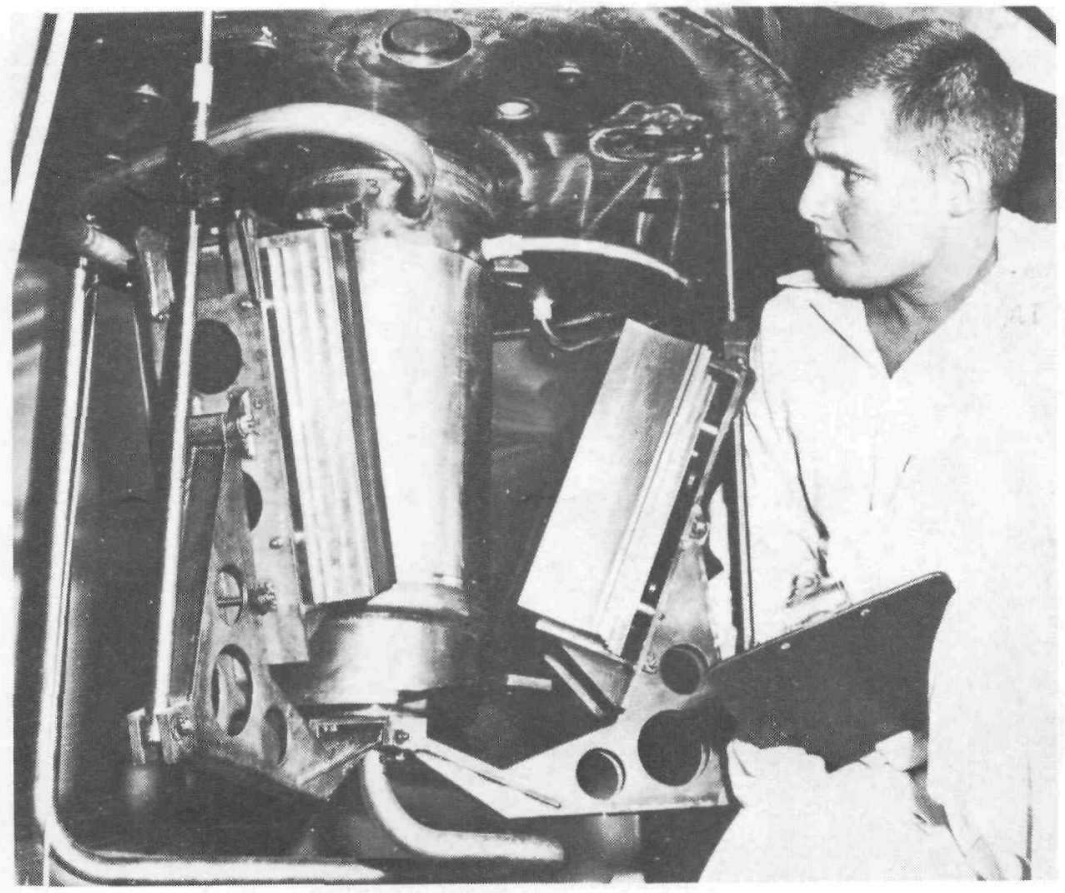

Figure II-74. SNAP 10A/2 Experimental Reactor 
core vessel. The SER operated at a peak power of $50 \mathrm{kw}$ thermal at an outlet temperature of $1200^{\circ} \mathrm{F}$. During one test the reactor was operated continuously for 1000 hours at these conditions. During the test program the SER produced an integral energy output of 225,000 kwt-hr. Of the 6035 total test hours, 3300 were at temperatures in excess of $900^{\circ} \mathrm{F}$ and 1900 hours were at $1200^{\circ} \mathrm{F}$ outlet.

The next step in the SNAP reactor development was the fabrication and testing of a second generation design which more nearly approximated the detailed requirements of a space reactor. This reactor, the SNAP Development Reactor (SDR), began operation in April 1961 and the test program was completed in December 1962, see Figure II-75. The SDR core consisted of 37 fuel elements of $1-1 / 4$ in. diameter containing 10 wt \% uranium and $6.5 \times 10^{23} \mathrm{H}_{2}$ atoms/cc. The core geometry was similar to

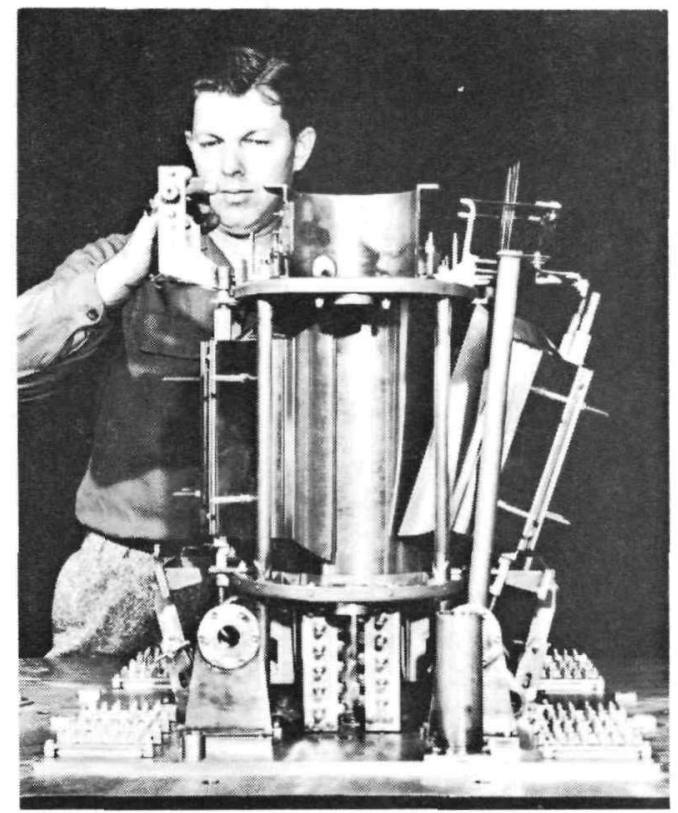

$7550-2091$

Figure II-75. SNAP 2 Development Reactor 


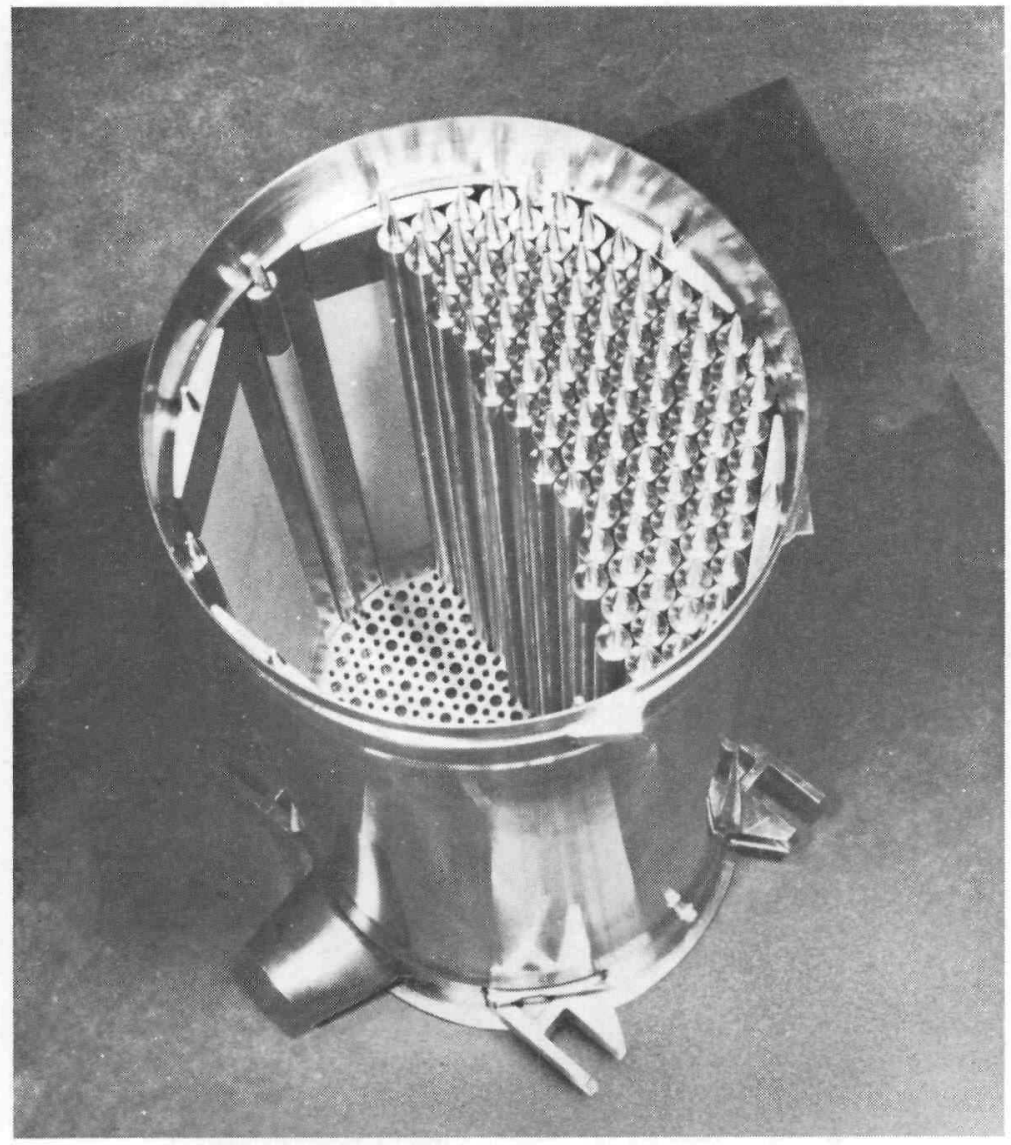

Figure II-76. Internal View of the SNAP 8 Core 
SER and the coolant was NaK-78. The Be reflector region was about 3 in. thick and was divided into two fall-away safety elements and two rotatable half-cylinder control elements. The SDR operated at a power of $50 \mathrm{kwt}$ and a maximum outlet temperature of $1200^{\circ} \mathrm{F}$. During the test program the SDR produced an integral energy output of $273,000 \mathrm{kwt}-\mathrm{hr}$. Of the total 11,300 test hours, 6,900 were at temperatures in excess of $900^{\circ} \mathrm{F}$ and 2,100 hours were at $1200^{\circ} \mathrm{F}$ outlet. The SDR program also included an endurance test of 1,100 hours at $50 \mathrm{kwt}$ and $1200^{\circ} \mathrm{F}$ outlet.

\section{c. SNAP 8 Reactor}

In addition to forming the basis for the reactor heat source for SNAP 10A, the SER and SDR provided the necessary fuel, physics, and reactor operating experience to design the high power (1600 kwt) and high temperature $\left(1300^{\circ} \mathrm{F}\right)$ reactor for SNAP 8. In order to achieve the higher power and temperature of SNAP 8, the core size was increased and the fuel diameter reduced. An internal view of the SNAP 8 core is shown in Figure II-76. The SNAP 8 Experimental Reactor (S8ER) operated during the period of May 1963 to April 1965. A photograph of the S8ER ground test reactor assembly prior to installation in the test facility is shown in Figure II-77. The S8ER operated for over 11,000 hours of wh1ch 1 year was at $1300^{\circ} \mathrm{F}$ outlet temperature and power levels between 400 to $600 \mathrm{kwt}$. During the test program the reactor sustained an uninterrupted power run of 5000 hours. The S8ER produced $5.1 \times 10^{6} \mathrm{kwt}-\mathrm{hr}$ of energy, the integral design energy requirement of the SNAP 8 system.

After shutdown in April 1965, the reactor was subjected to a complete post-mortem evaluation. About $85 \%$ of the fuel elements were found to have cracked as a result of radiationinduced reduction in cladding ductility and inadequate design clearance to accommodate the normal fuel swelling. The critical materials phenomena have been defined through extensive in-pile experiments and analysis of the S8ER results. On the basis of 


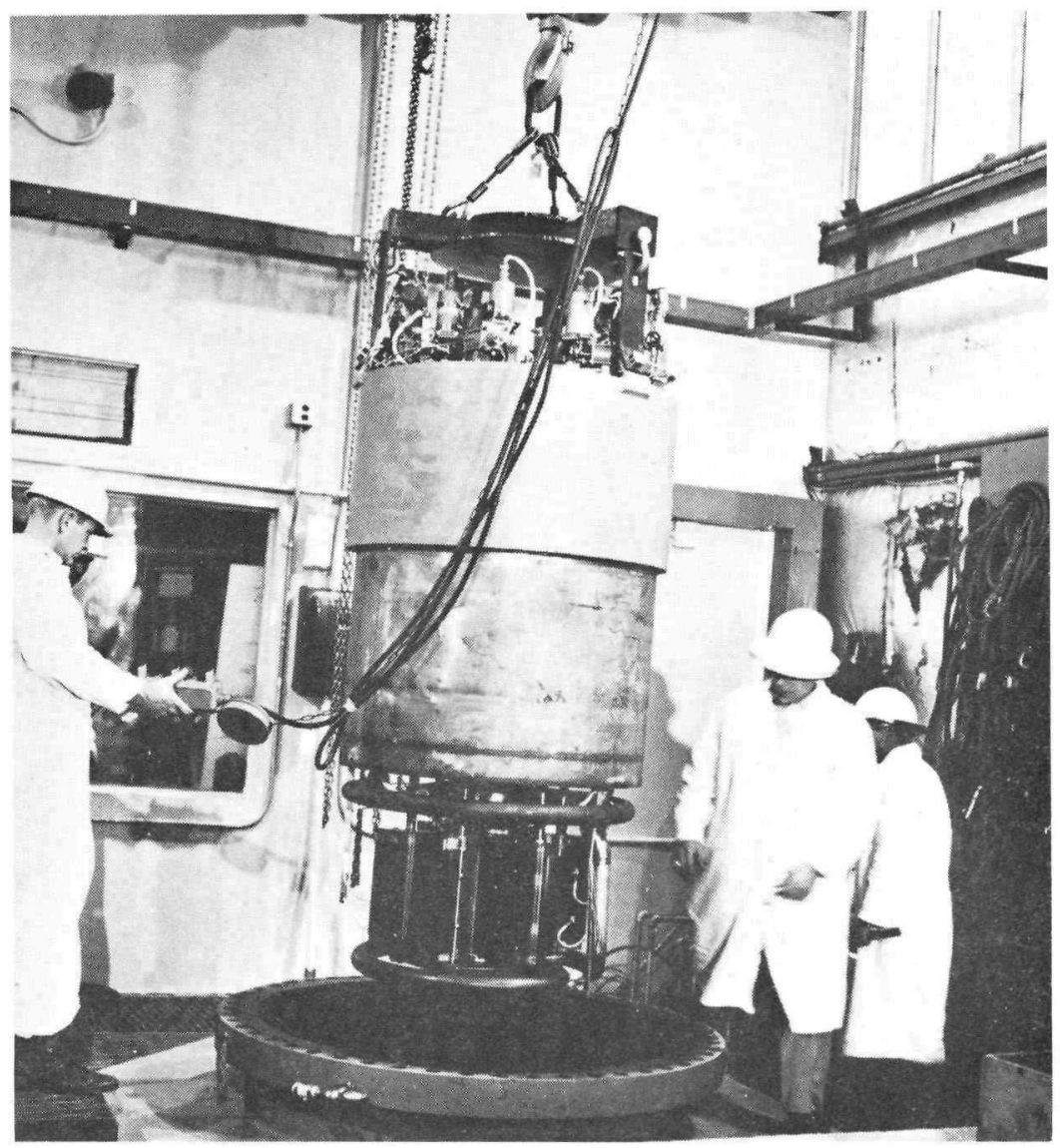

$7568-56110 \mathrm{CN}$

Figure II-77. SNAP 8 Experimental Reactor Test Assembly Being Lowered into the Test Facility 
these data, the design has been established for the next SNAP 8 reactor test, S8DR. This reactor is scheduled to begin operation in the spring of 1968 and will demonstrate the reactor capability envelope shown in Figure II-78. An engineering development mockup of the flight design SNAP 8 reactor and shield is shown in Figure II-79.

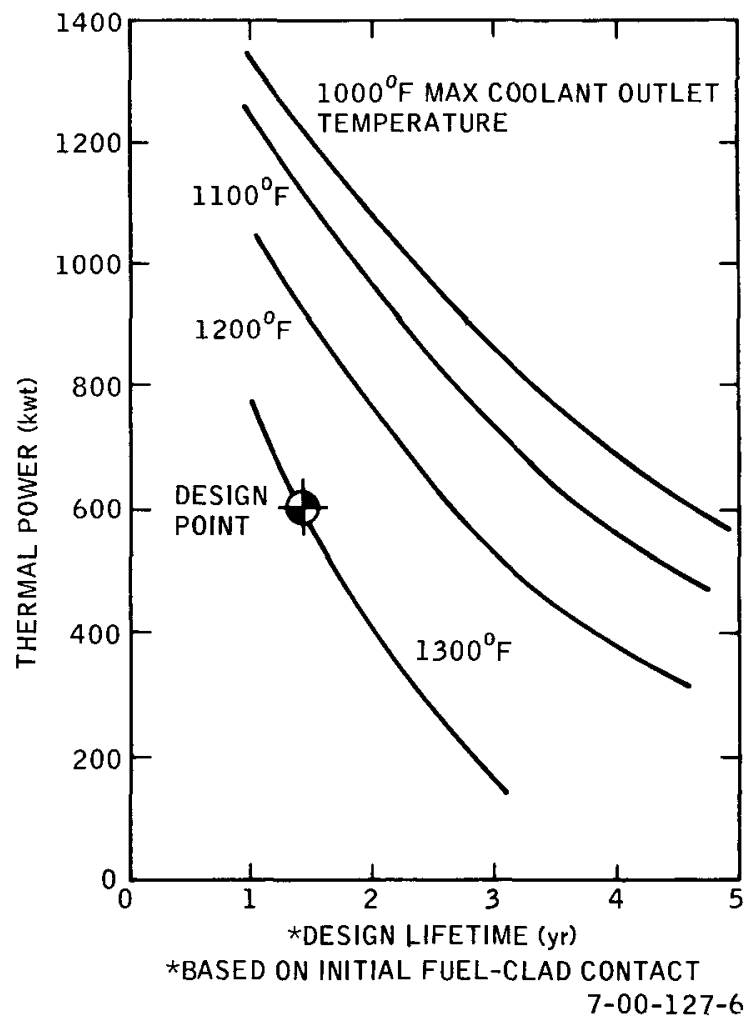

Figure II-78. Current SNAP 8 Reactor Performance Tradeoffs 


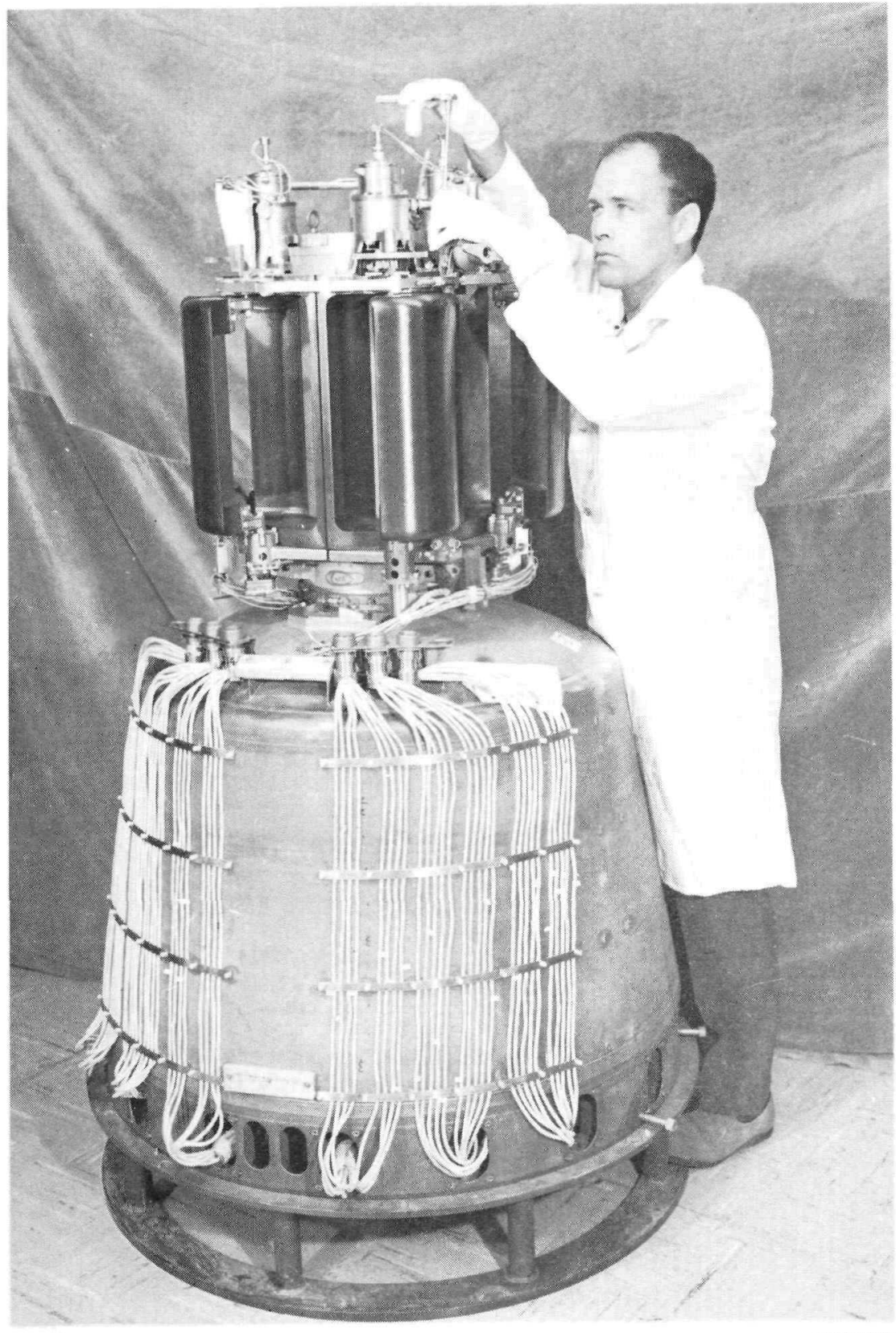

Figure II-79. SNAP 8 Reactor and Shield 


\section{d. SNAP 10A Reactor}

The specific reactor for SNAP IOA is illustrated in Figures II- 73 and II-80. The core consists of 37 fuel moderator elements of 1-1/4 in. diameter by $13 \mathrm{in}$. long located on a 1.26-1n. close packed triangular matrix contained within a 9-in. diameter core vessel. Each fuel element contains 10 wt $\%$ uranium and $6.3 \times 10^{23} \mathrm{H}_{2}$ atoms/cc. The fuel moderator elements are clad in 0.015-in. wall Hastelloy tubing with an internal hydrogen retention barrier. The NaK coolant flows axially through the core in the interstitial region between fuel elements. The region between the resulting hexagonal parallelopiped and the cylindrical core vessel is filled by beryllium metal. The average radial beryllum reflector thickness is $2.5 \mathrm{in}$. The bulk of the radial reflector is located outside of and is completely removable from the core vessel without modifying the integrity of the sealed NaK coolant system. With the reflector removed the reactor is far subcritical which provides a large safety margin for all handling, shipping, checkout, and installation activities.

The reactor control elements are four half-cylundrical sections of the radial reflector region. Rotation of these sections changes their proximity to the core and provides reactor control by leakage variation. The complete radial reflector control subassembly is split into two integral sections. These sections are held in place by a retention band which can be severed by command or by reentry heatıng. Stored energy in springs ejects the reflector halves when the band is released. 


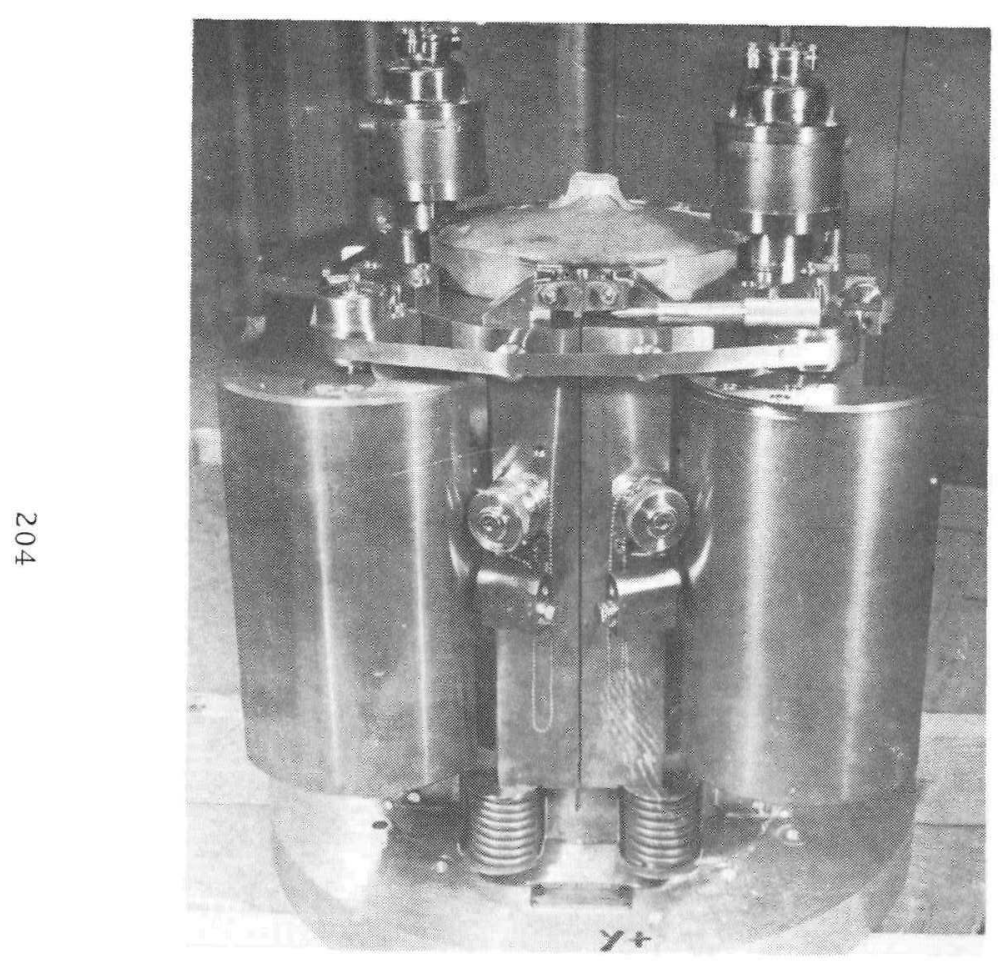

$7-5-63$
SIDE VIEW

TOP VIEW

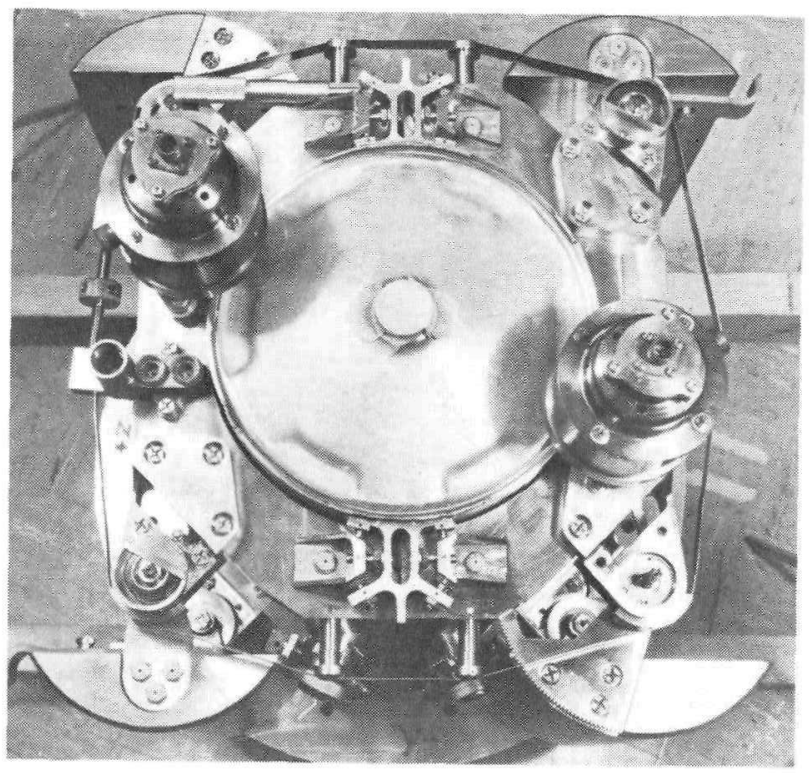

$7561-0517$

Figure II-80. SNAP 10A/2 Reactor 
During startup, release of the control elements by means of an explosive pin puller by ground radro command allows the elements to move inward to add reactivity. Two elements are driven in rapidly by spring energy and bring the reactor within a fraction of a dollar of cold critical. The remaining two elements are driven in slowly by electrical stepper motors. These elements add reactivity continually at a slow rate until the reactor reaches operating temperature. The ramp reactivity insertion rate is slow enough to maintain the initial power overshoot within design limits. After the initial burst, reactor temperature increases at the rate necessary to off set the reactivity insertion through the isothermal temperature coefficient. Reactor power is initially determined by the combination of reactor and system heat capacity and the equilibrium system heat rejection at the average source temperature.

The thermomechanical performance of the SNAP 10A reactor hardware under simulated conditions of checkout, launch, and orbital operation was verified by means of a Development Mockup Reactor (DRM), see Figure II-81. This assembly was successfully tested with electrical heat at the SNAP $10 A$ temperature conditions in a vacuum of $10^{-6}$ tor $r$ for 90 days of continuous operation.

Two reactors were operated as part of the SNAP $10 \mathrm{~A}$ program. The ground test system l0FS-3 was a replica of the flight system and began operation in January 1965 in a shielded vacuum, $10^{-3}-10^{-4}$ torr, chamber. The system startup, control, and operation was prototypical of that used on the subsequent flight test. The performance of lOFS-3 is shown in Figure II-82. After startup, control was maintained for three days to compensate for initial reactivity drifts like xenon. The system then operated for $\mathbf{l}$ year with no active control. The temperature drifted downward to compensate for residual reactivity losses. At the end of 1 year, the controls were activated to perturb the equilibrium and raise the operating temperature. The system 


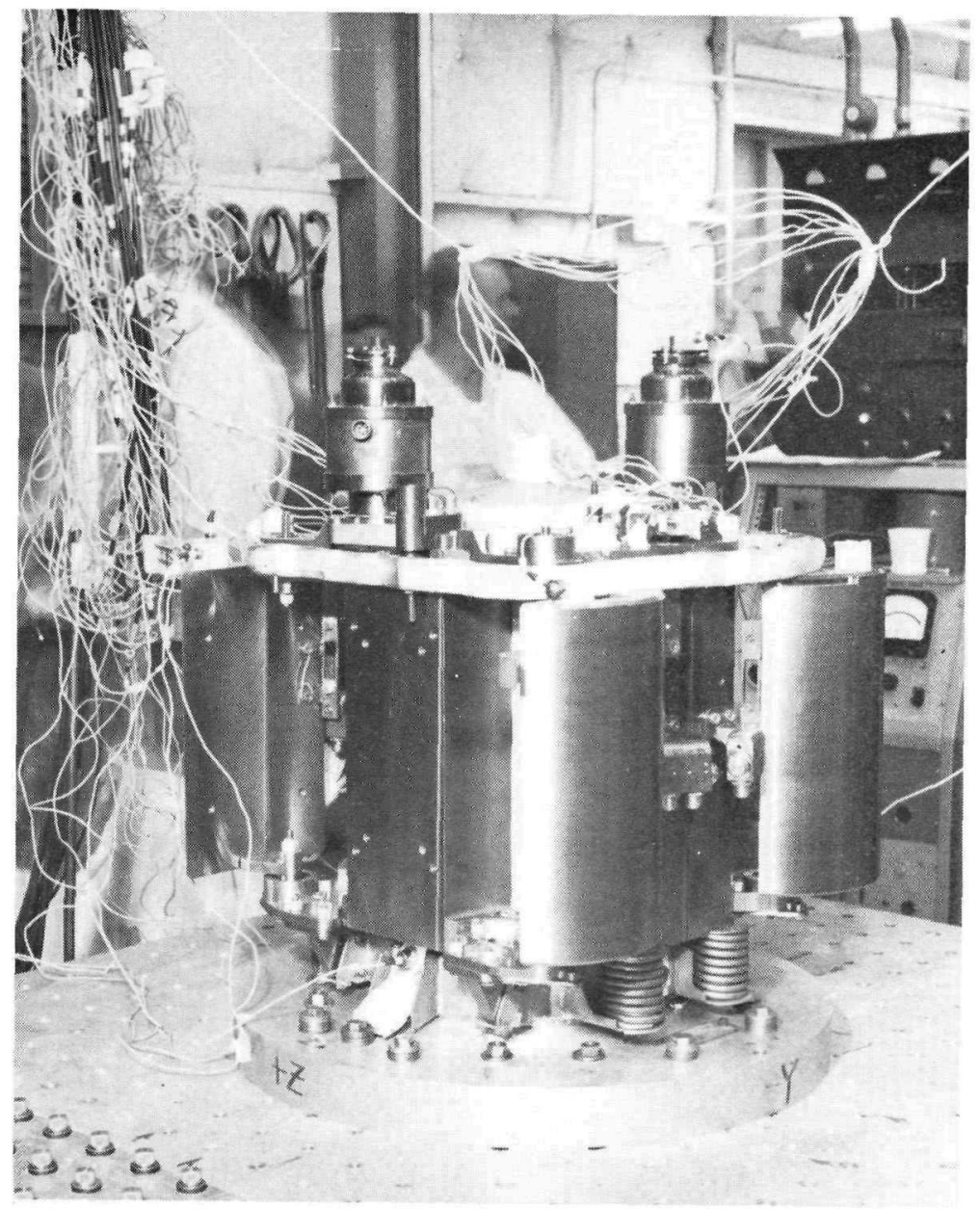

5-15-62

7580-5259C

Figure II-8I. Launch Shock and Vibration Environment Qualification of the SNAP 10A Reactor 


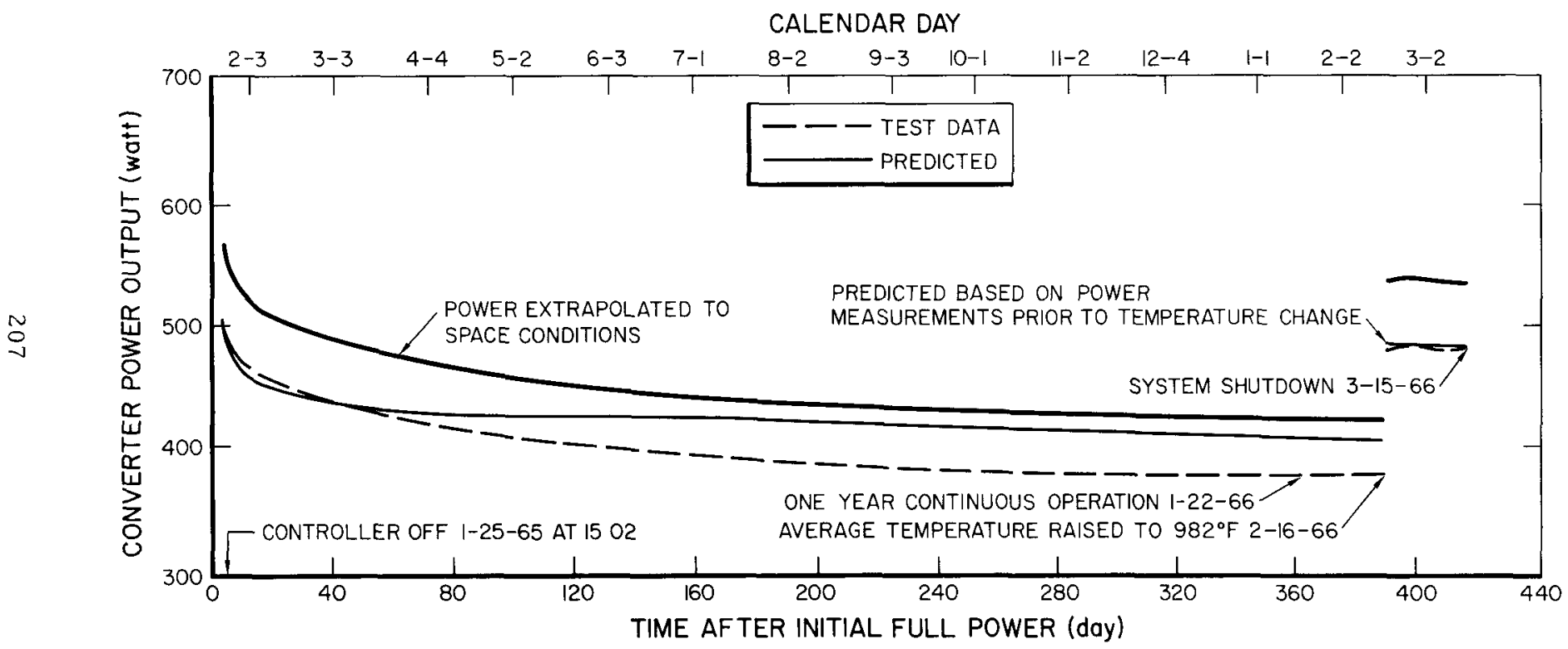

$5-16-66$

Figure II-82. SNAP 10A FS-3 Converter Power Output 
TAB LE III-19

SNAP HYDRIDE REACTORS

\begin{tabular}{|c|c|c|c|c|}
\hline & SER & S2DR & S8ER & SNAP IOA \\
\hline \multicolumn{5}{|l|}{ FUEL-MODERATOR ELEMENTS } \\
\hline DIAMETER $(\mathrm{cm} / / \mathrm{n})$ & $254 / 10$ & $318 / 125$ & I $42 / 056$ & $318 / 125$ \\
\hline LENGTH $(\mathrm{cm} / \mathrm{in})$ & $356 / 140$ & $330 / 130$ & $368 / 145$ & $330 / 130$ \\
\hline$N_{H}\left(H\right.$ atom $\left./ \mathrm{cm}^{3} \times 10^{-22}\right)$ & 60 & 65 & 60 & 65 \\
\hline W/O U FULLY ENRICHED U-235 & 7 & 10 & 10 & 10 \\
\hline NO OF ELEMENTS & 61 & 37 & 211 & 37 \\
\hline TOTAL U-235 (kg) & 29 & 43 & 656 & 43 \\
\hline CLADDING THICKNESS (mın/ın) & $025 / 0010$ & $033 / 0013$ & $025 / 0010$ & $038 / 0015$ \\
\hline \multicolumn{5}{|l|}{ REACTOR } \\
\hline CORE VESSEL DIAMETER $(\mathrm{cm} / \mathrm{ln})$ & $241 / 95$ & $228 / 90$ & $248 / 975$ & $227 / 894$ \\
\hline HEIGHT-NOMINAL $(\mathrm{cm} / \mathrm{/n})$ & $406 / 16$ & $406 / 16$ & $534 / 21$ & $396 / 156$ \\
\hline Be REFLECTOR THICKNESS-NOMINAL $(\mathrm{cm} / \mathrm{In})$ & $76 / 3$ & $58 / 23$ & $76 / 3$ & $51 / 2$ \\
\hline NO OF CONTROL DRUMS-SAFETY ELEMENTS & $3-3$ & $2-2$ & 6 & 4 \\
\hline CORE VOLUME (meter $\left.3 / \mathrm{ft}^{3}\right)$ & $074 \times 10^{-2} / 026$ & $068 \times 10^{-2} / 024$ & $159 \times 10^{-2} / 054$ & $085 \times 10^{-2} / 03$ \\
\hline WEIGHT WITH REFL CONTROL ASSEMBLY $(\mathrm{kg} / \mathrm{lb})$ & $159 / 350$ & $137 / 300$ & $250 / 550$ & $114 / 250$ \\
\hline FUEL ELEMENT SPACING-TRIANGULAR MATRIX $(\mathrm{cm} / \mathrm{ln})$ & $258 / 1015$ & $320 / 126$ & $145 / 057$ & $320 / 126$ \\
\hline \multicolumn{5}{|l|}{ OPERATING CHARACTERISTICS } \\
\hline COOLANT FLOW (1,ters $\left.\mathrm{sec}^{-1} / \mathrm{gpm}\right)$ & $17 / 23$ & $17 / 23$ & $88 / 140$ & $083 / 131$ \\
\hline INLET TEMPERATURE (C/F) & $538 / 1000$ & $538 / 1000$ & $593 / 1100$ & $482 / 900$ \\
\hline OUTLET TEMPERATURE (C/F) & $649 / 1200$ & $649 / 1200$ & $704 / 1300$ & $543 / 1010$ \\
\hline MAXIMUM FUEL TEMPERATURE (C/F) & $702 / 1295$ & $693 / 1280$ & $788 / 1450$ & $585 / 1085$ \\
\hline POWER (kwth) & 50 & 50 & 600 & 34 \\
\hline POWER DENSITY-AVG (Mwth liter ${ }^{-1} / \mathrm{Mwh} \mathrm{ft}^{-3}$ ) & $000675 / 0192$ & $000735 / 0206$ & $00378 / 111$ & $00040 / 0113$ \\
\hline $\begin{array}{l}\text { HEAT FLUX-AVG (kcal } \mathrm{n}^{-2} \mathrm{hr}^{-1} / \mathrm{Btu} \mathrm{ft}^{-2} \mathrm{hr}^{-1} \text { ) } \\
\text { NEUTRON FLUX-AVG }\left(\mathrm{n} / \mathrm{cm}^{2} / \mathrm{sec}\right)\end{array}$ & $\begin{array}{l}35,000 / 12,800 \\
31 \times 10^{11}\end{array}$ & $\begin{array}{l}48,000 / 17,600 \\
32 \times 10^{11}\end{array}$ & $\begin{array}{l}150,000 / 55,000 \\
23 \times 10^{12}\end{array}$ & $\begin{array}{l}28,000 / 10,200 \\
17 \times 10^{11}\end{array}$ \\
\hline
\end{tabular}


was again returned to inherent self-control and operated until shutdown after 10,000 hours of continuous and uninter rupted operation. The reactor was subsequently subjected to a postmortem examination in a hot cell. No evidence of incipient fallure was observed and the reactor could most likely have continued to operate for 5 years or more.

The flight test reactor 10A-FS-4 was launched on April 3, 1965. The reactor sustained the launch shock and vibration, was started remotely in space, was under active control for 6 days to minimize subsequent drift, and operated continuously for 43 days. The orbital test was terminated by a spacecraft fallure, which produced an erroneous signal, which shut down the reactor. The space test behaved almost identically with the ground test.

\section{e. SNAP Hydride Reactor Summary}

The design conditions of the five SNAP hydride reactors that have been tested to date are shown in Table II-19, and a summary of the operating history to date is given in Table II-20.

\section{B. SNAP IOA \\ a. System Description}

The objective of the SNAP l0A program was to develop a nuclear reactor power unit capable of producing a minimum of 500 electrical watts for a period of 1 year in a space environment. SNAP IOA is the first reactor-powered electrical system to have been flight tested in earth orbit.

SNAP IOA employs a ( $\mathrm{ZrH})$ thermal reactor coupled to an integral $S_{1}-G e$ thermoelectric converter-radiator which converts NaK-transported fission heat to electrical power. The unit is approximately conical in configuration with a base diameter of $5 \mathrm{ft}$ and an overall height of $11 \mathrm{ft}$. The first flight system will have a shielded weight of $950 \mathrm{lb}$. The $225-\mathrm{lb}$ shield will restrict the radiation dose at the base to about $4 \times 10^{12}$ nvt of fast neutrons 
TAB LE II - 20

SNAP REACTOR OPERA TING EXPERIENCE

\begin{tabular}{|c|c|c|c|c|c|}
\hline Reactor & $\begin{array}{l}\text { Date Critical/ } \\
\text { Shutdown }\end{array}$ & $\begin{array}{c}\text { Thermal } \\
\text { Power } \\
\text { (kwt) }\end{array}$ & $\begin{array}{c}\text { Teinper- } \\
\text { ature } \\
\left({ }^{\circ} \mathrm{F}\right)\end{array}$ & $\begin{array}{c}\text { Thermal } \\
\text { Energy } \\
(k w t-h r)\end{array}$ & $\begin{array}{l}\text { Time at Power } \\
\text { and Temperature }\end{array}$ \\
\hline SER & $\begin{array}{l}\text { September } 1959 / \\
\text { December } 1960\end{array}$ & 50 & 1200 & 225,000 & $\begin{array}{l}1800 \mathrm{hr} \text { at } 1200^{\circ} \mathrm{F} \\
3500 \mathrm{hr} \text { above } 900^{\circ} \mathrm{F}\end{array}$ \\
\hline SDR & $\begin{array}{l}\text { April } 1961 / \\
\text { December } 1962\end{array}$ & 65 & 1200 & 273,000 & $\begin{array}{l}2800 \mathrm{hr} \text { at } 1200^{\circ} \mathrm{F} \\
7700 \mathrm{hr} \text { above } 900^{\circ} \mathrm{F}\end{array}$ \\
\hline S8ER & $\begin{array}{l}\text { May 1963/ } \\
\text { April } 1965\end{array}$ & 600 & 1300 & $5,100,000$ & $\begin{array}{l}\mathrm{l} \mathrm{yr} \text { at } 1300^{\circ} \mathrm{F} \text { and } \\
400 \text { to } 600 \mathrm{kwt}\end{array}$ \\
\hline$S 10 A-F S-3$ & $\begin{array}{l}\text { January } 1965 / \\
\text { March } 1966\end{array}$ & 38 & 1000 & 382,944 & $10,005 \mathrm{hr}$ \\
\hline$S 10 A-F S-4$ & $\begin{array}{l}\text { April 1965/ } \\
\text { May } 1965\end{array}$ & 43 & 1000 & 41,000 & 43 days \\
\hline
\end{tabular}


and $1 \times 10^{7} \mathrm{r}$ of gammas during the 1 -year lifetime. After orbital startup on ground command, the unit operates without the need for active control, without moving parts, and without attitude orientation requirements. Because of the negligible heat rejection variation during the orbital sun-shade transient, SNAP 10A provides a continuous source of at least 500 watts without the cyclic limitations of secondary batteries. On a production basis, the SNAP 10A unit should cost about one million dollars.

The SNAP 10A system configuration is virtually dictated by minimum weight shielding requirements. The critical shielding problem is the elimination of neutron scattering around the shadow shield. As a result, the system configuration is conical, with a base diameter established by the vehicle payload, the upper diameter determined by the effective area of the reactor source, and its length determined by the total radiator area requirement. Figure II-83 is an illustration of the powerplant configuration. Basic design operating conditions of the system

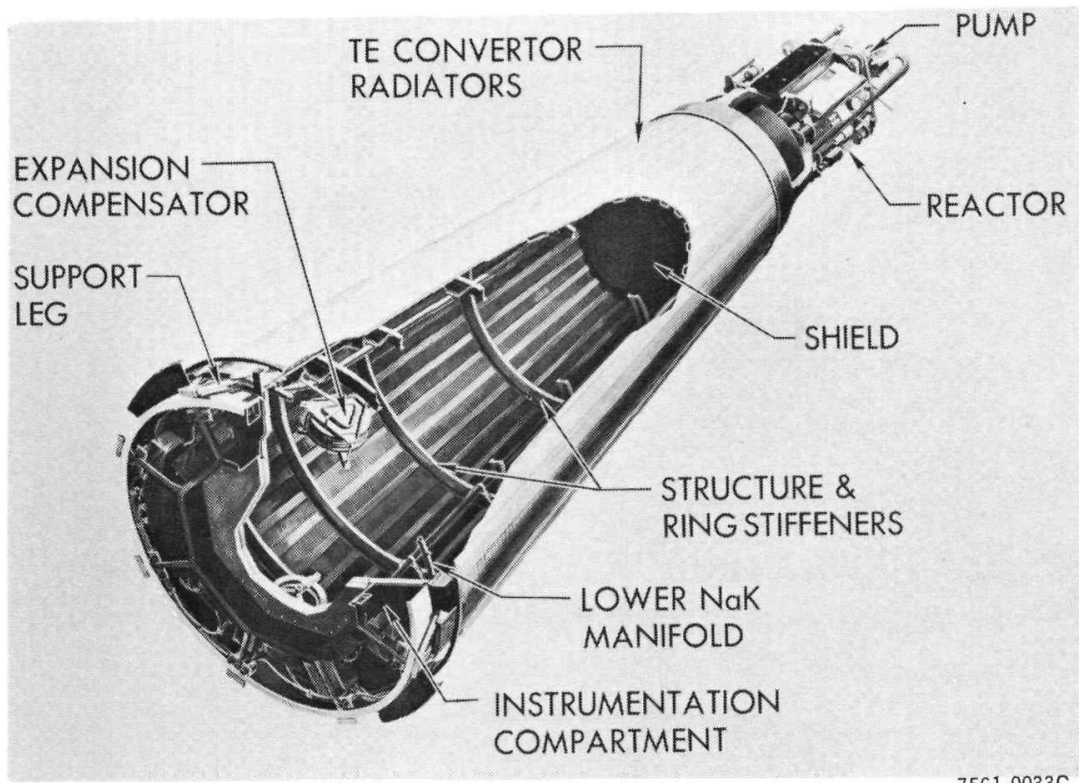

Figure II-83. SNAP-IOA System 


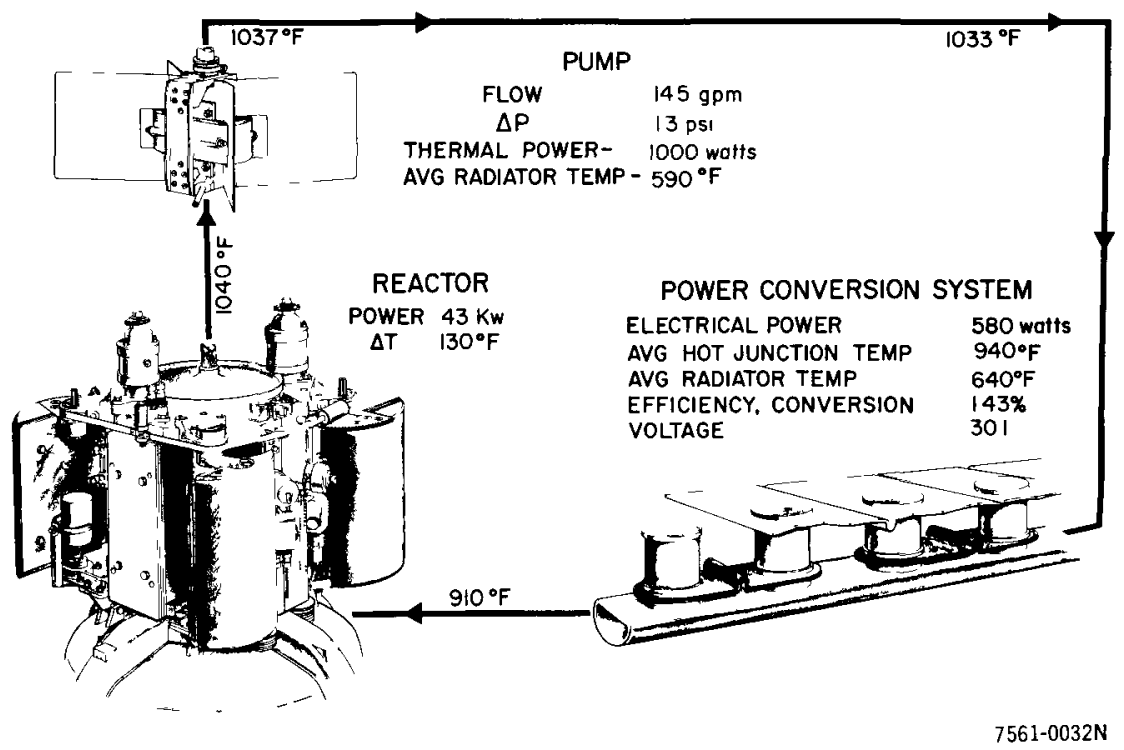

Figure II-84. SNAP 10A Performance Characteristics

TAB LE II - 21

SNAP IOA WEIGHT

(lb)

\begin{tabular}{l|r}
\hline \hline Reactor & 275 \\
Shield & 217 \\
Converter & 154 \\
Pump & 20 \\
Expansion Compensator & 28 \\
Piping and NaK & 45 \\
Structure & 83 \\
Instrumentation and Compartment & 106 \\
Heat Shield & 32 \\
& \\
\hline
\end{tabular}


are shown in Figure II-84. The system weight breakdown is given in Table II-21.

An exploded view of the reactor shield combination is shown in Figure II-85. The fission heat is transferred from the reactor to the thermoelectric power converter by means of a liquid metal (NaK-78) coolant loop. The liquid metal is circulated by a dc Faraday conduction pump which derives its current from a shorted $\mathrm{PbTe}$ thermocouple operating between the NaK outlet temperature and a cold junction determined by a small pump radiator. The pump magnetic field is supplied by a permanent magnet. A photograph of the flight design pump is shown in Figure II-86. The NaK flow is divided among 40 parallel tubes arranged axially along the unit's conical surface.

Each NaK tube is a series fluid connection of three thermoelectric modules. Thus, the full 500-watt converter is made up of 120 modules of about 4 to 5 watts each. A detalled drawing of a module is shown in Figure II-87 and an actual module photograph is shown in Figure II-88. Seventy-two cylindrical pellets of $\mathrm{N}$ - and P-doped Ge-Si alloy thermoelectric material are alternately spaced along the length of each NaK tube. The pellets are electrically insulated from the NaK tube by means of thin alumina disks. The pellets are electrically connected in series with copper straps at the hot end and aluminum combination strapradiators at the cold end. Each aluminum radiator platelet is electrically insulated from adjacent platelets by a clearance gap. All material interfaces from the NaK tube through the aluminum radiator are either metallurgically bonded or brazed. Each material stack from $\mathrm{NaK}$ tube to radiator is capable of supporting 200 to $300 \mathrm{lb}$ in tension. The radiator platelets have an emissivity of about 0.9 and are sized to maintain the thermocouple cold junction at an average temperature of about $600^{\circ} \mathrm{F}$. The total radiator area (1ncluding gaps) is approximately $65 \mathrm{ft}^{2}$. 


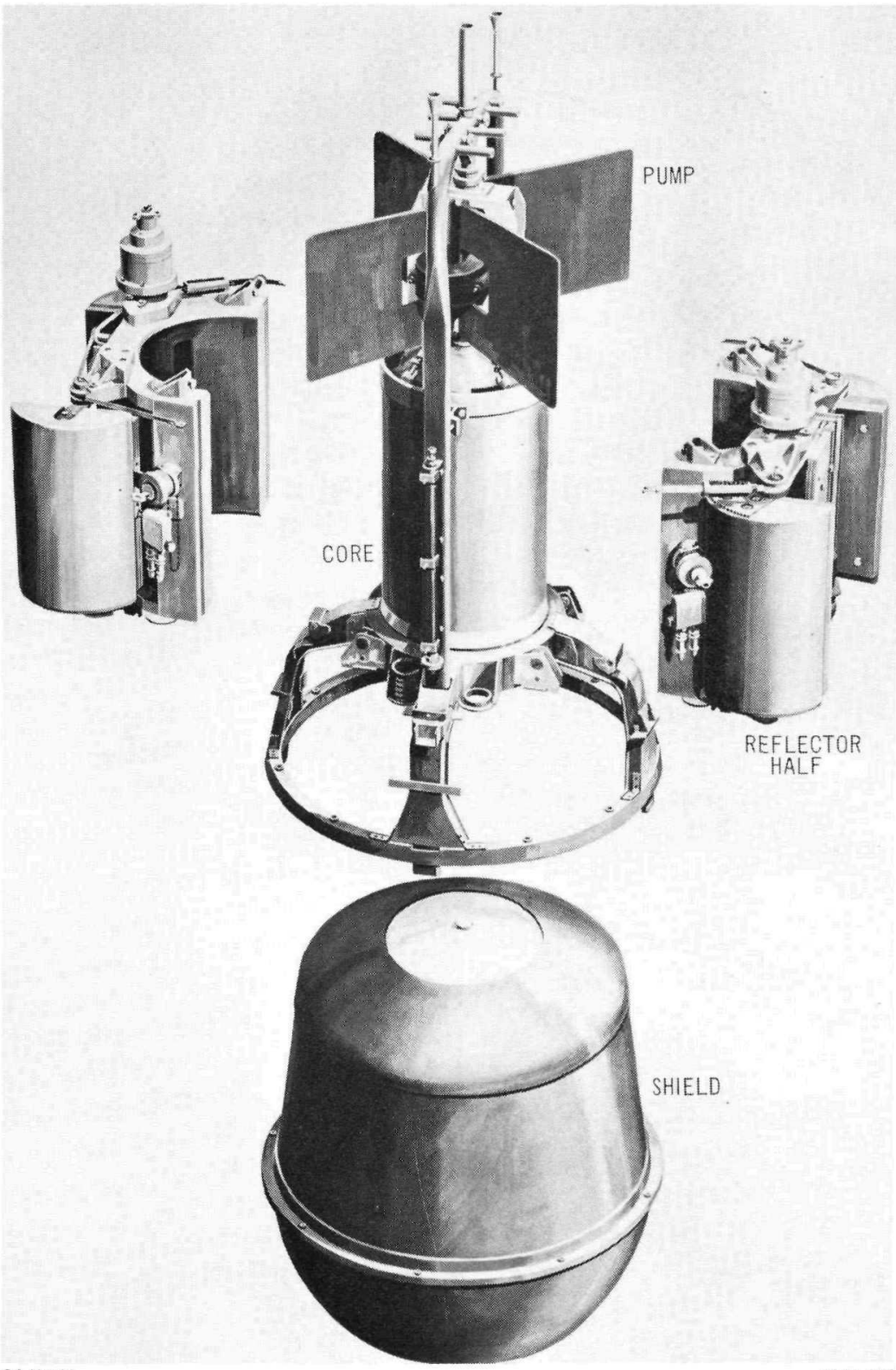

7-27-64 UNCL

7623-0019C

Figure II-85. Exploded View of SNAP 10A/2 Reactor and Shield 


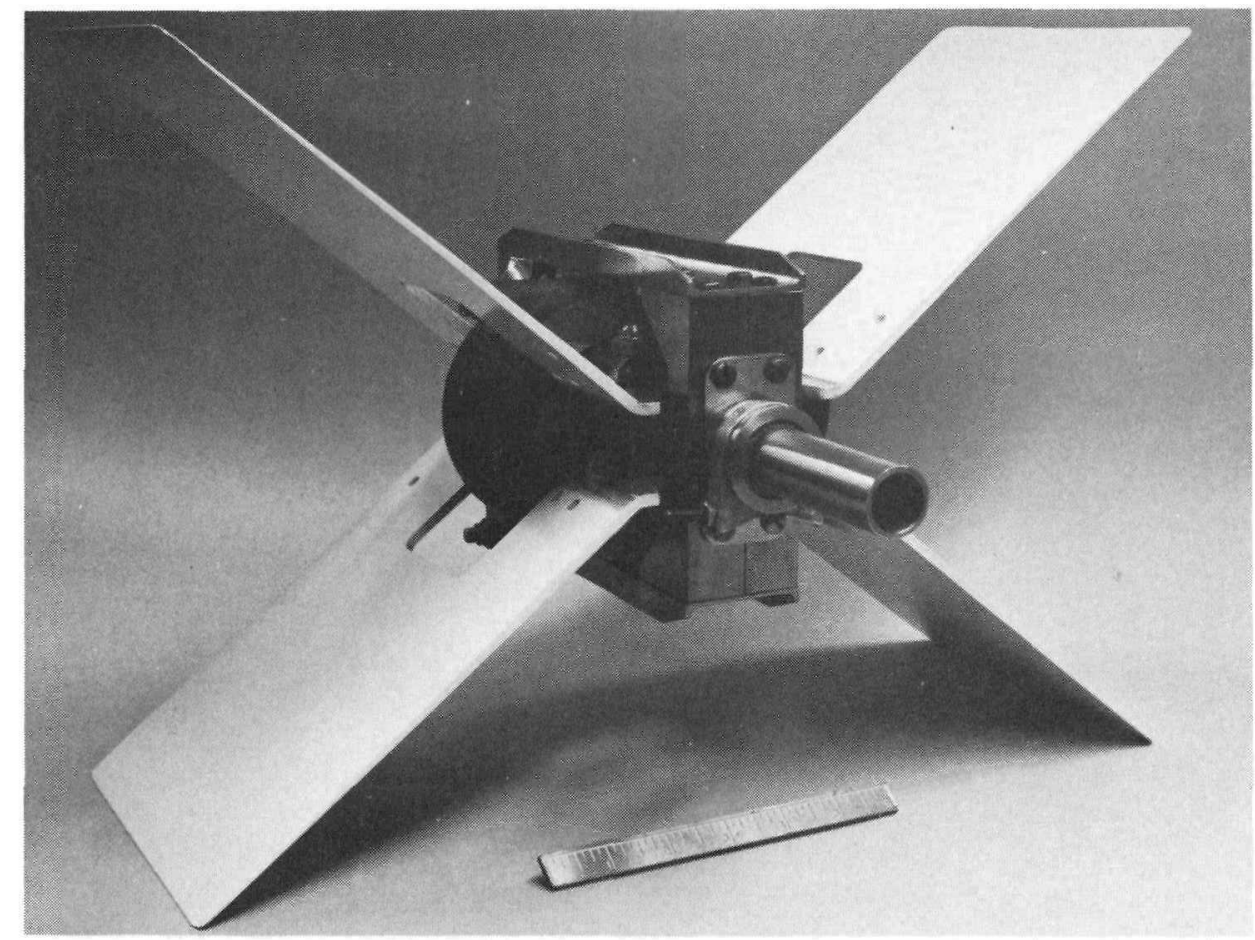

Figure II-86. SNAP 10A Thermoelectric Powered NaK Pump 


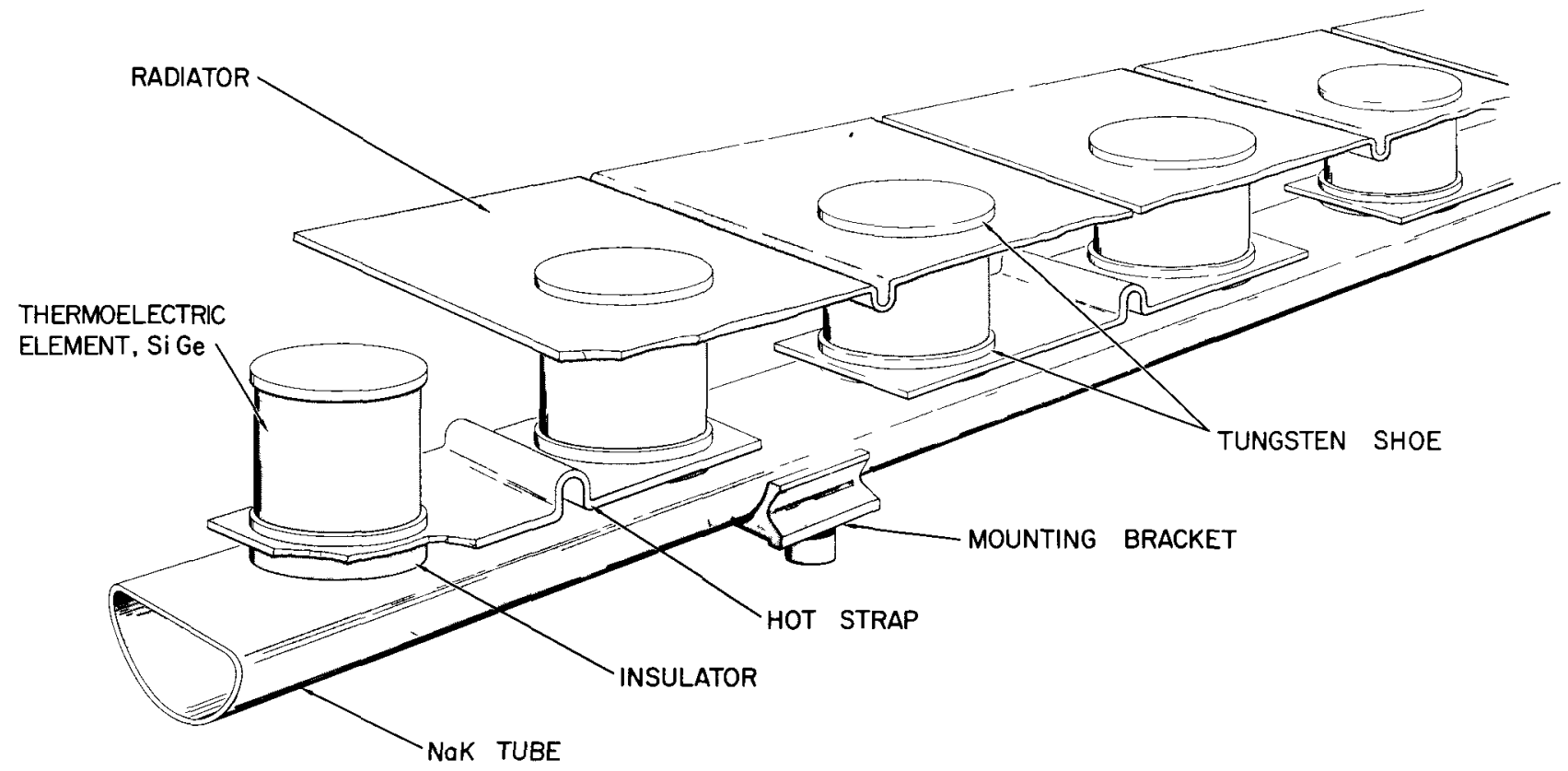




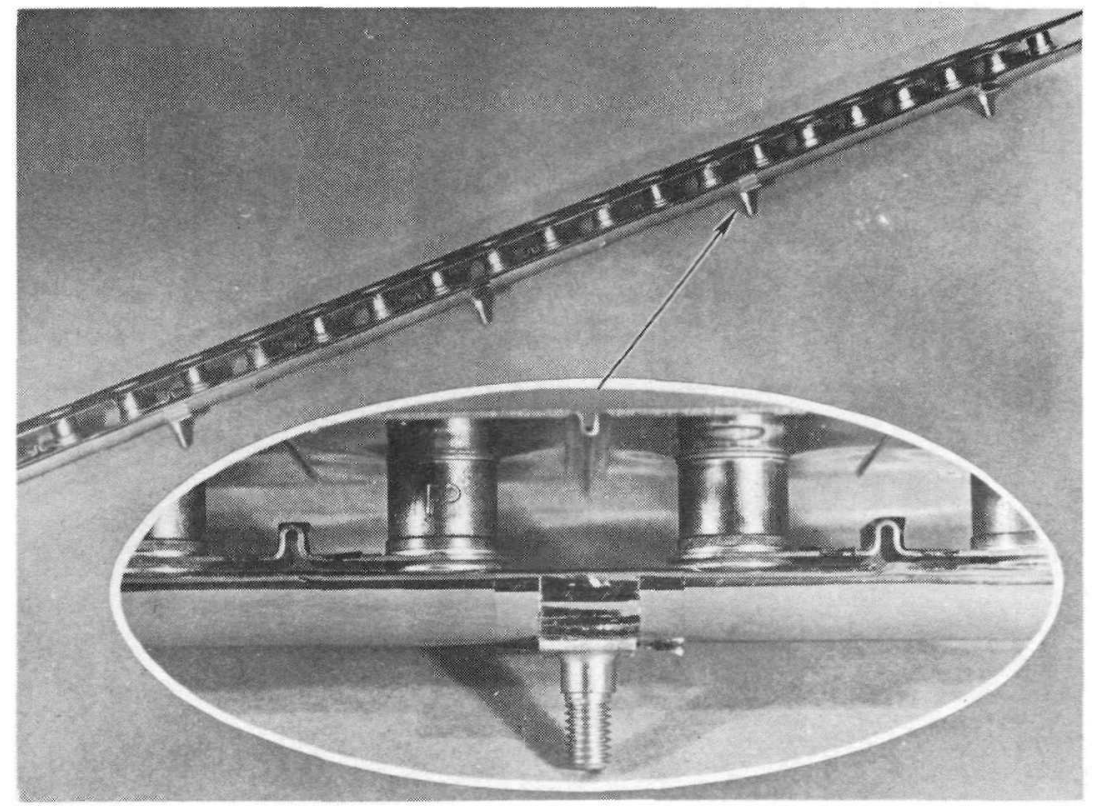

$7-16-62$

7561-5102Ba

Figure II-88. SNAP 10A Thermoelectric Converter Module

The series string of couples on each NaK tube is mated with an adjacent string by electrical cross connection at each radiator platelet along the length of the $\mathrm{NaK}$ tube. These adjacent pairs of series-parallel-connected couples are then connected in series. This connection has eliminated the possibility of one open couple causing a system failure. The open circuit failure rate during launch was less than one open per thousand elements. The converter reliability should be in excess of $99 \%$. The SNAP IOA thermoelectric generator has an open circuit voltage of about 60 volts and a generator resistance of about 1.6 ohms.

A conical corrugated titanium structure supports the reactor shield mass and forms a mounting bed for the thermoelectric modules. A photo of this structure is shown in Figure II- 89. The structural adequacy of the SNAP 10 A system was demonstrated by subjecting structural and mass mockup systems to 

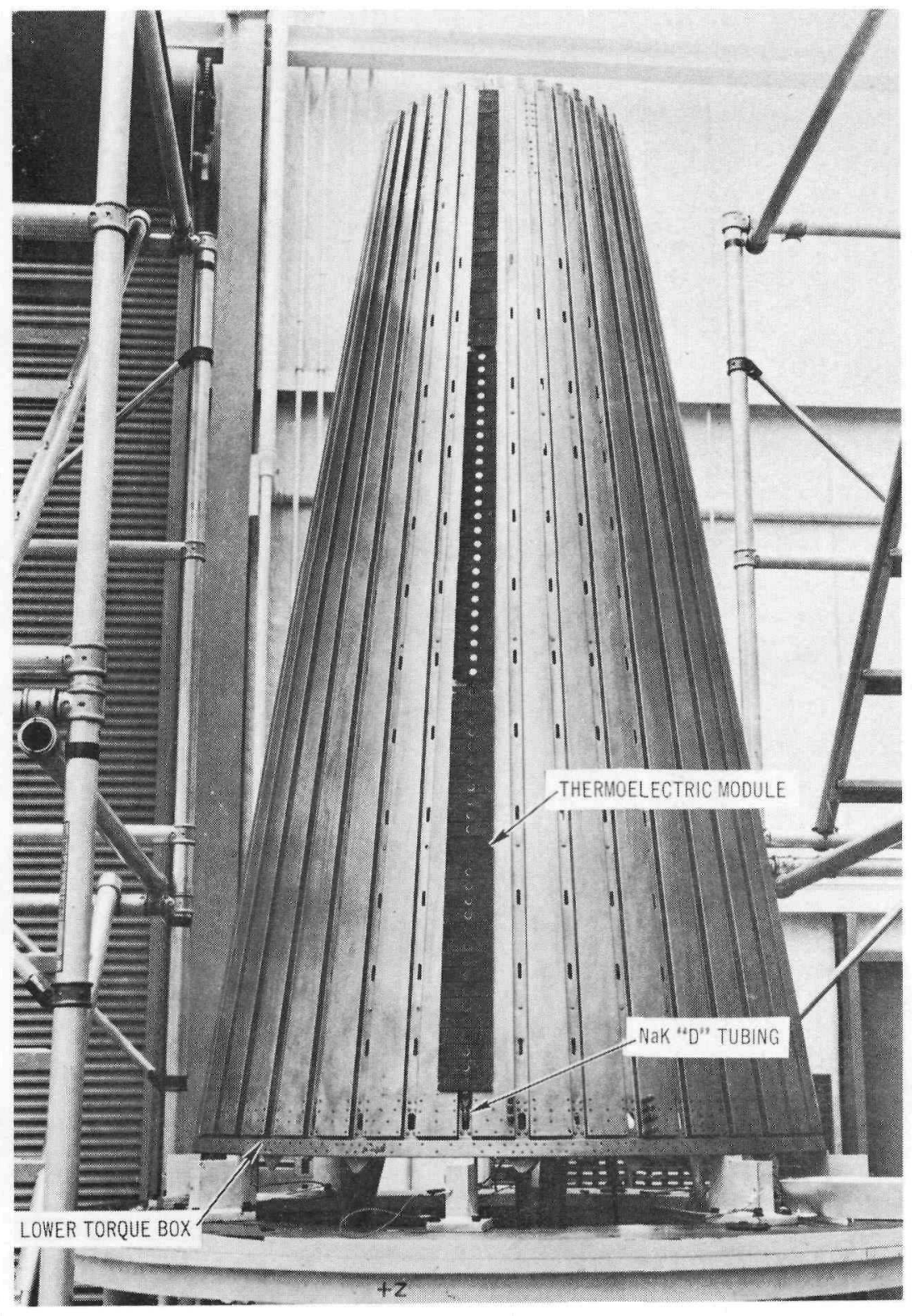

Figure II-89. SNAP 10A Titanium Structure 
the simulated launch environment. A system structural qualification test is shown in Figure II -90 .

A prototype system was subjected to thermal performance test ing in a vacuum chamber. The test employed electrical heat to simulate the reactor output. The test setup is shown in Figure II-9l. This test verified overall thermal compatibility of the system.

Two flight-design systems were fabricated for preflight qualification testing. A photo of one of these units is shown in Figure II-92. This specific unit was tested with an electrical heater simulating the reactor. The second unit was tested with a live reactor.

For the flight test, the SNAP 10A unit was placed in orbit by means of an Atlas-Agena boost vehicle. The overall flight test integration of SNAP $10 \mathrm{~A}$ and the booster is shown in Figure II-93. During launch the power unit is protected by an aerodynamic nose fairing which is ejected at the end of the Atlas boost phase. A heat shield surrounds the thermoelectric converter in order to prevent liquid metal freezing in orbit prior to startup. The heat shield is ejected during system startup.

The electrical interface to the Agena flight test vehicle was verified by means of a combined electrical mockup test. The SNAP 10A mockup mated to an Agena mockup is shown in Figure II-94.

In-flight operation of the SNAP I0A unit is initiated by a single ground command. This ground command releases the locking pins holding the four control drums, applies power to the startup controller, and arms the heat shield eject temperature switch circuit. Two of the control drums are spring loaded and immediately drive to the full-in position, the two remaining drums are automatically inserted by the control system at a rate of $1 / 2 \% / 150 \mathrm{sec}$. Approximately 7 hours are required to reach criticality, during which time the system remains at space ambient temperature with the heat shields in place protecting the liquid metal circuit from freezing. 


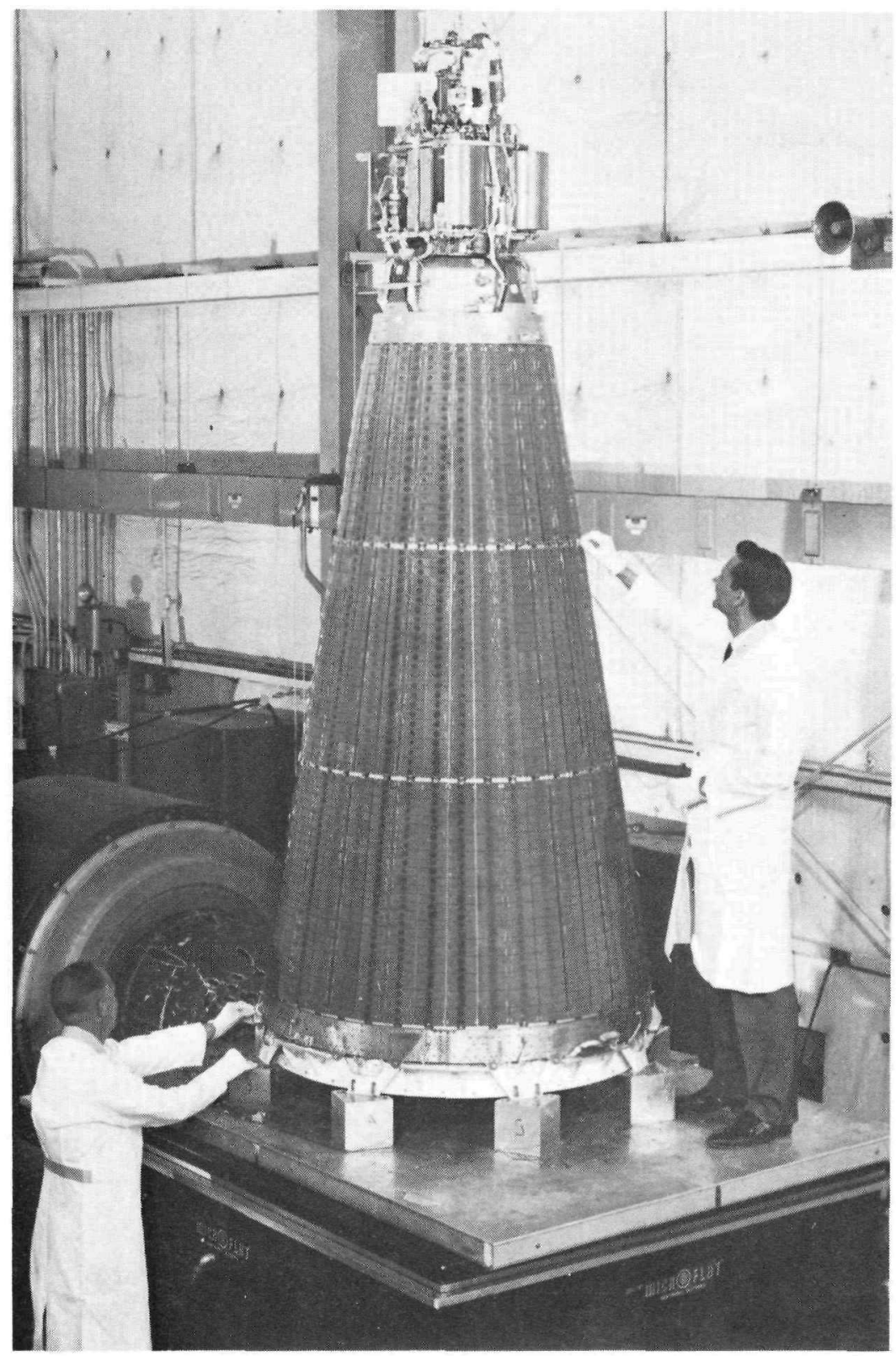

7561-0753

Figure II-90. SNAP 10A System Preflight Qualification Test-Shock and Vibration 


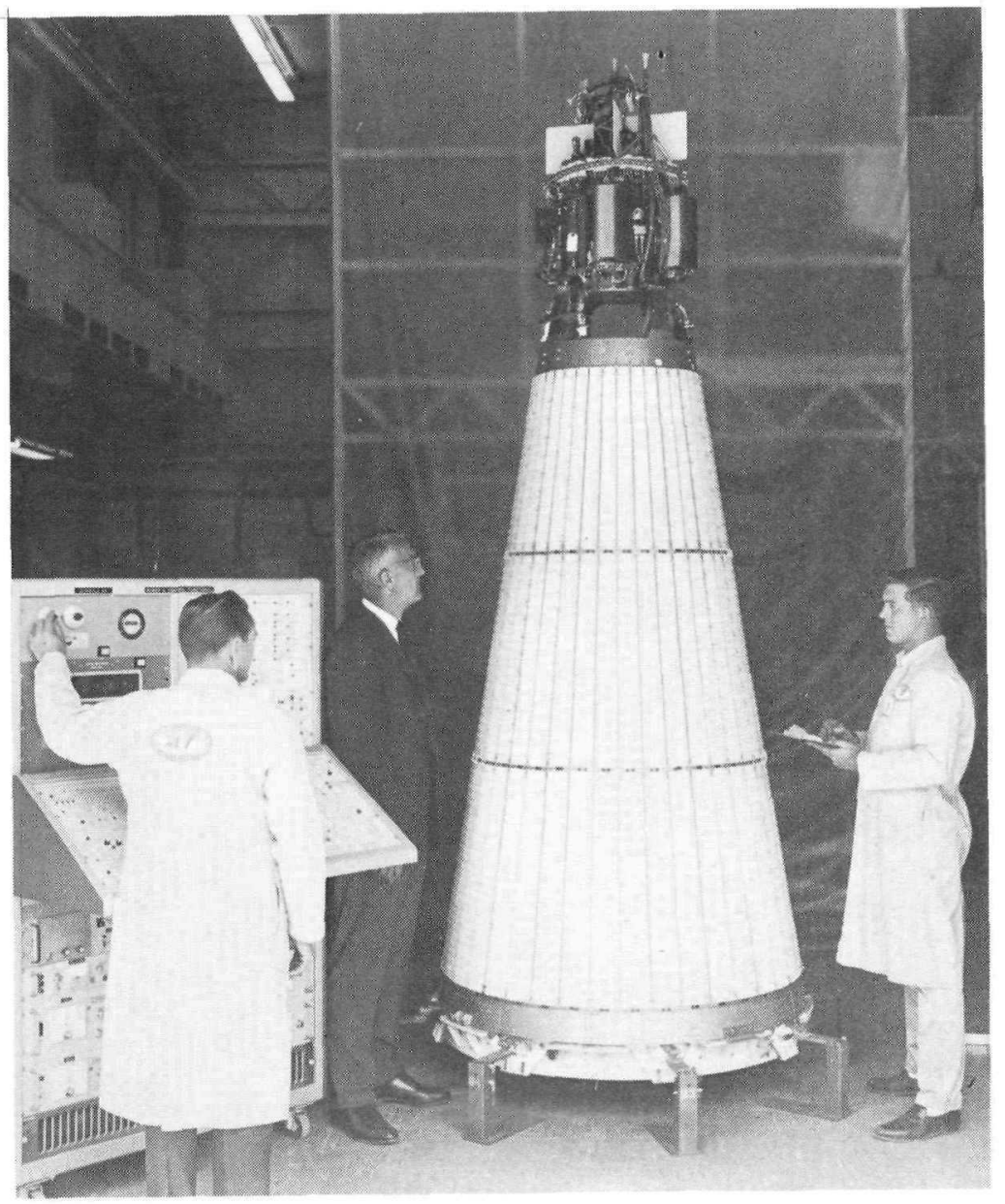

Figure II-92. SNAP 10A Flight System 


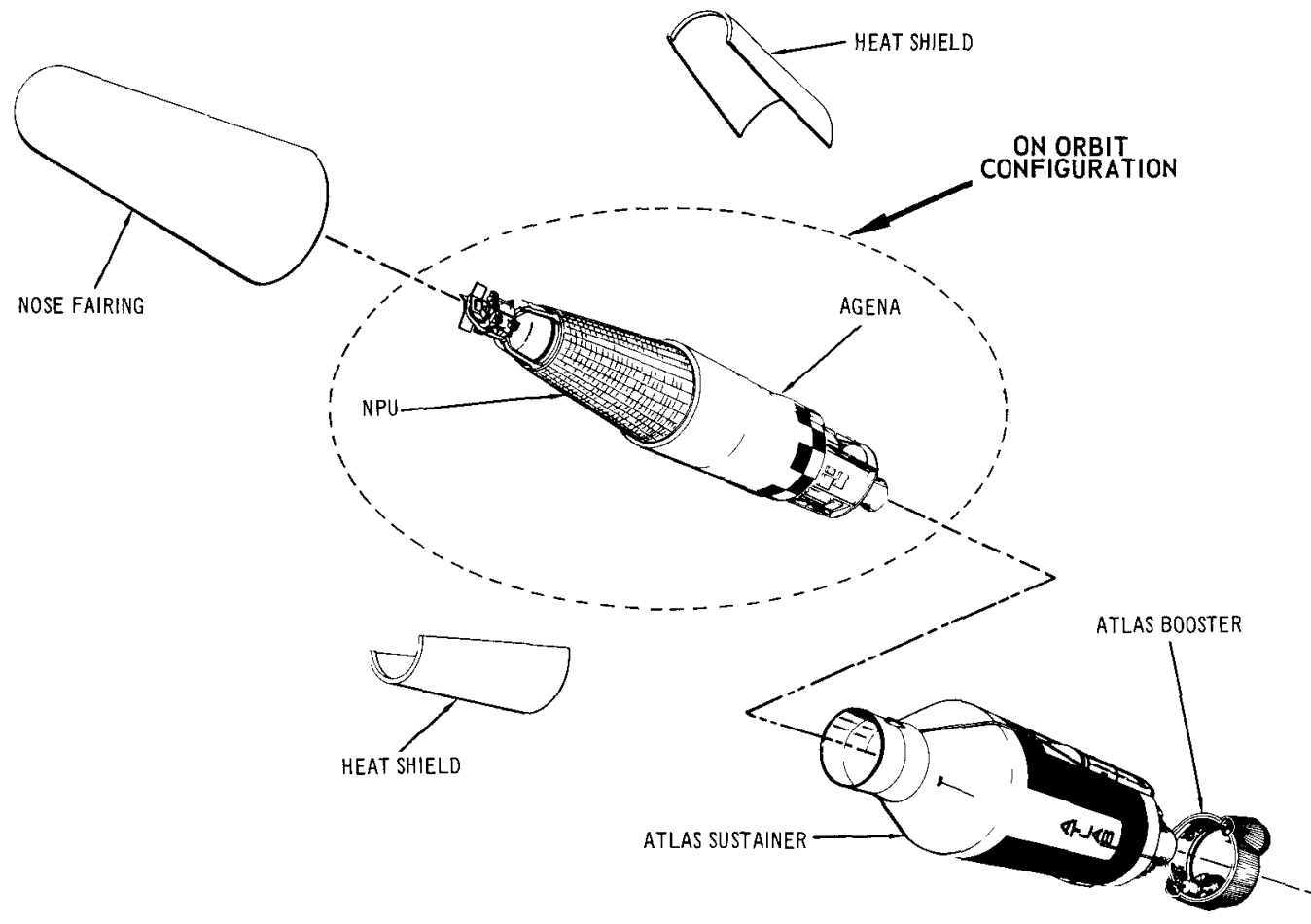

Figure II-93. SNAP 10A NPU Integration with Atlas-Agena 


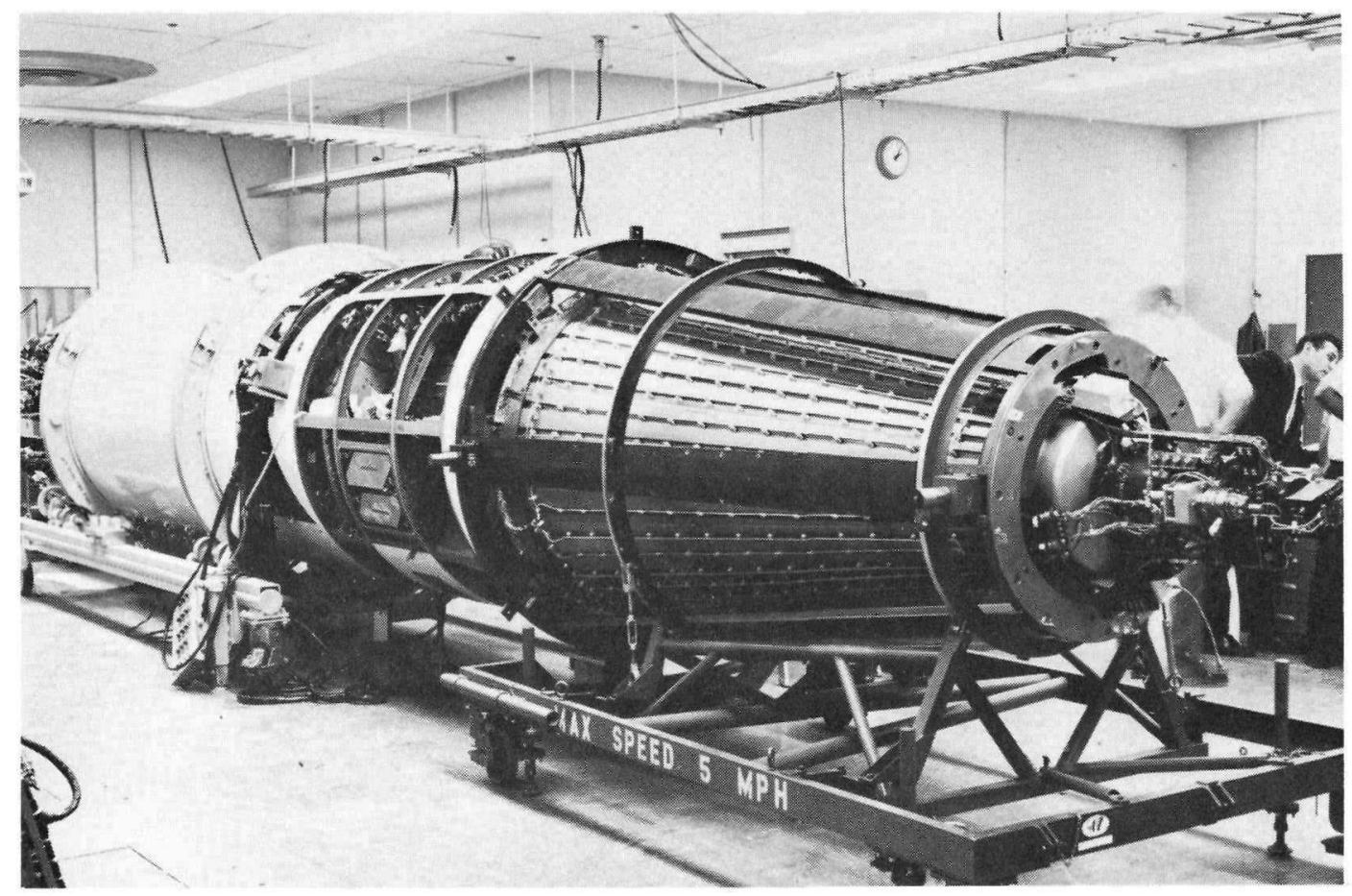

Figure II-94. SNAP 10A System Electrical Simulator Compatibility Test with an Agena Mockup 
Shortly after criticality the reactor experiences a nominal power excursion which is terminated by the inherent negative temperature coefficient of the reactor. With the continued insertion of reactivity, the reactor gradually approaches full power. At $275^{\circ} \mathrm{F}$ liquid metal temperature, the thermal heat shield switches release the heat shield halves and preloaded sorings effect their ejection from the powerplant. The thermoelectric pump, located on the reactor vessel head, bootstraps flow immediately upon an increase in reactor temperature. When the system reaches full operating temperature, the ramp insertion of reactivity is terminated by the action of a temperature switch which senses reactor outlet temperature. The temperature switch functions like a thermostat by allowing an occasional reactivity insertion to maintain the reactor outlet temperature above the switch setpoint. After initial equilibrium, the control system is turned off and no further control actions are required. The reactor behaves like a constant temperature source of power.

\section{b. SNAPSHOT Flight Experience}

Prior to shipment from Atomics International to the launch site, the SNAP 10A system was thoroughly checked out during acceptance tests. The reactor was brought critical both at ambient temperature and at full design temperature to check the reactor nuclear data and to ensure adequate reactivity. The system was operated in a simulated space environment, using electrical heaters, at the full design temperature to verify operation of the power conversion system, pump, instrumentation, and other portions of the unit. The nuclear reactor was not operated at full power since doing so would add little additional technical verification and would have built up a fission product inventory which would inhibit later access to the unit. Following final acceptance testing, the unit required no subsequent manipulation of the reactor fuel and the liquid metal coolant circuit remained 
sealed at all times. The unit was transported from Atomics International, located at the northwestern outskirts of Los Angeles, to Vandenberg Air Force Base by commercial truck carrier.

Launch pad activities were the same as with any payload. The SNAP 10A system was mated with the Agena vehicle in the gantry, was functionally exercised through the vehicle to verify commands and instrumentation readouts (the reactor, however, was not made critical), and all subsystems operated. The SNAP 10A-Agena system was put through an electronically simulated flight as further verification of flight readiness. The actual launch was conducted with a normal countdown procedure and with no unusual delays or difficulties.

With respect to operations through launch, the following observations can be made

1) Launch pad time to mate, perform functional check out, and complete booster payload preparation for launch was similar to that experienced with other payloads. No unique operations were introduced by the reactor power system.

2) No unusual handling tools or other elaborate aerospace ground equipment were required to support the launch.

3) Since the unit was nonradioactive, normal working access on the gantry, to exther the payload or the launch vehicles, was not restricted.

The SNAP IOA flight system was launched by an Atlas-Agena vehicle at 1.24 p.m., PST, on April 3, 1965. The target orbit was $700 \mathrm{n}$. mi circular, the vehicle actually achieved an orbit with an apogee of $717 \mathrm{n} . \mathrm{ml}$ and a perigee of $699 \mathrm{n} . \mathrm{mi}$. The orbital parameters and the vehicle status were adequately determined by the second orbit and the start command was given at 505 p.m. The reactor reached criticality at approximately 1115 p.m. that evenıng and was operatıng at full power at 1.45 a.m. on April 4. 
The startup sequence was activated by a single ground command with all other functions performed by an on-board control subsystem. The ejectable heat shields remained over the power conversion unit until the reactor was producing sufficient power to hold the liquid metal at or above $275^{\circ} \mathrm{F}$. Prior to heat shield ejection, duxing the time period from launch to sensible heat, the presence of the heat shields caused a net solar heat input to the system and the NaK temperatures were approximately $35^{\circ} \mathrm{F}$ higher at the time of criticality than at launch. The automatic insertion of the control drums was terminated by thermal switches when the reactor reached power.

The behavior of the system during startup and the initial stabilization period is given in Figure II-95. The saw-tooth characteristic of the temperature is due to the automatic actuation of the control system inserting reactivity into the reactor. During the initial control period, maximum power output reached approximately 650 watts, or about $12 \%$ over design. The reactor reached power with a total of 200 steps on each of the two fine control drums, or within four steps of that predicted. Initial liquid metal flow provided by the pump was $14.3 \mathrm{gpm}$, also at or above the design value. Reactor thermal power was $42 \mathrm{kw}$. Temperature distributions around the SNAP $10 \mathrm{~A}$ system in orbit, system heat balances, and operation of all principal components were as predicted through ground tests.

Approximately 6 days after reaching full power, the onboard automatic control system was deactivated by ground command. The SNAP IOA unit was then controlled solely by the inherent negative temperature coefficient of the reactor heat source itself. In this mode of operation, no electronic devices or mechanical moving parts are required to function for the balance of the system life. Without further active control, the inherent reactor characteristics (for example, hydrogen redistribution, hydrogen losses from the fuel, fission product poisons, etc.) cause a decrease in the system average temperature with 

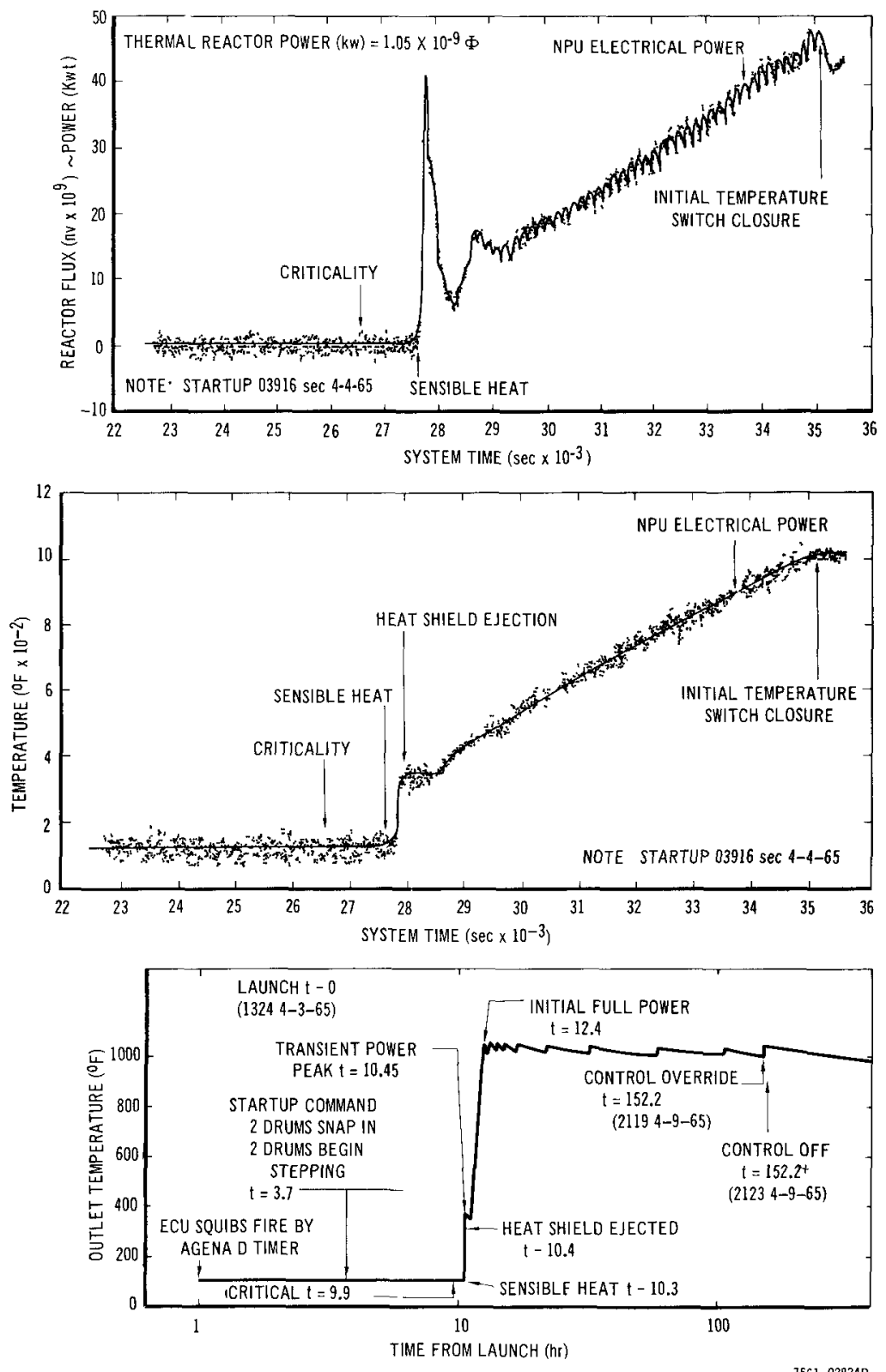

Figure II-95. SNAP 10A Orbital Startup Sequence 
time which reduces the power output from the unit. At the end of 43 days at power, the SNAP $10 \mathrm{~A}$ unit was producing about 535 watts. Based on the ground test reactor performance and, in the absence of unusual space environmental effects, it was predicted that the SNAP $10 \mathrm{~A}$ unit would put out about 510 watts at the end of 90 days and about 475 watts at 1 year. The principal time-dependent operating characteristics are shown superimposed on the ground test system data curves in Figure II-96.

On May 16, 43 days after startup of the reactor powerplant, contact with the spacecraft was lost. Failure of the spacecraft

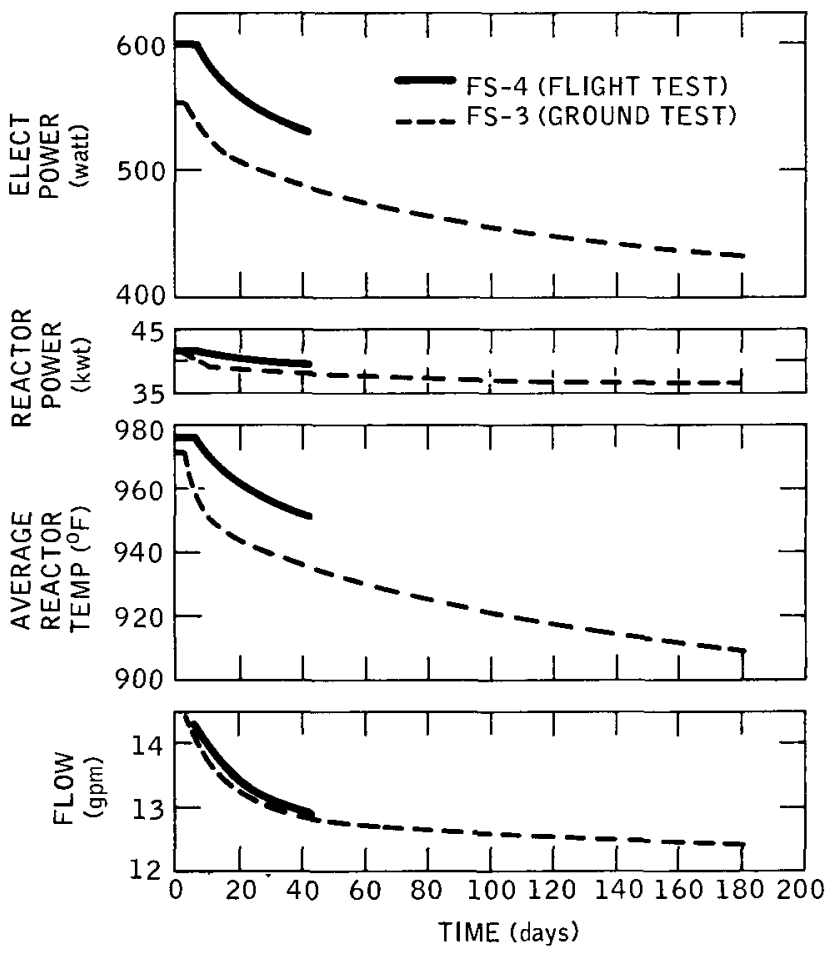

7-S7-192-93

Figure II-96. SNAP 10A Ground Test and Flight Test Performance 
was abrupt.' Operation was entirely normal at Rev. 552. Neither tracking nor telemetry signals were observed from that time until 40 hours later when, on Rev. 574, the vehicle was acquired with a telemetry transmitter broadcasting. The telemetry data revealed (1) the spacecraft was apparently operating from the reserve battery supply, (2) the command system was inoperative, (3) the redundant $115 \mathrm{v}$ ac power system was dead, (4) the reactor reflectors had been ejected, and (5) SNAP 10A electrical power output was zero. All system temperature and NaK flow data were absent, since this portion of the telemetry system requires ac power. The control moment gyros vehicle attitude system also lost ac power; however, indications were that the vehicle maintained its Earth-center orientation and did not tumble.

The data which are available indicate the SNAP 10A system did not fail but rather responded in a normal manner to spurious signals emanating from failed electronic components on board the spacecraft. Ground simulation tests have shown that the voltage regulator in the Agena most probably failed and allowed a voltage increase which overheated the command receiver and caused false commands.

A capsule evaluation of the major operating parameters is as follows:

1) The entire reactor subsystem performed properly and very predictably based on ground test data. Criticality was achieved at the reactivity levels expected, which indicates no adverse effect of the launch environment on the reactor. There was no sign of self-welding problems or failures in the high-temperature bearings of the reactor control elements.

2) The thermoelectric power conversion system was reliable; its generator characteristics (voltage, resistance 
and current) agree with ground test data. The space heat sink is slightly more efficient than predicted causing an increase in power output at $3 \%$ over that expected.

3) The liquid metal subsystem (pump, piping, expansion compensators) all behaved in accordance with the ground test data. The system integrity was not affected by the launch vibration. There was no apparent micrometeoroid puncture of the 20-mil liquid metal tubing and no detectable deterioration of applied emissivity control surfaces.

4) The launch shock and vibration loads were a factor of approximately 1.5 under the qualification levels. There were no signs of any high-amplitude vibration about the SNAP 10A lateral fundamental frequency of approximately 16 cps.

5) The instrumentation system, including the high temperature transducers, functioned very adequately. Of the total of 105 in-orbit channels, 103 were still transmitting useful data after 30 days. The 17 launch-to-startup instruments all performed satisfactorily.

6) The heat transfer from the high temperature SNAP 10A system to the Agena, as well as other interface variables, correlate closely with ground test experience. The heat load to the Agena was less than 130 watts.

7) Radiation levels about the system were carefully measured. The neutron flux immediately adjacent to the outer surface of the beryllium reactor reflector was about $4 \times 10^{10} \mathrm{n} / \mathrm{cm}^{2} / \mathrm{sec}$. At the mating plane between the SNAP 10A system and the Agena vehicle, the fast neutron flux was about $10^{6} \mathrm{n} / \mathrm{cm}^{2} / \mathrm{sec}$ on the vehicle centerline and $3 \times 10^{6} \mathrm{n} / \mathrm{cm}^{2} / \mathrm{sec}$ on the outer surface. These values are about a factor of 4 higher on the vehicle centerline and a factor of 4 lower on the outer vehicle surface than predicted. 
The gamma dose rate at the interface was approximately $630 \mathrm{r} / \mathrm{hr}$. The gamma flux toward the aft end of the vehicle is about 2 orders of magnitude less than quoted above and the fast neutron flux about 2-1/2 orders of magnitude less than at the interface. These are less than would be predicted by a simple inverse square law relationship and are a result of scattering and attenuation by the vehicle structure and components. There was no sign of premature radiationinduced component failures of any of the in-orbit equipment.

The spacecraft failure which shut down the orbital test of SNAP 10A after 43 days prevented a system reliability demonstration in space. However, the duplicate ground test system FS- 3 operated continuously for 10,000 hours in a shielded vacuum chamber. The post-shutdown examination of FS-3 revealed no wear-out modes or incipient failures, and it was concluded that a SNAP IOA system could probably operate for 5 years or more.

\section{SNAP 2}

The SNAP 2 concept was the result of a 1956 preliminary study which evaluated the state of the art of reactor and power conversion technology as well as projected space vehicle and mission requirements. As previously discussed, the SNAP hydride reactor concept, which was chosen for SNAP 2, has become the basic heat source for SNAP 10A and SNAP 8. The SNAP 2 program was initially oriented toward the development of a specific 3-kwe mercury rankine cycle system. This specific objective has been dropped in favor of a broader technology development program in order to demonstrate the engineering feasibility of larger multikilowatt mercury rankine cycle systems for space. The development work to date on the SNAP 2 program has indicated a concept performance capability at 5 kwe of about 200 to $250 \mathrm{lb} / \mathrm{kwe}$ and 25 to $30 \mathrm{ft}^{2} / \mathrm{kwe}$. The power conversion development progress on SNAP 2 has demonstrated the feasibility 
of hermetically sealed and working fluid lubricated rotating machinery for reliable operation of 1 year or longer in space. The basic concept of a hydride reactor and a mercury rankine power conversion system should be applicable into the 100 - to 200-kwe range.

A system schematic for a 5-kwe SNAP 2 unit is shown in Figure II-97. A liquid metal ( $\mathrm{NaK}-78$ ) heat transfer fluid is circulated through the reactor core and the mercury boller superheater by a dc conduction EM pump which derives its current from a chromel-constantan thermocouple operating between the reactor outlet temperature and the boller preheat temperature. In the boiler superheater the reactor heat is transferred

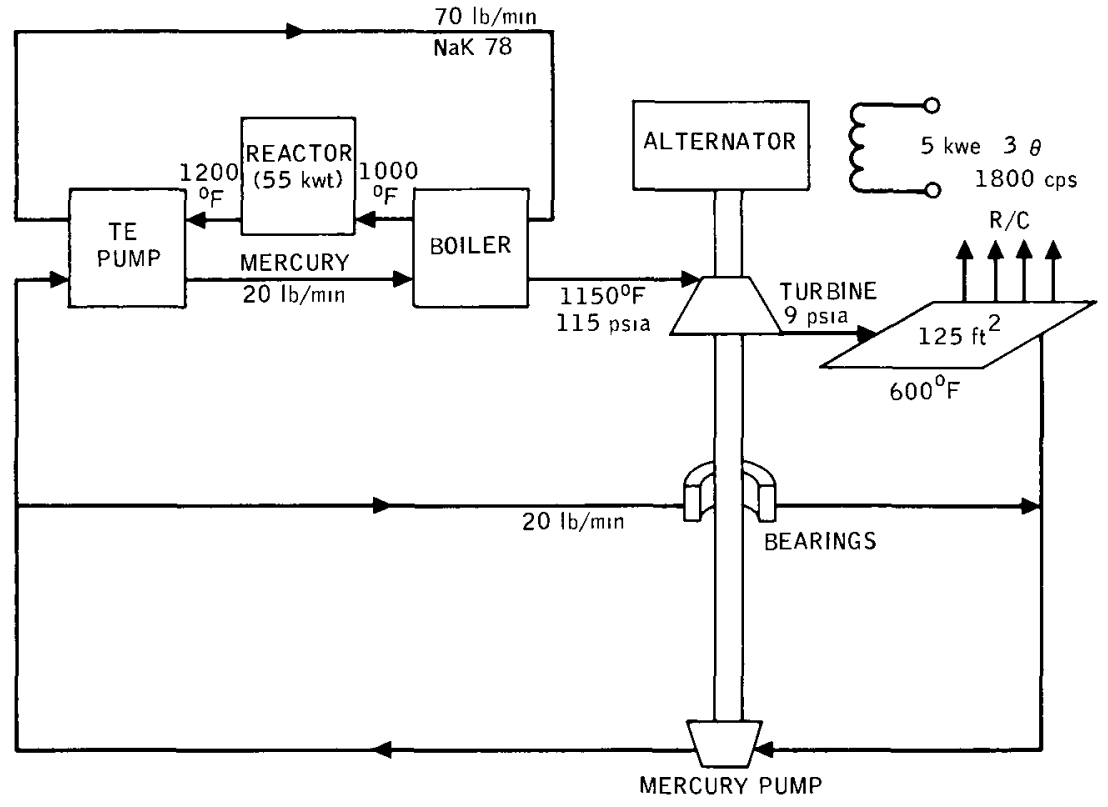

Figure II-97. 5-kwe Mercury Rankine Schematic 


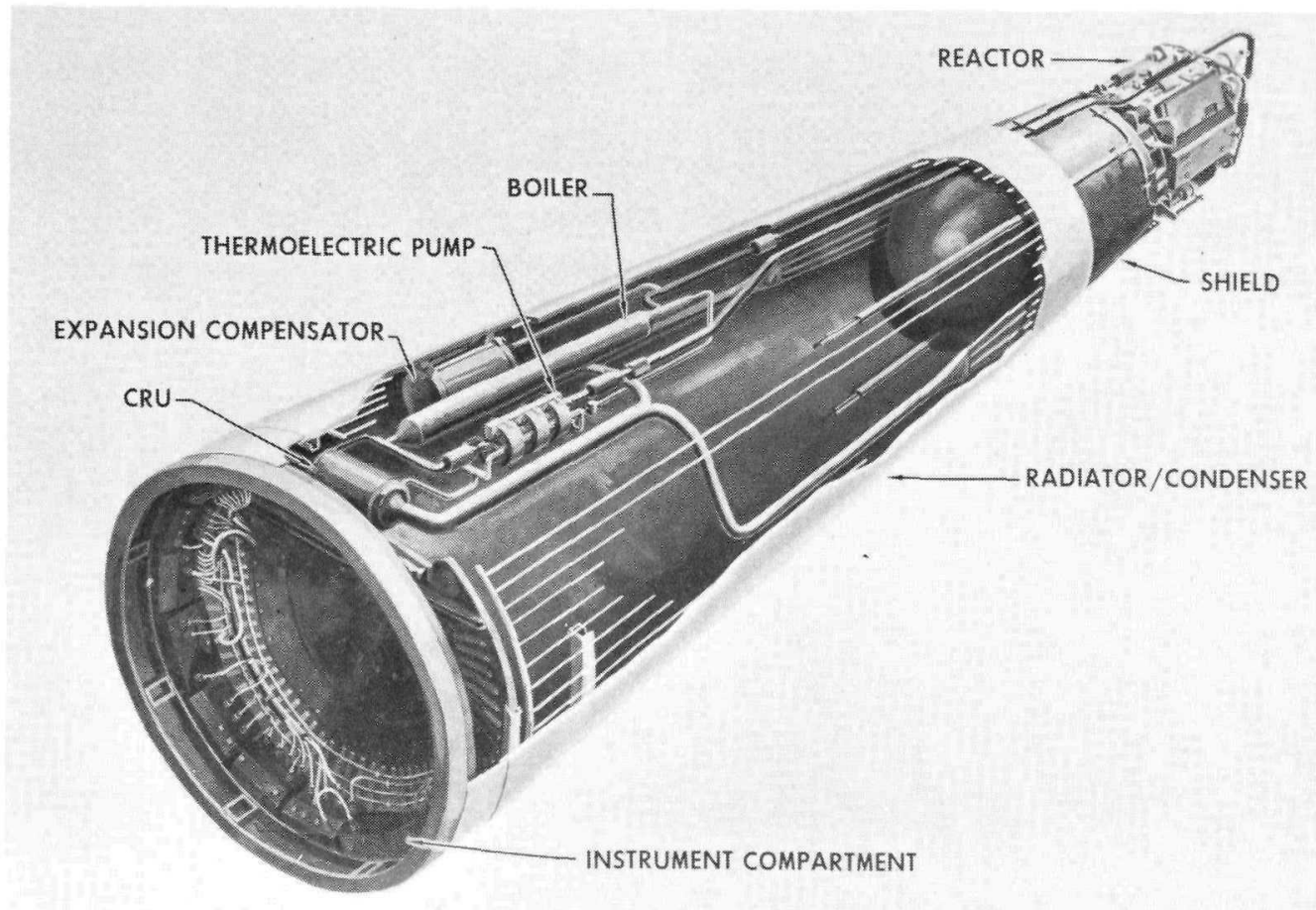

Figure II-98. 5-kwe Mercury Rankine System 
from the primary reactor coolant to the mercury working fluid of the rankıne power conversion cycle. The reactor heat converts liquid mercury into superheated vapor which is expanded through a turbine. The resulting mechanical power output of the turbine is converted to electrical power by the alternator. The mercury vapor exhaust from the turbine is condensed in the radiator-condenser which is part of the outer skin of the space vehicle. The mercury condensate is returned to the boiler by a boiler feedpump. The system configuration is shown in Figure II-98.

All the power conversion system rotating components are mounted on a single common shaft component which is called the combined rotating unit (CRU). Thus, the entire SNAP 2 power conversion system has only one moving part which is supported on liquid mercury lubricated bearings. The entire assembly of rotating machinery is enclosed within a hermetic housing which prevents the loss of the mercury working fluid during the system life. The CRU is shown in Figure II-99. The individual components of the rotating shaft include (1) the mercury turbine which is a two-stage axial flow impulse machine, (2) the alternator which is a permanent magnet machine with a sealed stator and delivers $5 \mathrm{kw}$ at $1800 \mathrm{cps}$, and (3) the mercury pump which is a conventional but miniature centrifugal pump supplying pressurized mercury to the boiler and to the bearings.

A photo of the CRU test assembly is shown in Figure II-100. The power conversion rotating machinery development has progressed to a level of test experience in excess of 30,000 hours. One machine has operated for an integral time of about 5,000 hours, and several machines have operated beyond 2,000 hours. Individual machine components are shown in Figure II-101. 


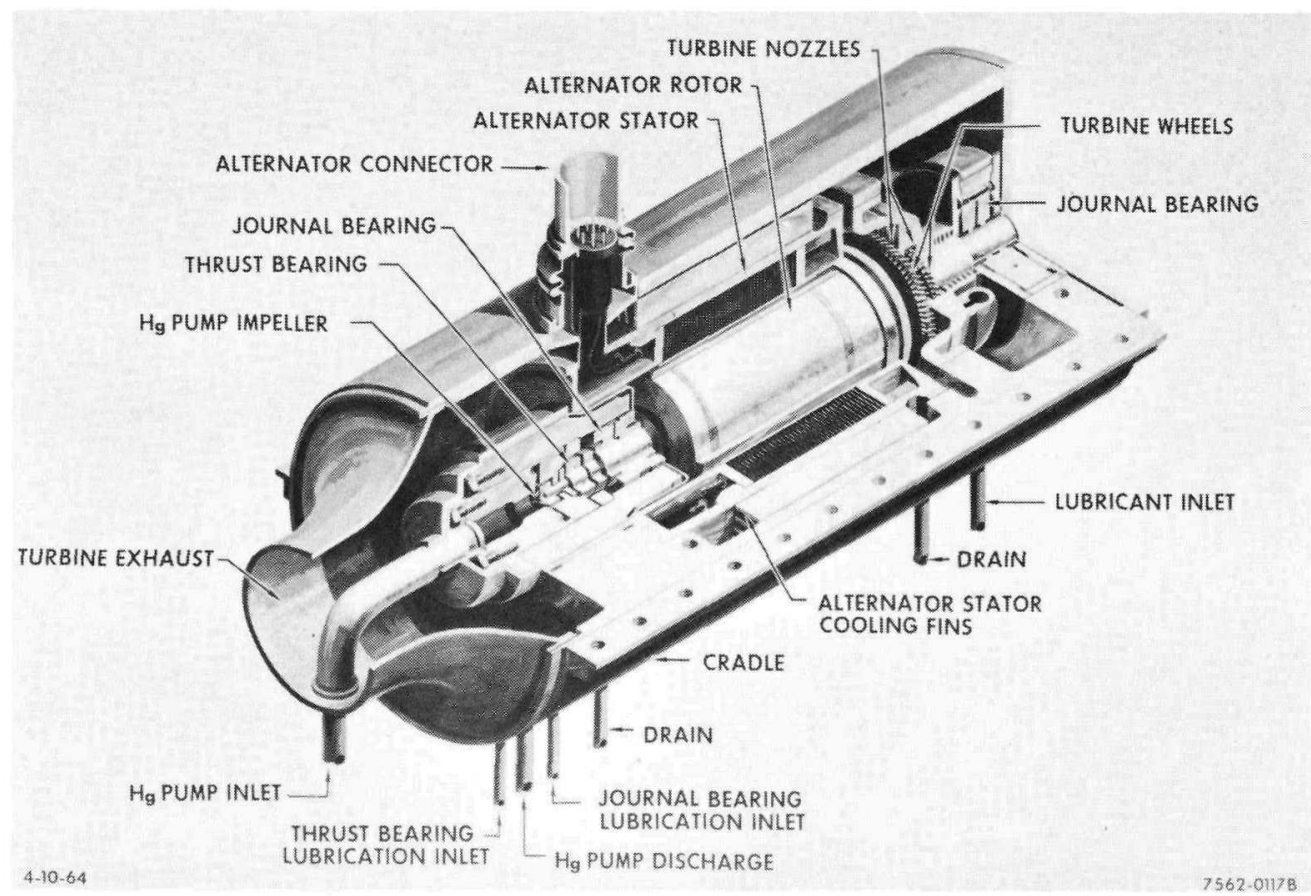

Figure II-99. Combined Rotating Unit - V 


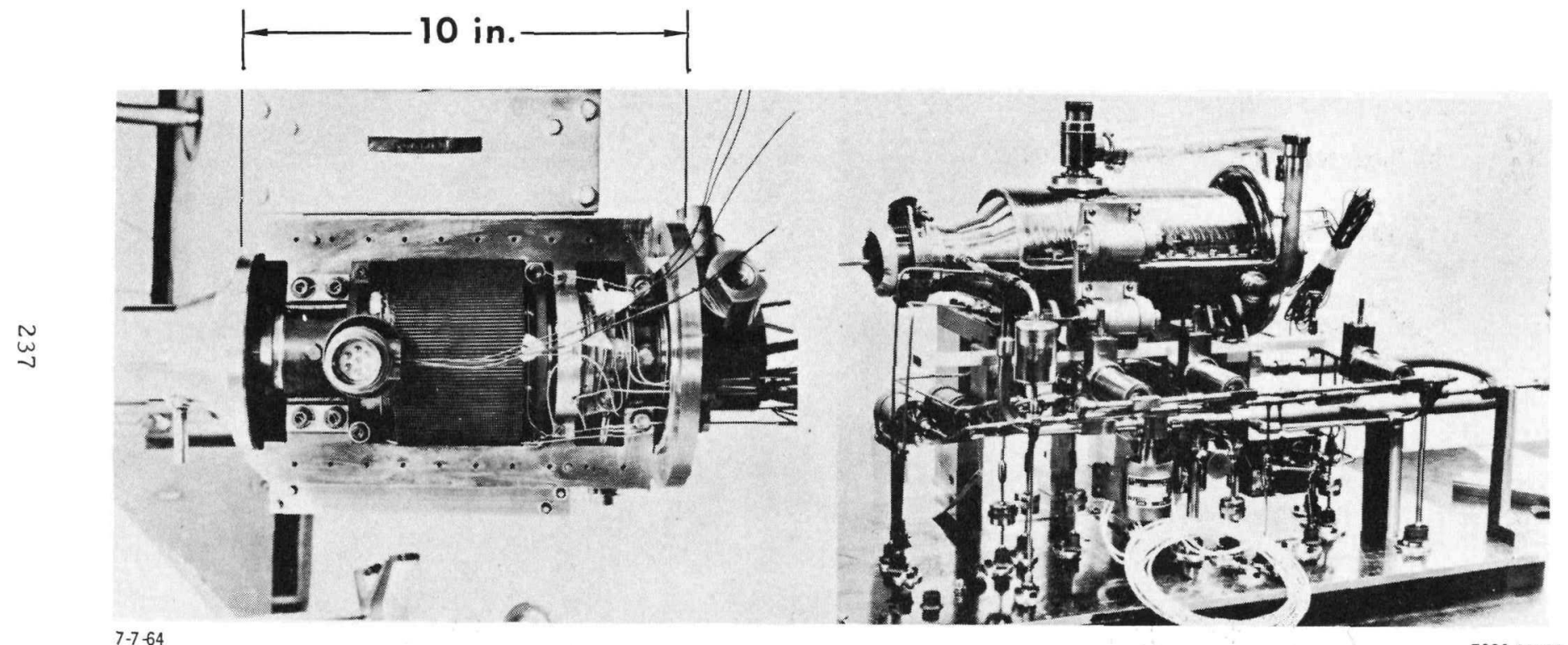

Figure II-100. Combined Rotating Unit Hardware-CRU V 


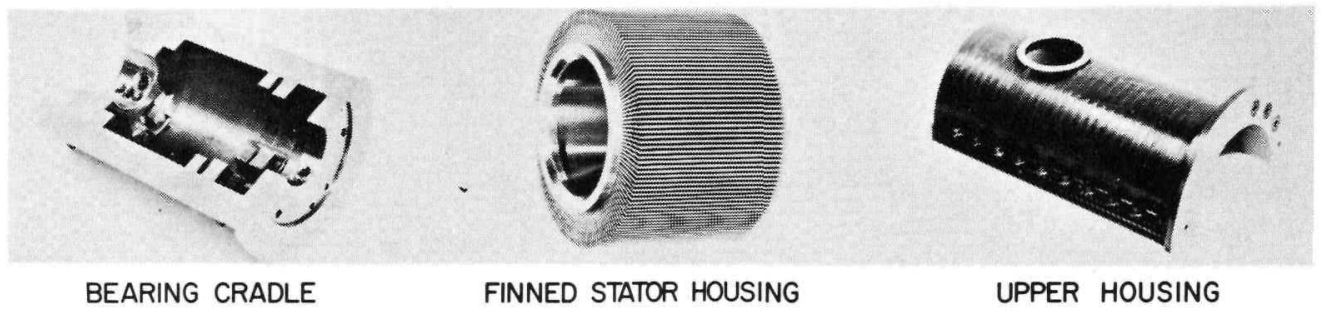

$\underset{\infty}{\sim}$

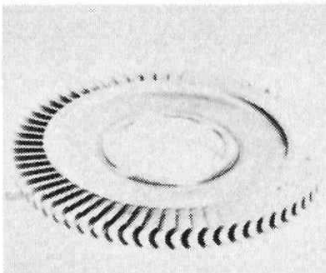

FIRST STAGE WHEEL

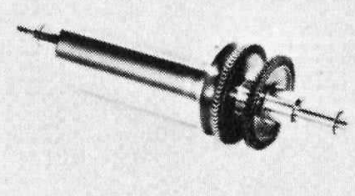

CRU $\square$ SHAFT

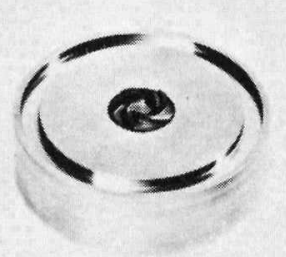

Hg PUMP IMPELLER

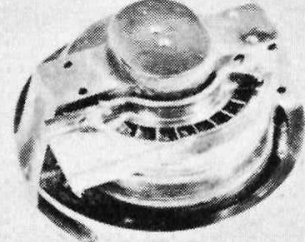

TURBINE HOUSING

$7636-0456$

Figure II-101. CRU Components 
The mercury boiler-superheater is a tube and shell, counterflow, once-through boller with $\mathrm{NaK}$ in the shell and mercury in the tubes. The boiler is in a coiled tube configuration which provides local high accelerations such that the boiler operation is not affected by zero gravity.

The cycle rejection heat is radiated to space by a combined radiator-condenser which forms part of the outer structural skin of the space vehicle. Mercury condensation takes place at $600^{\circ} \mathrm{F}$ and 6 psia within a number of small-diameter parallel tubes which are attached to a high thermal conductivity skin which in turn radiates the heat of condensation to space. The total area necessary to radiate the cycle waste heat and subcool the pump inlet is about $125 \mathrm{ft}^{2}$ and welghs 200 to $250 \mathrm{lb}$. A developmental radiator-condenser is shown in Figure II-102.

The major system components, the reactor heat source and the power-conversion package for a 5-kwe system have been demonstrated at the required design point.

In addition to the development of components extensive system development was initiated under the SNAP 2 program to evaluate the important thermal, hydraulic, and structural interactions between system components and to establish system operating modes for startup, steady state, shutdown, and restart. The electrically heated thermal performance test system is shown in Figure II-103. This mockup of a complete SNAP 2 system demonstrated overall system performance compatibılity and stability. A second mockup system demonstrated the structural integrity of the concept. A third system, devised to determine the zero gravity steady-state and startup characteristics was tested in the Mercury Rankine Program. In this test system, the influence of gravity is minimized by locating the radiator condenser tubes and all critical hydraulic components in one horizontal plane. The CRU is started by "blowdown" upon injection of liquid mercury into a preheated boiler. The mercury injection continues until the integrated heat of condensation has 


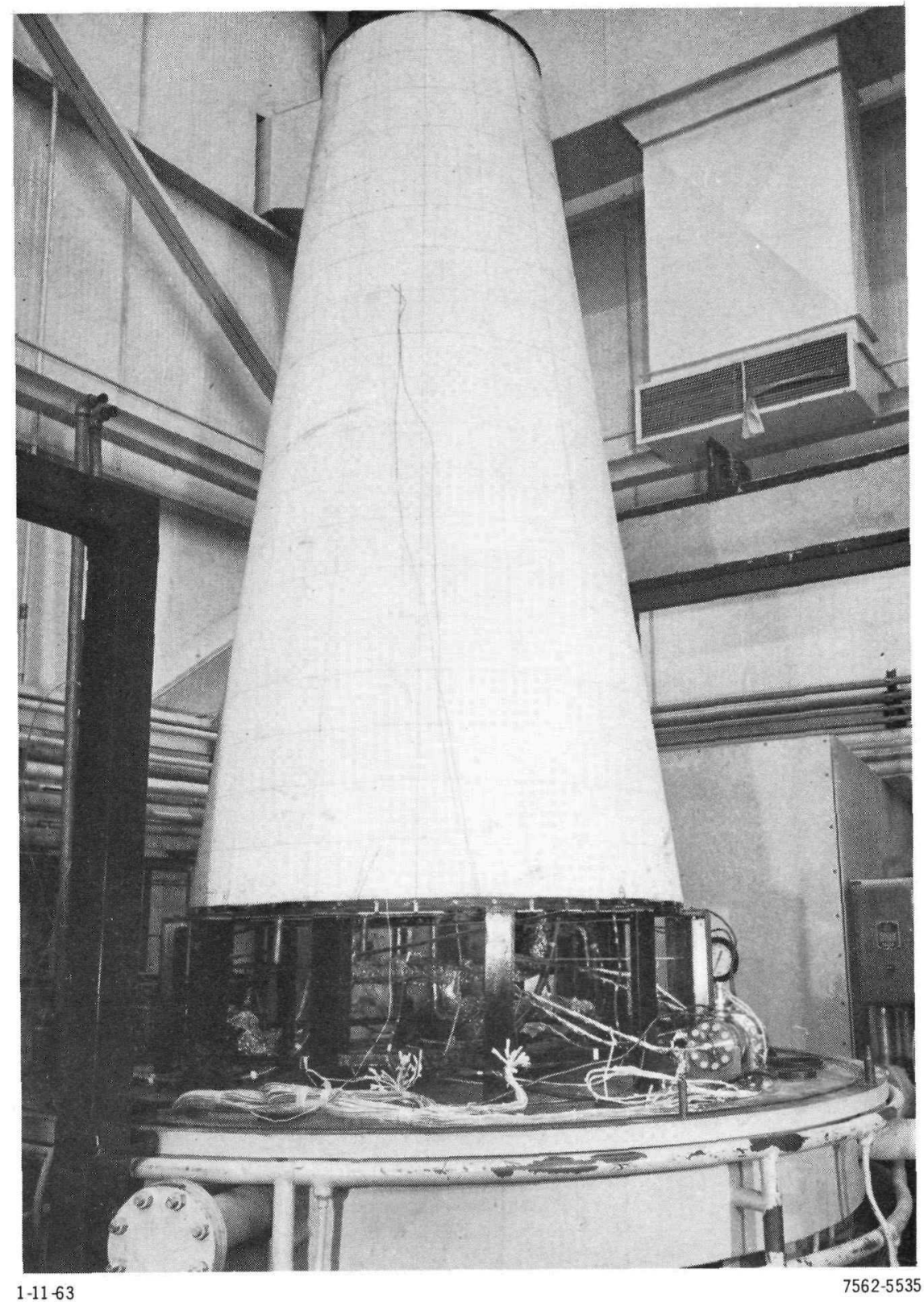

Figure II-102. Developmenta1 Mercury Rankine Radiator Condenser 


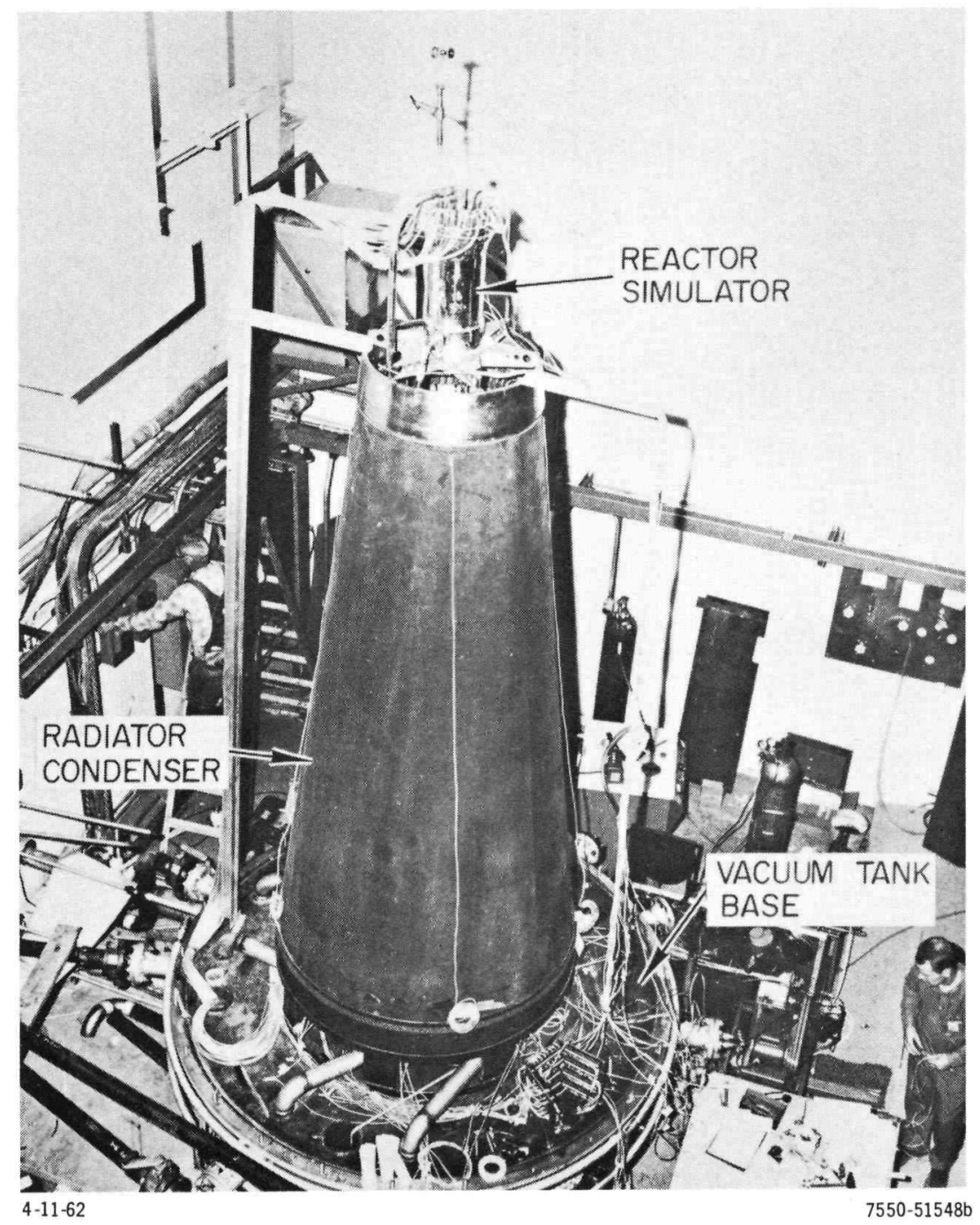

Figure II-103. Mercury Rankine Performance Test System 


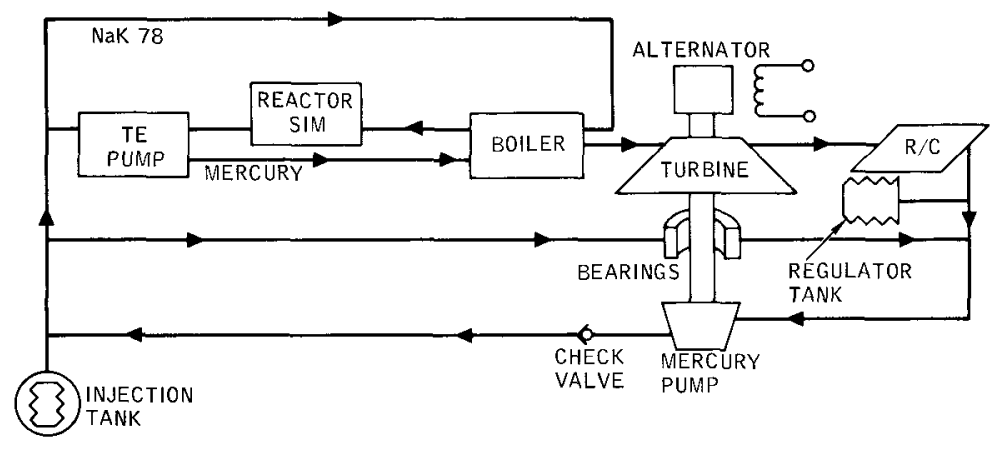

1 NaK PUMP ENERGIZED AT 5\% FLOW

2. UPON COMMAND, SIMULATED REACTOR TRANSIENT PREHEATS NaK

3. AT $1200^{\circ} \mathrm{F}$ BOILER TEMPERATURE, Hg INJECTED INTO BOILER AND CRU BEARINGS

4 Hg VAPOR FROM BOILER SPINS UP CRU AND COLLECTS IN RC

5 RC PRESSURE BUILDS UP (PREHEAT) UNTIL Hg PUMP PRIMES AT ABOUT $6 \mathrm{psIa}$
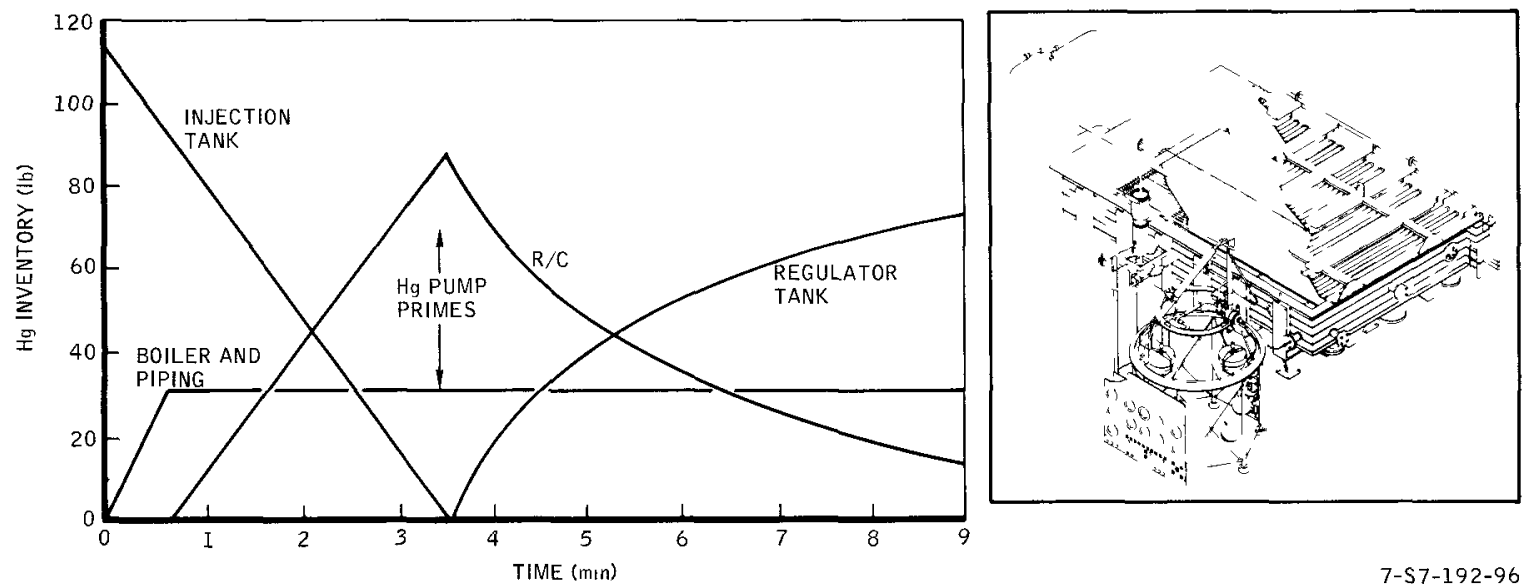

Figure II-104. SNAP 2 Startup and Test 
raised the condenser temperature sufficiently to provide the condenser exhaust pressure necessary to prime the CRU mercury pump and complete the closed cycle. At this point mercury injection is ceased and excess mercury in the condenser is discharged into a constant pressure volume until the system equilibrates at the desired condensing temperature and pressure. In over 200 start cycles this system has demonstrated the vapor liquid interface stability of the parallel tube condenser, the ability to start and achieve equilibrium without condenser preheat, the ability to prime the CRU mercury feed pump and operate a closed cycle, and general system startup and steady state stability. The startup schematic and a picture of the test system are shown in Figure II- 104.

Potential applications for the mercury Rankine system include provision of electrical power for manned laboratories, manned space stations, lunar bases, communication satellites, military satellites, and space probes. Both isotope and reactor heat sources can be used with present hardware.

\section{SNAP 8}

The SNAP 8 Program has been under active development by NASA/AEC since 1960. It consists of a compact zurconium hydride-moderated thermal reactor and a rankine power conversion system with mercury as the working fluid. Its present objectives are tallored to a 35-kwe flight test system capable of 10,000-hour contınuous space operation. Space startup and shutdown is an objective.

A schematic of the SNAP 8 system is shown in Figure II- 105. The reactor primary coolant ( $\mathrm{NaK}-78$ ) is circulated through the mercury boiler by a rotating pump/motor assembly (NaK PMA). Hot mercury vapor from the boller is then expanded through a four-stage turbine contained within the turbine/alternator assembly (TAA) and is condensed in an indirect condenser. Mercury is then returned to the boiler by the mercury pump/motor assembly (Hg PMA). A NaK-78 heat rejection loop is used to cool the 


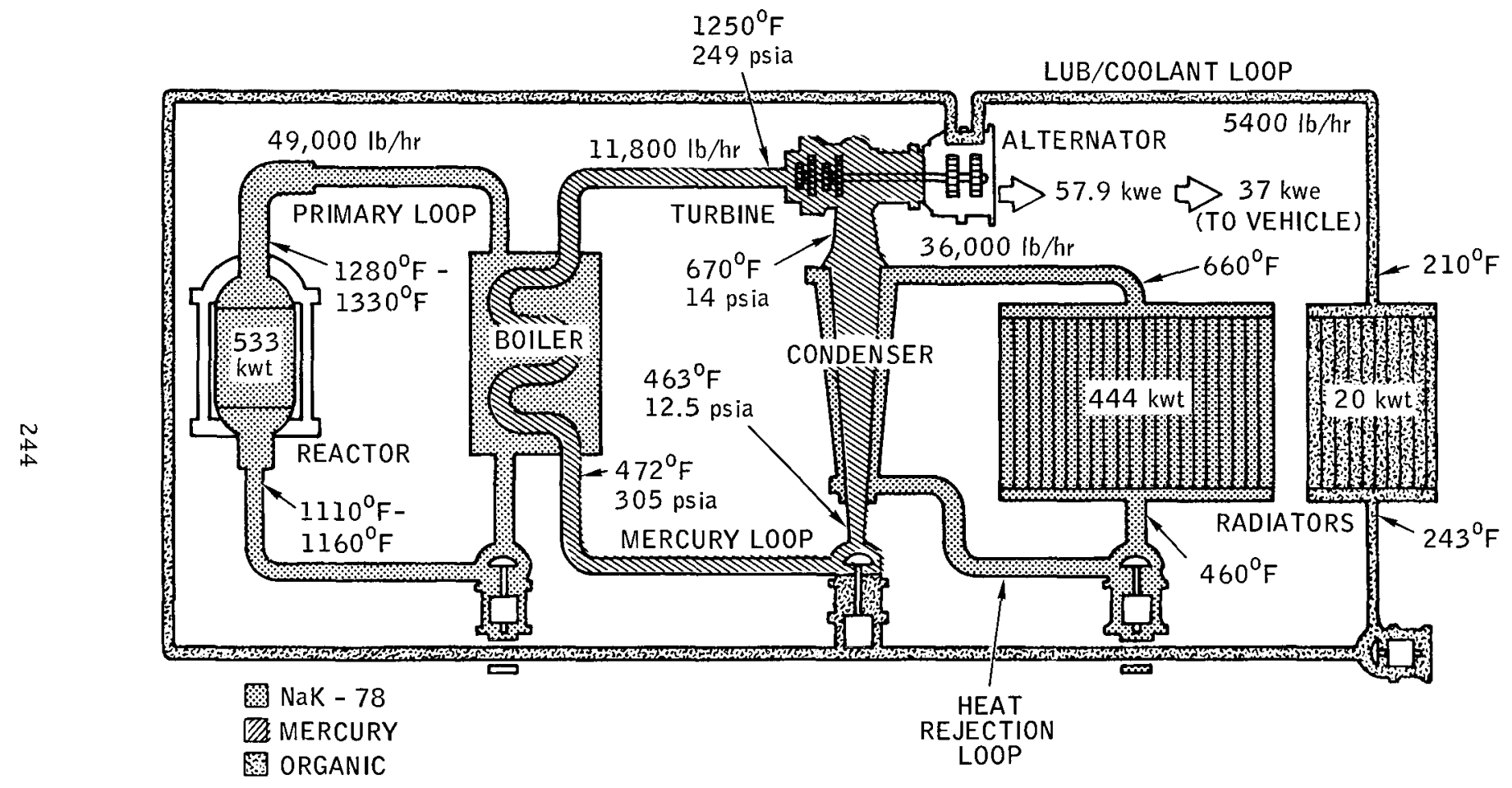

Figure II-105. SNAP 8 Electric Generating System 
condenser and heat is rejected from this loop by means of a direct radiator. The significant difference between the SNAP 8 and the Mercury Rankine (SNAP 2) equipment is the use of oll-lubricated ball bearings on SNAP 8 rotating equipment. This requires the use of an auxiliary lube/coolant loop with its separate pump (L/C PMA) and radiator. Another difference from the Mercury Rankıne system is the use of an indirect radiator concept with the NaK heat rejection loop. Developmental testing to date has concentrated on design and performance integrity tests of the prime components. Tests have, in general, reached the several thousand hour stage. The program is scheduled to demonstrate reliability and endurance of components integrated into complete systems. Both nuclear and nonnuclear system tests are planned.

The TAA is shown in Figure II-106. The TAA contains a space seal which is designed to limit oil and mercury leakage to space. Seal leak test data has been correlated to analytical predictions. A remaining developmental problem is formation of cavitation zones in the vicinity of lands on the visco (screw) space seal. Material and seal configuration changes have been made to minimize cavitation problems.

The mercury boller assembly has been tested and current development problems includes boiler "deconditioning" and potentially inadequate containment material. Boiler "deconditioning," as in the Mercury Rankine Program, produces poor heat transfer and seems to be associated with boller surface contamination by air, water, oll, etc. Development is continuing on control of the environment and improvement in the boiler design to make it more insensitıve to "deconditioning."

Other components such as the condenser, NaK PMA, Hg PMA, and L/C PMA have undergone performance testing.

Potential applications for SNAP 8 include manned and unmanned satellites, lunar and planetary bases, interplanetary missions, communications, navigation, and remote terrestrial mobile energy depots. The typical integration of a SNAP 8 unmanned flight unit (without a radiator) is shown in Figure II- 107. 
TURBINE TYPE - 4 IMPULSE STAGES MERCURY FLOW RATE - 11,200 LBS/HR

\section{PRESSURE \\ INLET - 265 PSIA \\ OUTLET - 20 PSIA}

POWER OUTPUT - $51 \mathrm{KW}$

EFFICIENCY

$-60 \%$

SPEED

- 12,000 RPM

$\sim$

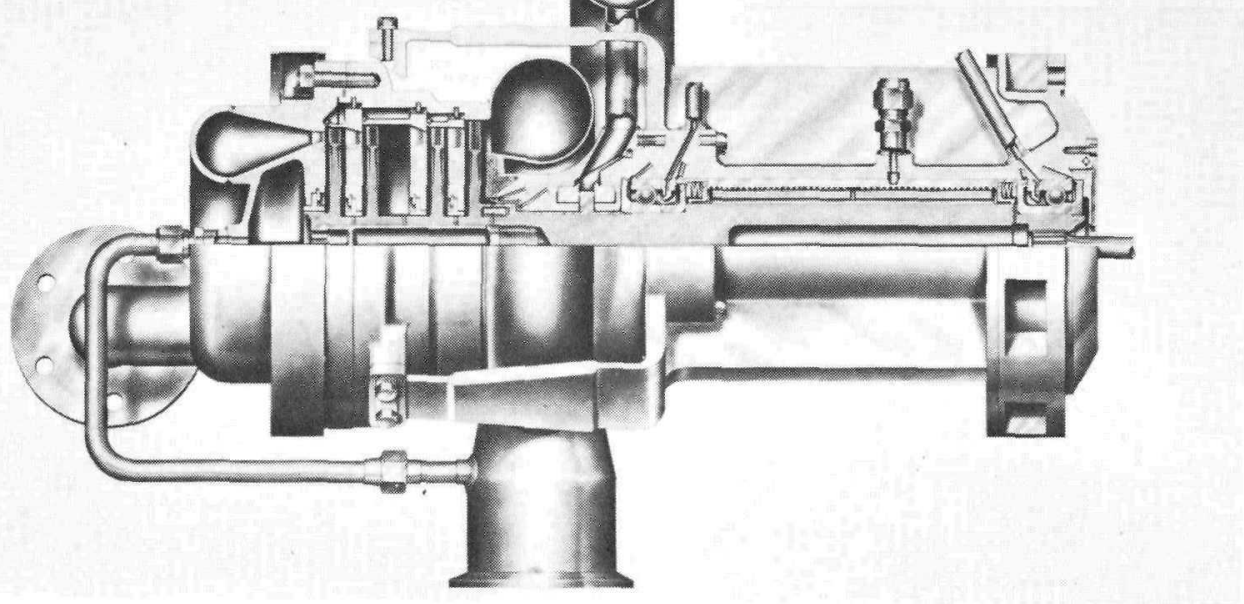

7-57-192-97

Figure II-106. SNAP 8 Turbine Assembly 


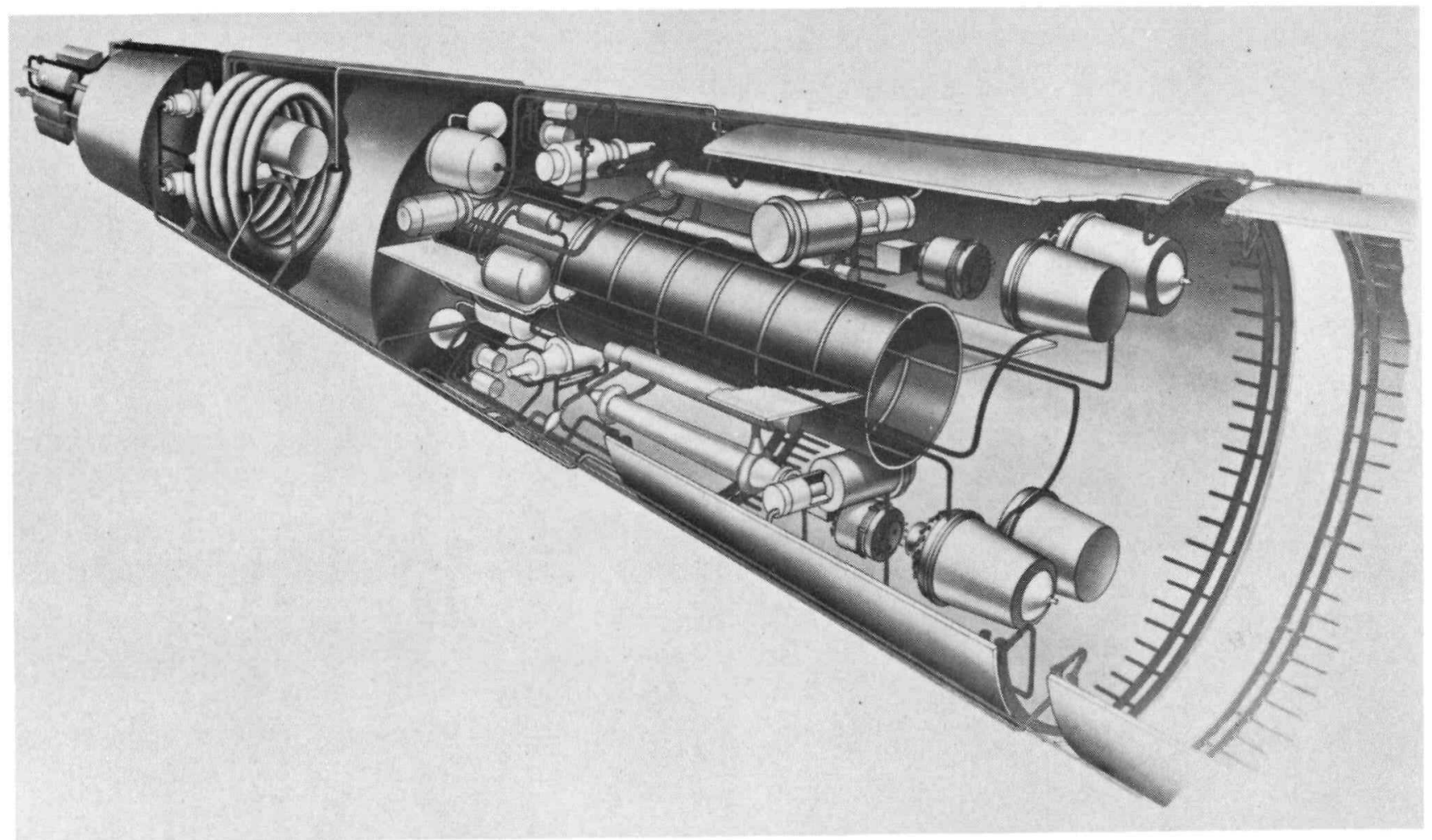




\section{BIBLIOGRAPHY}

1. The Elements of Nuclear Reactor Theory, Glasstone and Edlund, D. Van Nostrand Company, 1952

2. Principles of Nuclear Reactor Engineering, S. Glasstone, D. Van Nostrand Company, 1955

3. The Physical Theory of Neutron Chain Reactors, Weinberg and Wigner, University of Chicago Press, 1958

4. Reactor Handbook, Interscience, 1960

5. Propulsion Systems for Space Flight, W. R. Corliss, McGraw-Hill, 1960

6. Space Power Systems, Edited by N. W. Snyder, Progress in Astronautics and Rocketry, Vol. 4, Academic Press, 1961

7. "The Practical Application of Space Nuclear Power in the 1960's," J. R. Wetch, H. M. Dieckamp, and Lt. Col. G. M. Anderson (USAF), Presented to the International Astronautical Congress, August 1960, Stockholm, Sweden

8. "Advanced Reactor Concepts for Nuclear Propulsion," F. E. Rom, Astronautics Journal, October 1956

9. "A 20,000 kw Nuclear Turboelectric Power Supply for Manned Space Vehicle," NASA MEMO-2-20-59E, March 1959

10. "Compact Reactors for Space Power," H. M. Dieckamp, R. Balent, and J. R. Wetch, Nucleonics, April 1961, p 73

11. "The SNAP Program Present Status and Future Prospects," Lt. Col. G. M. Anderson, Presented at Atomic Industrial Forum, December 1960

12. "Atoms in Space," Nucleonics, July 1960

13. "Megawatt Power Levels Sought for Space," P. J. Klass, Aviation Week, August 22, 1960, p 69

14. "SNAP lA Targeted for 1961 Operation," Aviation Week, January 30, 1961, p 71

15. "Systems Must be Flight Tested Soon," Missiles and Rockets, March 20, 1961, p 32 
16. 'Nuclear Space Power Systems Utılızıng Magnetohydrodynamics Vortices," Lewellen and Grabowsky, ARS Paper $1738-61$

17. "Hazards Associated with Nuclear Power Reactors in Space Applications," P. G. Lafyatis, ARS Paper 1754-61

18. Nuclear Flight, Lt. Col. K. E. Grantz, Duell, Sloan and Pearce, 1960

19. "Forecasts for Flight Vehicle Power,"G. W. Sherman, SAE 297C, March 1961

20. "A Binary Vapor Nuclear Power Plant," D. R. Sawle and J. Salisbury, ASME-60-WA-309, November 1960

21. "Summary of SNAP Nuclear Space Power Systems," E. B. Baumeister, ARS-61-SA-52, June 1961

22. "The Potential of the SNAP Reactors with Advanced Mercury Rankine Energy Conversıon Systems," V. Kovacık, Presented at ANS Nucleonics in Flight Symposium, March 1961

23. "300 kw Thermionic Generator by 67,"W. Beller, Missiles and Rockets, October 3, 1960, p 30

24. "Megawatt Electrical Power in Space," P. Ross, E. Ray, I. Taylor, Astronautics, December 1960, p 26

25. "Trends in Turboelectric Power Generation for Space Vehicles," P. Ross and E. Ray, ASME, May 20, 1960

26. "Space Nuclear Power Conversion System," C. E. Johnson, M. G. Coombs, and R. L. Hirsch, NAE-CON-IRE, May 2, 1959

27. "Earth Satellite Mission for Electrical Propulsion," by E. E. Dangle, J. D. Gossett, and W. D. Hibbard, Jr., paper No. 2225-61, American Rocket Society, Space Flight Report to the Natıon, October 9-15, 1961

28. "SNAP 8 - The First Electric Propulsion Power System," by P. I. Wood, D. L. Forrest, and B. M. Wilner, paper No. 2050-61, American Rocket Socrety, Space Flight Report to the Nation, October 9-15, 1961

29. "Performance of Nuclear Electrıc Propulsion Systems in Space Exploration," by Evelyn W. Speiser, paper 2224-61, American Rocket Society, Space Flight Report to the Nation, October 9-15, 1961 
30. "Test-Bed Configurations for Flight Testing of SNAP 8 Powered Electrical Propulsion Systems," JPL Technical Report No. $32-190$

31. "Systems Engineering of a Nuclear Electric Spacecraft," by R. J. Beale, paper No. 2257-61, American Rocket Society, Space Flight Report to the Nation, October 9-15, 1961

32. "Space Reactor Power," by J. R. Wetch and M. G. Coombs, paper 2167-61, American Rocket Society, Space Flight Report to the Nation, October 9-15, 1961

33. "Nuclear Reactor Space Power Systems," by J. R. Wetch and M. G. Coombs, AIEE Aerospace Technical Conference, June 17-22, 1962, Denver, Colorado

34. "Reactor Power for Communications Satellites," by A. B. Martin, AIF Conference, November 6-8, 1961, Chicago, Illinois

35. G. Safanov, "Survey of Reacting Mixtures Employing U-235, $\mathrm{Pu}-239$, and $\mathrm{U}-233$ for Fuel and $\mathrm{H}_{2} \mathrm{O}, \mathrm{D}_{2} \mathrm{O}, \mathrm{C}, \mathrm{Be}$, and $\mathrm{BeO}$ for Moderators," R-259, January 1954

36. "Status of The SNAP 2 Reactor," by R. D. Keen and R. R. Eggleston, presented at the ARS Space Power Systems Conference, Santa Monica, California, September 27-30, 1960

37. "SNAP 2 Power-Conversion Status," by D. L. Southam, presented at the Space Power Systems Conference, Santa Monica, California, September 27-30, 1960

38. "SNAP 2 Radiative-Condenser Design," by M. G. Coombs and R. A. Stone, presented at the Space Power Systems Conference, September 27-30, 1960

39. "SNAP 2 System and Vehicle Integration,"D. J. Cockeram and R. L. Wallerstedt, presented at the Space Power Systems Conference, Santa Monica, California, September $27-30,1960$

40. "SNAP Thermoelectric Systems," by A. W. Thiele and M. G. Coombs, presented at the Space Power Systems Conference, Santa Monica, California, September 27-30, 1960

41. "The Application of SNAP Units in Current Space Vehicles," by J. R. Wetch and J. G. Lundholm, Jr., presented at the Space Power Systems Conference; Santa Monica, California, September $27-30,1960$ 
42. "Problems Associated with the Development of a Thermionic Conversion Reactor," by R. L. Hirsch and J. W. Holland, presented at the Space Power Systems Conference, Santa Monica, California, September 27-30, 1960

43. "Dynamic Versus Direct Conversion," by K. P. Johnson, presented at the Space Power Systems Conference, Santa Monica, California, September 27-30, 1960

44. "Boiling vs Non-Boiling Liquid Metal Cooled Reactors in Rankine Cycle Space Power Plants," by D. L. Cochran and Keith E. Buck, presented at the Space Power Systems Conference, Santa Monica, California, September 27-30, 1960

45. "Redesigned SNAP 8," Nucleonics, Vol 21, No. 7, July 1963

46. 'Nuclear Reactor Systems," G. M. Anderson, Astronautics and Aerospace Engineering, May 1963

47. "Space Electrical Power," W. Scott and F. Schulman, Astronautics and Aerospace Engineering, May 1963

48. "Orbital Testing of SNAP Systems," Lt. Col. G. E. Austin, USAF, Astronautics and Aerospace Engineering, May 1963

49. "Thermionic Converters for Space Power," V. C. Wilson and R. C. Hamilton, Astronautics and Aerospace Engineering, May 1963

50. "Dynamic Energy Conversion," V. P. Kovacik, Astronautics and Aerospace Engineering, May 1963

51. "SNAP 8 Reactor and Shield," by C. E. Johnson and C. A. Goetz, AIAA Electric Propulsion Conference, presented at Colorado Springs, Colorado, March 1963

52. "Application of a SNAP Reactor Power Plant to a Manned Orbiting Space Station," by H. N. Rosenberg, AI-MEMO7845, October 1962

53. "Nuclear Space Power Systems - The SNAP Reactors," by R. E. Wimmer, AIEE Winter Meeting, New York, February 1962

54. "Power Supply Aspects of the Mars Mission," by R. Balent and J. R. Wetch, presented at the American Astronautical Society, Symposium on the Exploration of Mars, Denver, June 1963

55. "Thermal Radiators for Space Vehicle Environmental Control Systems," by R. M. Wright, et al., Report No. STC-20-R, AiResearch Manufacturing Division, July 1962 
56. "Radiator Heat-Transfer Analysis for Space Vehicles," by J. A. Stevenson and J. C. Grafton, ASD61-119

57. "Space Radiator Analysis and Design," by D. B. Mackay and C. P. Bacha, ASD Technical Report 61-30, October 1961, Part 1

58. "Prediction of Space Radiator Performance," by B. T. Lubin and R. B. Trusch, ASME Paper 63-AHGT-85, 1963

59. "The SNAP 2 Radiative Condenser Analysis," by M. G. Coombs, R. A. Stone, and T. Kapus, NAA-SR-5317

60. "The Influx Rate of Meteors in the Earth's Atmosphere," by G. S. Hawkins and E. K. L. Upton, Astrophysical Journal, $128,3,1958$

61. "Results of an Artificial Iron Meteoroid at $10 \mathrm{~km} / \mathrm{sec}$," by R. E. McCrosky and R. K. Soberman, Smithsonian Contributions to Astrophysics, 1962

62. "Luminous Efficiency of Iron and Stone Asteroidal Meteors," by A. F. Cook, L. G. Jacchia, and R. E. McCrosky, Smithsonian Contributions to Astrophysics, 1962

63. "SPUR High-Temperature Space Radiator," by K. O. Parker and R. A. Stone, ARS Paper 2549-62, Space Power Systems Conference, September 1962

64. "The Meteoric Risk to Space Vehicles," by F. L. Whipple, Vistas in Astronautics, 115-124, Pergamon Press, 1958

65. Between the Planets, by F. G. Watson, Harvard University Press, Cambridge, Massachusetts, 1956

66. "The Meteoritic Hazard of Environment of a Satellite," by J. E. Duberg, NASA-TN-D-1248, 1962

67. "Radar Determination of Meteor Orbits," by G. S. Hawkins, Research Report No. 13 of the Harvard College Radio Meteor Project, 1962

68. "High Speed Impact of Metal Projectiles in Targets of Various Materials," by J. L. Summers and A. C. Charters, Proc. 3rd Symposium on Hypervelocity Impact, Armour Research Foundation, Chicago, 1959

69. "Effects of a Meteoroid Impact on Steel and Aluminum in Space," by R. L. Bjork, Tenth International Astronautical Congress, London, England, August 1959 
70. "Effects of Meteoroid Impacts on Space Vehicles," by R. J. Eichelberger and J. W. Gehring, ARS Paper No. 2030-61, October 1961

71. "Analysis of a Megawatt Level Direct Condenser-Radiator," by R. P. Krebs, D. M. Winch, and S. Lieblein, ARS Paper 2545-62, Space Power Systems Conference, Santa Monica, California, September 1962

72. "Large Space Radiators," by M. G. Coombs and R. A. Stone, 18th Annual Propulsion Meeting, IAS, March 1963

73. Design of Space Power Plants, Donald B. Mackay, Prentice Hall, 1963

74. "The Application of the Recuperated Brayton Cycle to Space Power Conversion Systems," by L. W. Norman, AiResearch Mfg. Co., SY $-5527 R$

75. "Thermionic Conversion," by N. S. Rasor, Space Power Systems - Short Course, George Washington University, October 1963

76. "Space Power Systems State of the Art," by George Szego, AIAA Paper No. 64-525

77. "Reactor Direct Conversion Units," by H. Dieckamp, Third United Nations Conference on the Peaceful Uses of Atomic Energy, Geneva, Switzerland, September 1964

78. "Design of a Multiple-Unit SNAP 2 Power Supply for a Manned Space Station," by W. V. Botts, AIAA Meeting, Washington, D.C., June 1964

79. "An Evaluation of Systems for Nuclear Auxiliary Power," by U.S. Atomic Energy Commission, TID-20079, January 1964 
BLANK 


\section{CHAPTER III \\ Y RADIOISOTOPE SPACE POWER}

1. PHYSICS OF RADIOISOTOPES

Radioisotopes are isotopes that are radioactive. They are in an excited state, thus they decay by emittıng charged particles and/or by spontaneous fission. This is usually followed by gamma ray emission. In the case of spontaneous fission, neutrons are also emitted.

Radiolsotopes differ from nuclear reactors in that they decay exponentially in time at a rate which is not affected by outside forces, 1.e., the decay rate cannot be altered. As a result of this property, the heat generation from the decay of radioisotopes cannot be turned off. This last feature makes radioisotopes very reliable heat sources and very attractive for some applications. However, in some cases, it is desirable to have a capability of shutting down the heat source.

\section{A. CLASS OF RADIOISOTOPES}

In the decay process of radioisotopes, the nuclear particles and/or gamma rays are absorbed in the fuel material and as a result they generate heat. Radiolsotopes may be classed according to their main source of heat generation. Table III-1 presents the potential radiolsotopes with some of their important properties. (1)

\section{a. Beta Emitters}

In the case of beta emitters, not all of the decay energy is avallable for heat generation. The average beta particle possesses only about one third of the decay beta energy, the rest is given off as a neutrino. Part of the gamma ray energy which usually accompanies beta decay and which is absorbed in the fuel region has to be added to the beta energy in order to determine the total energy deposited per disintegration. The following 
TAB LE III- 1

CHARAC TERISTICS OF RADIOISOTOPIC HEAT SOURCES

\begin{tabular}{|c|c|c|c|c|c|c|c|c|c|c|c|}
\hline \multirow{2}{*}{$\begin{array}{r}\text { CLASS OF EMITTER } \\
\text { RADIOISOTOPE }\end{array}$} & \multirow{2}{*}{\begin{tabular}{|c|} 
GAMMA \\
$\mathrm{Co}_{0} 60$ \\
\end{tabular}} & \multicolumn{6}{|c|}{ BETA } & \multicolumn{4}{|c|}{ ALPHA } \\
\hline & & $\mathrm{Sr}^{90}$ & $\mathrm{Ru}^{106}$ & $\mathrm{Cs}^{137}$ & $C \mathrm{e}^{144}$ & $\mathrm{Pm}^{147}$ & $\mathrm{Tm}^{170}$ & $\mathrm{Po}^{210}$ & $\mathrm{Pu}^{238}$ & $\mathrm{Cm}^{242}$ & $\mathrm{Cm}^{244}$ \\
\hline \multicolumn{12}{|l|}{ HALF LIFE YEARS } \\
\hline DECAY & 5.3 & 27.7 & 1.0 & 30 & 0.78 & 2.6 & 0.35 & 0.38 & 86 & 0.45 & 18 \\
\hline SPONTANEOUS FISSION & - & - & - & - & - & - & - & - & $4.9 \times 10^{10} \mid$ & $7.2 \times 10^{6}$ & $1.4 \times 10^{7}$ \\
\hline \multicolumn{12}{|l|}{ PRINCIPAL DECAY (Mev) } \\
\hline$\alpha$ & - & - & - & - & - & - & - & 5.3 & 5.49 & 6.11 & 5.80 \\
\hline$\beta$ & 0.31 & 2.24 & & 0.529 & 1.321 & 0.223 & 0.96 & & & & \\
\hline$\gamma$ & 1.17 & 1.734 & & 0.66 & 2.18 & 0.121 & 0.084 & 0.8 & 0.044 & 0.04 & 0.04 \\
\hline FUEL FORM & METAL & $\mathrm{SrTiO}_{3}$ & METAL & $\mathrm{CsCl}$ & $\mathrm{CeO}_{2}$ & $\mathrm{Pm}_{2} \mathrm{O}_{3}$ & $\mathrm{Tm}_{2} \mathrm{O}_{3}$ & GdPo & $\mathrm{PuO}_{2}$ & $\mathrm{Cm}_{2} \mathrm{O}_{3}$ & $\mathrm{Cm}_{2} \mathrm{O}_{3}$ \\
\hline DENSITY $\left(\mathrm{gm} / \mathrm{cm}^{3}\right)$ & 8.7 & 3.7 & 12.2 & 3.6 & 6.6 & 6.6 & 8.5 & 9.9 & 10 & & 9 \\
\hline WATTS/GRAM (PURE) & 17.4 & 0.95 & 33.1 & 0.42 & 25.6 & 0.33 & 12.1 & 79.5 & 0.56 & 120 & 2.65 \\
\hline ISOTOPIC PURITY $(\%)$ & 10 & 50 & 3.3 & 35 & 18 & 95 & 10 & 95 & 80 & 90 & 95 \\
\hline $\begin{array}{l}\text { POWER DENSITY } \\
\quad \mathrm{w} / \mathrm{cm}^{3} \text { FUEL }\end{array}$ & 15.2 & 0.94 & 13.4 & 0.42 & 25.3 & 1.8 & 9.1 & 815 & 3.9 & 882 & 20.4 \\
\hline SHIELDING REQUIRED & HEAVY & HEAVY & HEAW & HEAVY & HEAVY & MINOR & MODERATE & MINOR & MINOR & MINOR & MODERATE \\
\hline CURIES/WATT & 65 & 148 & 102 & 207 & 126 & 2788 & 500 & 32 & 30 & 28 & 29 \\
\hline MELTING POINT $\left({ }^{\circ} \mathrm{C}\right)$ & 1480 & 1900 & 2450 & 646 & 2680 & 2270 & 2300 & 1675 & 2280 & 1950 & 1950 \\
\hline $\begin{array}{l}\text { MPC IN AIR } \\
\quad\left(\mathrm{Ci} / \mathrm{cm}^{3}\right)\end{array}$ & $3 \times 10^{-9}$ & $10^{-10}$ & $2 \times 10^{-9}$ & $5 \times 10^{-9}$ & $2 \times 10^{-4}$ & $2 \times 10^{-8}$ & $10^{-8}$ & $7 \times 10^{-11}$ & $7 \times 10^{-13}$ & $4 \times 10^{-11}$ & $3 \times 10^{-12}$ \\
\hline $\begin{array}{l}\text { ESTIMATED FUTURE COST } \\
\text { (\$/watt) }\end{array}$ & 16 & 21 & 5 & 24 & 2 & 220 & 10 & 20 & 540 & 17 & 64 \\
\hline
\end{tabular}


expression presents the curies per watt required of a radioisotope as a function of energy avallable per disintegration,

$$
C=\frac{168.6}{E}
$$

where,

$$
\begin{aligned}
C= & \text { Curies per watt required (curie }=3.7 \times 10^{10} \\
& \text { disintegrations } / \text { sec) } \\
E= & \text { Total energy available per disintegration } \\
& (\text { Mev/disintegration) }
\end{aligned}
$$

The above expression applies to all radiolsotopes.

\section{b. Alpha Emitters}

Alpha emitting radioisotopes are usually the heavy isotopes. All the alpha particle energy is deposited in the fuel. Part of the gamma ray energy that usually accompanies alpha decay is absorbed in the fuel similar to beta emitters.

\section{c. Gamma Emitters}

Gamma emitters are similar to beta emitters except that the main source of heat is the gamma rays. Cobalt-60 is a typical gamma emitter. It emits a 0.31 -Mev beta particle, followed by two gamma rays in series, 1.17 and 1.33 Mev respectively. Gamma emitters usually have high gamma ray energies which are very penetrating. Thus great care has to be taken to determine the energy absorbed in the fuel per disintegration.

\section{B. FORMATION OF RADIOISOTOPES}

Radioisotope fuels used for heat generation are artificially made from fission products or by irradiation in reactors.

\section{a. Fission Products}

Fission products result from the fission of fissile materials. In the unseparated form, mixed fission products (MFP) have a very low power density, about 0.05 watts $/ \mathrm{cm}^{3}$ or less. Separated 
fission products are more attractive since they have fairly high power densities. Typical separated fission products are $\mathrm{Sr}^{90}$, $\mathrm{Ru}^{106}, \mathrm{Ce}^{144}, \mathrm{Cs}^{137}$, and $\mathrm{Pm}{ }^{147}$. All are beta emitters and have power densities greater than 0.4 watts $/ \mathrm{cm}^{3}$.

\section{b. Reactor Produced Fuels}

Reactor produced fuels may be divided into two classes those that absorb one neutron and those that absorb more than one neutron. In the first class, a stable isotope captures one neutron and thus becomes radioactive. In this class are the following radiolsotopes $\mathrm{Co}^{60}, \mathrm{Tm}^{170}$, and $\mathrm{Po}^{210}$.

In the second class, a stable isotope (or isotope with a very long half-life) absorbs more than one neutron until it ends up as the desired radioisotope. In this class are the following radio1sotopes $\mathrm{Pu}^{238}, \mathrm{Cm}^{242}$, and $\mathrm{Cm}^{244}$. Tables III-2 to III-4 present the methods by which these radioisotopes are formed. Since these radiolsotopes require more than one neutron, and further more, since they have to be chemically separated from their parent isotope, they are very costly.

\section{SOURCES OF RADIATION}

The radiation from heat producing radiolsotopes can emanate directly from the natural decay scheme of the radioactive nuclide, or it can result from the interaction with other materials. Direct radiation is in the form of gamma rays, alpha particles, beta particles, and neutrons, with each radioisotope having a typical spectrum of each radiation form. The direct radiation from many nuclides is primarily restricted to only one or two forms, while in others there are significant contributions from three or more decay modes. Only gamma rays and neutrons are important in the shielding analysis since they are more penetrating than both beta and alpha particles. The following are the main sources of radiation from radioisotopes 
TAB LE III - 2

PRODUCTION OF Pu 238

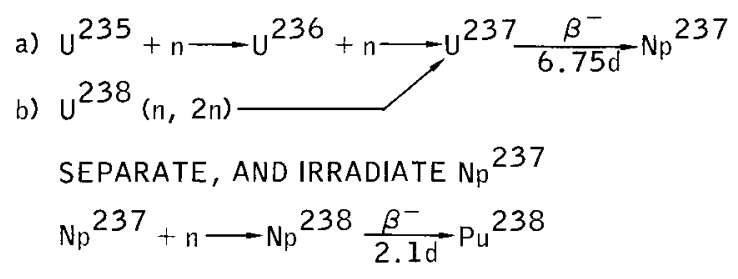

(THIS SECOND STEP AVOIDS PROBLEM OF SEPARATING $\mathrm{Pu}^{238} \mathrm{FROM} \mathrm{Pu}{ }^{239}$ )

TAB LE III- 3

PRODUCTION OF $\mathrm{Cm}^{242}$

$\mathrm{U}^{238}+n \rightarrow \mathrm{U}^{239} \frac{\beta^{-}}{23.5 m} \mathrm{~Np}^{239} \frac{\beta^{-}}{2.35 d} \mathrm{Pu}^{239}+\mathrm{n} \longrightarrow \mathrm{Pu}^{240}+\mathrm{n} \longrightarrow \mathrm{Pu}^{241}$

(STORE PLUTONIUM IN SPENT FUEL FOR A FEW YEARS TIME TO PRODUCE Am 241,

$\mathrm{Pu}^{241} \frac{\beta^{-}}{13 \mathrm{y}} \mathrm{Am}^{241}$

SEPARATE Am ${ }^{241}$, AND IRRADIATE

$A m^{241}+n \longrightarrow A m^{242} \frac{\beta^{-}}{16 h} \mathrm{Cm}^{242}$ 


\section{TAB LE III-4 \\ PRODUC TION OF $\mathrm{Cm}^{244}$}

MAKE $\mathrm{Pu}^{241}$ AS IN TABLE III-3, BUT LEAVE Pu ${ }^{241}$ IN

REACTOR AND CONTINUE TO IRRADIATE

$\mathrm{Pu}^{241}+\mathrm{n} \longrightarrow \mathrm{Pu}^{242}+\mathrm{n} \longrightarrow \mathrm{Pu}^{243} \frac{\beta^{-}}{4.98 \mathrm{~h}} \mathrm{Am}^{243}$

$\mathrm{Am}^{243}+\mathrm{n} \longrightarrow \mathrm{Am}^{244} \frac{\beta^{-}}{10 \mathrm{~h}} \mathrm{Cm}^{244}$

1. Gamma Rays

a) Gamma rays emitted in the decay of the radioisotope.

b) Gamma rays resulting from bremsstrahlung.

c) Gamma rays from impurities found in the radioisotope.

d) Gamma rays resulting from the alpha neutron $(\alpha, n)$ reaction with low atomic weight elements in the immediate vicinity of the radioisotope.

e) Prompt fission and fission product decay gamma rays due to spontaneous fission of the parent radioisotope.

f) Prompt fission and fission product decay gamma rays due to the induced fissioning of the parent radioisotope.

2. Neutrons

a) Neutrons from spontaneous fission of the parent radioisotope.

b) Neutrons due to the induced fissioning of the parent radioisotope. 
c) Neutrons from the $(\alpha, n)$ reaction with low atomic weight elements in the immediate vicinity of the radioisotope.

Some of the sources of radiation 1isted above are primary sources and others are secondary. The primary sources are inherent of the particular radioisotope and they cannot be altered. The secondary sources are due to primary sources acting upon the surrounding medium. In some instances the secondary sources may become more important than the primary ones. This situation should be avoided or reduced in magnitude if economically acceptable. The following sections discuss in more detail secondary sources and their importance.

\section{a. Alpha-Neutron Reaction}

When alpha emitters are used, care should be taken to include the secondary neutron source from the $(\alpha, n)$ reaction. For instance, when $\mathrm{Pu}^{238}$ is used in the oxide form $\left(\mathrm{Pu}^{238} \mathrm{O}_{2}\right)$, the neutron source increases from the existing spontaneous fission source of $\mathrm{Pu}{ }^{238}$ by a factor of $11 .^{(2)}$ This $1 \mathrm{~s}$ due to the $(\alpha, \mathrm{n})$ reaction with oxygen. The $(\alpha, n)$ reaction is a process whereby the target nucleus absorbs an alpha particle and subsequently emits a neutron. This is a threshold reaction, which means that this process takes place only if the energy of the alpha particle is greater than the threshold for the $(\alpha, n)$ reaction of the target nucleus. Natural oxygen contains three isotopes, namely, ${ }_{0} 16$, $0^{17}$, and $0^{18}$ in the following abundances $99.759 \%, 0.037 \%$, and $0.204 \%$ respectively. The thresholds for $(\alpha, n)$ reaction for $0^{16}$, $0^{17}$, and $0^{18}$ are $15.2 \mathrm{Mev}, 0$, and $0.86 \mathrm{Mev}$ respectively. In the alpha decay of $\mathrm{Pu} 238$ the maxımum energy of the alpha particle is $5.5 \mathrm{Mev}$. This means that the $(\alpha, \mathrm{n})$ reaction can take place only with $0^{17}$ and $0^{18}$ isotopes. Thus by reducing the $0^{17}$ and $0^{18}$ content un oxygen, the neutron source from the $(\alpha, n)$ reaction can be reduced. This has been shown experimentally. $(3,4)$ 
However, for a particular application, the economics will determine if additional neutron shielding should be used or if $\mathrm{Pu}^{238}$ oxide with oxygen depleted in $0^{17}$ and $0^{18}$ should be used.

For $\mathrm{Cm}_{2}^{244} \mathrm{O}_{3}$, the neutron source from the $(\alpha, n)$ reaction with oxygen represents a very small fraction, (5) about $3 \%$, and the rest is from the spontaneous fission of $\mathrm{Cm}^{244}$. In this case, use of oxygen depleted in $0^{17}$ and $0^{18}$ wall not affect the total neutron source.

Another important example is $\mathrm{Po}^{210}$ where all the neutron source is from the $(\alpha, n)$ reaction with impurities. If the concentration of these impurities is reduced, the neutron source would be reduced also. As a result, the neutron shield required would be reduced in thickness and weight. For space application where weight is at a premium, it may be economically worth purifying the Po ${ }^{210}$ of all impurities thus eliminating the necessity of using a neutron shield.

\section{b. Importance of Isotopic Purity}

Another important secondary source is that from impurities found in the main radioisotope. This applies to radioisotopes which emit very low energy gamma rays and in very small abundance. Since these radioisotopes can be very easily shielded, the presence of impurities even in very small quantities becomes very important. Two such radioisotopes are $\mathrm{Pm}^{147}$ and $\mathrm{Pu}^{238}$.

Pm 147 decays by beta emission to either the ground state of $\mathrm{Sm}^{147}$ or to an excited state of $\mathrm{Sm}^{147}$ which in turn decays to the ground state of $\mathrm{Sm}^{147}$ by emitting a 0.121 -Mev gamma ray. Additional very low energy bremsstrahlung gamma rays are emitted due to the $0.225-\mathrm{Mev}$ beta partıcle from the decay of $\mathrm{Pm}^{147}$. It may be noted that the gamma ray energies from Pm 147 are very low and can be very easily shielded using about $0.150 \mathrm{~m}$. of steel. However, Pm ${ }^{147}$ contains small amounts of $\mathrm{Pm}^{146}$, which is formed by the $(\mathrm{n}, 2 \mathrm{n})$ reaction with $\mathrm{Pm}^{147}$. Since this is a threshold reaction, the Pm ${ }^{146}$ content will vary 
with various reactors used to produce Pm ${ }^{147}$. The higher the medium fission energy, the higher the $\operatorname{Prm}^{146^{\circ}}$ content. The Pm ${ }^{146}$ content varies from 0.25 up to 4 ppm depending on the reactor. Pm 146 decays by emitting $0.75-\mathrm{Mev}$ gamma rays and for a $\mathrm{Pm}^{147}$ heat source, the $\mathrm{Pm}^{146}$ dictates the shield thickness required. Since $\mathrm{Pm}^{147}$ and $P \mathrm{~m}^{146}$ have half lives of 2.6 and 5.5 years, respectively, aging will not reduce the Pm ${ }^{146}$ content. Thus one has to shield the radiation from Pm 146 .

A somewhat similar condition exists for $\mathrm{Pu}^{238}$ which contains small amounts (about $1.2 \mathrm{ppm})^{(2)}$ of $\mathrm{Pu}{ }^{236} \cdot \mathrm{Pu}^{238}$ is formed by the irradiation of $\mathrm{Np}^{237}$ in a reactor, see Table III- 2 . $\mathrm{Pu}^{236}$ is also formed by the irradiation of $\mathrm{Np}^{237}$ in a reactor. However, it is formed by the $\mathrm{Np}^{237}(\mathrm{n}, 2 \mathrm{n}) \mathrm{Np}^{236}$ reaction which decays by beta emission to $\mathrm{Pu}^{236}$. It should be stressed that there is no way of avoiding the formation of $\mathrm{Pu}^{236}$ in a reactor. Table III-5 shows the decay chain of $\mathrm{Pu}^{236}$ to $\mathrm{Pb}^{208}$ which is a stable isotope. In this chain, $\mathrm{T}^{208}$ and $\mathrm{B}^{212}$ emit high energy

TAB LE III - 5

DECAY CHAIN OF Pu ${ }^{236}$
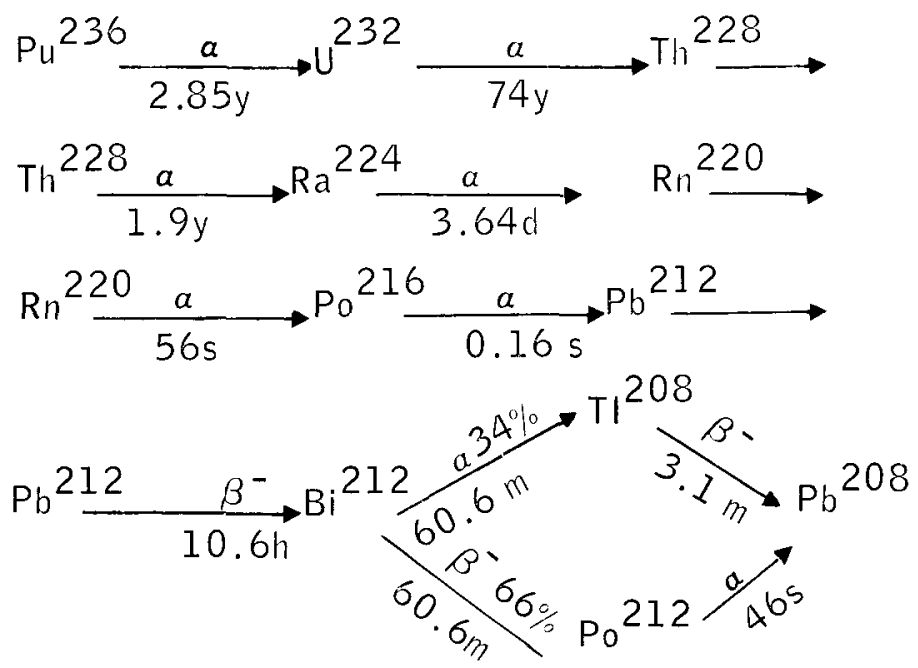
gamma rays and the dose rate resulting from these two isotopes is greater than the unshelded dose rate from $\mathrm{Pu}^{238}$, 2.1 years after the formation of $\mathrm{Pu}^{236}$, see Figure III-1. Since the half-

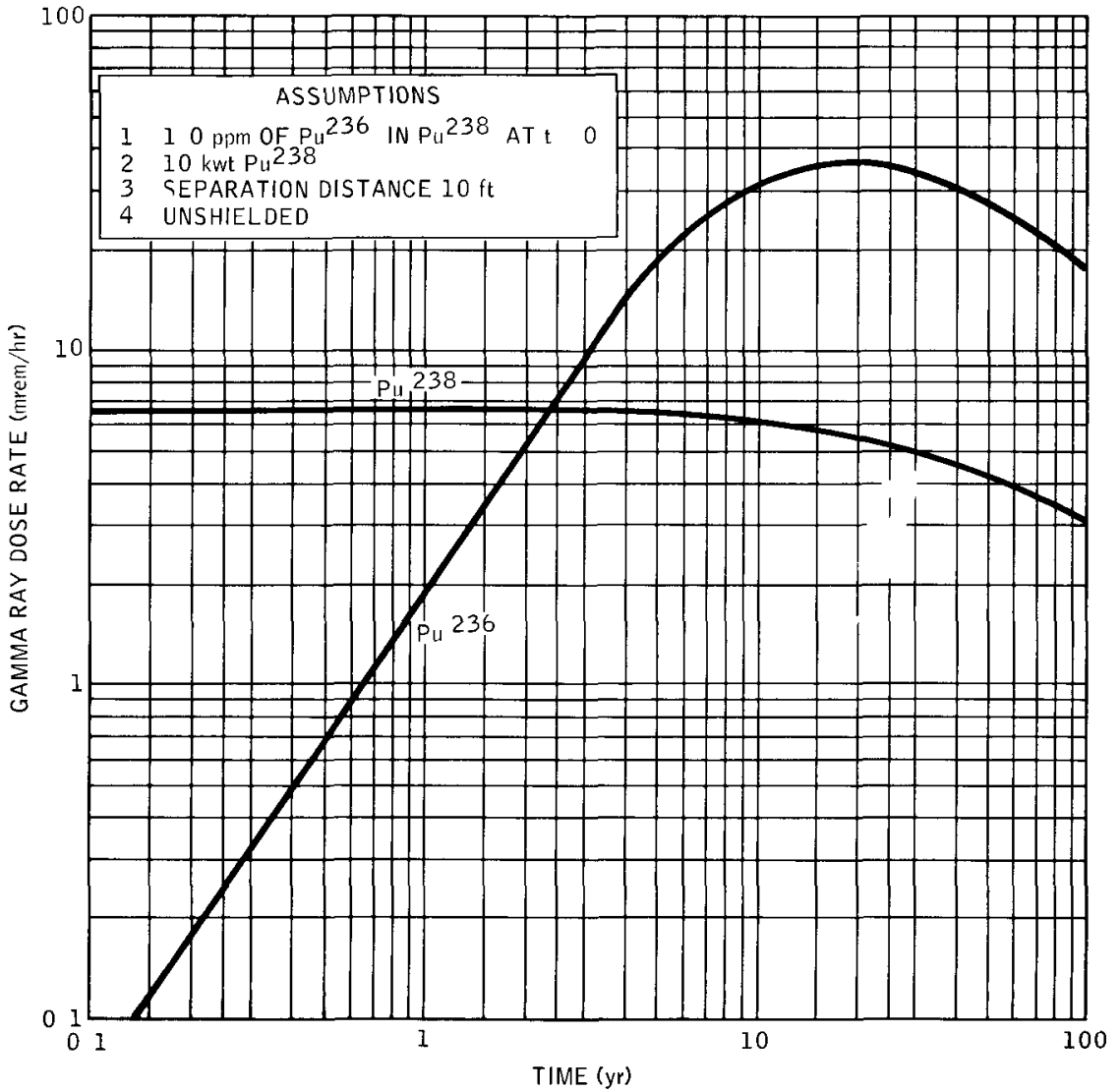

$7 \$ 192132$

Figure III-1. Unshielded Dose Rate as a Function of Time from $1.0 \mathrm{ppm}$ of $\mathrm{Pu}^{236}$ in $\mathrm{Pu} 238$

lives of isotopes in the decay chain beyond $\mathrm{Th}^{228}$, shown in Table III-5, are short in comparison to the preceding ones, the activity of both $\mathrm{Tl}^{208}$ and $\mathrm{Br}^{212}$ is dependent on the activity of $\mathrm{Th}^{228}$. The average energy of gamma rays from $\mathrm{Pu}^{238}$ is less than $0.9 \mathrm{Mev}$, whereas that resultang from $\mathrm{Pu}{ }^{236}$ is equal to 
2.6 Mev. Thus since the gamma rays from $\mathrm{Pu}^{236}$ are more penetrating than those from $\mathrm{Pu}^{238}$, for a shielded system, the ratio of the dose rate resulting from $\mathrm{Pu}^{236}$ to $\mathrm{Pu}^{238}$ will be greater than that shown in Figure III- 1 .

c. Criticality of $\mathrm{Pu}^{238}$ and $\mathrm{Cm}^{244}$

Another important source is due to the induced fissioning of the radioisotope fuel. This applies only to $\mathrm{Pu}^{238}$ and $\mathrm{Cm}^{244}$. Under the condition of subcriticality, the subcritical multiplication factor or the ratio of neutrons emitted in a multiplying medium to the number of neutrons originally present in the medium is equal to

$$
\frac{n}{n_{o}}-\frac{1}{1-k}=1+\frac{k}{1-k}
$$

where,

$\mathrm{n}=$ number of neutrons emitted in a multiplying medium $\mathrm{n}_{\mathrm{o}}=$ number of neutrons originally present in the medium $\mathrm{k}=$ neutron multiplication factor $(\mathrm{k}<1)$

This means that a radiolsotope heat source which has a " $k$ " less than one, is safe as far as criticality is concerned. How ever, for shielding calculations, the number of neutrons emitted from the radiolsotope heat source is not "n," the number of neutrons present in the medium or the neutron source experimentally determined using very small sources, where " $k$ " is much less than 0.01 , instead, the correct source is " $n$," the number of neutrons emitted in the multiplying medium. For example, in a radiolsotope heat source with a $\mathrm{k}=0.8, \mathrm{n} / \mathrm{n}_{\mathrm{o}}-5,1$. e., the number of neutrons requiring shielding because of the multiplying medium is 5 times that originally present.

In order to determine the "k" of the radiolsotope heat source, criticality calculations have to be performed. There is presently a lack of experimental data on neutron cross sections for Pu 238 and especially $\mathrm{Cm}^{244}$. Cross sections for both $\mathrm{Pu}^{238}$ and $\mathrm{Cm}^{244}$ 
have been generated theoretically using a non-spherical optical model. ${ }^{(6)}$ The agreement with a few experimentally measured points for $\mathrm{Pu}^{238}$ is good. Using these cross sections, Figure III-2 presents the critical mass of base spheres of $\mathrm{Pu}^{238}$ and $\mathrm{Cm}^{244} \cdot(7,8)$ It may be noted that $\mathrm{Pu}^{238}$ has a slightly lower critical mass than $\mathrm{Pu}^{239}$ and $\mathrm{Cm}^{244}$ has a higher critical mass than $\mathrm{Pu}^{239}$.

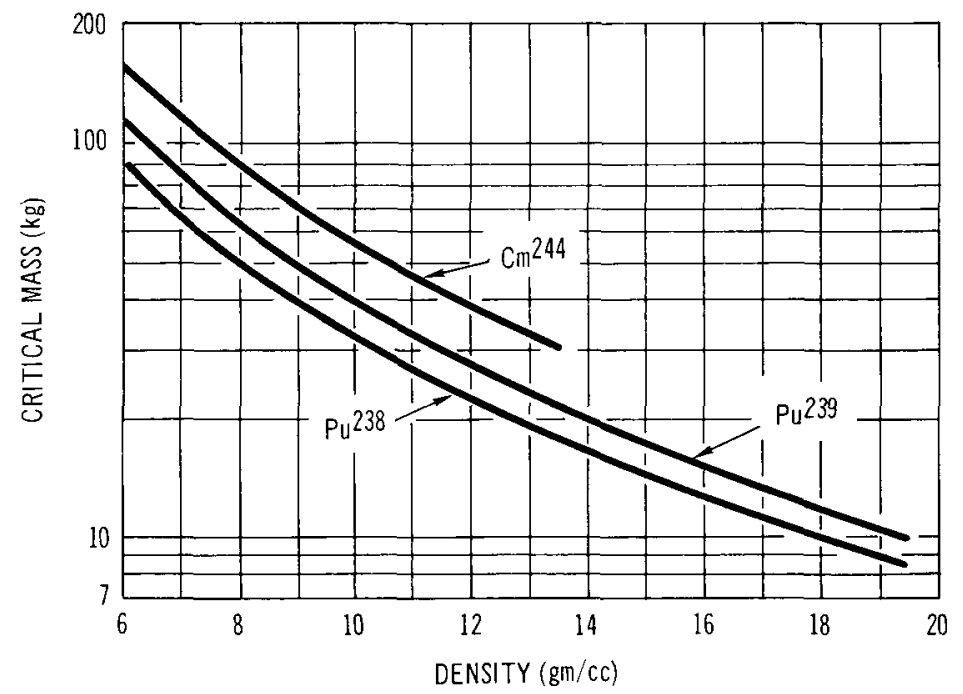

$11-2-65$

Al-65-02021

Figure III-2. Critical Mass of Unreflected $\mathrm{Cm}^{244}$ and $\mathrm{Pu} 238$ Spheres

\section{SHIELDING}

The weight of the nuclear shield required to protect equipment or personnel from the radiolsotope source radiations is a major consideration in the utilization of radioisotope heat sources. The weight of the shield required is a function of the following

1) Radioisotope source characteristics

2) Form the radioisotope is in, 1. e., oxide, etc. 
3) Impurities in the radioisotope heat source.

4) System geometry.

5) Dose criteria.

\section{a. Radiation Sources for Shielding Analysis}

For detailed shielding calculations, Table III- 6 presents the gamma ray source strength per thermal watt of the radionsotope for various photon energies. Table III- 7 presents the neutron source strengths for the neutron emitters. For detailed analysis, fuel self-shielding and intercapsule shielding should be considered. Figure III- 3 presents the ratio of self-shielded to unshielded gamma ray dose rate for $\mathrm{Po}^{210}, \mathrm{Pu}^{238}$, and $\mathrm{Cm}^{244}$ capsules. It may be noted that the capsule self-shielding is important, furthermore, dose rate as a function of angle with capsule axis is also important.

\section{TABLE III-7}

NEUTRON SOURCE STRENGTH

\begin{tabular}{c|c|c}
\hline Radioisotope & $\begin{array}{c}\text { Source Strength } \\
\text { (n/sec-watt) }\end{array}$ & $\begin{array}{c}\% \text { of Neutrons } \\
\text { from }(\alpha, \mathrm{n})\end{array}$ \\
\hline $\mathrm{Po}^{210}$ & $3.22 \times 10^{3}$ & 100 \\
$\mathrm{Pu}^{238}$ & $5.18 \times 10^{4}$ & 91 \\
$\mathrm{Cm}^{242}$ & $4.0 \times 10^{5}$ & 46.5 \\
$\mathrm{Cm}^{244}$ & $4.55 \times 10^{6}$ & 3 \\
\hline
\end{tabular}

\section{b. Shield Analysis}

For preliminary work, Figures III-4 through III- 8 present parametric shielding curves for $\mathrm{Co}^{60}, \mathrm{Sr}^{90}, \mathrm{Pm}^{147}, \mathrm{Po}^{210}$, and $\mathrm{Pu}^{238}$. These curves do not include self-shielding, thus they should give conservative shield thicknesses. These curves present the thickness of uranium (or other shield materials) and 1ithium hydride as a function of a shielding parameter $D S^{2} / P$, and in 
TABLE III- 6

GAMMA RAY SOURCE SPECTRUM

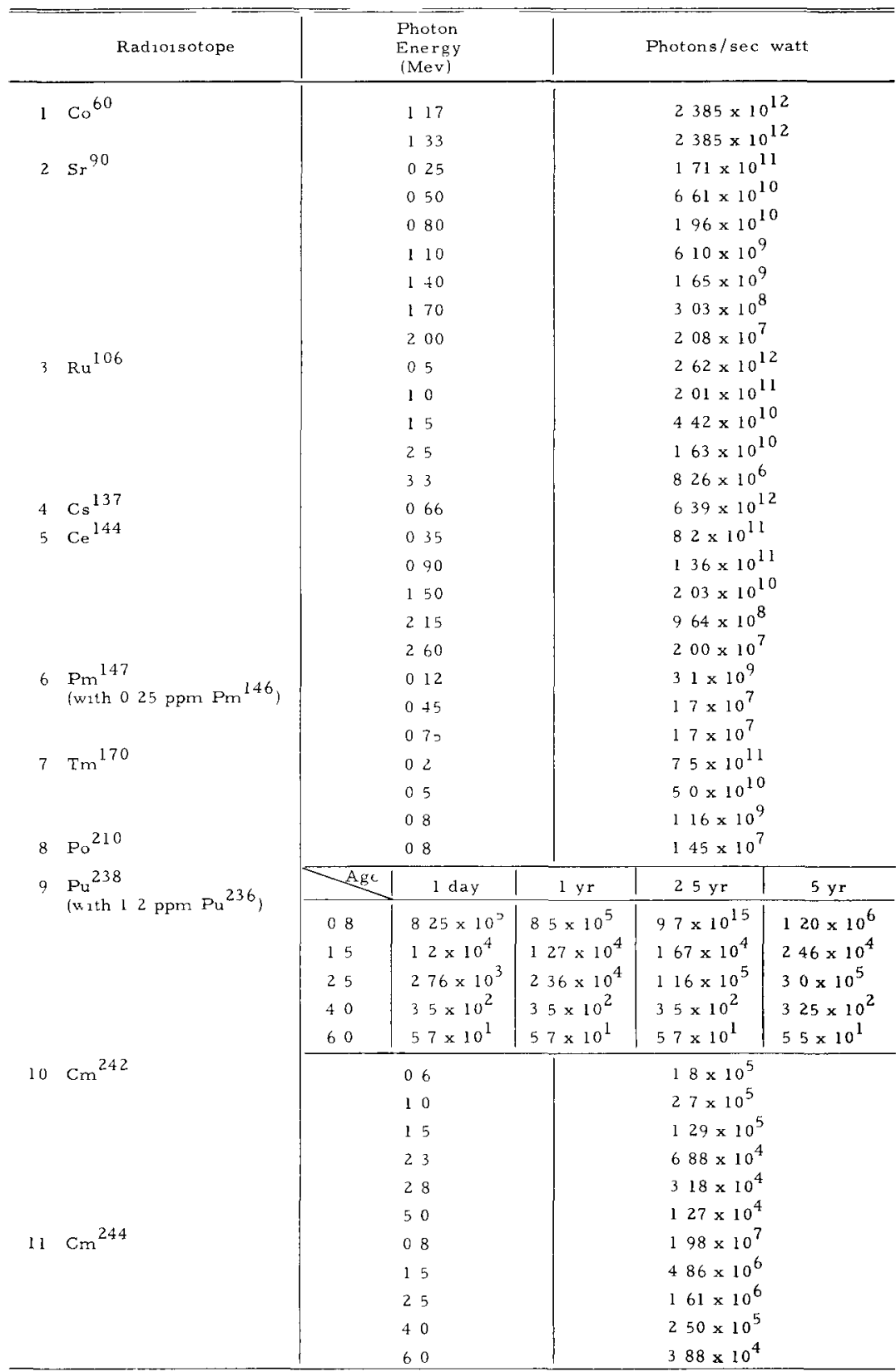




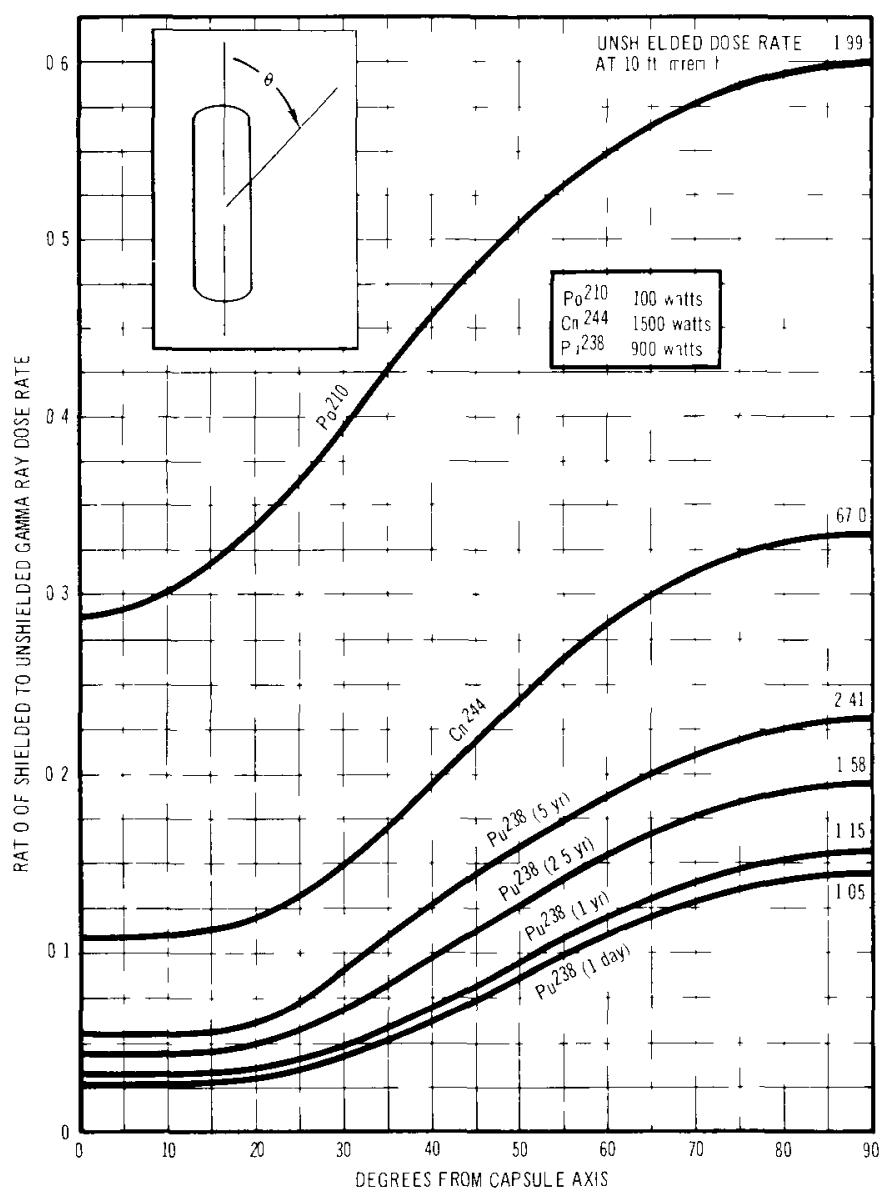

7 S7 192133

Figure III-3. Effect of Self-Shiclding on Gamma Ray Dose Rate for Capsules 


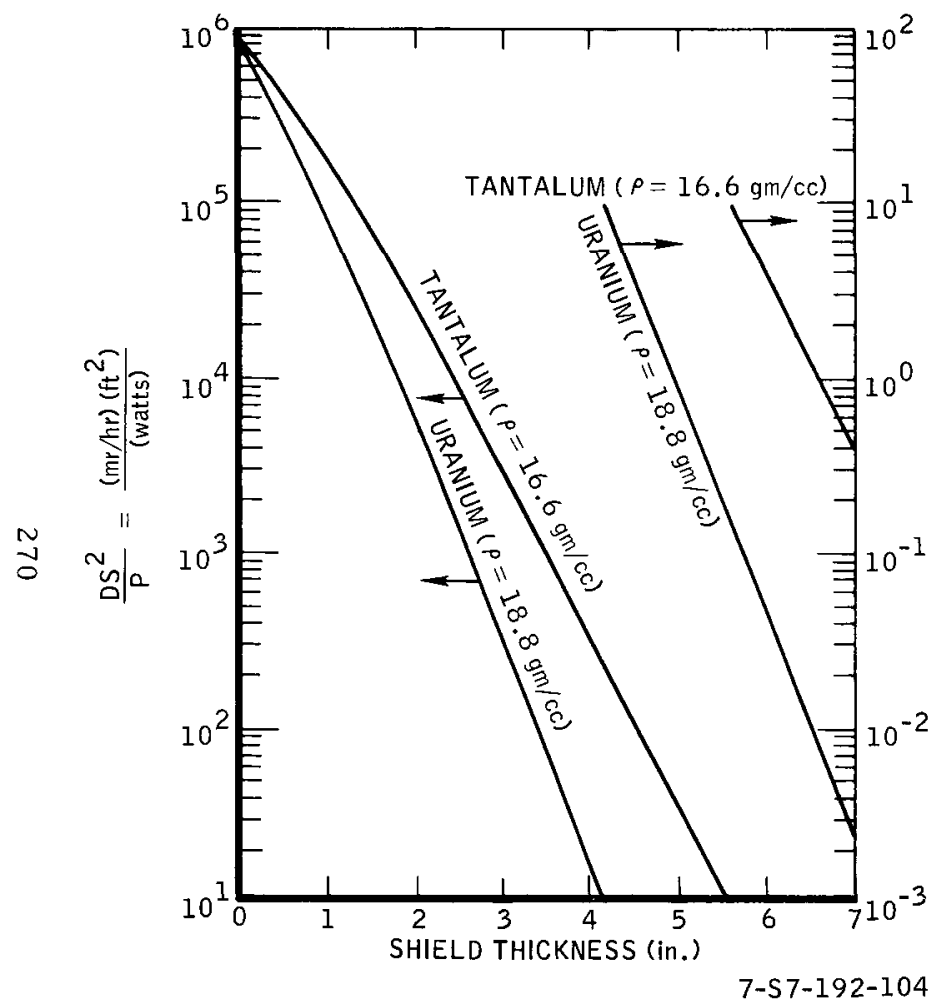

Figure III-4. Parametric Shielding for Co 60

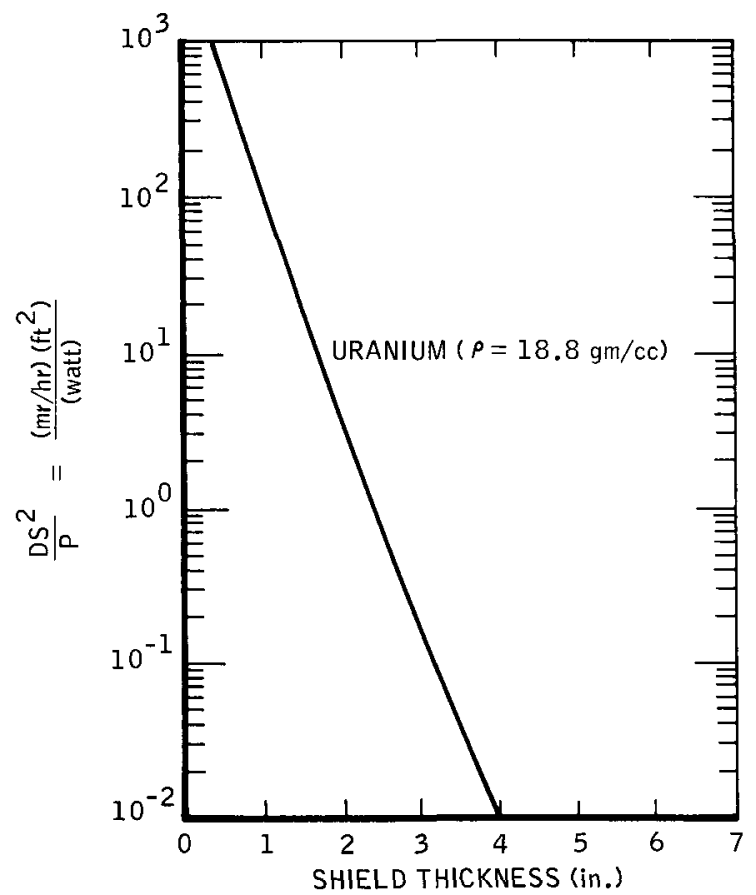

7-S7-192-105

Figure III-5. Parametric Shielding for $\mathrm{Sr}^{90}\left(\mathrm{SrTiO}_{3}\right)$ 


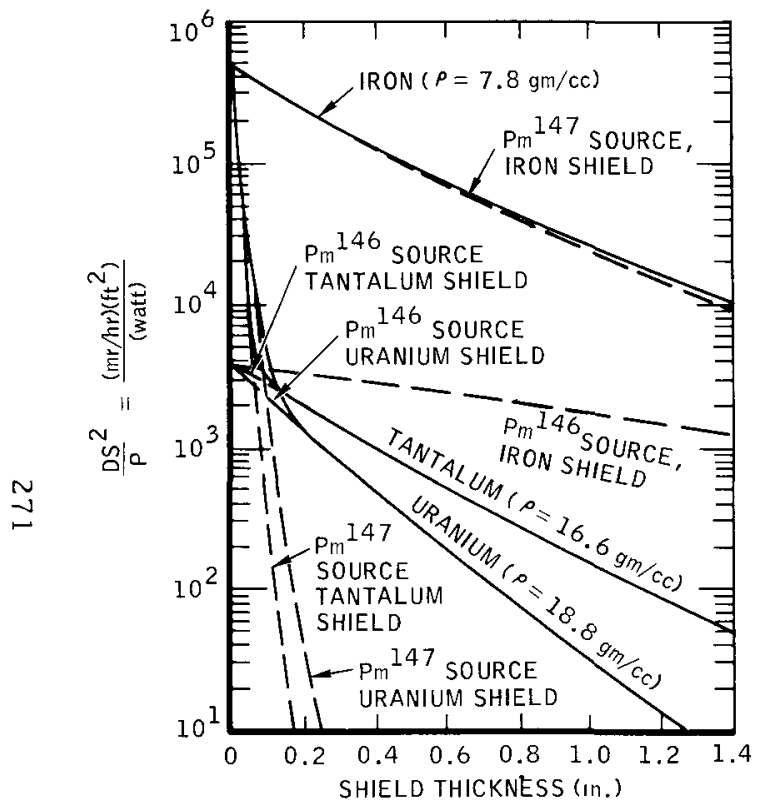

7-S7-192-106

Figure III-6. Parametric Shielding for Pml47 with 0.25 ppm Pml46, No $\operatorname{Pm} 148$

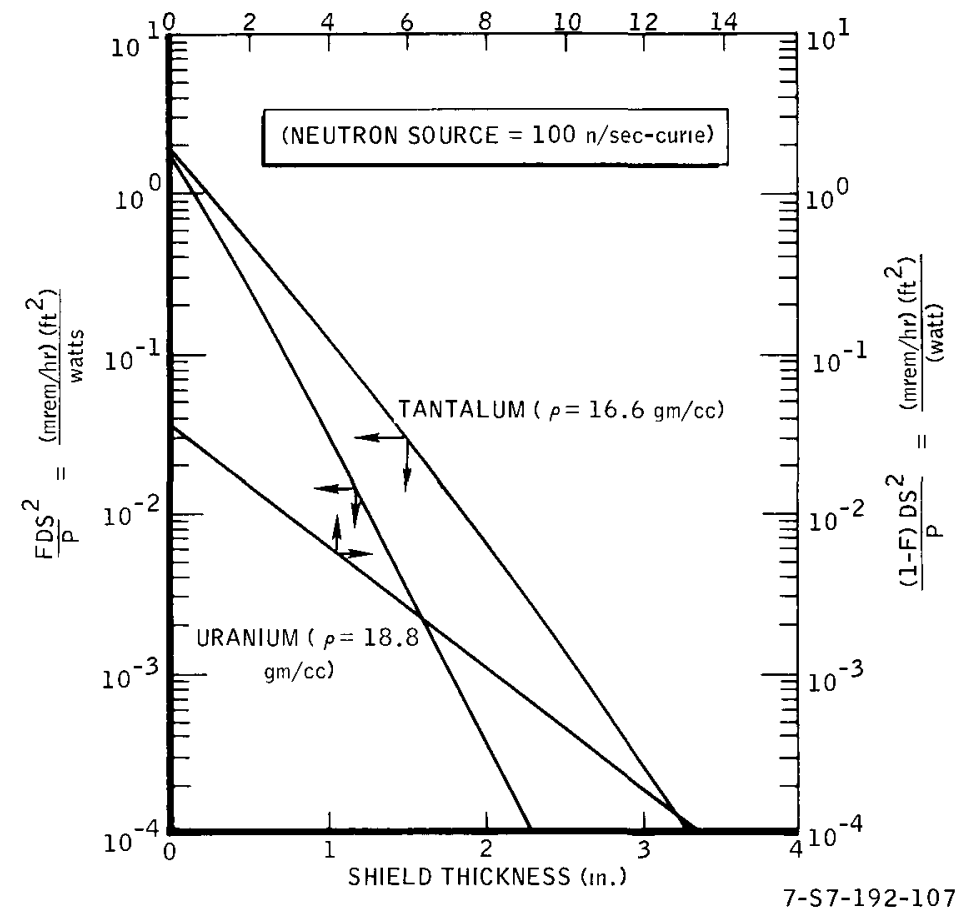

Figure III-7. Parametric Shielding for $\mathrm{Po}^{210}$ 


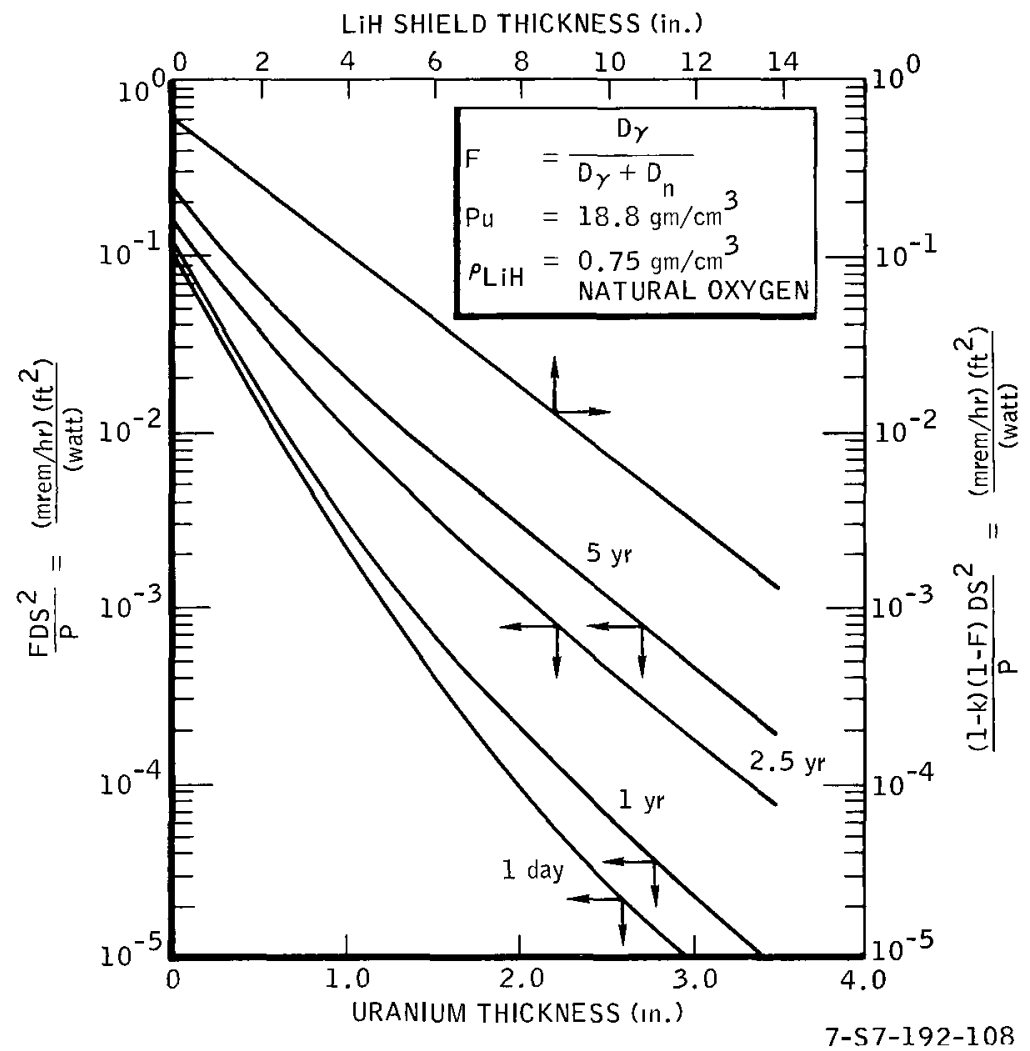

Figure III-8. Parametrıc Shieldıng for $\mathrm{Pu}^{238}$ 
some cases this parameter is multiplied by, F or $(1-F)$ and $(1-k)$. The terms are defined below

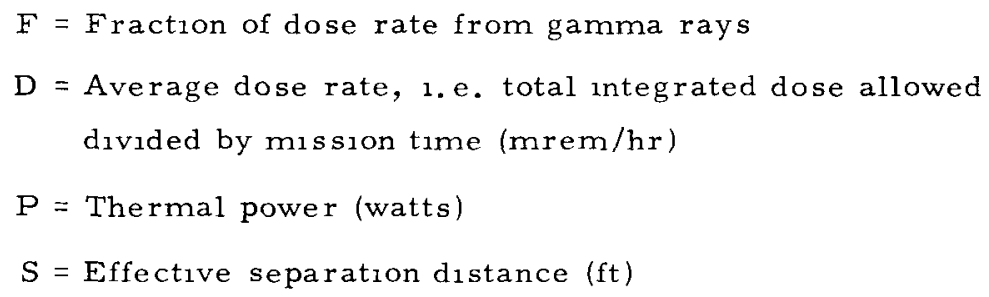

The $(1-k)$ term is only used for the $\mathrm{Pu}^{238}$ neutron shield thickness, and it is the correction for the neutron subcritical multiplication factor. It is not used for the gamma shield thickness since the dose rate from spontaneous fission and induced fission gamma rays is much less than that of decay gamma rays of $\mathrm{Pu}^{238}$ (and $\mathrm{Pu}^{236}$ ).

For both $\mathrm{Po}^{210}$ and $\mathrm{Pu} 238$, using a value of 0.7 for "F" will result in a minimum weight. This applies for slab geometry and could be used for preliminary work. Figures III-4 to III-8 may be used as follows

1) For $\mathrm{Co}^{60}, \mathrm{Sr}^{90}, \mathrm{Pm}^{147}$, calculate $\mathrm{DS}^{2} / \mathrm{P}$, and from Figures III-4 to III-6, the shield required may be determined. 2a) For Po 210 , using Figure III-7 calculate $(1-F) D S^{2} / P$, where $F$ is equal to 0.70 . If this quantity is less than $3.9 \times 10^{-2}$, then calculate $\mathrm{FDS}^{2} / \mathrm{P}$ and determine the uranium 
(or tantalum) and the lithium hydride shield thicknesses required.

2b) If the expression (1-F)DS $\mathrm{S}^{2} / \mathrm{P}$ is greater than $3.9 \times 10^{-2}$, and the expression $D S^{2} / P$ is less than 1.9 , then no lithium hydride shield will be required, and the uranium for tantalum) shield thickness should be determined by calculating the following

$$
\frac{F^{1} D S^{2}}{P}=\frac{D S^{2}}{P}-3.9 \times 10^{-2}
$$

where,

$$
\mathrm{F}^{1}=\text { effective fraction of dose from gamma rays. }
$$

Then using this expression in Figure III-7 will yield the uranium (or tantalum) shield thickness required.

2c) If $\mathrm{DS}^{2} / \mathrm{P}$ is greater than 1.937 , no shield will be required at all.

3a) For $\mathrm{Pu}^{238}$, calculate $\mathrm{FDS}^{2} / \mathrm{P}$, again $\mathrm{F}$ is equal to 0.7 . If this quantity is less than 0.17 (assuming $\mathrm{Pu}^{238}$ aging of 2.5 years), then use Figure III-8.

3b) If the expression $F D S^{2} / P_{1 s}$ greater than 0.17 and the expression $(1-k) D S^{2} / P$ is less than 0.61 , then no uranium shield will be required. The lithium hydride shield thickness should be determined by calculating the following

$$
(1-k)\left(1-F^{l}\right) \frac{D S^{2}}{P}=(1-k)\left(\frac{D S^{2}}{P}-0.17\right)
$$

Then using this expression in Figure III- 8 will yield the 1ithıum hydride thickness.

$3 c)$ If the expression $(1-k) D s^{2} / P$ is greater than 0.78 , no shield will be required at all. 


\section{c. Shield Example}

An example is presented to show the comparative shielding required for these radioisotopes. Consider a 5000-watt radioisotope heat source, where the dose rate requirement is $5 \mathrm{mrem} / \mathrm{hr}$ at a separation distance of $10 \mathrm{ft}$. Using Figures III-4 to III-8, Table III- 8 presents the shielding required.

TABLE III-8

SHIELD REQUIREMENT FOR A 5000-WATT SOURCE AND $5 \mathrm{mrem} / \mathrm{hr}$ AT $10 \mathrm{FEET}$

\begin{tabular}{l|c|c|c|c|c}
\hline \hline & $\mathrm{Co}^{60}$ & $\mathrm{Sr}^{90}$ & $\mathrm{Pm}^{147}$ & $\mathrm{Po}^{210}$ & $\mathrm{Pu}^{238}$ \\
\hline $\begin{array}{c}\text { Uranium Thickness } \\
\begin{array}{c}\text { (1n.) } \\
\text { LiH Thickness } \\
(1 \mathrm{n} .)\end{array}\end{array}$ & 5.73 & 3.2 & 0.74 & 0.8 & 0.29 \\
\hline
\end{tabular}

Th1s applies to $\mathrm{Pu}^{238}$ aged 2.5 years. If it 1 s aged 1 day, 1 year and 5 years, the uranium shielding required will be $0.13,0.15$, and $0.48 \mathrm{in}$. respectively.

It may be noted that natural oxygen was used for the $\mathrm{Pu}^{238} \mathrm{O}_{2}$. If pure $\mathrm{O}^{16}$ were used to make $\mathrm{Pu}^{238} \mathrm{O}_{2}^{16}$, then the lithium hydride shield of $6.9 \mathrm{~m}$. could be reduced to only $0.9 \mathrm{~m}$.

\section{E. CRITERIA FOR RADIOISOTOPE SELECTION}

Criteria used in the selection of a radioisotope for a heat source will depend on the application. Screening through various radioisotopes, selecirion will be based on the following.

\section{a. Half-Life}

The half-life of the radioisotope has to be sufficiently long such that the beginning-of-1ife (BOL) power is not much greater than the end-of-1ife (EOL) thermal power required. If short halflife radioisotopes are used, power (or temperature) flattening devices will be required, which will reduce the reliability of the heat source. Stockpiling, encapsulation time, and mission delay time in addition to the mission time should be considered. 


\section{b. Power Density}

High power density is preferred since low fuel volume results in a low weight shield. The power density should be greater than 0.1 watts $/ \mathrm{cm}^{3}$ in order to keep the heat source to a reasonable size.

\section{c. Fuel Composition}

A fuel form should be selected that is non-corrosive, compatible with structural materıals, stable in time, and insoluble in water. It should also exhibit good chemical stability and good engineering properties at high temperatures. Table III-1 presents the recommended fuel forms for the candidate radioisotopes.

\section{d. Physical Properties}

The fuel has to have a high melting point, dimensional stability, and low vapor pressure. These properties are important both during operation and for safety analysis.

\section{e. Shielding Requirement}

Depending on the application, 1.e., manned or unmanned, the shielding requirement is extremely important. The radioisotope selection should consider the shielding required, since this could vary by a few orders of magnitude in weight. Again, economic factors and weight constraints may influence the selection of the radioisotope.

\section{f. Cost and Avarlability}

The radioisotope must be avallable in quantities desired, at the time specified and at a reasonable cost. Fuel costs must be competitive with other energy sources. The cost of radioisotopes is high per watt, however, when one considers more farrly the cost per watt-year, and, further, when the rellability is also taken into account, a more accurate assessment of radioisotopes can be made. 


\section{g. Nuclear Safety Considerations}

Radiolsotope heat sources represent a potential ha 2 ard since large quantities of radioactive materials must be concentrated in a small volume. Due to this potential danger, one of the most important parts of the radioisotope heat source design is to eng1neer the capsules such that a solution of the nuclear safety problem is achieved.

When a radiolsotope is to be selected for a particular application, all the above criteria have to be considered. Screening through the various potential radiolsotopes, one is selected which 1s usually a compromise. Once the radioisotope is selected, then the engineering should include the nuclear safety aspects.

\section{F. NUCLEAR SAFETY}

Safety considerations play an important part in the choice of radiorsotopes for space, land, or underwater applications. An accidental release of radioisotopes might create a hazardous condition in localized areas. Because of this potential danger, an important part of every radioisotope heat source program is to evaluate and solve the nuclear safety problem.

There are three basic general design guidelines

1) Design for complete containment of radioisotope under all conditions.

2) If complete containment is impossible, design for dispersal and diffusion of the fuel such that it is within the maximum permissible concentration.

3) Define an exclusion area where accidental releases may occur (such as launch pads).

For space missions, nuclear safety analysis has to be performed from the time the radiolsotope is produced up to the time capsules are disposed of. 


\section{a. Fuel Capsule Fabrication}

This phase includes the radioisotope production to the final acceptance testing of the capsules. During all this period it has to be assured that personnel do not receive high radiation levels during normal and accidental conditions (such as spills). In this period, the radioisotope is purified, usually pressed and sintered, encapsulated, welded, leak checked, and acceptance tested.

\section{b. Transportation to Launch Site}

When the capsules are transported to the launch site, safety analysis is performed to assure that in case of an accident or fire, the capsules will not break, releasing the fuel.

\section{c. Prelaunch Activities}

Again, safety analysis is made for each phase of transporting the capsules from the vehicle that brought them to their installation in powe $r$ generator. The major potential accident modes are

a) Loss of fuel capsule cooling.

b) Inadvertent dropping of fuel capsules.

c) Bending of fuel capsule in the loading device, resulting in abnormal mechanical forces which could rupture the capsules.

\section{d. Flight Safety}

Flight safety includes from launch to post reentry of fuel capsules. In the launch to orbit injection phase, the major potential accidents are

a) Propellant explosion and/or fire

b) Corrosive attack by missile fuel, fresh water, salt water, or atmosphere

c) Thermal shock 
d) Mechanical shock due to high-velocity impact on a varıety of surfaces like concrete, soll, rock, water, etc.

e) Atmospheric heating and ablation forces.

f) Excessively high fuel capsule temperature due to earth or vehicle debris insulation.

In the mission operational phase, the principal operational accident mode for fuel capsule rupture is loss of cooling.

In the reentry phase, two principal accident modes must be considered.

a) Loss of fuel material containment during reentry due to aerodynamic heating and ablation forces, with significant loss of fuel material to the atmosphere prior to impact.

b) Loss of fuel material containment at impact.

In the post-reentry phase, assuming fuel containment through impact, the next major concern is the possibility of subsequent capsule rupture due to more long-term post-impact effects such as corrosion of capsule wall on land or sea, and soll burial.

All the above have to be analyzed with probabilities of mal functions and fuel released, the quantities and locations of fuels that might be released, and their blological impact on the population. If maximum permissible concentration (MPC) of that par ticular radioisotope in air and water has not been exceeded, furthermore if the dose to the general population has not exceeded the maximum permissible exposures to external radiation, then the mission will be acceptable. 


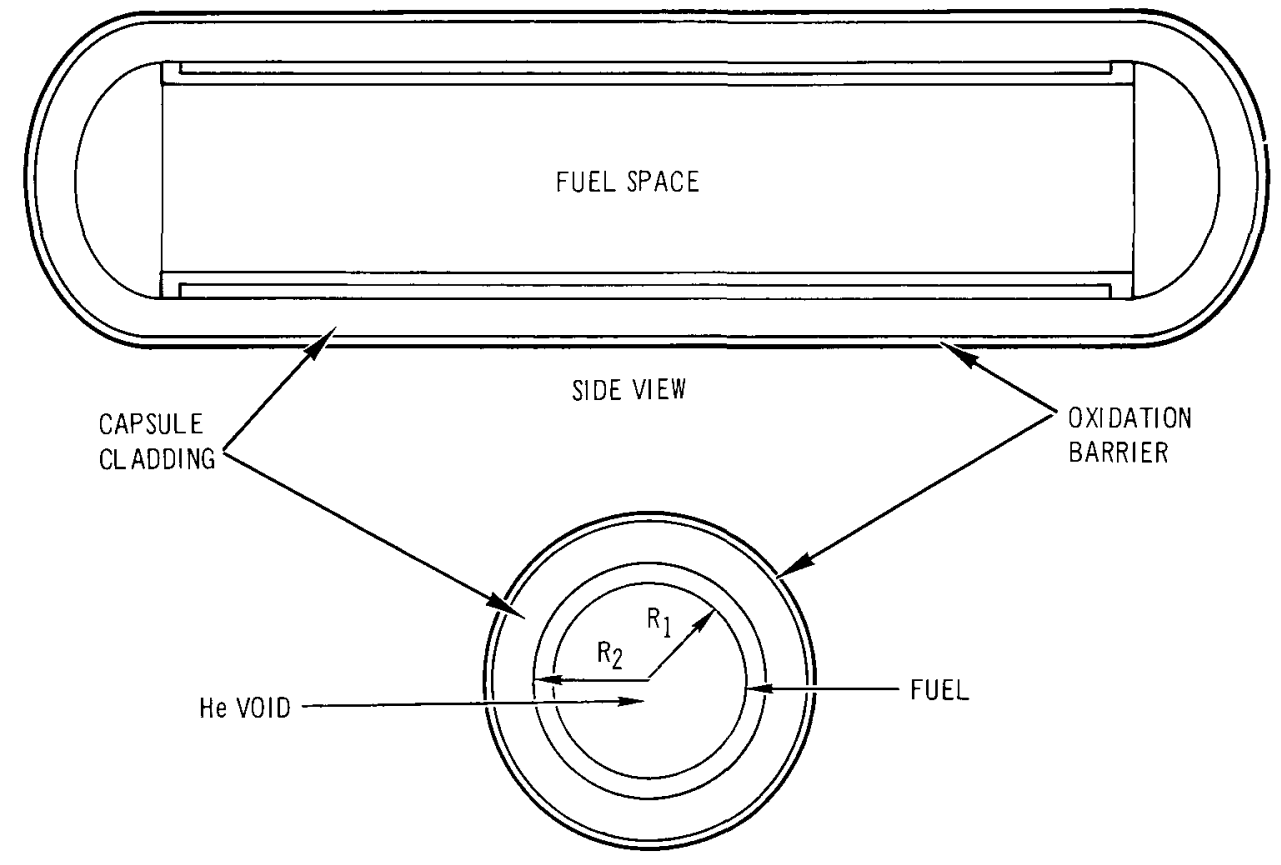

FRON T VIEW

Figure III-9. Typical Capsule 


\section{RADIOISOTOPE CAPSULE DESIGN}

The practical application of radioisotopes requires containment of the radioactive material. This containe $r$ is referred to as a capsule. The major goal of the capsule analysis and design is to evolve a minimal weight design that satisfies the environmental requirements.

A typical capsule is comprised of the fuel, a cladding designed to contain it, and a protector for the cladding as shown in Figures III-9 and III-10. For high temperature advanced heat sources, refractory metals are used for the claddings, and the protector serves as an oxidation barrier for the se readily oxidized materials. Because of the possible material solubility of the refractory metals and the oxidation protector, a barrier is

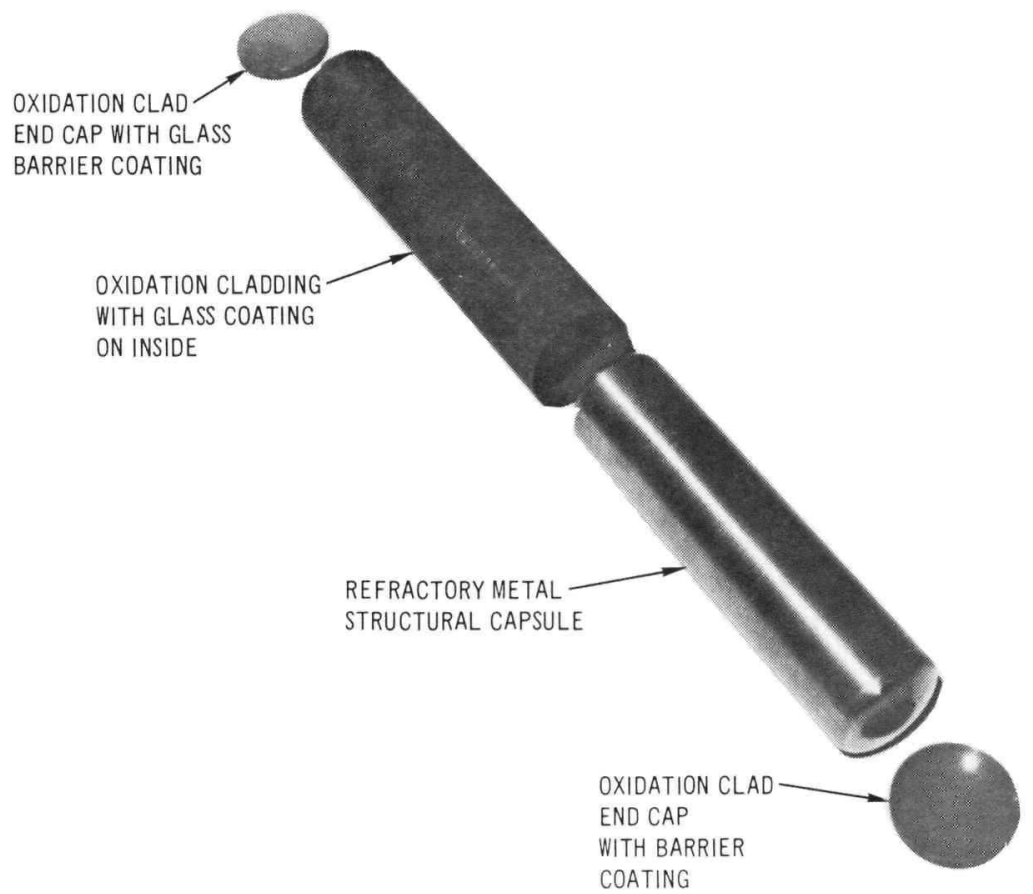

6090-5196 CNA

Figure III-10. Prototype Refractory Alloy Capsule 


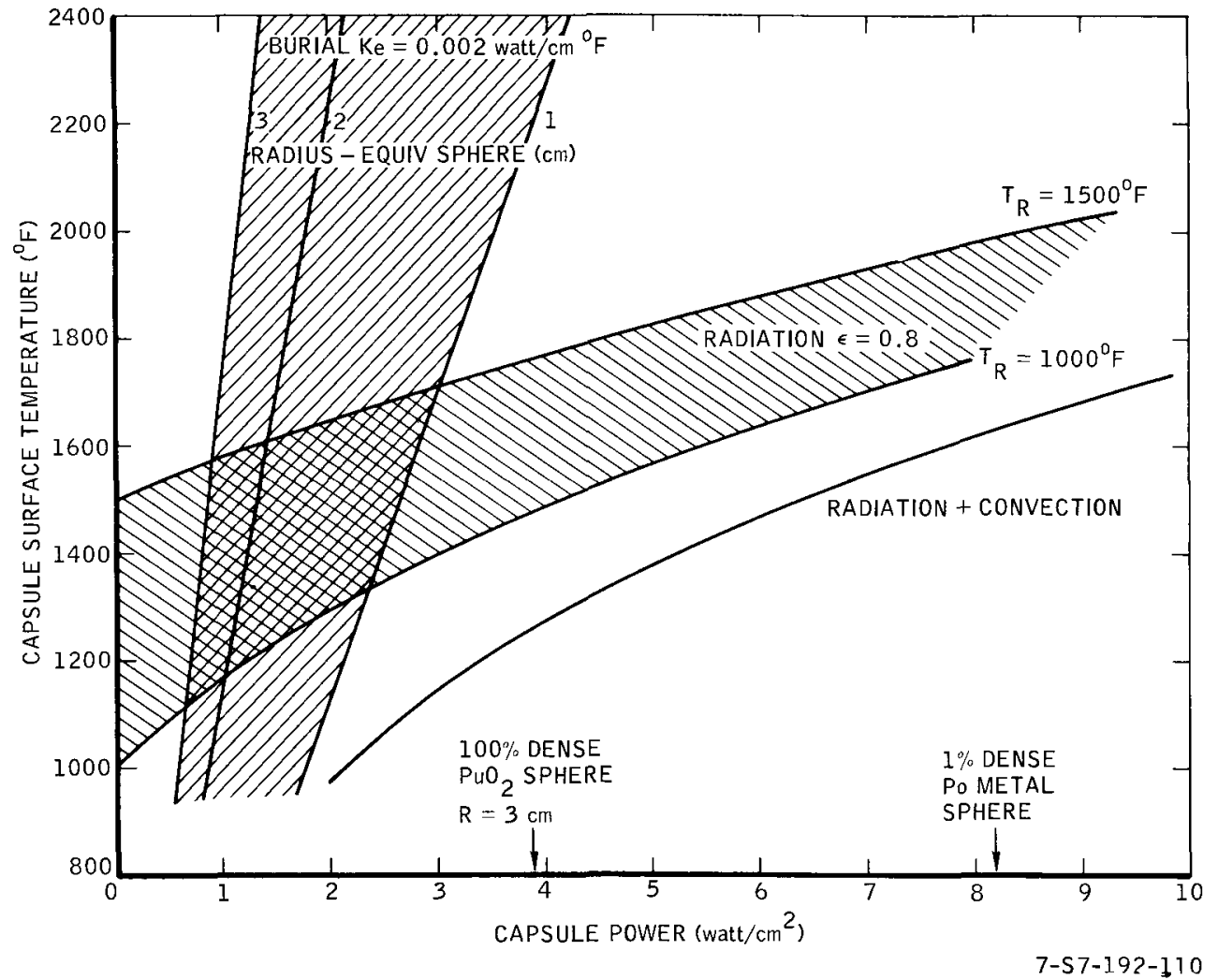

Figure III-11. Capsule Temperature as a Function of Surface Power Density 
placed between the two to prevent interdiffusion. For normal operation, the helium buildup is the most important single factor in determining capsule weight. This weight is dependent on vold-tofuel volume, capsule size, mission duration, and operating temperature.

\section{A. THERMAL ENVIRONMENT \\ 1. Normal Operation}

In large radiolsotope systems, operational requirements to load and unload the fuel during system checkout, delivery, and launch pad operations require radiative coupling between the fuel capsules and the heat receiving member of the power conversion subsystem. The capsule operatıng temperature is determined by the power density, the surface emissivity, and the conversion cycle recelver temperature (for example, the boile $r$ in a rankine cycle). The operating temperature can be calculated from the familiar

$$
\frac{\mathrm{P}}{\mathrm{A}}=\sigma \epsilon\left(\mathrm{T}_{\mathrm{C}}^{4}-\mathrm{T}_{\mathrm{R}}^{4}\right)
$$

where

$$
\begin{aligned}
\mathrm{P} & =\text { capsule power } \\
\mathrm{A} & =\text { capsule area } \\
\sigma & =\text { Boltzmann constant } \\
\epsilon & =\text { emissivity } \\
\mathrm{T}_{\mathrm{C}} & =\text { capsule temperature } \\
\mathrm{T}_{\mathrm{R}} & =\text { receiver temperature. }
\end{aligned}
$$

Capsule temperature as a function of surface power density for recelver temperatures of 1000 and $1500^{\circ} \mathrm{F}$ is shown in Figure III-11. This sample calculation used a surface emissivity of 0.8 which is representative of metal oxides at the indicated temperatures. 


\section{In Air}

During ground handling operations, when exposed to convective cooling to the atmosphere, the capsule temperatures will be less than the normal operating temperature defined by radiative heat transfer. In the atmosphere the temperature is determined from

$$
\frac{P}{A}=\sigma \epsilon\left(T_{C}^{4}-T_{R}^{4}\right)+h\left(T_{C}-T_{a}\right)
$$

where

$$
\begin{aligned}
& h=\text { convective heat transfer coefficient } \\
& T_{a}=\text { ambient temperature. }
\end{aligned}
$$

Capsule temperature as a function of surface power density is shown in Figure III-11 for a representative natural convection heat transfer coefficient of $1 \mathrm{Btu} / \mathrm{hr}-\mathrm{ft}^{2}-{ }^{\circ} \mathrm{F}$.

Even though the capsule temperature is lower in air than in vacuum, the temperature limit of the capsule materials can be equally or more restrictive due to consideration of oxidation.

\section{Earth Burial}

In the event of capsule impact into soft soil at terminal velocity, the capsule will bury to distances of a few inches to a few feet depending upon the capsule velocity, its attitude, and the properties of the soil. Since dry soil has a low thermal conductivity, of the order of $0.2 \mathrm{Btu} / \mathrm{hr}-\mathrm{ft}-{ }^{\circ} \mathrm{F}$, the capsule is virtually insulated and its surface temperature rises accordingly.

It is impossible to obtain a closed analytical solution for the surface temperature of a buried finite cylindrical isotope capsule. It is, however, simple to obtain the solution for a buried sphere. The temperature distribution around an infinitely buried source of power, $P$, is:

$$
T(r)=\frac{P}{4 \pi K r}+T_{a}
$$


where

$$
\begin{aligned}
T(r) & =\text { soil temperature at distance } r \\
r & =\text { distance from source } P
\end{aligned}
$$

The surface temperature of a spherical capsule of radius $R_{C}$ is:

$$
\mathrm{T}_{\mathrm{C}}=\frac{\mathrm{P}}{4 \pi \mathrm{KR}_{\mathrm{C}}}+\mathrm{T}_{\mathrm{a}}
$$

The temperature of spherical capsules of various radii are shown as a function of capsule surface power density in Figure III- 11.

The temperature distribution around a finite cylinder has been calculated by means of a digital computer code. The normalized temperature distribution around a typical Po ${ }^{210}$ capsule is shown in Figure III-12. It was found that the temperature of an equivalent sphere, i.e., same area and power, agreed with

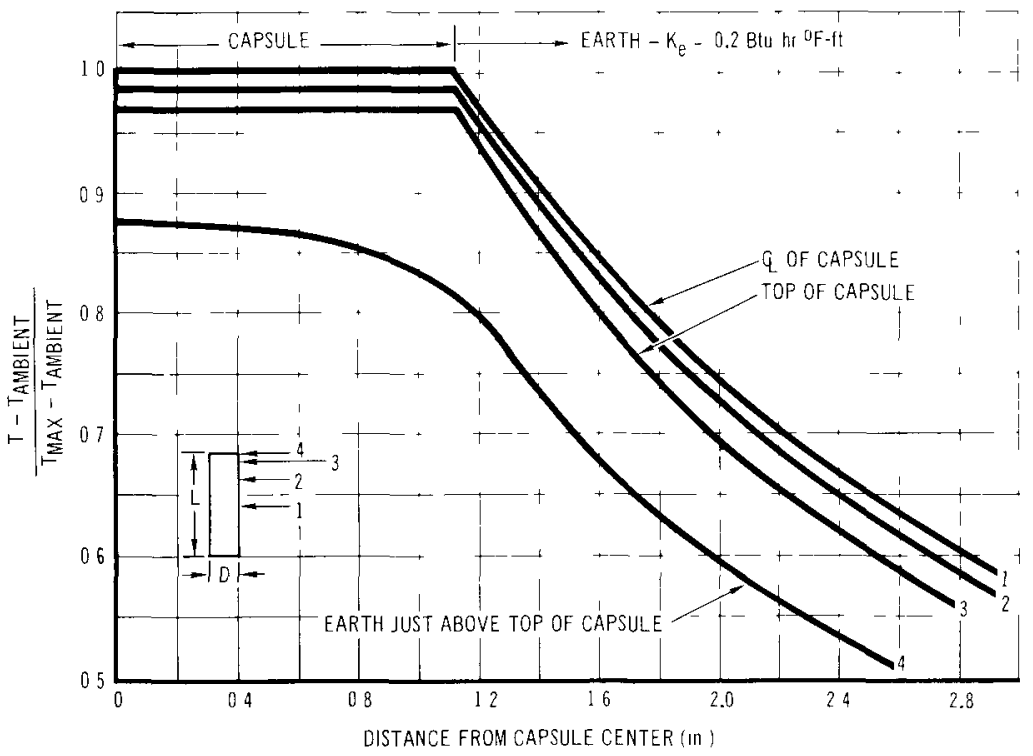

7-\$7-192-135

Figure III-12. Normalized Temperature Distribution Around Infinitely Buried Capsule - Computer Results 
the computer results for cylindrical capsules when reduced by a factor of 1.15. The agreement without the normalization is probably a lesser error than in the soil conductivity variations.

The high temperatures that can result from earth burial introduce critical requirements for capsule compatibility with near molten soll, and for oxidation resistance.

\section{Summary}

It can be seen from Figure III- 1 that the most stringent thermal environment can be imposed in the event of capsule burial in dry soil. The temperature and compatibility limits of the applicable materials can restrict allowable capsule surface power density if earth burial is a credible accident. The penalty is not great in the case of the low power density $\mathrm{Pu}^{238}$ but is a severe restriction on the allowable loading for $\mathrm{Po}^{210}$.

\section{B. PRESSURE BUILDUP}

The alpha particle is an energetic, ionized helium atom, thus energy release through alpha decay results in He gas generation and pressure bullup in a closed container. Since the alpha emittıng 1 sotopes are all of about the same energy, 1.e., about $5.5 \mathrm{Mev}$, the helium production per watt-sec of energy is about the same. Since $1 \mathrm{Mev}=1.6 \times 10^{-13}$ watt-sec, each alpha partrcle yields about $8.8 \times 10^{-13}$ watt-sec

or

$$
1 \text { watt-sec } \cong 1.15 \times 10^{12} \text { helium atoms }
$$

or

$$
1 \text { watt-sec }=\frac{1.15 \times 10^{12}}{6.03 \times 10^{23}}=1.9 \times 10^{-12} \text { moles of He gas }
$$

The integral of energy produced by a radioisotope source of power $P_{0}$ at time zero is

$$
\int P(t) d t=\int P_{0} e^{-t / \tau} t=P_{0} \tau\left(1-e^{-t / \tau}\right)
$$


where

$$
\begin{aligned}
\tau & =\frac{T_{1 / 2}}{0.6925} \text { sec } \\
T_{1 / 2} & =\text { the 1sotope half-life-sec. }
\end{aligned}
$$

The gas production, $n(t)$, per watt as a function of time is

$$
\frac{n(t)}{P_{o}}=\left(1.9 \times 10^{-12}\right) \tau\left(1-e^{-t / \tau}\right) \text { moles }
$$

and the total gas evolution at $t>\tau$ is

$$
\frac{{ }^{n}}{\mathrm{P}_{0}}=\left(1.9 \times 10^{-12}\right) \tau \text { moles of } \mathrm{He} \text {. }
$$

The pressure as a function of time can be calculated from the gas law

$$
P V_{\mathrm{V}}=\mathrm{nRT}_{\mathrm{C}}
$$

where

$$
\begin{aligned}
\mathrm{P} & =\text { capsule pressure } \\
\mathrm{V}_{\mathrm{V}} & =\text { capsule vold volume } \\
\mathrm{R} & =\text { gas constant } \\
\mathrm{n}(\mathrm{t}) & =\text { moles of He generated at time }(\mathrm{t}) \\
\mathrm{T}_{\mathrm{C}} & =\text { capsule temperature. }
\end{aligned}
$$

The gas generation rate is directly proportional to the capsule power and is essentially independent of the alpha isotope considered. The total gas generated by a given initial capsule power is, however, directly proportional to the isotope half-life.

Since the atom density of the various fuel forms is about the same, the total gas generated per unit volume of fuel is about the same. Specifically 


$$
\frac{n(t)}{V_{F}}-\left(1.9 \times 10^{-12}\right) S \tau\left(1-e^{-t / \tau}\right)
$$

where

$$
\begin{aligned}
S & =\text { fuel power density (watts/cc) } \\
V_{F} & =\text { fuel volume }
\end{aligned}
$$

The pressure buildup $\mathrm{P}(\mathrm{t})$ as a function of time at standard temperature $\left(273^{\circ} \mathrm{K}\right)$ is then

$$
P(t) \cong \frac{S \tau\left(1-F_{V}\right) V_{O}\left(1.9 \times 10^{-12}\right)}{F_{V}}\left(1-e^{-t / \tau}\right)
$$

where

$$
\begin{aligned}
& \mathrm{V}_{\mathrm{O}}=\text { molal volume at } 273^{\circ} \mathrm{K}, 22,400 \mathrm{cc} \\
& \mathrm{F}_{\mathrm{V}}=\text { capsule vold fraction }
\end{aligned}
$$

For plutonium

$$
P(t)=650 \frac{\left(1-F_{V}\right)}{F_{V}}\left(1-e^{-t / \tau}\right) \text { atmos. }
$$

The ultimate pressure is almost independent of the alpha 1sotope in question but is a direct function of the capsule void fraction. The time to reach the ultimate pressure is proportional to the isotope half-life (1. e. about $3 \tau)$.

C. TEMPERATURE AND PRESSURE VS TIME

The power, temperature, and pressure of the capsule are all varyıng with time. For example, since

$$
\frac{P}{P_{0}}=e^{-t / \tau}
$$

the temperature, under radiative heat rejection is 


$$
\frac{T}{T_{0}}=\left(\frac{P}{P_{0}}\right)^{1 / 4}=e^{-t / 4 \tau}
$$

and the gas buildup is

$$
\frac{n}{n_{\infty}}=1-e^{-t / \tau}
$$

The capsule pressure is proportional to nT or

$$
\frac{P}{P_{\infty}}=\frac{n}{n_{\infty}} \frac{T}{T_{0}}=\left(1-e^{-t / \tau}\right) e^{-t / 4 \tau}
$$

This function reaches a maximum at $t / \tau=1.6$. The time dependent behavior of a radiatively cooled capsule is illustrated in Figure III-13. A conduction cooled capsule reaches maximum pressure at $t / \tau=1$.

\section{FUEL FRACTION}

The capsule temperature is a function of cooling regime and fuel loading. The capsule gas pressure buildup is primarily a function of fuel or void fraction. The relative importance of the temperature and pressure dependence on fuel fraction can be 11 lustrated by the example shown in Figure III-14. The capsule is a $3-\mathrm{cm}$ radius sphere with a wall thickness of $0.5 \mathrm{~cm}$. As discussed earlier, the ultimate pressure is almost independent of fuel form, i.e. $\mathrm{Pu}^{238}$ or Po 210 . The power density difference, however, has a marked effect on the allowable fuel fraction for a given temperature limit. From Figure III-14 it can be seen that $\mathrm{Po}^{210}$ capsules are strongly heat transfer limited and pressure buildup is not a severe problem. However, $\mathrm{Pu}^{238}$ capsules are probably pressure limited. It should be noted that the long halflife of $\mathrm{Pu}^{238}$ also results in pressure at high temperature for at least a factor of 100 longer duration than in the case of a $P_{0} 210$ capsule. 


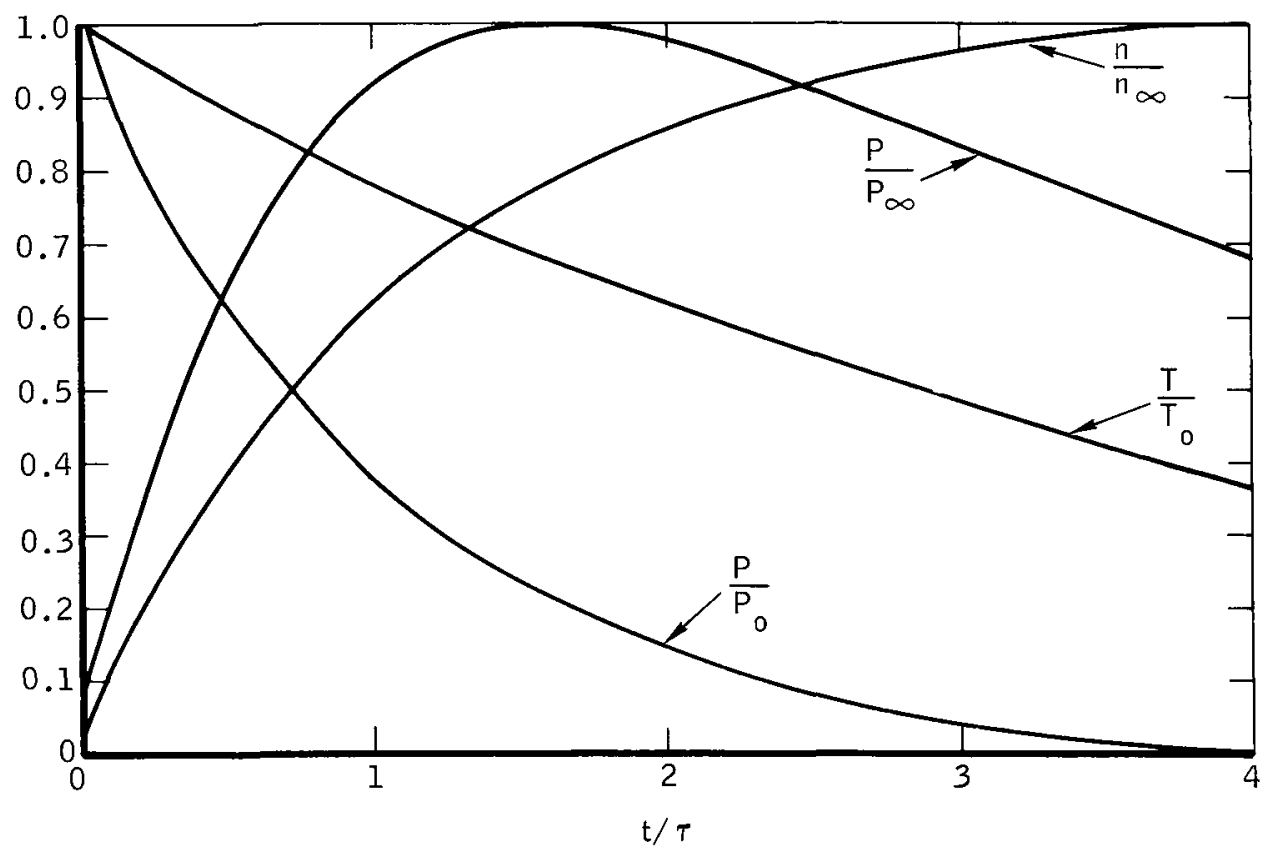

7-57-192-111

Figure III-13. Time Dependent Behavior of a Radiatively Cooled Reactor 


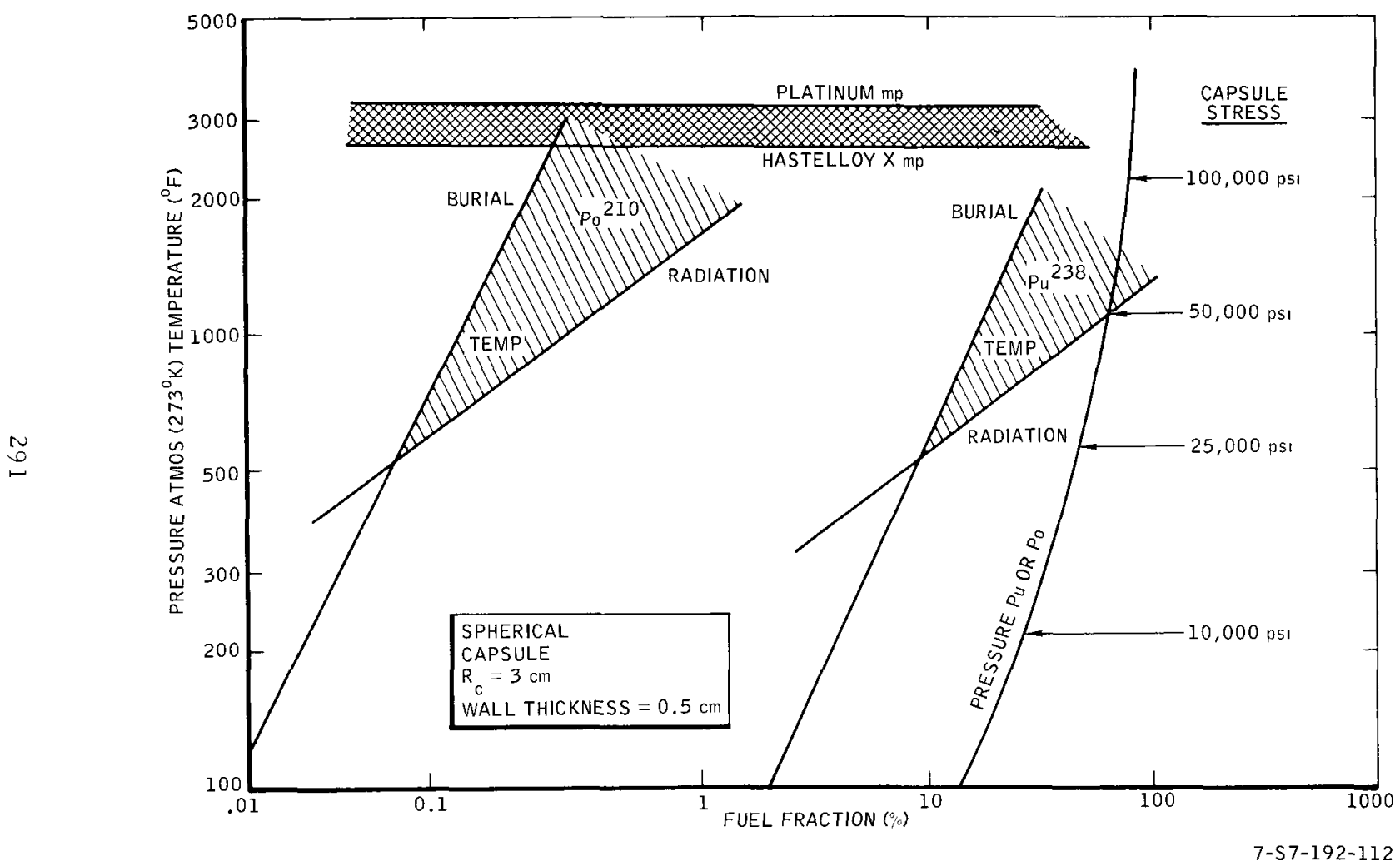

Figure III-14. Temperature and Pressure Dependence on Fuel Fraction 


\section{E. CONTAINER MATERIAL EVALUATION AND SELECTION}

The cladding which is to contain the fuel must meet all the performance and environmental requirements. Because of the high stress and temperatures that must be withstood for long periods of time, only the refractory metals are reasonable choices for the structural cladding. Since these metals oxidize rapidly, they must be protected by an oxidation-resistant alloy at all times. The use of a secondary cladding relieves the structural cladding of the oxidation and corrosion environmental requirements, but imposes a compatibility requirement with fuel and outer cladding. The outer cladding thus assumes the burden of resisting all the hostile environments and must remain intact even after impact on granite. This separation of the basic requirements will be used as the basis for the selection of the two claddings.

\section{Structural Container}

The bulldup of helium pressure at the operating temperature produces high stresses that must be withstood for at least the period of normal operation. At temperatures above about $1500^{\circ} \mathrm{F}$, the stress levels, temperature, and times eliminate all materials except alloys of the refractory metals columbium, molybdenum, tantalum, and tungsten. Tungsten alloys are all brittle, hard to fabricate and difficult to weld, and, since the re are no commercially available tungsten alloys in the required configuration, they can be eliminated from further consideration. $\mathrm{Cb}-752(\mathrm{Cb}-10 \mathrm{~W}$ $2.5 \mathrm{Zr}), \mathrm{TZM}(\mathrm{Mo}-0.5 \mathrm{~T}-0.08 \mathrm{Zr})$, and $\mathrm{T}-222(\mathrm{Ta} 9.6 \mathrm{~W}-2.4 \mathrm{Hf})$ are the best commercially avallable alloys of $\mathrm{Cb}, \mathrm{Mo}$, and $\mathrm{Ta}$, respectively, and the selection of a structural material is Iimited to a cholce between these three materials.

\section{a. Materials Properties}

Ultimate tensile strengths of the se three alloys are shown in Figure III-1 5 plotted against temperature in both the stressrelieved condition and the recrystallized condition. Both TZM 


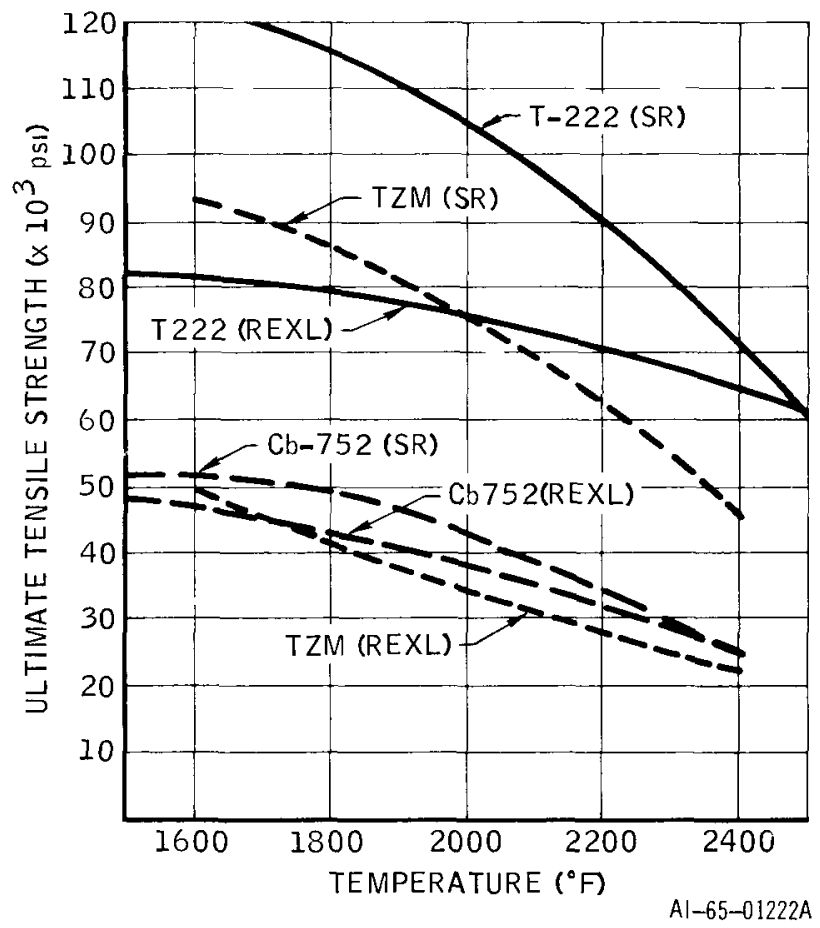

Figure III-15. Ultimate Tensile Strengths of $\mathrm{Cb}-752, \mathrm{TZM}$, and $\mathrm{T}-222$

and $\mathrm{T}-222$ are stronger than $\mathrm{Cb}-752$. This, plus the tendency for metallurgical instability of columbium alloys during longterm service, eliminated $\mathrm{Cb}-752$ from further consideration. Columbium alloys generally precipitate a second phase after long times at high temperatures. This second phase strengthens the alloy but also makes it brittle so that it probably would fracture on impact.

Long-term stress rupture strength, however, is much more important to capsule design than short-time ultimate tensile strength. Avallable stress rupture data for TZM and T-222 (plus forerunner alloys $\mathrm{Ta}-10 \mathrm{~W}$ and $\mathrm{T}-111$ ) have been obtained in from 10- to $1000-\mathrm{hr}$ tests at 2600 to $3000^{\circ} \mathrm{F}$. The Larson-Miller parameter can be used to extrapolate this data to the region of interest for fuel capsule design. A best fit to the existing data 
for TZM is shown in Figure III-16 and for T-222 in Figure III-17. From these figures, the stress rupture strength per unit weight for TZM and T-222 can be estimated. These estimates for 5.5year life are shown in Figure III-18.

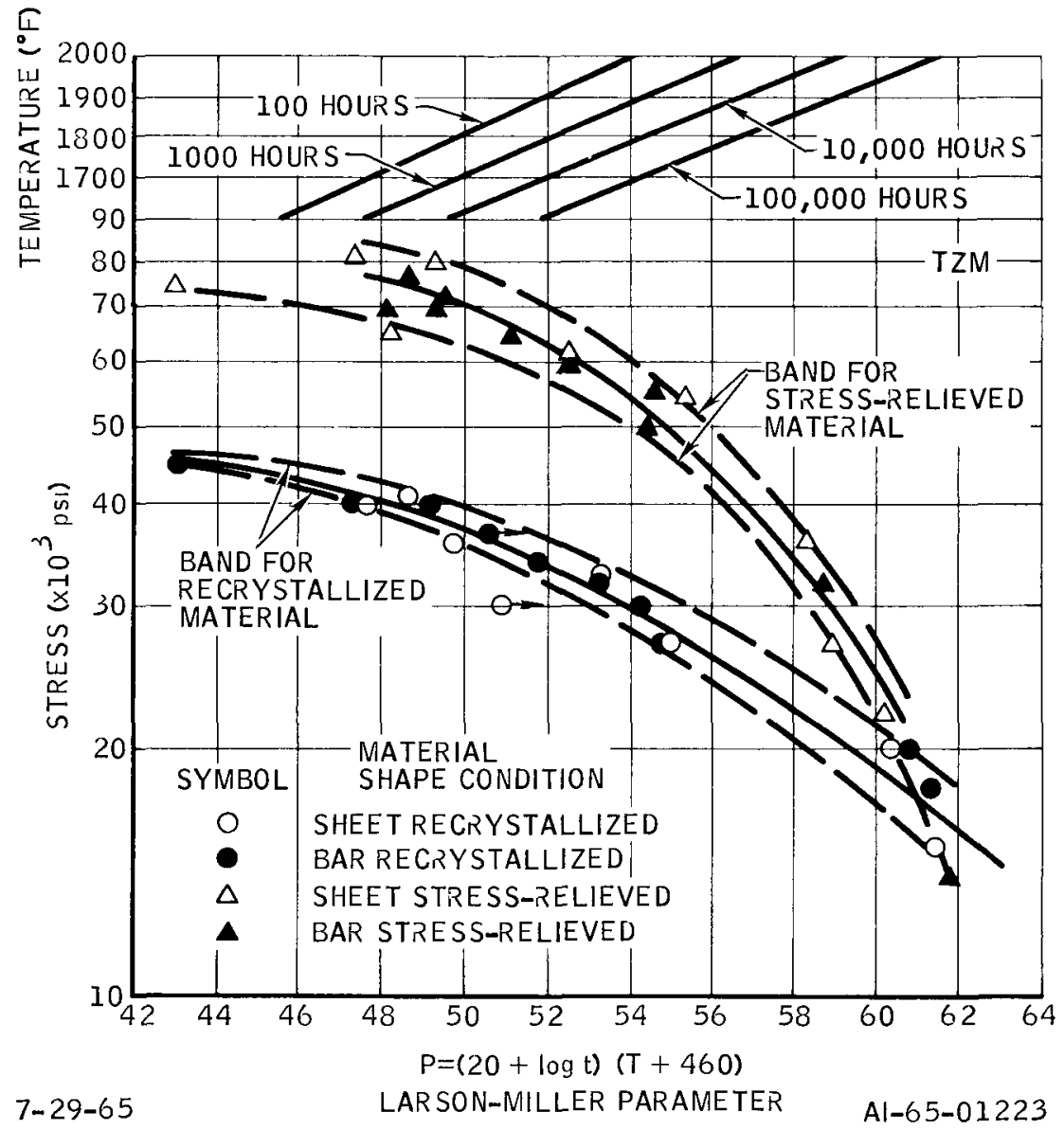

Eigure III-16. Larson-Miller Relationship for Stress Rupture of TZM $(\mathrm{C}=20)$

No impact data are available for either TZM or T-222. However, based on impact data for Ta-10W, the impact ductile-brittle transition temperature for $\mathrm{T}-222$ is around $-320^{\circ} \mathrm{F}$. Based on data for TZC (Mo-1.2 T1-0.25 Zr-0.25C) and Mo-0.5 Tr, the $1 \mathrm{~m}-$ pact ductile-brittle transition temperature for cold-worked TZM 
should be in the range 600 to $700^{\circ} \mathrm{F}$. Welding could raise the transition temperature of either material approximately 0 to $300^{\circ} \mathrm{F}$.

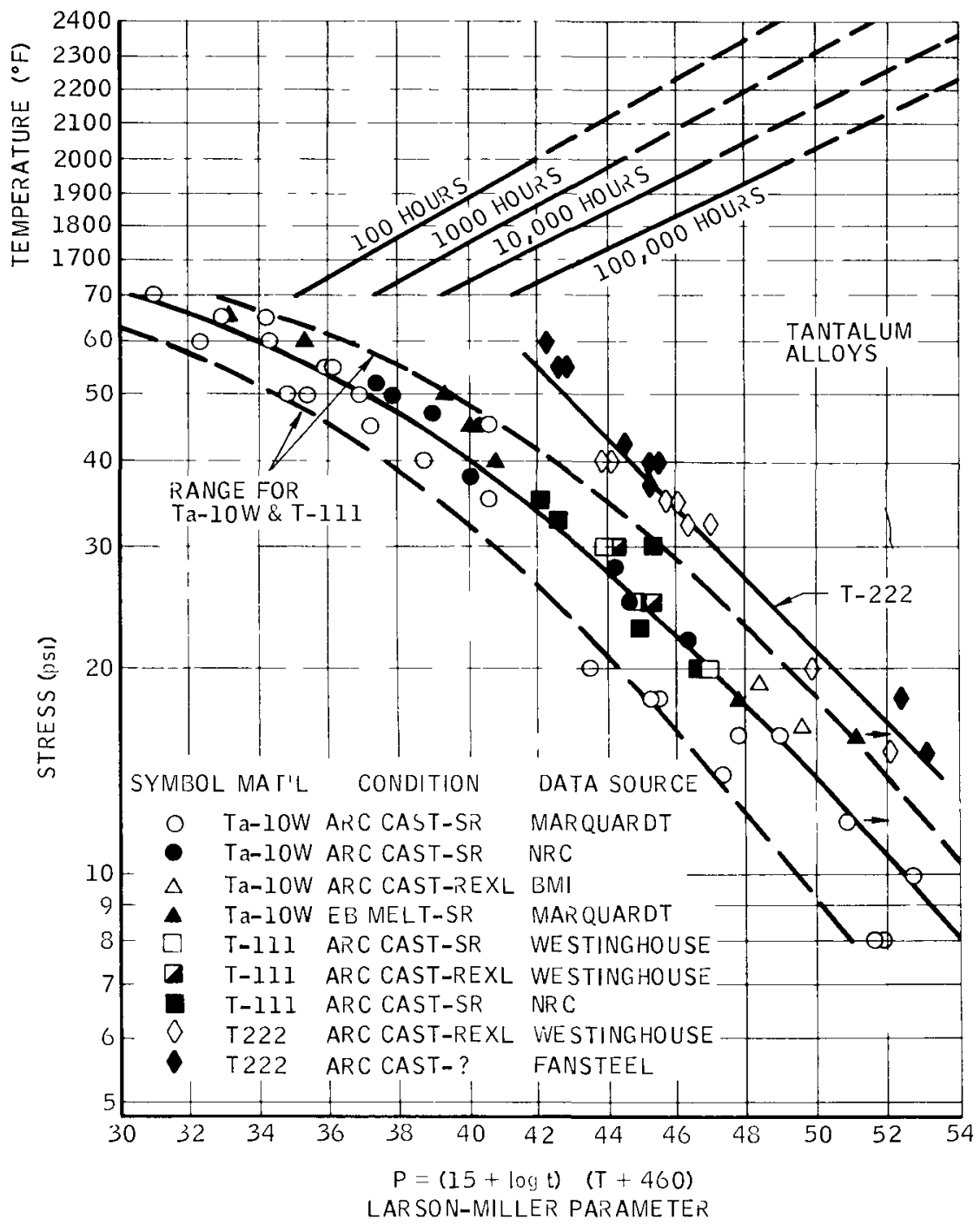

Figure III-17. Larson-Miller Relationship for Stress Rupture of Tantalum Alloys $(\mathrm{C}=15)$ 


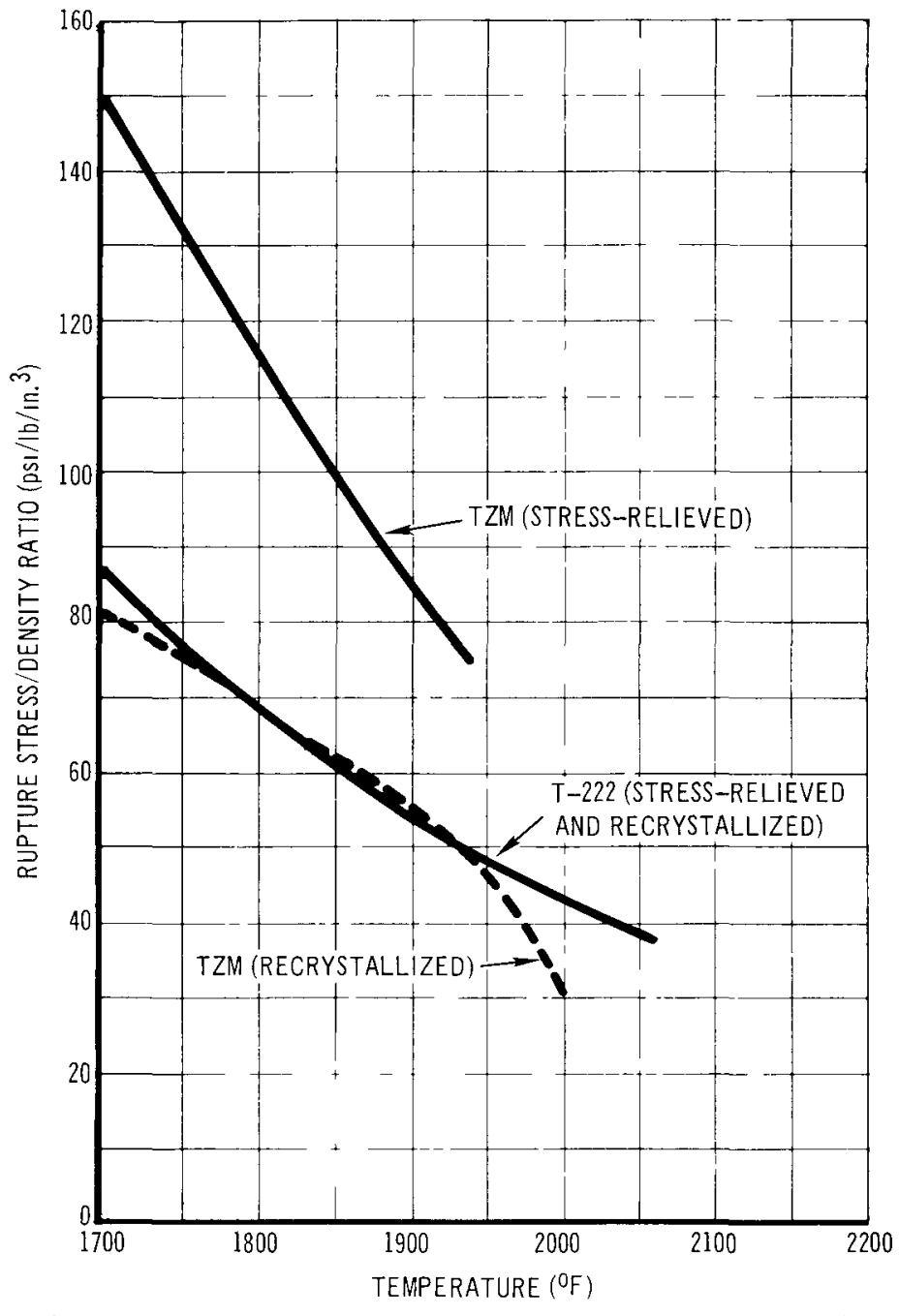

$7-29-65$

Ai $-65-01225$

Figure III-18. Rupture-Stress-to-Density Ratio for $T Z M$ and $T-222$ at 5.5 Years 


\section{b. Materials Selection}

The structural cladding must

1) Withstand the buildup of helium pressure during operation, during the peak reentry heating period, and during bur1al of Po capsules,

2) Survive impact without cracking, and

3) Be compatible with the fuel and outer container.

Based on the Larson-Miller extrapolation, as shown in Figure III-18, T-222 (either stress-relieved or recrystallized) is equivalent to recrystallized $\mathrm{TZM}$ but weake $\mathrm{r}$ per unit weight than stress-relleved $\mathrm{TZM}$ at $1800^{\circ} \mathrm{F}$ for 5 years. As the temperature increases during reentry or burial the $T-222$ becomes superior to even stress-relleved TZM.

The fuel capsule must contain at least one weld and this weld will have properties and microstructure of recrystallized material. This weld area must be of sufficient thickness to have the same load-bearing properties as the body of the capsule (stressrelieved) or the design must be based on recrystallized properties throughout. Thickening the weld area for TZM complicates the internal design of the capsule or increases the overall capsule diameter and complicates attachment of an outer cladding.

To produce welds with the highest quality, minimum weld area, and minimum heat-affected zone, electron-beam welding of TZM is required but TIG welding of $T-222$ is satisfactory. The EB weld process is more costly and slower, while TIG welding allows a greater freedom in part geometry and fixturing.

In summary, T-222 and recrystallized TZM are comparable for impact (above the brittle-ductile transition temperature) and for long-term stress weight. Stress-relieved TZM is superıor but loses its advantage if the operating temperature is increased. TZM is more difficult to fabricate. TZM is more costly and less reliable to weld. 


\section{Oxidation Cladding Materials Evaluation and Selection}

All refractory metals require a barrier to prevent oxidation. Three groups of metals possess satisfactory oxidation resistance at temperatures to $2000^{\circ} \mathrm{F}$. The first are the $\mathrm{Fe}-\mathrm{Cr}-\mathrm{Al}$ alloys used as heating elements. Second are the nickel-based alloys, primarily Nichrome and Hastelloy-X. Third are the precious metals, primarily platinum and rhodium. The Fe-Cr-Al alloys are very hard and brittle and would not survive impact on granite.

The nickel alloys, including both Nichrome and Hastelloy-X, become metallurgically unstable after extended times at temperature. The agglomeration and growth of the carbide second phase in these alloys has been noted by several investigators at temperatures as low as $1750^{\circ} \mathrm{F}$ for times as short as 1000 hours. The growth of these carbides is accompanied by a silght decrease in room-temperature tensile strength and a marked reduction in ductility. Typically, after 2500 hours at $1750^{\circ} \mathrm{F}$, the elongation of Hastelloy-X had dropped from 41 to $29 \%$. The low melting point of nickel-based alloys and cobalt-based alloys such as Haynes -25 cause a significant decrease in allowable burial power level. The oxidation resistance of these alloys is virtually lost above $2000^{\circ} \mathrm{F}$.

The precious metals possess the required oxidation resistance, higher melting points, excellent ductility, and fabricability. Data reported by Krier and Jaffee show that, of the precious metals, platinum and rhodium have the lowest surface recession rates in air by several orders of magnitude. The oxidation of platinum and rhodium is shown in Figure III-19. Because of their excellent ductility, the precious metals should survive impact without fracture. Impact gauging can be accommodated by using a metal thickness greatcr than the gauge depth. The seawater corrosion of the precious metals is reported as exccllent but quantitative data are lacking. The compatibility of platinum and rhodium with molten rock must be excellent since both occur as native metal in igneous rocks. 


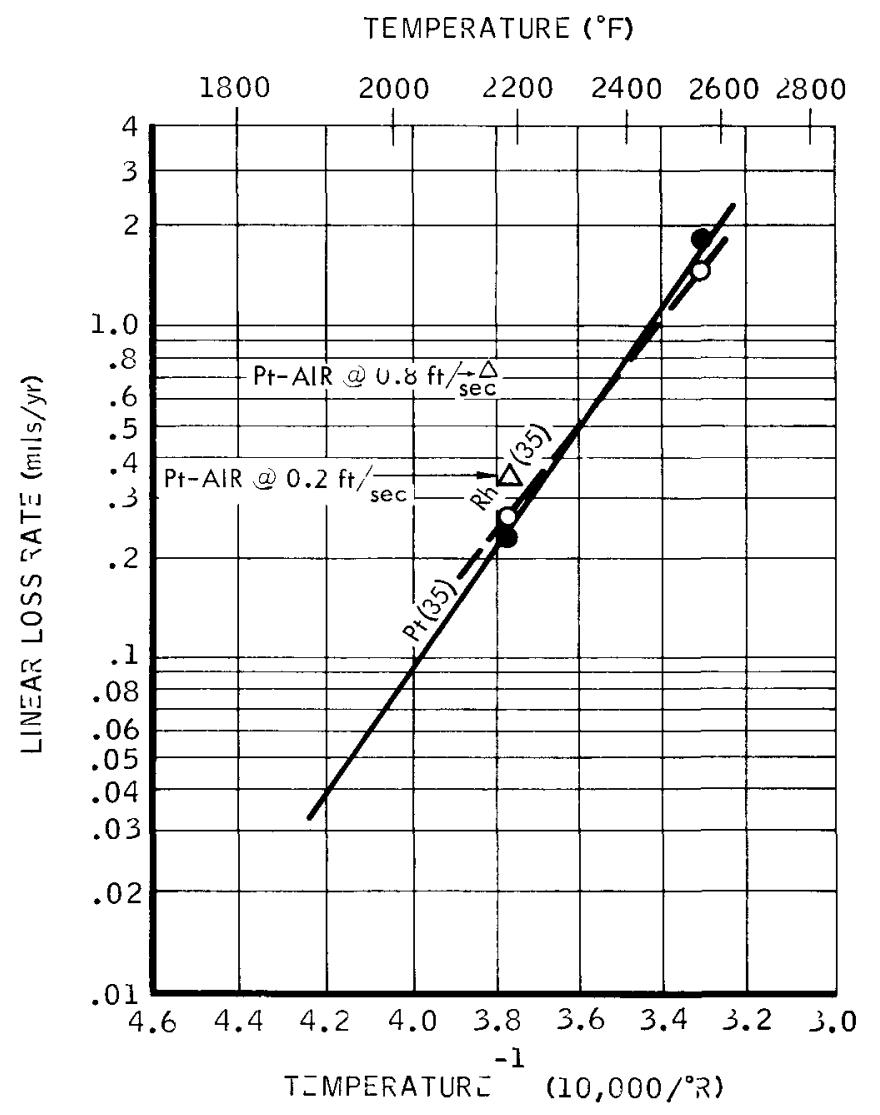

72965 UNCL

Al 6501233

Figure III-19. Air Oxidation of Platinum and Rhodıum

Rhodium is superıor to platınum in all respects but it is also very limited in supply. The current annual U.S. production of Rh is 10,000 to 20,000 troy oz. This would be sufficient to clad about 1000 to 2000 capsules with a 0.020-in. layer.

Pure platinum has the disadvantage of being relatively low melting compared to T-222 and most of the other precious metals. The melting point can be raised by alloying with $\mathrm{Rh}$, Ir, Os, or Ru without changing its environmental inertness markedly. 


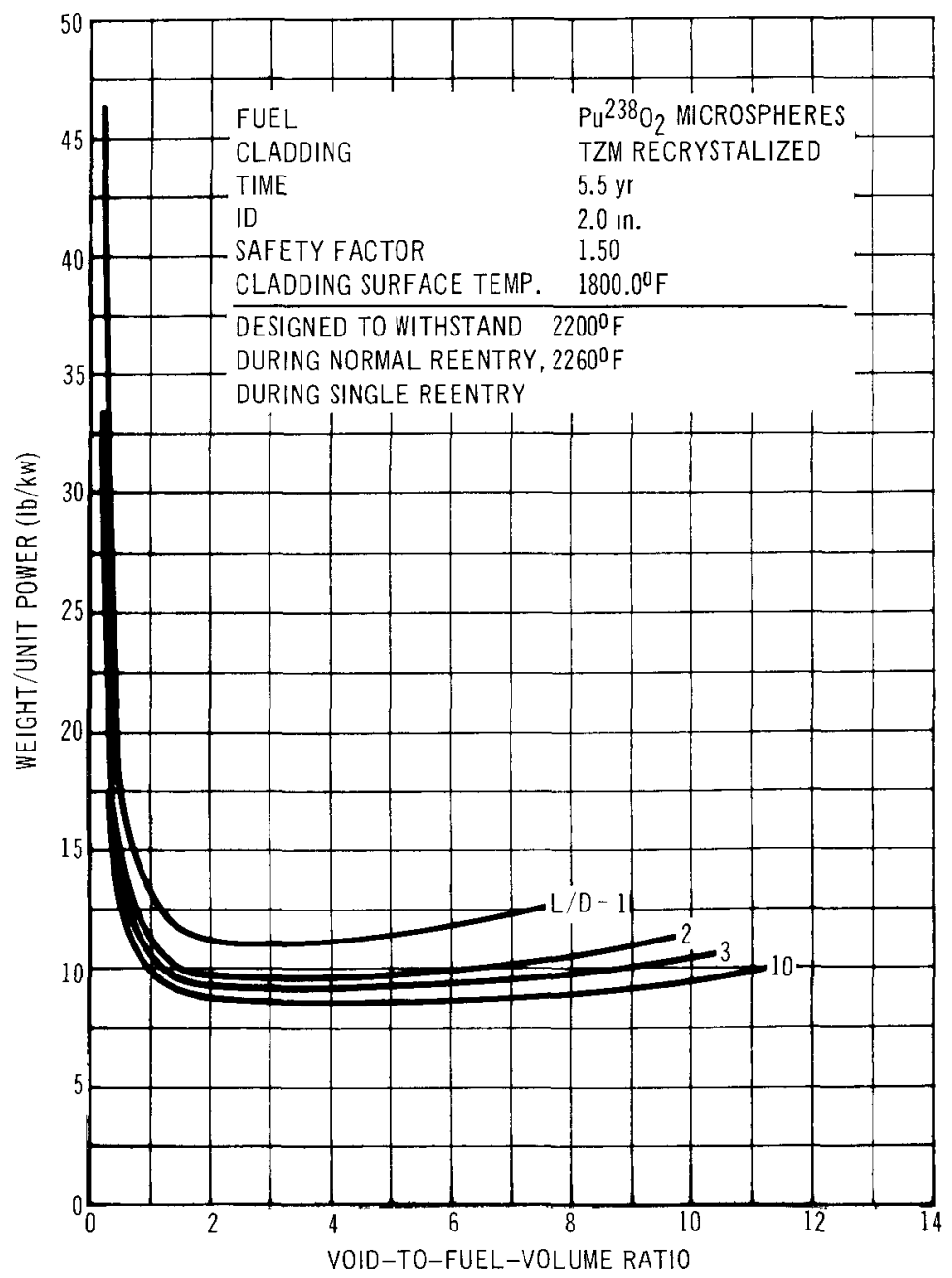

7-\$7 192-136

Figure III-20. Specific Weight vs Vold-to-FuelVolume Ratıo, $\mathrm{Pu}^{238} \mathrm{O}_{2}$ Microspheres, Recrystallized TZM Cladding 
Alloying has the additional advantage of increasing the hardness of the Pt cladding.

Precıous metals alloys are only rarely used in high-temperature structural applications, but rather for special uses such as thermocouples, crucibles, and oxıdation protection. Therefore, almost no high-temperature strength data are avallable for the precious metal alloys.

\section{F. CAPSUle STRUCTURAL DESiGN}

The pressure containment requirements of the capsule are established by the environmental temperature conditions and the helium gas pressure buildup. The wall thickness and weight of the required capsule are determined by the allowable materials properties that can be used over the temperature and stress lifetime of the capsule. The objective is to design a minimum weight container that meets the requirements.

The detaled discussion of the necessary pressure vessel design is beyond the scope of these notes. Such designs are normally conducted with the ald of digital computer codes which systematically vary all of the key parameters and seek an optimum design. The results of such calculations are shown in Figures III-20 and III-21 for typical cylindrical capsules designed for a 5.5-year infe at $1800^{\circ} \mathrm{F}$. The optimum capsule weight is in the region of $101 \mathrm{~b}$ per thermal kilowatt. The capsule weight asymptotically approaches a minimum as the L/D 1 s increased to about 10. The minimum weight also occurs in the region of $1 / 3$ vold and 2/3 fuel for $\mathrm{PuO}_{2}$.

\section{G. IMPACT ANALYSIS}

When an isotope-filled capsule impacts on a hard, unyıelding surface, after accidental reentry, two possıble stress fallure modes exist. The first is due to the localized stresses generated near the point of impact. The second fallure mode is due to bending and shear stresses in the body of the capsule. The stress 


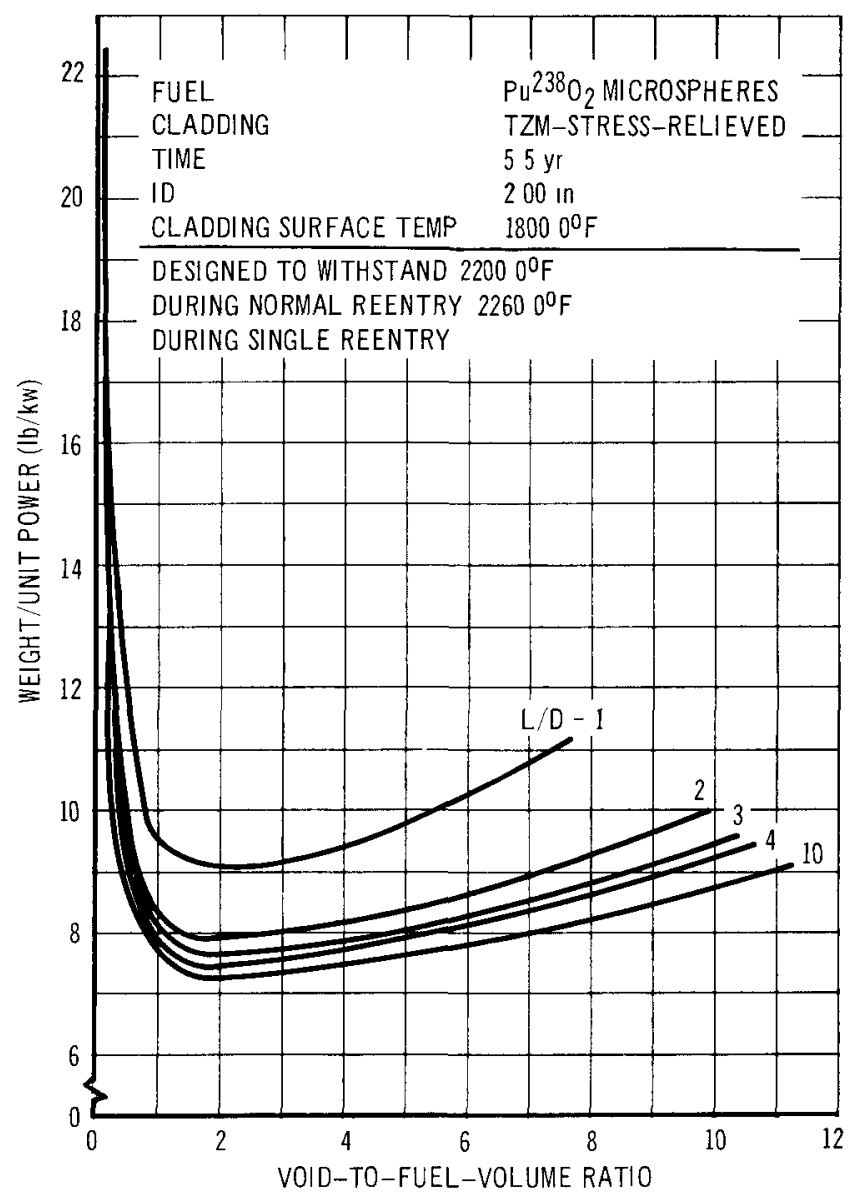

7 S7 192137

Figure III-21. Specific Power vs Void-to-FuelVolume Ratio for $\mathrm{Pu}^{238} \mathrm{O}_{2}$ Microspheres, TZM Stress-Relieved Cladding 
levels for this mode are a function of impact angle and length/ drameter of the capsule.

The plastic stress wave analysis required to predict the system behavior is complex and time-consuming due to the constantly changing geometry during impact. Because of the rigorous analysis difficulties, the approach taken is to conduct statistical tests of typical capsules filled with simulated fuel.

A typical impact test program conducted by Sandia is reported in SC-RR-65-9. Test capsules of the general design shown in Figure III-22 were impacted on granite over a range of velocity in order to determine the fallure frequency as a function of velocity. A typical fallure frequency vs velocity plot is shown in Figure III-23. An extensive survey involving hundreds of capsules allowed the correlation of wall thickness vs L/D ratio for varying velocities with a $100_{0}^{\circ}$ fallure probability. These results are shown in Figure III-24.

Photos of some typical refractory metal capsules with an outer oxidation barrier are shown in Figure III-25.

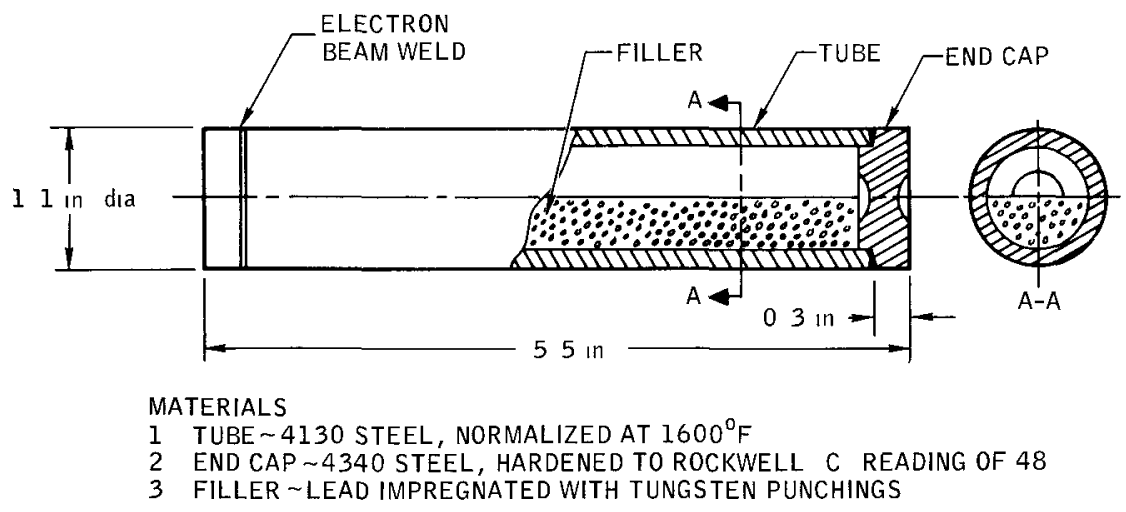

7-57-192-113

Figure III-22. Typical Test Specimen, Containment Capsule Impact Safety Study 


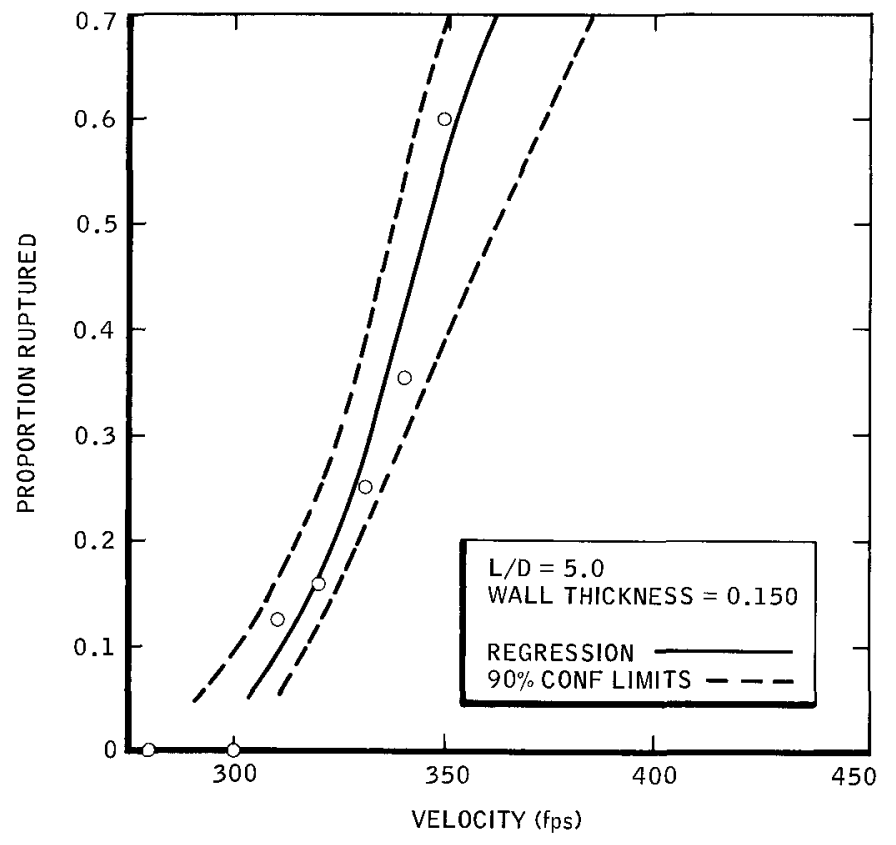

7-S7-192-114

Figure 23. Velocity vs Proportion Ruptured, S I Capsules (Parallel Analysis)

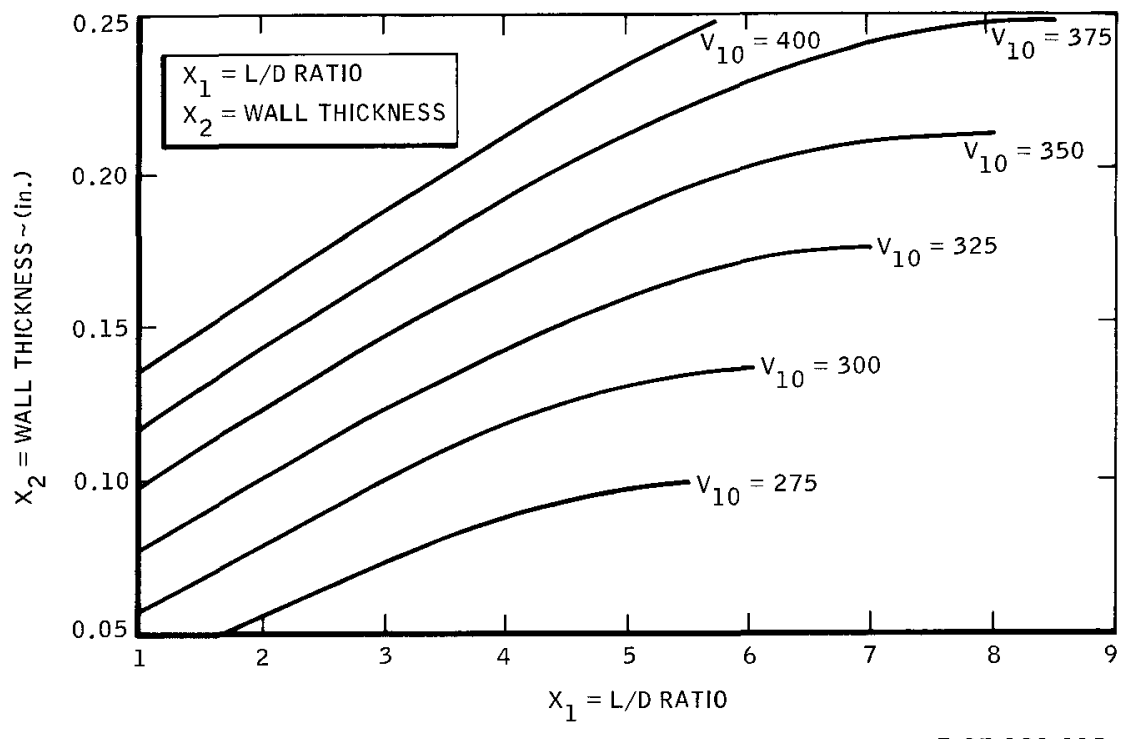

7-S7-192-115

Figure III-24. L/D Ratio vs Wall Thickness, Probit Analysis 


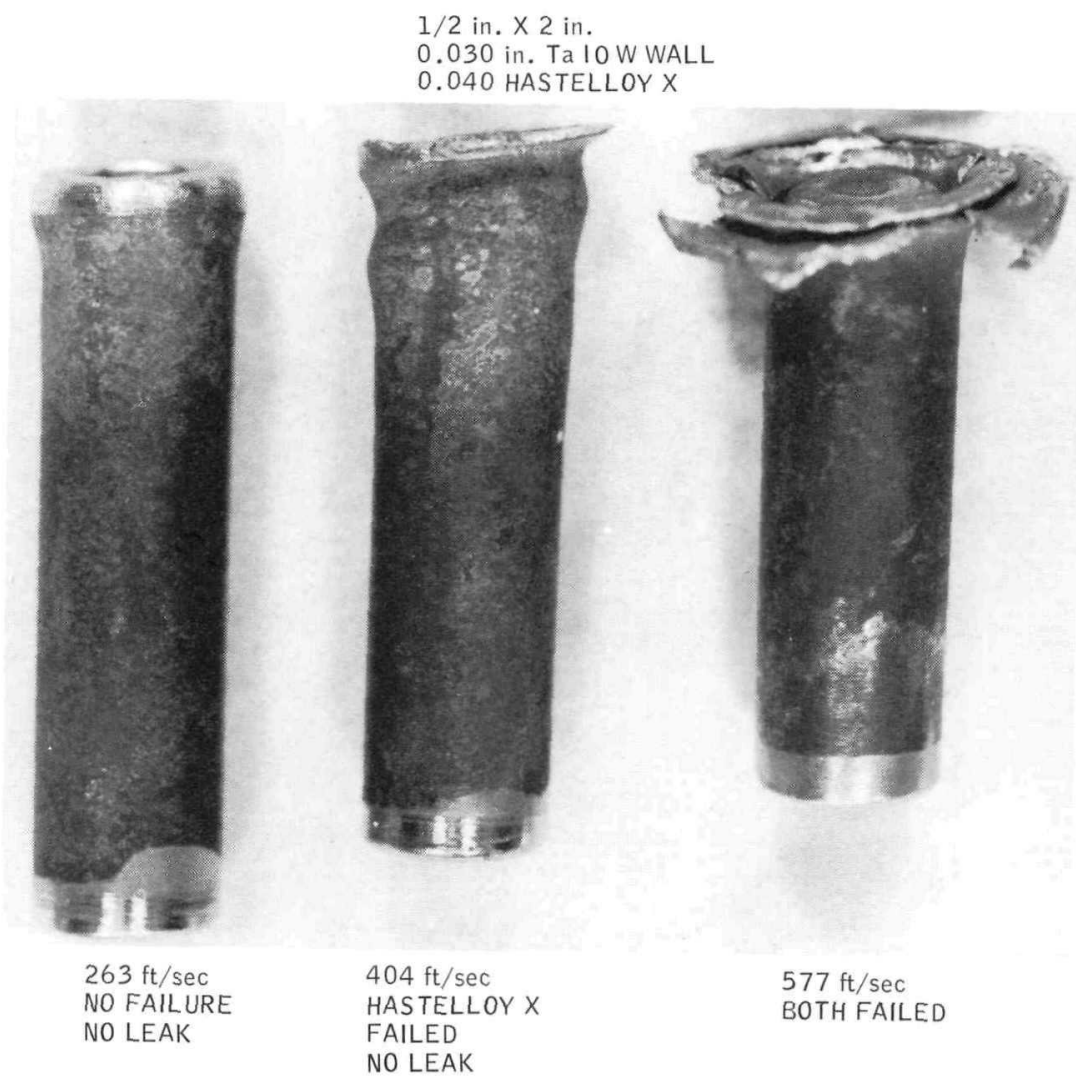

6090-2522

Figure III-25. Refractory Metal Capsule Specimens 


\section{RADIOISOTOPE POWER SYSTEMS FOR MANNED APPLICATIONS}

\section{A. GENERAL CHARACTERISTICS}

Radioisotope energy sources are of major interest for prolonged manned space missions because of their high power density with relatively low nuclear shielding requirements. This unique heat producing capability leads to lightweight systems having much flexibility in the areas of integration and growth potentıal.

The excellent performance of this type of system is realized only when adequate safeguards are provided. The fuel material itself if released to the biosphere can in some cases be a serious hazard. Therefore, attention must be pald to safety requirements from the onset of system design.

The specific advantages and characteristics of radioisotope power systems alc shown below

Low total weight

Low radiator area

Low volume requirement

Ease of vehicle integration

No consumables required

No orientation requirements

Minimum projected drag arca

Large growth potential in both power and duration

Unaffected by variations in solar constant

Development costs comparable with other power systems

System delivery costs comparable with other power systems

Availability presently comparable with mission requirement schedules 
The key characteristics for earth orbital applications are low radiation area, no consumables and no orientation requirements. These all contribute to mission flexibility, ease of integration and mission life extension.

For the above reasons, radiolsotope systems have recently achieved a wide interest by the mission planner. The following detailed considerations for fuel and power conversion selection are presented in light of the above.

\section{B. ISOTOPE FUEL SELECTION}

The selection of an isotope for a particular application is based on considerations related to the is otope half-life, power density, availability, and safety. For manned applications, safety includes radiological shielding for the crew as well as protection of operating personnel and the general public from the consequences of handling, launch, or re-entry accidents.

Screening of the chart of the nuclides identıfies approximately 12 (See Table III-1) radiolsotopes with power densities sufficiently high to warrant consideration for power system applicatıons. These isotopes can be categorızed as alpha, beta, or gamma emitters. The gamma emitters can be eliminated from consideration for manned space applications because of the excessive shield weights which would be associated with their use. The beta emitters, with the exception of $\mathrm{Pm}^{147}$ and $\mathrm{Tm}^{170}$, can likewise be eliminated on the basis of shielding considerations. The alpha emitters, $\mathrm{Po}^{210}, \mathrm{Pu}^{238}, \mathrm{Cm}^{242}$, and $\mathrm{Cm}^{244}$, should all be considered.

The above six isotopes are the primary contenders for manned applicatıons. To roughly define regimes of applicability, the se isotopes can be grouped as short-lived, those with a halflife of one year or less, and long-lived. 
The short-lived isotopes are $\mathrm{Po}^{210}, \mathrm{Tm}^{170}$, and $\mathrm{Cm}^{242}$. Of these, polonium, $\mathrm{Po}^{210}$, has received the bulk of the attention to ate. $P^{210}$ is an alpha emitting isotope with a half-life of 138 days. It has a high power density and relatively low shielding requirements. The AEC plans to produce large quantities of Po 210 and availability should not be a problem. Cost should be in the $\$ 10$ to $\$ 30$ per watt range. The preferred fuel form for $P_{0}^{210}$ is a rare earth polonide (e.g. DyPo). While this fuel form provides relatively low vapor pressures at anticipated operating conditions, it will not retain the highly volatile $P^{210}$ sufficiently to preclude radiological hazards. Therefore, safety requirements require demonstration of $\mathrm{Po}^{210}$ fuel capsule integrity under all postulated accident conditions. Because of the large amount of data available on Po ${ }^{210}$, its availability, low shielding requirements and relatively low cost, Po 210 must be considered the primary candidate for short-lived applications.

The thulium isotope $\mathrm{Tm}^{170}$ is a beta emitter with a half-life of 129 days. Its power density is much lower than Po ${ }^{210}$ but adequate for power system applications. $\operatorname{Tm}^{170}$ is very easy to produce and availability at about $\$ 10 /$ watt should be no problem. The gammas associated with $\mathrm{Tm}^{170}$ decay lead to a shield weight penalty when compared to $P^{210}$ and introduce the considerations of direct dose in the safety analyses. However, there is no helium containment problem with $\mathrm{Tm}^{170}$ (as there is with alpha emitters). Also, the preferred fuel form, $\operatorname{Tm}_{2} \mathrm{O}_{3}$, has a very low vapor pressure which when combined with the higher MPC a could eliminate the strict containment requirements which are necessary with $\mathrm{Po}^{210}$. Tm ${ }^{170}$, therefore, should be considered as an alternate for $\mathrm{Po}^{210}$ if weight does not become limiting in a particūlar mission.

The final short-lived isotope, $\mathrm{Cm}^{242}$, has a half-life of 164 days. Its performance is not unfavorable; however, the re are no plans now to produce it in quantity and, in any event, its 
price would be 3 to 10 times that of Po ${ }^{210}$ or $\operatorname{Tm}^{170}$. Therefore, $\mathrm{Cm}^{242}$ does not appear particularly attractive when compared to the other two short-lived 1sotopes.

\section{b. Long-Lived Isotopes}

The long-lived isotopes are $\mathrm{Pu}{ }^{238}, \mathrm{Cm}^{244}$, and $\mathrm{Pm}^{147}$. Of these, the plutonium isotope, $\mathrm{Pu}^{238}$, has undergone the greatest development. $P u^{238}$ is an alpha emitter with a half-life of 87.6 years. The isotope has a low power density which leads to relatively large heat sources, however, it requires very intle shielding. The avallability of $\mathrm{Pu}^{238}$ is presently limited. Quantities in the tens of kwt per year are planned in the 1970 time period. In addition, the very long half-life does allow stockpiling. Pu 238 will also be expensive, on the order of $\$ 500 /$ watt. Leasing ar rangements, with return of the $\mathrm{Pu}^{238}$ after the mission, would substantially reduce this cost. The preferred fuel form for $\mathrm{Pu}^{238}$ is $\mathrm{PuO}_{2}$ in the form of plasma fired microspheres. The microspheres are not of respirable size and the vapor pressure of the fuel form is sufficiently low that release of the fuel under any conditions other than extreme temperature does not cause an inhalation hazard. The microspheres may also be chemically inert and could reduce potential contamination problems. Containment of the fuel under most accident conditions is required. Because of its advanced state of development, reasonable avallability, low shielding requirements and excellent fuel form, $\mathrm{Pu}^{238}$ in the form of $\mathrm{PuO}_{2}$ microspheres must be considered the leading candidate for long-lived applications.

The curium isotope $\mathrm{Cm}^{244}$ has a half-life of 18.4 years. It requires more shielding than $\mathrm{Pu}^{238}$ and would lead to heavier power systems. The preferred fuel form for $\mathrm{Cm}^{244}$ is $\mathrm{Cm}_{2} \mathrm{O}_{3}$ microspheres which should exhibit properties similar to the $\mathrm{PuO}_{2}$ microspheres. Cost and avallability are comparable to the cost and avalability of $\mathrm{Pu}^{238} \cdot \mathrm{Cm}^{244}$ in the form of $\mathrm{Cm}_{2} \mathrm{O}_{3}$ microspheres is recommended for consideration as an alternate to 
$\mathrm{Pu}^{238}$ for applications where weight is not critical and/or the avallability of Pu ${ }^{238}$ proves restrictive.

The final long-lived candidate is Pm 147 with a half-life of 2.62 years. $\mathrm{Pm}^{147}$ has the advantage of being a beta emitter (1.e., no helium containment), and in the form of $\mathrm{Pm}_{2} \mathrm{O}_{3}$ has the low vapor pressure desirable from safety considerations. The shielding requirements for $\mathrm{Pm}^{147}$ are midway between $\mathrm{Pu}{ }^{238}$ and $\mathrm{Cm}^{244}$. The cost of $\mathrm{Pm}{ }^{147}$ should be reduced to $\$ 93 /$ watt in the 1970's. However, the availability is very poor. Quantities on the order of $30 \mathrm{kwt} /$ year could be obtained in the late 1970's but the shorter half-life makes stockplling more difficult than with $\mathrm{Pu}^{238}$ or $\mathrm{Cm}^{244}$. $\mathrm{Pm}^{147}$ is an attractive candidate from the standpoint of performance and cost and as such should be evaluated for individual missions, but the immited avallability should prove very restrictive for the larger power systems.

\section{c. Fuel Selection Summary}

The characteristics of the primary candidate isotope fuels are summarized in Table III-9. On the basis of its advanced development, avallability and performance, $P_{0} 210$ is recommended as primary contender for short missions. Tm 170 offers some potential safety advantages for short-lived missions which are not weight limited. It should be seriously considered.

For the longer missions, the performance of $\mathrm{Pu}^{238}$ is the most attractive. $\mathrm{Cm}^{244}$ offers a backup for $\mathrm{Pu}^{238}$ where weight is not restrictive and $P^{147}$ offers potential cost advantages where its limited availability is not restrictive. In a high usage program Pm 147 cannot be considered generally because of this avallability problem. Further, $\mathrm{Cm}^{244}$ is not a good backup to $\mathrm{Pu}^{238}$ as the power level grows because of the compounding of the shield weight penalty with increasing size.

A last alternative exists for the intermediate and long earth orbital mission. This is a heat source resupply approach. Such a concept might be attractive if resupply of either men or material 
TABLE III-9

ISOTOPE FUEL CHARACTERISTICS

\begin{tabular}{|c|c|c|c|c|}
\hline Fuel & Half-Life & $\begin{array}{l}\text { Power } \\
\text { Density } \\
(w / c c)\end{array}$ & $\begin{array}{l}\text { Shield } \\
\text { Thickness } \\
\text { (cm) }\end{array}$ & $\begin{array}{c}\text { Approximate } \\
\text { Fuel Costs } \\
(\$ / \text { watt })\end{array}$ \\
\hline $\mathrm{Po}^{210}$ & 138 (days) & 1150 & 1.4 & 10 to 30 \\
\hline $\mathrm{Pu}^{238}$ & 87.6 (yr) & 3.6 & $\left(N_{1} l\right)$ & 545 \\
\hline $\mathrm{Cm}^{244}$ & $18.4(\mathrm{yr})$ & 22.5 & 3.5 & 480 \\
\hline $\operatorname{Pm}^{147}$ & $2.62(\mathrm{yr})$ & 1.8 & 1.4 & 93 \\
\hline $\operatorname{Tr} 170$ & 129 (days) & 7.9 & 5.1 & 10 \\
\hline
\end{tabular}

Thickness required to reduce dose of 1000 -watt source to $10 \mathrm{mr} / \mathrm{hr}$ at 1 meter

is required independent of the power system. If this is the case it would be possible to launch new source blocks periodically and through proper docking equipment make an automatic source transfer. The primary advantage is in fuel availability and use of low cost fuel for long missions.

The above considerations lead to fuel selections for various time periods. For early missions, a choice of $\mathrm{Tm}^{170}$ or Poll ${ }^{21 s}$ dictated by both the mission duration and avallability. In the 1971 time period $\mathrm{Pu}^{238}$ appears to be improved relative to availability but still borderline. Therefore, Po 210 with resupply, might serve an interim need. The ultimate isotope system at high power would utilise $\mathrm{Pu}{ }^{238}$ based on mission duration and good avallability after the mid-1970's.

\section{HEAT SOURCE CONCEPTS}

The heat source provides the functions of fuel encapsulation, reentry protection, and heat transfer. Geometrical shape of fuel encapsulation components is largely dependent on the reentry protection concept. The reentry protection concept, in turn, affects the heat source size and shape. The various reentry 
concepts which have been investigated can be divided into four categories, as follows

1) Reentry of the fuel in separate capsules.

2) Reentry of the fuel enclosed in a lightweight sheet which oscıllates lıke a falling leaf and lands softly.

3) Reentry of the fuel enclosed in a lenticular lifting body aerodynamıcally designed to seek a stable attıtude and glıde to a soft landing.

4) Reentry of the complete heat source assembly in a package that is equipped with devices for controlled reentry and recovery.

These four concepts are discussed below. Table III- 10 gives typical characteristics of each conceptual configuration.

\section{a. Individual Capsule Reentry}

An attractive method of providing reentry protection, from the point of view of simplicity and reliability, is to provide individual aerodynamic heating protection for each fuel capsule. If the capsules are designed to spin and tumble during the period of intense aerodynamic heatıng, the heat input rates are distributed over the outer surfaces of the capsules. It has been found that this minimi 2 es the amount of heat-protection material required, as compared with the amount needed for reentry in a stable attıtude.

A typical polonium-fueled capsule with individual reentry protection material is shown in Figure III-26. The outer surface of the capsule is coated with approximately 0.2 in. of zinc oxide, held in a matrix of molybdenum wire. Zinc oxide sublimes at a temperature of approximately $2800^{\circ} \mathrm{F}$, and the end othermic heat of sublimation limits the temperature of the capsule wall to about this same value during reentry.

The capsule in Figure III-26 is shown with external fins which will ensure that the capsule spins during reentry. It is 
TABLE III- 10

SUMMARY OF REENTRY BODY CONCEPTS

\begin{tabular}{|c|c|c|c|c|c|c|}
\hline Concept & \multicolumn{2}{|c|}{$\begin{array}{l}\text { Individual } \\
\text { Capsules }\end{array}$} & \multirow{2}{*}{$\begin{array}{l}\begin{array}{c}\text { Lightweight } \\
\text { Sheet }\end{array} \\
\text { Po }\end{array}$} & \multirow{2}{*}{$\frac{\begin{array}{c}\text { Gliding } \\
\text { Body }\end{array}}{P_{0}}$} & \multicolumn{2}{|c|}{$\begin{array}{c}\text { Recoverable } \\
\text { Package }\end{array}$} \\
\hline Isotope Fuel & Po & $P_{u}$ & & & Po & Pu \\
\hline $\begin{array}{l}\text { Ballistıc Coefficient, } \\
\text { W/C } \mathrm{C}_{\mathrm{D}} \mathrm{A}(\mathrm{lb} / \mathrm{ft} 2)\end{array}$ & 30 & 110 & 10 & 26 & - & 145 \\
\hline $\begin{array}{l}\text { Life/Drag Ratio } \\
\text { (subsonic) }\end{array}$ & 0 & 0 & 0 & 2 & 0 & 0 \\
\hline $\begin{array}{l}\text { Terminal Velocity } \\
(\mathrm{ft} / \mathrm{sec})\end{array}$ & 160 & 305 & 92 & 30 & 10 & 10 \\
\hline $\begin{array}{l}\text { Probability of Burial } \\
\text { at Impact }\end{array}$ & high & high & moderate & low & low & low \\
\hline $\begin{array}{l}\text { Peak Aerodynamic } \\
\left.\text { Rate (Btu } / \mathrm{ft}^{2}-\mathrm{sec}\right)\end{array}$ & 200 & 400 & 50 & 80 & - & 125 \\
\hline $\begin{array}{l}\text { Heat-Protection } \\
\text { Material }\end{array}$ & & & & & & \\
\hline Broad faces & & & & & & \\
\hline Type & $\mathrm{ZnO}+\mathrm{Mo}$ & $\mathrm{AlF}_{3}+\mathrm{Mo}$ & Ceramic & Ceramic & & $\begin{array}{l}\text { Phenolac } \\
\text { Refrasil }\end{array}$ \\
\hline Thickness (1n.) & 0.21 & 1.05 & 0.10 & 0.10 & & 2.0 \\
\hline Edges & & & & & & \\
\hline Type & Same & Same & $\mathrm{ZnO}+\mathrm{Mo}$ & $\mathrm{ZnO}+\mathrm{Mo}$ & & - \\
\hline Thrckness (1n.) & 0.4 & 1.3 & 1.0 & 1.0 & & - \\
\hline Specific Weights (1b/kwt) & & & & & & \\
\hline $\begin{array}{l}\text { Fuel and reentry } \\
\text { protection }\end{array}$ & 5.0 & 43.2 & 1.0 & 1.2 & 38.6 & 76.8 \\
\hline $\begin{array}{l}\text { Heat exchanger and } \\
\text { structure }\end{array}$ & 5.4 & 5.4 & 5.4 & 5.4 & 10.0 & 10.0 \\
\hline Unshrelded & 10.4 & 48.6 & 6.4 & 6.6 & 48.6 & 86.8 \\
\hline Shreld & 8.8 & 10.0 & 6.0 & 6.5 & 17.6 & 33.7 \\
\hline Shrelded & 19.2 & 58.6 & 12.4 & 13.1 & 66.2 & 120.5 \\
\hline
\end{tabular}

With parachute

expected that these fins will sublime fairly early during the reentry heat pulse, but the angular momentum which they impart to the capsule will remain. A circular cylinder of this type has a natural trim position with its major axis at a right angle with respect to the relative wind, but its degree of stability is very low. Therefore, the initial spin rate will be practically undamped during the reentry period.

The capsule is designed to survive the shock of impact at terminal velocity and/or earth burial without release of the isotope fuel. 


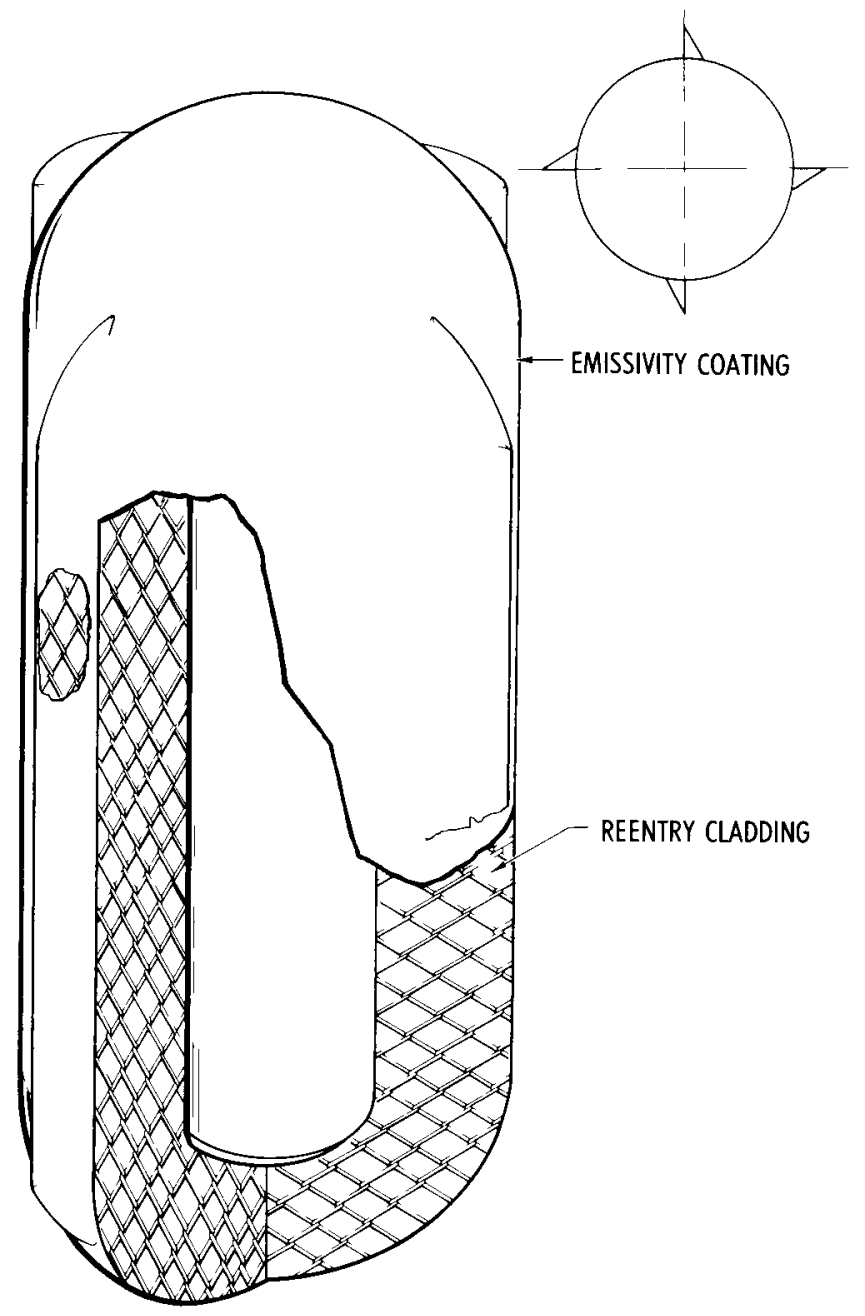

757192138

Figure III-26. Reentry Clad Polonium Isotope Capsule 
A plutonium-fueled capsule is larger than the poloniumfueled capsule for a given thermal power output. It s ballistic coefficient is higher, as shown in Table III-10. This increases the intensity of the aerodynamic heating pulse. Consequently, more reentry protection material is required. Because the relatıve vold volume allowed for containment of helium evolved during decay is less for the plutonium-fueled capsule, it is deemed preferable to limit the masimum temperature during reentry to a lower value than with polonium. Thus a material such as aluminum trifluoride, which sublimes at about $2100^{\circ} \mathrm{F}$, would be used for the plutonium capsule.

\section{b. Reentry of Lightweight Sheets}

Another approach to the problem of insuring intact reentry of cylindrical fuel capsules $1 \mathrm{~s}$ to enclose an array of slender capsules in a body having a large surface area-to-mass ratio. Such a design is shown in Figure III-27. Curving the block by about $30^{\circ}$ of arc will tend to insure a preferential orientation during reentry with its convex face forward. This curvature will not completely suppress oscillation and tumbling of the sheet, and the edges will require application of a rim of heat-protective material.

An alternate design would be to curve the sheet in the shape of a segment of a sphere. This would give added stability in the face-forward attitude, but would require that the heat exchanger be less compact than would be possible with singly curved sheets.

The greater part of the volume of the lightweight sheet shown in Figure III-27 consists of a honeycomb or foamed structure, made of a superalloy. The outer surfaces of the sheet are also of a superalloy. The fuel is enclosed in slender capsules of a refractory alloy, with a platinum oxidation barrier.

Terminal velocity for an oscillating sheet of this type could be as low as $92 \mathrm{ft} / \mathrm{sec}$ at sea level. The honeycomb and outer structure would absorb much of the shock on impact, and the 

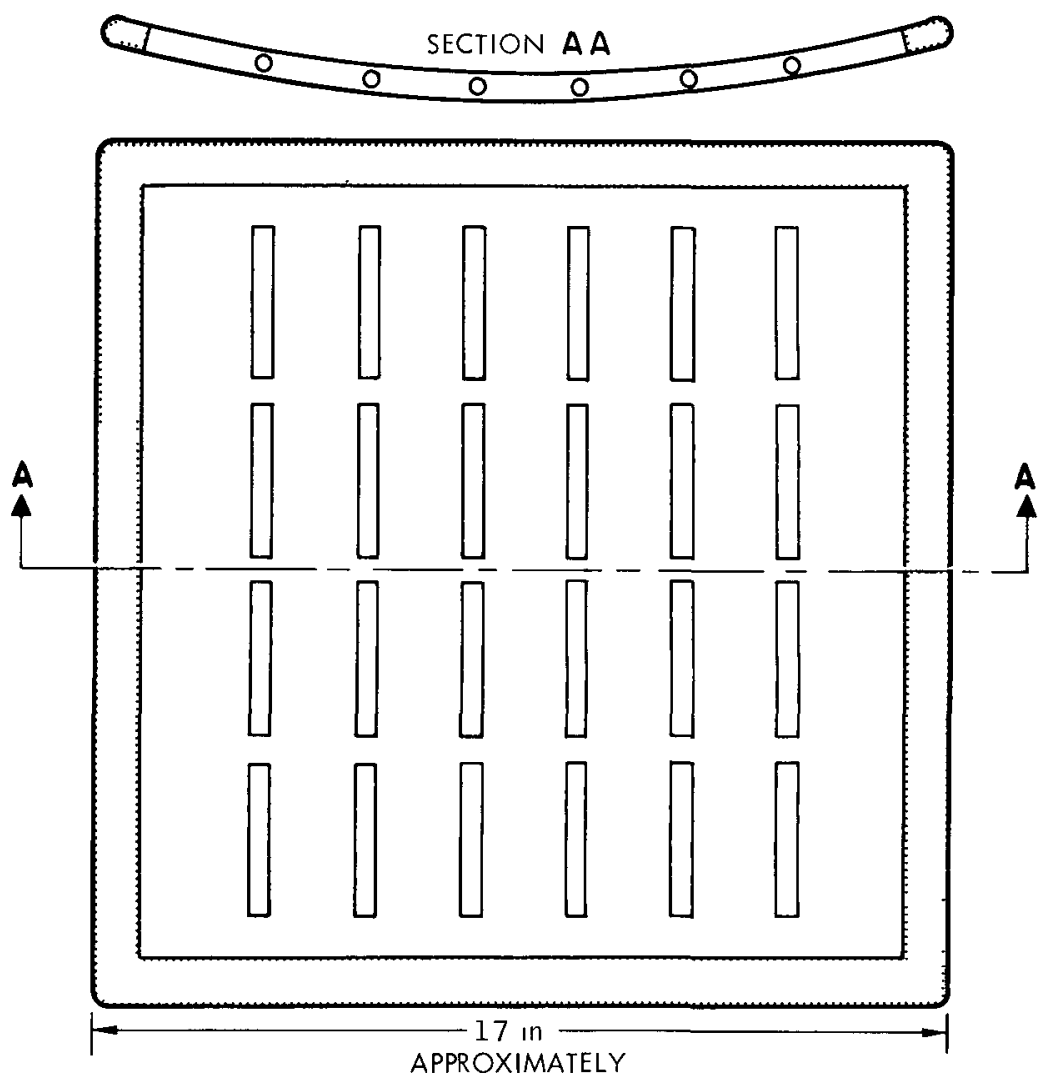

$7 \$ 7192139$

Figure III-27. Lightweight Sheet

fuel tubes are designed not to rupture. Burial would not occur upon impact with most surface solls.

\section{c. Reentry of Lenticular Lifting Body}

A lighter landing can be accomplished if the body is shaped so that it has some aerodynamic lift in the subsonic flight regime just prior to impact. A design incorporating this concept is shown in Figure III-28. The underside of this body has a large spherical radius which shifts the CG down and stabilizes the body with this side down in the hypersonic flight regime during which maximum heating occurs. 


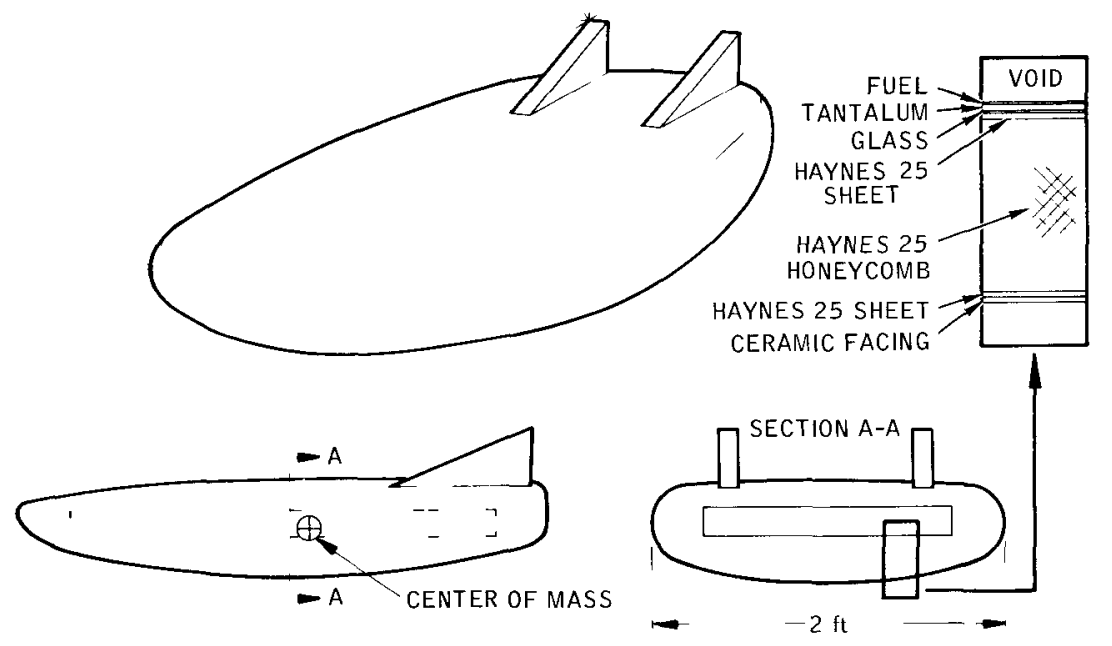

7 S7 $192 \quad 140$

Figure III-28 Lenticular Lifting Body, Concept 3

After the body has slowed to subsonic velocities, it loses its tendency to stabilize face-forward, and tends to oscillate over wider limits. The center of mass can be offset horizontally, and stabilizing fins provided will tend to make the body fall on a gliding path. The fins will be on the lee side of the body during the period of high heating, and thus will not melt away.

The predicted specific weight, in pounds per kilowatt ther mal of this lifting body, is very nearly the same as that of the lightweight curved plate. The additional weight of the stabilizing fins is partially offset by the possibility of saving some weight of protective material due to the flight attitude being more closely controlled

A novel feature shown in Figure III-28 is the enclosure of the fuel in a flat sheet, rather than in separate tubes. Thus the reentry body has the form of a sandwich, with a honeycomb main structure. Heat transfer from the inner surface, surrounding the fuel, to the outer surface is by thermal radiation and conduction through the cells of the honeycomb. It is estimated that a 


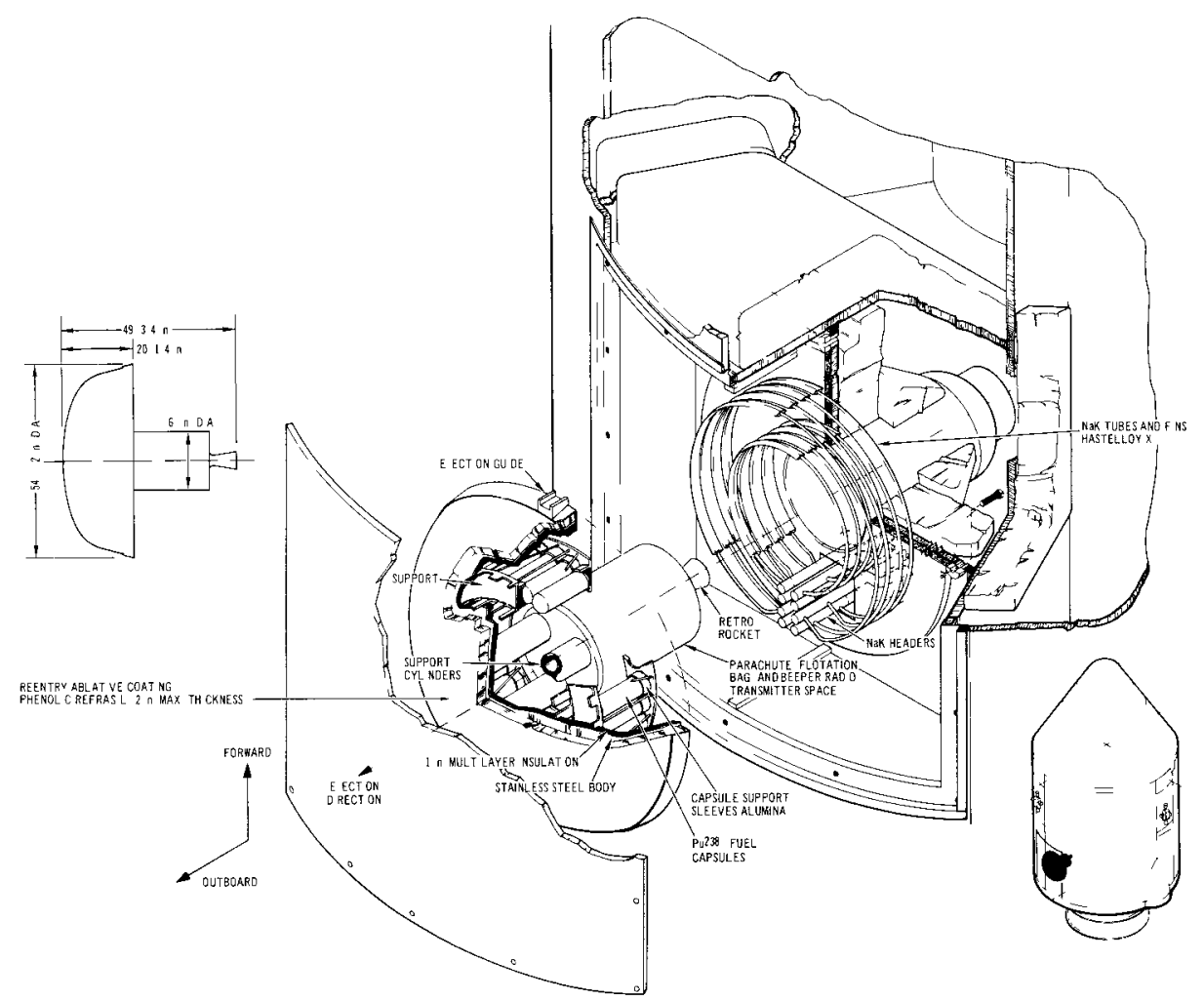

7 S7 192141

Figure III-29. 45-kwt Reentry Package Heat Source, Pu ${ }^{238}$ Fueled 
temperature difference of about $100^{\circ} \mathrm{F}$ will exist between the inner and outer plates when the outer plate is at $1800^{\circ} \mathrm{F}$. This design puts a crushable cushion between the fuel containe $r$ and the point of impact, which helps to absorb the impact energy. This same concept could also be used in the lightweight sheet design, but is not practical for $\mathrm{Pu}^{238}$ sources.

The major advantage of this body is the high nonburial probability that its stable and low-velocity landing mode provides.

d. Controlled Reentry of Complete Heat Source

A controlled reentry and recovery of the heat source assembly is highly desirable if the fuel is valuable enough to offset the resultant high cost in equipment weight. One concept for recovery is shown in Figure III-29. The point of reentry is controlled by firing a retrorocket at a selected point in the orbit.

The reentry package is shaped to be stable in one particular attitude, like a manned reentry capsule. Heat protective material is used on the forward face. At the end of the heating period, a parachute is deployed, reducing the sink rate to a few feet per second. The capsule can then be recovered in the air, or it can be allowed to reach the carth where it can be picked up. Flotation gear would be used for recovery in water.

The concept of controlled reentry has a disadvantage with respect to the weight of the equipment required for a given the rmal power, when it is compared with the other concepts discussed previously. Its use may be mandatory for plutonium fuel, which has a long half-life, giving a greater incentive for recovery. For polonium-fueled capsules, the other concepts are preferable.

e. Effect of Reentry Mode on Heat Source Design

The major effect that the reentry mode has on heat source design is on the specific weight, as can be seen from an inspection of Table $\amalg I-10$. For polonum, the fuel and reentry protection weights vary from $1.0 \mathrm{lb} / \mathrm{kwt}$ for a lightweight sheet and 
$5.0 \mathrm{lb} / \mathrm{kwt}$ for individual capsules to almost $40 \mathrm{lb} / \mathrm{hwt}$ for a reentry body. Heat exchanger and structural specific weights are the same order of magnitude as the encapsulated fuel weights, so the unshielded heat source specif $\downarrow$ c weights vary the same as do the fuel weights. It is readily seen that a recoverable package suffers a substantial weight penalty and its use must be justified on the basis of economics. It is more likely to be used with $\mathrm{Pu}^{238}$ than Po 210 systems.

Integration of either of the source block concepts into a kilowatt thermal heat source wlll require more than one block per heat source. For large manned systems, the area required for a single source block design is impractical from the shielding and vehicle integration standpoint, but a group of 2 to 5 blocks can be integrated into a compact, cubical array. As an example, six source blocks roughly 17 by 17 by $1 \mathrm{in}$. would provide the equivalent of $80.6 \mathrm{kwt}$.

Finally, heat sources comprised of source blocks can be made more compact than with capsules. Because of their compact design, the source block heat source shielded areas are less than those for capsules, and both the shield weights and shielded heat source weights are lower. Thus a lightweightsheet heat source offers a $35 \%$ weight saving over individual capsule designs. However, it presents a probability of burial which must be considered. The stabilized gliding body on the other hand offers a slightly lower weight savings, $32 \%$, but has a lower probability of burial. Because of this, it is a very promising approach.

\section{SHIELD}

The nuclear sheld required to reduce the isotope source radiation is a major consideration for manned systems. The shield is comprised of two parts, a gamma-ray shield and a neutron shield. The design of the shield depends upon 
1) Is otope source characteristics,

2) Isotope form (1.e., oxide, nitride, etc.),

3) Impurities associated with the isotopes,

4) Heat source geometry,

5) Vehicle geometry,

6) Mission operational constraints,

7) Allowable dose rates.

Without farrly specific information on each of the items above, no firm estimate of shielding requirements can be made. However, an insight into the weight of shielding can be gained by considering certain applications which have been investigated in detall. Figure III-30 shows the shield weight as a function of source thermal

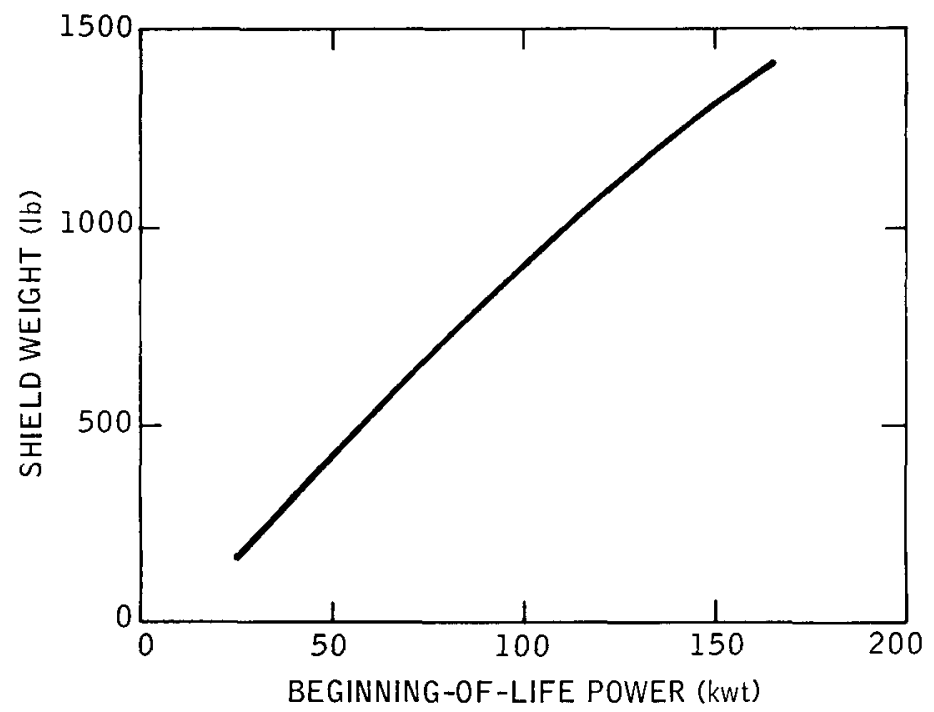

60902508

Figure III-30. Po 210 Shield Weight vs

Beginning-of-Life Power 
power for a $10-\mathrm{ft}$-diameter dose plane at $16 \mathrm{ft}$. The weights are based on a dose of 10 rem per 90 days. If the separation distances decrease, and/or the shield angle increases, the shield weight will, of course, increase. The shield weights for $\mathrm{Pu}^{238}$ systems are typically of the same order of magnitude as for Po 210 systems; in all cases at will be found that the shield weight is a substantial portion of total system weight for manned isotope applications.

\section{E. THERMOELECTRIC SYSTEMS}

Thermoelectric space power systems offer advantages in several areas. Most notable of the se is the ability to convert thermal energy directly to electrical energy without rotating or other moving parts. In systems to be operated in remote regions such as space environments, this advantage contributes significantly to overall system reliability.

With the advent of semiconductors the use of thermoclectrics for energy conversion has become competitive with other conversion systems. Studies and research over a 10 -year period have established that the most desirable semiconductor candidates fall into two classes: the silicon-germanium ( $\left.\mathrm{S}_{1} \mathrm{Ge}_{\mathrm{c}}\right)$ alloys, and lead telluride $(\mathrm{PbTc})$.

Figure III-31 shows the schematic of a typical Po 210 isotopic thermoelectric space power system. In this system the the rmal energy released by the isotope heat source is carried to a compact thermoelectric converter by the primary heat transfer loop. The medium used is the eutectic mixture of potassium and sodium, NaK-78. The wasto thermal power of the converter is transported to a radiator by the secondary loop, also containing NaK-78. A de conduction punp with an integral thermoelectric power source provides the pumping power for both primary and secondary loops.

With the use of short-lived 1sotopes such as Po 210 , a substantial decay of heat source power can occur over the mission 


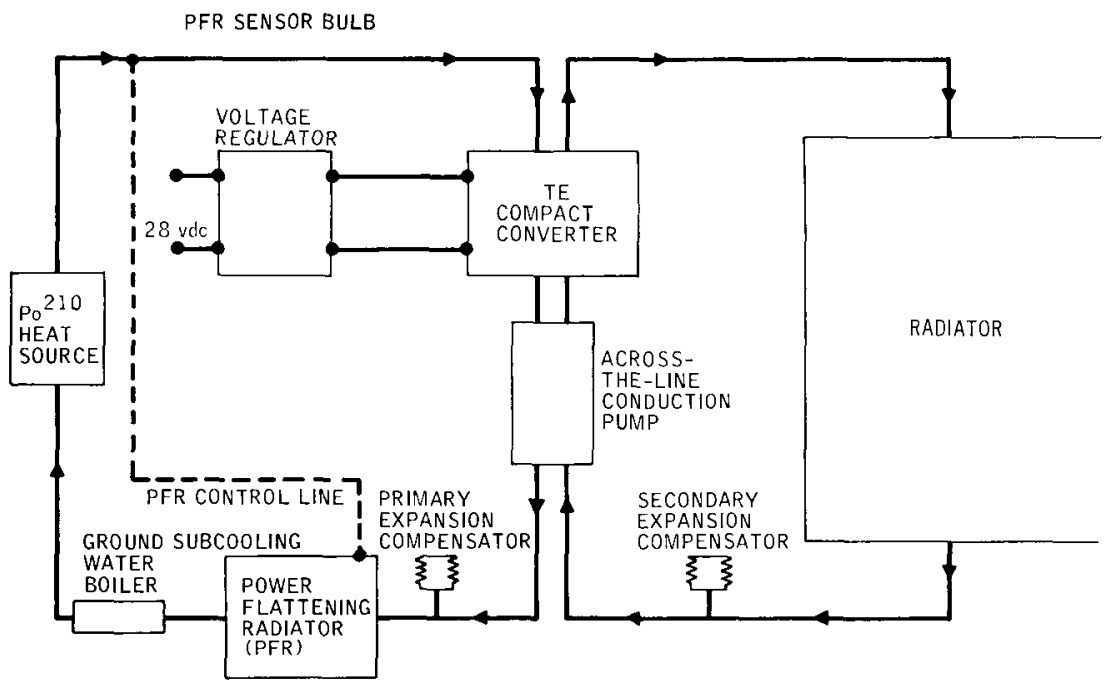

72966 UNCL

60902510

Fisure III-31. Po 210 SiGe System Schematic

IIfe and optimum system performance requires the use of a power-flattening device. In the system illustrated in Figure III-31, a power-flattening radiator is used to maintain a near-constant thermal input to the converter.

Each heat transfer loop is suppled with an expansion compensator to provide for changes in the volume of the heat transfer medium and to provide system pressurization. This is typically an all-metal bellows device.

The electrical output from the converter is delivered to a voltage regulator which conditions the power in response to load demands. The waste energy delivered to the radiator is in turn rejected to space. The specific types of thermoelectric systems of interest are discussed below.

a. SiGe Thermoelectric Systems

Of the various semiconductors investigated to date, alloys of silıcon and germanium ( $\mathrm{S}_{1} \mathrm{Ge}$ ) offer significant advantages over most of the others. SiGe can be hot-pressed and compacted into 
shapes having good structural strength; is stable in air, vacuum, and most gases; and can be metallurgically bonded to other materials. The degradation rate for $\mathrm{S}_{1} \mathrm{Ge}$ is generally lower than for other thermoelectric materials.

A typical compact converter is shown in Figure III-32. It consists of four submodules, each of which is a complete powerconverting device. The submodules can be connected in various parallel and series arrangements, both hydraulically and electrically, to provide considerable flexibility in their use and installation.

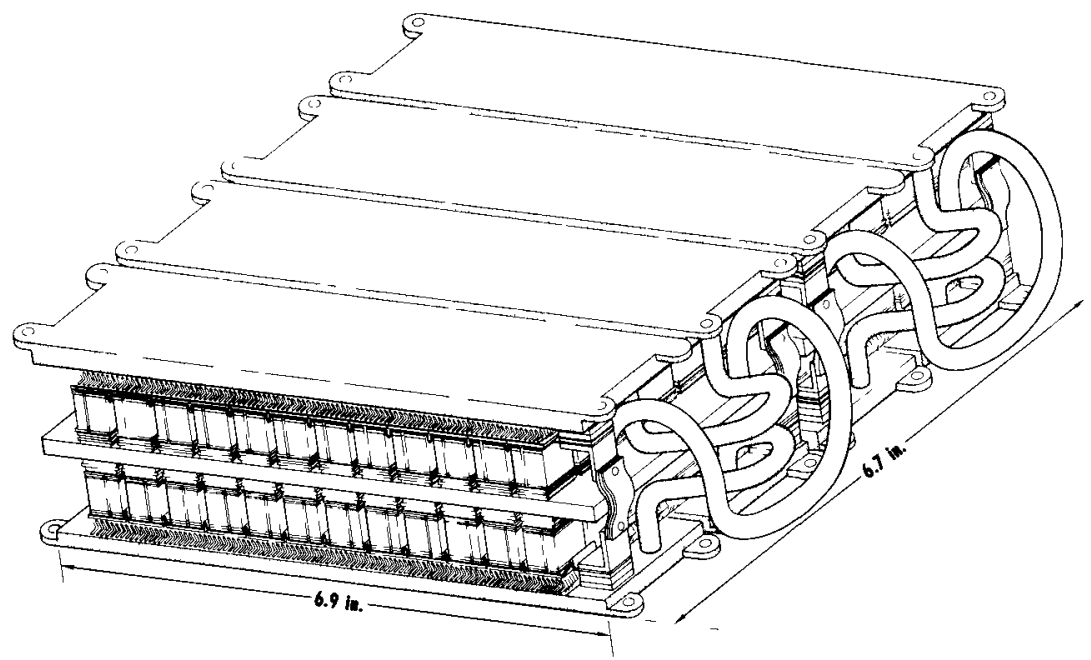

$6096-2506$

Figure III-32. SiGe Compact Converter Module

The criteria used for selection and sizing of the various components involve a tradeoff between the minimum system weight, maximum system efficiency, and minimum radiator area. All of these items are important to any space mission and the careful consideration of each is necessary. 
In general the system tradeoffs are made on the basis of minimum weight and the decision is then tempered by radiator area requirements and efficiency. Figure III-33 shows the effect of Carnot efficiency on system weight, system efficiency, and radiator area for a $P_{0} 210$-fueled system desirned for a 90-day mission. The system net output is 2.1 kwe. A design goal of 0.98 full-power reliability was used. The shielding critc rion is 10 rem over a $10-\mathrm{ft}$ diameter dose plane at $16 \mathrm{ft}$. The efficiency shown is the overall system efficiency at the end of life. As indicated, the system efficiency increases with increasing Carnot

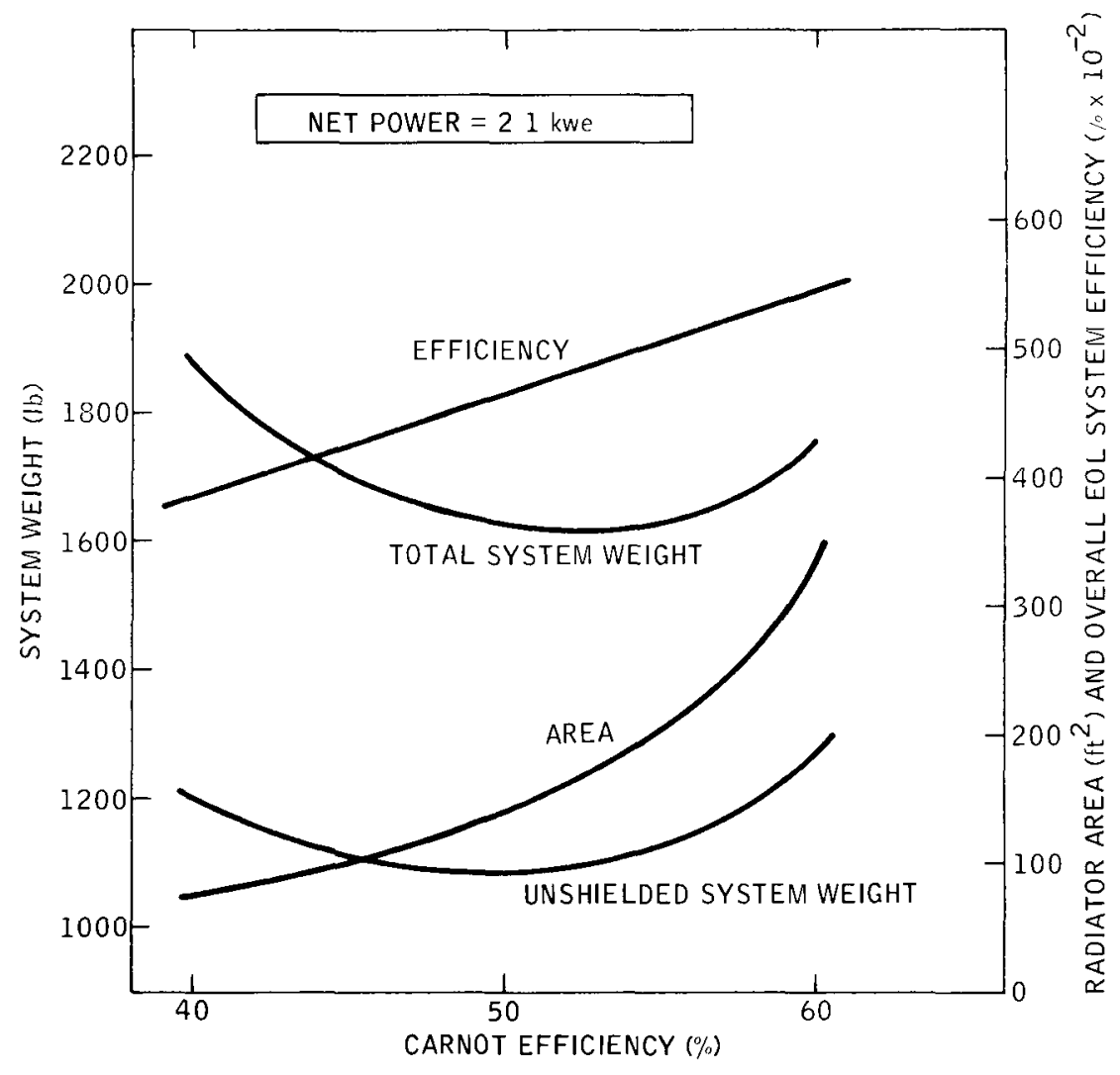

72966 UNCL

Figure III-33. Po ${ }^{210}$ SiGe System Performance vs Carnot Efficiency 
efficiency (decreasing radiator temperature for fixed heat source temperature). The system radiator area increases exponentially with increasing Carnot efficiency due to the decreasing radiator temperature and the $\mathrm{T}^{4}$ relationship for heat rejection from the radiator.

Both shielded and unshielded system weights are shown in the figure. The weights decrease, pass through a minimum, and then increase with increasing Carnot efficiency. The initial weight decrease is due primarly to decreases in the source and shield weight as the system efficiency increases. As the Carnot efficiency continues to increase, the radiator weight begins to dominate and turns the weight curves up. For this $\mathrm{SiGe}_{\mathrm{G}}$ system the minimum system weight of $16101 \mathrm{~b}$ occurs at a $52 \%$ Carnot efficiency. However, for Carnot efficiencies in the range of 45 to $60 \%$, the system weight varies little. In situations where the radiator area available is limited, one would pick an operating point at a Carnot efficiency somewhat less than $52 \%$ to reduce the radiator area required. A slight weight penalty is incurred and the system efficiency drops. On the other hand, if isotope fuel inventory is critical and adequate radiator area is available, an operating point at a Carnot efficiency greater than $52 \%$ would be chosen.

The performance of a SiGe system using $\mathrm{Pu}{ }^{238}$ for the heat source is shown in Figure III-34. The design life of this system is 2 years, with a 2.1 -kwe output and with a full power reliability of 0.98 . The trends of the various parameters as a function of Carnot efficiency are the same as described for the Po 210 system. However, it will be noted that the weights of the $\mathrm{Pu}^{238}$ system are almost a factor of two higher than those of the Po 210 systems. The heavier weight of the $\mathrm{Pu}^{238}$ system can be attributed almost completely to the increased weight of the heat source and associated shielding. The Pu ${ }^{238}$ heat sources are more than four times heavier than the $\mathrm{Po}^{210}$ sources for the same thermal power. Of 


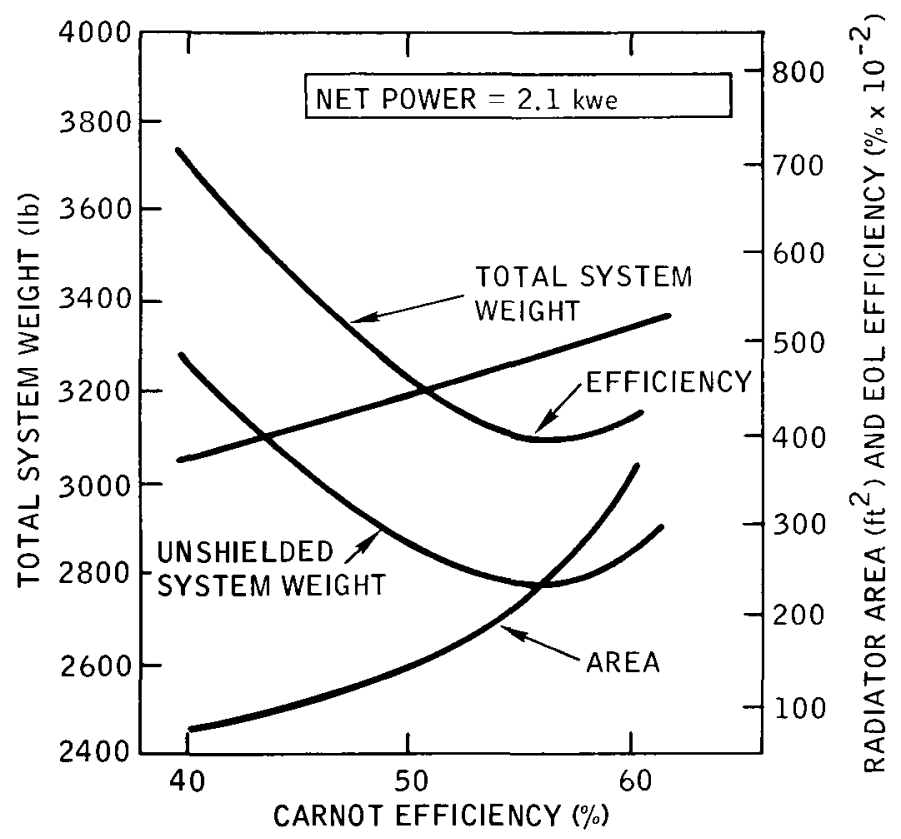

72966 UNCL

60902519

Figure III-34. Pu ${ }^{238}$ SiGe System Performance vs Carnot Efficiency

course the Pu ${ }^{238}$ has a much longer life, thus increasing substantially the specific energy of the system.

The increased specific weights of the $\mathrm{Pu}^{238}$ heat sources place a premium on system efficiency and account for the minimum Pu 238 system weight occurring at a higher Carnot efficiency than for the Po 210 systems. The higher Carnot efficiency results in the minimum-weight $\mathrm{Pu}^{238}$ system having larger radiators than the corresponding $\mathrm{Po}^{210}$ system.

The effect of output power on system weight is shown in Figures III-35 and III-36. The weights shown are total shielded system werghts based on the overall end-of-life system effuciencies shown. It will be noted that the relationship of the system weight curves for various efficiencies corresponds to the shape of the system weight as a function of Carnot efficiency, which has been 


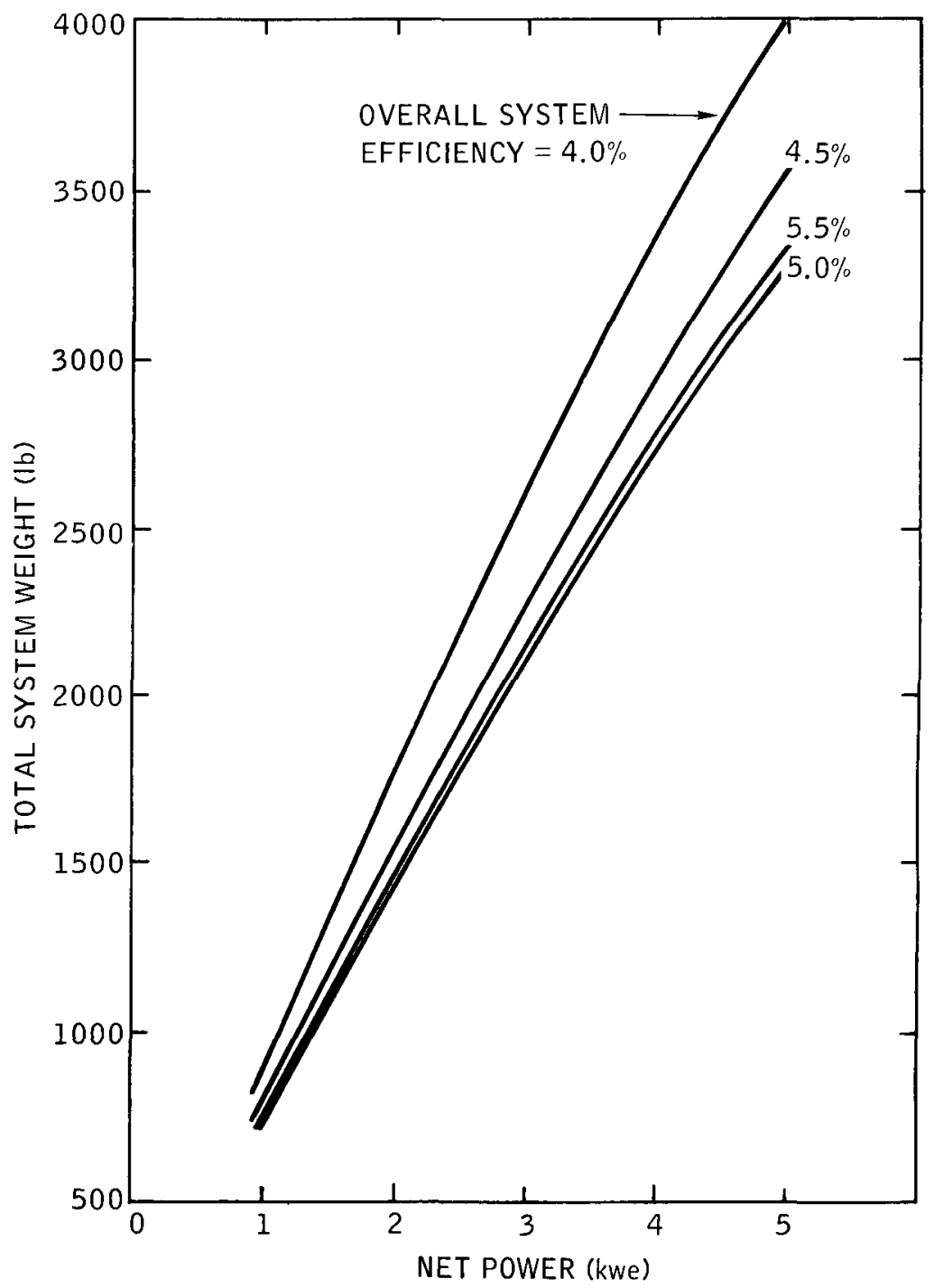

7-29-66 UNCL

6090-2517

Figure III-35. Po ${ }^{210}$ SiGe System Weight vs Power 


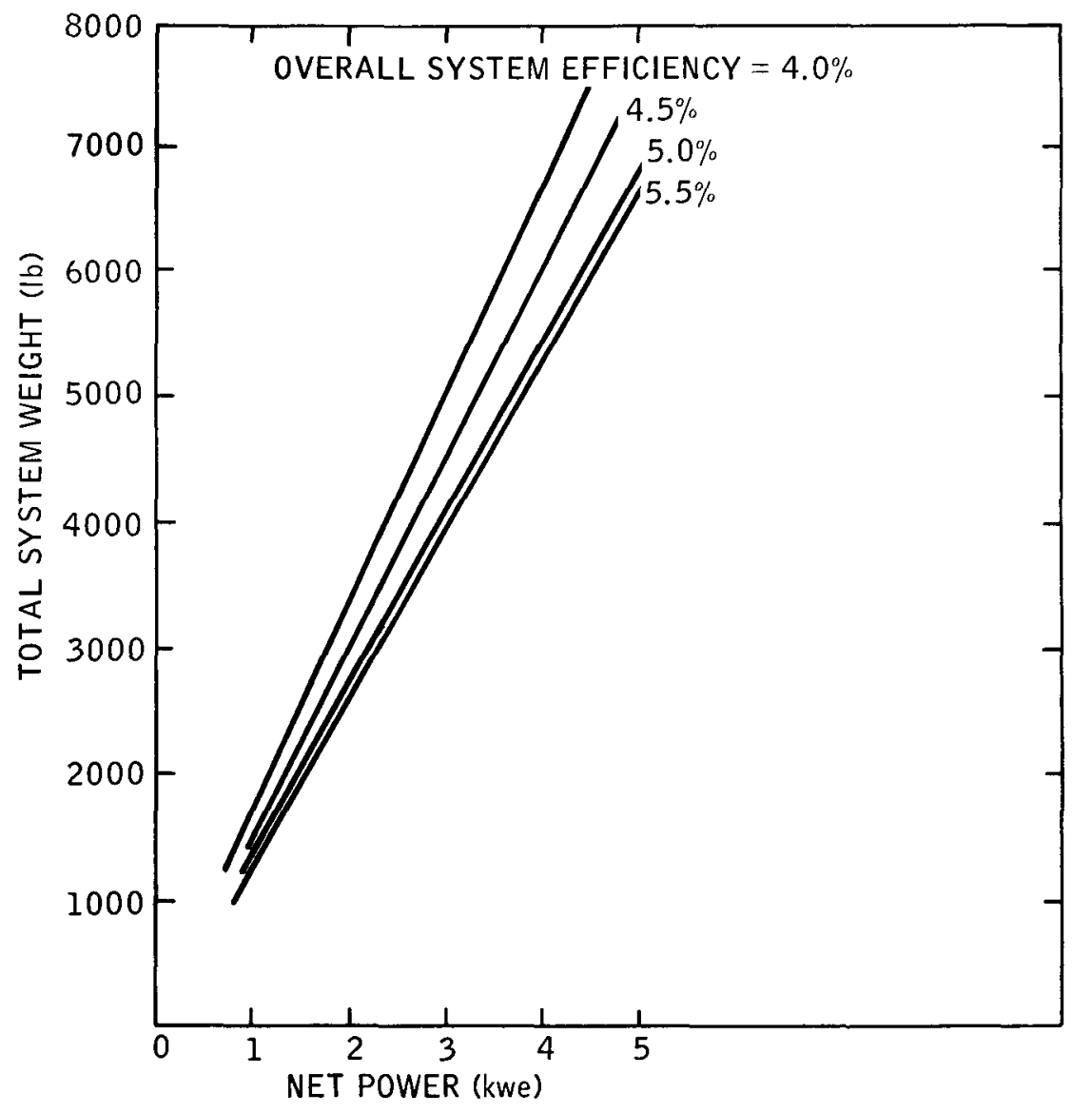

6-11-66 UNLL

60902518

Figure III-36. Pu ${ }^{238}$ SiGe System Weight vs Power 
shown previously. That is, the total system weight decreases as the system efficiency is increased, and then increases again.

As indicated in the figures, the system weight as a function of power for constant system efficiency is nearly a straight line. This is due to the fact that for constant efficiency, the source weight, converter weight, and radiator weight vary directly with system power. And, while the shield weight does not vary exactly as power, the relationship is close to linear because of the increasing size of the heat source. The component weights which do not vary directly with power are the pump, piping, and structure weights. However, the se are a fairly small part of the total system weight.

The variation of radiator area with power is linear for constant efficiency since at a given efficiency the radiator temperature is fixed and the radiator area varies directly with the thermal power rejected. For a given efficiency the radiator area for 2.1 kwe can be taken from Figure III-35 or III-36 and scaled directly for the power output of interest.

Variations in the hot-junction temperature can produce significant changes in the performance of the power systems. In general, increasing the allowable hot-junction temperature results in decreased system weight, decreased radiator area, and increased system efficiency. However, increasing the hotjunction temperature generally results in an increased degradation rate which partially offsets the gains to be made in efficiency and weight and area reductions.

As the average primary temperature is increased, the Carnot efficiency of the minimum-weight system increases. This is due to the reduced fraction of the total system weight that the radiator comprises. This shift makes the fuel weight more significant and results in a tendency to reduce the fraction that the fuel weight comprises by operating the converter at a higher efficiency. In general, the higher operating temperatures would be 
selected consistent with system reliability goals and the anticipated state of converter development.

The ability to fabricate the $S_{1} G e$ in structurally sound shapes allows significant simplification in the geometric arrangement of the elements. Cylindrical as well as rectangular geometries have both been used in the construction of the thermoelectric converters.

Since $\mathrm{S}_{1} \mathrm{Ge}$ is little affected by most gases at high temperatures, no special provisions have to be made to provide gas exclusion from the thermoelements. This further reduces the design problem and alleviates stresses which might be transmitted from the hot-junction plate to the cold-junction plate through containment members.

The ability of the SiGe to operate at temperatures in excess of $1500^{\circ} \mathrm{F}$ takes advantage of the high temperature capability of the isotopic heat sources and allows heat transfer between the heat source and the thermoelectric converter to be accomplished efficiently by radiation. The converter efficiency varies from 4 to $6 \%$. The ability to operate at high temperature results in a fairly high radiator temperature, 400 to $600^{\circ} \mathrm{F}$, and reasonably low radiator areas.

Design summaries for typical $2.1-k w e P o^{210}$ and $\mathrm{Pu}^{238} \mathrm{SiGe}$ systems are shown in Table III-11. The se data are typical of systems using either the compact or direct-radiating thermoelectric converter configuration. For a 5-kwe system using a SiGe converter with Po ${ }^{210}$ having a mission 1 fe of 90 days, the weight will be about $3250 \mathrm{lb}$. For a 5 -kwe system using a $\mathrm{S}$ Ge converter with $\mathrm{Pu}^{238}$ having a mission life of 2 years, the weight will be around $6800 \mathrm{lb}$.

b. PbTe Thermoelectric Systems

The other class of thermoelectrics currently being used for space power systems is the lead-telluride (PbTe). Also a semiconductor, PbTe has a material efficiency up to $50 \%$ higher than 


\begin{tabular}{|c|c|c|}
\hline & $\mathrm{Po}^{210}$ & $\mathrm{Pu}^{238}$ \\
\hline Net Power (kwe) & 2.1 & 2.1 \\
\hline Life $(y \mathbf{r})$ & 0.25 & 2 \\
\hline Full Power Reliability & 0.98 & 0.98 \\
\hline Dose Criteria (rem/90 day) & 10 & 10 \\
\hline Dose Plane Diameter (ft) & 10 & 10 \\
\hline Separation Distance $(\mathrm{ft})$ & 16 & 16 \\
\hline End-of-Life Efficiency $(\%)$ & 4.5 & 4.45 \\
\hline $\begin{array}{l}\text { Beginning-of-Life Thermal Power } \\
\text { (kwt) }\end{array}$ & 78.7 & 46.9 \\
\hline Radiator Area $\left(\mathrm{ft}^{2}\right)$ & 120 & 135 \\
\hline Specific Weight (1b/kwe) & 792 & 1555 \\
\hline \multicolumn{3}{|l|}{ Weight Summary (1b) } \\
\hline Heat source & 496 & 2230 \\
\hline Shield & 574 & 375 \\
\hline Pump & 65 & 65 \\
\hline $\mathrm{TE}$ converter & 66 & 60 \\
\hline $\mathrm{NaK}$ & 35 & 60 \\
\hline Expansion compensator & 54 & 70 \\
\hline Radiator and piping & 240 & 275 \\
\hline Power flattener & 19 & - \\
\hline Structure & 45 & 60 \\
\hline Instrumentation and control & 70 & 70 \\
\hline Total & 1664 & 3265 \\
\hline
\end{tabular}

SiGe and for this reason considerable effort is being expended to perfect converters using this material. Current $\mathrm{PbTe}$ devices are generally limited to below $1100^{\circ} \mathrm{F}$. Above this level serious degradation occurs, making the use of $\mathrm{PbTe}$ impractical. In addition, the tensile strength of $\mathrm{PbTe}$ is extremely low and all converter designs investigated to date have some method of holding the thermoelements in compression. In spite of these 
limitations, the high efficiency makes the material competitive with $S_{1} G e$ and further work is in progress to overcome the limitations.

A compact $\mathrm{PbTe}$ converter is currently being developed for the AEC. Figure III-37 shows the cross section of one of these devices. It is composed of two concentric cylinders, between which the $\mathrm{PbTe}$ elements are placed. The heat is carried to the elements by the hot fluid which passes through the inner tube. The cold fluid surrounding the outer tube carries away the waste energy. The thermoelements are connected in series internally and the electrical connections to the submodule are made at the ends of the device.

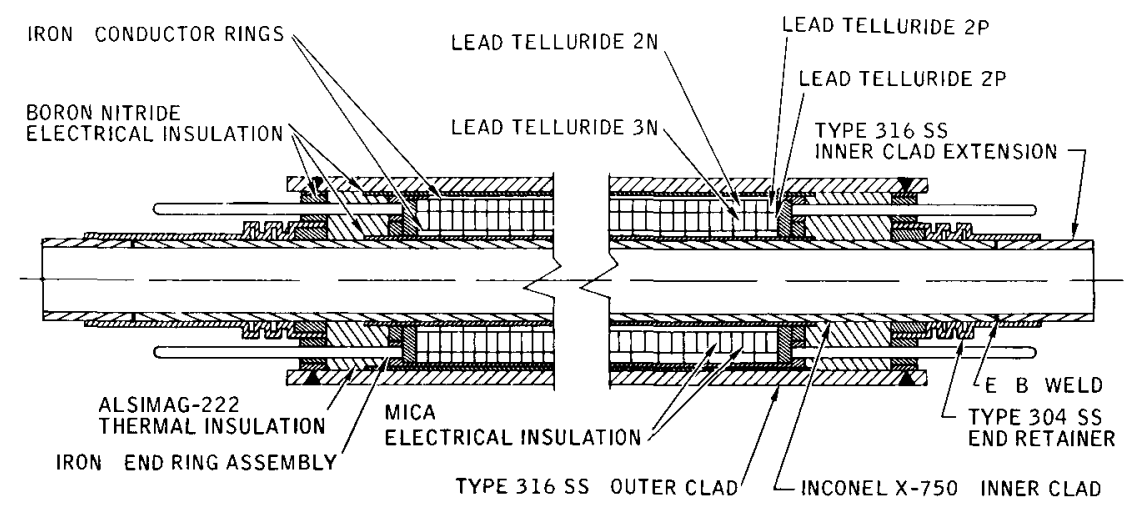

These submodules are then connected together to form the converter. The number of submodules used determines the power output and other characteristics of the converter. The submodules may be connected together in series or parallel, both hydraulically and electrically.

This type of compact converter module has been fabricated and is currently undergoing development testing. While the temperature capability, stability, and reliability of this compact 
converter design are still to be demonstrated, extensive development and life testing and, for lower powered systems, operational orbital use of other $\mathrm{PbTe}$ converter configurations, assure the avallability of $\mathrm{PbTe}$ thermoelectric converters.

For purposes of comparison with systems using a SiGe converter, the $\mathrm{PbTe}$ system will be illustrated on the same basis as in the previous section. Figure III-38 shows the overall system characteristics for a $\mathrm{Po}^{210}$-fueled system using a $\mathrm{PbTe}$

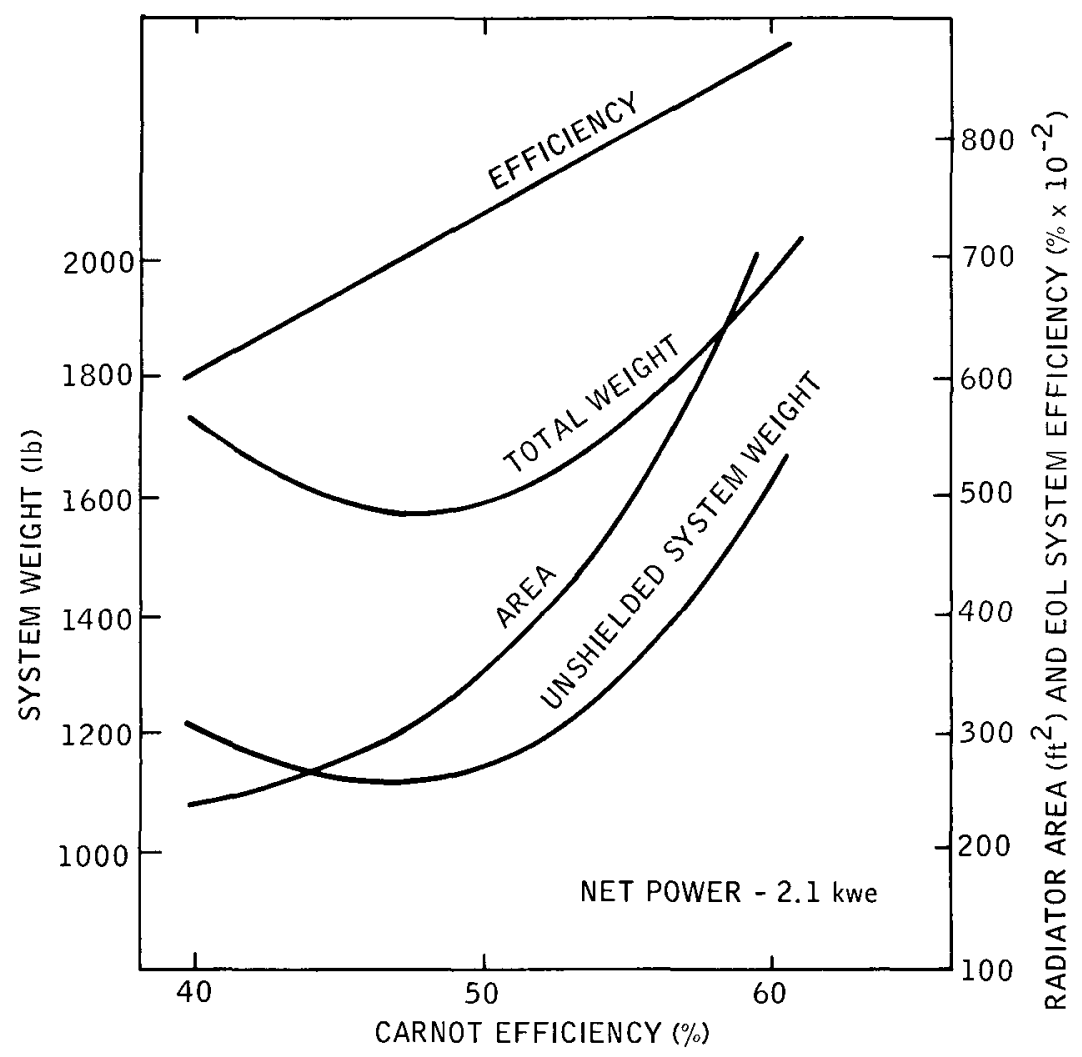

72966 UNCL

Figure III-38. Po 210 PbTe System Performance
vs Carnot Efficiency 
converter. The mission lifetime is 90 days and the net electrical output power is $2.1 \mathrm{kw}$. The design reliability goal $1 \mathrm{~s} 0.98$. The trends of weight, area, and efficiency as a function of Carnot efficiency are the same as discussed for systems using a SiGe converter. The radiator areas for the PbTe systems are greater than for the $\mathrm{S}_{1} \mathrm{Ge}$ systems, approximately $40 \%$ greater for minimum-weight systems, because of the lower operating temperatures of the PbTe systems. The system weight curves are again relatively flat, varying $10 \%$ over the range of 40 to $50 \%$ Carnot efficiency. The total system weights are about the same for the $\mathrm{PbTe}$ and $\mathrm{SiGe}$, with the $\mathrm{PbTe}$ showing a slight advantage. However, the system efficiency of the $\mathrm{PbTe}$ system is substantially higher than the SiGe system, about $25 \%$ higher at the minımum weight point. Figure III-39 shows the weight, area, and efficiency for the 2.1-kwe $\mathrm{Pu}^{238}$ system using the PbTe converter.

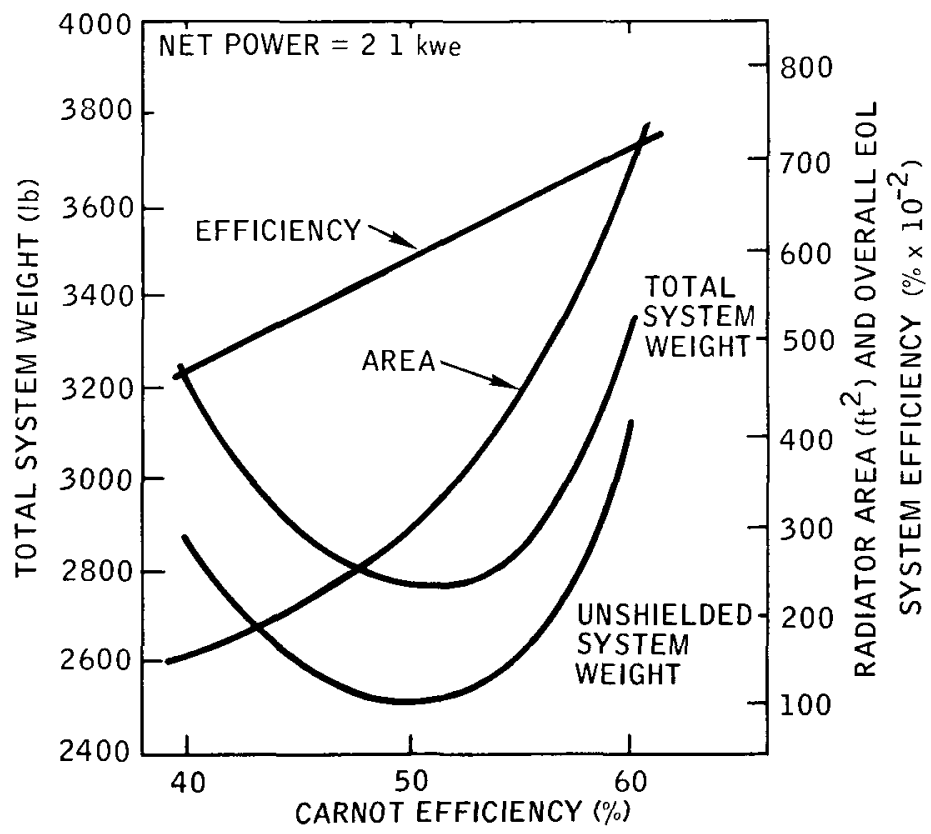

72966 UNCL

60902515

Figure III-39. Pu ${ }^{238}$ PbTe System Performance vs Carnot Efficiency 
The effect of net electrical power on system weight is shown in Figure III-40 for the Po 210 system, and in Figure III-4I for the Pu ${ }^{238}$ system. Again the same characteristics apply to the variation in system weight and radiator area with net electrical power for the $\mathrm{PbTe}$ systems as for the $\mathrm{S}_{1} \mathrm{Ge}$ systems. Also, the effects of hot-junction temperature on system weight, efficiency, and radiator area discussed previously again apply.

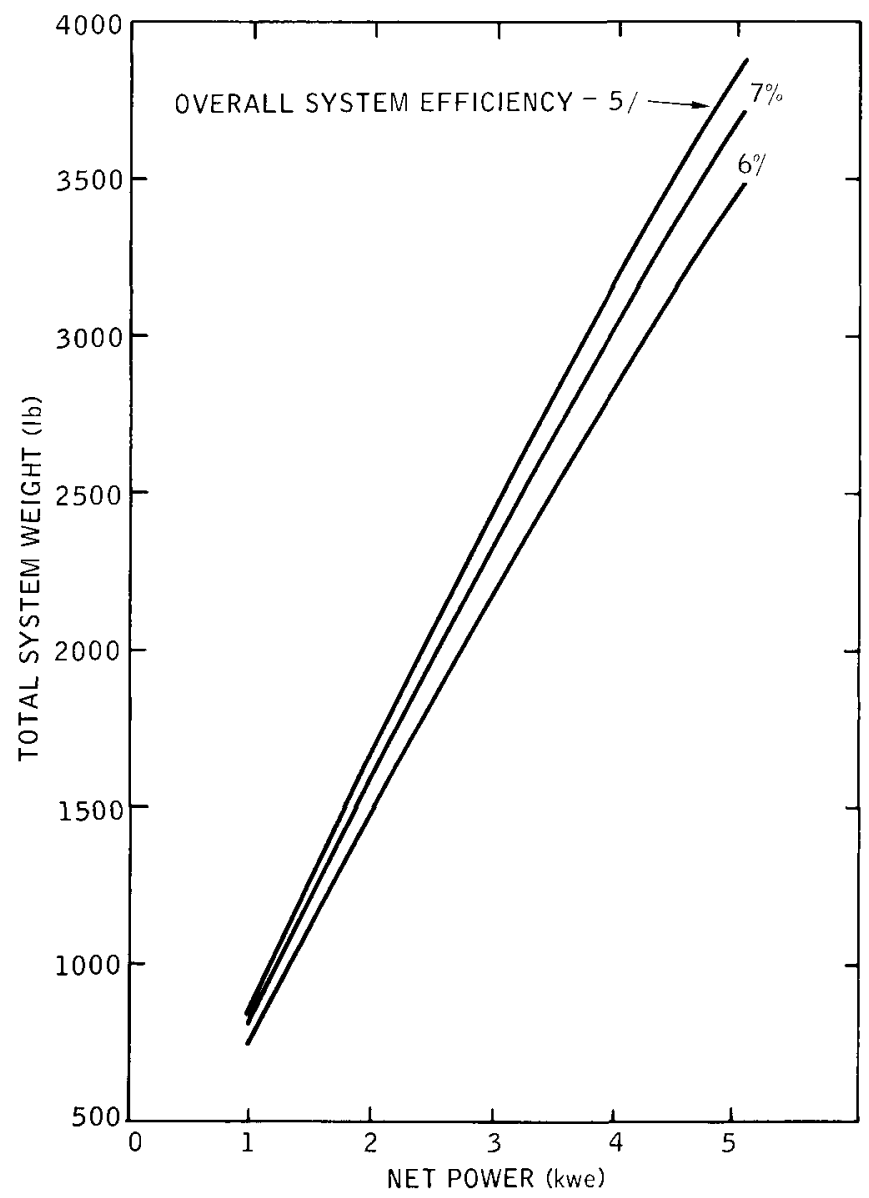

72966 UNCL

Figure III-40. Po ${ }^{210}$ PbTe System Weight vs Power 


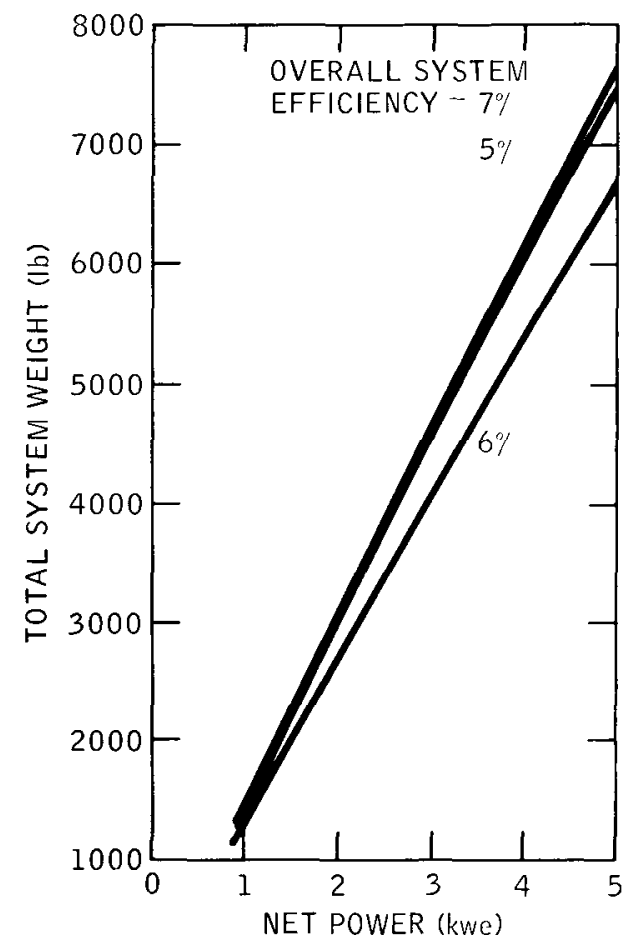

72966 UNCL

60902514 Figure III-41. Pu ${ }^{238}$ PbTe System
Weight vs Power

The use of PbTe for thermoelectric converters is desirable due to its materials efficiency being about $50 \%$ above the next best material. However, limitations on the use of $\mathrm{PbTe}$ exist, both in temperature and in the structural integrity of the material, which pose fabrication difficulties and contribute to a fairly high degradation rate.

The advantage of the PbTe occurs in systems where fuel weight and shield weight are significant fractions of the total system weight. In these cases, the high efficiency of the PbTe results in fuel inventory reduction and therefore also in shield weight reduction. For Po 210 -fueled systems, the difference between the systemweights using $\mathrm{PbTe}$ and $\mathrm{SiGe}$ converters is small, 
but for $\mathrm{Pu}^{238}$-fueled systems the $\mathrm{PbTe}$ results in a weight savings of about $10 \%$ due to the reduction in fuel weight.

Table III-12 presents system design summaries for 2.1-kwe $\mathrm{PbTe}$ systems. A 5-kwe system fueled by $\mathrm{Po}^{210}$ having a 90-day life would weigh about $3400 \mathrm{lb}$ with a $\mathrm{PbTe}$ converter. The same system fueled with $\mathrm{Pu}^{238}$ and having a 2-year life would weigh $6700 \mathrm{lb}$.

TABLE III- 12

2.1-kwe ISOTOPE PbTe SYSTEMS

\begin{tabular}{|c|c|c|}
\hline & $P_{0}^{210}$ & $\mathrm{Pu}^{238}$ \\
\hline Net Power (kwe) & 2.1 & 2.1 \\
\hline Life (yr) & 0.25 & 2 \\
\hline Full Power Reliability & 0.98 & 0.98 \\
\hline Dose Criterion (rem/90 day) & 10 & 10 \\
\hline Dose Plane Drameter (ft) & 10 & 10 \\
\hline Separation Distance $(\mathrm{ft})$ & 16 & 16 \\
\hline End-of-Life Efficiency $(\%)$ & 5.95 & 5.7 \\
\hline Beginning-of-Life Thermal Power (kwt) & 59.6 & 35.5 \\
\hline Radiator Area $\left(\mathrm{ft}^{2}\right)$ & 200 & 250 \\
\hline Specıf $1 \mathrm{c}$ Weıght (lb/kwe) & 809 & 1435 \\
\hline \multicolumn{3}{|l|}{ Weight Summary (lb) } \\
\hline Heat source & 367 & 1660 \\
\hline Shreld & 440 & 270 \\
\hline Pump & 65 & 65 \\
\hline TE converter & 165 & 150 \\
\hline $\mathrm{NaK}$ & 35 & 75 \\
\hline Expansion compensator & 31 & 40 \\
\hline Radiator and piping & 375 & 480 \\
\hline Power flattener & 30 & \\
\hline Structure & 40 & 60 \\
\hline Instrumentation and control & 70 & 70 \\
\hline Total & 1618 & 2870 \\
\hline
\end{tabular}




\section{c. Cascaded Thermoelectric System}

The two thermoelectric systems discussed above are based on the use of single converter units. The different thermoelectric materials have different optımum operating temperatures. The use of both the $\mathrm{PbTe}$ and $\mathrm{S}_{1} \mathrm{Ge}$ converters in the same system will yield a higher system efficiency and hence lower fuel inventory, lower system weight, and lower radiator area. Significant improvements are possıble for the se parameters.

Figure III-42 shows the flow schematic of a cascaded thermoelectric system capable of using both of the compact converters at their optimum temperature points. It can be seen that the coupling between converters is accomplished by a pumped liquidmetal loop similar to the normal system hot and cold loops discussed previously.

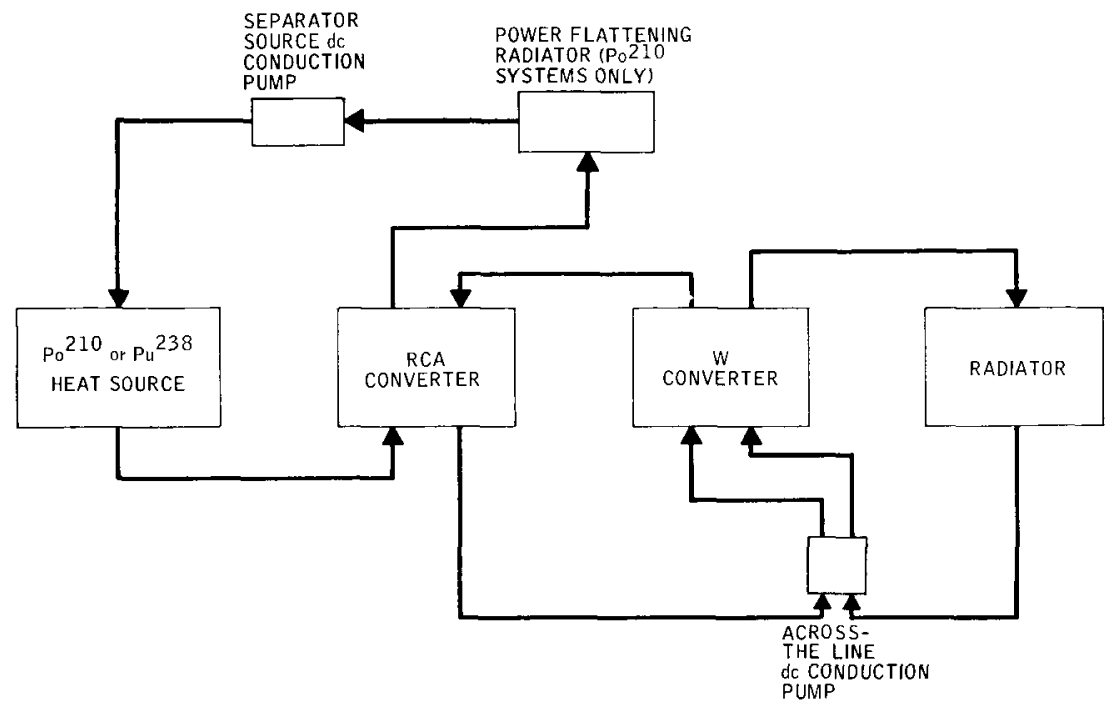

72966 UNCL

Figure III-42. Cascaded System Schematic 


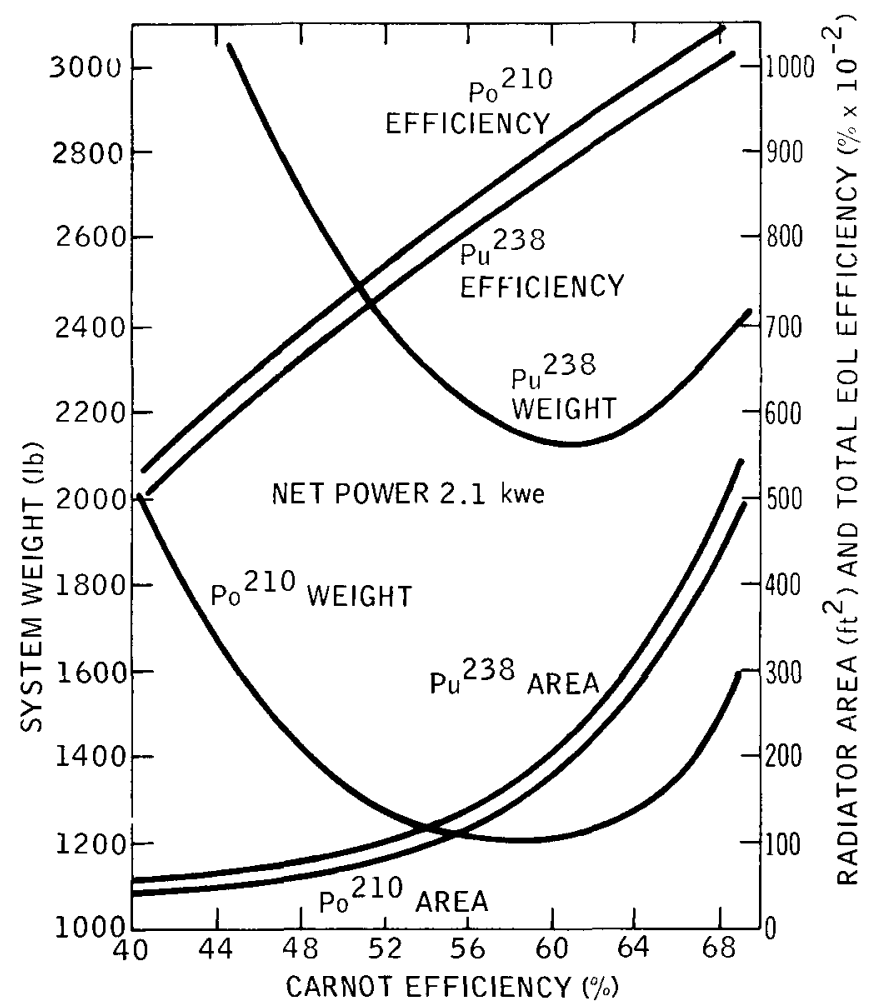

$7-29-66$

$6090-2511$

Figure III-43. Cascaded System Performance

TABLE III- 13

CASCADED TE SYSTEM PERFORMANCE

\begin{tabular}{c|c|c|c|c}
\hline \multirow{2}{*}{$\begin{array}{c}\text { Power } \\
\text { (kwe) }\end{array}$} & \multicolumn{2}{|c|}{ Po $^{210}$} & \multicolumn{2}{|c}{$\mathrm{Pu}^{238}$} \\
\hline 2.1 & $\begin{array}{c}\text { Area } \\
\left(\mathrm{ft}^{2}\right)\end{array}$ & $\begin{array}{c}\text { Weight } \\
(1 \mathrm{~b})\end{array}$ & $\begin{array}{c}\text { Area } \\
\left(\mathrm{ft}^{2}\right)\end{array}$ & $\begin{array}{c}\text { Weight } \\
(1 \mathrm{~b})\end{array}$ \\
\hline 5.0 & 100 & 1,210 & 150 & 2,200 \\
10.0 & 240 & 2,875 & 355 & 2,840 \\
15.0 & 780 & 5,560 & 710 & 8,880 \\
\hline
\end{tabular}

The above weights are based on a 10 -rem allowable radiation dose at a distance of $16 \mathrm{ft}$ per each 90 days of the mission. 
The cascaded system is composed of components identical to those of the single converter systems above. The compact converters are operated in the same temperature ranges as are the liquid-metal ( $\mathrm{NaK}$ ) loops. It can be concluded that the heat source requires the most effort but is technologically capable of a low-risk development cycle.

The use of a cascade system is dependent on the development of both compact converter designs.

Figure III-43 shows the performance characteristics, 1.e., area, weight, efficiency, of the cascaded system as radiator temperature (Carnot efficiency) is varied. It can be seen that the minimum weight occurs at about $60 \%$ Carnot. The data shown are for 2.1 -kwe systems for 90 days with $\mathrm{Po}^{210}$, and 2 years with $\mathrm{Pu}^{238}$. The weight varies almost linearly with power, as does area. Therefore, this system at 5, 10, and 15 kwe has the characteristıcs shown in Table III-13.

\section{F. MERCURY RANKINE SYSTEM}

The radioisotope mercury Rankine system offers a lightweight, low specific area electrical power system for space applications. Modules of 3 to 5 kwe, presently in an advanced state of development, can be used in multiples to produce up to 30 or 40 kwe of power. High reliability of such systems can be achieved since it is relatively simple to include additional standby modules for redundancy.

Figure III-44 is a schematic of a typical radioısotope mercury Rankine system employing two basic power conversion modules. This could depict either two active power conversion modules or one active and one standby module. Also shown is one method of achleving power per module or higher module reliability through the use of redundant CRU's in a given module. Mercury is boiled and superheated in a single-tube, once-through boiler which receives its heat by the rmal radiation from the isotope heat source. 'The superheated vapor drives a turboalternator 


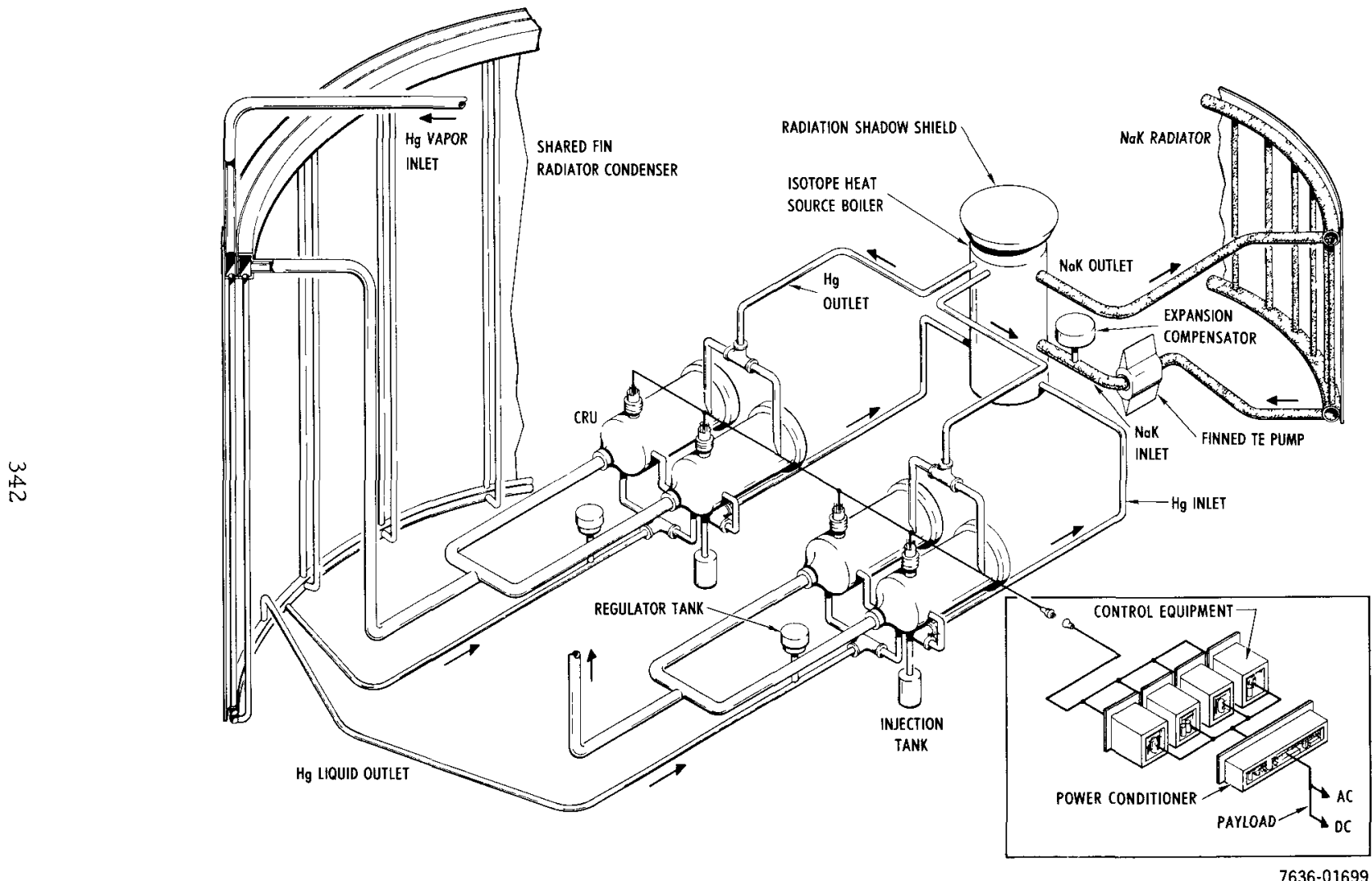

Figure III-44. Multiple PCS Mercury Rankine System with Isotope Heat Source 
unit, is then condensed in a multitube radiator-condenser (RC) and the condensate is pumped back to the boller. The turboalternator Combined Rotating Unit (CRU), shown in Figure III-45, is a single-shaft, hermetically sealed unit which contains all the system rotating components, 1.e., the turbine, alternator, and condensate pump, and is supported by liquid-mercury-lubricated journal and thrust bearings. The turbine back pressure (condenser pressure) is maintained constant by a passive bellows pressure regulator tank at the condenser exit, which accepts $R C$ inventory changes at constant pressure. A cavitating venturi is used to passively maintain constant flow to the boller.

An auxiliary $\mathrm{NaK}$ coolant loop provides cooling for the 1sotope prior to startup of the system, as well as during any shutdown periods. This loop also acts to remove the excess heat required to maintain constant turbine inlet temperature as the isotope decays.

System startup is achieved by injecting pressuriced liquid mercury from a bellows injection tank into the hot boiler (and simultaneously through the CRU bearings). Vapor from the boiler passes through the turbine spinning up the CRU and is condensed in the cool radiator condenser. This injection process continues until the CRU mercury pump primes and takes over the system flow. The pump will prime as soon as the RC pressure builds up sufficiently as a result of preheat from the continued vapor condensation. During the preheat process, liquid mercury collects in the RC both from condensation and from the bearing drain flow. When the pressure builds up to the design point, the regulator tank becomes effectıve and accepts this excess mercury from the $\mathrm{RC}$ and the system reaches steady state. The excess inventory in the regulator tank can be simply transferred back to the injection tank to provide restart capability. This startup process can be utilized for an orbital startup or slightly modified for prelaunch startup. 


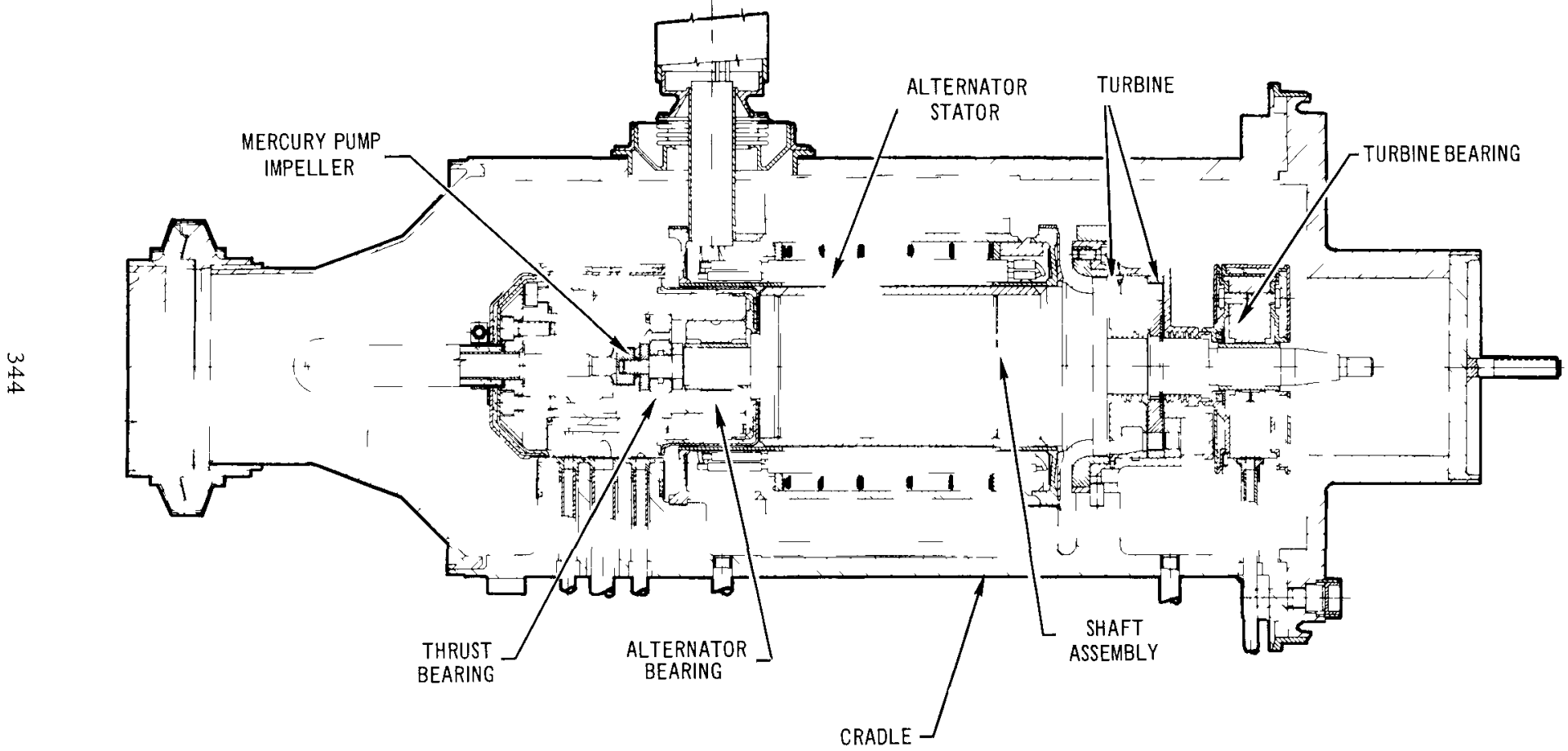

Figure III-45. Turboalternator Assembly - CRU-V 
a State of Development

The Mercury Rankıne Program (MRP) hardware is all in an advanced state of development. In addition, a great deal of complete developmental prototype system development testing has also been achieved on the program. Table III-14 summarizes some of the more pertinent test experience on the program.

TABLE III- 14

MRP EXPERIENCE SUMMARY

\begin{tabular}{l|r}
\hline \hline Operation of CRU Machinery (hr) & \\
Accumulated SNAP 2/MRP machinery & 30,000 \\
Accumulated prototype CRU-V machinery & 20,000 \\
One machine & 6,600 \\
Operation of Mercury-Lubricated Bearing (hr) & 150,000 \\
Component Test Experience (hr) & \\
NaK-Hg boiler & 26,000 \\
NaK TE pump & 17,000 \\
Radiator-condenser & 2,000 \\
Load control & 30,000 \\
Integrated System Experience (hr) & 550 \\
PSM-1 (flight configuration) & 1,200 \\
PSM-3 (orbital simulation) &
\end{tabular}

The CRU has recerved by far the most development effort in the MRP. Two models have been under test in the past few years the CRU-IVM, a developmental prototype, and the CRU-V, the flight prototype. Over 20,000 hours of testing have been achleved with the CRU-V unit. Thus far, seven units have operated for more than 1000 hours, s1x units have operated more than 2500 hours, and one unit (which 1 s still on test) has operated in excess of 4200 hours. An intensive design review and subsequent modifications in the unit were effective in eliminating the infant mortality failure modes of the unit. 


\section{b. System Performance}

The overall power system performance for the radiolsotope Rankine system is a function of bolling temperature (source temperature), the heat rejection temperature, and the mission duration (type of isotope). The various tradeoffs to be considered are efficiency, area, and weight. For isotope systems, and in particular for the mercury Rankıne system because of its low area requirements, the maximum efficiency system (also close to the minimum weight system) is usually selected at an increased area penalty to minimize system (isotope inventory) cost.

For potential manned mission requirements, power systems of from 5 to $15 \mathrm{kwe}$ can be made up of from one to three 5 -kwe power conversion modules operating in parallel from the isotope heat source. Additional redundant modules can also be added as required from reliability aspects. The basic 5-kwe module would operate with the performance characteristics listed in Table III- 15 .

TABLE III- 15

\section{5-kwe NET MODULE DESIGN POINT}

\begin{tabular}{l|l}
\hline Gross Electrical Power (kwe) & 5.75 \\
Turbine Inlet Temperature $\left({ }^{\circ} \mathrm{F}\right)$ & 1300 \\
Boiling Temperature $\left({ }^{\circ} \mathrm{F}\right)$ & 1065 \\
Turbine Inlet Pressure (psia) & 250 \\
Turbine Discharge Temperature $\left({ }^{\circ} \mathrm{F}\right)$ & 578 \\
Turbine Discharge Pressure (psia) & 5.1 \\
Turbine Efficiency $(\%)$ & 55 \\
R-C Pressure Drop (psi) & 1.6 \\
Pump Inlet Pressure (psıa) & 3.5 \\
Pump Discharge Pressure (psia) & 450 \\
Net System Efficiency (\%) & 11.3 \\
RC Area Per Module (ft ${ }^{2}$ ) & 109 \\
End-of-Life Thermal Power (kwt) & 44.4 \\
Flow (lb/min) & 17.4 \\
\hline
\end{tabular}

The 5.75-kwe gross power to achieve 5 kwe net includes an allowance of $5 \%$ for system degradation and $10 \%$ for power conditioning. 
A reference 5-kwe radioısotope mercury Rankine power system description is presented below.

This power conversion system would be made up of 1 active 5-kwe net module and a standby redundant module for increased reliability. This redundant module shares the radiator fin area of the active unit without imposing an additional area penalty.

A single isotope heat source radiates to the boiler tube-fin arrangement. The specific isotope heat source to be employed would depend on the mission duration. Polonium $\mathrm{x} 11$ be used for the shorter ( $\sim 90-\mathrm{day})$ missions and plutonium for the longer duration missions. Shielding requirements for the se two isotopes differ significantly and will require modification with specific mission variations.

A single auxiliary $\mathrm{NaK}$ loop is provided for cooling of the 1sotope prior to startup and during any shutdown periods, and also to reject the excess isotope decay heat to mantain constant turbine inlet conditions. The loop consists of a set of coolant tubes mounted on the boller tube-fin arrangement, a directradiating thermoelectric NaK pump and a high-temperature NaK radiator. This auxiliary cooling function could also be accomplished by radiation to space with a variable-shutter type of control.

Table III-16 lists the overall system performance characteristics of the 5-kwe mercury Rankine power system, and Table III-1 7 gives the system weight breakdown.

\section{c. Summary of Characteristics}

To a first approximation, all of the various power conversion system concepts exhibit approximately the same weight per kilowatt when they are compared under the same ground rules. The real differences among the systems can only be seen in cycle efficiency, radiator area, inherent system reliability, and the state of development. 
TABLE III- 16

5-kwe MERCURY RANKINE POWER SYSTEM PERFORMANCE CHARACTERISTICS

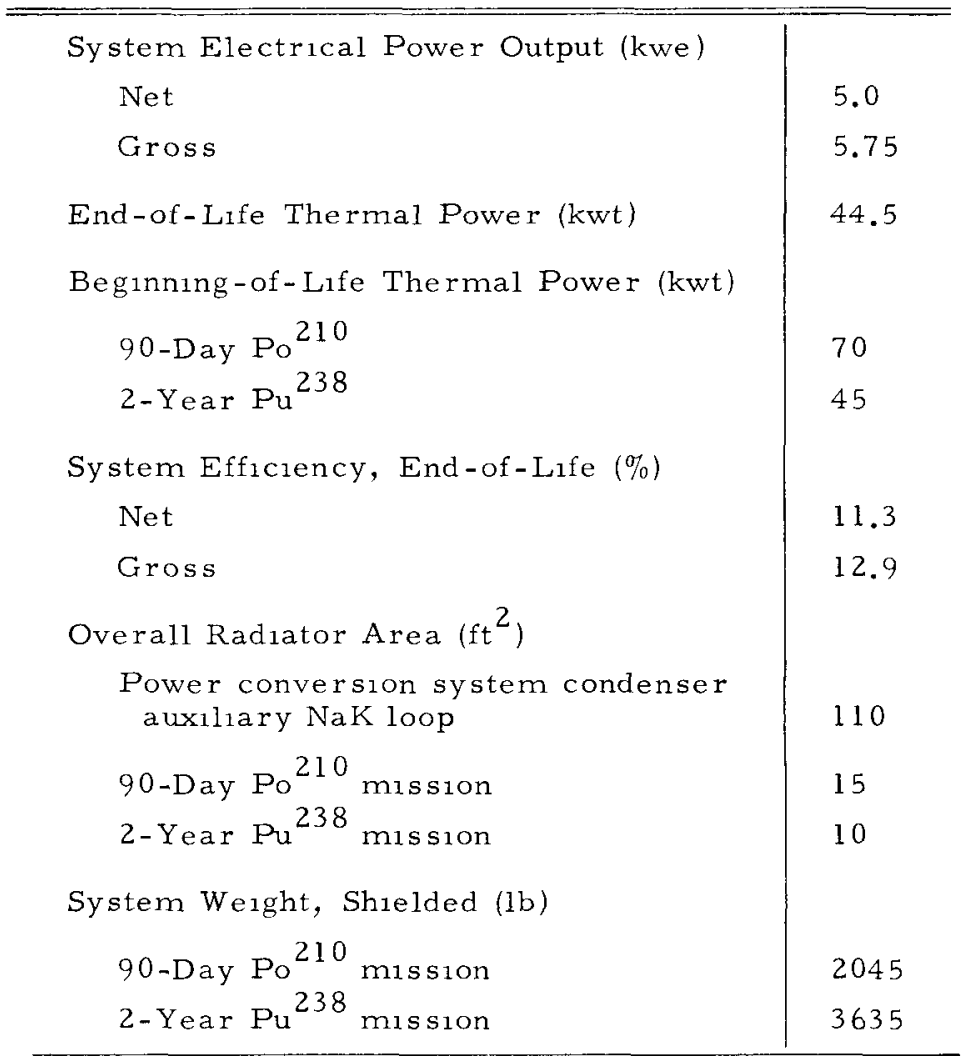


TABLE III- 17

5-kwe NET MERCURY RANKINE SYSTEM WEIGHT BREAKDOWN (lb)

\begin{tabular}{|c|c|c|}
\hline & Active & Standby \\
\hline \multicolumn{3}{|l|}{ Basic Power Conversion System } \\
\hline CRU modules & 90 & 90 \\
\hline Boilers & 25 & 25 \\
\hline Radiator condensers & 175 & 35 \\
\hline Startup equipment & 75 & 160 \\
\hline $\begin{array}{l}\text { Electrical and power- } \\
\text { conditioning equipment }\end{array}$ & 100 & 100 \\
\hline Structure & 75 & 75 \\
\hline Miscellaneous & 50 & 50 \\
\hline Active module weight & 590 & \\
\hline \multirow[t]{2}{*}{ Redundant Standby 5-kwe Module } & & 535 \\
\hline & $90-$ day $P_{0}^{210}$ & 2 -year $\mathrm{Pu}^{238}$ \\
\hline \multicolumn{3}{|l|}{ Auxilıary NaK Loop } \\
\hline Thermoelectric pump & 20 & 20 \\
\hline Radiator & 40 & 30 \\
\hline Miscellane ous & 10 & 10 \\
\hline \multirow[t]{2}{*}{ Subtotal } & 70 & 60 \\
\hline & $90-$ day $P_{0}^{210}$ & 2 -year $\mathrm{Pu}^{238}$ \\
\hline \multicolumn{3}{|l|}{ Heat Source } \\
\hline Isotope heat source & 450 & 2200 \\
\hline Shielding ${ }^{\dagger}$ & 510 & 360 \\
\hline Subtotal & 960 & 2560 \\
\hline Total Shrelded Weight & 2045 & 3635 \\
\hline Total Unshielded Weight & 1535 & 3275 \\
\hline
\end{tabular}

Includes ablative reentry protection

†Using 10 rem per 90 days at a mean separation of $16 \mathrm{ft}$ 


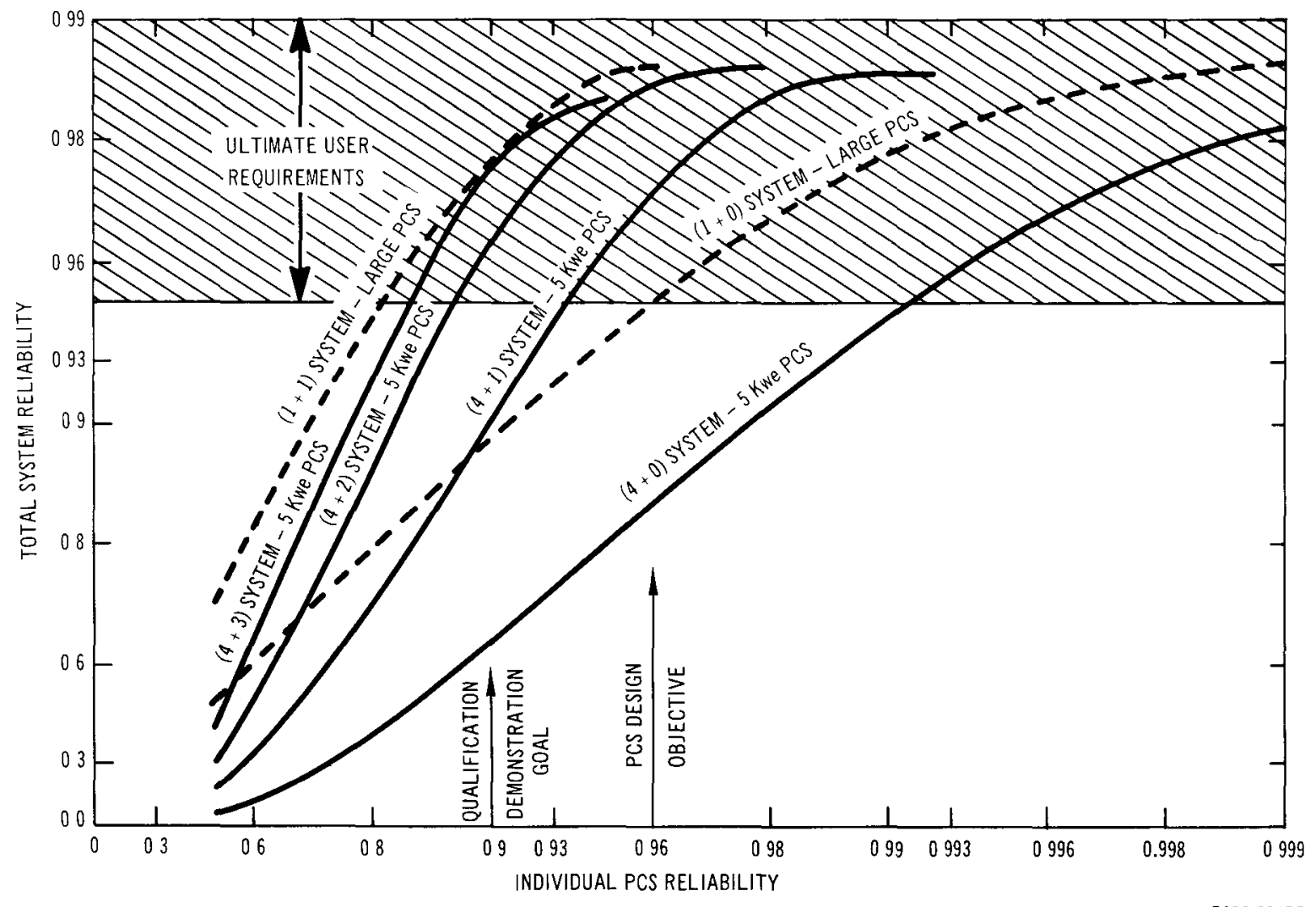

Figure $\amalg-46$. Effect of PCS Redundancy on Total System Reliability, 20-kwe System 
The mercury Rankine system is characterized by a moderately high efficiency $(8$ to $14 \%$ ) and a low specıfic area requirement ( 15 to $25 \mathrm{ft}^{2}$ per net electrical kilowatt). To achieve the higher efficiencies one must sacrifice radiator area, and 1ıkewise minimum-area systems exhibit the lower efficiencies.

Another important aspect of the Rankine cycle is its relative insensitivity to effective sink temperature variations because of the high $\left(\sim 600^{\circ} \mathrm{F}\right)$ heat rejection temperature. One disadvantage is the requirement to maintain vapor-liquid interfaces in the boile $r$ and condenser under orbital zero-g, and also in most cases, small negative-g loads. Whereas this represents a disadvantage, there are straightforward design procedures avallable to overcome this problem. In the boller where large pressure drops can be tolerated, this is accomplished by simply swirling the two-phase mixture to achieve high internal body forces. Methods - while not as straightforward and simple are also avallable to achieve stable interfaces in the radiator condenser through control of the tube diameter, the relative friction loss to momentum gain (entrance loss and condensing length), and the velocity gradient at the interface itself. In the present design, one to two psi of pressure drop in the RC is sufficient to protect against interface instability up to about a tenth of a "g" negative acceleration.

The inherent reliability of the mercury Rankıne conversion system is essentially that of any of the rotating machinery concepts. Whereas this is lower than that achievable with a static thermoelectric system, very high overall system reliabilities can be achieved through the use of redundant standby units. This can be seen from the solid lines of Figure III-46 as a function of individual module reliability and the degree of redundancy employed. The system depicted is a 20 -kwe system made up of four active 5-kwe net modules. The turnover portion at the top of the curve is the reliability limit imposed by the remainder of the system, 1. e., heat source, etc. From this curve, one can 
see that for a demonstrated module reliability of 0.9 with no redundancy, one can demonstrate a system reliability of only about 0.63 . With one redundant unit this increases to 0.9 , with two redundant to 0.96 , etc. This is the approach taken with the mercury Rankine cycle. Each additional redundant 5-kwe module costs about $400 \mathrm{lb}$ in weight but does not add a significant area penalty since it shares the area already provided for the active units, requiring only an added set of tubes.

\section{G. MANNED BRAYTON SYSTEMS}

The Brayton cycle offers a conversion system with the highest potential performance of the three systems discussed. The efficiejcy of the Brayton systems is a factor of two greater than that of the mercury Rankine systems and a factor of 2 to 4 greater than that of the thermoelectric systems. The Brayton systems are amenable to modular development, as with the mercury Rankine systems. Again 4 to 6 kwe modules can be used to obtain the desired power level. Also, redundant power conversion system modules can be used in order to obtain a high overall system reliability.

The Brayton cycle ideally is composed of two reversible constant-pressure processes and two adiabatic reversible processes. The real cycle requires modification to the ideal cycle diagram since the compression and expansion processes cannot be isentropic. The cycle efficiency can be increased greatly if the energy rejection from the cycle can be conserved. This is accomplished regeneratively by a recuperator. Waste thermal energy is transferred from the turbine exhaust to the compressor discharge gas, the reby retaining this energy in the cycle.

The fluid cycle schematıc for a single-shaft, radial-flow power conversion system is depicted in Figure III-47. The working gas is heated by the radiolsotope heat source heat exchanger and passes through the radial turbine. It then enters the recuperator, regeneratıvely heatıng the return flow to the heat exchanger. 


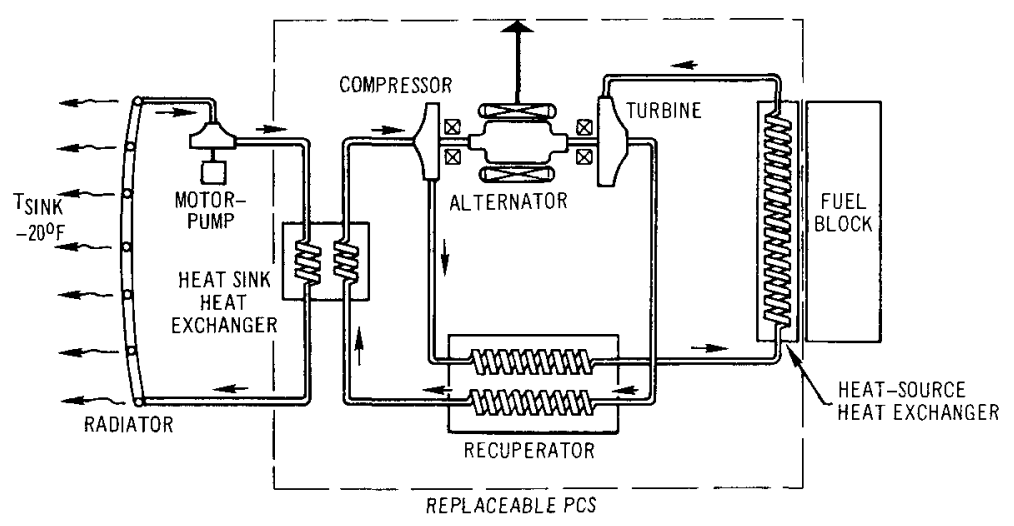

Al 6501117

Figure III-47. Brayton Cycle System Schematic

The gas is then cooled in the radiator, compressed in the radial compressor, regeneratively heated in the recuperator, and returned to the heat-source heat exchanger to complete the cycle. A motor-driven jacking-gas compressor may be provided to increase gas pressure in the bearings of the combined rotating unit for increased load-carrying capability under launch conditions.

The alternator provides raw electrical power to the powerconditioning system. A parasitic load associated with each PCS maintains constant output power despite normal fluctuations in the load demand.

The heat-rejection loop removes waste heat from the heatsink heat exchangers and cools the alternator and bearings. A coolant reservoir is provided to accommodate thermal expansion of the fluid and minor system leaks. In addition, this reservoir is preloaded to ensure the required NPSH at the pump inlet.

An isometric drawing of a typical isotope Brayton cycle power system designed for a manned space station is shown in Figure III-48. Two completely independent heat source/PCS units operate in parallel to provide station power. $\mathrm{Pu}^{238} \mathrm{O}_{2}-$ filled fuel capsules are located in a graphite fuel block. The fuel 


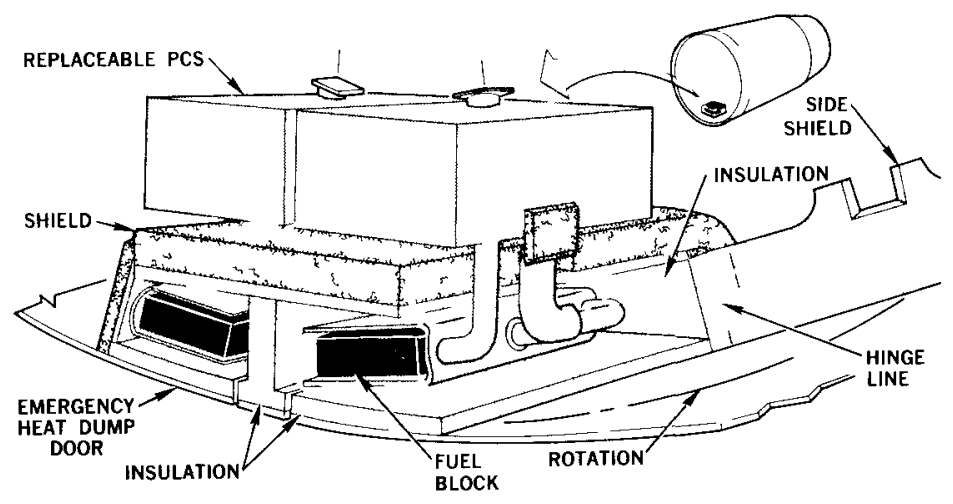

Al 660021

Figure III-48. Pu ${ }^{238}$ Brayton Cycle System

blocks are shaped to function as a reentry body and provide intact reentry of the fuel capsules in the event of a mission abort. Heat is transferred by radiation from the fuel blocks to the Ushaped heat exchanger. The argon gas working fluid is heated in the heat exchanger and flows to the Brayton cycle rotating assembly which is located above the lithium-hydride shield.

Insulation completely surrounds the fuel block and heat exchanger to limit thermal losses. Emergency heat dump doors are located beneath each fuel block to allow direct fuel block radiation to space in the event of a PCS failure. Upon replacement of the falled PCS module, the heat dump door may be closed and normal operation resumed.

The lithum-hydride shadow shield has a movable side portion - shown in the open position - to permit PCS replacement. The shield is designed to limit direct- and scatterradiation dose to the crew. Shield thickness is contoured to minimize system weight.

a. State of Development

The most detailed study to date of a radio1sotope Brayton cycle power system for a manned spacecraft application has been 
the study conducted by the joint AI/Douglas Aircraft/AiResearch team for NASA-Langley. This study defined an 11 -kwe power system for MORL. The system proved to be very attractive. Major conclusions from this study are given below.

The results of the CRU studies indicate that the performance and life goals required to meet the overall MORL requirements can be obtained, and no unique development problems have been introduced into the design.

The efficiencies of the compressor, turbine, and Rice alternator selected for the CRU can be obtained with a high degree of confidence. Based on experience using argon, compressors and turbines have been designed and tested and have closely demonstrated the desired performance at typical system conditions (specific speed, inlet pressure, etc.). A careful loss analysis of the Rice alternator design indicates a performance margin of $2 \%$ over that required to meet MORL system performance and weight estimates. Since similar Rice machines have been desıgned and tested, high confidence in the accuracy of the loss and efficiency analysis exists.

Stress analyses of the turbine rotor indicate that for over 50,000 hours of operation, the growth of the rotor will be less than $1 \%$ (according to data on conventional materials in air) and will not exceed normal operating clearances. Thus, it is expected that the turbine rotor can satisfy life requirements. Stresses in the compressor and alternator rotors are not critical.

The design efficiency of the turbine is 0.873 , based on totalto-total pressure ratio. A turbine configuration similar to the MORL turbine has been designed and tested as part of a Braytoncycle research package for NASA. This turbine, with a 6.00-in. rotor, has been tested in argon by NASA and has shown a design point efficiency of 0.90 based on total-to-total pressure ratio.

Test data were avallable on a 5-kwe 85,000-rpm Rice motor which had been operated as an alternator. The MORL alternator 


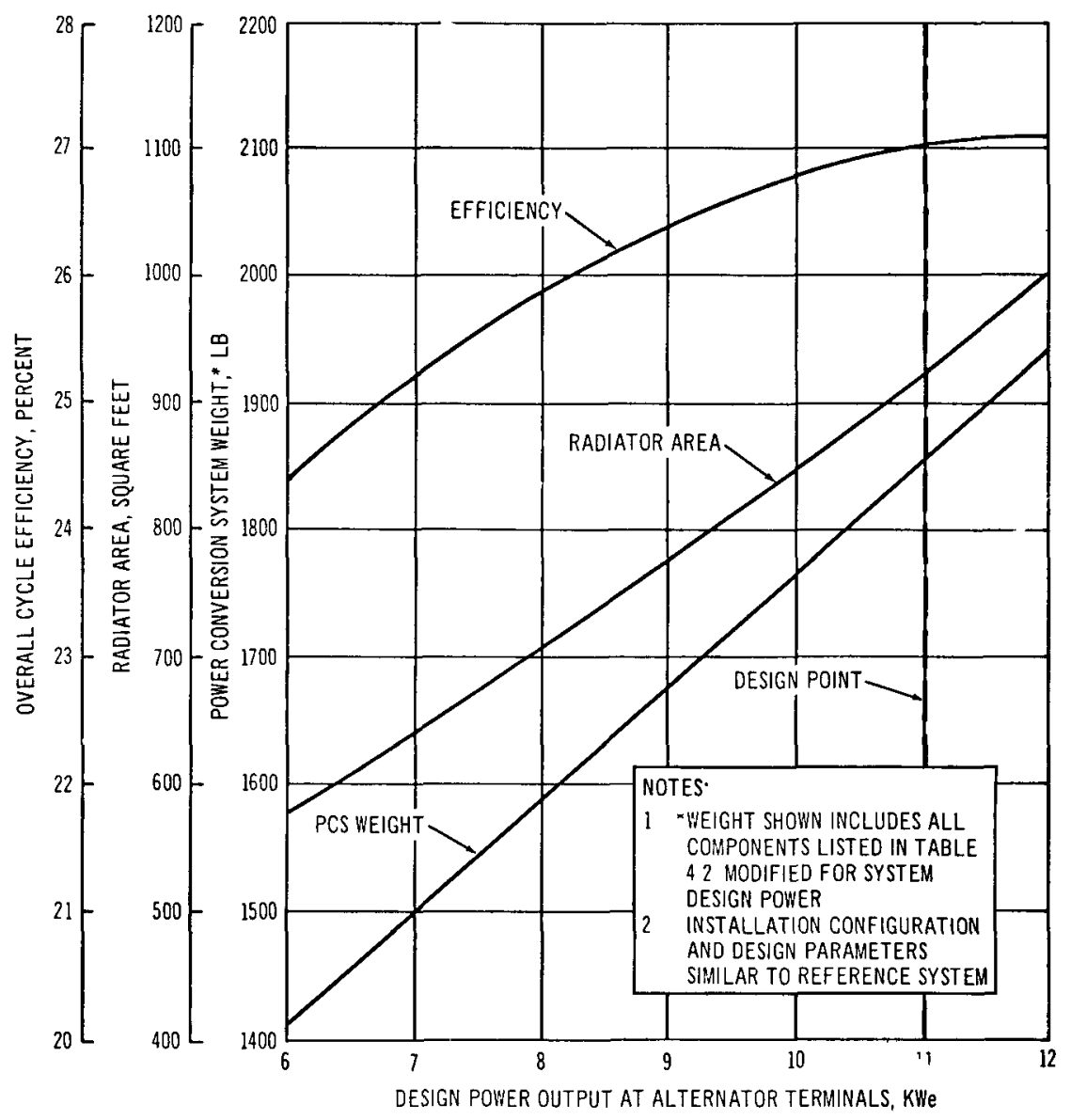

Figure III-49. PCS Weight as a Function of Power Rating 
design represented a minor uprating of this machine to 7 kwe using design and fabrication techniques proven at the 5-kwe level.

b. System Performance

The data shown below were developed specifically for the MORL application. The data are valid, however, for any manned spacecraft of the MORL category.

Power conversion system weight, radiator area, and overall cycle efficiency vs electrical power output are shown in Figure III-49. Overall cycle efficiency vs turbine inlet and compressor inlet temperature are shown in Figure III-50. The strong dependence of efficiency upon compressor inlet temperature is demonstrated. The MORL reference design point is shown. Fuel block and shield weight vs fuel block thermal power are shown in Figure III-5I.

Figure III-52 shows typical radioisotope Brayton cycle power system parameters optimized to provide $11 \mathrm{kwe}$ to a manned spacecraft payload. Selection of the fundamental PCS design parameters involved an overall analysis and optimization of heat source, PCS, and radiator requirements with respect to performance, weight, and physical size. From this optimization, a turbine inlet temperature of $1640^{\circ} \mathrm{F}$ and a radiator surface area of $920 \mathrm{ft}^{2}$, corresponding to a compressor inlet temperature of $65^{\circ} \mathrm{F}$, were selected.

Each of the two fuel blocks is designed to produce a thermal power output of $20.35 \mathrm{kw}$ at the end of a 5 -year mission. This output corresponds to an initial installed capacity of $21.15 \mathrm{kwt}$ when isotope decay is considered. This power level includes an allowance of $1.15 \mathrm{kwt}$ for each PCS to account for heat losses from the system. The fuel-block surface operates at a maximum temperature of $1800^{\circ} \mathrm{F}$, radiative heat transfer across an effective heat transfer surface area of $9.5 \mathrm{ft}^{2}$ (each fuel block) raises argon gas temperature to $1640^{\circ} \mathrm{F}$ in $1 \mathrm{ts}$ passage through the heat source heat exchanger. The combined rotating unit (CRU) design 


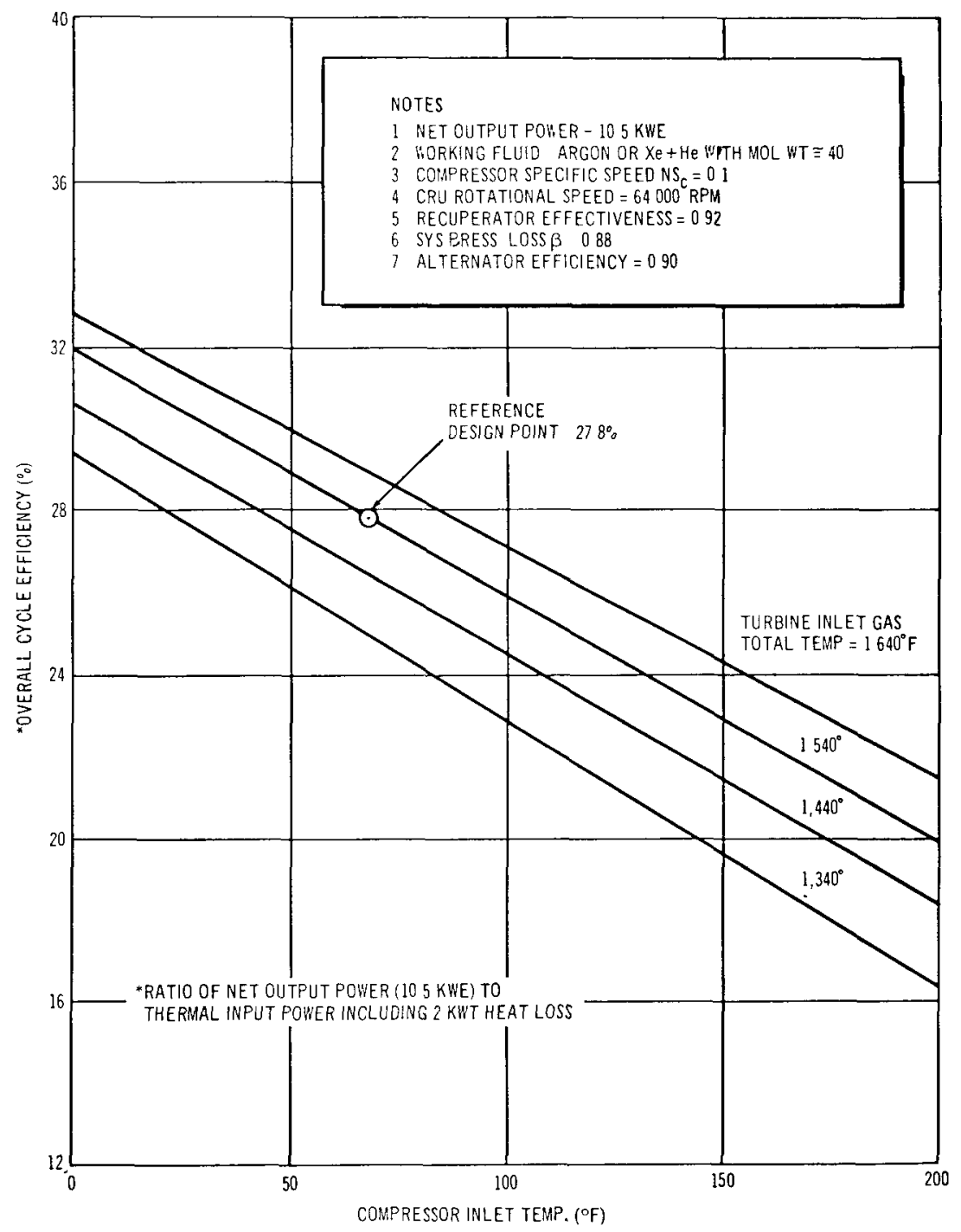

7-27-66 UNCL

Figure III-50. Compressor Inlet Temperature vs Overall Efficiency 


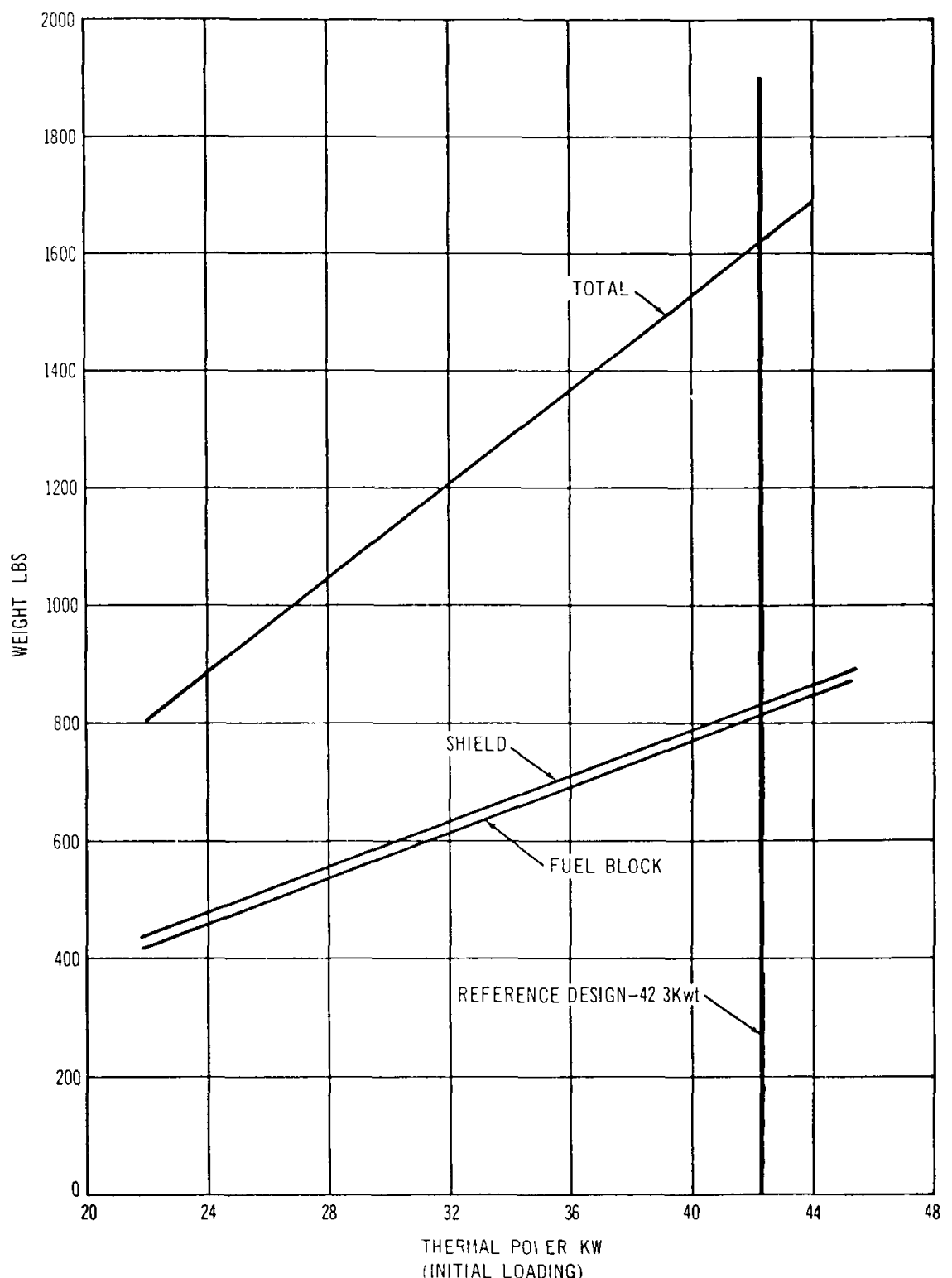

60902504

Figure III-51. We1ght vs Power 


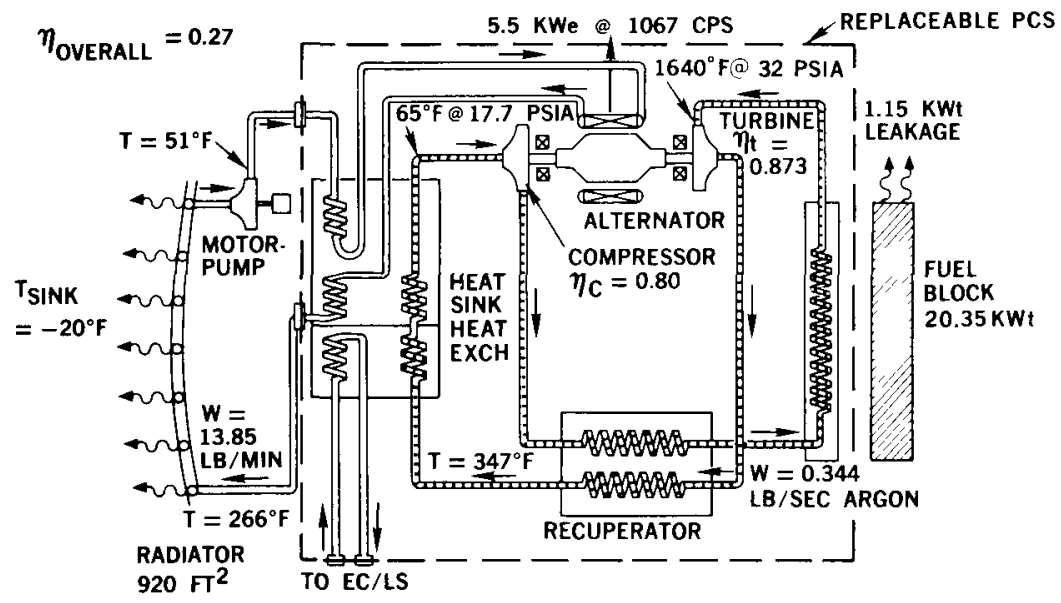

Al-66-0013

Figure III-52. PCS Schematic

is based on a turbine efficiency of $87.3 \%$ and a compressor efficiency of $80 \%$. Operation of the CRU at $64,000 \mathrm{rpm}$ results in an optimum pressure of 17.7 psia at the compressor inlet. Gas expansion through the turbine expends about $88 \%$ of the total head developed by the compressor; the remainder is allotted to pressure drop through heat exchange components and ducts.

The gas flow from the recuperator enters the heat sink heat exchanger at $347^{\circ} \mathrm{F}$, where provision is made for removing up to 2.42 kwt by the environmental control/1ife support (EC/LS) coolant.

The remainder of the waste heat load is transferred to the heat-rejection system. After passage through the compressor, the gas flow is regeneratively heated from 265 to $1203^{\circ} \mathrm{F}$ in the recuperator. The high recuperator effectiveness (0.92), combined with optimized cycle operating conditions, results in a cycle efficiency of $27 \%$ under design conditions.

In each of the heat-rejection systems the coolant, FC-75, removes waste heat from the sink heat exchanger and the alternator. Under design conditions, flow through the radiator 
reduces coolant temperature from 266 to $51^{\circ} \mathrm{F}$ by the rmal radiation to a design average heat sink temperature of $-20^{\circ} \mathrm{F}$.

System design parameters are shown in Table III- 18.

\section{TABLE III- 18}

SYSTEM DESIGN PARAMETERS

\begin{tabular}{l|l}
\hline Net Electrical Output Power & $11 \mathrm{kwe}$ \\
Design Orbital - Sink Temperature & $-20^{\circ} \mathrm{F}$ \\
Radıator Area & $920 \mathrm{ft}^{2}$ \\
Workıng Fluıd & Argon \\
Turbine Inlet Temperature & $1640^{\circ} \mathrm{F}$ \\
Compressor Inlet Temperature & $65^{\circ} \mathrm{F}$ \\
Shaft Speed & $64,000 \mathrm{rpm} \pm 1.25 \%$ \\
Recuperator Effectiveness & 0.92 \\
Total Pressure Loss Factor ( $\left.\mathrm{r}_{\mathrm{t}} / \mathrm{r}_{\mathrm{C}}\right)$ & 0.88 \\
Compressor Specifıc Speed & 0.092 \\
Alternator Efficıency & 0.90 \\
Total Heat Leakage & $2.3 \mathrm{kwt}$ \\
Bearıng Loss/CRU & $500 \mathrm{watts}$ \\
System Thermal Efficiency & 0.27 \\
(kwe/fuel kwt) & \\
\hline
\end{tabular}

Total for 2 PCS packages, at alternator terminals

The launch weight of the Plutonium Brayton Cycle (PBC) system is presented in Table III-19.

The very high efficiency of an isotope Brayton cycle system minımizes fuel block and shield weight and simplifies system design and integration into a manned spacecraft.

Minimizing the required isotope inventory is also important due to the current limited supply and high cost of the more attractive long-1ived isotopes. 


\begin{tabular}{|c|c|c|}
\hline & \multicolumn{2}{|c|}{$\begin{array}{l}\text { Weight } \\
(1 b)\end{array}$} \\
\hline Fuel Block and Radiation Shreld & & 1653 \\
\hline Power Conversion Unit & & 1344 \\
\hline Brayton cycle PCS (2) & 1124 & \\
\hline Coolant motor and pump as sembly (4) & 90 & \\
\hline Evaporator subsystem & 130 & \\
\hline Power Conditioning and Control & & 1089 \\
\hline Transformer and rectıfiers & 116 & \\
\hline Inverters & 119 & \\
\hline Varıable frequency 'nverters & 200 & \\
\hline Battery and battery charger & 430 & \\
\hline $\begin{array}{l}\text { Speed control, relays, breakers, and } \\
\text { miscellaneous }\end{array}$ & 224 & \\
\hline Vehicle Integration & & 1381 \\
\hline Interstage extension & 355 & \\
\hline Structural and mechanical & 665 & \\
\hline EC/LS system & 64 & \\
\hline Insulation and attachment & 133 & \\
\hline Radiator & 164 & \\
\hline Total system weight & & 5467 \\
\hline Extrapolated system weight & 4967 & \\
\hline
\end{tabular}

Excludes 519 lb for distribution and protection equipment.

Heat transfer from the fuel block to the working gas heat exchanger by direct radiation results in a very attractive system. The entire power conversion system can be replaced simply by disconnecting the radiator lines, the alternator electrical plug, and the mechanical support latches. No gas connections need be broken or resealed, and no difficult assembly operations are required. 
The very low radiator temperatures required for efficient cycle operation can be a problem in some applications, however, in large manned space station applications, the radiators usually integrate very well into the vehicle. The low heat rejection temperature allows use of space station skin itself for a radiating surface, and also minimizes thermal interfaces with the crew living quarters. Aluminum radiator tubes can be used and the spacecraft skin provides the required meteoroid protection without an additional weight penalty. Difficult radiator fabrication, corrosion, or plugging problems are not encountered. The use of argon in the primary loop eliminates corrosion problems within the PCS.

The Brayton $\mathrm{CRU}$ and $1800^{\circ} \mathrm{F}$ heat source represent the long lead items with this system. Neither are presently involved in a full development effort. The principal potential problem with the CRU is the thermal and mechanical integration of each shaft and housing component into a reliable and operable unit.

\section{H. RADIOISOTOPE SYSTEMS SUMMARY}

Based on the fuel, heat source, and power conversion considerations discussed previously, the final selection 1 s based on the combinations which best meet the requirements of a particular mission. The mission parameters which influence system selection are

Parameter

. Mission Life........... Fuel selection PCS selection

- Weight Constraints....... Fuel selection

- Area Constraints........ PCS selection

- Mission Type.......... Heat source configuration

. Mission Date.......... Fuel selection

. Volume Constrants........ Fuel, heat source, and PCS

A summary of the major elements is presented in the following. 
The power conversion equipment falls in one of two classes: static conversion or dynamic conversion. Static conversion includes thermoelectrics and thermionics. Dynamic power conversion includes Rankine and Brayton rotating machinery. For early programs thermionics cannot be considered. This leaves thermoelectrics and dynamic systems for consideration.

\section{a. Thermoelectric Conversion}

Thermoelectric (TE) conversion is characterized by simplicity, high reliability, and static operations. However, this is achieved at the expense of system conversion efficiency. There are two principal TE materials which must be considered. These are $\mathrm{SiGe}$ and $\mathrm{PbTe}$. Either of the se materials can provide an operating efficiency in the range of 5 to $6 \%$. The $\mathrm{PbTe}$ has a slight edge on efficiency but has a lower operating temperature and larger radiation area. SiGe has a lower inherent materials efficiency; however, its higher temperature capability helps to provide nearly equal system efficiency. The SiGe also requires about $1 / 4$ the radiation area of $\mathrm{PbTe}$.

The $\mathrm{PbTe}$ and SiGe thermoelectrics have both undergone extensive development. SNAP 10A utilized a direct radiating SiGe module which has now demonstrated several years of life at about $1300^{\circ} \mathrm{F}$. $\mathrm{PbTe}$ has also been used extensively. However, until recently it was never put through the exhaustive technology and device development effort through which SiGe has passed.

Presently both $\mathrm{PbTe}$ and SiGe are being developed in the configuration of a compact converter. The compact converters both utilize heat transfer from a hot liquid metal loop through the TE material to a cold liquid metal loop. These two concepts could result in demonstrated conversion devices in time for the early flights. Table III-20 shows design characteristics of several TE systems.

Based on current technology status only, TE conversion can be considered for the 1968-1970 time period. None of the dynamic 
TABLE III-20

RADIOISOTOPE SYSTEMS PERFORMANCE SUMMARY

(Manned applications; power $=5$ kwe)

\begin{tabular}{l|c|c|c|c}
\hline \multicolumn{1}{|c|}{ System } & $\begin{array}{c}\text { System } \\
\text { Life }\end{array}$ & $\begin{array}{c}\text { System } \\
\text { Weight } \\
(1 \mathrm{~b})\end{array}$ & $\begin{array}{c}\text { Radiator } \\
\text { Area } \\
(\mathrm{ft} 2)\end{array}$ & $\begin{array}{c}\text { Overall } \\
\text { Efficiency } \\
(\%)\end{array}$ \\
\hline $\mathrm{Po}^{210}$ Thermoelectric & 90 days & 3550 & 225 & 4.5 \\
$\mathrm{Po}^{210}$ Mercury Rankine & 90 days & 2045 & 125 & 11.3 \\
$\mathrm{Po}^{210}$ Brayton & 90 days & 2570 & 460 & 25 \\
$\mathrm{Pu}^{238}$ Thermoelectric & 1 to 5 years & 6800 & 395 & 5.0 \\
$\mathrm{Pu}^{238}$ Mercury Rankine & 1 to 2 years & 3635 & 120 & 11.3 \\
$\mathrm{Pu}$ & 1 to 3 years & 2910 & 445 & 25 \\
\hline
\end{tabular}

systems can be ready for this early flight. The choices the refore are the $\mathrm{PbTe}$ compact converter, the SiGe compact converter, or the SiGe direct radiating converter. Any of these can be available. The direct radiating SiGe module has the most advanced developmental status with both compact designs requiring additional development.

During the 1969-1970 time period, the TE power conversion represents the only potential approach. Cascading is a unique possible system which contains a developed SiGe compact converte $\mathbf{r}$ combined with a compact $\mathrm{PbTe}$ converter to provide an 8 to $9 \%$ system efficiency, low weight and area. This system has the best chance of being ready by 1969-1970 because the problems of cascading $\mathrm{PbTe}$ with $\mathrm{SiGe}$ have been eliminated by use of an intermediate loop. Further, the improved efficiency reduces the fuel availability problem. From a practical standpoint the re is little time between now and 1969 to carry through even the Po 210 TE system. However, this system does stand the best chance of meeting such a flight date. 


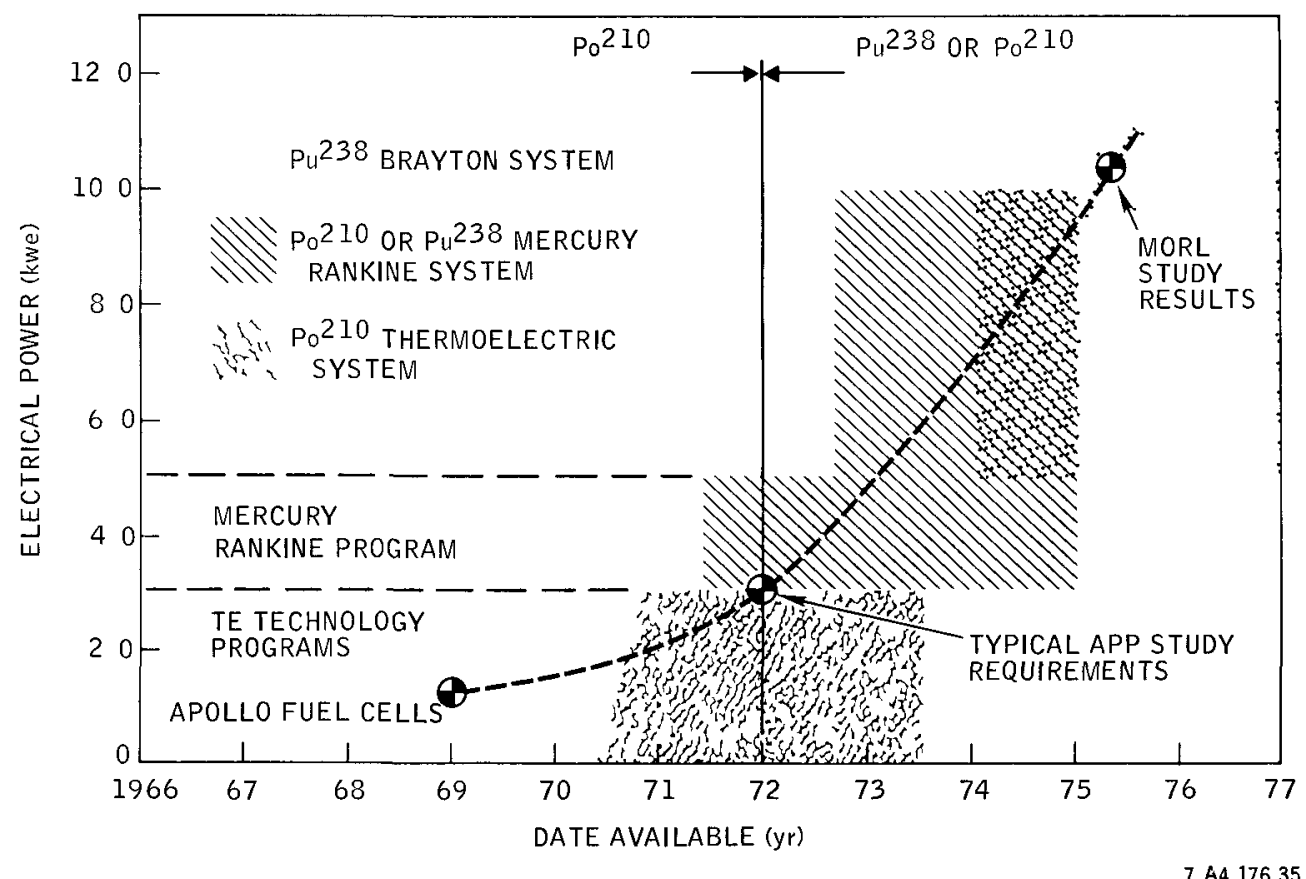

Figure III-53. Power Conversion Selection and Avaılability 


\section{b. Dynamic Power Conversion}

The two dynamic systems which are of interest are the mercury Rankine system and the Brayton system. The mercury Rankine system has been under development for about 8 years, while development of the Brayton machinery has just recently been initiated.

The mercury system represents the most advanced dynamic system in existence. Presently unit operation is in excess of 5000 hours with an objective of 10,000 as the next step. The mercury system can provide an overall efficiency of between 10 and $12 \%$. Another advantage of the mercury Rankine approach is the low area requirement. Generally a specific area of $20 \mathrm{ft}^{2} /$ kwe will result.

The Brayton cycle system is the most efficient space power approach which can be considered. It should provide net efficiencies of between 20 and $22 \%$. With isotope heat sources this is a major advantage. The Brayton system does, however, require large radiation areas. The specific radiator area of about 90 to $100 \mathrm{ft}^{2} / \mathrm{kwe}$ would be expected.

The Brayton system has only just entered the developmental cycle. For this reason its use cannot be considered before the early $1970^{\prime} \mathrm{s}$.

Table III-20 shows typical system characteristics for the Rankine and Brayton system along with the single module TE system. This data is based on a 5-kwe power output in an Apollo hardware mission.

Based on the status of development, it is projected that the mercury system can be available for the 1972 time period with the Brayton available in 1973.

The potential availability times are shown for each system type in Figure III-53. It can be seen from this figure that the projected power-time relationship for each type of system is quite consistent with early manned programs. 
vs FUEL CELLS

- LOW SYSTEM WEIGHT

- NO CONSUMABLES REQUIRED

- LOW VOLUME REQUIREMENT

- LARGE GROWTH POTENTIAL IN BOTH LIFE AND POWER LEVEL

$\underset{\infty}{\omega}$ vs SOLAR CELLS

- LOW RADIATOR AREA

- SURVIVABILITY

- INTEGRATION FLEXIBILITY

- INDEPENDENT OF ORIENTATION OR SUNSHADE EFFECTS

- NO RESTRICTION ON IN-ORBIT MANEUVERS
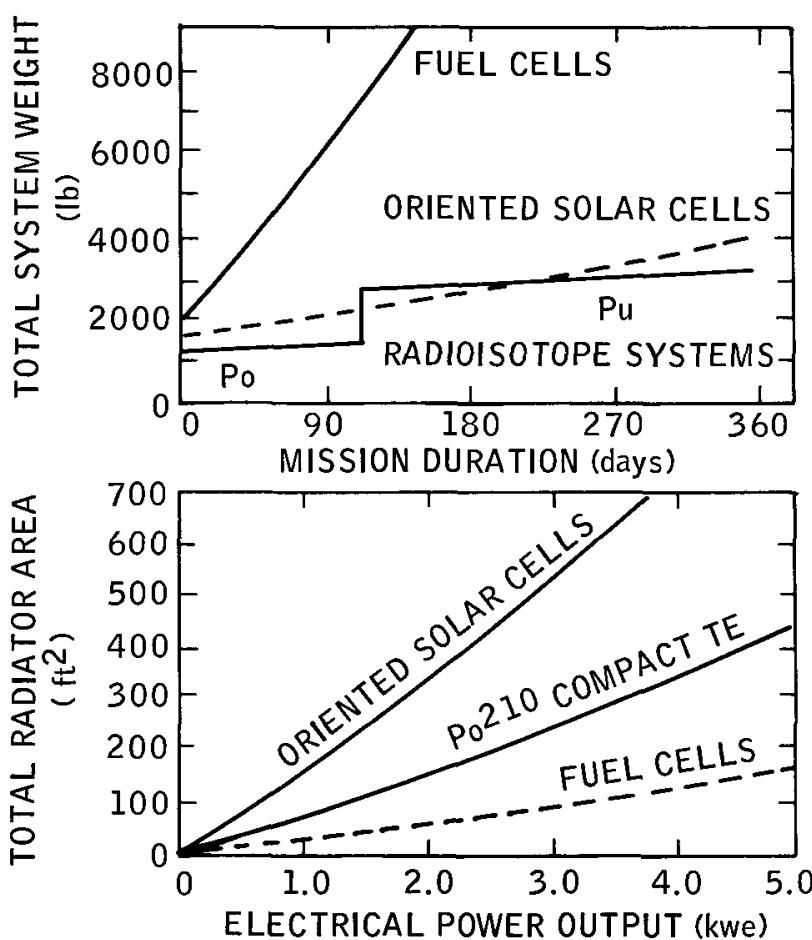

7-A4-176-30

Figure III-54. Power Systems Performance Comparison 
The question of fuel availability is one of the most important questions which must be answered. Based on many studies it is concluded that adequate fuel can be produced if a requirement is established.

\section{c. Selection Summary}

In summary, it can be concluded that the isotope fuels Po 210 , $\mathrm{Tm}^{170}, \mathrm{Pu}^{\bar{C} 88}$, and possibly $\mathrm{Cm}^{244}$ are applicable. The se can be coupled with TE, mercury Rankine, and Brayton power conversion subsystems and be available at the times indicated in Figure III-53.

The most logical application of isotope systems appears to be with initial selection of TE power conversion coupled to either $\mathrm{Tm}^{170}$ or $\mathrm{Po}^{210}$. At the second stage of use the TE conversion can be retained and technology growth incorporated which will yield modest performance improvement. Along with this growth TE conversion subsystem, the capability for using either the short life fuel or $\mathrm{Pu}{ }^{238}$ will become available. With the introduction of $\mathrm{Pu}{ }^{238}$ in 1971-1972, the growth to 1973 + should be by a step change to either $\mathrm{Pu}^{238}$ Rankine or Brayton. By retaining both PCS systems the selection can be made much later and on a better demonstration basis.

\section{d. Mission Applications}

Currently three types of power systems are being considered for early manned space missions. These are the solar cell system, fuel cell system, and radioisotope systems. The fuel cell system is being used on the short-life mission Apollo.

Figure III-54 shows a comparison of the typical performance of fuel cells, solar cells, and radioisotope systems. The comparisons shown are at $3 \mathrm{kwe}$ and in the case of the area comparison at 90-day life. It can be seen from this figure that the radioisotope system weight vs mission duration is generally less than either solar cells or fuel cells. As mission time increases the 


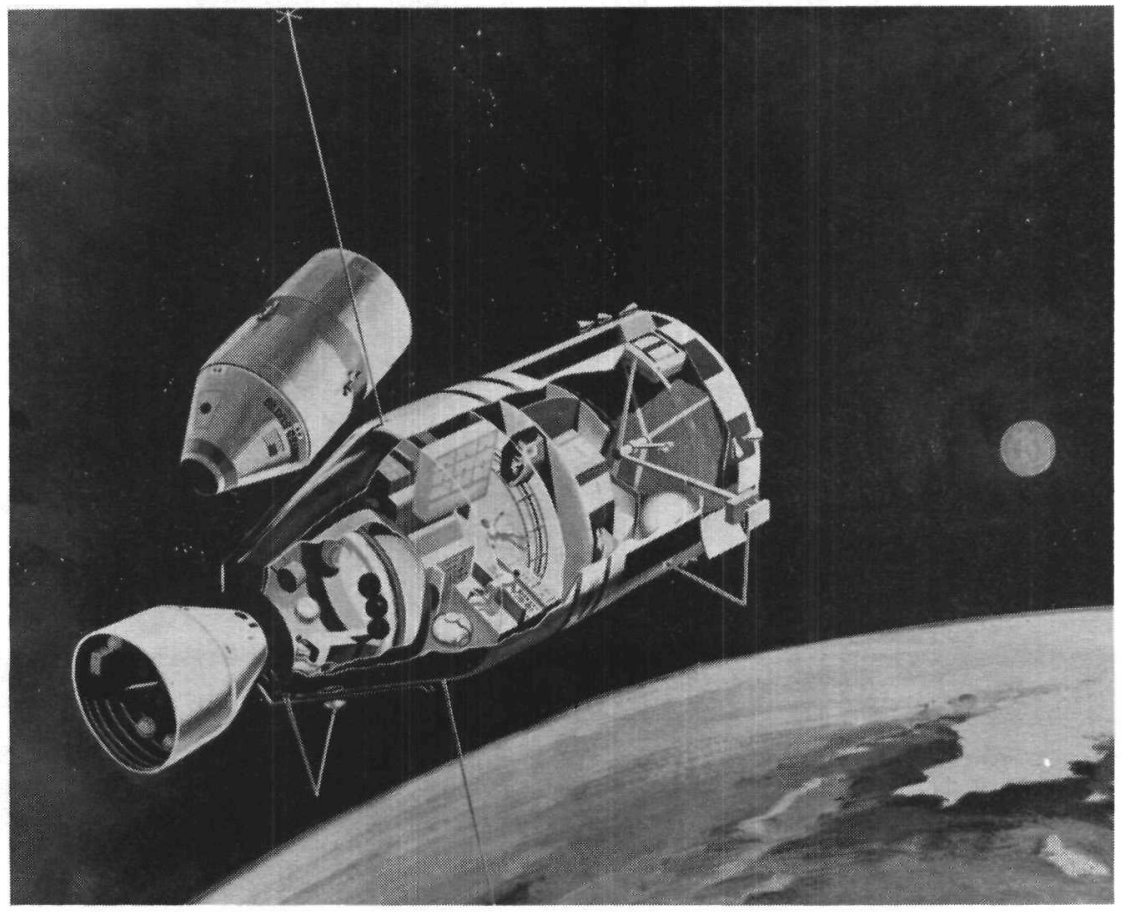

5-18-66 UNCL

Figure III-55. Manned Orbiting Research Laboratory 
fuel cells become prohibitive in weight. In a generalized mission such as is represented by Figure III-54, the solar cell system is quite close in weight to the radioisotope system.

In the area comparison shown in Figure III-54, it can be seen that the fuel cell is the lowest area system of the three. However, the more significant relationship is between solar cells and radioisotope. Using a Po $210 \mathrm{TE}$ system as the base, the solar cells required at least twice the deployed area. In addition this must be oriented to the sun at all times.

In summary, it is clear from Figure III-54 that the radioisotope system offers the best performance of the three candidates in the kilowatt electrical range for missions over about 45to 90-day duration.

Typical examples of the missions for which radioisotope systems are being considered are shown in Figures III- 55 and III-56. These are discussed below.

\section{MORL (Manned Orbiting Research Laboratory)}

Figure III-55 shows an artist's sketch of the MORL spacecraft. It is designed as a long-life (up to 5 years) space station capable of supporting up to 10 men. The power system studied for this mission was the $\mathrm{Pu}{ }^{238}$ Brayton cycle system. It is integrated directly into the spacecraft and uses the surface as a low temperature radiator.

\section{MOL (Manned Orbiting Laboratory)}

Typical of a small manned space station, the MOL is shown in Figure III-56. This type of application would require two to three men and a power level of 3 to 5 kwe. A Po ${ }^{210}$ or $\mathrm{Pu} 238$ heat source coupled with either straight thermoelectrics or a cascade thermoelectric subsystem would make an attractive power supply for this class of mission. 


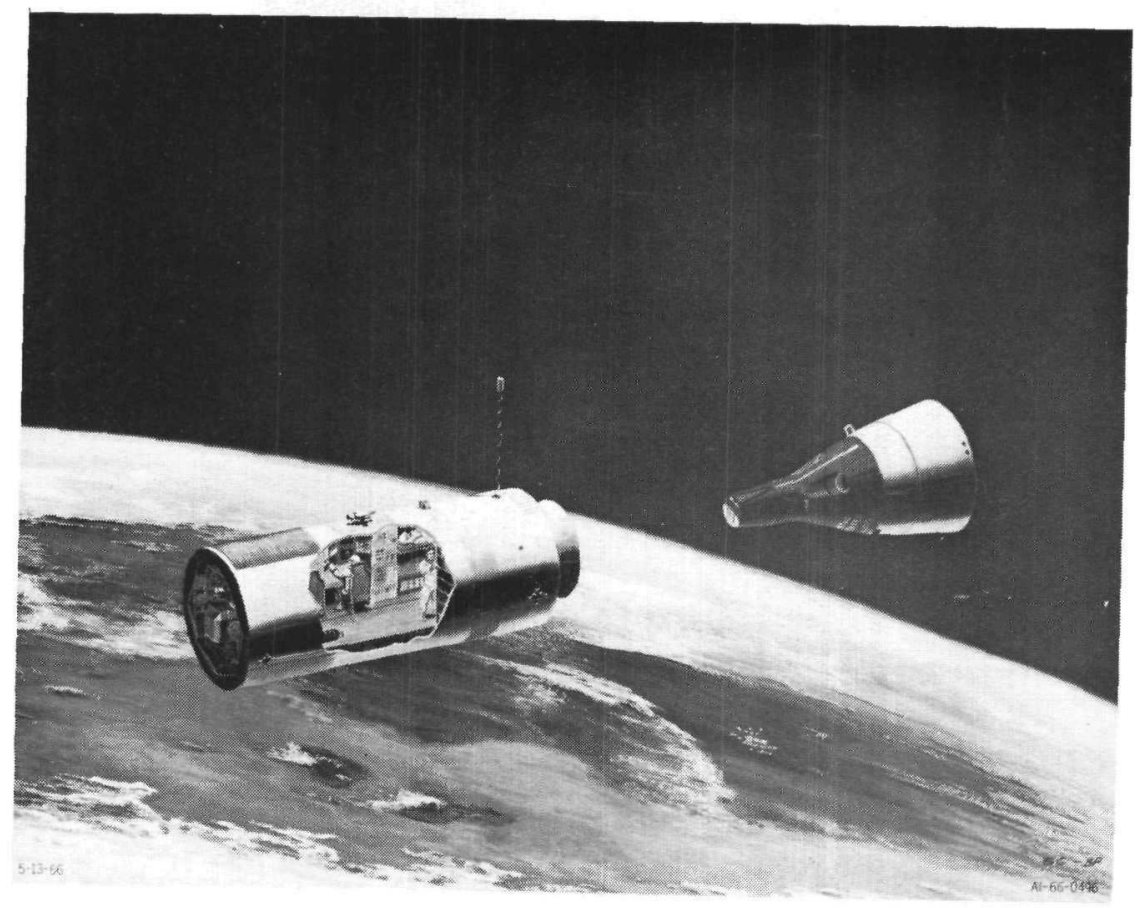

5-13-66 UNCL

Al-66-0496

Figure III-56. Manned Orbiting Laboratory 


\section{UNMANNED RADIOISOTOPE SYSTEMS}

\section{A. INTRODUCTION}

There are a growing number of space missions requiring varying amounts of electric power for which solar cells and batteries look unattractive. These missions include interplanetary and galactic probes where solar energy is reduced to very low levels and where relatively long lifetimes are required. For these applications radioisotopes using long half-life fuel, such as $\mathrm{Pu}^{238}$, offer significant advantages. The use of radioisotopes in a variety of unmanned earth orbital missions also offers significant advantages over the use of solar cells and batteries. These advantages include:

1) High inherent reliability, which offers a power supply lifetime capability considerably in excess of that obtainable with oriented solar cells and batteries.

2) Low weight, which permits the installation of excess redundant electrical equipment in a given spacecraft to increase useful mission life.

3) The capability of using waste heat for spacecraft temperature control and to further increase electronic equipment lifetimes.

4) Cost effectiveness through increased mission lifetimes.

5) Low heat rejection area requirements.

6) Radioisotope performance independent of orientation.

The heat from the radioisotopes can be converted to electrical energy by the use of either thermoelectric converters or by use of dynamic systems utilizing the Rankine or Brayton cycle. Since dynamic systems are less reliable and since efficiency is less important at low pressure, thermoelectric systems are more attractive for unmanned missions. 


\section{B. RADIOISOTOPE THERMOELECTRIC SYSTEMS}

An RTG consists basically of the radioisotope heat source, a thermoelectric converter, a radiator to reject waste heat, and the thermal coupling between these components. There are many ways of physically and thermally coupling the components of an RTG. The methods of heat transfer between the major system components are limited to conduction, forced convection, and radiation.

For lower power levels, i.e., under a few hundred watts, the optimum design appears to be one where the radioisotope capsule transfers heat to the TE converter, which in turn transfers to fins, which radiate to space. Since this type of a system uses no moveable parts, it is extremely reliable, and has been used for most of the systems built up to this time. The following RTG's for space use this type of a design: SNAP's 3B, 9A, 19, and 27.

For higher power levels, a one-loop convection system appears to be more attractive. Figure III-57 presents a one-loop convection system. In this design, the radioisotope capsules transfer heat to the TE converters by radiation. The liquid metal coolant then transfers the heat by convection from the TE converter to the radiator which radiates to space. In Figure III-57, a power flattening device is shown for radioisotopes with short half-life, such as $\mathrm{Tm}^{170}$ and $\mathrm{PO}^{210}$. If a long halflife radioisotope, such as $\mathrm{Pu}{ }^{238}$, is used, the power flattening device will not be necessary.

The following presents some details on RTG's that have already been placed in orbit, or are under development.

SNAP 3 B

The SNAP 3 program had its beginning in early 1958. Its fundamental purpose was to investigate the potentialities of direct conversion as applied to radioisotopes in space. Po 210 and $\mathrm{Pu} 238$ fuel were used in their ground experiments. 


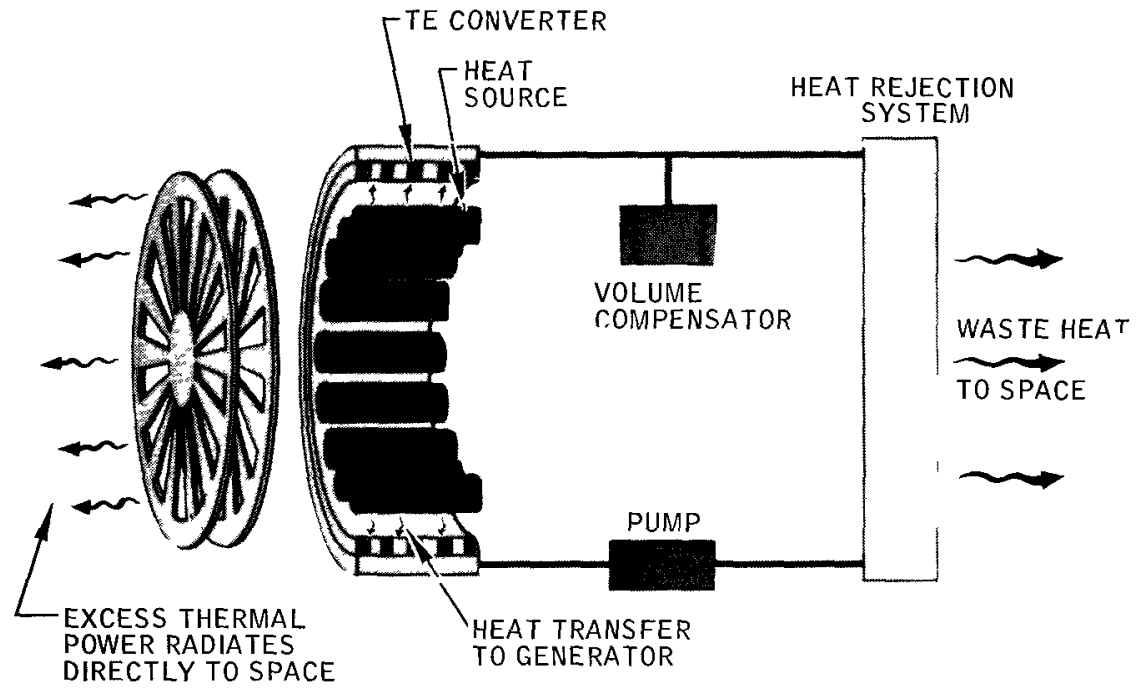

7679-0178B

Figure III-57. Typical One-Loop Convection System

However, the flight systems SNAP 3 B 7 and SNAP 3 B 8 used $\mathrm{Pu}^{238}$. Table III-21 presents pertinent data regarding SNAP $3 \mathrm{~B}$. SNAP $3 B 7$ was the first nuclear power plant to be used in space. It was installed aboard the Transit $4 \mathrm{~A}$ Navigational Satellite, and was successfully launched on June 29, 1961. The SNAP 3B8 was launched aboard the Transit $4 \mathrm{~B}$ on November 15, 1961. The Transit satellites transmit constant frequency radio waves to earth. Through precision doppler-shift techniques and knowledge of the satellite's position, ships at sea can make accurate navigational fixes. Since long life and stable operation are mandatory for Transit power supplies, $\mathrm{Pu}^{238}$ was used as the heat source since it has a long (86 years) half-life. Figure UI-58 presents the SNAP 3B fuel capsule drawing using $\mathrm{Po}^{210}$. The $\mathrm{Pu}^{238}$ capsule which was used for the flight system is very similar. Note the three capsules are placed one within the other to assure 


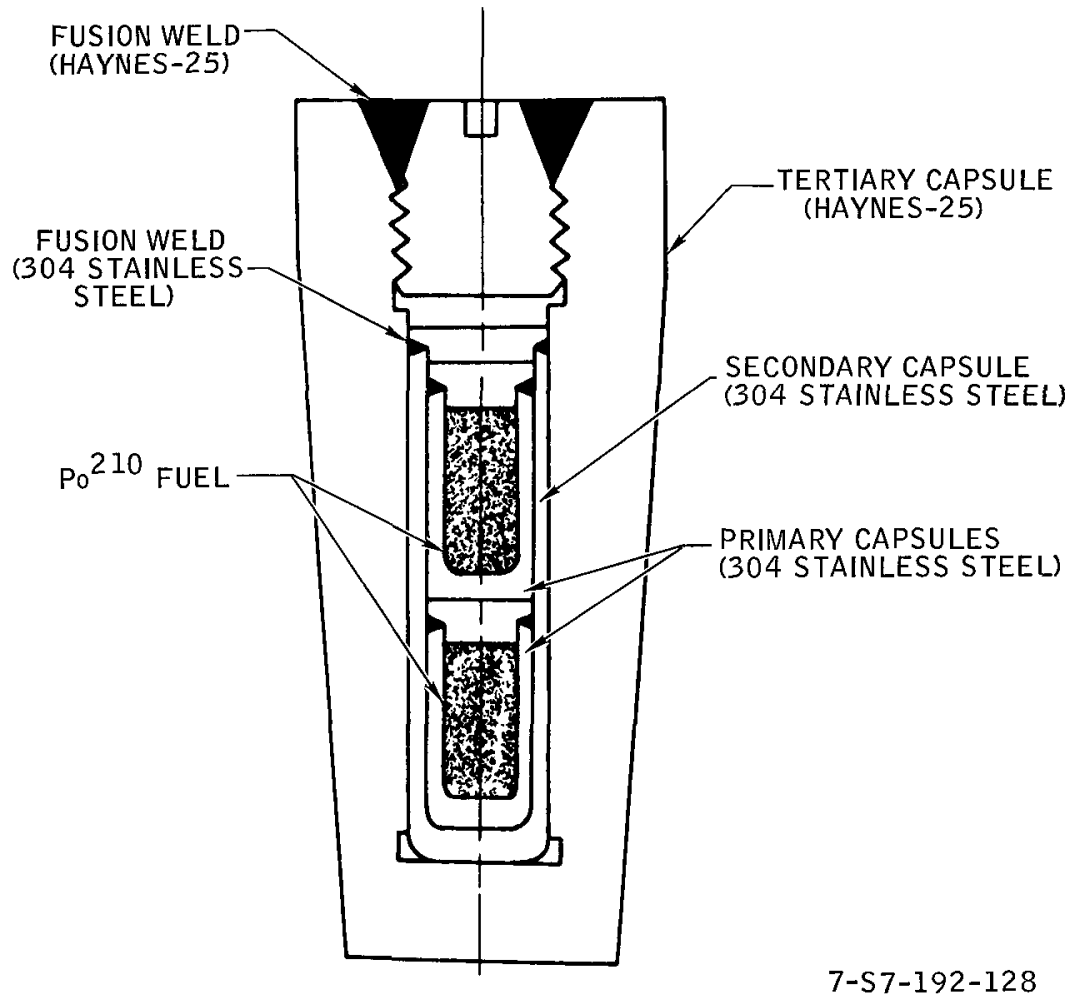

Figure III-58. SNAP 3B Fuel Capsule Drawing 
TABLE III-21

CHARACTERISTICS OF SNAP 3B7, 8, AND SNAP 9A

\begin{tabular}{l|l|c}
\hline \multicolumn{1}{c|}{ Characteristic } & SNAP 3B7 and 8 & SNAP 9A \\
\hline Fuel & $\mathrm{Pu}^{238}$ & $\mathrm{Pu}^{238}$ \\
Fuel Loading (gm) & 95 & 1000 \\
Fuel Capsule Material & Haynes-25 & \\
Converter Material & PbTe & \\
Number of Couples & 54 & \\
Insulation Material & Min-K & \\
Generator Shell & Copper & \\
Output Voltage (volts) & 3.5 & 25 \\
Maximum Electrical Power & 2.7 & $\sim 550$ \\
(watts) & 52 & $\sim 4.6$ \\
Thermal Power (watts) & 5.2 & 12.3 \\
Generator Efficiency (\%) & 2.1 & 1963 \\
Mass (kg) & 1961 & \\
Operational Date & & \\
\hline
\end{tabular}

that the radioisotope will not be released in case of an accident. Figure III-59 presents a cross section of the SNAP $3 \mathrm{~B}$ generator with the fuel capsule placed at the center.

SNAP 9A

The SNAP 9A RTG was a direct outgrowth of the successful SNAP SB's. Table III- 21 also presents pertinent data about the SNAP 9A. It also used $\mathrm{Pu}^{238}$ fuel; however, the electrical output was 25 watts instead of 3 watts for the SNAP $3 B$. The first SNAP 9A was successfully launched aboard a DOD satellite for the Navy in September 1963, and operated at a power lower than the design power. The second SNAP $9 A$ was also successfully launched in December 1963. It also operated at a lower power than the design power. The third SNAP 9A was launched in April 1964. However, due to a malfunction, it did not go into orbit and was burned upon reentry. Figure III- 60 presents an 


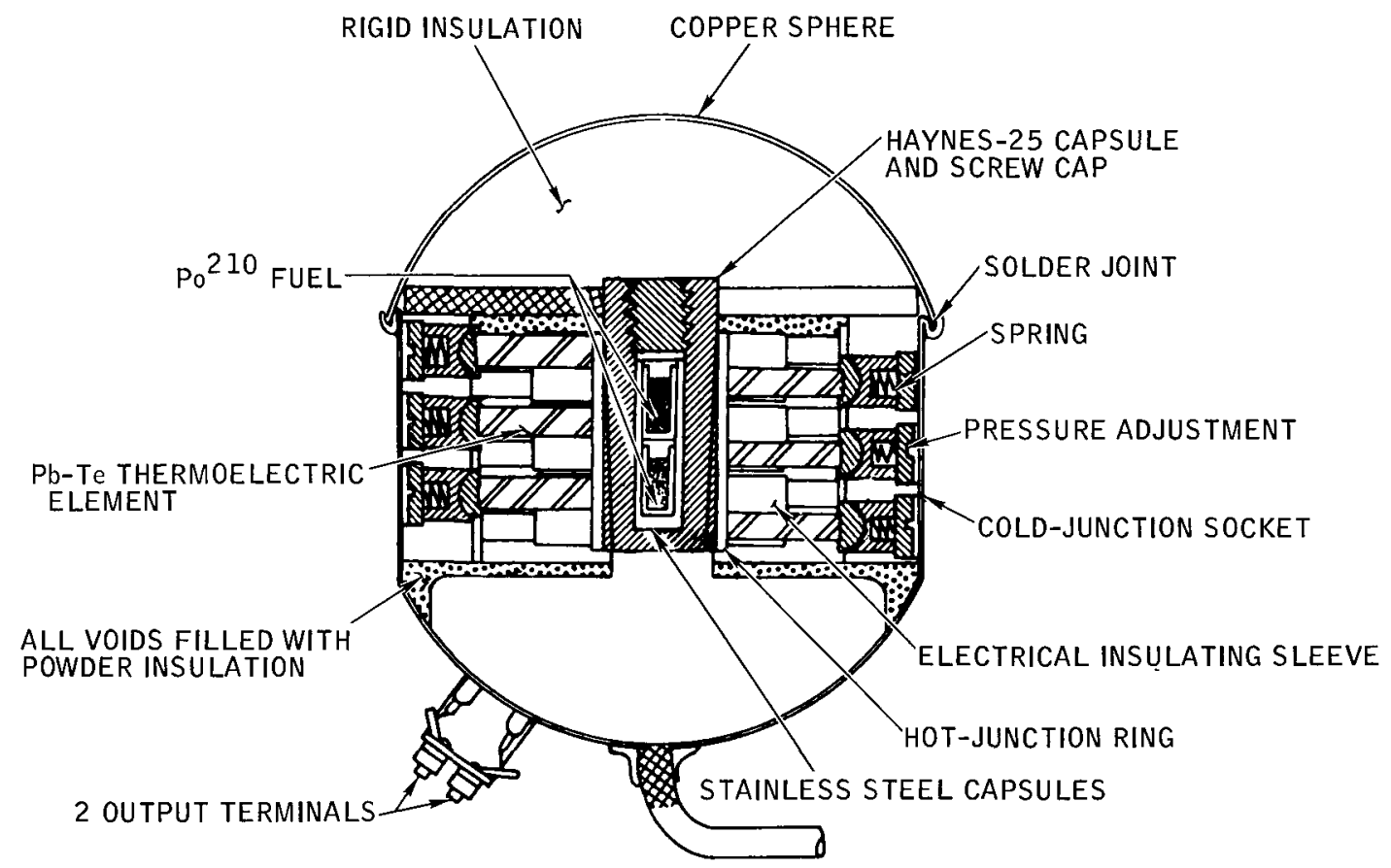

Figure III-59. Cross Section of SNAP 3 B Generator 


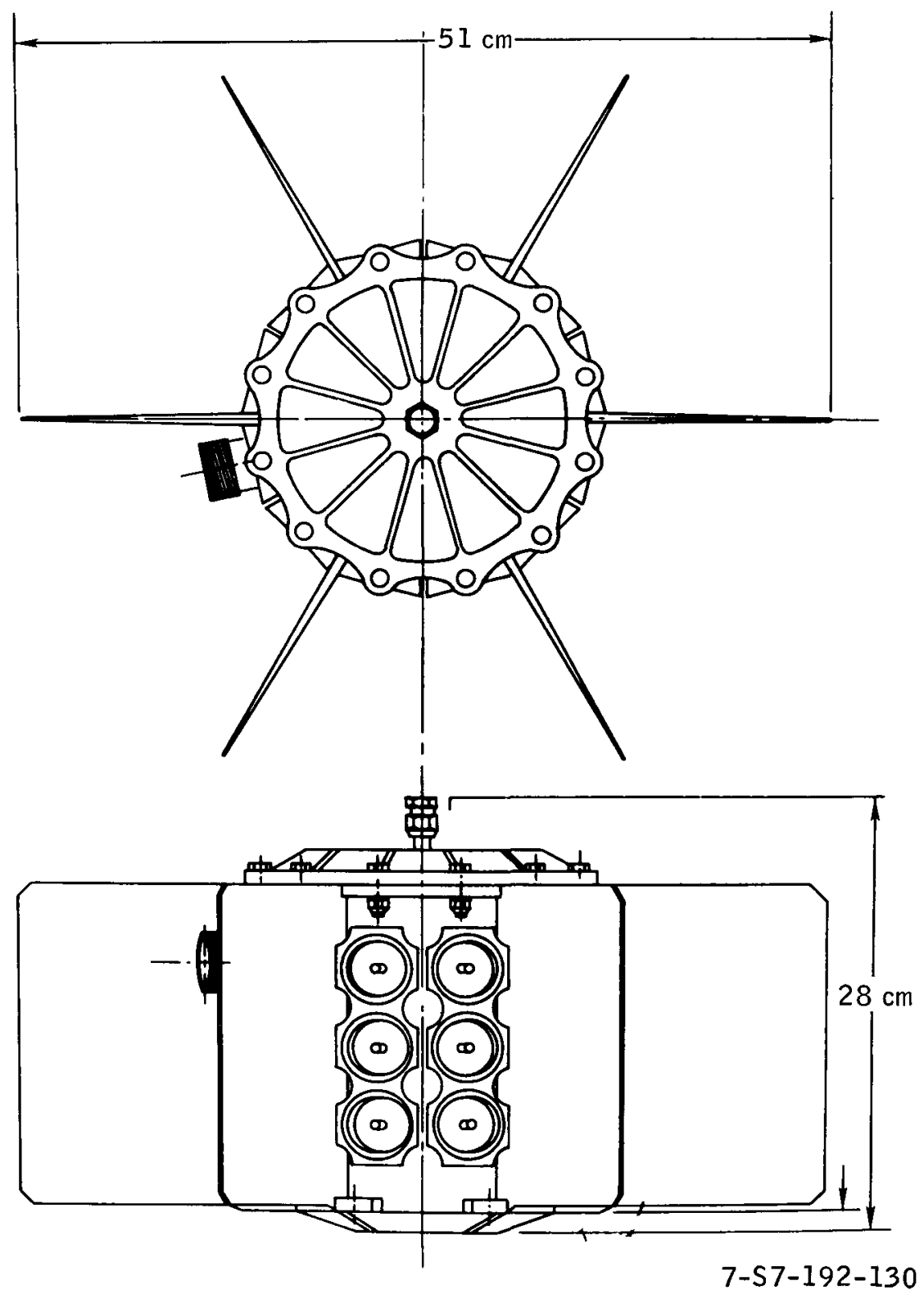

Figure III-60. Exterior Drawing of the SNAP 9A Generator 
exterior drawing of the SNAP 9A generator. Note the size of the fins used to radiate the excess heat.

$\underline{\text { SNAP } 19}$

SNAP 19 is a 25-watt RTG system which will be used to augment the solar array and batteries on the Nimbus B spacecraft.

Nimbus B is one of a family of meteorological satellites for research and development. The basic Nimbus spacecraft is equipped with a solar array and battery power supply which provides an average of 185 watts. On Nimbus B spacecraft, this will be augmented by two SNAP 19's which will furnish a continuous power of 50 watts for the in-flight mission life of one year.

The SNAP 19 power supply for Nimbus B consists of three subsystems: a generator subsystem, a power conditioner, and a telemetry conditioner. The generator subsystem is mounted on top of the spacecraft main structure - the sensory ring. The power conditioner and the telemetry signal conditioner are housed in standard Nimbus modules and are installed in a bay of the sensory ring just below the generator subsystem. The generator subsystem includes two SNAP 19 generators mounted in tandem. Each generator has an output of approximately 27 watts at 2.5 to 2.8 volts, and each generator weighs $301 \mathrm{~b}$. The generator housing is 6-1/2 in. in diameter and 10-3/4 in. in height. Six fins are used to increase radiator surface. These fins give the unit an overall diameter of 22 in. Maximum radiator temperature is $350^{\circ} \mathrm{F}$, and thermal radiation to the spacecraft surfaces is negligible. This is an important consideration since the Nimbus sensory ring includes a precise thermal control system. The generators are fueled with plutonium-238 and utilize PbTe converters. Sensors are provided for measurement of internal pressure and internal and external temperatures. The support structure contains provisions for structural dynamic isolation and for minimization of heat conduction to the spacecraft. The generator subsystem is designed for high altitude dispersal of the radioisotope fuel upon reentry. 
The power conditioner contains two dc-to-dc converters, instrumentation, and relays which connect or disconnect the ind 1 vidual generator outputs and the spacecraft regulated bus. The converter design provides minimum heat dissipation within the module in the event of a no-load condition at the output.

The telemetry conditioner transforms raw analog measurements from various sensors into analog signals that are compatible with the analog-to-digital converter of the spacecraft telemetry subsystem. The conditioner provides for 30 analog measurements, and requires less than 2 watts for operation.

Power conditioners and electrically heated generators have been built and subjected to developmental environmental tests, and integration and safety studies are in progress. Performance testing has been conducted. The final phase of the program has been initiated. This phase provides for delivery of complete systems to be tested and integrated with a Nimbus B model and flight spacecraft. The major objective for SNAP 19 is to assess the operational capability of radioisotope power for long-life meteorological satellites.

\section{SNAP 27}

SNAP 27 is a 56-watt radio1sotope-fueled thermoelectric generator used with the Apollo program. The SNAP 27 systems will be used as the power supply of the Apollo Lunar Surface Experiment Package (ALSEP). However, the SNAP 27 could be used for unmanned systems in space, and the performance characteristics would be slightly different.

Plutonium-238 is used as the radiolsotope fuel for the SNAP 27. The SNAP 27 generator consists of three subsystems the generator and the fuel capsule heat source, a storage cask, and a fuel capsule handling tool which is used for fueling the generator on the lunar surface. The SNAP 27 generator assembly, with fuel capsule in place, is shown in Figure III-61. Table III-22 presents pertinent data about the SNAP 27. Heat generated by 


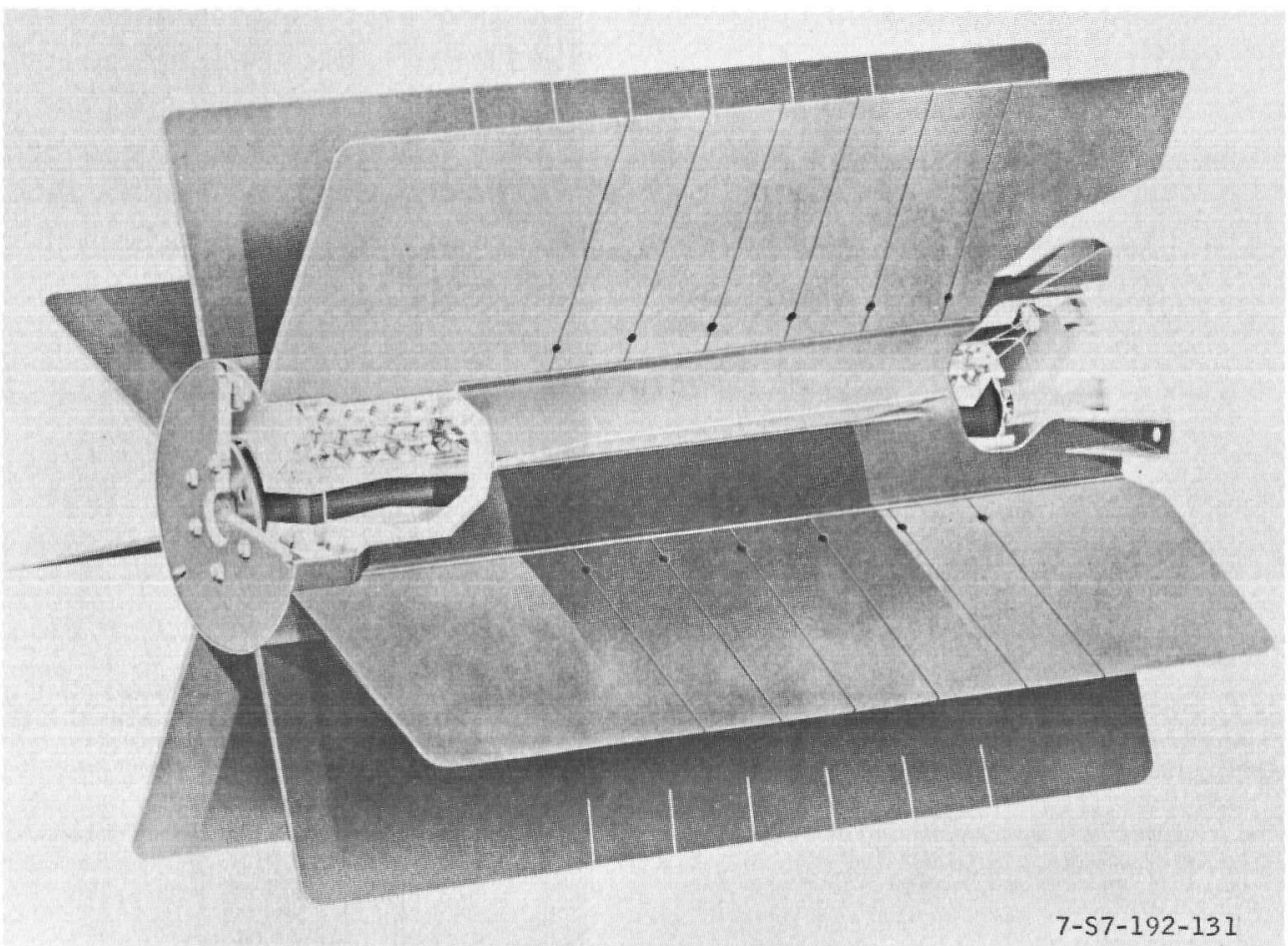

Figure III-61. SNAP 27 System 


\begin{tabular}{|c|c|}
\hline \multicolumn{2}{|l|}{ Generator Performance } \\
\hline Output power (test) & 73.3 watts \\
\hline Output power (minımum) & $\begin{array}{l}63.5 \text { watts } \\
\text { (end of mission) }\end{array}$ \\
\hline Output voltage (nominal) & 16 volts $\mathrm{dc}$ \\
\hline Current (nominal) & 4 amps \\
\hline $\begin{array}{l}\text { Maximum hot junction } \\
\text { temperature }\end{array}$ & $\begin{array}{l}1130^{\circ} \mathrm{F} \\
\text { (lunar day) }\end{array}$ \\
\hline $\begin{array}{l}\text { Maximum cold junction } \\
\text { temperature }\end{array}$ & $\begin{array}{l}525^{\circ} \mathrm{F} \\
\text { (lunar day) }\end{array}$ \\
\hline \multicolumn{2}{|l|}{ General Design Characteristics } \\
\hline $\begin{array}{l}\text { Overall generator diameter } \\
\text { (over fins) }\end{array}$ & $15.7 \mathrm{in.}$ \\
\hline Overall generator length & $18.1 \mathrm{in.}$ \\
\hline Number of fins & 8 \\
\hline Fin radial length & $5.0 \mathrm{in}$. \\
\hline \multicolumn{2}{|l|}{ Heat Source Characteristics } \\
\hline Number of fuel capsules & 1 \\
\hline Capsule OD & $2.50 \mathrm{in}$. \\
\hline Capsule length & $15.6 \mathrm{in}$ \\
\hline Thermal output (nominal) & 1450 watts \\
\hline \multicolumn{2}{|l|}{ Weight } \\
\hline $\begin{array}{l}\text { Generator as sembly } \\
\text { (Includes cable, connec- } \\
\text { tor, and instrumentation) }\end{array}$ & $28.2 \mathrm{lb}$ \\
\hline Fuel capsule as sembly & $14.51 \mathrm{~b}$ \\
\hline Total IPU weight & $42.71 \mathrm{~b}$ \\
\hline
\end{tabular}

decay of the fuel is transferred by radiation to a cylindrical hot frame in the generator. The thermoelectric elements, mounted radially around the hot frame, convert heat directly to electrical power, and the waste heat is rejected by a set of radiating fins. The thermoelectric elements are of the lead-telluride type, 
spring-loaded and sealed in an inert atmosphere. For maximum reliability, the elements are connected in a series-parallel ladder arrangement. A spherical seat is used at the outer end of each element to reduce problems of misalignment under thermal cycling (lunar day-night variation). Wherever possible, beryllium is used as the main structural material to minimize system weight. Other materials include Inconel and Haynes superalloys for strength at high temperature.

Assembly of the first engineering model of the generator was completed in October 1966, and this unit has successfully completed development tests. Qualification models will be completed and placed on test early in 1967. Delivery of four flight-qualified systems and one backup system will begin in July 1967.

SNAP 29

SNAP 29 is a 500 -watt RTG system for unmanned space application. For potential applications requiring multiples of 500 watts, modular arrays of SNAP 29 systems have been proposed.

Polonium-210 has been selected as the radioisotope fuel for the SNAP 29. Its high power density capability is ideal for space application and its short half-life is satisfactory since the required heat source lifetime is 144 days; this time span includes prelaunch-hold potential and mission time. At the end of the operational period, the net electrical power output of the SNAP 29 generator must be at least 500 watts with high reliability.

The SNAP 29 generator consists of five subsystems: the heat source subsystem, the thermoelectric modules subsystem, the thermal control subsystem, the heat rejection subsystem, and the structure, shielding, and instrumentation subsystem.

The polonium-210 fuel is contained in capsules. The heat source is designed for intact reentry into the earth's atmosphere through impact, and containment of the fuel during normal as well as abort conditions. 
The thermoelectric module is the basic power-producing component of the generator. The thermoelectric elements selected are of the $\mathrm{PbTe}$ type, fully bonded into couples with positive pressure provided by spring-loaded pistons. The hot plate which receives radiant heat from the heat source is stiffened by ribs to counteract the spring and internal gas pressure forces acting on the hot plate. Heat is removed at the cold end of the module by NaK coolant circulating in parallel flow channels inside the cold end parts. SNAP 29 is basically a one-loop convection system, as shown in Figure III-57. The modules are connected in series to yield 500 watts net power at approximately 28 volts. In addition, the power for the electromagnetic pump is obtained from a separate circuit in each module. All pump circuits are parallel connected to yield low voltage, high current power for the EM pump.

The thermal control subsystem consists of thermal shutters, a mechanical linkage connecting the shutters, and an actuating device which is thermally coupled to the module hot plate through thermal sensing tubes containing NaK fluid. Basically, the expansion and contraction of a constrained NaK volume, due to changes in temperature of the thermoelectric module hot plate, actuate a bellows-piston device, which in turn drives the mechanical linkage of the shutter.

The coolant heat rejection subsystem consists of the NaK coolant, EM pump, $\mathrm{NaK}$ accumulator, and space radiator. Eutectrc NaK coolant ( $78 \% \mathrm{~K}$ by weight) was selected as the fluid to transport the waste thermal energy from the thermoelectric module to the radiator. The space radiator consists of coolant tubes and associated meteorold protection armor joined to an aluminum skin. The configuration of the radiator is variable and will be dependent upon the vehicle into which the power system is being integrated. A high emissivity coating on the radiator surface will be used to improve the heat radiation characteristics in vacuum operation. 
The major subsystems (exclusive of the radiator) are brought together to form a basic thermoelectric power conversion generator. The common component which ties these subsystems together is the generator housing. The basic RTG subsystems are unitized for maintainability and interchangeability. For example, the $T E$ conversion modules are mounted to a common frame which attaches to the generator housing. Thus, the TE module assemblies can be removed or replaced. The thermal control system, consisting of the thermal shutters, mechanical linkage, $\mathrm{NaK}$ actuator, and thermal sensing tubes, is also mounted on a common frame which attaches to the generator housing. The thermal sensing tubes can be removed from the hot plate of the modules through holes in the top of the generator. Finally, the heat source is installed in the generator through an opening in the side of the housing. In addition, the housing is designed to break apart during reentry to enable the heat source to re-enter separately.

The major development objectives for SNAP 29 are as follows:

$\begin{array}{ll}\text { Power Level } & 500 \mathrm{w}(\mathrm{e}) \\ \text { Power/Weight Ratio } & \mathrm{l} \text { watt/1b } \\ \text { Reliability } & \text { High } \\ \text { Lifetime - Fueled Generator } & 144 \text { days }\end{array}$

The design features of the SNAP 29 system enable its application to a broad variety of mission requirements. Power levels below 500 watts can be accommodated and, as indicated previously, higher power levels can be achieved by modular arrays of the basic generator. 


\section{REFERENCES}

1. E. D. Arnold, "Handbook of Shielding Requirements and Radiation Characteristics of Isotopic Power Sources for Terrestrial, Marine and Space Applications," ORNL-3576, April 1964

2. D. H. Stoddard and E. L. Albenesius, "Radiation Properties of $\mathrm{Pu} 238$ Produced for Is otopic Power Generators," DP-984, July 1965

3. V. Keshishian and K. M. Broom, "Use of Oxygen-16 with Plutonium-238 to Reduce Neutron Yield, "Paper presented at ANS, June 1966

4. W. M. Rutherford, et al., "Preparation and Neutron Counting of Pu23802 Depleted in O18," Nuclear Applications, $\underline{2}$, 366 (1967)

5. D. H. Stoddard, et al., "Radiation Properties of $\mathrm{Cm}^{244}$ Produced for Isotopic Power Generators, " D P-939, November 1964

6. C. L. Dunford and H. Alter, "Neutron Cross Sections for Plutonıum-238, Pluton1um-242 and Cur1um-244," NAA-SR12271, May 1967

7. V. Keshishian, et al., "The Criticality of $\mathrm{Cm}^{244}$, "Paper presented at ANS Meeting, November 1965

8. E. H. Ottewitte and V. Keshishian, "Need for $\mathrm{Pu}^{238}$ and Cm244 Cross Section Data," CONF-660303, March 1966

\section{ADDITIONAL REFERENCES}

W. D. Corliss and D. G. Harvey, "Radıoısotopic Power Generation," Prentice-Hall, Inc., 1964

W. H. Steigelman, "Radioısotope Shieldıng Desıgn Manual," NYO-10721, July 1963

K. W. Foster, "Gamma Shielding Requirements for Plutonium238 and Polonıum-210 (Revised), " MLM-1315, January 21, 1966

H. T. Fullam and H. H Van Tuyl, "Promethium Isotopic Data Sheets," BNWL-363, February 1967 
H. L. Davis, "Isotope Costs and Availability," Nucleonics, March 1963, p 61

D. G. Harvey, et al., "Isotope-Generator Reliability and Safety," Nucleonics, April 1963, p 56

F. Schulman, "Generator Performance and Mission Prospects," Nucleonics, September 1963, p 54

J. F. Stehn, "Table of Radioactive Nuclides," Nucleonics, November 1960, p 186

"Gamma Emitters by Half-Life and Energy," Nucleonics, November 1960 , p 196

"Large Scale Production and Application of Radioisotopes," DP-1066, Volumes I and II, March 1966

"Advances in Energy Conversion Engincering, " Papers presented at 1967 Intersociety Enorgy Conversion Engineering Conference, August 13, 1967, Miami, Florida

"Industrial Applications for Isotopic Power Generators," International Symposium A.E.R. E. Harwell, Scplember 1966 
